\title{
THREE-DIMENSIONAL MULTIPLE SCATTERING OF ELASTIC WAVES BY SPHERICAL INCLUSIONS
}

by ZUNPING LIU

M.S., University of Arizona, 2002

\section{AN ABSTRACT OF A DISSERTATION}

submitted in partial fulfillment of the requirements for the degree of DOCTOR OF PHILOSOPHY

Department of Mechanical and Nuclear Engineering College of Engineering

KANSAS STATE UNIVERSITY

Manhattan, Kansas

2007 


\section{Abstract}

A computational system is built for conducting deterministic simulations of threedimensional multiple scattering of elastic waves by spherical inclusions.

Based on expansion expression of elastic wave fields in terms of scalar and vector spherical harmonics, analytically exact solutions of single scattering and multiple scattering are obtained, implemented and verified. The verification is done by using continuities of displacement and surface traction at the interface between an inclusion and host medium, energy conservation and published results.

The scatterer polymerization methodology is extended to three-dimensional multiple scattering solution. By using this methodology, an assemblage of actual scatterers can be treated as an abstract scatterer. This methodology is verified by using different approaches, with or without scatterer polymerization, to solve a physically the same multiple scattering problem.

As an application example, band gap formation process for elastic wave propagation in cubic lattice arrangements of spherical scatterers is observed through a series of numerical simulations. Along the direction of the incident wave, scatterer arrangements are viewed as comprising layers of scatterers, within which scatterers form a square grid. Starting from one layer and by increasing the number of layers, near-field forward wave propagation spectra are computed as the number of layers increases. These simulations also demonstrates that the computational system has the capability to simulate multiple scattering solutions of elastic waves in three-dimension. 
Thesis Committee:

Dr. Liang-Wu Cai (Committee Chairman, Assistant Professor, Mechanical Engineering)

Dr. Talat S. Rahman (University Distinguished Professor, Physics, currently with the University of Central Florida)

Dr. Daniel V. Swenson (Professor, Mechanical Engineering)

Dr. Sameer I. Madanshetty (Associate Professor, Mechanical Engineering)

Dr. Youqi Wang (Associate Professor, Mechanical Engineering) 


\section{THREE-DIMENSIONAL MULTIPLE SCATTERING OF ELASTIC WAVES BY SPHERICAL INCLUSIONS}

by

\section{ZUNPING LIU}

M.S., University of Arizona, 2002

\section{A DISSERTATION}

submitted in partial fulfillment of the requirements for the degree of DOCTOR OF PHILOSOPHY

Department of Mechanical and Nuclear Engineering College of Engineering

KANSAS STATE UNIVERSITY

Manhattan, Kansas

2007

Approved by:

Major Professor

Liang-Wu Cai 


\section{Abstract}

A computational system is built for conducting deterministic simulations of threedimensional multiple scattering of elastic waves by spherical inclusions.

Based on expansion expression of elastic wave fields in terms of scalar and vector spherical harmonics, analytically exact solutions of single scattering and multiple scattering are obtained, implemented and verified. The verification is done by using continuities of displacement and surface traction at the interface between an inclusion and host medium, energy conservation and published results.

The scatterer polymerization methodology is extended to three-dimensional multiple scattering solution. By using this methodology, an assemblage of actual scatterers can be treated as an abstract scatterer. This methodology is verified by using different approaches, with or without scatterer polymerization, to solve a physically the same multiple scattering problem.

As an application example, band gap formation process for elastic wave propagation in cubic lattice arrangements of spherical scatterers is observed through a series of numerical simulations. Along the direction of the incident wave, scatterer arrangements are viewed as comprising layers of scatterers, within which scatterers form a square grid. Starting from one layer and by increasing the number of layers, near-field forward wave propagation spectra are computed as the number of layers increases. These simulations also demonstrates that the computational system has the capability to simulate multiple scattering solutions of elastic waves in three-dimension. 
Thesis Committee:

Dr. Liang-Wu Cai (Committee Chairman, Assistant Professor, Mechanical Engineering)

Dr. Talat S. Rahman (University Distinguished Professor, Physics, currently with the University of Central Florida)

Dr. Daniel V. Swenson (Professor, Mechanical Engineering)

Dr. Sameer I. Madanshetty (Associate Professor, Mechanical Engineering)

Dr. Youqi Wang (Associate Professor, Mechanical Engineering) 


\section{Table of Contents}

Table of Contents vii

List of Figures $\quad$ X

List of Tables $\quad$ xvi

Acknowledgements $\quad$ xvii

1 Thesis Introduction 1

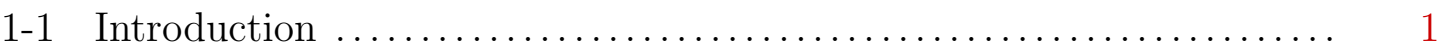

1-1.1 Single Scattering by a Spherical Inclusion ................ 2

1-1.2 Multiple Scattering by Spherical Inclusions ............... 3

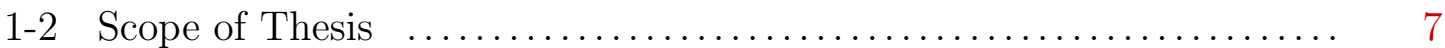

2 Single Scattering Solution 9

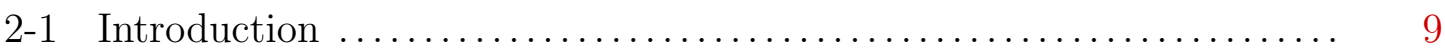

2-2 Equations of Elastic Wave Motion ........................ 10

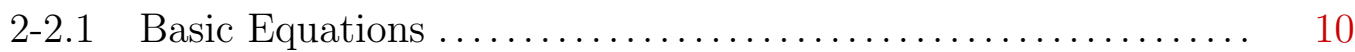

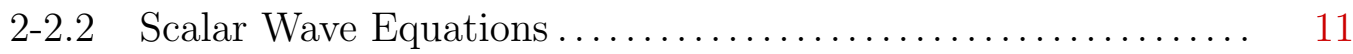

2-2.3 Steady-State Solution to Wave Equations................. 13

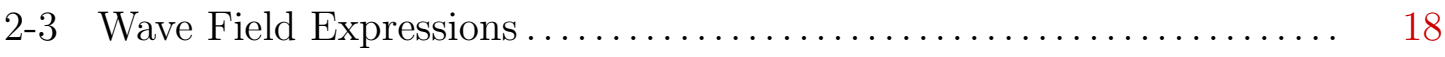

2-3.1 Wave Fields in Terms of Spherical Harmonics .............. 19

2-3.2 Wave Fields in Terms of Vector Spherical Harmonics .......... 23

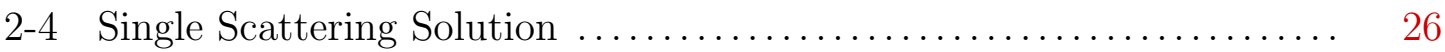

2-4.1 Displacement Continuity Equations................... 28

2-4.2 Stress Continuity Equations.......................... 30

2-4.3 Solution of Continuity Equations and Mode Conversion ...... 30

2-4.4 T-Matrix for Single Spherical Scatterer ................ 36

2-4.5 Displacements and Stresses ...................... 39

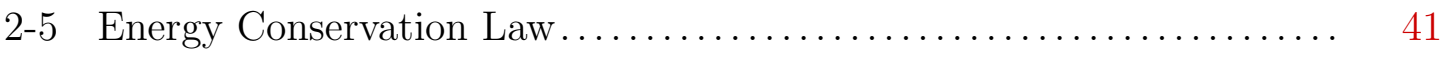

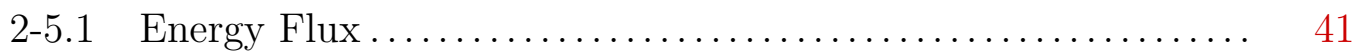

2-5.2 Numerical Integration Over a Closed Spherical Surface....... 43 
2-6 Numerical Examples of Single Scattering Solution ............... 45

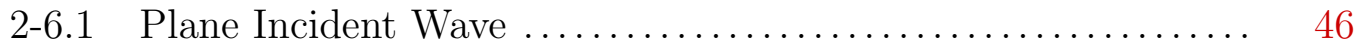

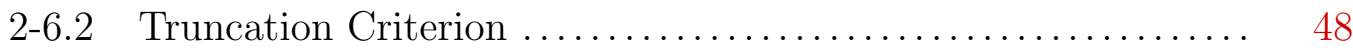

2-6.3 Wave Fields and Field Continuity ..................... 49

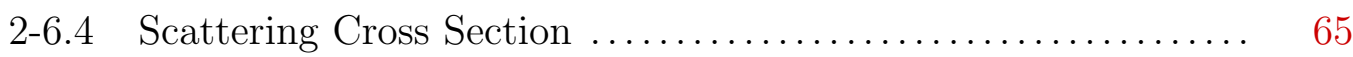

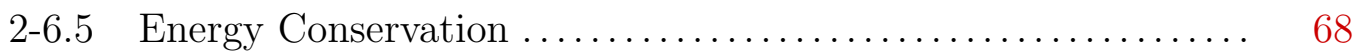

3 Multiple Scattering Solution $\quad 72$

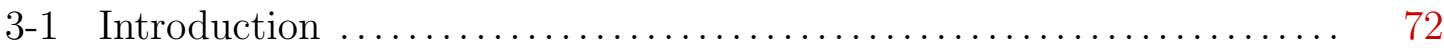

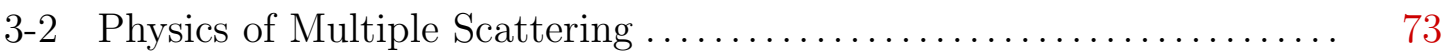

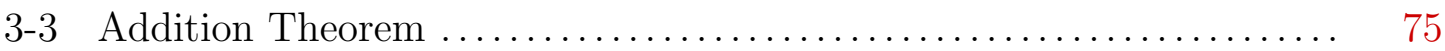

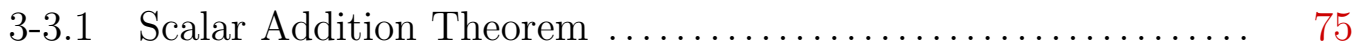

$3-3.2$ Vector Addition Theorem $\ldots \ldots \ldots \ldots \ldots \ldots \ldots \ldots \ldots \ldots \ldots, \quad 80$

3-4 Formulation of Multiple Scattering Solution................... 88

3-4.1 Formulation Based on Waves at Various Orders ............. 89

3-4.2 Formulation Based on Single Scattering Solution ............ 94

3-4.3 Displacements and Stresses ........................... 94

4 Implementation of Multiple Scattering Solution 101

4-1 Introduction ............................................... 101

4-2 Implementation of Addition Theorems ........................ 102

4-2.1 Transformation of Incident Wave Fields ................ 105

4-2.2 Transformation of Scattered Wave Fields ................ 111

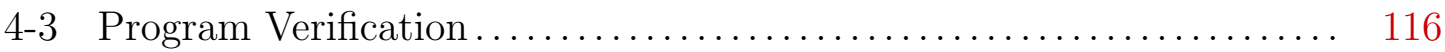

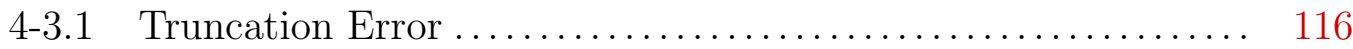

4-3.2 Comparison with a Published Result ................... 117

$4-3.3 \quad$ Energy Conservation ................................ 120

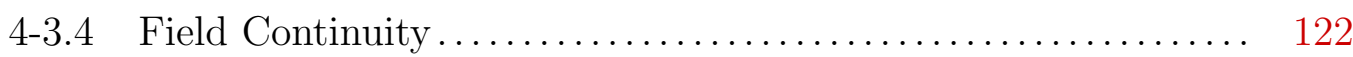

4-4 Concluding Remarks ...................................... 125

5 Scatterer Polymerization $\quad 129$

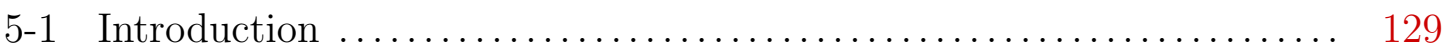

5-2 Idea of Scatterer Polymerization Methodology ................... 130

5-3 Algorithm of Scatterer Polymerization ....................... 133

5-3.1 Mathematics of Scatterer Polymerization ................. 134

5-3.2 Core Region and Territory of An Abstract Scatterer ......... 136

$5-4 \quad$ Verification............................................ 139 
5-4.1 Description of Numerical Procedure ..................... 139

5-4.2 Comparison of Numerical Results ..................... 142

$5-5 \quad$ Concluding Remarks .................................. 157

6 Application Example $\quad 159$

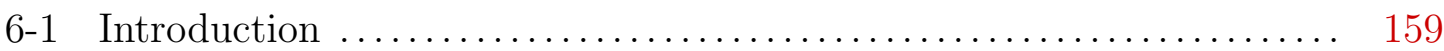

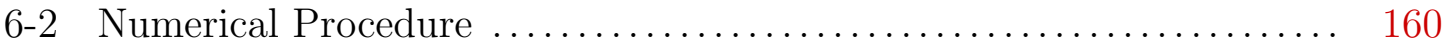

6-2.1 Sixteen Scatterers in A Layer ........................ 160

6-2.2 Thirty-Two Scatterers in Two Layers .................... 161

6-2.3 Sixty-Four Scatterers in Four Layers ..................... 162

6-3 Truncation Number and the Error due to Coordinate Transformation 164

6-4 Simulation Results ..................................... 169

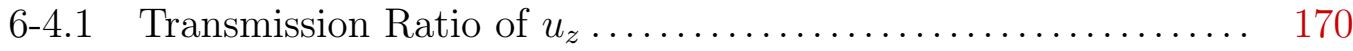

6-4.2 Displacements for Different Number of Layers ............. 174

6-4.3 Stresses for Different Number of Layers ................... 199

6-5 Concluding Remarks ................................ 220

7 Thesis Conclusion $\quad 221$

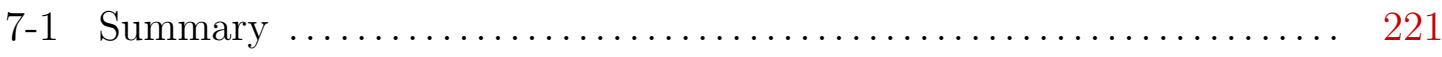

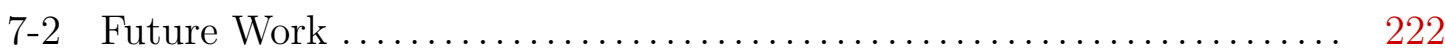

$\begin{array}{ll}\text { Bibliography } & 230\end{array}$

A Orthogonal Curvilinear Coordinate System 231

B Reduce Navier's Equation to Three Scalar Wave Equations 235

C Spherical Coordinate System and Field Operators 241

D Coordinate Transformation for Displacements, Strains and Stresses between Spherical and Cartesian Coordinate Systems 244

E Definition of Functions $\mathfrak{E}_{p q}^{s t}(r, n)$ and $\mathfrak{E}_{p q}^{s t}(\mathbf{r}, n) \quad 249$

F Vector Spherical Harmonics $\quad 252$

G Normalized Vector Spherical Harmonics 261

H Vector Wave Functions in Terms of Vector Spherical Harmonics 265 


\section{List of Figures}

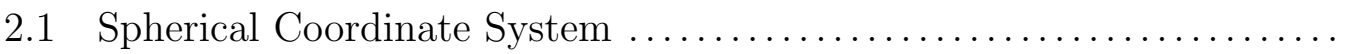

2.2 Truncation number for truncation error $\epsilon=10^{-8}$ and $10^{-5}$ : (a) using

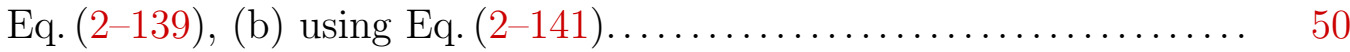

2.3 Continuity of displacements $u_{x}$ at the scatterer's boundary in the $y=0$ plane. ............................................. 52

2.4 Continuity of displacements $u_{y}$ at the scatterer's boundary in the $y=0$

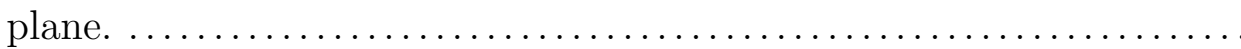

2.5 Continuity of displacements $u_{z}$ at the scatterer's boundary in the $y=0$ plane. ............................................ 53

2.6 Continuity of displacements $u_{r}$ at the scatterer's boundary in the $y=0$ plane.

2.7 Continuity of displacements $u_{\theta}$ at the scatterer's boundary in the $y=0$ plane.

2.8 Continuity of displacements $u_{\varphi}$ at the scatterer's boundary in the $y=0$ plane.

2.9 Continuity of stresses $\sigma_{r r}$ at the scatterer's boundary in the $y=0$

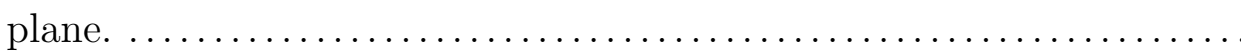

2.10 Continuity of stresses $\sigma_{r \theta}$ at the scatterer's boundary in the $y=0$ plane.

2.11 Continuity of stresses $\sigma_{r \varphi}$ at the scatterer's boundary in the $y=0$ plane.............................................. 56

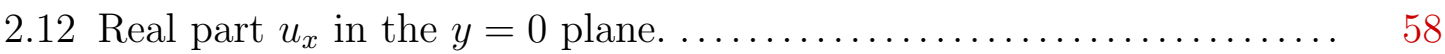

2.13 Real part of $u_{z}$ in the $y=0$ plane. ....................... 58

2.14 Real part of $u_{r}$ in the $y=0$ plane. .......................... 59

2.15 Real part of $u_{\theta}$ in the $y=0$ plane. ......................... 59

2.16 Real part of $\sigma_{r r}$ in the $y=0$ plane......................... 60

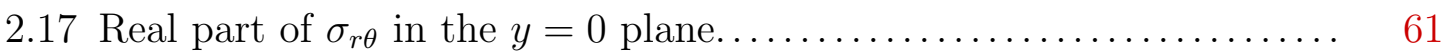

2.18 Real part of $\sigma_{\theta \theta}$ in the $y=0$ plane.......................... 61

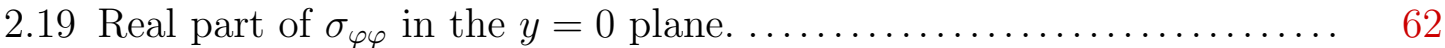

2.20 Real part of $\sigma_{x x}$ in the $y=0$ plane. ....................... 63 


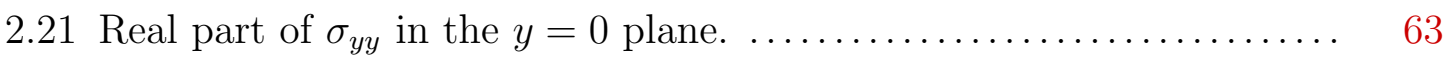

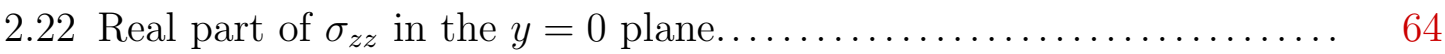

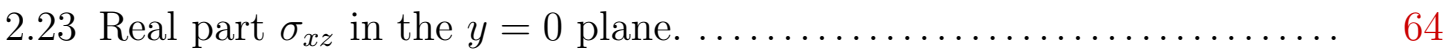

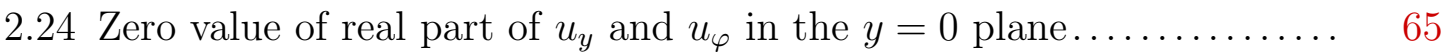

2.25 Zero value of real part of $\sigma_{x y}$ and $\sigma_{r \varphi}$ in the $y=0$ plane $\ldots \ldots \ldots \ldots .66$

2.26 Zero value of real part of $\sigma_{y z}$ and $\sigma_{\theta \varphi}$ in the $y=0$ plane $\ldots \ldots \ldots \ldots .66$

2.27 Comparison of normalized scattering cross section of a beryllium sphere in a polyethylene matrix. $\ldots \ldots \ldots \ldots \ldots \ldots \ldots \ldots \ldots \ldots \ldots \ldots \ldots \ldots . \ldots \ldots$

3.1 Multiple scattering of a single wave between two scatterers . . . . . . . $\quad 74$

3.2 Multiple scattering among a group of scatterers .................. 74

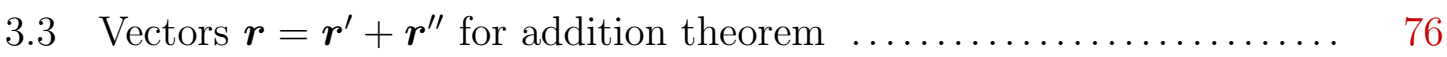

3.4 Two coordinate systems for addition theorem ................ 77

4.1 Vectors $\boldsymbol{r}=\boldsymbol{r}^{\prime}+\boldsymbol{r}^{\prime \prime}$ for addition theorem. . . . . . . . . . . . . . 102

4.2 Significant figures of displacements and stresses due to incident wave

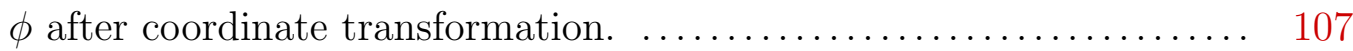

4.3 Significant figures of displacements and stresses due to incident wave $\psi$ after coordinate transformation. ......................... 109

4.4 Significant figures of displacements and stresses due to incident wave $\psi$ after coordinate transformation.

4.5 Significant figures of the transformed results of displacement and stress due to the scattered waves for incident wave $\phi \ldots \ldots \ldots \ldots \ldots \ldots \ldots . \ldots 114$

4.6 Significant figures of the transformed results of displacement and stress due to the scattered waves for incident wave $\psi \ldots \ldots \ldots \ldots \ldots \ldots \ldots . \ldots 114$

4.7 Significant figures of the transformed results of displacement and stress due to the scattered waves for incident wave $\chi \ldots \ldots \ldots \ldots \ldots \ldots$

4.8 Significant figures of the right hand side of Eq. (4-22) with truncation

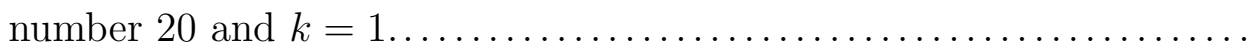

4.9 Comparison with published result of multiple scattering of $5-\mathrm{MHz}$ longitudinal plane wave incident on a pair of 1.0-mm diameter quartz spheres in ice. Two spheres are $2.0-\mathrm{mm}$ away and sit at $Z$-axis sym-

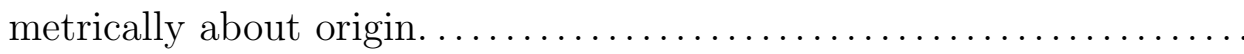

4.10 Significant figures of incident and scattered wave fields due to coordinate transformation. 
4.11 Displacement continuity is shown through $\left|u_{x}\right|,\left|u_{y}\right|$ and $\left|u_{z}\right|$ for the multiple scattering of a longitudinal plane wave incident by a pair of

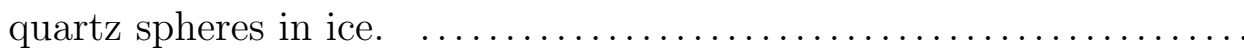

4.12 Displacement continuity is shown through $\left|u_{r}\right|,\left|u_{\theta}\right|$ and $\left|u_{\varphi}\right|$ in the $y=0$ plane for the multiple scattering of a longitudinal plane wave incident by a pair of quartz spheres in ice. .....................

4.13 Surface traction continuity is shown through $\left|\sigma_{r_{1} r_{1}}\right|,\left|\sigma_{r_{1} \theta_{1}}\right|$ and $\left|\sigma_{r_{1} \varphi_{1}}\right|$ in the $y=0$ plane for the multiple scattering of a longitudinal plane wave incident by a pair of quartz spheres in ice.................

4.14 Surface traction continuity is shown through $\left|\sigma_{r_{2} r_{2}}\right|,\left|\sigma_{r_{2} \theta_{2}}\right|$ and $\left|\sigma_{r_{2} \varphi_{2}}\right|$ in the $y=0$ plane for the multiple scattering of a longitudinal plane wave incident by a pair of quartz spheres in ice..................

5.1 An example of scatterer polymerization $\ldots \ldots \ldots \ldots \ldots \ldots \ldots \ldots$

5.2 Position $O$ is chosen as the origin of the global coordinate system for 4 scatterers. . . . . . . . . . . . . . . . . . . . . . . . . . . .

5.3 Core and territory of an abstract scatterer made up of 4 scatterers. .. 136

5.4 Core and territory of an abstract scatterer made up of 3 abstract scat-

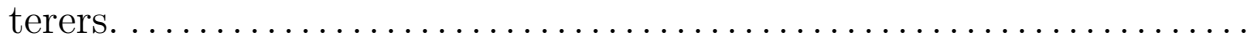

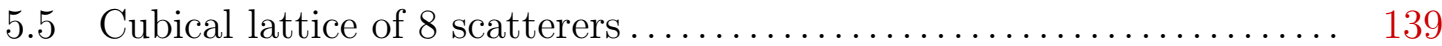

5.6 Five approaches to solve the multiple scattering problem of 8 scatterers. 141

5.7 Modulus of $u_{x}$ of scattered waves at the $y=0$ plane, for the multiple scattering problem of a longitudinal plane wave $(\kappa a=0.5)$ by 8 scatterers of low-carbon steel spheres in epoxy matrix..............

5.8 Modulus of $u_{y}$ of scattered waves at the $y=0$ plane, for the multiple scattering problem of a longitudinal plane wave $(\kappa a=0.5)$ by 8 scatterers of low-carbon steel spheres in epoxy matrix.............

5.9 Modulus of $u_{z}$ of scattered waves at the $y=0$ plane, for the multiple scattering problem of a longitudinal plane wave $(\kappa a=0.5)$ by 8 scatterers of low-carbon steel spheres in epoxy matrix...............

5.10 Modulus of $\sigma_{x x}$ of scattered waves at the $y=0$ plane, for the multiple scattering problem of a longitudinal plane wave $(\kappa a=0.5)$ by 8 scatterers of low-carbon steel spheres in epoxy matrix.............. 149

5.11 Modulus of $\sigma_{y y}$ of scattered waves at the $y=0$ plane, for the multiple scattering problem of a longitudinal plane wave $(\kappa a=0.5)$ by 8 scatterers of low-carbon steel spheres in epoxy matrix.............. 
5.12 Modulus of $\sigma_{z z}$ of scattered waves at the $y=0$ plane, for the multiple scattering problem of a longitudinal plane wave $(\kappa a=0.5)$ by 8 scatterers of low-carbon steel spheres in epoxy matrix.............

5.13 Modulus of $\sigma_{x y}$ of scattered waves at the $y=0$ plane, for the multiple scattering problem of a longitudinal plane wave $(\kappa a=0.5)$ by 8 scatterers of low-carbon steel spheres in epoxy matrix............

5.14 Modulus of $\sigma_{x z}$ of scattered waves at the $y=0$ plane, for the multiple scattering problem of a longitudinal plane wave $(\kappa a=0.5)$ by 8 scatterers of low-carbon steel spheres in epoxy matrix.............

5.15 Modulus of $\sigma_{y z}$ of scattered waves at the $y=0$ plane, for the multiple scattering problem of a longitudinal plane wave $(\kappa a=0.5)$ by 8 scatterers of low-carbon steel spheres in epoxy matrix...............

6.1 Sixteen actual scatterers in a square arrangement in a layer. ......... 161

6.2 Thirty-two actual scatterers in a cubic arrangement. .............. 162

6.3 Sixty-four actual scatterers in a cubic arrangement. .............. 163

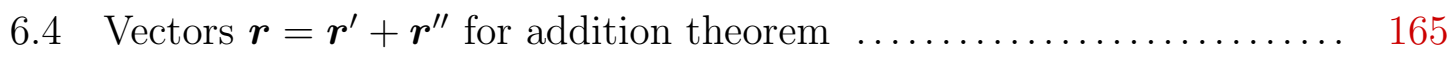

6.5 Relation of truncation number, significant figures, the ratio $r^{\prime \prime} / r^{\prime}$ and three orientations of vector $\mathbf{r}^{\prime \prime}$ during coordinate transformation. . . . 166

6.6 Relation of wave number and significant figure after transformation between two abstract scatterers .......................... 169

6.7 Comparison of $u_{z}$ modulus for different layers ................. 171

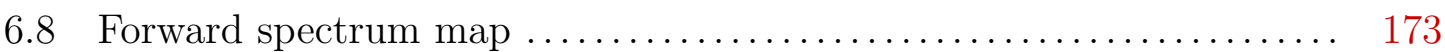

6.9 Geometric relation of scatterer arrangement and the $y=0$ plane. ... 174

6.10 modulus of $u_{y}$ in the $y=0$ plane when $\kappa a=1.35$ and there are sixty-

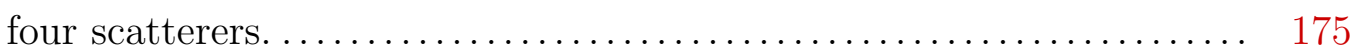

6.11 Modulus of $u_{z}$ in the $y=0$ plane of total waves for the case of one layer of 16 scatterers. . . . . . . . . . . . . . . . . . . . . .

6.12 Real part of $u_{z}$ in the $y=0$ plane of total waves for the case of one

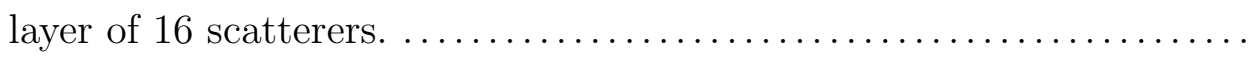

6.13 Modulus of $u_{z}$ in the $y=0$ plane of scattered waves for the case of one

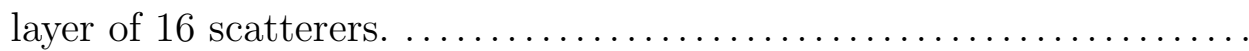

6.14 Real part of $u_{z}$ in the $y=0$ plane of scattered waves for the case of one layer of 16 scatterers.

6.15 Modulus of $u_{x}$ in the $y=0$ plane of total waves for the case of one layer of 16 scatterers. 
6.16 Real part of $u_{x}$ in the $y=0$ plane of total waves for the case of one

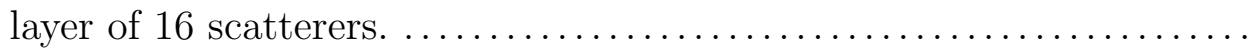

6.17 Modulus of $u_{z}$ in the $y=0$ plane of total waves for the case of two

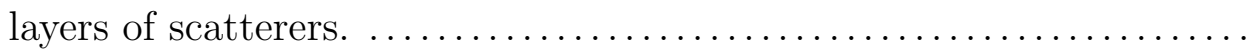

6.18 Real part of $u_{z}$ in the $y=0$ plane of total waves for the case of two

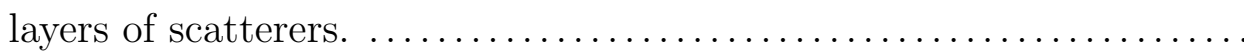

6.19 Modulus of $u_{z}$ in the $y=0$ plane of scattered waves for the case of two

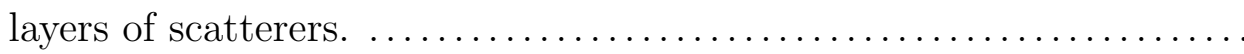

6.20 Real part of $u_{z}$ in the $y=0$ plane of scattered waves for the case of two layers of scatterers.

6.21 Modulus of $u_{x}$ in the $y=0$ plane of total waves for the case of two

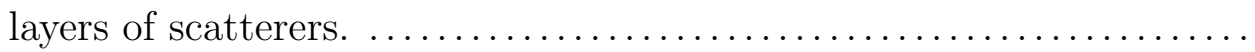

6.22 Real part of $u_{x}$ in the $y=0$ plane of total waves for the case of two

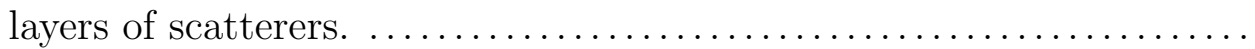

6.23 Modulus of $u_{z}$ in the $y=0$ plane of total waves for the case of four layers of 64 scatterers.

6.24 Real part of $u_{z}$ in the $y=0$ plane of total waves for the case of four

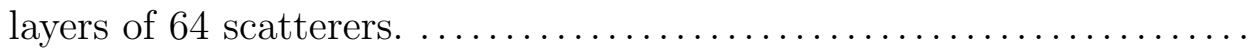

6.25 Modulus of $u_{z}$ in the $y=0$ plane of scattered waves for the case of four layers of 64 scatterers.

6.26 Real part of $u_{z}$ in the $y=0$ plane of scattered waves for the case of

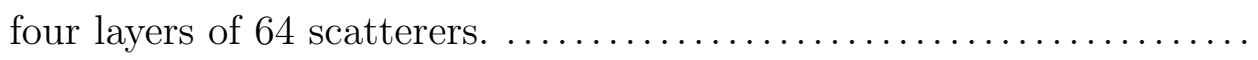

6.27 Modulus of $u_{x}$ in the $y=0$ plane of total waves for the case of four

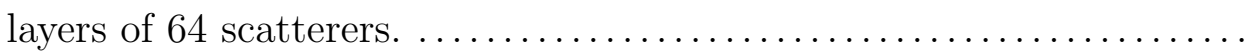

6.28 Real part of $u_{x}$ in the $y=0$ plane of total waves for the case of four

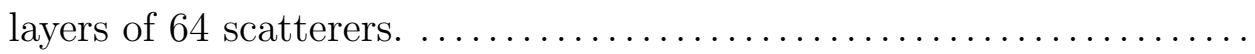

6.29 Modulus of stresses $\sigma_{x x}, \sigma_{y y}$ and $\sigma_{z z}$ in the $y=0$ plane of total waves at $\kappa a=0.3$ and for three cases of one, two and four layers. ........

6.30 Real part of stresses $\sigma_{x x}, \sigma_{y y}$ and $\sigma_{z z}$ in the $y=0$ plane of total waves at $\kappa a=0.3$ and for three cases of one layer, two and four layers. . . . 202

6.31 Modulus of stresses $\sigma_{x x}, \sigma_{y y}$ and $\sigma_{z z}$ in the $y=0$ plane of scattered waves at $\kappa a=0.3$ and for three cases of one layer, two and four layers.

6.32 Real part of stresses $\sigma_{x x}, \sigma_{y y}$ and $\sigma_{z z}$ in the $y=0$ plane of scattered waves at $\kappa a=0.3$ and for three cases of one layer, two and four layers.

6.33 Modulus of stress $\sigma_{x z}$ in the $y=0$ plane of total waves at $\kappa a=0.3$ and for three cases of one layer, two and four layers. 
6.34 Real part of stress $\sigma_{x z}$ in the $y=0$ plane of total waves at $\kappa a=0.3$ and for three cases of one layer, two and four layers. ............ 205

6.35 Modulus of stresses $\sigma_{x x}, \sigma_{y y}$ and $\sigma_{z z}$ in the $y=0$ plane of total waves at $\kappa a=0.75$ and for three cases of one layer, two and four layers. . . 207

6.36 Real part of stresses $\sigma_{x x}, \sigma_{y y}$ and $\sigma_{z z}$ in the $y=0$ plane of total waves at $\kappa a=0.75$ and for three cases of one layer, two and four layers. ... 208

6.37 Modulus of stresses $\sigma_{x x}, \sigma_{y y}$ and $\sigma_{z z}$ in the $y=0$ plane of scattered waves at $\kappa a=0.75$ and for three cases of one layer, two and four layers. 209

6.38 Real part of stresses $\sigma_{x x}, \sigma_{y y}$ and $\sigma_{z z}$ in the $y=0$ plane of scattered waves at $\kappa a=0.75$ and for three cases of one layer, two and four layers.

6.39 Modulus of stress $\sigma_{x z}$ in the $y=0$ plane of total waves at $\kappa a=0.75$ and for three cases of one layer, two and four layers. ............ 212

6.40 Real part of stress $\sigma_{x z}$ in the $y=0$ plane of total waves at $\kappa a=0.75$ and for three cases of one layer, two and four layers. ........... 212

6.41 Modulus of stresses $\sigma_{x x}, \sigma_{y y}$ and $\sigma_{z z}$ in the $y=0$ plane of total waves at $\kappa a=1.2$ and for three cases of one layer, two and four layers. . ...

6.42 Real part of stresses $\sigma_{x x}, \sigma_{y y}$ and $\sigma_{z z}$ in the $y=0$ plane of total waves at $\kappa a=1.2$ and for three cases of one layer, two and four layers. ....

6.43 Modulus of stresses $\sigma_{x x}, \sigma_{y y}$ and $\sigma_{z z}$ in the $y=0$ plane of scattered waves at $\kappa a=1.2$ and for three cases of one layer, two and four layers. 216

6.44 Real part of stresses $\sigma_{x x}, \sigma_{y y}$ and $\sigma_{z z}$ in the $y=0$ plane of scattered waves at $\kappa a=1.2$ and for three cases of one layer, two and four layers. 217

6.45 Modulus of stress $\sigma_{x z}$ in the $y=0$ plane of total waves at $\kappa a=1.2$ and for three cases of one layer, two and four layers. ........... 218

6.46 Real part of stress $\sigma_{x z}$ in the $y=0$ plane of total waves at $\kappa a=1.2$ and for three cases of one layer, two and four layers. ............ 218

C.1 Spherical and Cartesian Coordinate Systems. ..................... 241

D.1 Spherical and Cartesian Coordinate Systems. .................. 244 


\section{List of Tables}

2.1 Material Properties of Beryllium and Polyethylene (after [1]) ...... 45

2.2 Data of Normalized Scattering Cross Section in Figure 2.27 ....... 69

2.3 Single scattering: normalized average energy flux across a closed sur-

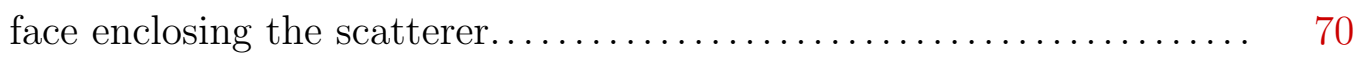

2.4 Single scattering: normalized average energy flux across a closed surface enclosing no scatterer. ................................ 71

4.1 Coordinates of Ten Positions in the Local System. .............. 103

4.2 Material Properties of Low Carbon Steel (After [2]) and Ice (After [3]). 105

4.3 Material Properties of Quartz and Ice...................... 118

4.4 Normalized Average Energy Flux through Closed Surfaces ......... 121

5.1 Comparison of Problem Sizes of Scatterer Polymerization Methodology

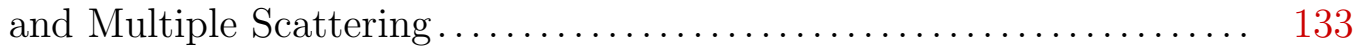

5.2 Material Properties of Low-Carbon Steel and Epoxy (after [2, 4]) ... 140

5.3 Modulus of $u_{x}$ of scattered waves from five computational approaches 143

5.4 Modulus of $u_{y}$ of scattered waves from five computational approaches 143

5.5 Modulus of $u_{z}$ of scattered waves from five computational approaches 147

5.6 Modulus of $\sigma_{x x}$ of scattered waves from five computational approaches 148

5.7 Modulus of $\sigma_{y y}$ of scattered waves from five computational approaches 148

5.8 Modulus of $\sigma_{z z}$ of scattered waves from five computational approaches 155

5.9 Modulus of $\sigma_{x y}$ of scattered waves from five computational approaches 155

5.10 Modulus of $\sigma_{x z}$ of scattered waves from five computational approaches 156

5.11 Modulus of $\sigma_{y z}$ of scattered waves from five computational approaches 156

5.12 Comparison of normalized average energy flux(NAEF) from five ap-

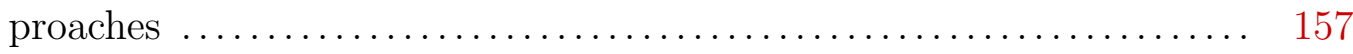

6.1 Coordinates of Ten Positions in the Local System. ............... 168 


\section{Acknowledgments}

First of all, I would like to say thanks to my wife, Suping Fan, for her support, encouragement and share of my endeavors of continuing journey through work and education for the last 13 years.

I would like to say thanks to my academic advisor, Dr. Liang-Wu Cai, for his support and valued advice during my years in the Mechanical and Nuclear Engineering Department of Kansas State University.

I would like also to say thanks to my doctoral committee members, Dr. Youqi Wang, Dr. Daniel V. Swenson, Dr. Talat S. Rahman and Dr. Sameer I. Madanshetty, for their guidance, help and caring about me in academic or non-academic area.

I would like also to say thanks to Dr. Timothy Edwin Doyle and Dr. Weng Cho Chew, for providing valued information to strengthen my confidence in my coding in $\mathrm{C}++$.

I would like also to say thanks to all of my friends, not named here, for going through my thesis and my presentation, and for the enjoyable moments with them.

I would like also to say thanks to staffs in the Mechanical and Nuclear Engineering Department of Kansas State University, for their cooperation with me. 


\section{Chapter 1}

\section{Thesis Introduction}

\section{1-1 Introduction}

Elastic waves include longitudinal wave and two shear waves in perpendicular polarization orientations. Various applications involve the subject of scattering of elastic waves. For examples, the subject of scattering of elastic waves can be found in nondestructive evaluations (NDE) of materials ${ }^{[5,6]}$, geophysics ${ }^{[7]}$ and investigations

of phononic materials (elastic wave band gap materials) ${ }^{[8,9]}$. There are two types of problems in the scattering of elastic waves. When mechanical properties and geometrical parameters of elastic inclusions and the host media are known and used to predict the scattered wave fields ${ }^{[8,9]}$, the problem is the direct problem of scattering of elastic waves. When mechanical properties and geometrical parameters of inclusions or defects are extracted from measured scattering data, the problem is the inverse problem of scattering of elastic waves. Interpretation of measured results of the inverse problem relies on the understanding of wave phenomena of the direct problem. The direct problem is the focus in the thesis.

This thesis is concerned with the scattering of elastic waves by spherical inclusions 
in an unbounded linear elastic medium. Spherical or nearly spherical shape is a typical shape of inclusions in engineered materials, such as concrete, rubber tires and nanocomposites. An unbounded medium is considered in this thesis and the effects due to the edge of the host medium are neglected. This thesis intends to develop a computational system to simulate three-dimensional multiple scattering of elastic waves by large number of inclusions, via a combination of theoretical and computational tools.

Previous work related to the scattering of elastic waves by spherical inclusions is reviewed in this section. The review is divided into two parts: single scattering by a spherical inclusion and multiple scattering by spherical inclusions. Investigations of scattering of electromagnetic or acoustic waves are not reviewed here.

\section{1-1.1 Single Scattering by a Spherical Inclusion}

Single scattering implies that a scattered wave is not further scattered. Here, single scattering is the scattering by one inclusion located in an infinite medium. Problems of single scattering of elastic waves by a spherical inclusion have been treated extensively in the literature. The spherical inclusion could be rigid, elastic or void. The earliest investigation of the scattering of longitudinal elastic wave by a rigid sphere was conducted in 1872 by Rayleigh $\left(J\right.$. W. Strutt) ${ }^{[10]}$. The more general treatment of the scattering of elastic waves by a spherical inclusion was not accomplished until 1927 by Sezawa ${ }^{[11]}$. He assumed a general isotropic, homogeneous, elastic solid as the host medium, with a circular cylinder, an elliptical or a sphere inclusion of either rigid or vacuous material as the scatterer. The scattering of a plane longitudinal wave by an elastic sphere was investigated in 1956 by Ying and Truell ${ }^{[1]}$, in which the scalar spherical harmonics (the general solution of Helmholtz equation in a spherical coordinate system when the function in Helmholtz equation is a scalar function) is 
used. The scattering by a rigid sphere is further studied in 1963 by Pao and Mow ${ }^{[12]}$ by additionally considering rigid body translation and the mass density of the sphere. This study showed that the solution in [1] was a special case in which the mass density of the sphere is infinite (so that the sphere is immovable).

The scattering of a plane shear wave by a spherical inclusion was investigated in 1960 by Einspruch, Witterholt and Truell ${ }^{[13]}$, in which a set of vector spherical harmonics are used. Vector spherical harmonics ${ }^{[14]}$ are a set of vector functions that can be used as alternative form of the general solution to the Helmholtz equation when the unknown for the Helmholtz equation is a vector. By using vector spherical harmonics, Norris ${ }^{[15]}$ in 1986 derived the solution for scattering of both longitudinal and shear elastic waves by a spherical inclusion. By using scalar spherical harmonics, Hinders $^{[16]}$ in 1991 derived the solution for scattering of both longitudinal and shear waves by a spherical inclusion.

For an inclusion of arbitrary shape, the $T$-matrix theory for the scattering of acoustic waves was presented in 1969 by Waterman ${ }^{[17]}$. The $T$-matrix theory was extended to the scattering of elastic waves in 1976 by Varadan (Varatharajulu) and Pao ${ }^{[18]}$ and by Waterman ${ }^{[19]}$. The $T$-matrix represents a linear transformation between scattered and incident waves. Properties of the $T$-matrix are discussed in detail in [18] and [19]. When the T-matrix is obtained for a scatterer, the problem of single scattering of elastic waves is solved.

\section{1-1.2 Multiple Scattering by Spherical Inclusions}

When there is more than one inclusion in a medium, multiple scattering occurs. When inclusions in the medium are far away from each other, the effects of multiple scattering may be neglected. In this way, the scattered wave fields are the summation of the scattered wave fields of single scattering from each inclusion. By using such 
a single scattering approximation, Norris ${ }^{[15]}$ and Hinders ${ }^{[16]}$ solved scattering by dilute concentrations of multiple spherical inclusions by neglecting multiple scattering effect. Sayers and Smith ${ }^{[20]}$ in 1983 compared the predicted results with measurements, by Kindra, et al. ${ }^{[21]}$ in 1982, of the scattering of ultrasonic incident waves by lead spheres embedded in an epoxy matrix. Reasonable agreement between prediction and experiments is obtained for a volume concentration of $5 \%$. For a volume concentrations of $15 \%$, the prediction by this approximate method deviates significantly from experimental results. Thus more rigorous analyses are required.

Twersky ${ }^{[22]}$ in 1952 proposed that the scattered waves can be decomposed as various orders of scattered waves, known as ordered scattering. For example, the first order scattered waves are those waves scattered by each inclusion separately when it is excited by the incident waves. When the first order scattered waves by one inclusion is then re-scattered by other inclusions, the second order scattered waves are generated. This procedure is infinitely continued. Boundary-value problem is solved at each order of scattering. A formal solution for the scattering of a plane electromagnetic or acoustic wave by an arbitrary configuration of parallel cylinders is presented in [22]. Twersky ${ }^{[23]}$ in 1962 extended the approach to multiple scattering of a plane electromagnetic or acoustic wave by arbitrary configurations in three-dimensional space.

For multiple scattering of elastic waves by spherical inclusions in arbitrary arrangement, Doyle ${ }^{[24]}$ in 2006 used an iterative method (based on ordered scattering approach) for calculating wave fields in the elastic system. Calculation is carried out at each order of scattering. In two successive orders, numerical results are compared for the iteration to arrive at a convergent solution for the multiply scattered fields. Due to the virtual memory requirement in a computer, computation and storage of the matrix for coordinate transformation between coordinate systems of every two 
scatterers are limiting factors for this method ${ }^{[24]}$. The convergence of solutions during iterations is another limiting factor for this method. For example ${ }^{[24]}$, for longitudinal wave field at $600 \mathrm{kHz}$ and for $600 \mu \mathrm{m}$ glass beads in polymer with $30 \%$ volume fraction, numerical results do not converge after 10 iteration steps so that this method fails at this case.

Boström $^{[25]}$ in 1980 extended the $T$-matrix formulation of the scattering of elastic waves for the case of single inclusion by Varadan and $\mathrm{Pao}^{[18]}$ and by Waterman ${ }^{[19]}$ to the case of an arbitrary number of inclusions. Waves impinging upon one scatterer include the incident waves and the scattered waves due to all other scatterers. In this approach, the total waves impinging upon a scatterer and the resulting scattered waves is related by the $T$-matrix of the scatterer. The multiple scattering solution is represented by a set of linear equations that can be solved by many numerical solvers.

For multiple scattering of elastic waves by periodic arrays of spherical inclusions, Liu et al. ${ }^{[9]}$ and Psarobas et al. ${ }^{[26]}$ in 2000 independently developed formalisms for calculating band structure (relation between the wave frequency and the wave vector, which is also known as the dispersion relation), reflection and transmission of elastic waves. Both formalisms are based on the $T$-matrix approach for exact solutions of multiple scattering problems. The periodic arrays of spherical inclusions are viewed as a sequence of planes of spherical inclusions, parallel to each other and having the two-dimensional periodicity in each plane. For example, a face-centered cubic lattice (such that five spheres in a plane and four spheres in the adjacent plane) is used in both papers. Finite number of planes were used in examples of calculation.

Computation time and the storage required for the linear equation system are main limiting factors for the $T$-matrix approach. For example, when the $T$-matrix of a scatterer has a size of $(\mathcal{M} \times \mathcal{M})$ and there are $\mathcal{N}$ scatterers in a multiple scattering problem, the matrix for solving the multiple scattering problem has a size of $(\mathcal{N} \mathcal{M} \times$ 
$\mathcal{N} \mathcal{M})$. The CPU memory runs out quickly as the number of scatterers, $\mathcal{N}$, increases.

Numerical methods such as finite element method, finite difference method, and boundary element method have also been applied to multiple scattering problems ${ }^{[27-30]}$, such as scattering by a scatterer lattices where the microstructure can be simplified with repeating structural elements ${ }^{[28,29]}$ or by a few scatterers ${ }^{[30]}$. The major drawback of these methods is the large number of degree of freedom. An enormous number of elements, cells or gridpoints that approximate the microstructure is required. A finer mesh will provide a higher fidelity solution, but at the cost of an increased computational burden.

Based on statistical or effective medium assumptions, approximate solutions of the direct problem have also been developed. For examples, Waterman and Truell ${ }^{[31]}$ used a configurational average procedure of Foldy ${ }^{[32]}$ to average wave fields in a statistical sense; Gaunaurd and Überall ${ }^{[33]}$ treated the scattering region, the smallest space enclosing all scatterers, as an effective medium with effective material properties such as moduli. Both assumptions neglect localized variations. The effective medium assumption also constrains the range of wave frequency. These solutions are not sufficiently accurate inside the scattering region.

Little work has been reported for simulating three-dimensional multiple scattering of elastic waves by an arbitrary number of spherical inclusions of random distribution. It is possibly because of complexity of the scattering of elastic waves. Elastic waves include both longitudinal wave and transverse wave, and mode conversion exists between longitudinal and transverse wave during scattering. The mode conversion (conversion between the longitudinal and shear waves) occurs through continuities of displacement and surface traction at the interface between inclusion(s) and the host medium.

For computation of multiple scattering of elastic waves in two-dimensional space, 
a $T$-matrix-based scatterer polymerization method has been introduced by Cai and Williams ${ }^{[34]}$. The scatterer polymerization method treats an assemblage of actual scatterers as an abstract scatterer that has its own T-matrix. "Full-scale" scattering of horizontally polarized shear wave were simulated ${ }^{[35]}$. "The qualifying phrase fullscale implies that the region occupied by the deterministic model is of macroscopic dimension, the scale in which the composite might be viewed as homogeneous." ${ }^{[35]}$ In numerical simulations, scatterer polymerization method is used to reduce the number of actual scatterers to a lesser number of abstract scatterers.

\section{1-2 Scope of Thesis}

The present thesis extends the scatterer polymerization method to threedimensional scattering of elastic waves by an arbitrary number of spherical inclusions in arbitrary arrangement. The number of actual scatterers is reduced to a lesser number of abstract scatterers, so that the limiting factor of matrix size for solving multiple scattering problems of large number of actual scatterers can be released. A computational system is built for three-dimensional multiple scattering of elastic waves by spherical inclusions. This is achieved by a combination of theoretical development and computer implementation. The process involves the following steps:

1. Derive analytical expression of elastic wave fields in terms of the scalar and vector spherical harmonics;

2. Formulate analytically exact solution for single scattering of elastic waves by a spherical inclusion in a solid medium;

3. Implement and verify the single scattering solution;

4. Formulate analytically exact solution of three-dimensional multiple scattering of elastic waves in a solid medium by using $T$-matrix approach; 
5. Implement and verify the multiple scattering solution;

6. Reformulate multiple scattering solution of elastic waves with scatterer polymerization method;

7. Implement and verify the reformulated solution of multiple scattering.

The computer code was written in $\mathrm{C}++$ language. It is assumed in the solution that the contact between a scatterer and the host medium is never lost during scattering process.

After the computer implementation is verified, it is used to study the scattering of longitudinal incident waves by a finite number of scatterers arranged in cubic lattice, and to observe the band gap formation process. Numerical examples also serve to demonstrate that the computational system has the capability to simulate multiple scattering solutions of elastic waves in three-dimensional space. 


\section{Chapter 2}

\section{Single Scattering Solution}

\section{2-1 Introduction}

Scattering of elastic waves by a spherical inclusion embedded in an infinite medium is considered in this chapter. As stated in Chapter 1 , solutions to problems of single scattering of elastic waves by a spherical inclusion have been found in terms of either scalar spherical harmonics or vector spherical harmonics. Since solutions of single scattering problem serve as the basic building block used in the formulation of multiple scattering solution, it is necessary to review single scattering solutions that will be used for multiple scattering solution.

In this chapter, solutions to the single scattering problem are derived in terms of both scalar and vector spherical harmonics. Basic equations in terms of scalar spherical harmonics can be found in [36]. A modified form of vector spherical harmonics is given, so that the coefficients of wave expansion in terms of both scalar and vector spherical harmonics can be easily converted in an analytical manner.

The structure of this chapter is as the following: Section 2-2 introduces notations and equations for wave motion in linear elastic solids; Section 2-3 reviews two sets 
of wave field expressions; Section 2-4 focuses on solutions of steady-state governing equations; in Section 2-5, the energy conservation law is discussed; and in Section 2-6, numerical examples of single scattering solution are presented and discussed.

\section{2-2 Equations of Elastic Wave Motion}

A brief review of the basic equations, concepts and notations relating to elastic wave motion is presented in this section. Interested readers can find more comprehensive theoretical presentations in references [14, 36, 37].

\section{2-2.1 Basic Equations}

In an ideal elastic medium, stress and strain in Einstein's tensor notation can be written as ${ }^{[37]}$

$$
\begin{gathered}
\sigma_{i j, j}=\lambda \varepsilon_{k k} \delta_{i j}+2 \mu \varepsilon_{i j}, \\
\varepsilon_{i j}=\frac{1}{2}\left(u_{i, j}+u_{j, i}\right)
\end{gathered}
$$

respectively; where index $i, j$ or $k$ takes on values 1,2 or 3 denoting the axes in a Cartesian coordinate system, $\sigma_{i j}$ and $\varepsilon_{i j}$ are stress and strain on the surface normal to $i$ axis and along $j$ axis direction at a field point, $u_{i}$ is displacement along $i$ axis direction at the field point, $\lambda$ and $\mu$ are Lamé elasticity constants of the material, and $\delta_{i j}$ is the Kronecker delta:

$$
\delta_{i j}=\left\{\begin{array}{c}
0 \text { when } i \neq j, \\
1 \text { when } i=j .
\end{array}\right.
$$

The equation of motion in the elastic medium can be written as ${ }^{[37]}$

$$
\rho \ddot{u}_{i}=\sigma_{i j, j}+\rho f_{i}
$$


where $\rho$ is mass density and $f_{i}$ is the externally applied body force per unit volume of the medium (along $i$ axis direction).

By utilizing the stress-strain and strain-displacement relations in Eqs. (2-1) and $(2-2)$, Eq. $(2-4)$ can be written as

$$
\rho \ddot{u}_{i}=\mu u_{i, j j}+(\lambda+\mu) u_{j, j i}+\rho f_{i}
$$

The equation of motion can also be written as ${ }^{[37]}$

$$
\rho \ddot{\boldsymbol{u}}=\mu \nabla^{2} \boldsymbol{u}+(\lambda+\mu) \nabla(\nabla \cdot \boldsymbol{u})+\rho \boldsymbol{f}
$$

For the case in which body force vanishes, Eq. (2-6) is known as Navier's equation:

$$
\rho \ddot{\boldsymbol{u}}=\mu \nabla^{2} \boldsymbol{u}+(\lambda+\mu) \nabla(\nabla \cdot \boldsymbol{u})
$$

In this thesis, only cases in which the body forces vanish are considered.

\section{2-2.2 Scalar Wave Equations}

According to Helmholtz's theorem of decomposition of a vector field ${ }^{[14]}$, displacement vector $\boldsymbol{u}$ can be written in terms of displacement potentials, as follows:

$$
\boldsymbol{u}=\nabla \phi+\nabla \times \boldsymbol{\psi}
$$

where $\phi$ is called the scalar displacement potential, and $\boldsymbol{\psi}$ is called the vector displacement potential of $\boldsymbol{u}$. The first term on the right hand side of Eq. (2-8), $\nabla \phi$, gives the dilatational or longitudinal part of the displacement $\boldsymbol{u}$. The vector $\boldsymbol{\psi}$ in Eq. (2-8) gives rise to shear part of displacement $\boldsymbol{u}$ so that it can further be decomposed into two parts. It is suggested in [14] that

$$
\boldsymbol{u}_{\text {due to shear wave }}=\nabla \times \boldsymbol{\psi}=\nabla \times\left(w \psi \boldsymbol{e}_{3}\right)+\nabla \times \nabla \times\left(\ell w \chi \boldsymbol{e}_{3}\right)
$$

where $\ell$ is an arbitrary constant length scale so introduced to keep two unspecified scalar potentials $\psi$ and $\chi$ having the same dimension, $w$ is a function of $\xi_{3}$ with $\xi_{3}$ 
as one of three coordinates $\left(\xi_{1}, \xi_{2}, \xi_{3}\right)$ in an orthogonal curvilinear coordinate system (Appendix A), and $\boldsymbol{e}_{3}$ is one of three unit vectors $\boldsymbol{e}_{1}, \boldsymbol{e}_{2}$ and $\boldsymbol{e}_{3}$ corresponding to coordinates $\xi_{1}, \xi_{2}$ and $\xi_{3}$, respectively. Substituting Eq. (2-9) into Eq. (2-8) gives

$$
\boldsymbol{u}=\nabla \phi+\nabla \times\left(w \psi \boldsymbol{e}_{3}\right)+\nabla \times \nabla \times\left(\ell w \chi \boldsymbol{e}_{3}\right)
$$

Longitudinal wave is often called the $\mathrm{P}$ wave. Shear waves can be divided into $\mathrm{SH}$ and SV waves ${ }^{[14]}$, denoting horizontally polarized and vertically polarized shear waves, respectively. The two cases gain their names by the direction of the displacement. Recall that in the Cartesian coordinate system in seismic wave analyses, the $y$-axis customarily points to the sky (the vertical). For an SV wave, the displacement is in the vertical direction. For an $\mathrm{SH}$ wave, the displacement is parallel to the ground (the horizontal). In this way, the displacement due to the $\psi$ component is normal to $\boldsymbol{e}_{3}$ direction, which would become an SV wave in a Cartesian coordinate system. The displacement due to $\chi$ is along the $\boldsymbol{e}_{3}$ direction, which would become an $\mathrm{SH}$ wave in a Cartesian coordinate system. (Note that for other coordinate systems, the shear wave generally cannot be separated into SH and SV components.)

By choosing $w=\xi_{3}$ in Eq. (2-9) and substituting Eqs. (2-8) and (2-9) into Navier's equation in Eq. (2-7), the equation of motion can be reduced to the following three uncoupled equations of scalar displacement potentials (details of reducing Navier's equation to three equations can be found in Appendix B):

$$
\begin{aligned}
& \ddot{\phi}=c_{L}^{2} \nabla^{2} \phi \\
& \ddot{\psi}=c_{S}^{2} \nabla^{2} \psi \\
& \ddot{\chi}=c_{S}^{2} \nabla^{2} \chi
\end{aligned}
$$

where

$$
c_{L}=\sqrt{\frac{\lambda+2 \mu}{\rho}} \quad \text { and } \quad c_{S}=\sqrt{\frac{\mu}{\rho}}
$$

are two different wave speeds, representing two different types of waves in the solids. 


\section{2-2.3 Steady-State Solution to Wave Equations}

Consider the steady state in which all waves in the field have the same temporal factor $\mathrm{e}^{-\hat{\imath} \omega t}$ where $\omega$ is the circular frequency. Then the wave equations in $(2-11 \mathrm{a})$ through (2-11c) become the following set of Helmholtz equations

$$
\begin{aligned}
& \nabla^{2} \phi+\kappa^{2} \phi=0 \\
& \nabla^{2} \psi+k^{2} \psi=0 \\
& \nabla^{2} \chi+k^{2} \chi=0
\end{aligned}
$$

where

$$
\kappa=\frac{\omega}{c_{L}} \quad \text { and } \quad k=\frac{\omega}{c_{S}}
$$

are the wave numbers for the longitudinal and shear waves, respectively.

In the following analysis, eqn. (2-13b) ( $\psi$-equation) is used to illustrate the solution process in the spherical coordinate system $(r, \theta, \varphi)$ where $r \in[0, \infty), \theta \in[0, \pi]$, and $\varphi \in[0,2 \pi]$ (Fig. 2.1). Detailed expressions for various vector operators are listed in Appendix C.

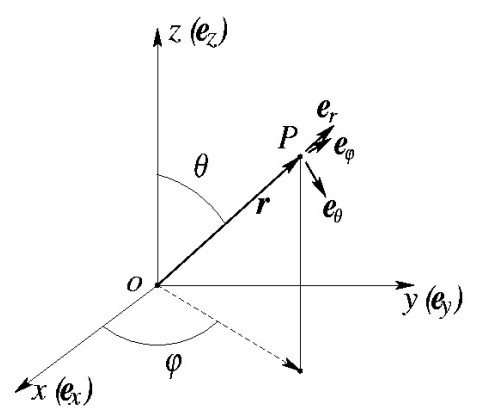

Figure 2.1: Spherical Coordinate System

The expanded Helmholtz equation in the spherical coordinate system is

$$
\frac{1}{r^{2}} \frac{\partial}{\partial r}\left(r^{2} \frac{\partial \psi}{\partial r}\right)+\frac{1}{r^{2} \sin \theta} \frac{\partial}{\partial \theta}\left(\sin \theta \frac{\partial \psi}{\partial \theta}\right)+\frac{1}{r^{2} \sin ^{2} \theta} \frac{\partial^{2} \psi}{\partial \varphi^{2}}+k^{2} \psi=0
$$


By assuming the variable-separated form of the solution as

$$
\phi(r, \theta, \varphi)=R(r) \Theta(\theta) \Phi(\varphi)
$$

equation (2-15) becomes

$$
\frac{1}{r^{2}}\left(r^{2} R^{\prime}\right)^{\prime} \Theta \Phi+\frac{1}{r^{2} \sin \theta}\left(\sin \theta \Theta^{\prime}\right)^{\prime} R \Phi+\frac{1}{r^{2} \sin ^{2} \theta} R \Theta \Phi^{\prime \prime}+k^{2} R \Theta \Phi=0 .
$$

Multiplying both sides by $r^{2} /(R \Theta \Phi)$ gives

$$
\frac{1}{R}\left(r^{2} R^{\prime}\right)^{\prime}+\frac{1}{\Theta} \frac{1}{\sin \theta}\left(\sin \theta \Theta^{\prime}\right)^{\prime}+\frac{1}{\Phi} \frac{1}{\sin ^{2} \theta} \Phi^{\prime \prime}+k^{2} r^{2}=0
$$

The variable $r$ is now separated: the first and the last terms contain only $r$ and two terms in the middle do not contain $r$. This leads to two equations connected by an arbitrary constant $p$

$$
\begin{array}{r}
\left(r^{2} R^{\prime}\right)^{\prime}+\left(k^{2} r^{2}-p^{2}\right) R=0 \\
\frac{1}{\Phi} \Phi^{\prime \prime}+\frac{\sin \theta}{\Theta}\left(\sin \theta \Theta^{\prime}\right)^{\prime}+(p \sin \theta)^{2}=0
\end{array}
$$

Equation (2-19b) can be further separated by another arbitrary constant $q$

$$
\begin{aligned}
\frac{1}{\sin \theta}\left(\sin \theta \Theta^{\prime}\right)^{\prime}+\left(p^{2}-\frac{q^{2}}{\sin ^{2} \theta}\right) \Theta & =0 \\
\Phi^{\prime \prime}+q^{2} \Phi & =0
\end{aligned}
$$

In summary, the three separated equations are

$$
\begin{aligned}
\left(r^{2} R^{\prime}\right)^{\prime}+\left(k^{2} r^{2}-p^{2}\right) R & =0 \\
\frac{1}{\sin \theta}\left(\sin \theta \Theta^{\prime}\right)^{\prime}+\left(p^{2}-\frac{q^{2}}{\sin ^{2} \theta}\right) \Theta & =0 \\
\Phi^{\prime \prime}+q^{2} \Phi & =0
\end{aligned}
$$

\section{Ф-equation:}

Equation (2-21c) is solved first. The solution is $\Phi(\varphi)=\mathrm{e}^{\hat{\imath} q \varphi}$, where $\hat{\imath}=\sqrt{-1}$. In order to maintain the single-valuedness of the solution, that is $\Phi(0)=\Phi(2 \pi), q$ has to be an integer, denoted as $m$. Then

$$
\Phi(\varphi)=\mathrm{e}^{\hat{\imath} m \varphi} \quad \text { where } m=0, \pm 1, \pm 2, \cdots
$$




\section{$\Theta-e q u a t i o n:$}

Denote

$$
p^{2}=\nu(\nu+1), \quad v=\cos \theta \quad(-1 \leq v \leq 1)
$$

since

$$
\frac{d}{d \theta}=\frac{d}{d v} \frac{d v}{d \theta}=-\sin \theta \frac{d}{d v} \quad \text { and } \quad \sin ^{2} \theta=1-v^{2}
$$

eqn. (2-21b) can be written as

$$
-\frac{d}{d v}\left[-\left(1-v^{2}\right) \frac{d \Theta}{d v}\right]+\left[\nu(\nu+1)-\frac{m^{2}}{1-v^{2}}\right] \Theta=0
$$

or

$$
\left(1-v^{2}\right) \frac{d^{2} \Theta}{d v^{2}}-2 v \frac{d \Theta}{d v}+\left[\nu(\nu+1)-\frac{m^{2}}{1-v^{2}}\right] \Theta=0
$$

Equation (2-24) is the associated Legendré equation, and its solution is known as the associated Legendré function, generally denoted as $P_{\nu}^{m}(v)$ where $\nu$ is called the degree and $m$ is called the order. (When $m=0$, the equation is called the Legendré equation, the function is called the Legendré function and is denoted as $P_{\nu}(v)$.) It is also known that only when $\nu$ is an integer, denoted as $n$, the associated Legendré function converges at $v= \pm 1$. Convergence at $v= \pm 1$ is required for the physical problem at hand because $v= \pm 1$ is at $\theta=0$ or $\pi$. There is another solution, generally denoted as $Q_{\nu}^{m}(v)$, that is also a solution to the Legendré equation, but this function does not generally converge. Therefore the only physically admissible solution is the associated Legendré function of integer degree $n$ and integer order $m, P_{n}^{m}(v)$. That is

$$
\Theta(\theta)=P_{n}^{m}(\cos \theta) \quad \text { where } n=0,1,2, \cdots
$$

Usually, the associated Legendré function of integer order and integer degree is called the associated Legendré polynomial, and when $m=0$, it is denoted as $P_{n}(v)$ and called the Legendré polynomial. 


\section{$R$-equation:}

Now it is known that

$$
p^{2}=n(n+1)
$$

and denote

$$
R(r)=\frac{f(r)}{\sqrt{k r}}
$$

then

$$
\begin{aligned}
\frac{d R}{d r} & =\frac{1}{\sqrt{k r}} f^{\prime}-\frac{1}{2} k(k r)^{-3 / 2} f=\frac{1}{\sqrt{k r}} f^{\prime}-\frac{1}{2 r} \frac{1}{\sqrt{k r}} f \\
r^{2} \frac{d R}{d r} & =\frac{r^{3 / 2}}{k^{1 / 2}} f^{\prime}-\frac{r^{1 / 2}}{2 k^{1 / 2}} f \\
\frac{d}{d r}\left(r^{2} \frac{d R}{d r}\right) & =\frac{r^{3 / 2}}{k^{1 / 2}} f^{\prime \prime}+\frac{3}{2} \frac{r^{1 / 2}}{k^{1 / 2}} f^{\prime}-\frac{r^{1 / 2}}{2 k^{1 / 2}} f^{\prime}-\frac{1}{2} \frac{r^{-1 / 2}}{2 k^{1 / 2}} f \\
& =\frac{r^{2}}{\sqrt{k r}} f^{\prime \prime}+\frac{r}{\sqrt{k r}} f^{\prime}-\frac{1}{4} \frac{1}{\sqrt{k r}} f
\end{aligned}
$$

Equation (2-21a) becomes

$$
\frac{r^{2}}{\sqrt{k r}} f^{\prime \prime}+\frac{r}{\sqrt{k r}} f^{\prime}+\left[k^{2} r^{2}-n(n+1)-\frac{1}{4}\right] \frac{f}{\sqrt{k r}}=0
$$

or,

$$
r^{2} f^{\prime \prime}+r f^{\prime}+\left[(k r)^{2}-\left(n+\frac{1}{2}\right)^{2}\right] f=0
$$

since

$$
n(n+1)+\frac{1}{4}=\left(n+\frac{1}{2}\right)^{2}
$$

Equation (2-28) is known as the Bessel equation, and the solution is the Bessel function of order $(n+1 / 2)$ :

$$
f(r)=Z_{n+\frac{1}{2}}(k r)
$$

where $Z_{\nu}(z)$ represents Bessel functions of various kinds, among which, $J_{\nu}(z)$, Bessel function of the first kind, and $H_{\nu}^{(1)}(z)$, Hankel function of the first kind, are most often used. 
Recall the definition for spherical Bessel functions ${ }^{[38]}$

$$
\begin{aligned}
j_{n}(z) & =\sqrt{\frac{\pi}{2 z}} J_{n+\frac{1}{2}}(z) \\
h_{n}^{(1)}(z) & =\sqrt{\frac{\pi}{2 z}} H_{n+\frac{1}{2}}^{(1)}(z)
\end{aligned}
$$

Hence the general solution for eqn. $(2-13 b)$ can be written as

$$
\psi(r, \theta, \varphi)=\sum_{n=0}^{\infty} \sum_{m=-n}^{n} A_{m n} \mathcal{Z}_{n}(k r) P_{n}^{m}(\cos \theta) \mathrm{e}^{\hat{\imath} m \varphi}
$$

where $\mathcal{Z}_{n}(\cdot)$ denotes a spherical Bessel function, either $j_{n}(\cdot)$ or $h_{n}^{(1)}(\cdot)$, and the limit of summation for $m$ is restricted by the value of $n$, because

$$
P_{n}^{m}(v)=0 \quad \text { for }|m|>n
$$

The combination of $P_{n}^{m}(\cos \theta)$ and $\mathrm{e}^{\hat{\imath} m \varphi}$ is often referred to as the spherical harmonics $[39,40]$ and is often denoted as $X_{n}^{m}(\theta, \varphi)$ :

$$
X_{n}^{m}(\theta, \varphi)=P_{n}^{m}(\cos \theta) \mathrm{e}^{\hat{\imath} m \varphi}
$$

Furthermore, it is often normalized and denoted as $Y_{n}^{m}(\theta, \varphi)$ :

$$
Y_{n}^{m}(\theta, \varphi)=\sqrt{\frac{2 n+1}{4 \pi} \frac{(n-m) !}{(n+m) !}} P_{n}^{m}(\cos \theta) \mathrm{e}^{\hat{\imath} m \varphi}
$$

Spherical harmonics have different names [40]: when $m=0$, it is called the zonal harmonics; when $m=n$, it is called sectorial harmonics; and when $m \neq n$, it is called tesseral harmonics.

Using the notation for the spherical harmonics, the general solution for eqn. $(2-13 b)$ can be written as

$$
\psi(r, \theta, \varphi)=\sum_{n=0}^{\infty} \sum_{m=-n}^{n} A_{m n} \mathcal{Z}_{n}(k r) Y_{n}^{m}(\theta, \varphi)
$$




\section{2-3 Wave Field Expressions}

Since $\xi_{3}=r$ in spherical coordinate system, the displacement can be written as

$$
\boldsymbol{u}=\nabla \phi+\nabla \times\left(r \psi \boldsymbol{e}_{r}\right)+\ell \nabla \times \nabla \times\left(r \chi \boldsymbol{e}_{r}\right)
$$

The displacement $\boldsymbol{u}$ can also be written as

$$
\boldsymbol{u}=u_{r} \boldsymbol{e}_{r}+u_{\theta} \boldsymbol{e}_{\theta}+u_{\varphi} \boldsymbol{e}_{\varphi}
$$

Therefore, displacement components $u_{r}, u_{\theta}$ and $u_{\varphi}$ can be written as

$$
\begin{aligned}
& u_{r}=\frac{\partial \phi}{\partial r}+\ell\left[\frac{\partial^{2}(r \chi)}{\partial r^{2}}-r \nabla^{2} \chi\right] \\
& u_{\theta}=\frac{1}{r} \frac{\partial \phi}{\partial \theta}+\frac{1}{r \sin \theta} \frac{\partial(r \psi)}{\partial \varphi}+\frac{\ell}{r} \frac{\partial^{2}(r \chi)}{\partial \theta \partial r} \\
& u_{\varphi}=\frac{1}{r \sin \theta} \frac{\partial \phi}{\partial \varphi}-\frac{1}{r} \frac{\partial(r \psi)}{\partial \theta}+\frac{\ell}{r \sin \theta} \frac{\partial^{2}(r \chi)}{\partial \varphi \partial r}
\end{aligned}
$$

In a spherical coordinate system the strain-displacement relations are ${ }^{[41]}$

$$
\begin{aligned}
\varepsilon_{r r} & =\frac{\partial u_{r}}{\partial r} \\
\varepsilon_{r \theta} & =\frac{1}{2}\left[\frac{1}{r} \frac{\partial u_{r}}{\partial \theta}-\frac{u_{\theta}}{r}+\frac{\partial u_{\theta}}{\partial r}\right] \\
\varepsilon_{r \varphi} & =\frac{1}{2}\left[\frac{1}{r \sin \theta} \frac{\partial u_{r}}{\partial \varphi}-\frac{u_{\varphi}}{r}+\frac{\partial u_{\varphi}}{\partial r}\right] \\
\varepsilon_{\theta \theta} & =\frac{1}{r} \frac{\partial u_{\theta}}{\partial \theta}+\frac{u_{r}}{r} \\
\varepsilon_{\varphi \varphi} & =\frac{1}{r \sin \theta} \frac{\partial u_{\varphi}}{\partial \varphi}+\frac{u_{r}}{r}+\frac{\cos \theta}{\sin \theta} \frac{u_{\theta}}{r} \\
\varepsilon_{\theta \varphi} & =\frac{1}{2}\left[\frac{1}{r} \frac{\partial u_{\varphi}}{\partial \theta}-\frac{\cos \theta}{\sin \theta} \frac{u_{\varphi}}{r}+\frac{1}{r \sin \theta} \frac{\partial u_{\theta}}{\partial \varphi}\right]
\end{aligned}
$$

Using strain-displacement relations in Eq. (2-40) and stress-strain relations in Eq. $(2-1)$ (Eq. $(2-1)$ can also be used in a spherical coordinate system $\left.{ }^{[41]}\right)$, and assuming Lamé elasticity constants as $\lambda$ and $\mu$, stresses in terms of displacement potentials can be found as [36]:

$$
\sigma_{r r}=\lambda \nabla^{2} \phi+2 \mu\left\{\frac{\partial^{2} \phi}{\partial r^{2}}+\ell \frac{\partial}{\partial r}\left[\frac{\partial^{2}(r \chi)}{\partial r^{2}}-r \nabla^{2} \chi\right]\right\}
$$




$$
\begin{aligned}
& \sigma_{r \theta}=\frac{2 \mu}{r}\left[\frac{\partial^{2} \phi}{\partial r \partial \theta}-\frac{1}{r} \frac{\partial \phi}{\partial \theta}\right]-\frac{\mu}{r}\left[\frac{1}{r \sin \theta} \frac{\partial(r \psi)}{\partial \varphi}-r \frac{\partial}{\partial r}\left(\frac{1}{r \sin \theta} \frac{\partial(r \psi)}{\partial \varphi}\right)\right] \\
& +\frac{\mu \ell}{r}\left[\frac{\partial}{\partial \theta}\left(\frac{\partial^{2}(r \chi)}{\partial r^{2}}-r \nabla^{2} \chi\right)-\frac{1}{r} \frac{\partial^{2}(r \chi)}{\partial r \partial \theta}+r \frac{\partial}{\partial r}\left(\frac{1}{r} \frac{\partial^{2}(r \chi)}{\partial r \partial \theta}\right)\right] \\
& \sigma_{r \varphi}=2 \mu\left[\frac{1}{r \sin \theta} \frac{\partial^{2} \phi}{\partial r \partial \varphi}-\frac{1}{r^{2} \sin \theta} \frac{\partial \phi}{\partial \varphi}\right]+\mu\left[\frac{2}{r^{2}} \frac{\partial(r \psi)}{\partial \theta}-\frac{1}{r} \frac{\partial^{2}(r \psi)}{\partial \theta \partial r}\right] \\
& +\mu \ell\left[\frac{1}{r \sin \theta} \frac{\partial}{\partial \varphi}\left(\frac{2 \partial^{2}(r \chi)}{\partial r^{2}}-r \nabla^{2} \chi\right)-\frac{2}{r^{2} \sin \theta} \frac{\partial^{2}(r \chi)}{\partial r \partial \varphi}\right] \\
& \sigma_{\theta \theta}=\lambda \nabla^{2} \phi+2 \mu\left\{\frac{1}{r} \frac{\partial \phi}{\partial r}+\frac{1}{r^{2}} \frac{\partial^{2} \phi}{\partial \theta^{2}}+\frac{1}{r} \frac{\partial}{\partial \theta}\left[\frac{1}{r \sin \theta} \frac{\partial(r \psi)}{\partial \varphi}\right]+\right. \\
& \left.+\ell\left[\frac{1}{r^{2}} \frac{\partial^{3}(r \chi)}{\partial \theta^{2} \partial r}+\frac{1}{r} \frac{\partial^{2}(r \chi)}{\partial r^{2}}-\nabla^{2} \chi\right]\right\} \\
& \sigma_{\varphi \varphi}=\lambda \nabla^{2} \phi+2 \mu\left\{\frac{1}{r \sin \theta} \frac{\partial}{\partial \varphi}\left(\frac{1}{r \sin \theta} \frac{\partial \phi}{\partial \varphi}\right)+\frac{1}{r} \frac{\partial \phi}{\partial r}+\frac{\cos \theta}{r^{2} \sin \theta} \frac{\partial \phi}{\partial \theta}\right. \\
& -\frac{\cos \theta}{r^{2} \sin ^{2} \theta} \frac{\partial(r \psi)}{\partial \varphi}+\frac{1}{r^{2} \sin \theta} \frac{\partial^{2}(r \psi)}{\partial \theta \partial \varphi} \\
& \left.+\ell\left[\frac{1}{r^{2} \sin ^{2} \theta} \frac{\partial^{3}(r \chi)}{\partial \varphi^{2} \partial r}+\frac{1}{r} \frac{\partial^{2}(r \chi)}{\partial r^{2}}-\nabla^{2} \chi+\frac{\cos \theta}{r^{2} \sin \theta} \frac{\partial^{2}(r \chi)}{\partial \theta \partial r}\right]\right\} \\
& \sigma_{\theta \varphi}=2 \mu\left[\frac{1}{r^{2} \sin \theta} \frac{\partial^{2} \phi}{\partial \theta \partial \varphi}-\frac{\cos \theta}{r^{2} \sin ^{2} \theta} \frac{\partial \phi}{\partial \varphi}\right] \\
& +\mu\left[\frac{\cos \theta}{r^{2} \sin \theta} \frac{\partial(r \psi)}{\partial \theta}-\frac{1}{r^{2}} \frac{\partial^{2}(r \psi)}{\partial \theta^{2}}+\frac{1}{r^{2} \sin ^{2} \theta} \frac{\partial^{2}(r \psi)}{\partial \varphi^{2}}\right] \\
& +2 \mu \ell\left[\frac{1}{r^{2} \sin \theta} \frac{\partial^{3}(r \chi)}{\partial r \partial \theta \partial \varphi}-\frac{\cos \theta}{r^{2} \sin ^{2} \theta} \frac{\partial^{2}(r \chi)}{\partial r \partial \varphi}\right]
\end{aligned}
$$

\section{2-3.1 Wave Fields in Terms of Spherical Harmonics}

It is known from Section 2-2.3 that, the general solutions of three Helmholtz equations in Eqs. (2-13a) through (2-13c) can be written as

$$
\begin{aligned}
& \phi(r, \theta, \varphi)=\sum_{n=0}^{\infty} \sum_{m=-n}^{n} a_{m n}^{\phi} \mathcal{Z}_{n}(\kappa r) Y_{n}^{m}(\theta, \varphi) \\
& \psi(r, \theta, \varphi)=\sum_{n=0}^{\infty} \sum_{m=-n}^{n} a_{m n}^{\psi} \mathcal{Z}_{n}(k r) Y_{n}^{m}(\theta, \varphi) \\
& \chi(r, \theta, \varphi)=\sum_{n=0}^{\infty} \sum_{m=-n}^{n} a_{m n}^{\chi} \mathcal{Z}_{n}(k r) Y_{n}^{m}(\theta, \varphi)
\end{aligned}
$$

where $a_{m n}^{\phi}, a_{m n}^{\psi}$ and $a_{m n}^{\chi}$ are wave expansion coefficients. 
Substituting Eq. (2-42) into Eq. (2-39) gives three displacement components in terms of spherical harmonics as follows.

$$
\begin{gathered}
u_{r}=\sum_{n=0}^{\infty} \sum_{m=-n}^{n} \frac{1}{r}\left[\kappa r a_{m n}^{\phi} \mathcal{Z}_{n}^{\prime}(\kappa r)+a_{m n}^{\chi} \ell \mathcal{Z}_{n}(k r) n(n+1)\right] Y_{n}^{m}(\theta, \varphi) \\
u_{\theta}=\sum_{n=0}^{\infty} \sum_{m=-n}^{n}\left\{\frac{1}{r}\left[a_{m n}^{\phi} \mathcal{Z}_{n}(\kappa r)+a_{m n}^{\chi} \ell\left(\mathcal{Z}_{n}(k r)+k r \mathcal{Z}_{n}^{\prime}(k r)\right)\right] \frac{\partial Y_{n}^{m}(\theta, \varphi)}{\partial \theta}\right. \\
\left.+\quad a_{m n}^{\psi} \mathcal{Z}_{n}(k r) \frac{1}{\sin \theta} \frac{\partial Y_{n}^{m}(\theta, \varphi)}{\partial \varphi}\right\} \\
u_{\varphi}=\sum_{n=0}^{\infty} \sum_{m=-n}^{n}\left\{\left[a_{m n}^{\phi} \mathcal{Z}_{n}(\kappa r)+a_{m n}^{\chi} \ell\left(\mathcal{Z}_{n}(k r)+k r \mathcal{Z}_{n}^{\prime}(k r)\right)\right] \frac{1}{r \sin \theta} \frac{\partial Y_{n}^{m}(\theta, \varphi)}{\partial \varphi}\right. \\
\left.\quad-a_{m n}^{\psi} \mathcal{Z}_{n}(k r) \frac{\partial Y_{n}^{m}(\theta, \varphi)}{\partial \theta}\right\}
\end{gathered}
$$

where $\mathcal{Z}_{n}^{\prime}(z)=d \mathcal{Z}_{n}(z) / d z$. Substituting Eq. (2-42) into Eq. (2-41) gives stress components in terms of spherical harmonics as follows.

$$
\begin{aligned}
& \sigma_{r r}=\frac{2 \mu}{r^{2}} \sum_{n=0}^{\infty} \sum_{m=-n}^{n}\left\{a_{m n}^{\phi}\left[\left(n^{2}-n-\frac{1}{2} k^{2} r^{2}\right) \mathcal{Z}_{n}(\kappa r)+2 \kappa r \mathcal{Z}_{n+1}(\kappa r)\right]\right. \\
& \left.+a_{m n}^{\chi} \ell n(n+1)\left[(n-1) \mathcal{Z}_{n}(k r)-k r \mathcal{Z}_{n+1}(k r)\right]\right\} Y_{n}^{m}(\theta, \varphi) \\
& \sigma_{r \theta}=\frac{\mu}{r} \sum_{n=0}^{\infty} \sum_{m=-n}^{n}\left\{\frac { 2 } { r } \left[a_{m n}^{\phi}\left[(n-1) \mathcal{Z}_{n}(\kappa r)-\kappa r \mathcal{Z}_{n+1}(\kappa r)\right]\right.\right. \\
& \left.+a_{m n}^{\chi} \ell\left[\left(n^{2}-1-\frac{1}{2} k^{2} r^{2}\right) \mathcal{Z}_{n}(k r)+k r \mathcal{Z}_{n+1}(k r)\right]\right] \frac{\partial}{\partial \theta} Y_{n}^{m}(\theta, \varphi) \\
& \left.+a_{m n}^{\psi}\left[(n-1) \mathcal{Z}_{n}(k r)-k r \mathcal{Z}_{n+1}(k r)\right] \frac{1}{\sin \theta} \frac{\partial}{\partial \varphi} Y_{n}^{m}(\theta, \varphi)\right\} \\
& \sigma_{r \varphi}=\frac{\mu}{r} \sum_{n=0}^{\infty} \sum_{m=-n}^{n}\left\{\frac { 2 } { r } \left[a_{m n}^{\phi}\left[(n-1) \mathcal{Z}_{n}(\kappa r)-\kappa r \mathcal{Z}_{n+1}(\kappa r)\right]\right.\right. \\
& \left.+\ell a_{m n}^{\chi}\left[\left(n^{2}-1-\frac{1}{2} k^{2} r^{2}\right) \mathcal{Z}_{n}(k r)+k r \mathcal{Z}_{n+1}(k r)\right]\right] \frac{1}{\sin \theta} \frac{\partial}{\partial \varphi} Y_{n}^{m}(\theta, \varphi) \\
& \left.-a_{m n}^{\psi}\left[(n-1) \mathcal{Z}_{n}(k r)-k r \mathcal{Z}_{n+1}(k r)\right] \frac{\partial}{\partial \theta} Y_{n}^{m}(\theta, \varphi)\right\}
\end{aligned}
$$




$$
\begin{aligned}
& \sigma_{\theta \theta}=\sum_{n=0}^{\infty} \sum_{m=-n}^{n}\left\{a _ { m n } ^ { \phi } \left[\left(-\lambda \kappa^{2} \mathcal{Z}_{n}(\kappa r)+\frac{2 \mu}{r} \kappa \mathcal{Z}_{n}^{\prime}(\kappa r)\right) Y_{n}^{m}(\theta, \varphi)\right.\right. \\
& \left.+\frac{2 \mu}{r^{2}} \mathcal{Z}_{n}(\kappa r) \frac{\partial^{2} Y_{n}^{m}(\theta, \varphi)}{\partial \theta^{2}}\right] \\
& +a_{m n}^{\chi}\left[\frac{2 \mu}{r^{2}} \ell\left(\mathcal{Z}_{n}(k r)+k r \mathcal{Z}_{n}^{\prime}(k r)\right) \frac{\partial^{2} Y_{n}^{m}(\theta, \varphi)}{\partial \theta^{2}}\right. \\
& \left.+\frac{2 \mu}{r^{2}} \ln (n+1) \mathcal{Z}_{n}(k r) Y_{n}^{m}(\theta, \varphi)\right] \\
& \left.+a_{m n}^{\psi}\left[-\frac{2 \mu}{r} \mathcal{Z}_{n}(k r) \frac{1}{\sin \theta} \frac{\partial^{2} Y_{n}^{m}(\theta, \varphi)}{\partial \theta \partial \varphi}+\frac{2 \mu}{r} \mathcal{Z}_{n}(k r) \frac{\cos \theta}{\sin ^{2} \theta} \frac{\partial Y_{n}^{m}(\theta, \varphi)}{\partial \varphi}\right]\right\} \\
& \sigma_{\varphi \varphi}=\sum_{n=0}^{\infty} \sum_{m=-n}^{n}\left\{a _ { m n } ^ { \phi } \left[\left(-\lambda \kappa^{2} \mathcal{Z}_{n}(\kappa r)+\frac{2 \mu}{r} \kappa \mathcal{Z}_{n}^{\prime}(\kappa r)\right) Y_{n}^{m}(\theta, \varphi)\right.\right. \\
& \left.+\frac{2 \mu}{r^{2}} \mathcal{Z}_{n}(\kappa r) \frac{1}{\sin ^{2} \theta} \frac{\partial^{2} Y_{n}^{m}(\theta, \varphi)}{\partial \varphi^{2}}+\frac{2 \mu}{r^{2}} \mathcal{Z}_{n}(\kappa r) \frac{\cos \theta}{\sin \theta} \frac{\partial Y_{n}^{m}(\theta, \varphi)}{\partial \varphi}\right] \\
& +a_{m n}^{\chi}\left[\frac{2 \mu}{r^{2}} \ell\left(\mathcal{Z}_{n}(k r)+k r \mathcal{Z}_{n}^{\prime}(k r)\right) \frac{1}{\sin ^{2} \theta} \frac{\partial^{2} Y_{n}^{m}(\theta, \varphi)}{\partial \varphi^{2}}\right. \\
& \left.+\frac{2 \mu}{r^{2}} \ln (n+1) \mathcal{Z}_{n}(k r) Y_{n}^{m}(\theta, \varphi)\right] \\
& \left.-a_{m n}^{\psi}\left[-\frac{2 \mu}{r} \mathcal{Z}_{n}(k r) \frac{1}{\sin \theta} \frac{\partial^{2} Y_{n}^{m}(\theta, \varphi)}{\partial \theta \partial \varphi}+\frac{2 \mu}{r} \mathcal{Z}_{n}(k r) \frac{\cos \theta}{\sin ^{2} \theta} \frac{\partial Y_{n}^{m}(\theta, \varphi)}{\partial \varphi}\right]\right\} \\
& \sigma_{\theta \varphi}=\sum_{n=0}^{\infty} \sum_{m=-n}^{n}\left\{a_{m n}^{\phi}\left[\frac{\mu}{r^{2}} \mathcal{Z}_{n}(\kappa r)\left(\frac{2}{\sin \theta} \frac{\partial^{2} Y_{n}^{m}(\theta, \varphi)}{\partial \theta \partial \varphi}-\frac{\cos \theta}{\sin ^{2} \theta} \frac{\partial Y_{n}^{m}(\theta, \varphi)}{\partial \varphi}\right)\right]\right. \\
& +a_{m n}^{\chi}\left[\frac{\mu}{r^{2}} \ell\left(\mathcal{Z}_{n}(k r)+\mathcal{Z}_{n}^{\prime}(k r)\right)\left(\frac{2}{\sin \theta} \frac{\partial^{2} Y_{n}^{m}(\theta, \varphi)}{\partial \theta \partial \varphi}-\frac{\cos \theta}{\sin ^{2} \theta} \frac{\partial Y_{n}^{m}(\theta, \varphi)}{\partial \varphi}\right)\right] \\
& \left.+a_{m n}^{\psi} \frac{\mu}{r} \mathcal{Z}_{n}(k r)\left[\frac{\cos \theta}{\sin \theta} \frac{\partial Y_{n}^{m}(\theta, \varphi)}{\partial \theta}-\frac{\partial^{2} Y_{n}^{m}(\theta, \varphi)}{\partial \theta^{2}}+\frac{1}{\sin ^{2} \theta} \frac{\partial^{2} Y_{n}^{m}(\theta, \varphi)}{\partial \varphi^{2}}\right]\right\}
\end{aligned}
$$

For notational simplicity, the displacements in Eqs. (2-43a) through (2-43c) can be written as:

$$
\begin{aligned}
u_{r} & =\sum_{n=0}^{\infty} \sum_{m=-n}^{n}\left[a_{m n}^{\phi} \mathfrak{E}_{1 q}^{1 t}(r, n)+a_{m n}^{\chi} \mathfrak{E}_{1 q}^{3 t}(r, n)\right] Y_{n}^{m} \\
u_{\theta} & =\sum_{n=0}^{\infty} \sum_{m=-n}^{n}\left\{\left[a_{m n}^{\phi} \mathfrak{E}_{2 q}^{1 t}(r, n)+a_{m n}^{\chi} \mathfrak{E}_{2 q}^{3 t}(r, n)\right]\left(Y_{, \theta}\right)_{n}^{m}+a_{m n}^{\psi} \mathfrak{E}_{2 q}^{2 t}(r, n)\left(Y_{, \varphi}\right)_{n}^{m}\right\}
\end{aligned}
$$




$$
u_{\varphi}=\sum_{n=0}^{\infty} \sum_{m=-n}^{n}\left\{\left[a_{m n}^{\phi} \mathfrak{E}_{3, q}^{1 t}(r, n)+a_{m n}^{\chi} \mathfrak{E}_{3 q}^{3 t}(r, n)\right]\left(Y_{, \varphi}\right)_{n}^{m}+a_{m n}^{\psi} \mathfrak{E}_{3 q}^{2 t}(r, n)\left(Y_{, \theta}\right)_{n}^{m}\right\}
$$

and the stresses in Eqs. (2-44a) through (2-44f) can be written as:

$$
\begin{aligned}
\sigma_{r r} & =\sum_{n=0}^{\infty} \sum_{m=-n}^{n}\left\{a_{m n}^{\phi} \mathfrak{E}_{4 q}^{1 t}(r, n)+a_{m n}^{\chi} \mathfrak{E}_{4 q}^{3 t}(r, n)\right\} Y_{n}^{m} \\
\sigma_{r \theta} & =\sum_{n=0}^{\infty} \sum_{m=-n}^{n}\left\{\left[a_{m n}^{\phi} \mathfrak{E}_{7 q}^{1 t}(r, n)+a_{m n}^{\chi} \mathfrak{E}_{7 q}^{3 t}(r, n)\right]\left(Y_{, \theta}\right)_{n}^{m}+a_{m n}^{\psi} \mathfrak{E}_{7 q}^{2 t}(r, n)\left(Y_{, \varphi}\right)_{n}^{m}\right\} \\
\sigma_{r \varphi} & =\sum_{n=0}^{\infty} \sum_{m=-n}^{n}\left\{\left[a_{m n}^{\phi} \mathfrak{E}_{8 q}^{1 t}(r, n)+a_{m n}^{\chi} \mathfrak{E}_{8 q}^{3 t}(r, n)\right]\left(Y_{, \varphi}\right)_{n}^{m}+a_{m n}^{\psi} \mathfrak{E}_{8 q}^{2 t}(r, n)\left(Y_{, \theta}\right)_{n}^{m}\right\} \\
\sigma_{\theta \theta} & =\sum_{n=0}^{\infty} \sum_{m=-n}^{n}\left\{a_{m n}^{\phi} \mathfrak{E}_{5 q}^{1 t}(\mathbf{r}, n)+a_{m n}^{\chi} \mathfrak{E}_{5 q}^{3 t}(\mathbf{r}, n)+a_{m n}^{\psi} \mathfrak{E}_{5 q}^{2 t}(\mathbf{r}, n)\right\} \\
\sigma_{\varphi \varphi} & =\sum_{n=0}^{\infty} \sum_{m=-n}^{n}\left\{a_{m n}^{\phi} \mathfrak{E}_{6 q}^{1 t}(\mathbf{r}, n)+a_{m n}^{\chi} \mathfrak{E}_{6 q}^{3 t}(\mathbf{r}, n)+a_{m n}^{\psi} \mathfrak{E}_{6 q}^{2 t}(\mathbf{r}, n)\right\} \\
\sigma_{\theta \varphi}= & \sum_{n=0}^{\infty} \sum_{m=-n}^{n}\left\{a_{m n}^{\phi} \mathfrak{E}_{9 q}^{1 t}(\mathbf{r}, n)+a_{m n}^{\chi} \mathfrak{E}_{9 q}^{3 t}(\mathbf{r}, n)+a_{m n}^{\psi} \mathfrak{E}_{9 q}^{2 t}(\mathbf{r}, n)\right\}
\end{aligned}
$$

In Eqs. (2-45a) through (2-46f),

$$
Y_{n}^{m}=Y_{n}^{m}(\theta, \varphi), \quad\left(Y_{, \theta}\right)_{n}^{m}=\frac{\partial Y_{n}^{m}(\theta, \varphi)}{\partial \theta}, \quad\left(Y_{, \varphi}\right)_{n}^{m}=\frac{1}{\sin \theta} \frac{\partial Y_{n}^{m}(\theta, \varphi)}{\partial \varphi} ;
$$

and a set of $\mathfrak{E}_{p q}^{s t}(r, n)$ and $\mathfrak{E}_{p q}^{s t}(\mathbf{r}, n)$ functions are defined in Appendix E by following Pao and Mow ${ }^{[36]}$. The subscript $p$ runs from 1 to 9 and is used to denote the component of displacement and stress. Particularly, $p=1$ for displacement $u_{r}, p=2$ for $u_{\theta}, p=3$ for $u_{\varphi}, p=4$ for $\sigma_{r r}, p=5$ for $\sigma_{\theta \theta}, p=6$ for $\sigma_{\varphi \varphi}, p=7$ for $\sigma_{r \theta}$, $p=8$ for $\sigma_{r \varphi}$ and $p=9$ for $\sigma_{\theta \varphi}$. The subscript $q=1,2$, refers to medium type, 1 for host medium and 2 for scatterer. It's noted that, $\kappa$ and $k$ are for host medium when $q=1$, while $\kappa$ and $k$ are for scatterer when $q=2$. The superscript $s$ runs from 1 to 3 and denotes wave-type $\phi, \psi$ and $\chi$ respectively. The superscript $t$, which equals 1 or 3 , is used to denote the kind of spherical Bessel function, $\mathcal{Z}_{n}(\cdot) . \mathcal{Z}_{n}(\cdot)=j_{n}(\cdot)$ corresponding to $t=1$ for incident and refracted waves. $\mathcal{Z}_{n}(\cdot)=h_{n}^{(1)}(\cdot)$ corresponding to $t=3$ for scattered wave. 
For the same wave in the same medium, there exist

$$
\begin{aligned}
& \mathfrak{E}_{2 q}^{1 t}(r, n)=\mathfrak{E}_{3 q}^{1 t}(r, n) \\
& \mathfrak{E}_{2 q}^{2 t}(r, n)=-\mathfrak{E}_{3 q}^{2 t}(r, n) \\
& \mathfrak{E}_{2 q}^{3 t}(r, n)=\mathfrak{E}_{3 q}^{3 t}(r, n) \\
& \mathfrak{E}_{7 q}^{1 t}(r, n)=\mathfrak{E}_{8 q}^{1 t}(r, n) \\
& \mathfrak{E}_{7 q}^{2 t}(r, n)=-\mathfrak{E}_{8 q}^{2 t}(r, n) \\
& \mathfrak{E}_{7 q}^{3 t}(r, n)=\mathfrak{E}_{8 q}^{3 t}(r, n)
\end{aligned}
$$

\section{2-3.2 Wave Fields in Terms of Vector Spherical Harmonics}

Alternatively, wave fields can be written in terms of vector spherical harmonics. The benefit of using vector spherical harmonics will be seen when boundary continuity equations are solved and when translation of wave fields is needed.

Write displacement vector such as:

$$
\mathbf{u}=u_{r} \mathbf{e}_{r}+u_{\theta} \mathbf{e}_{\theta}+u_{\varphi} \mathbf{e}_{\varphi}=\sum_{n=0}^{\infty} \sum_{m=-n}^{n} \mathbf{u}_{m n}
$$

Substituting $u_{r}, u_{\theta}$ and $u_{\varphi}$ in eqns. (2-45a) through (2-45c) into equation (2-49) gives

$$
\begin{aligned}
\mathbf{u}_{m n}= & {\left[a_{m n}^{\phi} \mathfrak{E}_{1 q}^{1 t}(r, n)+a_{m n}^{\chi} \mathfrak{E}_{1 q}^{3 t}(r, n)\right] Y_{n}^{m} \mathbf{e}_{r} } \\
& +\left[a_{m n}^{\phi} \mathfrak{E}_{2 q}^{1 t}(r, n)+a_{m n}^{\chi} \mathfrak{E}_{2 q}^{3 t}(r, n)\right]\left(Y_{, \theta}\right)_{n}^{m} \mathbf{e}_{\theta}+a_{m n}^{\psi} \mathfrak{E}_{2 q}^{2 t}(r, n)\left(Y_{, \varphi}\right)_{n}^{m} \mathbf{e}_{\theta} \\
& +\left[a_{m n}^{\phi} \mathfrak{E}_{3 q}^{1 t}(r, n)+a_{m n}^{\chi} \mathfrak{E}_{3 q}^{3 t}(r, n)\right]\left(Y_{, \varphi}\right)_{n}^{m} \mathbf{e}_{\varphi}+a_{m n}^{\psi} \mathfrak{E}_{3 q}^{2 t}(r, n)\left(Y_{, \theta}\right)_{n}^{m} \mathbf{e}_{\varphi} \\
= & {\left[a_{m n}^{\phi} \mathfrak{E}_{1 q}^{1 t}(r, n)+a_{m n}^{\chi} \mathfrak{E}_{1 q}^{3 t}(r, n)\right] Y_{n}^{m} \mathbf{e}_{r} } \\
& +\left[a_{m n}^{\phi} \mathfrak{E}_{2 q}^{1 t}(r, n)+a_{m n}^{\chi} \mathfrak{E}_{2 q}^{3 t}(r, n)\right]\left(Y_{, \theta}\right)_{n}^{m} \mathbf{e}_{\theta}+a_{m n}^{\psi} \mathfrak{E}_{2 q}^{2 t}(r, n)\left(Y_{, \varphi}\right)_{n}^{m} \mathbf{e}_{\theta} \\
& +\left[a_{m n}^{\phi} \mathfrak{E}_{2 q}^{1 t}(r, n)+a_{m n}^{\chi} \mathfrak{E}_{2 q}^{3 t}(r, n)\right]\left(Y_{, \varphi}\right)_{n}^{m} \mathbf{e}_{\varphi}-a_{m n}^{\psi} \mathfrak{E}_{2 q}^{2 t}(r, n)\left(Y_{, \theta}\right)_{n}^{m} \mathbf{e}_{\varphi} \\
= & {\left[a_{m n}^{\phi} \mathfrak{E}_{1 q}^{1 t}(r, n)+a_{m n}^{\chi} \mathfrak{E}_{1 q}^{3 t}(r, n)\right] Y_{n}^{m} \mathbf{e}_{r} } \\
& +\left[a_{m n}^{\phi} \mathfrak{E}_{2 q}^{1 t}(r, n)+a_{m n}^{\chi} \mathfrak{E}_{2 q}^{3 t}(r, n)\right]\left[\left(Y_{, \theta}\right)_{n}^{m} \mathbf{e}_{\theta}+\left(Y_{, \varphi}\right)_{n}^{m} \mathbf{e}_{\varphi}\right]
\end{aligned}
$$




$$
+a_{m n}^{\psi} \mathfrak{E}_{2 q}^{2 t}(r, n)\left[\left(Y_{, \varphi}\right)_{n}^{m} \mathbf{e}_{\varphi}-\left(Y_{, \theta}\right)_{n}^{m} \mathbf{e}_{\varphi}\right]
$$

A set of vector spherical harmonics were originally defined by Morse and Feshbach ${ }^{[14]}$, modifications are made in this work and detailed in Appendices F and G. Then, the normalized vector spherical harmonics $\mathfrak{P}_{m n}, \mathfrak{B}_{m n}$ and $\mathfrak{C}_{m n}$ can be written as

$$
\begin{aligned}
\mathfrak{P}_{m n}(\theta, \varphi) & =\mathbf{e}_{r} Y_{n}^{m}(\theta, \varphi) \\
\mathfrak{B}_{m n}(\theta, \varphi) & =\frac{r}{\sqrt{n(n+1)}} \nabla\left[Y_{n}^{m}(\theta, \varphi)\right] \\
& =\frac{\sqrt{n(n+1)}}{(2 n+1) \sin \theta}\left\{\mathbf{e}_{\theta}\left[\frac{n-m+1}{n+1} Y_{n+1}^{m}-\frac{n+m}{n} Y_{n-1}^{m}\right]+\mathbf{e}_{\varphi} \frac{m(2 n+1)}{n(n+1)} \hat{\imath} Y_{n}^{m}\right\} \\
& =\frac{1}{\sqrt{n(n+1)}} \mathbf{e}_{\theta}\left(Y_{, \theta}\right)_{n}^{m}+\frac{1}{\sqrt{n(n+1)}} \mathbf{e}_{\varphi}\left(Y_{, \varphi}\right)_{n}^{m} \\
\mathfrak{C}_{m n}(\theta, \varphi) & =\frac{1}{\sqrt{n(n+1)}} \nabla \times\left[\mathbf{e}_{r} r Y_{n}^{m}(\theta, \varphi)\right] \\
& =\frac{\sqrt{n(n+1)}}{(2 n+1) \sin \theta}\left\{\mathbf{e}_{\theta} \frac{m(2 n+1)}{n(n+1)} \hat{\imath} Y_{n}^{m}-\mathbf{e}_{\varphi}\left[\frac{n-m+1}{n+1} Y_{n+1}^{m}-\frac{n+m}{n} Y_{n-1}^{m}\right]\right\} \\
& =\frac{1}{\sqrt{n(n+1)}} \mathbf{e}_{\theta}\left(Y_{, \varphi}\right)_{n}^{m}-\frac{1}{\sqrt{n(n+1)}} \mathbf{e}_{\varphi}\left(Y_{, \theta}\right)_{n}^{m}
\end{aligned}
$$

Properties of $\mathfrak{P}_{m n}, \mathfrak{B}_{m n}$ and $\mathfrak{C}_{m n}$ can be found in Appendix $\mathrm{G}$.

Then Eq. (2-50) can be written as

$$
\begin{aligned}
\mathbf{u}_{m n}= & {\left[a_{m n}^{\phi} \mathfrak{E}_{1 q}^{1 t}(r, n)+a_{m n}^{\chi} \mathfrak{E}_{1 q}^{3 t}(r, n)\right] \mathfrak{P}_{m n}+a_{m n}^{\psi} \mathfrak{E}_{2 q}^{2 t}(r, n) \sqrt{n(n+1)} \mathfrak{C}_{m n} } \\
& +\left[a_{m n}^{\phi} \mathfrak{E}_{2 q}^{1 t}(r, n)+a_{m n}^{\chi} \mathfrak{E}_{2 q}^{3 t}(r, n)\right] \sqrt{n(n+1)} \mathfrak{B}_{m n} \\
= & a_{m n}^{\phi}\left[\mathfrak{E}_{1 q}^{1 t}(r, n) \mathfrak{P}_{m n}+\mathfrak{E}_{2 q}^{1 t}(r, n) \sqrt{n(n+1)} \mathfrak{B}_{m n}\right]+a_{m n}^{\psi} \mathfrak{E}_{2 q}^{2 t}(r, n) \sqrt{n(n+1)} \mathfrak{C}_{m n} \\
& +a_{m n}^{\chi}\left[\mathfrak{E}_{1 q}^{3 t}(r, n) \mathfrak{P}_{m n}+\mathfrak{E}_{2 q}^{3 t}(r, n) \sqrt{n(n+1)} \mathfrak{B}_{m n}\right]
\end{aligned}
$$

Therefore, the displacement in terms of normalized vector spherical harmonics can be written as

$$
\begin{aligned}
\mathbf{u}=\sum_{n=0}^{\infty} \sum_{m=-n}^{n} & \left\{a_{m n}^{\phi}\left[\mathfrak{E}_{1 q}^{1 t}(r, n) \mathfrak{P}_{m n}+\mathfrak{E}_{2 q}^{1 t}(r, n) \sqrt{n(n+1)} \mathfrak{B}_{m n}\right]\right. \\
& +a_{m n}^{\psi} \mathfrak{E}_{2 q}^{2 t}(r, n) \sqrt{n(n+1)} \mathfrak{C}_{m n} \\
& \left.+a_{m n}^{\chi}\left[\mathfrak{E}_{1 q}^{3 t}(r, n) \mathfrak{P}_{m n}+\mathfrak{E}_{2 q}^{3 t}(r, n) \sqrt{n(n+1)} \mathfrak{B}_{m n}\right]\right\}
\end{aligned}
$$


In spherical coordinates, definition of a set of vector wave functions can be found in [42-45]. Modifications are made in this work and detailed in Appendix H. Then, the vector wave functions can be written as

$$
\begin{aligned}
\boldsymbol{L}_{n m}(\kappa r) & =\mathfrak{E}_{1 q}^{1 t}(r, n) \mathfrak{P}_{n m}+\mathfrak{E}_{2 q}^{1 t}(r, n) \sqrt{n(n+1)} \mathfrak{B}_{n m} \\
\boldsymbol{M}_{n m}(k r) & =\mathfrak{E}_{2 q}^{2 t}(r, n) \sqrt{n(n+1)} \mathfrak{C}_{n m} \\
\boldsymbol{N}_{n m}(k r) & =\mathfrak{E}_{1 q}^{3 t}(r, n) \mathfrak{P}_{n m}+\mathfrak{E}_{2 q}^{3 t}(r, n) \sqrt{n(n+1)} \mathfrak{B}_{n m}
\end{aligned}
$$

By comparing Eq. (2-53) and Eq. (2-54), the displacement vector can take the following form

$$
\boldsymbol{u}=\sum_{n=0}^{\infty} \sum_{m=-n}^{n}\left\{a_{m n}^{\phi} \boldsymbol{L}_{n m}(\kappa r)+a_{m n}^{\psi} \boldsymbol{M}_{n m}(k r)+a_{m n}^{\chi} \boldsymbol{N}_{n m}(k r)\right\}
$$

On spherical surface of radius $r$, surface traction $\mathbf{t}$ can be written in the following form:

$$
\mathbf{t}=\sigma_{r r} \mathbf{e}_{r}+\sigma_{r \theta} \mathbf{e}_{\theta}+\sigma_{r \varphi} \mathbf{e}_{\varphi}=\sum_{n=0}^{\infty} \sum_{m=-n}^{n} \mathbf{t}_{m n}
$$

Substituting $\sigma_{r r}, \sigma_{r \theta}$ and $\sigma_{r \varphi}$ in Eqs. (2-46a) through (2-46c) into equation (2-56) gives

$$
\begin{aligned}
\mathbf{t}_{m n}= & {\left[a_{m n}^{\phi} \mathfrak{E}_{4 q}^{1 t}(r, n)+a_{m n}^{\chi} \mathfrak{E}_{4 q}^{3 t}(r, n)\right] Y_{n}^{m} \mathbf{e}_{r} } \\
& +\left[a_{m n}^{\phi} \mathfrak{E}_{7 q}^{1 t}(r, n)+a_{m n}^{\chi} \mathfrak{E}_{7 q}^{3 t}(r, n)\right]\left(Y_{, \theta}\right)_{n}^{m} \mathbf{e}_{\theta}+a_{m n}^{\psi} \mathfrak{E}_{7 q}^{2 t}(r, n)\left(Y_{, \varphi}\right)_{n}^{m} \mathbf{e}_{\theta} \\
& +\left[a_{m n}^{\phi} \mathfrak{E}_{8 q}^{1 t}(r, n)+a_{m n}^{\chi} \mathfrak{E}_{8 q}^{3 t}(r, n)\right]\left(Y_{, \varphi}\right)_{n}^{m} \mathbf{e}_{\varphi}+a_{m n}^{\psi} \mathfrak{E}_{8 q}^{2 t}(r, n)\left(Y_{, \theta}\right)_{n}^{m} \mathbf{e}_{\varphi} \\
= & {\left[a_{m n}^{\phi} \mathfrak{E}_{4 q}^{1 t}(r, n)+a_{m n}^{\chi} \mathfrak{E}_{4 q}^{3 t}(r, n)\right] Y_{n}^{m} \mathbf{e}_{r} } \\
& +\left[a_{m n}^{\phi} \mathfrak{E}_{7 q}^{1 t}(r, n)+a_{m n}^{\chi} \mathfrak{E}_{7 q}^{3 t}(r, n)\right]\left(Y_{, \theta}\right)_{n}^{m} \mathbf{e}_{\theta}+a_{m n}^{\psi} \mathfrak{E}_{7 q}^{2 t}(r, n)\left(Y_{, \varphi}\right)_{n}^{m} \mathbf{e}_{\theta} \\
& +\left[a_{m n}^{\phi} \mathfrak{E}_{7 q}^{1 t}(r, n)+a_{m n}^{\chi} \mathfrak{E}_{7 q}^{3 t}(r, n)\right]\left(Y_{, \varphi}\right)_{n}^{m} \mathbf{e}_{\varphi}-a_{m n}^{\psi} \mathfrak{E}_{7 q}^{2 t}(r, n)\left(Y_{, \theta}\right)_{n}^{m} \mathbf{e}_{\varphi} \\
= & {\left[a_{m n}^{\phi} \mathfrak{E}_{4 q}^{1 t}(r, n)+a_{m n}^{\chi} \mathfrak{E}_{4 q}^{3 t}(r, n)\right] Y_{n}^{m} \mathbf{e}_{r} } \\
& +\left[a_{m n}^{\phi} \mathfrak{E}_{7 q}^{1 t}(r, n)+a_{m n}^{\chi} \mathfrak{E}_{7 q}^{3 t}(r, n)\right]\left[\left(Y_{, \theta}\right)_{n}^{m} \mathbf{e}_{\theta}+\left(Y_{, \varphi}\right)_{n}^{m} \mathbf{e}_{\varphi}\right] \\
& +a_{m n}^{\psi} \mathfrak{E}_{7 q}^{2 t}(r, n)\left[\left(Y_{, \varphi}\right)_{n}^{m} \mathbf{e}_{\varphi}-\left(Y_{, \theta}\right)_{n}^{m} \mathbf{e}_{\varphi}\right]
\end{aligned}
$$




$$
\begin{aligned}
= & {\left[a_{m n}^{\phi} \mathfrak{E}_{4 q}^{1 t}(r, n)+a_{m n}^{\chi} \mathfrak{E}_{4 q}^{3 t}(r, n)\right] \mathfrak{P}_{m n}+a_{m n}^{\psi} \mathfrak{E}_{7 q}^{2 t}(r, n) \sqrt{n(n+1)} \mathfrak{C}_{m n} } \\
& +\left[a_{m n}^{\phi} \mathfrak{E}_{7 q}^{1 t}(r, n)+a_{m n}^{\chi} \mathfrak{E}_{7 q}^{3 t}(r, n)\right] \sqrt{n(n+1)} \mathfrak{B}_{m n} \\
= & a_{m n}^{\phi}\left[\mathfrak{E}_{4 q}^{1 t}(r, n) \mathfrak{P}_{m n}+\mathfrak{E}_{7 q}^{1 t}(r, n) \sqrt{n(n+1)} \mathfrak{B}_{m n}\right]+a_{m n}^{\psi} \mathfrak{E}_{7 q}^{2 t}(r, n) \sqrt{n(n+1)} \mathfrak{C}_{m n} \\
& +a_{m n}^{\chi}\left[\mathfrak{E}_{4 q}^{3 t}(r, n) \mathfrak{P}_{m n}+\mathfrak{E}_{7 q}^{3 t}(r, n) \sqrt{n(n+1)} \mathfrak{B}_{m n}\right] .
\end{aligned}
$$

Therefore, the surface traction $\mathbf{t}$ in terms of vector spherical harmonics can be written as

$$
\begin{aligned}
\mathbf{t}=\sum_{n=0}^{\infty} \sum_{m=-n}^{n} & \left\{a_{m n}^{\phi}\left[\mathfrak{E}_{4 q}^{1 t}(r, n) \mathfrak{P}_{m n}+\mathfrak{E}_{7 q}^{1 t}(r, n) \sqrt{n(n+1)} \mathfrak{B}_{m n}\right]\right. \\
& +a_{m n}^{\psi} \mathfrak{E}_{7 q}^{2 t}(r, n) \sqrt{n(n+1)} \mathfrak{C}_{m n} \\
& \left.+a_{m n}^{\chi}\left[\mathfrak{E}_{4 q}^{3 t}(r, n) \mathfrak{P}_{m n}+\mathfrak{E}_{7 q}^{3 t}(r, n) \sqrt{n(n+1)} \mathfrak{B}_{m n}\right]\right\}
\end{aligned}
$$

\section{2-4 Single Scattering Solution}

Assume a single scatterer embeded in an infinite medium is subjected to an incident wave. The scatterer is located at the origin of a spherical coordinate system. In general, the incident wave is representable as regular waves such that

$$
\begin{aligned}
\phi^{\text {inc }} & =\sum_{n=0}^{\infty} \sum_{m=-n}^{n} A_{m n}^{\phi} j_{n}(\kappa r) Y_{n}^{m}(\theta, \varphi), \\
\psi^{\text {inc }} & =\sum_{n=0}^{\infty} \sum_{m=-n}^{n} A_{m n}^{\psi} j_{n}(k r) Y_{n}^{m}(\theta, \varphi), \\
\chi^{\text {inc }} & =\sum_{n=0}^{\infty} \sum_{m=-n}^{n} A_{m n}^{\chi} j_{n}(k r) Y_{n}^{m}(\theta, \varphi) .
\end{aligned}
$$

The scattered waves are representable as singular waves as

$$
\begin{aligned}
\phi^{\mathrm{sct}} & =\sum_{n=0}^{\infty} \sum_{m=-n}^{n} B_{m n}^{\phi} h_{n}^{(1)}(\kappa r) Y_{n}^{m}(\theta, \varphi), \\
\psi^{\mathrm{sct}} & =\sum_{n=0}^{\infty} \sum_{m=-n}^{n} B_{m n}^{\psi} h_{n}^{(1)}(k r) Y_{n}^{m}(\theta, \varphi),
\end{aligned}
$$




$$
\chi^{\mathrm{sct}}=\sum_{n=0}^{\infty} \sum_{m=-n}^{n} B_{m n}^{\chi} h_{n}^{(1)}(k r) Y_{n}^{m}(\theta, \varphi) .
$$

The refracted waves are representable as regular waves as

$$
\begin{aligned}
\phi^{\mathrm{ref}} & =\sum_{n=0}^{\infty} \sum_{m=-n}^{n} C_{m n}^{\phi} j_{n}\left(\kappa_{1} r\right) Y_{n}^{m}(\theta, \varphi), \\
\psi^{\mathrm{ref}} & =\sum_{n=0}^{\infty} \sum_{m=-n}^{n} C_{m n}^{\psi} j_{n}\left(k_{1} r\right) Y_{n}^{m}(\theta, \varphi), \\
\chi^{\mathrm{ref}} & =\sum_{n=0}^{\infty} \sum_{m=-n}^{n} C_{m n}^{\chi} j_{n}\left(k_{1} r\right) Y_{n}^{m}(\theta, \varphi) .
\end{aligned}
$$

In Eqs. (2-59) through (2-61), subscripts inc, sct and ref stand for incident, scattered and refracted waves, respectively; and $\kappa_{1}$ and $k_{1}$ are wave numbers in the scatterer while $\kappa$ and $k$ are wave numbers in the host medium.

Assuming that the scatterer is perfectly bonded with the surrounding host medium, the boundary conditions require that all displacement components and three stress components involving a subscript $r$ be continuous across the boundary at $r=a$. This leads to the following equations:

$$
\begin{aligned}
& \left.\left(u_{r}^{\mathrm{inc}}+u_{r}^{\mathrm{sct}}\right)\right|_{r=a}=\left.u_{r}^{\mathrm{ref}}\right|_{r=a} \\
& \left.\left(u_{\theta}^{\mathrm{inc}}+u_{\theta}^{\mathrm{sct}}\right)\right|_{r=a}=\left.u_{\theta}^{\mathrm{ref}}\right|_{r=a} \\
& \left.\left(u_{\varphi}^{\mathrm{inc}}+u_{\varphi}^{\mathrm{sct}}\right)\right|_{r=a}=\left.u_{\varphi}^{\mathrm{ref}}\right|_{r=a} \\
& \left.\left(\sigma_{r r}^{\mathrm{inc}}+\sigma_{r r}^{\mathrm{sct}}\right)\right|_{r=a}=\left.\sigma_{r r}^{\mathrm{ref}}\right|_{r=a} \\
& \left.\left(\sigma_{r \theta}^{\mathrm{inc}}+\sigma_{r \theta}^{\mathrm{sct}}\right)\right|_{r=a}=\left.\sigma_{r \theta}^{\mathrm{ref}}\right|_{r=a} \\
& \left.\left(\sigma_{r \varphi}^{\mathrm{inc}}+\sigma_{r \varphi}^{\mathrm{sct}}\right)\right|_{r=a}=\left.\sigma_{r \varphi}^{\mathrm{ref}}\right|_{r=a}
\end{aligned}
$$

or in vector form,

$$
\begin{gathered}
\left.\left(\mathbf{u}^{\text {inc }}+\mathbf{u}^{\text {sct }}\right)\right|_{r=a}=\left.\mathbf{u}^{\text {ref }}\right|_{r=a} \\
\left.\left(\mathbf{t}^{\text {inc }}+\mathbf{t}^{\text {sct }}\right)\right|_{r=a}=\left.\mathbf{t}^{\text {ref }}\right|_{r=a}
\end{gathered}
$$




\section{2-4.1 Displacement Continuity Equations}

The displacements due to the incident, scattered and refracted waves, according to Eqs. (2-59) through (2-61), can be written respectively as:

$$
\begin{aligned}
\mathbf{u}^{\text {inc }}=\sum_{n=0}^{\infty} \sum_{m=-n}^{n} & \left\{A_{m n}^{\phi}\left[\mathfrak{E}_{11}^{11}(r, n) \mathfrak{P}_{m n}+\mathfrak{E}_{21}^{11}(r, n) \sqrt{n(n+1)} \mathfrak{B}_{m n}\right]\right. \\
& +A_{m n}^{\psi} \mathfrak{E}_{21}^{21}(r, n) \sqrt{n(n+1)} \mathfrak{C}_{m n} \\
& \left.+A_{m n}^{\chi}\left[\mathfrak{E}_{11}^{31}(r, n) \mathfrak{P}_{m n}+\mathfrak{E}_{21}^{31}(r, n) \sqrt{n(n+1)} \mathfrak{B}_{m n}\right]\right\} \\
\mathbf{u}^{\text {sct }}=\sum_{n=0}^{\infty} \sum_{m=-n}^{n}\{ & B_{m n}^{\phi}\left[\mathfrak{E}_{11}^{13}(r, n) \mathfrak{P}_{m n}+\mathfrak{E}_{21}^{13}(r, n) \sqrt{n(n+1)} \mathfrak{B}_{m n}\right] \\
& +B_{m n}^{\psi} \mathfrak{E}_{21}^{23}(r, n) \sqrt{n(n+1)} \mathfrak{C}_{m n}^{\mathrm{s}} \\
& \left.+B_{m n}^{\chi}\left[\mathfrak{E}_{11}^{33}(r, n) \mathfrak{P}_{m n}+\mathfrak{E}_{21}^{33}(r, n) \sqrt{n(n+1)} \mathfrak{B}_{m n}\right]\right\} \\
\mathbf{u}^{\text {ref }}=\sum_{n=0}^{\infty} \sum_{m=-n}^{n} & \left\{C_{m n}^{\phi}\left[\mathfrak{E}_{12}^{11}(r, n) \mathfrak{P}_{m n}+\mathfrak{E}_{22}^{11}(r, n) \sqrt{n(n+1)} \mathfrak{B}_{m n}\right]\right. \\
+ & C_{m n}^{\psi} \mathfrak{E}_{22}^{21}(r, n) \sqrt{n(n+1)} \mathfrak{C}_{m n} \\
& \left.+C_{m n}^{\chi}\left[\mathfrak{E}_{12}^{31}(r, n) \mathfrak{P}_{m n}+\mathfrak{E}_{22}^{31}(r, n) \sqrt{n(n+1)} \mathfrak{B}_{m n}\right]\right\}
\end{aligned}
$$

Applying the displacement continuity conditions at $r=a$, or eqns. (2-62a) through (2-62c), gives

$$
\begin{aligned}
& \sum_{n=0}^{\infty} \sum_{m=-n}^{n}\left\{A_{m n}^{\phi}\left[\mathfrak{E}_{11}^{11}(a, n) \mathfrak{P}_{m n}+\mathfrak{E}_{21}^{11}(a, n) \sqrt{n(n+1)} \mathfrak{B}_{m n}\right]\right. \\
&+A_{m n}^{\psi} \mathfrak{E}_{21}^{21}(a, n) \sqrt{n(n+1)} \mathfrak{C}_{m n} \\
&\left.+A_{m n}^{\chi}\left[\mathfrak{E}_{11}^{31}(a, n) \mathfrak{P}_{m n}+\mathfrak{E}_{21}^{31}(a, n) \sqrt{n(n+1)} \mathfrak{B}_{m n}\right]\right\} \\
&+\sum_{n=0}^{\infty} \sum_{m=-n}^{n}\left\{B_{m n}^{\phi}\left[\mathfrak{E}_{11}^{13}(a, n) \mathfrak{P}_{m n}+\mathfrak{E}_{21}^{13}(a, n) \sqrt{n(n+1)} \mathfrak{B}_{m n}\right]\right. \\
&+B_{m n}^{\psi} \mathfrak{E}_{21}^{23}(a, n) \sqrt{n(n+1)} \mathfrak{C}_{m n} \\
&\left.+B_{m n}^{\chi}\left[\mathfrak{E}_{11}^{33}(a, n) \mathfrak{P}_{m n}+\mathfrak{E}_{21}^{33}(a, n) \sqrt{n(n+1)} \mathfrak{B}_{m n}\right]\right\} \\
&-\sum_{n=0}^{\infty} \sum_{m=-n}^{n}\left\{C_{m n}^{\phi}\left[\mathfrak{E}_{12}^{11}(a, n) \mathfrak{P}_{m n}+\mathfrak{E}_{22}^{11}(a, n) \sqrt{n(n+1)} \mathfrak{B}_{m n}\right]\right.
\end{aligned}
$$




$$
\begin{aligned}
& +C_{m n}^{\psi} \mathfrak{E}_{22}^{21}(a, n) \sqrt{n(n+1)} \mathfrak{C}_{m n} \\
& \left.+C_{m n}^{\chi}\left[\mathfrak{E}_{12}^{31}(a, n) \mathfrak{P}_{m n}+\mathfrak{E}_{22}^{31}(a, n) \sqrt{n(n+1)} \mathfrak{B}_{m n}\right]\right\}=0
\end{aligned}
$$

Define

$$
\begin{aligned}
F_{m n}= & A_{m n}^{\phi} \mathfrak{E}_{11}^{11}(a, n)+A_{m n}^{\chi} \mathfrak{E}_{11}^{31}(a, n)+B_{m n}^{\phi} \mathfrak{E}_{11}^{13}(a, n)+B_{m n}^{\chi} \mathfrak{E}_{11}^{33}(a, n) \\
& -C_{m n}^{\phi} \mathfrak{E}_{12}^{11}(a, n)-C_{m n}^{\chi} \mathfrak{E}_{12}^{31}(a, n) \\
G_{m n}= & A_{m n}^{\phi} \mathfrak{E}_{21}^{11}(a, n)+A_{m n}^{\chi} \mathfrak{E}_{21}^{31}(a, n)+B_{m n}^{\phi} \mathfrak{E}_{21}^{13}(a, n)+B_{m n}^{\chi} \mathfrak{E}_{21}^{33}(a, n) \\
& -C_{m n}^{\phi} \mathfrak{E}_{22}^{11}(a, n)-C_{m n}^{\chi} \mathfrak{E}_{22}^{31}(a, n) \\
H_{m n}= & A_{m n}^{\psi} \mathfrak{E}_{21}^{21}(a, n)+B_{m n}^{\psi} \mathfrak{E}_{21}^{23}(a, n)-C_{m n}^{\psi} \mathfrak{E}_{22}^{21}(a, n)
\end{aligned}
$$

Then, eqn. (2-65) becomes

$$
\sum_{n=0}^{\infty} \sum_{m=-n}^{n}\left\{F_{m n} \mathfrak{P}_{m n}+G_{m n} \sqrt{n(n+1)} \mathfrak{B}_{m n}+H_{m n} \sqrt{n(n+1)} \mathfrak{C}_{m n}\right\}=0
$$

Multiply eqn. (2-67) with $\tilde{\mathfrak{P}}_{\mu \nu}$ to form a dot product, where the tilde indicates a complex conjugate, $\nu$ and $\mu$ are arbitrary integers in the range of $\nu \geq 0$ and $|\mu| \leq \nu$, and integrate the dot product over a spherical surface of unit radius. It gives

$$
\begin{aligned}
& \sum_{n=0}^{\infty} \sum_{m=-n}^{n} \int_{\Omega}\left\{F_{m n} \mathfrak{P}_{m n}+G_{m n} \sqrt{n(n+1)} \mathfrak{B}_{m n}+H_{m n} \sqrt{n(n+1)} \mathfrak{C}_{m n}\right\} \cdot \tilde{\mathfrak{P}}_{\mu \nu} d \Omega \\
& =0
\end{aligned}
$$

where $\Omega$ denotes the spherical surface of unit radius, $d \Omega=\sin \theta d \theta d \varphi$ and the integration is over the ranges $0 \leq \theta \leq \pi, 0 \leq \varphi \leq 2 \pi$. The vector spherical harmonics have properties of orthogonality as following (Appendix $G$ ):

$$
\begin{aligned}
\int_{\Omega} \mathfrak{B}_{m n} \cdot \tilde{\mathfrak{P}}_{\mu \nu} d \Omega & =\int_{\Omega} \mathfrak{C}_{m n} \cdot \tilde{\mathfrak{P}}_{\mu \nu} d \Omega=\int_{\Omega} \mathfrak{B}_{m n} \cdot \tilde{\mathfrak{C}}_{\mu \nu} d \Omega=0 \\
\int_{\Omega} \mathfrak{P}_{m n} \cdot \tilde{\mathfrak{P}}_{\mu \nu} d \Omega & =\int_{\Omega} \mathfrak{B}_{m n} \cdot \tilde{\mathfrak{B}}_{\mu \nu} d \Omega=\int_{\Omega} \mathfrak{C}_{m n} \cdot \tilde{\mathfrak{C}}_{\mu \nu} d \Omega=\frac{2}{\epsilon_{m}} \delta_{m \mu} \delta_{n \nu}
\end{aligned}
$$

where $\epsilon_{m}$ is a factor with values of $\epsilon_{0}=1, \epsilon_{m}=2(m \neq 0)$. Then, eqn. (2-68) reduces to $F_{m n}=0$ for each $m$ and $n$, or

$$
A_{m n}^{\phi} \mathfrak{E}_{11}^{11}(a, n)+A_{m n}^{\chi} \mathfrak{E}_{11}^{31}(a, n)+B_{m n}^{\phi} \mathfrak{E}_{11}^{13}(a, n)+B_{m n}^{\chi} \mathfrak{E}_{11}^{33}(a, n)
$$




$$
-C_{m n}^{\phi} \mathfrak{E}_{12}^{11}(a, n)-C_{m n}^{\chi} \mathfrak{E}_{12}^{31}(a, n)=0
$$

Multiply eqn. (2-67) with $\tilde{\mathfrak{B}}_{\mu \nu}$ to form a dot product, and follow the same procedure of finding eqn. (2-71), it can be found that

$$
\begin{gathered}
A_{m n}^{\phi} \mathfrak{E}_{21}^{11}(a, n)+A_{m n}^{\chi} \mathfrak{E}_{21}^{31}(a, n)+B_{m n}^{\phi} \mathfrak{E}_{21}^{13}(a, n)+B_{m n}^{\chi} \mathfrak{E}_{21}^{33}(a, n) \\
-C_{m n}^{\phi} \mathfrak{E}_{22}^{11}(a, n)-C_{m n}^{\chi} \mathfrak{E}_{22}^{31}(a, n)=0
\end{gathered}
$$

Multiply eqn. (2-67) with $\tilde{\mathfrak{C}}_{\mu \nu}$ to form a dot product, and follow the same procedure of finding eqn. (2-71), it can be found that

$$
A_{m n}^{\psi} \mathfrak{E}_{21}^{21}(a, n)+B_{m n}^{\psi} \mathfrak{E}_{21}^{23}(a, n)-C_{m n}^{\psi} \mathfrak{E}_{22}^{21}(a, n)=0
$$

\section{2-4.2 Stress Continuity Equations}

Following the same procedure of finding equations $(2-71),(2-72)$ and $(2-73)$, three stress continuity equations based on Eq. (2-63b) can be found:

$$
\begin{gathered}
A_{m n}^{\phi} \mathfrak{E}_{41}^{11}(a, n)+A_{m n}^{\chi} \mathfrak{E}_{41}^{31}(a, n)+B_{m n}^{\phi} \mathfrak{E}_{41}^{13}(a, n)+B_{m n}^{\chi} \mathfrak{E}_{41}^{33}(a, n) \\
-C_{m n}^{\phi} \mathfrak{E}_{42}^{11}(a, n)-C_{m n}^{\chi} \mathfrak{E}_{42}^{31}(a, n)=0 \\
A_{m n}^{\phi} \mathfrak{E}_{71}^{11}(a, n)+A_{m n}^{\chi} \mathfrak{E}_{71}^{31}(a, n)+B_{m n}^{\phi} \mathfrak{E}_{71}^{13}(a, n)+B_{m n}^{\chi} \mathfrak{E}_{71}^{33}(a, n) \\
-C_{m n}^{\phi} \mathfrak{E}_{72}^{11}(a, n)-C_{m n}^{\chi} \mathfrak{E}_{72}^{31}(a, n)=0 \\
A_{m n}^{\psi} \mathfrak{E}_{71}^{21}(a, n)+B_{m n}^{\psi} \mathfrak{E}_{71}^{23}(a, n)-C_{m n}^{\psi} \mathfrak{E}_{72}^{21}(a, n)=0
\end{gathered}
$$

\section{2-4.3 Solution of Continuity Equations and Mode Conver- sion}

By moving incident-wave terms to the right-hand side, displacement continuity equations (2-71) through (2-73), and stress continuity equations (2-74) through 
(2-76) can be rewritten as

$$
\begin{gathered}
B_{m n}^{\phi} \mathfrak{E}_{11}^{13}(a, n)+B_{m n}^{\chi} \mathfrak{E}_{11}^{33}(a, n)-C_{m n}^{\phi} \mathfrak{E}_{12}^{11}(a, n)-C_{m n}^{\chi} \mathfrak{E}_{12}^{31}(a, n) \\
=-A_{m n}^{\phi} \mathfrak{E}_{11}^{11}(a, n)-A_{m n}^{\chi} \mathfrak{E}_{11}^{31}(a, n) \\
B_{m n}^{\phi} \mathfrak{E}_{21}^{13}(a, n)+B_{m n}^{\chi} \mathfrak{E}_{21}^{33}(a, n)-C_{m n}^{\phi} \mathfrak{E}_{22}^{11}(a, n)-C_{m n}^{\chi} \mathfrak{E}_{22}^{31}(a, n) \\
=-A_{m n}^{\phi} \mathfrak{E}_{21}^{11}(a, n)-A_{m n}^{\chi} \mathfrak{E}_{21}^{31}(a, n) \\
B_{m n}^{\psi} \mathfrak{E}_{21}^{23}(a, n)-C_{m n}^{\psi} \mathfrak{E}_{22}^{21}(a, n)=-A_{m n}^{\psi} \mathfrak{E}_{21}^{21}(a, n) \\
B_{m n}^{\phi} \mathfrak{E}_{41}^{13}(a, n)+B_{m n}^{\chi} \mathfrak{E}_{41}^{33}(a, n)-C_{m n}^{\phi} \mathfrak{E}_{42}^{11}(a, n)-C_{m n}^{\chi} \mathfrak{E}_{42}^{31}(a, n) \\
=-A_{m n}^{\phi} \mathfrak{E}_{41}^{11}(a, n)-A_{m n}^{\chi} \mathfrak{E}_{41}^{31}(a, n) \\
B_{m n}^{\phi} \mathfrak{E}_{71}^{13}(a, n)+B_{m n}^{\chi} \mathfrak{E}_{71}^{33}(a, n)-C_{m n}^{\phi} \mathfrak{E}_{72}^{11}(a, n)-C_{m n}^{\chi} \mathfrak{E}_{72}^{31}(a, n) \\
=-A_{m n}^{\phi} \mathfrak{E}_{71}^{11}(a, n)-A_{m n}^{\chi} \mathfrak{E}_{71}^{31}(a, n) \\
B_{m n}^{\psi} \mathfrak{E}_{71}^{23}(a, n)-C_{m n}^{\psi} \mathfrak{E}_{72}^{21}(a, n)=-A_{m n}^{\psi} \mathfrak{E}_{71}^{21}(a, n)
\end{gathered}
$$

Values of functions $\mathfrak{E}_{4 q}^{s t}(a, n)$ and $\mathfrak{E}_{7 q}^{s t}(a, n)$ might be dramatically different from those of $\mathfrak{E}_{1 q}^{s t}(a, n)$ and $\mathfrak{E}_{2 q}^{s t}(a, n)$. The difference comes from the fact that shear modulus $\mu$ of either the host medium or the scatterer is involved in functions $\mathfrak{E}_{4 q}^{s t}(a, n)$ and $\mathfrak{E}_{7 q}^{s t}(a, n)$, which appears in Eqs. $(\mathrm{E}-1 \mathrm{j})$ through $(\mathrm{E}-1 \mathrm{r})$; but it is not involved in functions $\mathfrak{E}_{1 q}^{s t}(a, n)$ and $\mathfrak{E}_{2 q}^{s t}(a, n)$, which appears in Eqs. $(\mathrm{E}-1 \mathrm{a})$ through $(\mathrm{E}-1 \mathrm{i})$. In order to reduce the difference, left hand side and right hand side of eqns. (2-77d) through (2-77f) are divided by $\mu$ of the host medium. Then, Eq. $(2-77)$ can be written in 
matrix form as follows

$$
\begin{aligned}
& {\left[\begin{array}{cccccc}
\mathfrak{E}_{11}^{13}(a, n) & 0 & \mathfrak{E}_{11}^{33}(a, n) & -\mathfrak{E}_{12}^{11}(a, n) & 0 & -\mathfrak{E}_{12}^{31}(a, n) \\
0 & \mathfrak{E}_{21}^{23}(a, n) & 0 & 0 & -\mathfrak{E}_{22}^{21}(a, n) & 0 \\
\mathfrak{E}_{21}^{13}(a, n) & 0 & \mathfrak{E}_{21}^{33}(a, n) & -\mathfrak{E}_{22}^{11}(a, n) & 0 & -\mathfrak{E}_{22}^{31}(a, n) \\
\mathfrak{E}_{41}^{13}(a, n) / \mu & 0 & \mathfrak{E}_{41}^{33}(a, n) / \mu-\mathfrak{E}_{42}^{11}(a, n) / \mu & 0 & -\mathfrak{E}_{42}^{31}(a, n) / \mu \\
0 & \mathfrak{E}_{71}^{23}(a, n) / \mu & 0 & 0 & -\mathfrak{E}_{72}^{21}(a, n) / \mu & 0 \\
\mathfrak{E}_{71}^{13}(a, n) / \mu & 0 & \mathfrak{E}_{71}^{33}(a, n) / \mu-\mathfrak{E}_{72}^{11}(a, n) / \mu & 0 & -\mathfrak{E}_{72}^{31}(a, n) / \mu
\end{array}\right]} \\
& \left\{\begin{array}{c}
B_{m n}^{\phi} \\
B_{m n}^{\psi} \\
B_{m n}^{\chi} \\
C_{m n}^{\phi} \\
C_{m n}^{\psi} \\
C_{m n}^{\chi}
\end{array}\right\}=\left[\begin{array}{ccc}
-\mathfrak{E}_{11}^{11}(a, n) & 0 & -\mathfrak{E}_{11}^{31}(a, n) \\
0 & -\mathfrak{E}_{21}^{21}(a, n) & 0 \\
-\mathfrak{E}_{21}^{11}(a, n) & 0 & -\mathfrak{E}_{21}^{31}(a, n) \\
-\mathfrak{E}_{41}^{11}(a, n) / \mu & 0 & -\mathfrak{E}_{41}^{31}(a, n) / \mu \\
0 & -\mathfrak{E}_{71}^{21}(a, n) / \mu & 0 \\
-\mathfrak{E}_{71}^{11}(a, n) / \mu & 0 & -\mathfrak{E}_{71}^{31}(a, n) / \mu
\end{array}\right]\left\{\begin{array}{c}
A_{m n}^{\phi} \\
A_{m n}^{\psi} \\
A_{m n}^{\chi}
\end{array}\right\}
\end{aligned}
$$

For notational simplicity, define

$$
\left\{\boldsymbol{A}_{m n}\right\}=\left\{\begin{array}{c}
A_{m n}^{\phi} \\
A_{m n}^{\psi} \\
A_{m n}^{\chi}
\end{array}\right\},\left\{\boldsymbol{B}_{m n}\right\}=\left\{\begin{array}{c}
B_{m n}^{\phi} \\
B_{m n}^{\psi} \\
B_{m n}^{\chi}
\end{array}\right\},\left\{\boldsymbol{C}_{m n}\right\}=\left\{\begin{array}{l}
C_{m n}^{\phi} \\
C_{m n}^{\psi} \\
C_{m n}^{\chi}
\end{array}\right\} ;
$$

define two $3 \times 3$ matrices $\left[\boldsymbol{T}_{m n}\right]$ and $\left[\boldsymbol{R}_{m n}\right]$ such that

$$
\begin{array}{r}
\left\{\boldsymbol{B}_{m n}\right\}=\left[\boldsymbol{T}_{m n}\right]\left\{\boldsymbol{A}_{m n}\right\}, \\
\left\{\boldsymbol{C}_{m n}\right\}=\left[\boldsymbol{R}_{m n}\right]\left\{\boldsymbol{A}_{m n}\right\} ;
\end{array}
$$

and define

$$
\left[M_{L}\right]=
$$




$$
\begin{aligned}
& {\left[\begin{array}{cccccc}
\mathfrak{E}_{11}^{13}(a, n) & 0 & \mathfrak{E}_{11}^{33}(a, n) & -\mathfrak{E}_{12}^{11}(a, n) & 0 & -\mathfrak{E}_{12}^{31}(a, n) \\
0 & \mathfrak{E}_{21}^{23}(a, n) & 0 & 0 & -\mathfrak{E}_{22}^{21}(a, n) & 0 \\
\mathfrak{E}_{21}^{13}(a, n) & 0 & \mathfrak{E}_{21}^{33}(a, n) & -\mathfrak{E}_{22}^{11}(a, n) & 0 & -\mathfrak{E}_{22}^{31}(a, n) \\
\mathfrak{E}_{41}^{13}(a, n) / \mu & 0 & \mathfrak{E}_{41}^{33}(a, n) / \mu-\mathfrak{E}_{42}^{11}(a, n) / \mu & 0 & -\mathfrak{E}_{42}^{31}(a, n) / \mu \\
0 & \mathfrak{E}_{71}^{23}(a, n) / \mu & 0 & 0 & -\mathfrak{E}_{72}^{21}(a, n) / \mu & 0 \\
\mathfrak{E}_{71}^{13}(a, n) / \mu & 0 & \mathfrak{E}_{71}^{33}(a, n) / \mu-\mathfrak{E}_{72}^{11}(a, n) / \mu & 0 & -\mathfrak{E}_{72}^{31}(a, n) / \mu
\end{array}\right]} \\
& {\left[\boldsymbol{M}_{R}\right]=\left[\begin{array}{ccc}
-\mathfrak{E}_{11}^{11}(a, n) & 0 & -\mathfrak{E}_{11}^{31}(a, n) \\
0 & -\mathfrak{E}_{21}^{21}(a, n) & 0 \\
-\mathfrak{E}_{21}^{11}(a, n) & 0 & -\mathfrak{E}_{21}^{31}(a, n) \\
-\mathfrak{E}_{41}^{11}(a, n) / \mu & 0 & -\mathfrak{E}_{41}^{31}(a, n) / \mu \\
0 & -\mathfrak{E}_{71}^{21}(a, n) & 0 \\
-\mathfrak{E}_{71}^{11}(a, n) / \mu & 0 & -\mathfrak{E}_{71}^{31}(a, n) / \mu
\end{array}\right]}
\end{aligned}
$$

Then, Eq. (2-78) can be written as

$$
\left\{\begin{array}{l}
\left\{\boldsymbol{B}_{m n}\right\} \\
\left\{\boldsymbol{C}_{m n}\right\}
\end{array}\right\}=\left[\boldsymbol{M}_{L}\right]^{-1}\left[\boldsymbol{M}_{R}\right]\left\{\boldsymbol{A}_{m n}\right\}
$$

where $\left[\boldsymbol{M}_{L}\right]^{-1}\left[\boldsymbol{M}_{R}\right]$ is a $6 \times 3$ matrix. Comparing Eqs. (2-80) with (2-82) leads to

$$
\left[\begin{array}{c}
{\left[\boldsymbol{T}_{m n}\right]} \\
{\left[\boldsymbol{R}_{m n}\right]}
\end{array}\right]=\left[\boldsymbol{M}_{L}\right]^{-1}\left[\boldsymbol{M}_{R}\right]
$$

The entries of $\left[\boldsymbol{T}_{m n}\right]$ and $\left[\boldsymbol{R}_{m n}\right]$ are obtained using Eq. (2-83). 
Matrix equation (2-78) can be re-arranged as

$$
\begin{aligned}
& {\left[\begin{array}{cccccc}
\mathfrak{E}_{11}^{13}(a, n) & \mathfrak{E}_{11}^{33}(a, n) & -\mathfrak{E}_{12}^{11}(a, n) & -\mathfrak{E}_{12}^{31}(a, n) & 0 & 0 \\
\mathfrak{E}_{21}^{13}(a, n) & \mathfrak{E}_{21}^{33}(a, n) & -\mathfrak{E}_{22}^{11}(a, n) & -\mathfrak{E}_{22}^{31}(a, n) & 0 & 0 \\
\mathfrak{E}_{41}^{13}(a, n) / \mu & \mathfrak{E}_{41}^{33}(a, n) / \mu & -\mathfrak{E}_{42}^{11}(a, n) / \mu & -\mathfrak{E}_{42}^{31}(a, n) / \mu & 0 & 0 \\
\mathfrak{E}_{71}^{13}(a, n) / \mu & \mathfrak{E}_{71}^{33}(a, n) / \mu & -\mathfrak{E}_{72}^{11}(a, n) / \mu & -\mathfrak{E}_{72}^{31}(a, n) / \mu & 0 & 0 \\
0 & 0 & 0 & 0 & \mathfrak{E}_{21}^{23}(a, n) & -\mathfrak{E}_{22}^{21}(a, n) \\
0 & 0 & 0 & 0 & \mathfrak{E}_{71}^{23}(a, n) / \mu-\mathfrak{E}_{72}^{21}(a, n) / \mu
\end{array}\right]} \\
& \left\{\begin{array}{c}
B_{m n}^{\phi} \\
B_{m n}^{\chi} \\
C_{m n}^{\phi} \\
C_{m n}^{\chi} \\
B_{m n}^{\psi} \\
C_{m n}^{\psi}
\end{array}\right\}=\left[\begin{array}{ccc}
-\mathfrak{E}_{11}^{11}(a, n) & -\mathfrak{E}_{11}^{31}(a, n) & 0 \\
-\mathfrak{E}_{21}^{11}(a, n) & -\mathfrak{E}_{21}^{31}(a, n) & 0 \\
-\mathfrak{E}_{41}^{11}(a, n) / \mu-\mathfrak{E}_{41}^{31}(a, n) / \mu & \\
-\mathfrak{E}_{71}^{11}(a, n) / \mu-\mathfrak{E}_{71}^{31}(a, n) / \mu & 0 \\
0 & 0 & -\mathfrak{E}_{21}^{21}(a, n) \\
0 & 0 & -\mathfrak{E}_{71}^{21}(a, n) / \mu
\end{array}\right]\left\{\begin{array}{c}
A_{m n}^{\phi} \\
A_{m n}^{\chi} \\
A_{m n}^{\psi}
\end{array}\right\}
\end{aligned}
$$

Matrix equation (2-84) can be split into two matrix equations as the following:

$$
\begin{aligned}
& {\left[\begin{array}{cccc}
\mathfrak{E}_{11}^{13}(a, n) & \mathfrak{E}_{11}^{33}(a, n) & -\mathfrak{E}_{12}^{11}(a, n) & -\mathfrak{E}_{12}^{31}(a, n) \\
\mathfrak{E}_{21}^{13}(a, n) & \mathfrak{E}_{21}^{33}(a, n) & -\mathfrak{E}_{22}^{11}(a, n) & -\mathfrak{E}_{22}^{31}(a, n) \\
\mathfrak{E}_{41}^{13}(a, n) / \mu & \mathfrak{E}_{41}^{33}(a, n) / \mu-\mathfrak{E}_{42}^{11}(a, n) / \mu & -\mathfrak{E}_{42}^{31}(a, n) / \mu \\
\mathfrak{E}_{71}^{13}(a, n) / \mu & \mathfrak{E}_{71}^{33}(a, n) / \mu-\mathfrak{E}_{72}^{11}(a, n) / \mu & -\mathfrak{E}_{72}^{31}(a, n) / \mu
\end{array}\right]\left\{\begin{array}{c}
B_{m n}^{\phi} \\
B_{m n}^{\chi} \\
C_{m n}^{\phi} \\
C_{m n}^{\chi}
\end{array}\right\}} \\
& =\left[\begin{array}{cc}
-\mathfrak{E}_{11}^{11}(a, n) & -\mathfrak{E}_{11}^{31}(a, n) \\
-\mathfrak{E}_{21}^{11}(a, n) & -\mathfrak{E}_{21}^{31}(a, n) \\
-\mathfrak{E}_{41}^{11}(a, n) / \mu & -\mathfrak{E}_{41}^{31}(a, n) / \mu \\
-\mathfrak{E}_{71}^{11}(a, n) / \mu & -\mathfrak{E}_{71}^{31}(a, n) / \mu
\end{array}\right]\left\{\begin{array}{c}
A_{m n}^{\phi} \\
A_{m n}^{\chi}
\end{array}\right\} \\
& {\left[\begin{array}{cr}
\mathfrak{E}_{21}^{23}(a, n) & -\mathfrak{E}_{22}^{21}(a, n) \\
\mathfrak{E}_{71}^{23}(a, n) / \mu & -\mathfrak{E}_{72}^{21}(a, n) / \mu
\end{array}\right]\left\{\begin{array}{c}
B_{m n}^{\psi} \\
C_{m n}^{\psi}
\end{array}\right\}=\left[\begin{array}{c}
-\mathfrak{E}_{21}^{21}(a, n) \\
-\mathfrak{E}_{71}^{21}(a, n) / \mu
\end{array}\right] A_{m n}^{\psi}}
\end{aligned}
$$

Comparison of Eqs. (2-85a) and (2-85b) shows that a scattered $\phi$ wave can be 
generated from an incident $\chi$ wave; a scattered $\chi$ wave can be generated from an incident $\phi$ wave, which is known as mode conversion ${ }^{[36]}$; and there is no mode conversion between a $\psi$ wave and other waves in the single scattering solution.

A single scattering solution can be obtained by solving eqn. (2-78) or by solving eqns. (2-85a) and (2-85b). When $A_{m n}^{\phi}$ and $A_{m n}^{\chi}$ are zero, a single scattering solution can be obtained by solving eqn. (2-85b) rather than solving eqn. (2-78). When $A_{m n}^{\psi}$ is zero, a single scattering solution can be obtained by solving eqn. (2-85a) rather than solving eqn. (2-78).

When $\left\{\mathbf{B}_{m n}\right\}$ is obtained, scattered waves are known. The total wave fields outside the scatterer are the summation of incident and scattered wave fields in an ideally linear system. Total wave fields can be written as

$$
\begin{aligned}
& \phi^{\text {total }}=\phi^{\text {inc }}+\phi^{\text {sct }} \\
& \psi^{\text {total }}=\psi^{\text {inc }}+\psi^{\text {sct }} \\
& \chi^{\text {total }}=\chi^{\text {inc }}+\chi^{\text {sct }} \\
& u_{i}^{\text {total }}=u_{i}^{\text {inc }}+u_{i}^{\text {sct }} \\
& \sigma_{i j}^{\text {total }}=\sigma_{i j}^{\text {inc }}+\sigma_{i j}^{\text {sct }}
\end{aligned}
$$




\section{2-4.4 T-Matrix for Single Spherical Scatterer}

Define the following singular wave expansion basis in the form of column matrix

$$
\{\boldsymbol{h}(k ; \boldsymbol{r})\}=\left\{\begin{array}{c}
h_{0}^{(1)}(k r) Y_{0}^{0}(\theta, \varphi) \\
h_{1}^{(1)}(k r) Y_{1}^{-1}(\theta, \varphi) \\
h_{1}^{(1)}(k r) Y_{1}^{0}(\theta, \varphi) \\
h_{1}^{(1)}(k r) Y_{1}^{1}(\theta, \varphi) \\
h_{2}^{(1)}(k r) Y_{2}^{-2}(\theta, \varphi) \\
h_{2}^{(1)}(k r) Y_{2}^{-1}(\theta, \varphi) \\
\vdots
\end{array}\right\}
$$

That is, the entry at the $i$-th row is

$$
\{h(k ; \boldsymbol{r})\}_{i}=h_{n}^{(1)}(k r) Y_{n}^{m}(\theta, \varphi)
$$

where

$$
i=n^{2}+n+m \quad \Longleftrightarrow \quad n=[\sqrt{i}], m=i-n(n+1)
$$

and the square bracket represents the largest integer inside the bracket. It is noted that this is a particular way of packing a set of wave expansion basis functions with two indices (order $n$ and degree $m$ ) into a column matrix with a single index $i$. Note that $n$ runs from 0 to $\infty$, and $m$ runs from $-n$ to $n$.

The corresponding regular wave expansion basis can be denoted as

$$
\{\boldsymbol{j}(k ; \boldsymbol{r})\}=\{\operatorname{Rg} \boldsymbol{h}(k ; \boldsymbol{r})\}
$$

and is obtained by replacing the spherical Hankel function of the first kind $h_{n}(z)$ by the spherical Bessel function of the first kind $j_{n}(z)$. That is

$$
\{j(k ; \boldsymbol{r})\}_{i}=j_{n}(k r) Y_{n}^{m}(\theta, \varphi)
$$

By using the notation in Eq. (2-90), Eq. (2-59) for the incident waves can be 
rewritten as

$$
\begin{aligned}
\phi^{\text {inc }} & =\left\{\boldsymbol{A}^{\phi}\right\}^{T}\{\boldsymbol{j}(\kappa, \boldsymbol{r})\} \\
\psi^{\text {inc }} & =\left\{\boldsymbol{A}^{\psi}\right\}^{T}\{\boldsymbol{j}(k, \boldsymbol{r})\} \\
\chi^{\mathrm{inc}} & =\left\{\boldsymbol{A}^{\chi}\right\}^{T}\{\boldsymbol{j}(k, \boldsymbol{r})\}
\end{aligned}
$$

where $\left\{\boldsymbol{A}^{\phi}\right\},\left\{\boldsymbol{A}^{\psi}\right\}$ and $\left\{\boldsymbol{A}^{\chi}\right\}$ are the wave expansion coefficient (column) matrices for the three waves.

$$
\left\{\boldsymbol{A}^{\phi}\right\}_{i}=A_{m n}^{\phi}, \quad\left\{\boldsymbol{A}^{\psi}\right\}_{i}=A_{m n}^{\psi}, \quad \text { and } \quad\left\{\boldsymbol{A}^{\chi}\right\}_{i}=A_{m n}^{\chi}
$$

By using the notation in Eq. (2-87), Eq. (2-60) for the scattered waves can be rewritten as

$$
\begin{aligned}
& \phi^{\text {sct }}=\left\{\boldsymbol{B}^{\phi}\right\}^{T}\{\boldsymbol{h}(\kappa, \boldsymbol{r})\} \\
& \psi^{\text {sct }}=\left\{\boldsymbol{B}^{\psi}\right\}^{T}\{\boldsymbol{h}(k, \boldsymbol{r})\} \\
& \chi^{\text {sct }}=\left\{\boldsymbol{B}^{\chi}\right\}^{T}\{\boldsymbol{h}(k, \boldsymbol{r})\}
\end{aligned}
$$

where

$$
\left\{\boldsymbol{B}^{\phi}\right\}_{i}=B_{m n}^{\phi}, \quad\left\{\boldsymbol{B}^{\psi}\right\}_{i}=B_{m n}^{\psi}, \quad \text { and } \quad\left\{\boldsymbol{B}^{\chi}\right\}_{i}=B_{m n}^{\chi}
$$

Also, Eq. (2-61) for the refracted waves can be rewritten as

$$
\begin{aligned}
\phi^{\mathrm{ref}} & =\left\{\boldsymbol{C}^{\phi}\right\}^{T}\{\boldsymbol{j}(\kappa, \boldsymbol{r})\} \\
\psi^{\mathrm{ref}} & =\left\{\boldsymbol{C}^{\psi}\right\}^{T}\{\boldsymbol{j}(k, \boldsymbol{r})\} \\
\chi^{\mathrm{ref}} & =\left\{\boldsymbol{C}^{\chi}\right\}^{T}\{\boldsymbol{j}(k, \boldsymbol{r})\}
\end{aligned}
$$

where

$$
\left\{\boldsymbol{C}^{\phi}\right\}_{i}=C_{m n}^{\phi}, \quad\left\{\boldsymbol{C}^{\psi}\right\}_{i}=C_{m n}^{\psi}, \quad \text { and } \quad\left\{\boldsymbol{C}^{\chi}\right\}_{i}=C_{m n}^{\chi}
$$

With matrix notation, the general solution for a scattering problem is notationally simplified. The simplification amplifies the solution structure and the physical process (matrix manipulations have very intuitive meaning in a physical process). 
Because the system is linear, the scattered wave is related to the incident wave via a linear transformation matrix, which is conventionally called the $T$-matrix ${ }^{[19]}$. Equation (2-80a)suggests the following relation

$$
\begin{aligned}
\left\{\boldsymbol{B}^{\phi}\right\} & =\left[\boldsymbol{T}^{\phi \phi}\right]\left\{\boldsymbol{A}^{\phi}\right\}+\left[\boldsymbol{T}^{\phi \psi}\right]\left\{\boldsymbol{A}^{\psi}\right\}+\left[\boldsymbol{T}^{\phi \chi}\right]\left\{\boldsymbol{A}^{\chi}\right\} \\
\left\{\boldsymbol{B}^{\psi}\right\} & =\left[\boldsymbol{T}^{\psi \phi}\right]\left\{\boldsymbol{A}^{\phi}\right\}+\left[\boldsymbol{T}^{\psi \psi}\right]\left\{\boldsymbol{A}^{\psi}\right\}+\left[\boldsymbol{T}^{\psi \chi}\right]\left\{\boldsymbol{A}^{\chi}\right\} \\
\left\{\boldsymbol{B}^{\chi}\right\} & =\left[\boldsymbol{T}^{\chi \phi}\right]\left\{\boldsymbol{A}^{\phi}\right\}+\left[\boldsymbol{T}^{\chi \psi}\right]\left\{\boldsymbol{A}^{\psi}\right\}+\left[\boldsymbol{T}^{\chi \chi}\right]\left\{\boldsymbol{A}^{\chi}\right\}
\end{aligned}
$$

where, in the above $T$-matrices such as $\left[\boldsymbol{T}^{\chi \phi}\right]$, the first superscript represent the type of the scattered wave and the second superscript represents the type of the incident wave. Entries for individual $T$-matrices can be obtained using Eq. (2-83). Since there is no mode conversion between a $\psi$ wave and other waves, $\left[\boldsymbol{T}^{\phi \psi}\right],\left[\boldsymbol{T}^{\chi \psi}\right],\left[\boldsymbol{T}^{\psi \phi}\right]$ and $\left[\boldsymbol{T}^{\psi \chi}\right]$ are zero matrices:

$$
\left[\boldsymbol{T}^{\phi \psi}\right]=\left[\boldsymbol{T}^{\chi \psi}\right]=\left[\boldsymbol{T}^{\psi \phi}\right]=\left[\boldsymbol{T}^{\psi \chi}\right]=0
$$

For a more compact notation, define the following super-matrices

$$
\{\mathbb{A}\}=\left\{\begin{array}{c}
\left\{\boldsymbol{A}^{\phi}\right\} \\
\left\{\boldsymbol{A}^{\psi}\right\} \\
\left\{\boldsymbol{A}^{\chi}\right\}
\end{array}\right\} \text { and }\{\mathbb{B}\}=\left\{\begin{array}{c}
\left\{\boldsymbol{B}^{\phi}\right\} \\
\left\{\boldsymbol{B}^{\psi}\right\} \\
\left\{\boldsymbol{B}^{\chi}\right\}
\end{array}\right\}
$$

Then

$$
\{\mathbb{B}\}=[\mathbb{T}]\{\mathbb{A}\}
$$

where $[\mathbb{T}]$ is the super $T$-matrix for the scatterer,

$$
[\mathbb{T}]=\left[\begin{array}{ccc}
{\left[\boldsymbol{T}^{\phi \phi}\right]} & {\left[\boldsymbol{T}^{\phi \psi}\right]} & {\left[\boldsymbol{T}^{\phi \chi}\right]} \\
{\left[\boldsymbol{T}^{\psi \phi}\right]} & {\left[\boldsymbol{T}^{\psi \psi}\right]} & {\left[\boldsymbol{T}^{\psi \chi}\right]} \\
{\left[\boldsymbol{T}^{\chi \phi}\right]} & {\left[\boldsymbol{T}^{\chi \psi}\right]} & {\left[\boldsymbol{T}^{\chi \chi}\right]}
\end{array}\right] .
$$


Similarly, the following super matrices are defined

$$
\{\mathbb{C}\}=\left\{\begin{array}{c}
\left\{\boldsymbol{C}^{\phi}\right\} \\
\left\{\boldsymbol{C}^{\psi}\right\} \\
\left\{\boldsymbol{C}^{\chi}\right\}
\end{array}\right\}
$$

so that,

$$
\{\mathbb{C}\}=[\mathbb{R}]\{\mathbb{A}\}
$$

where

$$
[\mathbb{R}]=\left[\begin{array}{ccc}
{\left[\boldsymbol{R}^{\phi \phi}\right]} & {\left[\boldsymbol{R}^{\phi \psi}\right]} & {\left[\boldsymbol{R}^{\phi \chi}\right]} \\
{\left[\boldsymbol{R}^{\psi \phi}\right]} & {\left[\boldsymbol{R}^{\psi \psi}\right]} & {\left[\boldsymbol{R}^{\psi \chi}\right]} \\
{\left[\boldsymbol{R}^{\chi \phi}\right]} & {\left[\boldsymbol{R}^{\chi \psi}\right]} & {\left[\boldsymbol{R}^{\chi \chi}\right]}
\end{array}\right]
$$

Entries for individual $R$-matrices such as $\left[\boldsymbol{R}^{\phi \chi}\right]$ can be obtained using Eq. (2-83). Since there is no mode conversion between a $\psi$ wave and other waves, matrices $\left[\boldsymbol{R}^{\phi \psi}\right]$, $\left[\boldsymbol{R}^{\chi \psi}\right],\left[\boldsymbol{R}^{\psi \phi}\right]$ and $\left[\boldsymbol{R}^{\psi \chi}\right]$ are zero matrices:

$$
\left[\boldsymbol{R}^{\phi \psi}\right]=\left[\boldsymbol{R}^{\chi \psi}\right]=\left[\boldsymbol{R}^{\psi \phi}\right]=\left[\boldsymbol{R}^{\psi \chi}\right]=0
$$

Furthermore, the refracted wave inside the scatterer and the scattered wave can be related by

$$
\{\mathbb{C}\}=[\mathbb{G}]\{\mathbb{B}\}
$$

where

$$
[\mathbb{G}]=[\mathbb{R}][\mathbb{T}]^{-1} .
$$

\section{2-4.5 Displacements and Stresses}

Displacements and stresses of scattered waves and refracted waves in a spherical coordinate system are expressed in terms of wave expansion in this section. Assume wave expansion coefficients $\{\mathbb{B}\}$ and $\{\mathbb{C}\}$ are known. According to Eqs. (2-45) and 
(2-46), displacements and stresses of scattered waves can be written as

$$
\begin{aligned}
u_{r}^{s c t} & =\sum_{n=0}^{\infty} \sum_{m=-n}^{n}\left[B_{m n}^{\phi} \mathfrak{E}_{11}^{13}(r, n)+B_{m n}^{\chi} \mathfrak{E}_{11}^{33}(r, n)\right] Y_{n}^{m} \\
u_{\theta}^{s c t} & =\sum_{n=0}^{\infty} \sum_{m=-n}^{n}\left\{\left[B_{m n}^{\phi} \mathfrak{E}_{21}^{13}(r, n)+B_{m n}^{\chi} \mathfrak{E}_{21}^{33}(r, n)\right]\left(Y_{, \theta}\right)_{n}^{m}+B_{m n}^{\psi} \mathfrak{E}_{21}^{23}(r, n)\left(Y_{, \varphi}\right)_{n}^{m}\right\} \\
u_{\varphi}^{s c t} & =\sum_{n=0}^{\infty} \sum_{m=-n}^{n}\left\{\left[B_{m n}^{\phi} \mathfrak{E}_{31}^{13}(r, n)+B_{m n}^{\chi} \mathfrak{E}_{31}^{33}(r, n)\right]\left(Y_{, \varphi}\right)_{n}^{m}+B_{m n}^{\psi} \mathfrak{E}_{31}^{23}(r, n)\left(Y_{, \theta}\right)_{n}^{m}\right\} \\
\sigma_{r r}^{s c t} & =\sum_{n=0}^{\infty} \sum_{m=-n}^{n}\left\{B_{m n}^{\phi} \mathfrak{E}_{41}^{13}(r, n)+B_{m n}^{\chi} \mathfrak{E}_{41}^{33}(r, n)\right\} Y_{n}^{m} \\
\sigma_{r \theta}^{s c t} & =\sum_{n=0}^{\infty} \sum_{m=-n}^{n}\left\{\left[B_{m n}^{\phi} \mathfrak{E}_{71}^{13}(r, n)+B_{m n}^{\chi} \mathfrak{E}_{71}^{33}(r, n)\right]\left(Y_{, \theta}\right)_{n}^{m}+B_{m n}^{\psi} \mathfrak{E}_{71}^{23}(r, n)\left(Y_{, \varphi}\right)_{n}^{m}\right\} \\
\sigma_{r \varphi}^{s c t} & =\sum_{n=0}^{\infty} \sum_{m=-n}^{n}\left\{\left[B_{m n}^{\phi} \mathfrak{E}_{81}^{13}(r, n)+B_{m n}^{\chi} \mathfrak{E}_{81}^{33}(r, n)\right]\left(Y_{, \varphi}\right)_{n}^{m}+B_{m n}^{\psi} \mathfrak{E}_{81}^{23}(r, n)\left(Y_{, \theta}\right)_{n}^{m}\right\} \\
\sigma_{\theta \theta}^{s c t} & =\sum_{n=0}^{\infty} \sum_{m=-n}^{n}\left\{B_{m n}^{\phi} \mathfrak{E}_{51}^{13}(\mathbf{r}, n)+B_{m n}^{\chi} \mathfrak{E}_{51}^{33}(\mathbf{r}, n)+B_{m n}^{\psi} \mathfrak{E}_{51}^{23}(\mathbf{r}, n)\right\} \\
\sigma_{\varphi \varphi}^{s c t} & =\sum_{n=0}^{\infty} \sum_{m=-n}^{n}\left\{B_{m n}^{\phi} \mathfrak{E}_{61}^{13}(\mathbf{r}, n)+B_{m n}^{\chi} \mathfrak{E}_{61}^{33}(\mathbf{r}, n)+B_{m n}^{\psi} \mathfrak{E}_{61}^{23}(\mathbf{r}, n)\right\} \\
\sigma_{\theta \varphi}^{s c t}= & \sum_{n=0}^{\infty} \sum_{m=-n}^{n}\left\{B_{m n}^{\phi} \mathfrak{E}_{91}^{13}(\mathbf{r}, n)+B_{m n}^{\chi} \mathfrak{E}_{91}^{33}(\mathbf{r}, n)+B_{m n}^{\psi} \mathfrak{E}_{91}^{23}(\mathbf{r}, n)\right\}
\end{aligned}
$$

and displacements and stresses of refracted waves can be written as

$$
\begin{aligned}
u_{r}^{r e f} & =\sum_{n=0}^{\infty} \sum_{m=-n}^{n}\left[C_{m n}^{\phi} \mathfrak{E}_{12}^{11}(r, n)+C_{m n}^{\chi} \mathfrak{E}_{12}^{31}(r, n)\right] Y_{n}^{m} \\
u_{\theta}^{r e f} & =\sum_{n=0}^{\infty} \sum_{m=-n}^{n}\left\{\left[C_{m n}^{\phi} \mathfrak{E}_{22}^{11}(r, n)+C_{m n}^{\chi} \mathfrak{E}_{22}^{31}(r, n)\right]\left(Y_{, \theta}\right)_{n}^{m}+C_{m n}^{\psi} \mathfrak{E}_{22}^{21}(r, n)\left(Y_{, \varphi}\right)_{n}^{m}\right\} \\
u_{\varphi}^{r e f} & =\sum_{n=0}^{\infty} \sum_{m=-n}^{n}\left\{\left[C_{m n}^{\phi} \mathfrak{E}_{32}^{11}(r, n)+C_{m n}^{\chi} \mathfrak{E}_{32}^{31}(r, n)\right]\left(Y_{, \varphi}\right)_{n}^{m}+C_{m n}^{\psi} \mathfrak{E}_{32}^{21}(r, n)\left(Y_{, \theta}\right)_{n}^{m}\right\} \\
\sigma_{r r}^{r e f} & =\sum_{n=0}^{\infty} \sum_{m=-n}^{n}\left\{C_{m n}^{\phi} \mathfrak{E}_{42}^{11}(r, n)+C_{m n}^{\chi} \mathfrak{E}_{42}^{31}(r, n)\right\} Y_{n}^{m} \\
\sigma_{r \theta}^{r e f} & =\sum_{n=0}^{\infty} \sum_{m=-n}^{n}\left\{\left[C_{m n}^{\phi} \mathfrak{E}_{72}^{11}(r, n)+C_{m n}^{\chi} \mathfrak{E}_{72}^{31}(r, n)\right]\left(Y_{, \theta}\right)_{n}^{m}+C_{m n}^{\psi} \mathfrak{E}_{72}^{21}(r, n)\left(Y_{, \varphi}\right)_{n}^{m}\right\}
\end{aligned}
$$




$$
\begin{aligned}
\sigma_{r \varphi}^{r e f} & =\sum_{n=0}^{\infty} \sum_{m=-n}^{n}\left\{\left[C_{m n}^{\phi} \mathfrak{E}_{82}^{11}(r, n)+C_{m n}^{\chi} \mathfrak{E}_{82}^{31}(r, n)\right]\left(Y_{, \varphi}\right)_{n}^{m}+C_{m n}^{\psi} \mathfrak{E}_{82}^{21}(r, n)\left(Y_{, \theta}\right)_{n}^{m}\right\} \\
\sigma_{\theta \theta}^{r e f} & =\sum_{n=0}^{\infty} \sum_{m=-n}^{n}\left\{C_{m n}^{\phi} \mathfrak{E}_{52}^{11}(\mathbf{r}, n)+C_{m n}^{\chi} \mathfrak{E}_{52}^{31}(\mathbf{r}, n)+C_{m n}^{\psi} \mathfrak{E}_{52}^{21}(\mathbf{r}, n)\right\} \\
\sigma_{\varphi \varphi}^{r e f} & =\sum_{n=0}^{\infty} \sum_{m=-n}^{n}\left\{C_{m n}^{\phi} \mathfrak{E}_{62}^{11}(\mathbf{r}, n)+C_{m n}^{\chi} \mathfrak{E}_{62}^{31}(\mathbf{r}, n)+C_{m n}^{\psi} \mathfrak{E}_{62}^{21}(\mathbf{r}, n)\right\} \\
\sigma_{\theta \varphi}^{r e f} & =\sum_{n=0}^{\infty} \sum_{m=-n}^{n}\left\{C_{m n}^{\phi} \mathfrak{E}_{92}^{11}(\mathbf{r}, n)+C_{m n}^{\chi} \mathfrak{E}_{92}^{31}(\mathbf{r}, n)+C_{m n}^{\psi} \mathfrak{E}_{92}^{21}(\mathbf{r}, n)\right\}
\end{aligned}
$$

where superscript sct and ref denote scattered and refracted waves, respectively.

\section{2-5 Energy Conservation Law}

The energy conservation law is also known as energy balance principle in a scattering problem. In scattering problems, it states that energy flux across a closed surface should be zero when there is not energy generation or dissipation inside of the surface.

\section{2-5.1 Energy Flux}

The energy flux, $\dot{E}$, is defined as rate of energy flow through a cross section $A$.

$$
\dot{E}=\int_{A}(\mathbf{t} \cdot \dot{\mathbf{u}}) d A
$$

where $\mathbf{t}$ is the surface traction and $\mathbf{u}$ is the displacement at the point where the surface traction acts. The surface traction can be written as

$$
\mathbf{t}=\sigma_{i j} n_{j}
$$

where $n_{j}$ are the components of unit normal vector of the surface. Then, the energy flux can be written as

$$
\dot{E}=\int_{A} \tau_{i j} \dot{u}_{i} n_{j} d A
$$


Since either the real or the imaginary parts of $\sigma_{i j}$ and $u_{i}$ are taken to calculate the energy flux, $\frac{1}{2}\left(\sigma_{i j}+\bar{\sigma}_{i j}\right)$ and $\frac{1}{2}\left(\dot{u}_{i}+\overline{\dot{u}}_{i}\right)$ are used instead of $\sigma_{i j}$ and $\dot{u}_{i}$ in Eq. $(2-113)$, so that

$$
\dot{E}=\frac{1}{4} \int_{A}\left(\sigma_{i j} \dot{u}_{i}+\sigma_{i j} \overline{\dot{u}}_{i}+\bar{\sigma}_{i j} \dot{u}_{i}+\bar{\sigma}_{i j} \overline{\dot{u}}_{i}\right) n_{j} d A
$$

where the overbar denotes a complex conjugate.

In the case of steady-state waves in a linear elastic field, both $\sigma_{i j}$ and $u_{i}$ are harmonic functions of time,

$$
\begin{aligned}
\sigma_{i j} & =\sigma_{i j}^{*} e^{-\hat{\imath} \omega t} \\
u_{i} & =u_{i}^{*} e^{-\hat{\imath} \omega t}
\end{aligned}
$$

where $\sigma_{i j}^{*}$ and $u_{i}^{*}$ are complex amplitude. The energy flux averaged over one period $T=2 \pi / \omega$ is the average energy flux $\langle\dot{E}\rangle$. That is

$$
\begin{aligned}
\langle\dot{E}\rangle & =\frac{1}{T} \int_{0}^{T} \dot{E} d t \\
& =\frac{1}{T} \int_{0}^{T}\left\{\frac{1}{4} \int_{A}\left(\sigma_{i j} \dot{u}_{i}+\sigma_{i j} \overline{\dot{u}}_{i}+\bar{\sigma}_{i j} \dot{u}_{i}+\bar{\sigma}_{i j} \overline{\dot{u}}_{i}\right) n_{j} d s\right\} d t
\end{aligned}
$$

Because

$$
\begin{aligned}
\frac{1}{T} \int_{0}^{T} \sigma_{i j} \dot{u}_{i} d t & =\frac{1}{T} \int_{0}^{T} \sigma_{i j}^{*} e^{-\hat{\imath} \omega t} u_{i}^{*}(-\hat{\imath} \omega) e^{-\hat{\imath} \omega t} d t \\
& =\frac{-\hat{\imath} \omega}{T} \int_{0}^{T} \sigma_{i j}^{*} u_{i}^{*} e^{-\hat{\imath} 2 \omega t} d t=0 \\
\frac{1}{T} \int_{0}^{T} \bar{\sigma}_{i j} \overline{\dot{u}}_{i} d t & =\frac{1}{T} \int_{0}^{T} \bar{\sigma}_{i j}^{*} e^{\hat{\imath} \omega t} \bar{u}_{i}^{*}(\hat{\imath} \omega) e^{\hat{\imath} \omega t} d t=\frac{\hat{\imath} \omega}{T} \int_{0}^{T} \sigma_{i j}^{*} u_{i}^{*} e^{\hat{\imath} 2 \omega t} d t=0 \\
\frac{1}{T} \int_{0}^{T} \sigma_{i j} \overline{\dot{u}}_{i} d t & =\frac{1}{T} \int_{0}^{T} \sigma_{i j}^{*} e^{-\hat{\imath} \omega t} \bar{u}_{i}^{*}(\hat{\imath} \omega) e^{\hat{\imath} \omega t} d t=\hat{\imath} \omega \sigma_{i j}^{*} \bar{u}_{i}^{*} \\
\frac{1}{T} \int_{0}^{T} \bar{\sigma}_{i j} \dot{u}_{i} d t & =\frac{1}{T} \int_{0}^{T} \sigma_{i j}^{*} e^{\hat{\omega} \omega t} u_{i}^{*}(-\hat{\imath} \omega) e^{-\hat{\imath} \omega t} d t=-\hat{\imath} \omega \bar{\sigma}_{i j}^{*} u_{i}^{*}
\end{aligned}
$$

so that

$$
\langle\dot{E}\rangle=\frac{\hat{\imath} \omega}{4} \int_{A}\left(\sigma_{i j}^{*} \bar{u}_{i}^{*}-\bar{\sigma}_{i j}^{*} u_{i}^{*}\right) n_{j} d A
$$


Define

$$
\dot{e}=\frac{\hat{\imath} \omega}{4}\left(\sigma_{i j}^{*} \bar{u}_{i}^{*}-\bar{\sigma}_{i j}^{*} u_{i}^{*}\right) n_{j}
$$

then

$$
\langle\dot{E}\rangle=\int_{A} \dot{e} d A
$$

where $\dot{e}$ is sometimes called energy flux density.

\section{2-5.2 Numerical Integration Over a Closed Spherical Surface}

For a closed surface $S_{c}$, Eq. (2-119) can be written explicitly in spherical coordinates as follows:

$$
\begin{aligned}
\langle\dot{E}\rangle= & \frac{\hat{\imath} \omega}{4} \oint_{S_{c}}\left\{\left[n_{r} \sigma_{r r}^{*}+n_{\theta} \sigma_{r \theta}^{*}+n_{\varphi} \sigma_{r \varphi}^{*}\right] \bar{u}_{r}^{*}-\left[n_{r} \bar{\sigma}_{r r}^{*}+n_{\theta} \bar{\sigma}_{r \theta}^{*}+n_{\varphi} \bar{\sigma}_{r \varphi}^{*}\right] u_{r}^{*}\right. \\
& +\left[n_{r} \sigma_{\theta r}^{*}+n_{\theta} \sigma_{\theta \theta}^{*}+n_{\varphi} \sigma_{\theta \varphi}^{*}\right] \bar{u}_{\theta}^{*}-\left[n_{r} \bar{\sigma}_{\theta r}^{*}+n_{\theta} \bar{\sigma}_{\theta \theta}^{*}+n_{\varphi} \bar{\sigma}_{\theta \varphi}^{*}\right] u_{\theta}^{*} \\
& \left.+\left[n_{r} \sigma_{\varphi r}^{*}+n_{\theta} \sigma_{\varphi \theta}^{*}+n_{\varphi} \sigma_{\varphi \varphi}^{*}\right] \bar{u}_{\varphi}^{*}-\left[n_{r} \bar{\sigma}_{\varphi r}^{*}+n_{\theta} \bar{\sigma}_{\varphi \theta}^{*}+n_{\varphi} \bar{\sigma}_{\varphi \varphi}^{*}\right] u_{\varphi}^{*}\right\} d A
\end{aligned}
$$

Since $n_{r}=1, n_{\theta}=n_{\varphi}=0$ along the radius direction, eqn. (2-122) is reduced to the following one:

$$
\begin{aligned}
\langle\dot{E}\rangle & =\frac{\hat{\imath} \omega}{4} \oint_{S_{c}}\left\{\left[\sigma_{r r}^{*} \bar{u}_{r}^{*}-\bar{\sigma}_{r r}^{*} u_{r}^{*}\right]+\left[\sigma_{\theta r}^{*} \bar{u}_{\theta}^{*}-\bar{\sigma}_{\theta r}^{*} u_{\theta}^{*}\right]+\left[\sigma_{\varphi r}^{*} \bar{u}_{\varphi}^{*}-\bar{\sigma}_{\varphi r}^{*} u_{\varphi}^{*}\right]\right\} d A \\
& =\frac{\hat{\imath} \omega}{4} \oint_{S_{c}}\left\{\left[\sigma_{r r}^{*} \bar{u}_{r}^{*}+\sigma_{\theta r}^{*} \bar{u}_{\theta}^{*}+\sigma_{\varphi r}^{*} \bar{u}_{\varphi}^{*}\right]-\left[\bar{\sigma}_{r r}^{*} u_{r}^{*}+\bar{\sigma}_{\theta r}^{*} u_{\theta}^{*}+\bar{\sigma}_{\varphi r}^{*} u_{\varphi}^{*}\right]\right\} d A
\end{aligned}
$$

where, for a spherical surface of radius $R, d A=R^{2} \sin \theta d \theta d \varphi$. Equation (2-124) can be written as

$$
\langle\dot{E}\rangle=\oint_{S_{c}} \dot{e} d A
$$

where

$$
\dot{e}=\frac{\hat{\imath} \omega}{4}\left\{\left[\sigma_{r r}^{*} \bar{u}_{r}^{*}+\sigma_{\theta r}^{*} \bar{u}_{\theta}^{*}+\sigma_{\varphi r}^{*} \bar{u}_{\varphi}^{*}\right]-\left[\bar{\sigma}_{r r}^{*} u_{r}^{*}+\bar{\sigma}_{\theta r}^{*} u_{\theta}^{*}+\bar{\sigma}_{\varphi r}^{*} u_{\varphi}^{*}\right]\right\}
$$


Since $0 \leq \theta \leq \pi$ and $0 \leq \varphi \leq 2 \pi$, a $N \times 2 N$ mesh can be created for a spherical surface with $\delta \theta=\delta \varphi=\pi / N$. The spherical surface of radius $R$ is approximated by $N \times 2 N$ small planes. Therefore, Eq. $(2-125)$ can be approximated as

$$
\langle\dot{E}\rangle=\sum_{i=0}^{N-1} \sum_{j=0}^{2 N-1} \dot{e}\left(\theta_{i}, \varphi_{j}\right) R^{2} \sin \theta_{i} \delta \theta \delta \varphi
$$

where $\theta_{i}=(i+0.5) \delta \theta, \varphi_{j}=(j+0.5) \delta \varphi, \dot{e}\left(\theta_{i}, \varphi_{j}\right)$ is the energy flux density $\dot{e}$ at position of $\theta_{i}$ and $\varphi_{j}$.

Fibonacci numerical integration formula on a sphere by Hannay and Nye ${ }^{[46]}$ can be used to reduce the time for computation. According to Hannay and Nye ${ }^{[46]}$,

$$
\begin{aligned}
\oint_{S_{c}} f(\theta, \varphi) & R^{2} \sin \theta d \theta d \varphi=\int_{-R}^{R} \int_{0}^{2 \pi} f(z, \varphi) d \varphi d z \\
= & \frac{\pi \Delta z}{R} \sum_{i=0}^{F}\left[1+\cos \left(\pi z_{i} / R\right)\right]\left\{f\left(\frac{\pi i F^{\prime}}{F}, z_{i}+\frac{\sin \left(\pi z_{i} / R\right)}{\pi / R}\right)\right. \\
& \left.+f\left(\pi+\frac{\pi i F^{\prime}}{F}, z_{i}+\frac{\sin \left(\pi z_{i} / R\right)}{\pi / R}\right)\right\}
\end{aligned}
$$

where $z=R \cos \theta, f$ is a function with $f(z, \varphi)=f(\theta, \varphi)$ when $z=R \cos \theta, F$ is one of the Fibonacci numbers $1,1,2,3,5,8,13,21,34,55,89,144, \ldots$ (in this set each number is the sum of the pair before it); $F^{\prime}$ is the next smaller number than $F$ in the set, $\Delta z=2 R / F$ and $z_{i}=-R+i \Delta z$. The coordinates $\theta_{i}$ and $\varphi_{i}$ are chosen by

$$
\begin{aligned}
\theta_{i} & =\operatorname{acos}\left(\left[z_{i}+\frac{\sin \left(\pi z_{i} / R\right)}{\pi / R}\right] / R\right), \\
\varphi_{i} & =\frac{\pi i F^{\prime}}{F}
\end{aligned}
$$

According to Eq. (2-128), Eq. (2-124) can be written as

$$
\langle\dot{E}\rangle=\frac{-\hat{\imath} \omega}{4} \frac{\pi \Delta z}{R} \sum_{i=0}^{F}\left[1+\cos \left(\pi z_{i} / R\right)\right]\left\{\dot{e}\left(\varphi_{i}, \theta_{i}\right)+\dot{e}\left(\pi+\varphi_{i}, \theta_{i}\right)\right\}
$$

According to Purser and Swinbank ${ }^{[47]}$, the Fibonacci grids "possess virtually uniform and highly isotropic resolution, with an equal area for each grid point." The error using integration formula in Eq. $(2-128)$ goes as $(2 F)^{-3}[46]$. 


\section{2-6 Numerical Examples of Single Scattering So- lution}

The single scattering solution is implemented for the case of an elastic spherical scatterer in an infinite elastic host material. The scatterer can resemble a void inhomogeneity when its mechanical properties take near-zero values, and it can also resemble a rigid inclusion when its mechanical properties take extremely large values. In this section, computations are performed for the case of a beryllium sphere in a polyethylene matrix at room temperature.

The reason for selecting a beryllium sphere and a polyethylene matrix is to re-

produce the result of scattering cross section by Johnson and Truell ${ }^{[1]}$. The material properties of both materials are listed in Table 2.1 .

The sphere radius $a$ is a characteristic length of the problem. Normalized wave number $\kappa a$ is used in the numerical examples, since a particular value of radius $a$ is not important. However, it must be noted that this is not intended to be a comprehensive numerical analysis. It provides a glimpse into the main characteristics of single spherical scatterer models.

Table 2.1: Material Properties of Beryllium and Polyethylene (after [1])

\begin{tabular}{|c|c|c|}
\hline Property & Matrix (Polyethylene) & Sphere (Beryllium) \\
\hline Lamé constant $\lambda(\mathrm{GPa})$ & 2.897 & 15.789 \\
Lamé constant $\mu(\mathrm{GPa})$ & 0.262 & 147.458 \\
Density $\left(\mathrm{kg} / \mathrm{m}^{3}\right)$ & 900. & 1870. \\
P Wave Speed $(\mathrm{km} / \mathrm{s})$ & 1.95 & 12.89 \\
S Wave Speed $(\mathrm{km} / \mathrm{s})$ & 0.540 & 8.88 \\
\hline
\end{tabular}




\section{2-6.1 Plane Incident Wave}

A plane longitudinal incident wave is used for all numerical computation throughout this thesis. It can be represented by displacement potentials:

$$
\phi^{i n c}=\frac{\hat{\imath}}{\kappa} e^{-\hat{\imath} \kappa r \cos (\theta)}=\frac{\hat{\imath}}{\kappa} e^{-\hat{\imath} \kappa z}, \quad \psi^{i n c}=\chi^{i n c}=0 .
$$

Wave field information of the incident waves are summarized here for later use. The displacement potential $\phi$ can be expanded as ${ }^{[48]}$

$$
\phi^{i n c}=\sum_{n=0}^{\infty} \frac{(-\hat{\imath})^{n}}{\kappa} \sqrt{4 \pi(2 n+1)} j_{n}(\kappa r) Y_{n}^{0}(\theta, \varphi)
$$

The displacement components $u_{r}, u_{\theta}$ and $u_{\varphi}$ in Eq. (2-39) are repeated here

$$
\begin{aligned}
& u_{r}=\frac{\partial \phi}{\partial r}+\ell\left[\frac{\partial^{2}(r \chi)}{\partial r^{2}}-r \nabla^{2} \chi\right] \\
& u_{\theta}=\frac{1}{r} \frac{\partial \phi}{\partial \theta}+\frac{1}{r \sin \theta} \frac{\partial(r \psi)}{\partial \varphi}+\frac{\ell}{r} \frac{\partial^{2}(r \chi)}{\partial \theta \partial r} \\
& u_{\varphi}=\frac{1}{r \sin \theta} \frac{\partial \phi}{\partial \varphi}-\frac{1}{r} \frac{\partial(r \psi)}{\partial \theta}+\frac{\ell}{r \sin \theta} \frac{\partial^{2}(r \chi)}{\partial \varphi \partial r}
\end{aligned}
$$

In a spherical coordinate system the strain-displacement relations in Eq. (2-40) are repeated here

$$
\begin{aligned}
\varepsilon_{r r} & =\frac{\partial u_{r}}{\partial r} \\
\varepsilon_{r \theta} & =\frac{1}{2}\left[\frac{1}{r} \frac{\partial u_{r}}{\partial \theta}-\frac{u_{\theta}}{r}+\frac{\partial u_{\theta}}{\partial r}\right] \\
\varepsilon_{r \varphi} & =\frac{1}{2}\left[\frac{1}{r \sin \theta} \frac{\partial u_{r}}{\partial \varphi}-\frac{u_{\varphi}}{r}+\frac{\partial u_{\varphi}}{\partial r}\right] \\
\varepsilon_{\theta \theta} & =\frac{1}{r} \frac{\partial u_{\theta}}{\partial \theta}+\frac{u_{r}}{r} \\
\varepsilon_{\varphi \varphi} & =\frac{1}{r \sin \theta} \frac{\partial u_{\varphi}}{\partial \varphi}+\frac{u_{r}}{r}+\frac{\cos \theta}{\sin \theta} \frac{u_{\theta}}{r} \\
\varepsilon_{\theta \varphi} & =\frac{1}{2}\left[\frac{1}{r} \frac{\partial u_{\varphi}}{\partial \theta}-\frac{\cos \theta}{\sin \theta} \frac{u_{\varphi}}{r}+\frac{1}{r \sin \theta} \frac{\partial u_{\theta}}{\partial \varphi}\right]
\end{aligned}
$$

The stress-strain relation in Eq. (2-1), which can also be used in a spherical coordinate 
system ${ }^{[41]}$, is repeated here

$$
\sigma_{i j, j}=\lambda \varepsilon_{k k} \delta_{i j}+2 \mu \varepsilon_{i j}
$$

Substituting Eq. (2-131) into Eq. (2-133) and using Eqs. (2-134) and (2-135), the analytical expressions of displacements and stresses in spherical coordinates are

$$
\begin{aligned}
u_{r}^{i n c} & =\cos \theta e^{-\hat{\imath} \kappa r \cos \theta}, \\
u_{\theta}^{i n c} & =-\sin \theta e^{-\hat{\imath} \kappa r \cos \theta}, \\
\sigma_{r r}^{i n c} & =\left[\lambda(-\hat{\imath} \kappa)+2 \mu\left(-\hat{\imath} \kappa \cos ^{2} \theta\right)\right] e^{-\hat{\imath} \kappa r \cos \theta}, \\
\sigma_{r \theta}^{i n c} & =\mu(2 \hat{\imath} \kappa \sin \theta \cos \theta) e^{-\hat{\imath} \kappa r \cos \theta}, \\
\sigma_{\theta \theta}^{i n c} & =\left[\lambda(-\hat{\imath} \kappa)+2 \mu\left(-\hat{\imath} \kappa \sin ^{2} \theta\right)\right] e^{-\hat{\imath} \kappa r \cos \theta}, \\
\sigma_{\varphi \varphi}^{i n c} & =\lambda(-\hat{\imath} \kappa) e^{-\hat{\imath} \kappa r \cos \theta} ; \\
u_{\varphi}^{i n c} & =\sigma_{r \varphi}^{i n c}=\sigma_{\theta \varphi}^{i n c}=0 .
\end{aligned}
$$

Since $\psi^{i n c}=\chi^{i n c}=0$, Eq. $(2-37)$ can be written as

$$
\boldsymbol{u}=\nabla \phi,
$$

and then, using Eqs. (2-1) and (2-2), the analytical expressions of displacements and stresses in Cartesian coordinates are

$$
\begin{aligned}
& u_{z}^{i n c}=e^{-\hat{\imath} \kappa r \cos \theta}=e^{-\hat{\imath} \kappa z}, \\
& \sigma_{x x}^{i n c}=\lambda(-\hat{\imath} \kappa) e^{-\hat{\imath} \kappa z}, \\
& \sigma_{y y}^{i n c}=\lambda(-\hat{\imath} \kappa) e^{-\hat{\imath} \kappa z}, \\
& \sigma_{z z}^{i n c}=(\lambda+2 \mu)(-\hat{\imath} \kappa) e^{-\hat{\imath} \kappa z} . \\
& u_{x}^{i n c}=u_{y}^{i n c}=\sigma_{x y}^{i n c}=\sigma_{x z}^{i n c}=\sigma_{y z}^{i n c}=0
\end{aligned}
$$




\section{2-6.2 Truncation Criterion}

Prior to computing wave fields in single scattering problems, a criterion must be established to truncate the infinite series in the expansion expressions of wave fields, such as Eq. (2-60a) for the scattered waves and Eq. (2-59a) for the incident waves.

At first, consider Eq. (2-60a) for the scattered waves. The series can generally be truncated at order $N$ when magnitude of $B_{m(N+1)}^{\phi} h_{N+1}^{(1)}(\kappa r) Y_{N+1}^{m}(\theta, \varphi)$ is smaller than specified error $\epsilon$ for all $m$ in $[-N-1, N+1]$. Since the scattered waves are to be evaluated outside the scatterer, and the magnitude of $h_{n}^{(1)}(\kappa r)$ monotonically decreases when distance $r$ increases ${ }^{[38]}$, the criterion to truncate the series at order $N$ is

$$
\left|B_{m(N+1)}^{\phi} h_{N+1}^{(1)}(\kappa a) Y_{N+1}^{m}(\theta, \varphi)\right| \leq \epsilon .
$$

where $-N-1 \leq m \leq N+1$. When Eq. (2-139) is satisfied at order $N$, the series $B_{m n}^{\phi} h_{n}^{(1)}(\kappa r) Y_{n}^{m}(\theta, \varphi)$ are truncated at $N$. The number $N$ is the truncation number with the corresponding truncation error $\epsilon$.

When consider Eq. (2-60b) for the scattered waves, similarly, the criterion to truncation the series at order $N$ is

$$
\left|B_{m(N+1)}^{\psi} h_{N+1}^{(1)}(\kappa a) Y_{N+1}^{m}(\theta, \varphi)\right| \leq \epsilon .
$$

When consider Eq. (2-60c) for the scattered waves, similarly, the criterion to truncation the series at order $N$ is

$$
\left|B_{m(N+1)}^{\chi} h_{N+1}^{(1)}(\kappa a) Y_{N+1}^{m}(\theta, \varphi)\right| \leq \epsilon .
$$

Now, consider Eq. (2-59a) for an incident wave. Assume

$$
\left|A_{m(N+1)}^{\phi} j_{N+1}(\kappa r) Y_{N+1}^{m}(\theta, \varphi)\right| \leq \epsilon .
$$

For $R>r,\left|A_{m(N+1)}^{\phi} j_{N+1}(\kappa R) Y_{N+1}^{m}(\theta, \varphi)\right|$ may be larger than $\epsilon$, since $\left|j_{n}(\kappa r)\right|$ is not 
a decreasing function of distance $r^{[38]}$. The most convenient way to avoid this truncation error is to use an analytical expression, instead of wave expansion expression, for incident waves.

In a single scattering problem, a longitudinal plane wave in Eq. (2-131) impinges on the sphere. For a plane incident wave $\phi^{i n c}$, the scattered waves $\phi^{\text {sct }}$ and $\chi^{\text {sct }}$ are generated, and the scattered wave expansion coefficients $B_{m n}^{\phi}$ and $B_{m n}^{\chi}$ are obtained through the single scattering solution in Eq. (2-78). For the same wave frequency, $k / \kappa=\sqrt{(\lambda+2 \mu) / \mu}$ according to Eqs. (2-14) and (2-12). For a $\kappa a$ value, for a specified $\epsilon$, and for ten values of $\theta$ evenly distributed in $[0, \pi]$, assuming a number $N$ and for each $m$ in $[-N-1, N+1]$, check whether Eq. $(2-139)$ is satisfied or not. When Eq. (2-139) is satisfied, the number $N$ is the truncation number in Fig. 2.2(a). Similarly, for a $k a$ value, the truncation number in Fig. 2.2(b) is obtained using Eq. (2-141). In this example, $k / \kappa=3.6111$ for the same wave frequency. At the same wave frequency and for the same truncation error, the truncation number in Fig. 2.2(b) is not larger than the truncation number in Fig. 2.2(a). The relationships between truncation number and wave frequency for truncation error $\epsilon=10^{-8}$ and $10^{-5}$ are shown in Fig. 2.2(a). The truncation number generally increases when wave number $\kappa a$ increases for the same truncation error.

\section{2-6.3 Wave Fields and Field Continuity}

For the single scattering problem of a beryllium sphere in a polyethylene matrix, a longitudinal incident wave is used as an example. The longitudinal plane incident wave propagates along positive $Z$-axis direction, with an unit amplitude of the displacement and wave number $\kappa a=1.217$. The corresponding wave number $k a=4.395$. The truncation number is 11 in the computation, which corresponds to a truncation error $\epsilon=10^{-8}$ (Fig. 2.2). 


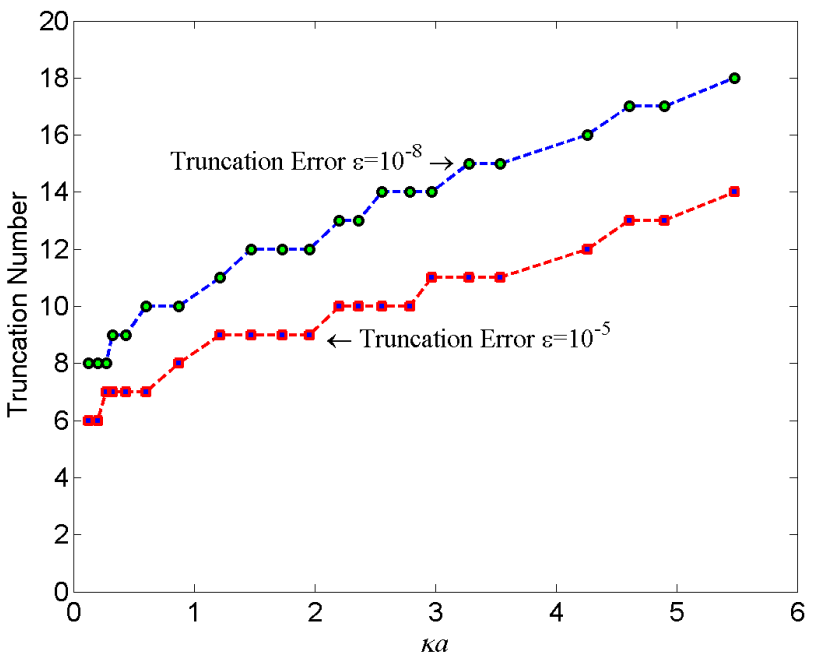

(a)

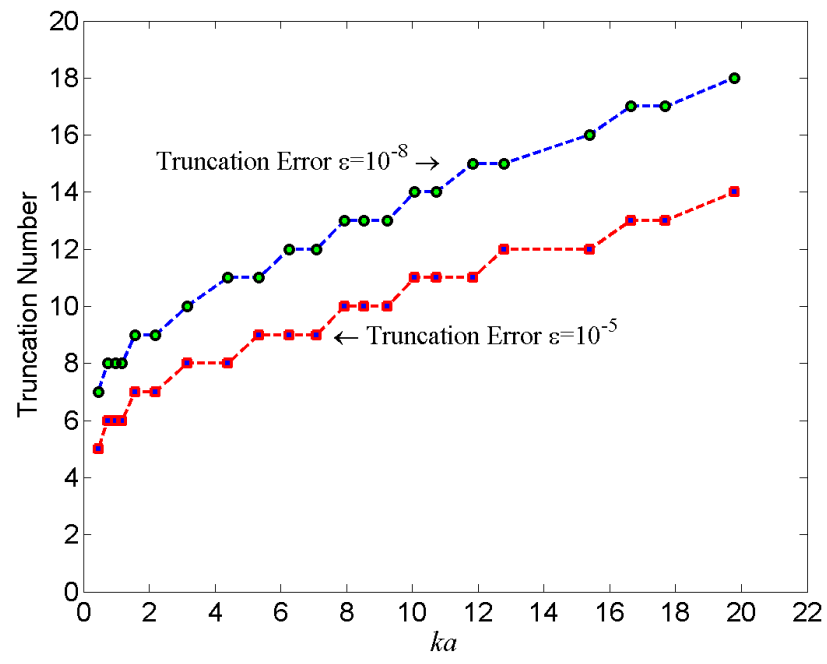

(b)

Figure 2.2: Truncation number for truncation error $\epsilon=10^{-8}$ and $10^{-5}$ : (a) using Eq. (2-139), (b) using Eq. (2-141); and $k / \kappa=3.6111$. 
By following Eq. (2-86), wave fields at a position outside the scatterer are evaluated from contributions of incident and scattered waves:

$$
u_{i}=u_{i}^{i n c}+u_{i}^{s c t}, \quad \sigma_{i j}=\sigma_{i j}^{i n c}+\sigma_{i j}^{s c t}
$$

where $u_{i}^{i n c}$ and $\sigma_{i j}^{i n c}$ are obtained by Eqs. $(2-136)$ and (2-138), $u_{i}^{s c t}$ and $\sigma_{i j}^{\text {sct }}$ in spherical coordinate system are obtained by Eqs. (2-45) and (2-46) after solving the single scattering problem, and $u_{i}^{s c t}$ and $\sigma_{i j}^{s c t}$ in Cartesian coordinate system can be obtained from those in spherical coordinate system by using coordinate transformation in Eqs. (D-8) and (D-10).

Wave fields at a position inside the scatterer are evaluated from refracted waves:

$$
u_{i}=u_{i}^{r e f}, \quad \sigma_{i j}=\sigma_{i j}^{r e f}
$$

where $u_{i}^{r e f}$ and $\sigma_{i j}^{r e f}$ in spherical coordinate system are obtained by Eqs. (2-45) and (2-46); and $u_{i}^{r e f}$ and $\sigma_{i j}^{r e f}$ in Cartesian coordinate system can be obtained from those in spherical coordinate system by using coordinate transformation in Eqs. (D-8) and (D-10).

Displacement continuities are required at the scatterer's boundary such that

$$
u_{i}^{i n c}+u_{i}^{s c t}=u_{i}^{r e f}
$$

Figure 2.3 shows real and imaginary parts of $u_{x}$ in the $y=0$ plane, Fig. 2.4 shows real and imaginary parts of $u_{y}$ in the $y=0$ plane, Fig. 2.5 shows real and imaginary parts of $u_{z}$ in the $y=0$ plane, Fig. 2.6 shows real and imaginary parts of $u_{r}$ in the $y=0$ plane, Fig. 2.7 shows real and imaginary parts of $u_{\theta}$ in the $y=0$ plane, and Fig. 2.8 shows real and imaginary parts of $u_{\varphi}$ in the $y=0$ plane. The black circle in each figure represents the scatterer boundary. The images in these figures are $200 \times 200$ pixels with a pixel size of $0.015 a \times 0.015 a$. At each position on the black circle, $u_{i}^{i n c}+u_{i}^{s c t}=u_{i}^{r e f}$. 


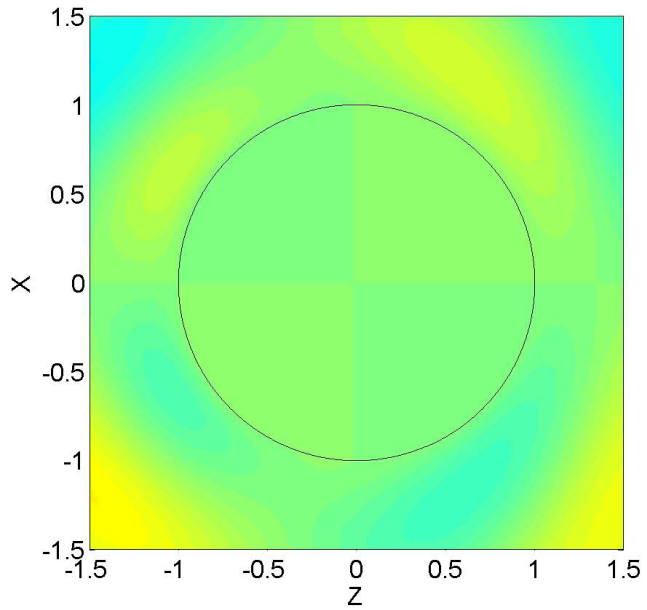

(a) Real part

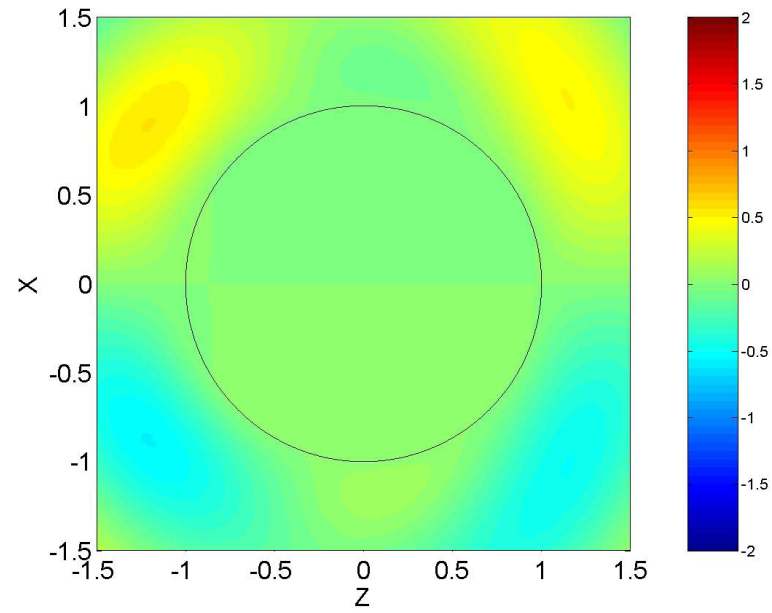

(b) Imaginary part

Figure 2.3: Continuity of displacements $u_{x}$ at the scatterer's boundary in the $y=0$ plane. The black circle in each figure represents the scatterer's boundary.

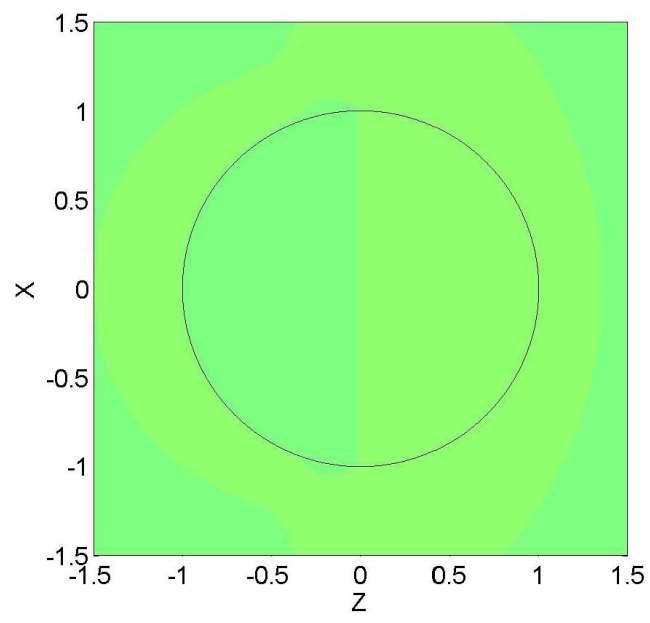

(a) Real part

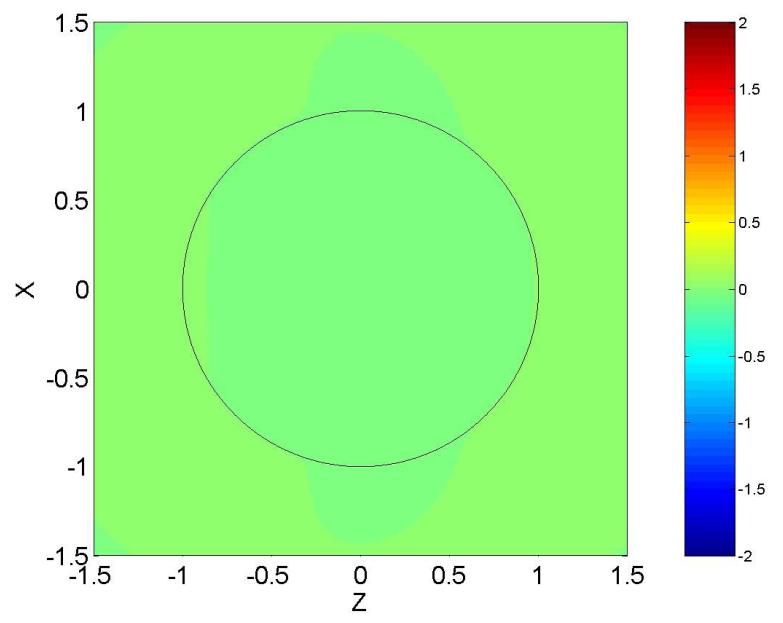

(b) Imaginary part

Figure 2.4: Continuity of displacements $u_{y}$ at the scatterer's boundary in the $y=0$ plane. The black circle in each figure represents the scatterer's boundary. 


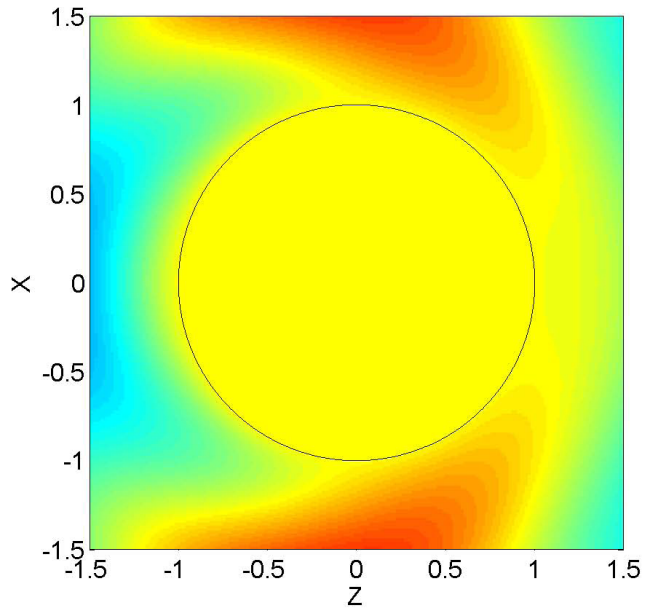

(a) Real part

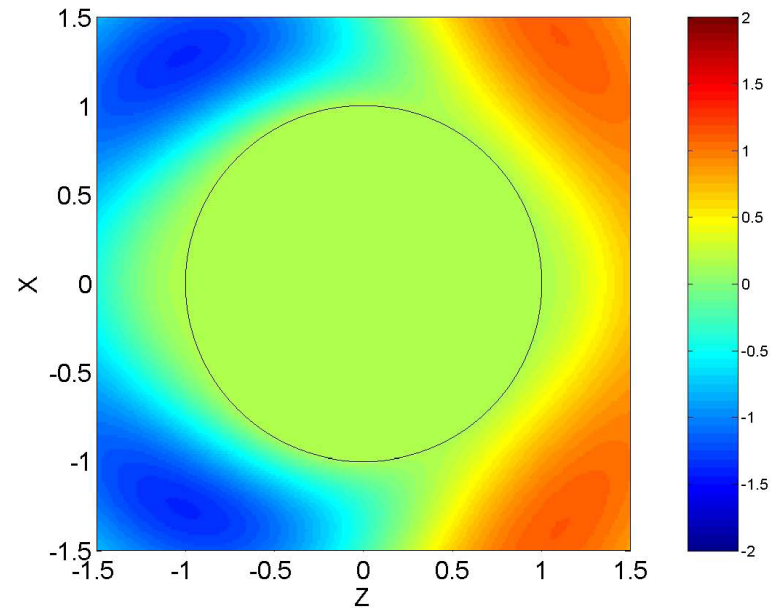

(b) Imaginary part

Figure 2.5: Continuity of displacements $u_{z}$ at the scatterer's boundary in the $y=0$ plane. The black circle in each figure represents the scatterer's boundary.

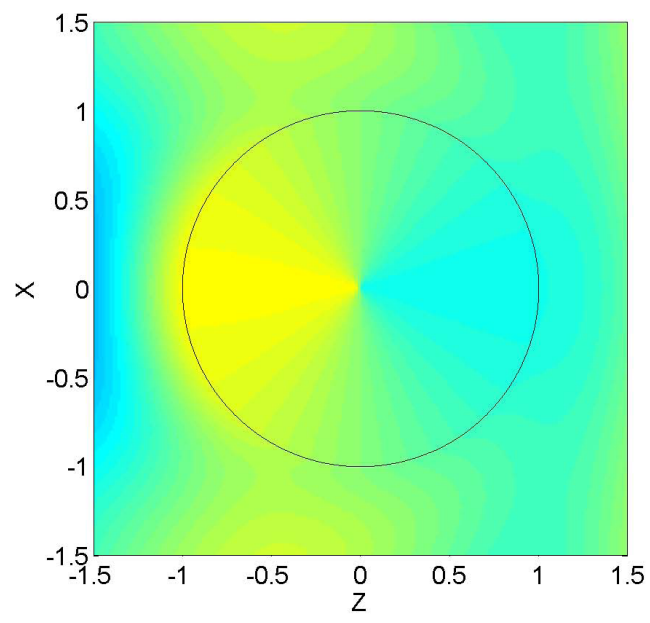

(a) Real part

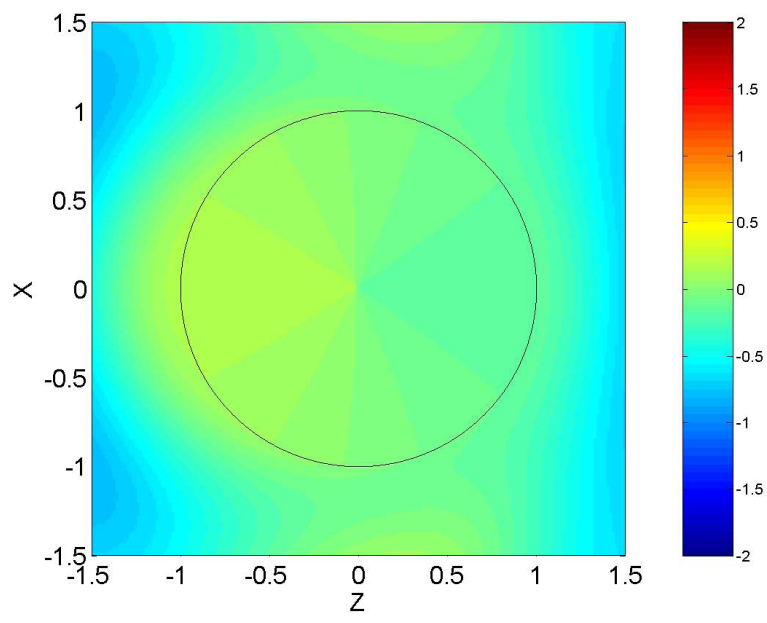

(b) Imaginary part

Figure 2.6: Continuity of displacements $u_{r}$ at the scatterer's boundary in the $y=0$ plane. The black circle in each figure represents the scatterer's boundary. 


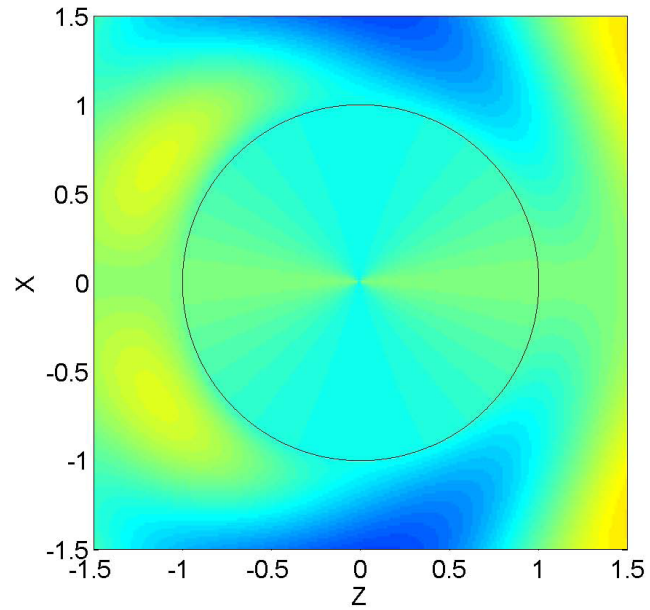

(a) Real part

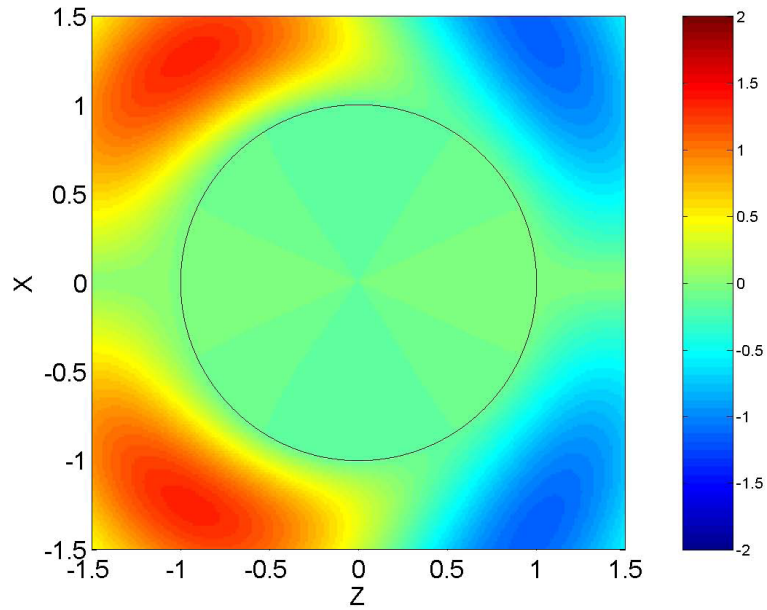

(b) Imaginary part

Figure 2.7: Continuity of displacements $u_{\theta}$ at the scatterer's boundary in the $y=0$ plane. The black circle in each figure represents the scatterer's boundary.

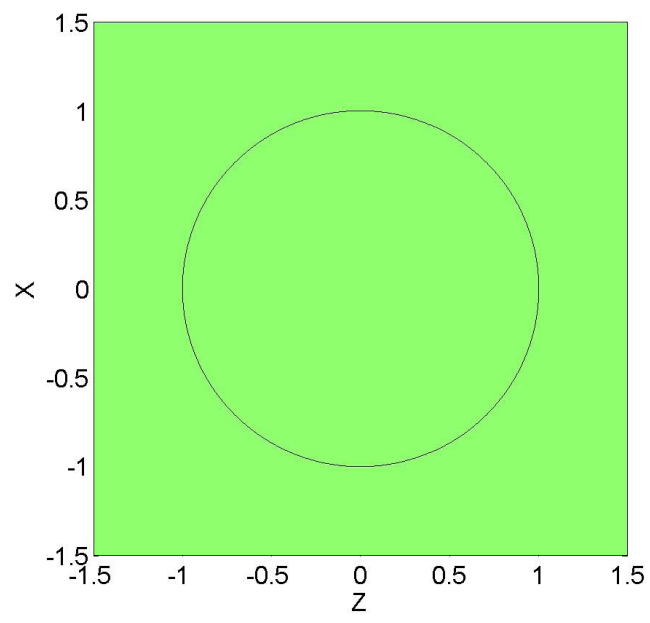

(a) Real part

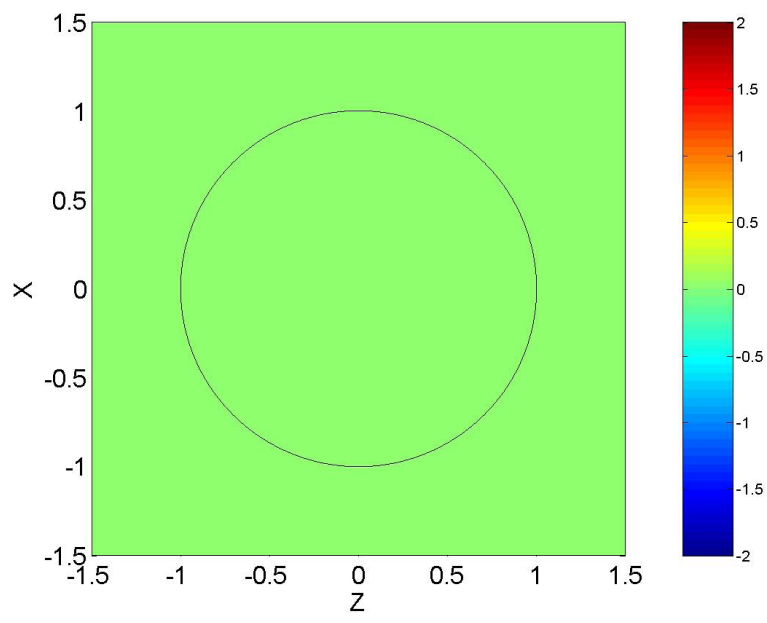

(b) Imaginary part

Figure 2.8: Continuity of displacements $u_{\varphi}$ at the scatterer's boundary in the $y=0$ plane. The black circle in each figure represents the scatterer's boundary. 
Surface traction continuity at the scatterer's boundary requires that

$$
\sigma_{r i}^{i n c}+\sigma_{r i}^{s c t}=\sigma_{r i}^{r e f},
$$

where $i$ denotes $r, \theta$ or $\varphi$. Figure 2.9 shows real and imaginary parts of $\sigma_{r r}$ in the $y=0$ plane, Fig. 2.10 shows real and imaginary parts of $\sigma_{r \theta}$ in the $y=0$ plane, and Fig. 2.11 shows real and imaginary parts of $\sigma_{r \varphi}$ in the $y=0$ plane. The images in these figures are $200 \times 200$ pixels with a pixel size of $0.015 a \times 0.015 a$. The black circle in each figure represents the scatterer boundary. At each position on the black circle, $\sigma_{r i}+\sigma_{r i}^{s c t}=\sigma_{r i}^{r e f}$.

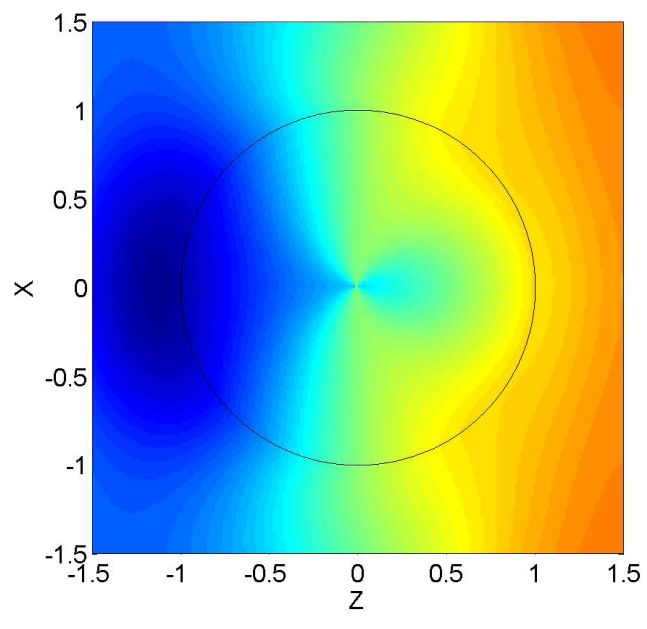

(a) Real part

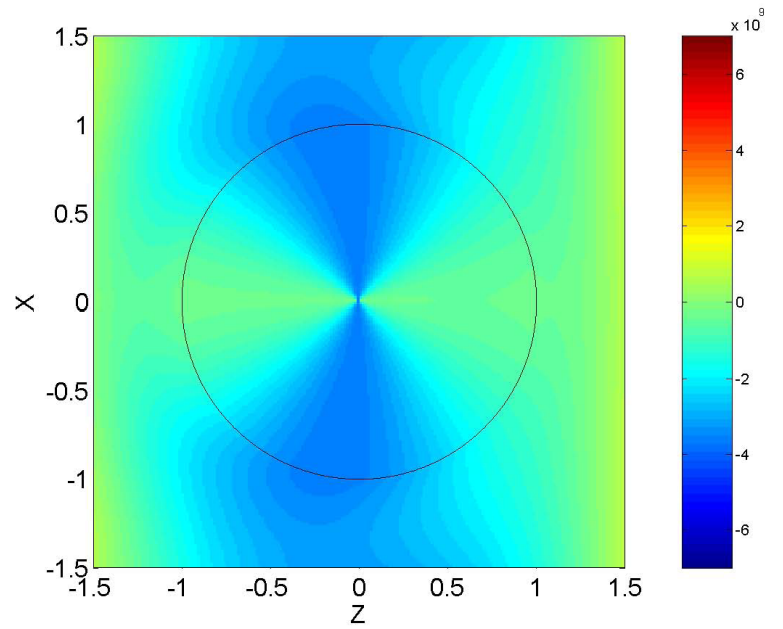

(b) Imaginary part

Figure 2.9: Continuity of stresses $\sigma_{r r}$ at the scatterer's boundary in the $y=0$ plane. The black circle in each figure represents the scatterer's boundary.

The coordinate $\theta$ has undetermined value at the origin so that $u_{r}, u_{\theta}, \sigma_{r r}$ and $\sigma_{r \theta}$ appear discontinuous at the origin. Equations (2-136a) through (2-136d) also show the discontinuity at the origin for the case of plane incident wave $\phi$. The continuities of $u_{x}, u_{y}$ and $u_{z}$ at origin proves the correctness of $u_{r}, u_{\theta}$ and $u_{\varphi}$ at origin, since $u_{x}$ and $u_{z}$ are obtained through coordinate transformation from $u_{r}$ and $u_{\theta}$.

By observing Figs. 2.3 through 2.9, it is concluded that the continuity conditions 


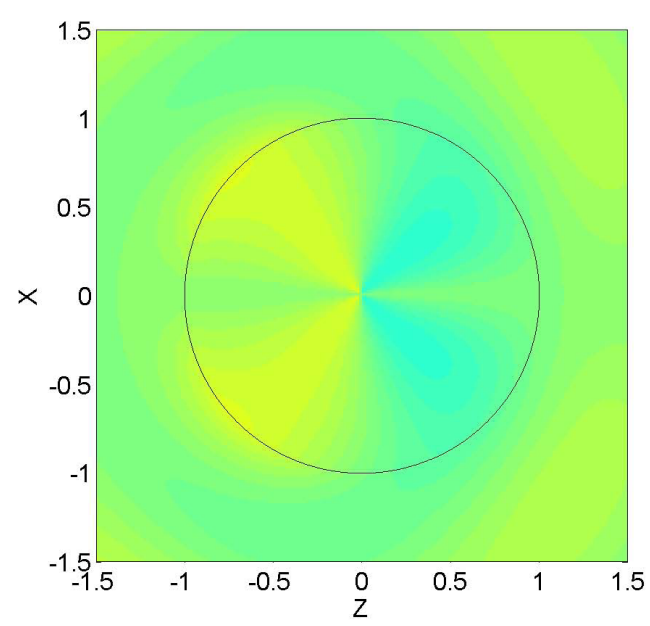

(a) Real part

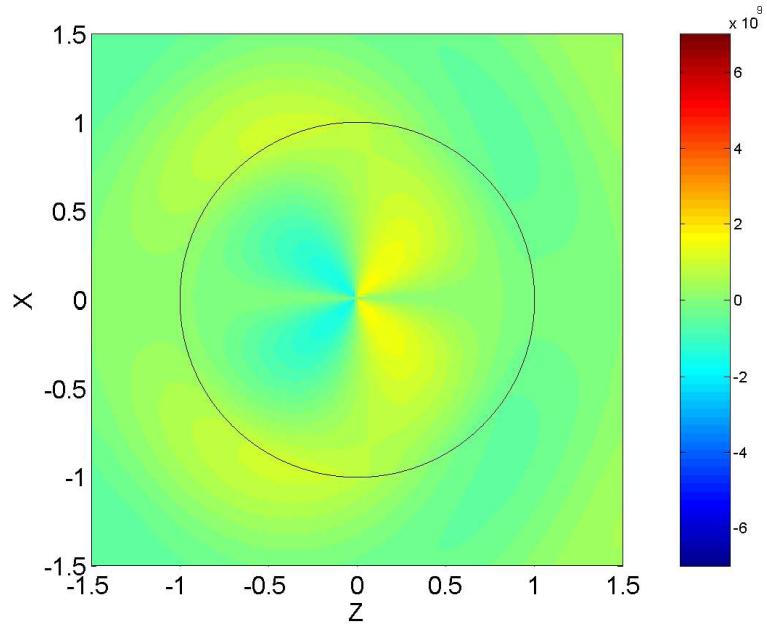

(b) Imaginary part

Figure 2.10: Continuity of stresses $\sigma_{r \theta}$ at the scatterer's boundary in the $y=0$ plane. The black circle in each figure represents the scatterer's boundary.

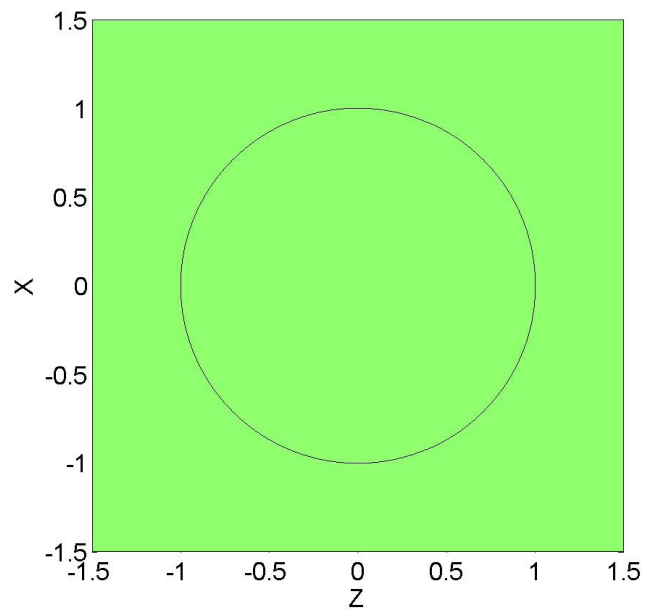

(a) Real part

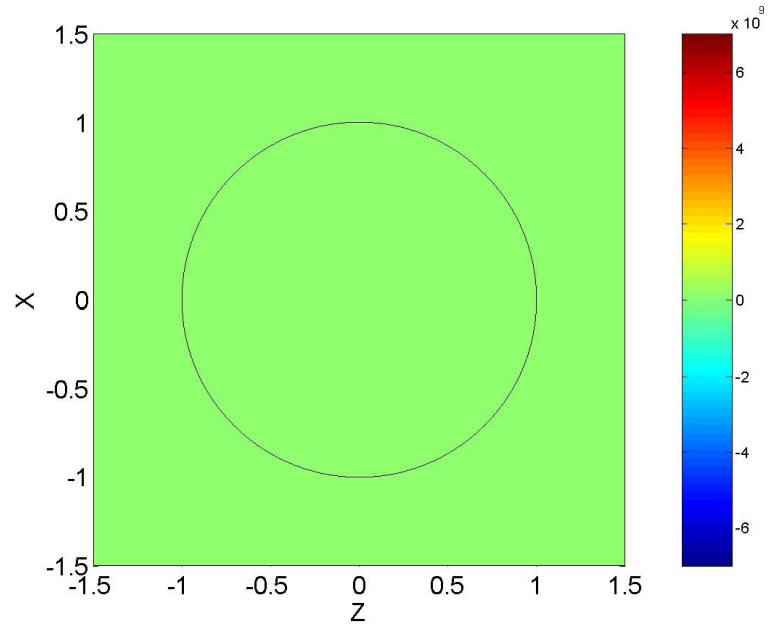

(b) Imaginary part

Figure 2.11: Continuity of stresses $\sigma_{r \varphi}$ at the scatterer's boundary in the $y=0$ plane. The black circle in each figure represents the scatterer's boundary. 
are satisfied.

To provide a glimpse into characteristics of the scattered waves, real part of each field due to the scattered waves and the total waves in the $y=0$ plane are shown in the next. Each of displacement components and stress components is a complex quantity. The real part of a complex quantity represents the quantity when its phase angle is zero. The imaginary part of a complex quantity represents the quantity when its phase angle is $\pi / 2$. The real part of a wave field is displayed. It must be noted that this is not intended to be a comprehensive analysis of the solved case. Images in Figs. 2.12 through 2.26 are $400 \times 400$ pixels with a pixel size of $0.05 a \times 0.05 a$. The black circle represents the scatterer's boundary. Wave fields inside the scatterer is obtained from refracted waves.

Real part of $u_{x}$ in the $y=0$ plane is shown in Fig. 2.12. The $u_{x}$ due to the scattered waves is also the $u_{x}$ due to total waves because the $u_{x}$ due to longitudinal incident wave is zero. Distribution of real part of $u_{x}$ in the $y=0$ plane is not symmetric about either $X$-axis or $Z$-axis. The peaks appear like circular ridges in each of four quarters in the $y=0$ plane. Real part of $u_{z}$ is shown in Fig. 2.13. Distribution of real part of $u_{z}$ due to the scattered waves appear like circular ridges with symmetry about $Z$-axis, and the value of $u_{z}$ approaches to zero when it is near $Z$-axis. Real part of $u_{r}$ is shown in Fig. 2.14. Distribution of real part of $u_{r}$ due to the scattered waves appear like circular ridges with symmetry about the $Z$-axis. The ridges in Fig. 2.14(a) are wider than those in Fig. 2.14(a). The spacing between ridges in Fig. 2.14(a) is larger than that in Fig. 2.13(a). There is no explanation for it yet. Real part of $u_{\theta}$ is shown in Fig. 2.15. Distribution of real part of $u_{\theta}$ due to the scattered waves appear like circular ridges with symmetry about $Z$-axis.

Real part of $\sigma_{r r}$ is shown in Fig. 2.16. Distribution of real part of $\sigma_{r r}$ due to the scattered waves in the $y=0$ plane appear like circular ridges with symmetry about 


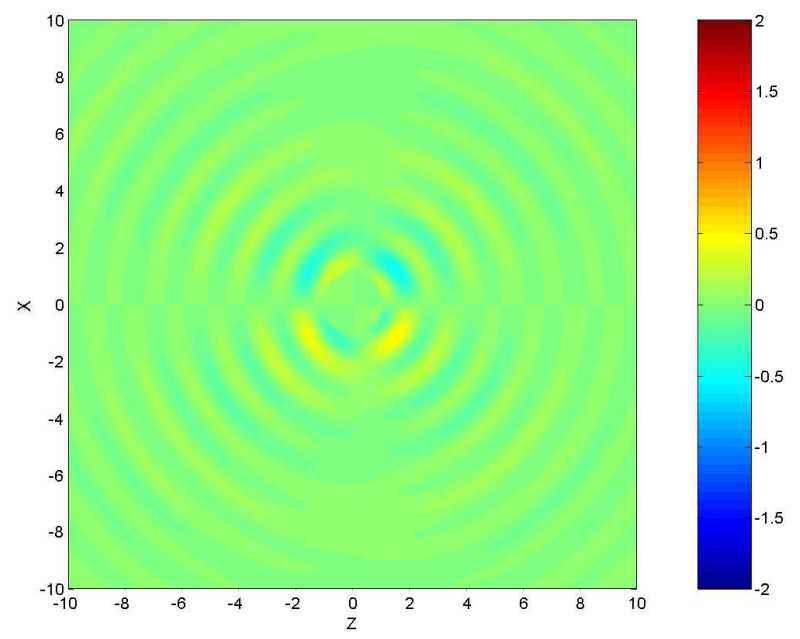

Figure 2.12: Real part $u_{x}$ in the $y=0$ plane. Displacement $u_{x}$ of longitudinal incident wave is zero so that the $u_{x}$ of scattered waves is also the $u_{x}$ of total waves. The black circle in the figure represents the scatterer's boundary. Displacements inside the scatterer is obtained from refracted waves instead of scattered waves.

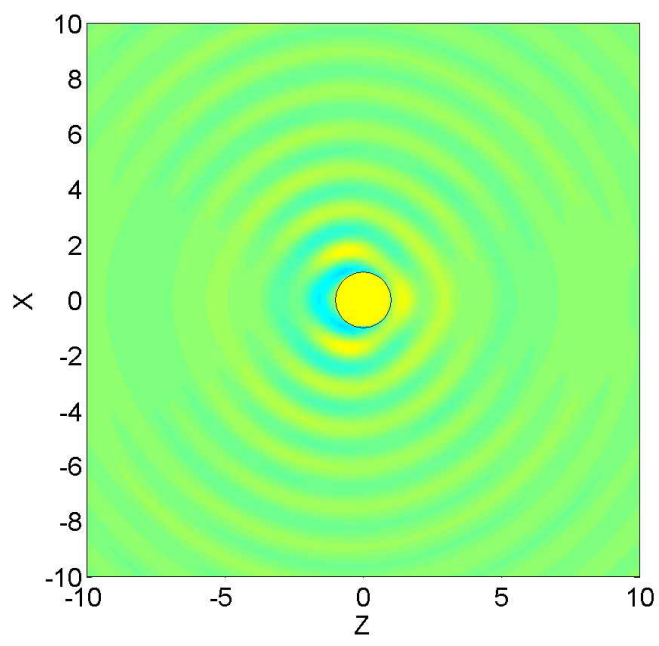

(a) scattered waves

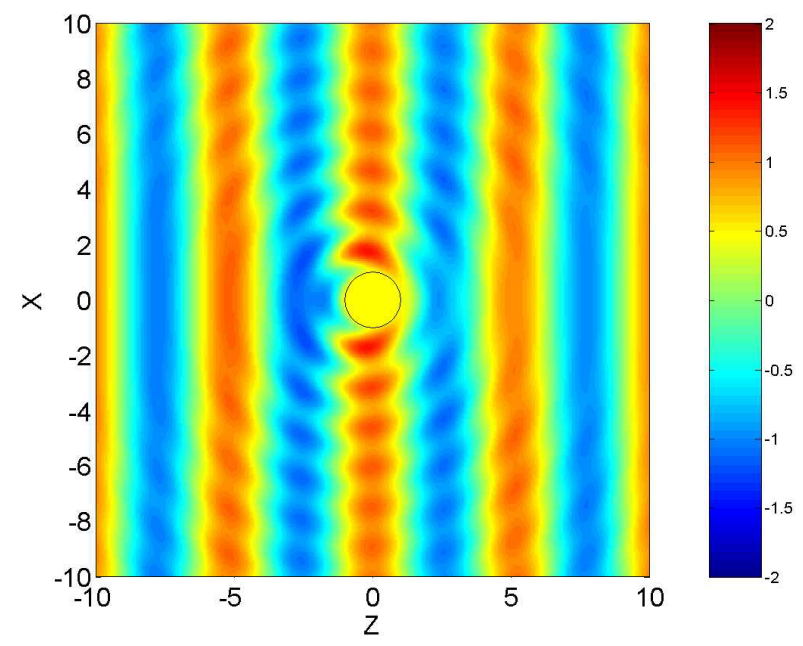

(b) total waves

Figure 2.13: Real part of $u_{z}$ in the $y=0$ plane. The black circle in each figure represents the scatterer's boundary. Displacements inside the scatterer is obtained from refracted waves instead of scattered waves, so that there is a discontinuity along the black circle in (a). 


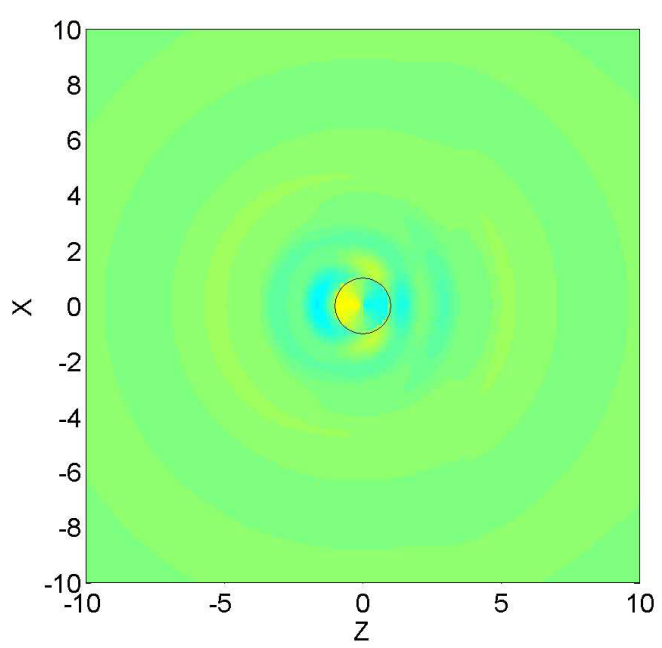

(a) scattered waves

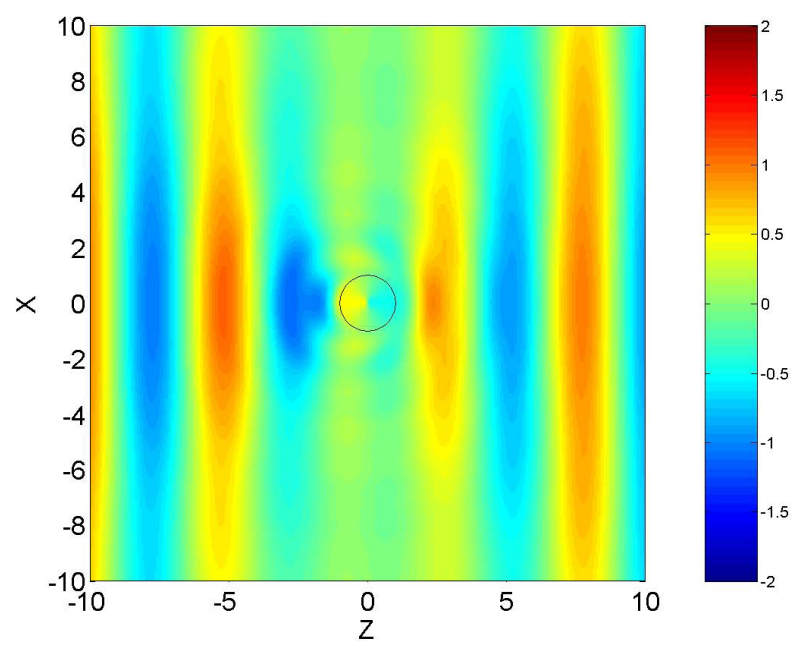

(b) total waves

Figure 2.14: Real part of $u_{r}$ in the $y=0$ plane. The black circle in each figure represents the scatterer's boundary. Displacements inside the scatterer is obtained from refracted waves instead of scattered waves, so that there is a discontinuity along the black circle in (a).

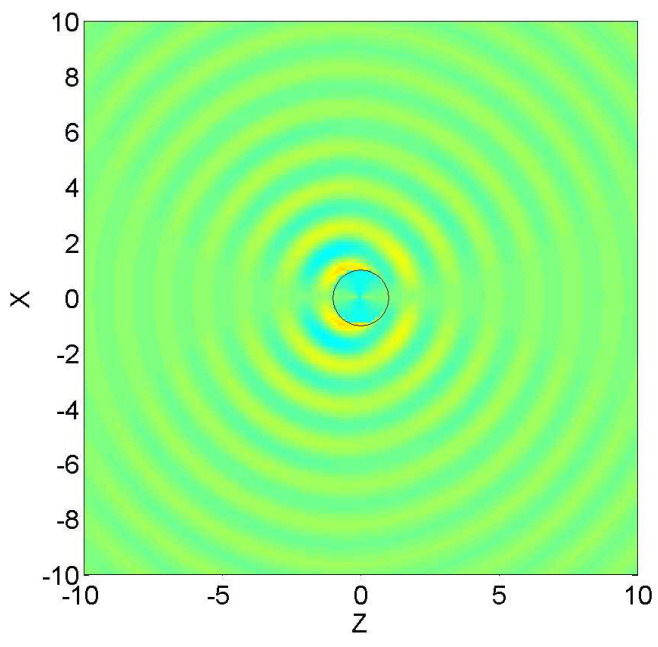

(a) scattered waves

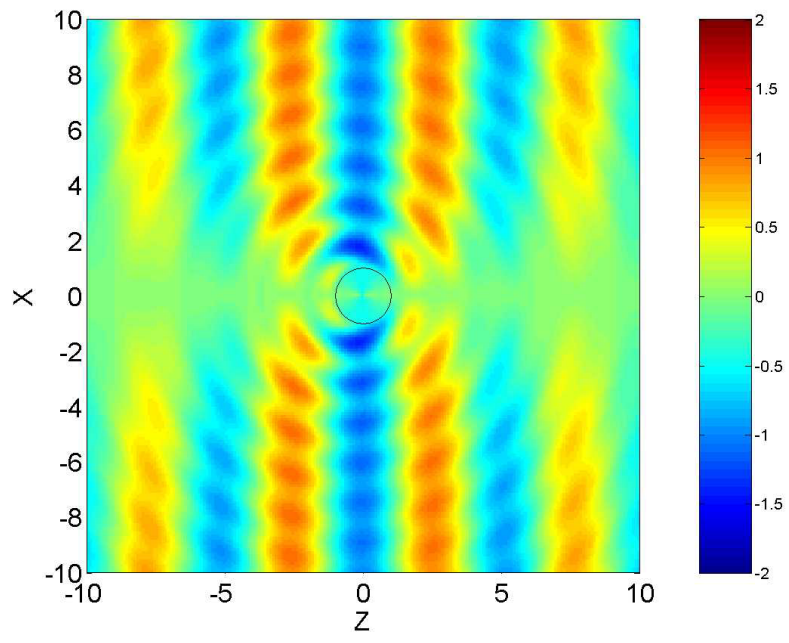

(b) total waves

Figure 2.15: Real part of $u_{\theta}$ in the $y=0$ plane. The black circle in each figure represents the scatterer's boundary. Displacements inside the scatterer is obtained from refracted waves instead of scattered waves, so that there is a discontinuity along the black circle in (a). 
$Z$-axis. Real part of $\sigma_{r \theta}$ is shown in Fig. 2.17. Distribution of real part of $\sigma_{r \theta}$ due to the scattered waves in the $y=0$ plane appear like circular ridges with symmetry about $Z$-axis. Real part of $\sigma_{\theta \theta}$ and $\sigma_{\varphi \varphi}$ are shown in Figs. 2.18 and 2.19. Distribution patterns of real part of $\sigma_{\theta \theta}$ and $\sigma_{\varphi \varphi}$ due to the scattered waves in the $y=0$ plane appear similar to the distribution pattern of real part of $\sigma_{r r}$ due to the scattered waves. Stress $\sigma_{r \varphi}$ in the $y=0$ plane has zero value (Fig. 2.11). Stresses $\sigma_{\theta \varphi}$ in the $y=0$ plane also has zero value. There is no explanation about why they have zero values.

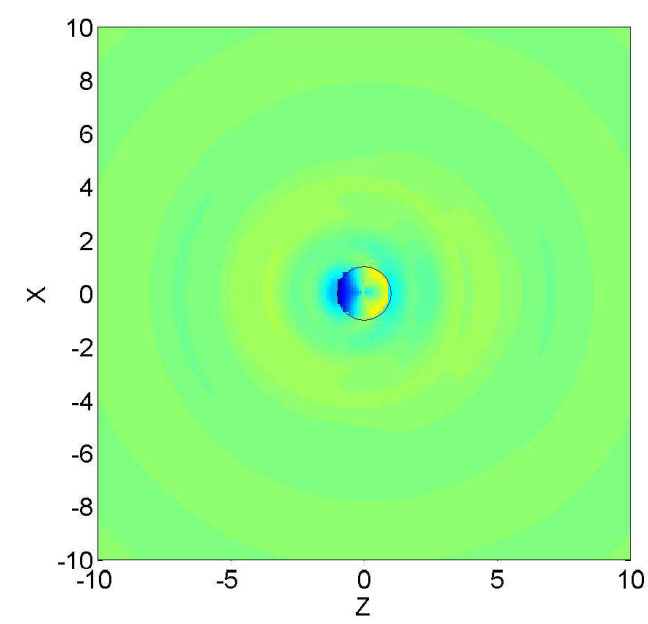

(a) scattered waves

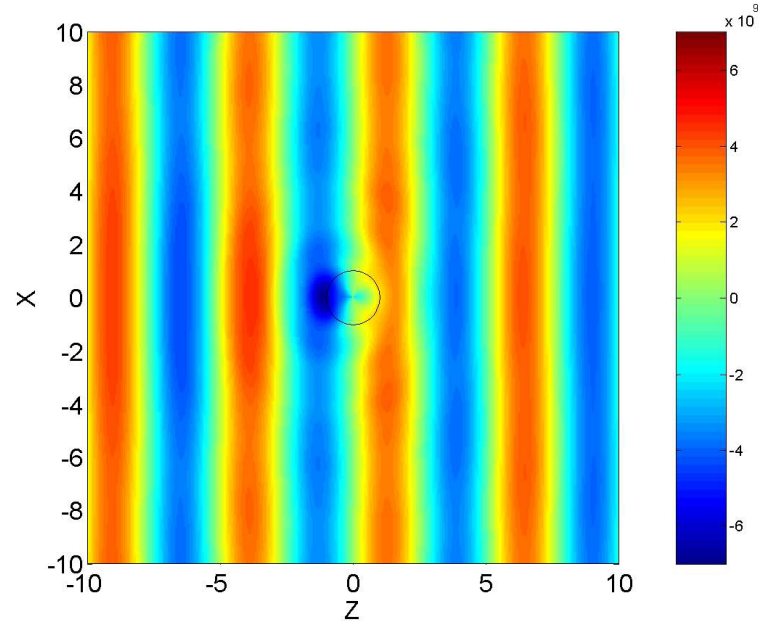

(b) total waves

Figure 2.16: Real part of $\sigma_{r r}$ in the $y=0$ plane. The black circle in each figure represents the scatterer's boundary. Stresses inside the scatterer is obtained from refracted waves instead of scattered waves, so that there is a discontinuity along the black circle in (a).

Real part of $\sigma_{x x}$ is shown in Fig. 2.20. Distribution of real part of $\sigma_{x x}$ due to the scattered waves in the $y=0$ plane appear like circular ridges with symmetry about $Z$-axis. Real part of $\sigma_{y y}$ and $\sigma_{z z}$ are shown in Figs. 2.21 and 2.22. Distribution patterns of real part of $\sigma_{y y}$ and $\sigma_{z z}$ due to the scattered waves in the $y=0$ plane appear similar to the distribution pattern of real part of $\sigma_{x x}$ due to the scattered 


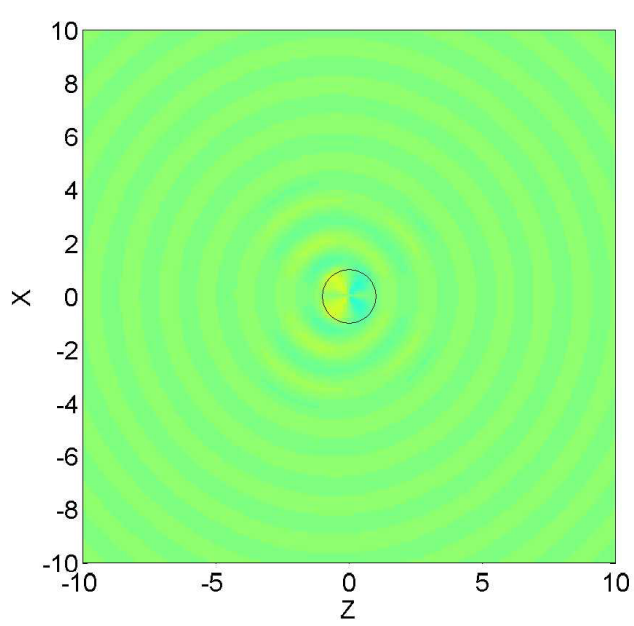

(a) scattered waves

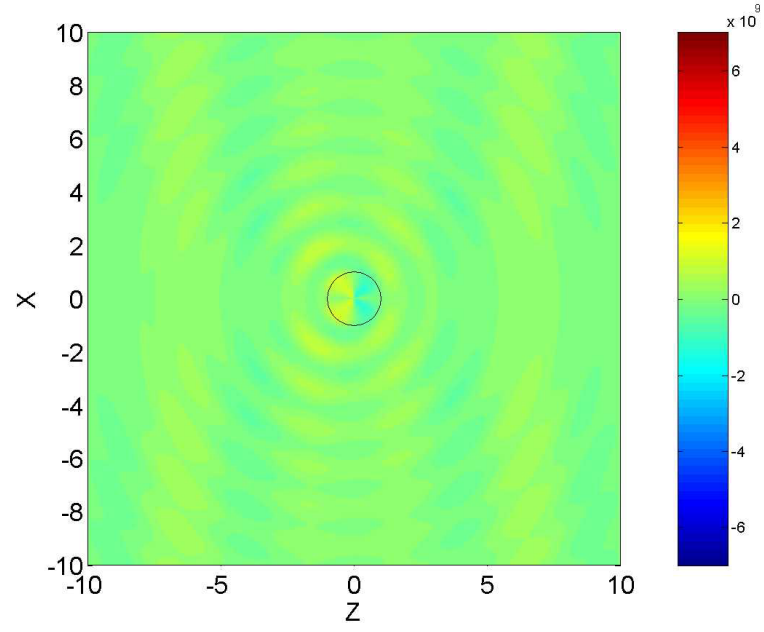

(b) total waves

Figure 2.17: Real part of $\sigma_{r q}$ in the $y=0$ plane. The black circle in each figure represents the scatterer's boundary. Stresses inside the scatterer is obtained from refracted waves instead of scattered waves, so that there is a discontinuity along the black circle in (a).

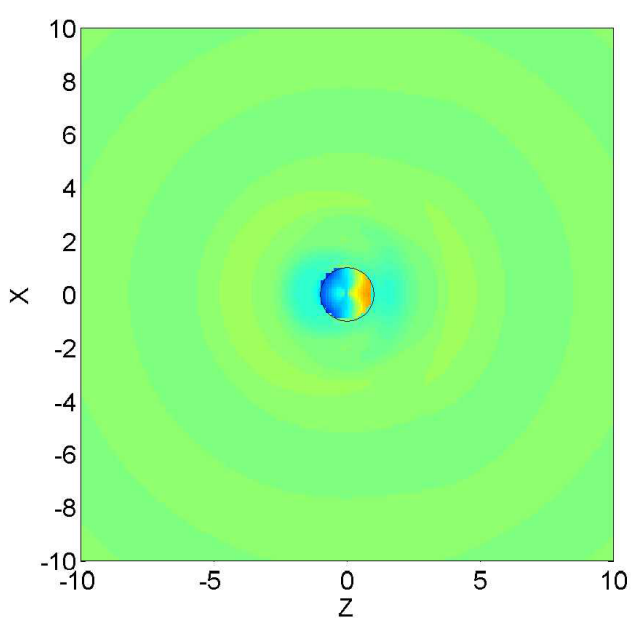

(a) scattered waves

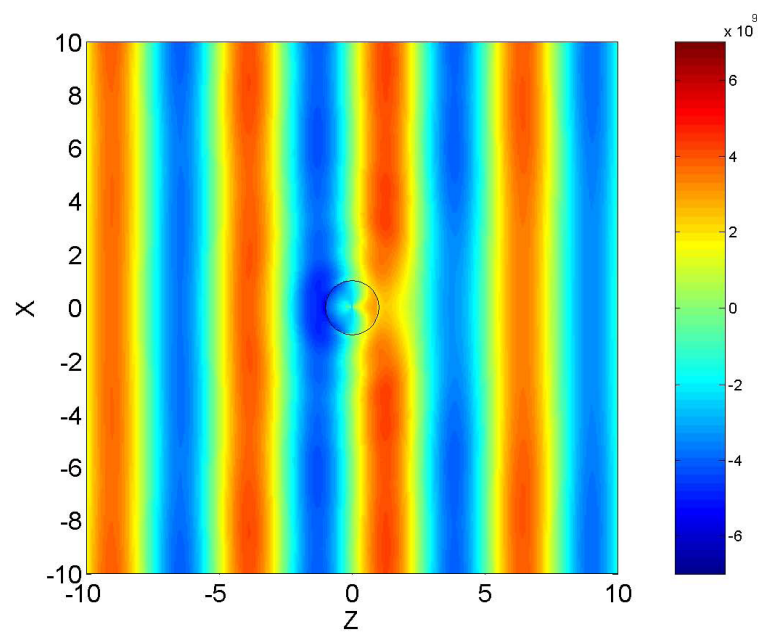

(b) total waves

Figure 2.18: Real part of $\sigma_{\theta \theta}$ in the $y=0$ plane. The black circle in each figure represents the scatterer's boundary. Stresses inside the scatterer is obtained from refracted waves instead of scattered waves, so that there is a discontinuity along the black circle in (a). 


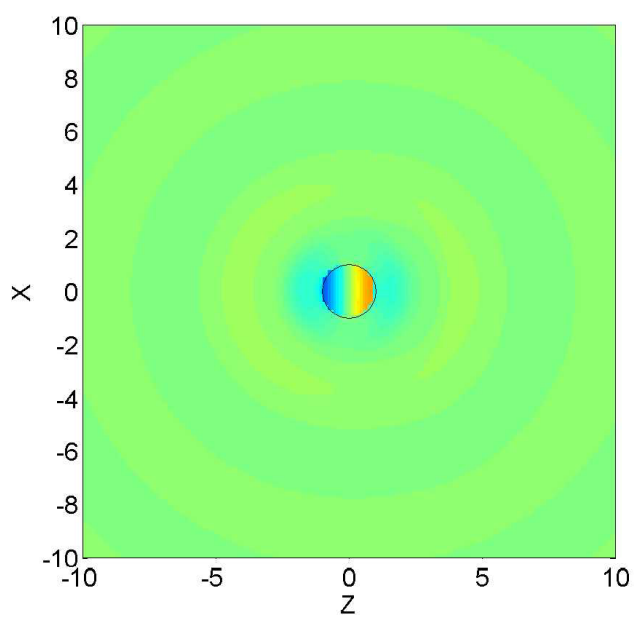

(a) scattered waves

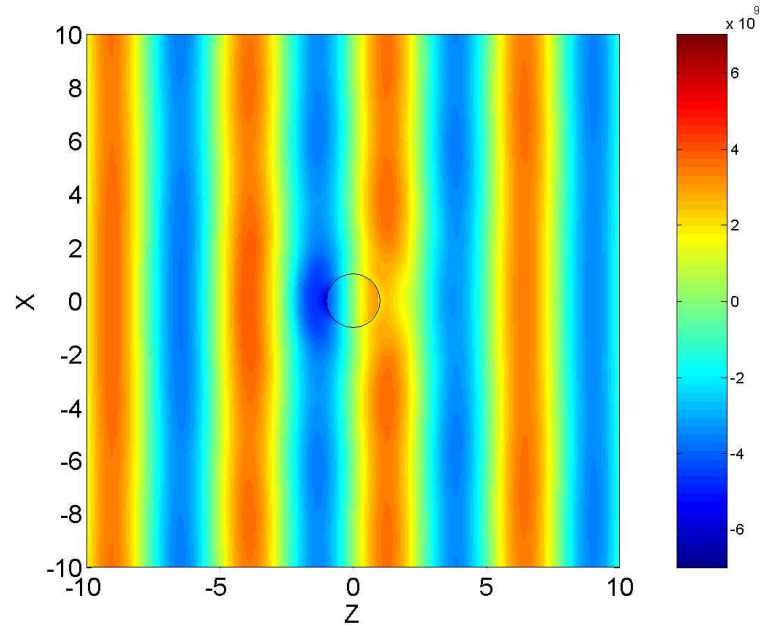

(b) total waves

Figure 2.19: Real part of $\sigma_{\varphi \varphi}$ in the $y=0$ plane. The black circle in each figure represents the scatterer's boundary. Stresses inside the scatterer is obtained from refracted waves instead of scattered waves, so that there is a discontinuity along the black circle in (a).

waves. Stress $\sigma_{x z}$ due to scattered waves is also the $\sigma_{x z}$ due to total waves because the $\sigma_{x z}$ due to the longitudinal incident wave is zero (Eq. (2-138e)). Real part of $\sigma_{x z}$ is shown in Fig. 2.23. Distribution of real part of $\sigma_{x z}$ is not symmetric about either $X$-axis or $Z$-axis.

As mentioned at the beginning of this section, wave field components in Cartesian coordinate system are obtained through coordinate transformation in Eqs. (D-8) and (D-10) from those in spherical coordinate system. In the $y=0$ plane, $\theta=\pi / 2$ and $\varphi=0$. Therefore, the matrix $\boldsymbol{T}_{r}$ for coordinate transformation in Eq. (D-5) can be written as

$$
\boldsymbol{T}_{r}=\left[\begin{array}{ccc}
1 & 0 & 0 \\
0 & 0 & -1 \\
0 & 1 & 0
\end{array}\right]
$$




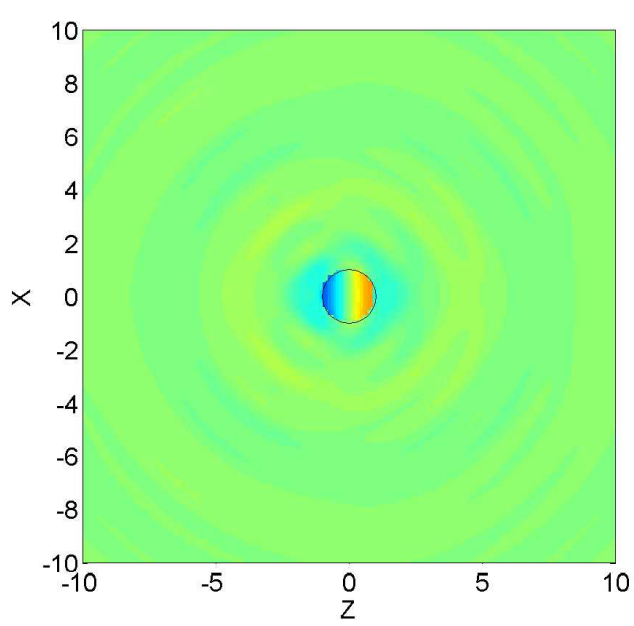

(a) scattered waves

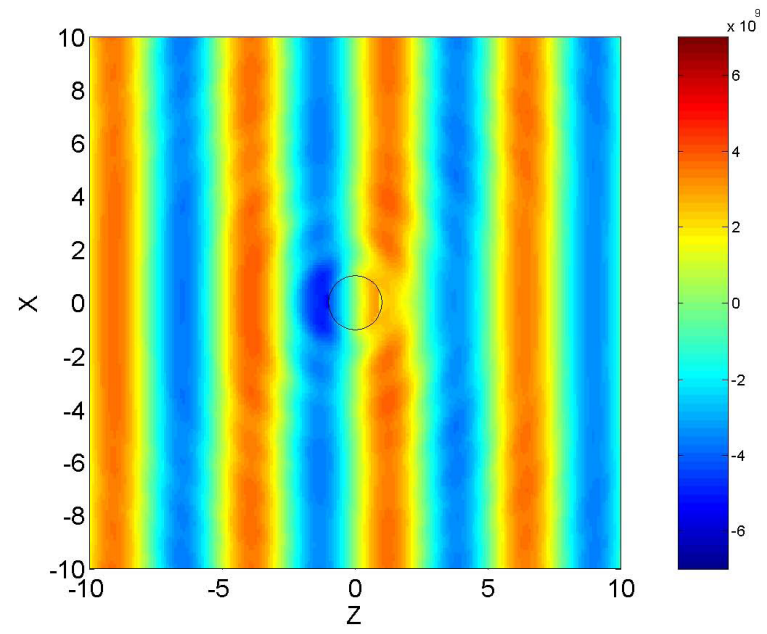

(b) total waves

Figure 2.20: Real part of $\sigma_{x x}$ in the $y=0$ plane. The black circle in each figure represents the scatterer's boundary. Stresses inside the scatterer is obtained from refracted waves instead of scattered waves, so that there is a discontinuity along the black circle in (a).

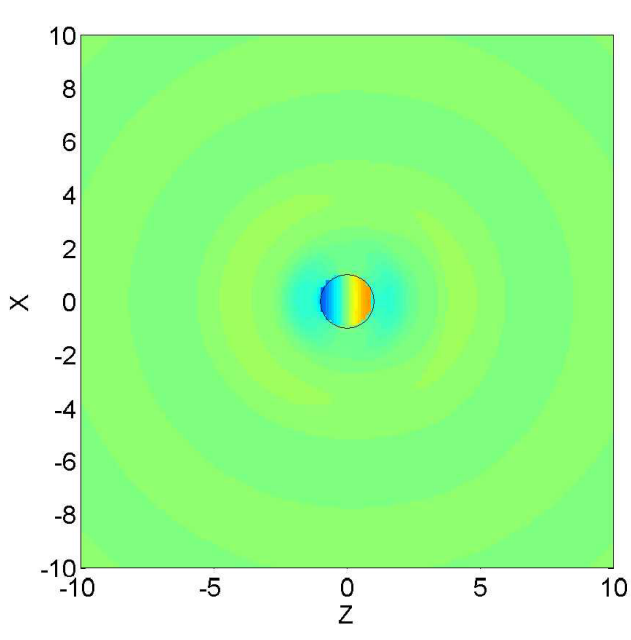

(a) scattered waves

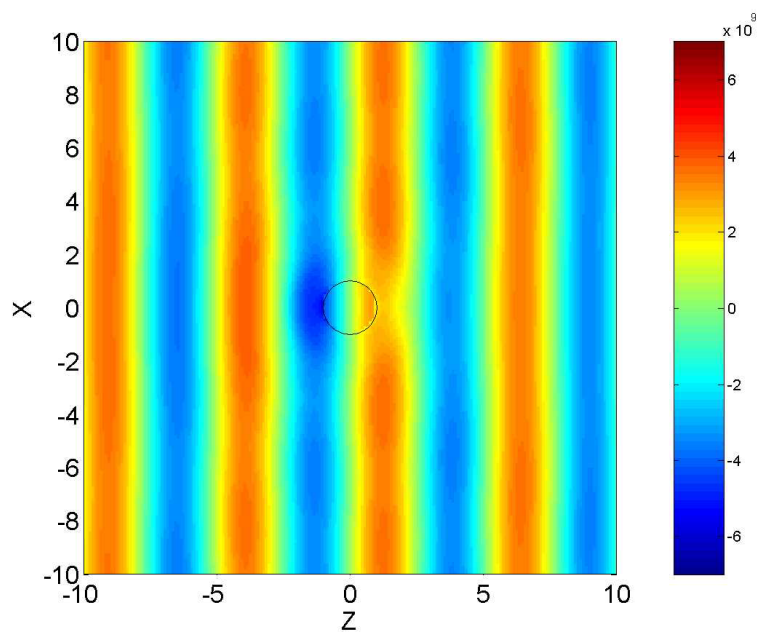

(b) total waves

Figure 2.21: Real part of $\sigma_{y y}$ in the $y=0$ plane. The black circle in each figure represents the scatterer's boundary. Stresses inside the scatterer is obtained from refracted waves instead of scattered waves, so that there is a discontinuity along the black circle in (a). 


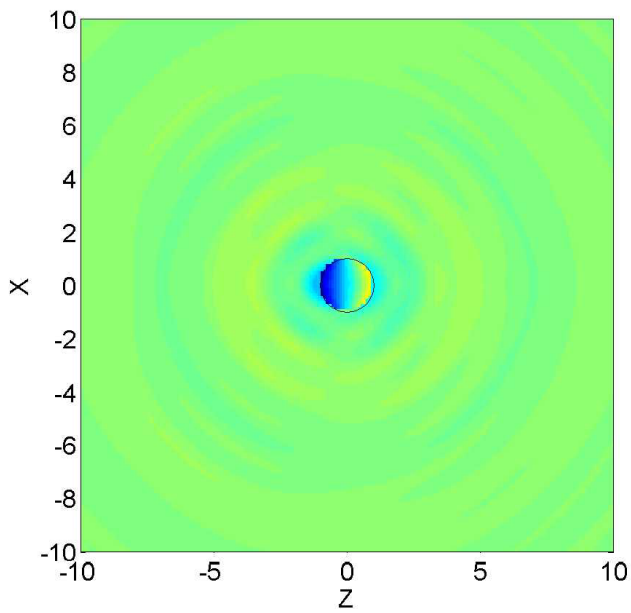

(a) scattered waves

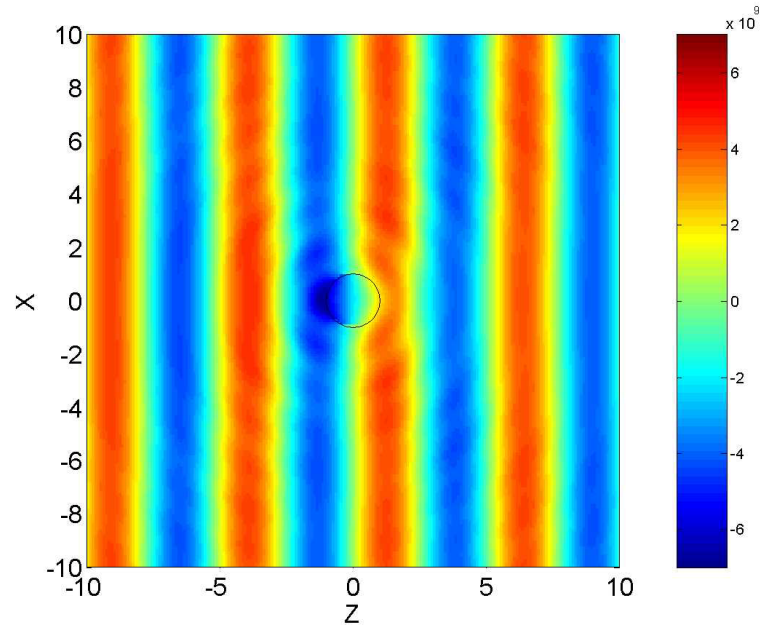

(b) total waves

Figure 2.22: Real part of $\sigma_{z z}$ in the $y=0$ plane. The black circle in each figure represents the scatterer's boundary. Stresses inside the scatterer is obtained from refracted waves instead of scattered waves, so that there is a discontinuity along the black circle in (a).

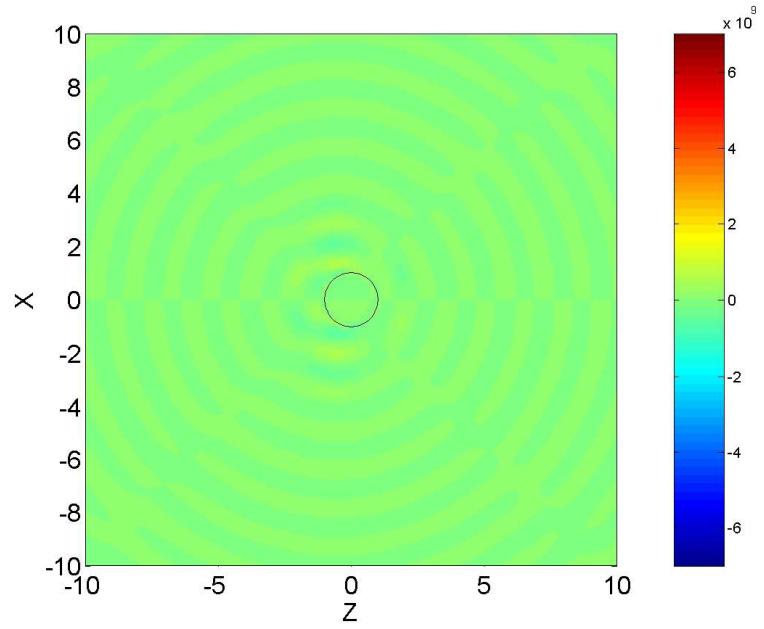

Figure 2.23: Real part $\sigma_{x z}$ in the $y=0$ plane. Stress $\sigma_{x z}$ of longitudinal incident wave is zero so that the $\sigma_{x z}$ of scattered waves is also the $\sigma_{x z}$ of total waves. The black circle in the figure represents the scatterer's boundary. Displacements inside the scatterer is obtained from refracted waves instead of scattered waves. 
By using Eqs. (D-8a) and (D-10a), it can be found that

$$
u_{y}=u_{\varphi}, \quad \sigma_{x y}=\sigma_{r \varphi}, \quad \sigma_{y z}=-\sigma_{\theta \varphi}
$$

In Fig. 2.24, numerical values of real part of $u_{\varphi}$ in the $y=0$ plane are exactly zero; numerical values of real part of $u_{y}$ in the $y=0$ plane are also expected to be zero but they are in the order $10^{-5}$. The values of real part of $\sigma_{x y}$ and $\sigma_{y z}$ in Figs. 2.25 and 2.26 are in the order of $10^{4}$, while the value of real part of $\sigma_{x x}$ in Fig. 2.20 is in the order of $10^{9}$. The ratio of peak values of real part of $\sigma_{x y}$ and $\sigma_{x x}$ implies that numerical errors for $\sigma_{x y}$ are in the order of $10^{-5}$. Similarly, numerical error for $\sigma_{y z}$ is also in the order of $10^{-5}$. This observation shows that extra numerical error is brought to wave fields in Cartesian coordinate system during coordinate transformation.

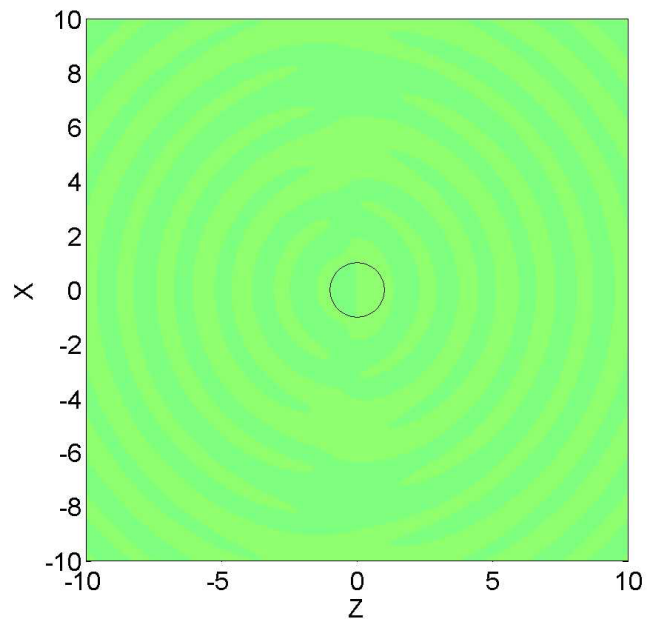

(a) Real part of $u_{y}$ (in the order of $10^{-5}$ )

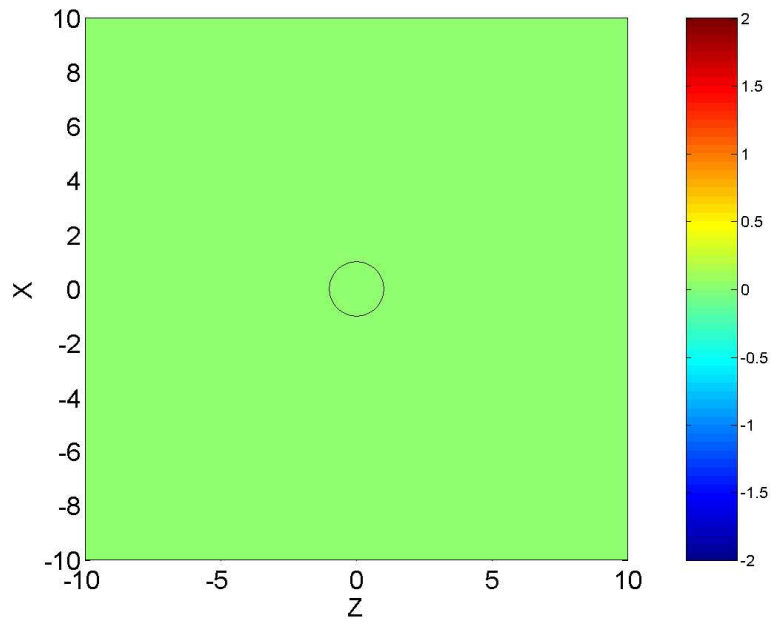

(b) Real part of $u_{\varphi}$ (exactly zero)

Figure 2.24: Zero value of $u_{y}$ and $u_{\varphi}$ in the $y=0$ plane. Numerical error of real part of $u_{y}$ is in the order of $10^{-5}$. The black circle in each figure represents the scatterer's boundary.

\section{2-6.4 Scattering Cross Section}

The scattering cross section is defined as the ratio of average energy flux of scattered waves to the energy flux density of the incident wave through a plane normal 


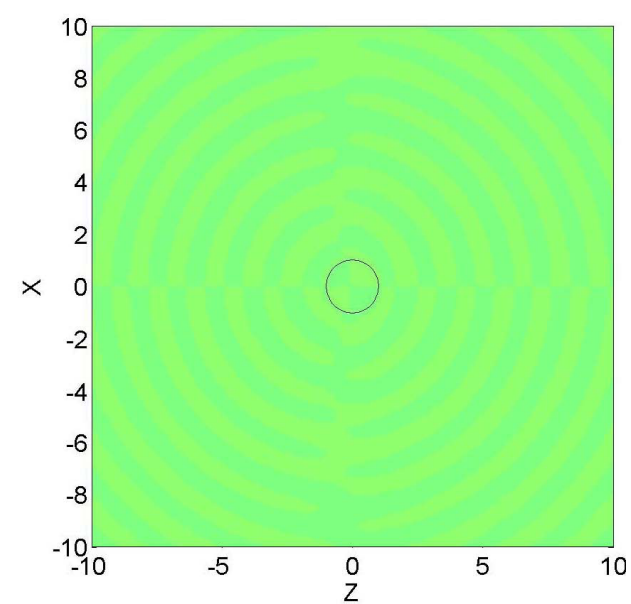

(a) Real part of $\sigma_{x y}$ (in the order of $\left.10^{4}\right)$

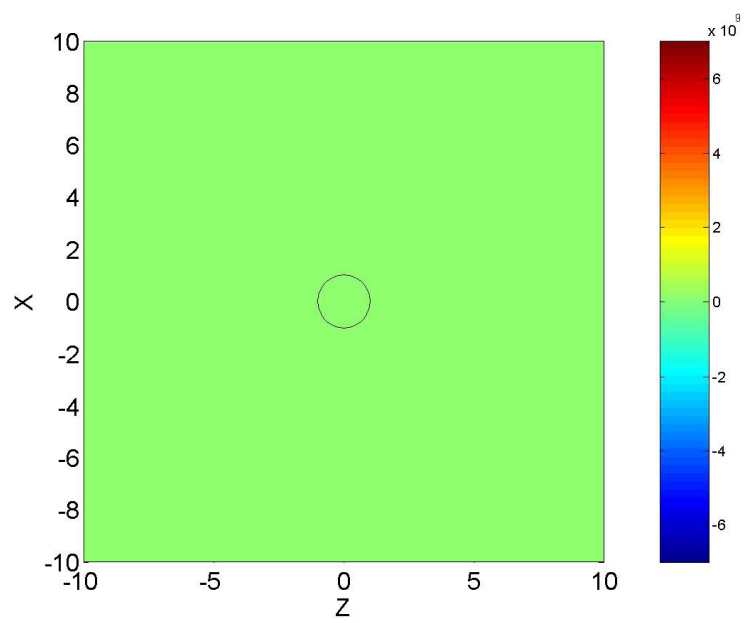

(b) Real part of $\sigma_{r \varphi}$ (exactly zero)

Figure 2.25: Zero value of real part of $\sigma_{x y}$ and $\sigma_{r \varphi}$ in the $y=0$ plane. The value of real part of $\sigma_{x y}$ is in the order of $10^{4}$ while the value of real part of $\sigma_{x x}$ is in the order of $10^{9}$. The ratio of peak values of real part of $\sigma_{x y}$ and $\sigma_{x x}$ implies that numerical errors for $\sigma_{x y}$ are in the order of $10^{-5}$. The black circle in each figure represents the scatterer's boundary.

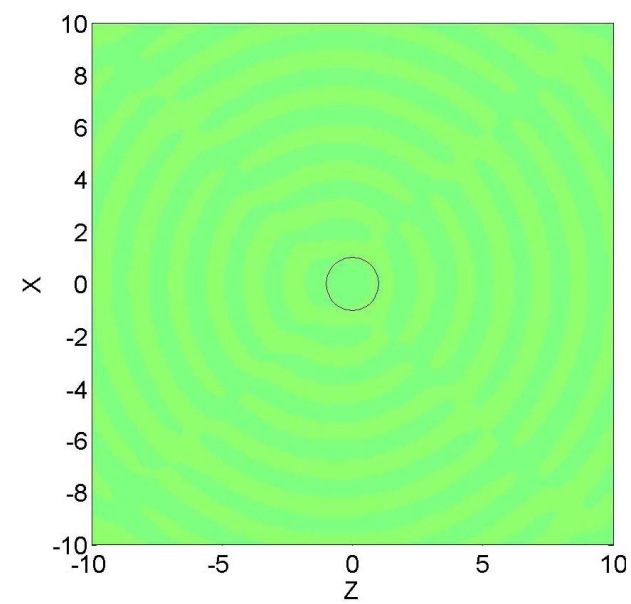

(a) Real part of $\sigma_{y z}$ (in the order of $\left.10^{4}\right)$

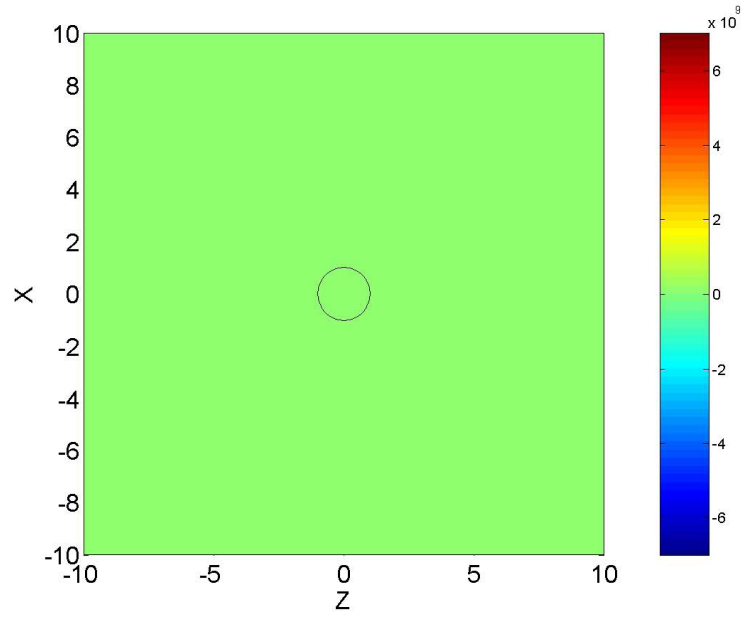

(b) Real part of $\sigma_{\theta \varphi}$ (exactly zero)

Figure 2.26: Zero value of real part of $\sigma_{y z}$ and $\sigma_{\theta \varphi}$ in the $y=0$ plane. The value of real part of $\sigma_{y z}$ is in the order of $10^{4}$ while the value of $\sigma_{x x}$ is in the order of $10^{9}$. The ratio of peak values of real part of $\sigma_{y z}$ and $\sigma_{x x}$ implies that numerical error of $\sigma_{y z}$ is in the order of $10^{-5}$. The black circle in each figure represents the scatterer's boundary. 
to the direction of the incident wave propagation ${ }^{[48]}$. The definition can be written as

$$
\gamma=\frac{\left\langle\dot{E}_{s c t}\right\rangle}{\dot{e}_{i n c}}
$$

where $\left\langle\dot{E}_{s c t}\right\rangle$ is the average energy flux of scattered waves and has the same expression as $\langle\dot{E}\rangle$ in Eq. (2-121), except that stresses and displacements are due to scattered waves; $\dot{e}_{i n c}$ is the energy flux density of the incident wave through a plane normal to the direction of propagation; and $\gamma$ has the dimension of an area. The $\left\langle\dot{E}_{s c t}\right\rangle$ can be computed using Eq. (2-127) or Eq. (2-130), except that the stresses and displacements are due to scattered waves. A normalized scattering cross section is defined such as ${ }^{[48]}$

$$
\gamma_{N}=\frac{1}{\pi a^{2}} \frac{\left\langle\dot{E}_{s c t}\right\rangle}{\dot{e}_{i n c}}
$$

where $a$ is the radius of the spherical scatterer.

For the plane incident wave $\phi$ in Eq. $(2-131)$,

$$
\begin{aligned}
\dot{e}_{i n c} & =\frac{\hat{\imath} \omega}{4} \sigma_{z z}^{*} \bar{u}_{z}^{*}=\frac{\hat{\imath} \omega}{4}(\lambda+2 \mu)(-\hat{\imath} \kappa) \\
& =\omega \kappa(\lambda+2 \mu) / 2=\rho \omega^{3} / 2 \kappa .
\end{aligned}
$$

Figure 4 of [48] is reproduced in Fig. 2.27 here. Figure 4 of [48] is digitized by using software DigXY version $1 .{ }^{[49]}$. By visually selecting a series of points along the curve, a set of $\kappa a$ and the normalized scattering cross section values are generated by the software DigXY, as listed in first two columns in Table 2.2. The numerical results of $\gamma_{N}$ in the third column in Table 2.2 is computed according to Eq. (2-147). The value of $\left\langle\dot{E}_{s c t}\right\rangle$ in Eq. (2-147) are produced numerically with Eq. (2-130) for each normalized wave number in the first column. Figure 2.27 shows a good agreement between numerical results by the present work and those by Johnson and Truell [48] For example, the difference is less than $0.8 \%$ at $\kappa a=5.47$.

Since Eq. (2-130) is used to produce $\left\langle\dot{E}_{s c t}\right\rangle$ for Fig. 2.27, Fig. 2.27 also shows that the Fibonacci numerical integration formula works well with $F=377$ for $\kappa a$ up to 


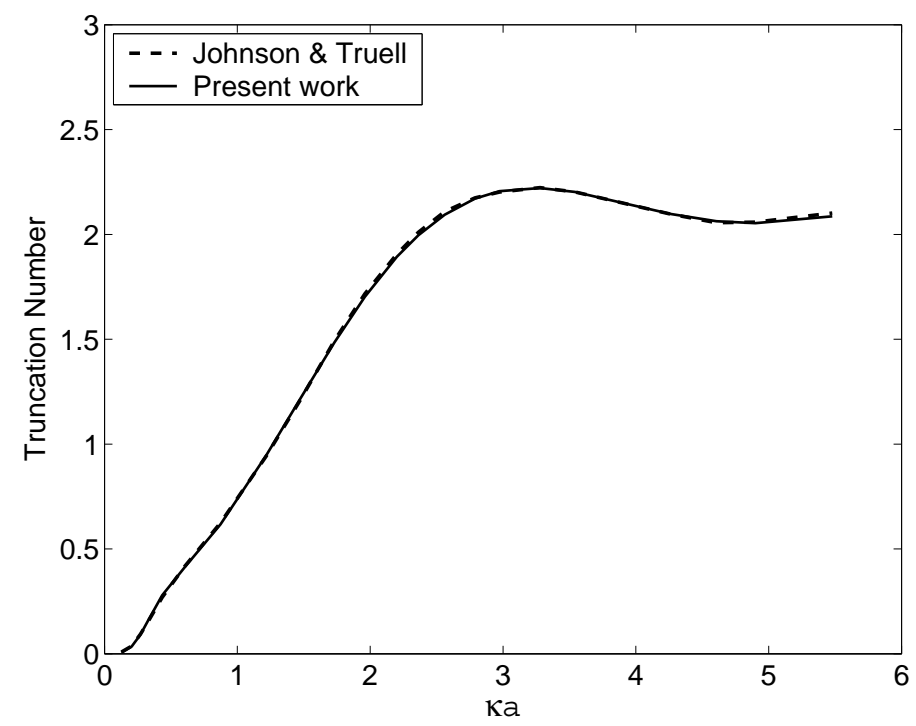

Figure 2.27: Comparison of normalized scattering cross section of a beryllium sphere in a polyethylene matrix.

\section{5 .}

\section{2-6.5 Energy Conservation}

When there is no energy dissipation or generation inside a closed surface, the energy flux across the surface should be zero, according to the energy conservation law. In this section, energy conservation law is used to verify the implementation of single scattering solution. The closed surface is chosen to either enclose the scatterer or not enclose the scatterer.

In analogy to the normalization for scattering cross section (Eq. (2-147)), the average energy flux $\langle\dot{E}\rangle$ can be normalized as

$$
\langle\dot{E}\rangle_{N}=\frac{1}{\pi R^{2}} \frac{\langle\dot{E}\rangle}{\dot{e}_{i n c}}
$$

where the subscript $N$ stands for normalization, $\dot{e}_{i n c}$ can be found in Eq. $(2-148)$ and $\langle\dot{E}\rangle$ can be computed through Eq. (2-130) for a spherical surface of radius $R$.

For the single scattering problem of a beryllium sphere in a polyethylene matrix, 
Table 2.2: Data of Normalized Scattering Cross Section in Figure 2.27

\begin{tabular}{|c|c|c|}
\hline$\kappa a$ & Johnson \& Truell $^{[48]}$ & Present Work \\
\hline 0.126882 & 0.00809786 & 0.00545173 \\
0.204763 & 0.0362266 & 0.0344299 \\
0.271038 & 0.0931373 & 0.0912684 \\
0.325382 & 0.150135 & 0.154953 \\
0.434124 & 0.268913 & 0.278371 \\
0.602849 & 0.415951 & 0.411953 \\
0.873699 & 0.62442 & 0.617054 \\
1.21739 & 0.942364 & 0.945554 \\
1.47701 & 1.21309 & 1.21990 \\
1.73078 & 1.49343 & 1.48485 \\
1.96003 & 1.71655 & 1.70236 \\
2.20084 & 1.90611 & 1.89303 \\
2.36311 & 2.01015 & 1.99614 \\
2.56112 & 2.10914 & 2.09416 \\
2.78858 & 2.17444 & 2.17065 \\
2.97384 & 2.20179 & 2.20641 \\
3.27837 & 2.22348 & 2.22135 \\
3.54065 & 2.20243 & 2.20244 \\
4.26136 & 2.09673 & 2.09781 \\
4.60694 & 2.05594 & 2.06311 \\
4.89932 & 2.05859 & 2.05387 \\
5.47854 & 2.10219 & 2.08619 \\
\hline
\end{tabular}


the longitudinal incident wave in Section 2-6.1 is used. The sphere has unit radius and is centered at the origin of global coordinate system. The normalized average energy flux in Eq. (2-149) is evaluated for two cases: first (referred as "Case 1"), the spherical surface is concentric with the scatterer; second (referred as Case 2), the spherical surface of unit radius is centered at the point with Cartesian coordinates $x=y=z=2$.

Table 2.3 shows $\langle\dot{E}\rangle_{N}$ values at different wave number $\kappa$ and radius of spherical integration surface for Case 1. TN dedicates truncation number. When the Fibonacci number $F \geq 377$ (used in Eq. $(2-130)$ ), $\langle\dot{E}\rangle_{N}$ converges to the values in Table 2.3. Table 2.4 shows $\langle\dot{E}\rangle_{N}$ values at different wave number $\kappa$ for Case 2 . When the Fibonacci number $F \geq 2584,\langle\dot{E}\rangle_{N}$ converges to the values in Table 2.4. The Fibonacci number $F$ in for Case 2 is larger than that for Case 1, possibly because value of the radius of the spherical surface in Case 2 is smaller than that in Case 1.

The $\langle\dot{E}\rangle_{N}$ is expected to be zero. The values in Tables 2.3 and 2.4 are "zero"s with acceptable numerical errors. Therefore, the energy conservation law is satisfied.

Table 2.3: Single scattering: normalized average energy flux across a closed surface enclosing the scatterer.

\begin{tabular}{|c|c|c|c|}
\hline $\mathrm{r}$ & \multicolumn{3}{|c|}{$\langle\dot{E}\rangle_{N}$} \\
\cline { 2 - 4 }$(m)$ & $\kappa=0.2\left(m^{-1}\right), T N=8$ & $\kappa=1.2\left(m^{-1}\right), T N=11$ & $\kappa=5.5\left(m^{-1}\right), T N=18$ \\
\hline 2 & $1.49634 \times 10^{-15}$ & $2.64886 \times 10^{-15}$ & $9.58003 \times 10^{-17}$ \\
3 & $9.43241 \times 10^{-16}$ & $1.30743 \times 10^{-15}$ & $4.01790 \times 10^{-17}$ \\
4 & $1.19168 \times 10^{-16}$ & $7.64833 \times 10^{-16}$ & $2.95576 \times 10^{-17}$ \\
5 & $3.45101 \times 10^{-16}$ & $1.23184 \times 10^{-16}$ & $4.28140 \times 10^{-17}$ \\
6 & $3.55080 \times 10^{-16}$ & $4.41349 \times 10^{-16}$ & $5.77322 \times 10^{-17}$ \\
7 & $2.33101 \times 10^{-17}$ & $5.87515 \times 10^{-17}$ & $6.54464 \times 10^{-17}$ \\
8 & $8.32186 \times 10^{-17}$ & $1.88870 \times 10^{-16}$ & $1.25140 \times 10^{-16}$ \\
9 & $9.13825 \times 10^{-17}$ & $4.08840 \times 10^{-18}$ & $4.28573 \times 10^{-17}$ \\
10 & $9.51467 \times 10^{-17}$ & $3.68709 \times 10^{-17}$ & $4.98138 \times 10^{-17}$ \\
\hline
\end{tabular}


Table 2.4: Single scattering: normalized average energy flux across a closed surface enclosing no scatterer.

\begin{tabular}{|c|c|c|}
\hline$\kappa\left(m^{-1}\right)$ & $T N$ & $\langle\dot{E}\rangle_{N}$ \\
\hline 0.2 & 8 & $6.05599 \times 10^{-17}$ \\
1.2 & 11 & $5.96005 \times 10^{-17}$ \\
5.5 & 18 & $2.20194 \times 10^{-17}$ \\
\hline
\end{tabular}




\section{Chapter 3}

\section{Multiple Scattering Solution}

\section{3-1 Introduction}

Multiple scattering occurs when a scattered wave is further scattered. In this thesis, multiple scattering is referred to the scattering process between multiple scatterers embedded in an infinite host medium. A plane time-harmonic incident wave propagates along an arbitrary known direction. The steady-state response at any location within the host medium is sought.

Several more assumptions are necessary for solving the problem: (1) It is assumed that all mechanical and geometrical properties of both the host medium and the scatterers are known. (2) It is assumed that the waves propagating inside the host medium and the scatterers are elastic waves. This assumption here is the same as that in Section 2-6. The scatterers can also be void or rigid. As mentioned in Section 2-6, by assigning near-zero values to material properties of the scatterer, it resembles a void inhomogeneity; and by assigning extremely large values to Lamés constants of the scatterer, it resembles a rigid inclusion. (3) It is assumed that the single scattering solution for each scatterer is known for any time-harmonic incident wave. A single 
scatterer is embedded in the same host medium as the multiple-scattering problem. The single scattering solution for each scatterer has been obtained in Chapter 2. Tmatrices for all scatterers are known, since a $T$-matrix contains all the mechanics regarding a scatterer.

\section{3-2 Physics of Multiple Scattering}

In this section, the physical process of multiple scattering in the view of ordered scattering $[22,50]$ is presented. The description in [50] is applicable to any waves and is thus adapted here. The adaptation includes a slightly different notation used to represent the waves.

Without loss of generality, a wave is denoted by a generic symbol $\Phi$, which contains 3 components: $\phi, \psi$ and $\chi$. An incident wave is denoted by $\Phi^{\text {inc }}$. The scattered wave by a scatterer, say $J$, at $p$-th order of ordered scattering process is denoted by $\Phi_{J}^{(p)}$.

The scattered wave $\Phi_{J}^{(p)}$ is called the $p$-th order scattered wave by Scatterer $J$. The term order signifies how many times an incident wave has encountered and been scattered by individual scatterers.

Fig. 3.1 depicts the incident wave and scattering at Scatterer $J$. When an incident wave $\Phi^{\text {inc }}$ impinges onto Scatterer $I$, the incident wave is scattered by Scatterer $I$ as if there were no other scatterers present. This is the first order scattered wave by Scatterer $I \Phi_{I}^{(1)}$. Every scatterer in the host medium generates a first order scattered wave. The excitation source at the first order scattering is always the incident wave. The wave $\Phi_{I}^{(1)}$ subsequently impinges onto a nearby Scatterer $J$, and then a second order scattered wave by Scatterer $J$ due to $\Phi_{I}^{(1)}$ is generated. Specifically, it is denoted as $\Phi_{J \text { due to } \Phi_{I}^{(1)}}^{(2)}$.

In the steady state, all the first order scattered waves, except the one scattered 


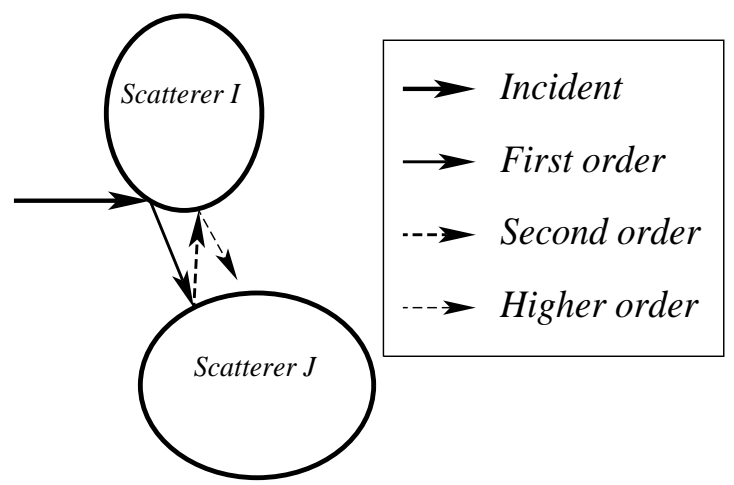

Figure 3.1: Multiple scattering of a single wave between two scatterers

by Scatterer $I$ itself, impinge upon Scatterer $I$. Fig. 3.2 presents a global view of the first two orders of scattering. Therefore, the (total) second order scattered wave by Scatterer $I$ is a summation of all the second order waves scattered by Scatterer $I$ :

$$
\Phi_{I}^{(2)}=\sum_{\substack{J=1 \\ J \neq I}}^{N} \Phi_{I \text { due to } \Phi_{J}^{(1)}}^{(2)}
$$

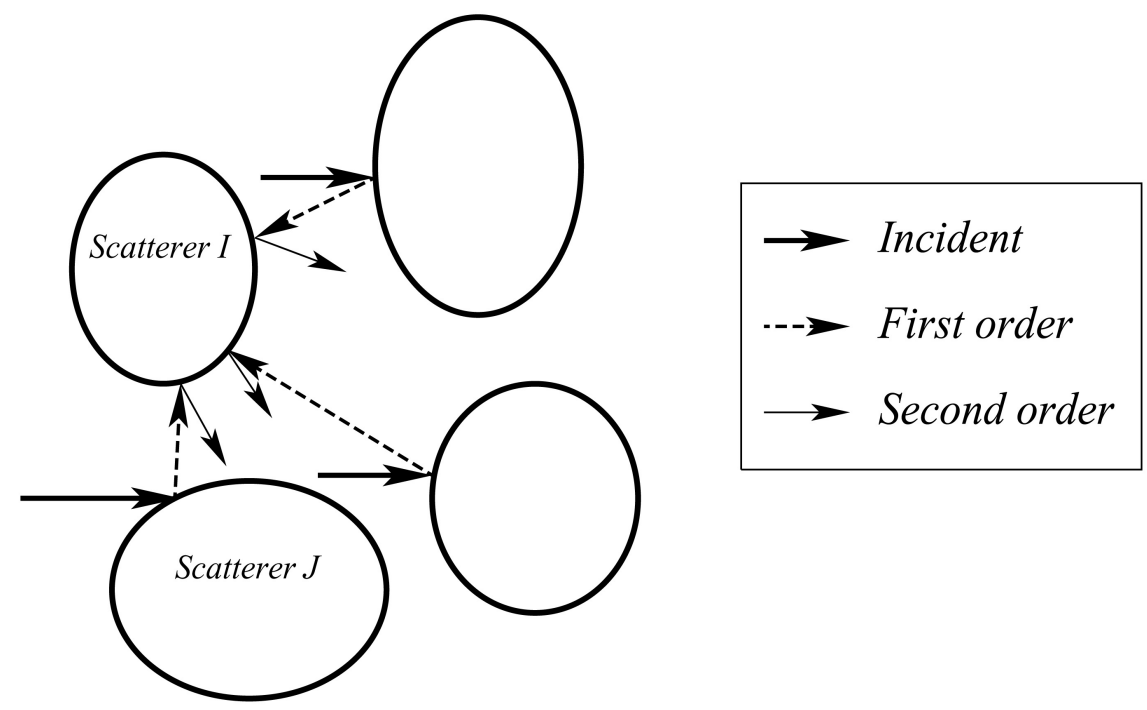

Figure 3.2: Multiple scattering among a group of scatterers

Generalization to any higher order is in the same manner. All the $(p-1)$-th order scattered waves, except the one scattered by Scatterer $I$ itself, impinge upon Scatterer 
I. The total $p$-th order scattered wave scattered by Scatterer $I$ can be found as

$$
\Phi_{I}^{(p)}=\sum_{\substack{J=1 \\ J \neq I}}^{N} \Phi_{I \text { due to } \Phi_{J}^{(p-1)}}^{(p)}
$$

\section{3-3 Addition Theorem}

In the formulation for the multiple scattering process, the basic idea is that each scattered wave is further scattered by other scatterers in the field, and each of this scattering process can be described by the super $T$-matrix of the scatterer that causes the scattering. Since the single scattering problem leading to the super $T$-matrix is defined in each scatterer's local coordinate system, it is necessary to convert the waves expressed in the local coordinate system of one scatterer, say Scatterer $I$, into the local coordinate system of anther scatterer, say Scatterer $J$. Before proceeding to the multiple scattering formulation, the coordinate transformation is discussed.

As shown in Fig. 2.1, a spherical coordinate system is built with reference to a Cartesian coordinate system. In this thesis, $X$ axes of all Cartesian systems are parallel, $Y$ axes of all Cartesian systems are parallel, and $Z$ axes of all Cartesian systems are parallel. Therefore, coordinate transformation involves translation only.

\section{3-3.1 Scalar Addition Theorem}

Figure 3.3 is used to introduce addition theorem. The position $O$ is the origin of the global coordinate system and the position $O^{\prime}$ is the origin of a local coordinate system. Three vectors $\boldsymbol{r}, \boldsymbol{r}^{\prime}$ and $\boldsymbol{r}^{\prime \prime}$ are related by

$$
\boldsymbol{r}(r, \theta, \varphi)=\boldsymbol{r}^{\prime}\left(r^{\prime}, \theta^{\prime}, \varphi^{\prime}\right)+\boldsymbol{r}^{\prime \prime}\left(r^{\prime \prime}, \theta^{\prime \prime}, \varphi^{\prime \prime}\right)
$$

where $\left(r^{\prime \prime}, \theta^{\prime \prime}, \varphi^{\prime \prime}\right)$ are coordinates of position $O^{\prime}$ in the global spherical coordinate system, $(r, \theta, \varphi)$ and $\left(r^{\prime}, \theta^{\prime}, \varphi^{\prime}\right)$ are coordinates of position $P$ in the global and local 


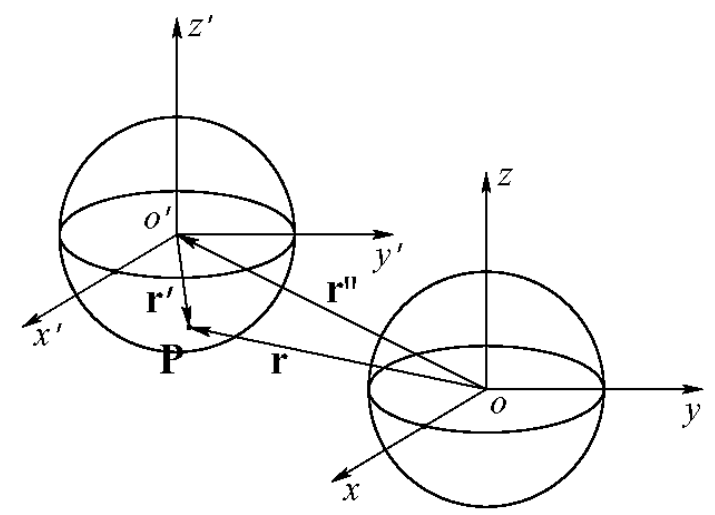

Figure 3.3: Vectors $\boldsymbol{r}=\boldsymbol{r}^{\prime}+\boldsymbol{r}^{\prime \prime}$ for addition theorem

spherical coordinate systems respectively.

The following relations for spherical wave functions exist ${ }^{[51]}$ :

$$
\mathcal{Z}_{n}(k r) Y_{n}^{m}(\theta, \varphi)= \begin{cases}\sum_{\nu=0}^{\infty} \sum_{\mu=-\nu}^{\nu} \alpha_{n m, \nu \mu}\left(\boldsymbol{r}^{\prime \prime}\right) j_{\nu}\left(k r^{\prime}\right) Y_{\nu}^{\mu}\left(\theta^{\prime}, \varphi^{\prime}\right) & \text { for } r^{\prime}<r^{\prime \prime} \\ \sum_{\nu=0}^{\infty} \sum_{\mu=-\nu}^{\nu} \beta_{n m, \nu \mu}\left(\boldsymbol{r}^{\prime \prime}\right) z_{\nu}\left(k r^{\prime}\right) Y_{\nu}^{\mu}\left(\theta^{\prime}, \varphi^{\prime}\right) & \text { for } r^{\prime}>r^{\prime \prime}\end{cases}
$$

where $\mathcal{Z}_{n}(\cdot)$ is any of the spherical Bessel functions: the spherical Bessel functions of the first kind $j_{n}(\cdot)$, the spherical Bessel functions of the second kind $y_{n}(\cdot)$, the spherical Bessel functions of the third kind $h_{n}^{(1)}(\cdot)$ and $h_{n}^{(2)}(\cdot)^{[38]}$; and

$$
\begin{aligned}
\beta_{n m, \nu \mu}\left(\boldsymbol{r}^{\prime \prime}\right)= & (-1)^{m} \hat{\imath}^{\nu-n} \sqrt{4 \pi(2 n+1)(2 \nu+1)} \cdot \sum_{s=0}^{\infty} \hat{\imath}^{s} \sqrt{2 s+1} \\
& \left(\begin{array}{ccc}
n & \nu & s \\
0 & 0 & 0
\end{array}\right)\left(\begin{array}{ccc}
n & \nu & s \\
-m & s & m-s
\end{array}\right) j_{s}\left(k r^{\prime \prime}\right) Y_{s}^{m-\mu}\left(\theta^{\prime \prime}, \varphi^{\prime \prime}\right) \\
\alpha_{n m, \nu \mu}\left(\boldsymbol{r}^{\prime \prime}\right)= & (-1)^{m} \hat{\imath}^{\nu-n} \sqrt{4 \pi(2 n+1)(2 \nu+1)} \cdot \sum_{s=0}^{\infty} \hat{\imath}^{s} \sqrt{2 s+1} \\
& \left(\begin{array}{lll}
n & \nu & s \\
0 & 0 & 0
\end{array}\right)\left(\begin{array}{ccc}
n & \nu & s \\
-m & \mu & m-\mu
\end{array}\right) z_{s}\left(k r^{\prime \prime}\right) Y_{s}^{m-\mu}\left(\theta^{\prime \prime}, \varphi^{\prime \prime}\right)
\end{aligned}
$$

The ensemble $\left(\begin{array}{ccc}j_{1} & j_{2} & j_{3} \\ m_{1} & m_{2} & m_{3}\end{array}\right)$ is called the Wigner 3- $j$ symbol ${ }^{[52]}$, which is defined 
as $[52]$

$$
\left(\begin{array}{ccc}
j_{1} & j_{2} & j_{3} \\
m_{1} & m_{2} & m_{3}
\end{array}\right)=\frac{(-1)^{j_{1}-j_{2}-m_{3}}}{\sqrt{2 j_{3}+1}}\left\langle j_{1} j_{2} m_{1} m_{2} \mid j_{1} j_{2} j_{3}\left(-m_{3}\right)\right\rangle
$$

The ensemble $\left\langle j_{1} j_{2} m_{1} m_{2} \mid j_{1} j_{2} j_{3}\left(-m_{3}\right)\right\rangle$ is called the Clebsch-Gordon coefficient, which is defined as ${ }^{[38]}$

$$
\begin{aligned}
\left\langle j_{1} j_{2} m_{1} m_{2}\right| & \left.j_{1} j_{2} j_{3}\left(-m_{3}\right)\right\rangle=\delta_{\left(-m_{3}\right)\left(m_{1}+m_{2}\right)} \cdot \sqrt{\frac{\left(j_{1}+j_{2}-j_{3}\right) !\left(j_{1}-j_{2}+j_{3}\right) !\left(-j_{1}+j_{2}+j_{3}\right) !\left(2 j_{3}+1\right)}{\left(j_{1}+j_{2}+j_{3}+1\right) !}} \\
& \cdot \sum_{l=0}^{\infty} \frac{(-1)^{l} \sqrt{\left(j_{1}+m_{1}\right) !\left(j_{1}-m_{1}\right) !\left(j_{2}+m_{2}\right) !\left(j_{2}-m_{2}\right) !\left(j_{3}+m_{3}\right) !\left(j_{3}-m_{3}\right) !}}{l !\left(j_{1}+j_{2}-j_{3}-l\right) !\left(j_{1}-m_{1}-l\right) !\left(j_{2}+m_{2}-l\right) !\left(-j_{2}+j_{3}+m_{1}+l\right) !\left(-j_{1}+j_{3}-m_{2}+l\right) !}
\end{aligned}
$$

Now, consider transformation between two local coordinate systems. Figure 3.4

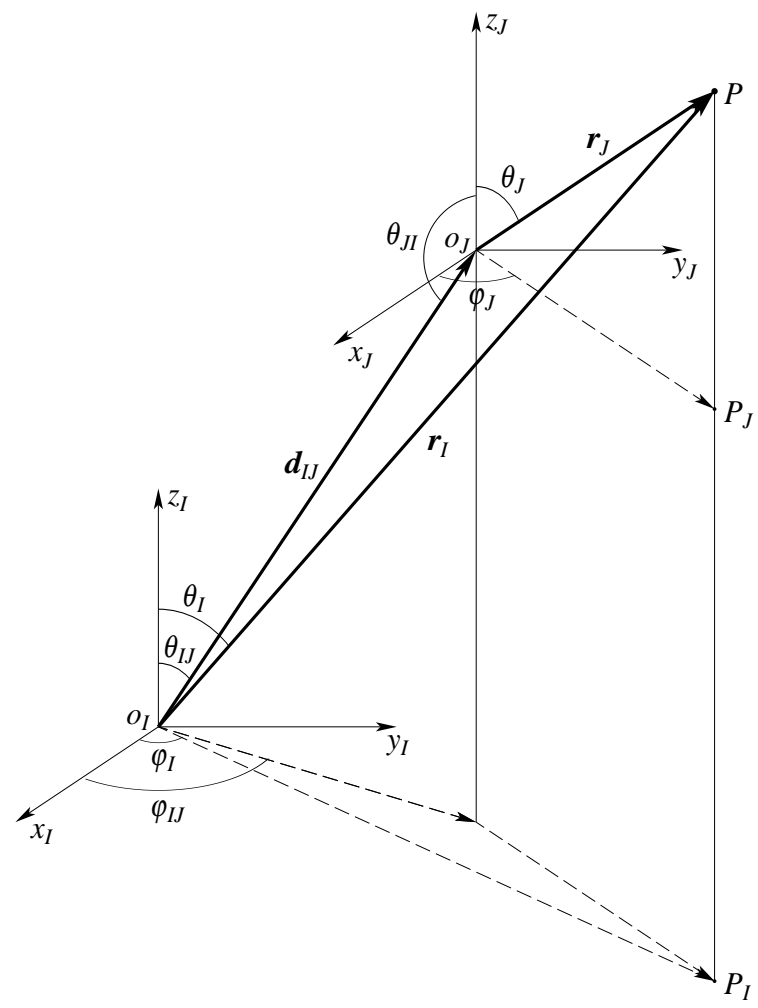

Figure 3.4: Local coordinate systems belonging to Scatterers $I$ and $J$ and arbitrary field point $P$. Dotted arrows represent projections of position vectors on horizontal planes

depicts the geometric relation between two local coordinate systems belonging to Scatterers $I$ and $J$ and an arbitrary field point $P$. For the illustration convenience, 
local Cartesian coordinate systems are also shown.

The origin of Scatterer I's local coordinate system is denoted as $o_{I}$. The origin of Scatterer $J$ 's local coordinate system is denoted as $o_{J}$, which can be located by vector $\boldsymbol{d}_{I J}\left(d_{I J}, \theta_{I J}, \varphi_{I J}\right)$ in the $o_{I}$ coordinate system. The field point $P$ can be located by either $\boldsymbol{r}_{I}\left(r_{I}, \theta_{I}, \varphi_{I}\right)$ in the $o_{I}$ coordinate system or $\boldsymbol{r}_{J}\left(r_{J}, \theta_{J}, \varphi_{J}\right)$ in the $o_{J}$ coordinate system. Let

$$
\boldsymbol{r}_{I}\left(r_{I}, \theta_{I}, \varphi_{I}\right)=\boldsymbol{r}, \quad \boldsymbol{r}_{J}\left(r_{J}, \theta_{J}, \varphi_{J}\right)=\boldsymbol{r}^{\prime}, \quad \text { and } \quad \boldsymbol{d}_{I J}\left(d_{I J}, \theta_{I J}, \varphi_{I J}\right)=\boldsymbol{r}^{\prime \prime}
$$

then, the scalar addition theorem in eqn. (3-4) becomes

$$
\begin{aligned}
j_{n}\left(k r_{I}\right) Y_{n}^{m}\left(\theta_{I}, \varphi_{I}\right) & =\sum_{\nu=0}^{\infty} \sum_{\mu=-\nu}^{\nu} \beta_{n m, \nu \mu}\left(\boldsymbol{d}_{I J}\right) j_{\nu}\left(k r_{J}\right) Y_{\nu}^{\mu}\left(\theta_{J}, \varphi_{J}\right) \\
h_{n}^{(1)}\left(k r_{I}\right) Y_{n}^{m}\left(\theta_{I}, \varphi_{I}\right) & =\sum_{\nu=0}^{\infty} \sum_{\mu=-\nu}^{\nu} \beta_{n m, \nu \mu}\left(\boldsymbol{d}_{I J}\right) h_{\nu}^{(1)}\left(k r_{J}\right) Y_{\nu}^{\mu}\left(\theta_{J}, \varphi_{J}\right), \text { for } r_{J}>d_{I J} \\
h_{n}^{(1)}\left(k r_{I}\right) Y_{n}^{m}\left(\theta_{I}, \varphi_{I}\right) & =\sum_{\nu=0}^{\infty} \sum_{\mu=-\nu}^{\nu} \alpha_{n m, \nu \mu}\left(\boldsymbol{d}_{I J}\right) j_{\nu}\left(k r_{J}\right) Y_{\nu}^{\mu}\left(\theta_{J}, \varphi_{J}\right), \text { for } r_{J}<d_{I J}
\end{aligned}
$$

where $\beta_{n m, \nu \mu}\left(\boldsymbol{d}_{I J}\right)$ and $\alpha_{n m, \nu \mu}\left(\boldsymbol{d}_{I J}\right)$ take the following forms:

$$
\begin{aligned}
\beta_{n m, \nu \mu}\left(\boldsymbol{r}^{\prime \prime}\right)= & (-1)^{m} \hat{\imath}^{\nu-n} \sqrt{4 \pi(2 n+1)(2 \nu+1)} \cdot \sum_{s=0}^{\infty} \hat{\imath}^{s} \sqrt{2 s+1} \\
& \left(\begin{array}{ccc}
n & \nu & s \\
0 & 0 & 0
\end{array}\right)\left(\begin{array}{ccc}
n & \nu & s \\
-m & s & m-s
\end{array}\right) j_{s}\left(k d_{I J}\right) Y_{s}^{m-\mu}\left(\theta_{I J}, \varphi_{I J}\right) \\
\alpha_{n m, \nu \mu}\left(\boldsymbol{d}_{I J}\right)= & (-1)^{m} \hat{\imath}^{\nu-n} \sqrt{4 \pi(2 n+1)(2 \nu+1)} \cdot \sum_{s=0}^{\infty} \hat{\imath}^{s} \sqrt{2 s+1} \\
& \left(\begin{array}{lll}
n & \nu & s \\
0 & 0 & 0
\end{array}\right)\left(\begin{array}{ccc}
n & \nu & s \\
-m & \mu & m-\mu
\end{array}\right) h_{s}^{(1)}\left(k d_{I J}\right) Y_{s}^{m-\mu}\left(\theta_{I J}, \varphi_{I J}\right)
\end{aligned}
$$

Note that for eqn. (3-8a) there is no restriction about its applicability since both expressions on the right-hand side of eqn. (3-4) are identical.

Use the packing method in eqn. (2-89) to pack two pairs of indices ( $n$ and $m, \nu$ 
and $\mu$ ) into two single indices ( $i$ and $j$ ):

$$
i=n^{2}+n+m, \quad j=\nu^{2}+\nu+\mu .
$$

Then, the wave expansion bases defined in Eqs. (2-87) and (2-90) can be transformed between two coordinate systems using the following matrix notation:

$$
\begin{aligned}
\left\{\boldsymbol{j}\left(k ; \boldsymbol{r}_{I}\right)\right\} & =\left[R g \boldsymbol{R}_{I J}\left(k ; \boldsymbol{d}_{I J}\right)\right]\left\{\boldsymbol{j}\left(k ; \boldsymbol{r}_{J}\right)\right\} \\
\left\{\boldsymbol{h}\left(k ; \boldsymbol{r}_{I}\right)\right\} & =\left\{\begin{array}{lll}
{\left[R g \boldsymbol{R}_{I J}\left(k ; \boldsymbol{d}_{I J}\right)\right]\left\{\boldsymbol{h}\left(k ; \boldsymbol{r}_{J}\right)\right\},} & \text { for } & r_{J}>d_{I J} \\
{\left[\boldsymbol{R}_{I J}\left(k ; \boldsymbol{d}_{I J}\right)\right]\left\{\boldsymbol{j}\left(k ; \boldsymbol{r}_{J}\right)\right\},} & \text { for } & r_{J}<d_{I J}
\end{array}\right.
\end{aligned}
$$

where entries for matrix $\left[\boldsymbol{R}_{I J}\left(k ; \boldsymbol{d}_{I J}\right)\right]$ and $\left[R g \boldsymbol{R}_{I J}\left(k ; \boldsymbol{d}_{I J}\right)\right]$ at the $i$-th row and the $j$-th column are

$$
\begin{aligned}
{\left[\boldsymbol{R}_{I J}\left(k ; \boldsymbol{d}_{I J}\right)\right]_{i j}=} & (-1)^{m} \hat{\imath}^{\nu-n} \sqrt{4 \pi(2 n+1)(2 \nu+1)} \cdot \sum_{s=0}^{\infty} \hat{\imath}^{s} \sqrt{2 s+1} . \\
& \left(\begin{array}{ccc}
n & \nu & s \\
0 & 0 & 0
\end{array}\right)\left(\begin{array}{ccc}
n & \nu & s \\
-m & \mu & m-\mu
\end{array}\right) Y_{s}^{m-\mu}\left(\theta_{I J}, \varphi_{I J}\right) h_{s}^{(1)}\left(k d_{I J}\right) \\
{\left[R g \boldsymbol{R}_{I J}\left(k ; \boldsymbol{d}_{I J}\right)\right]_{i j}=} & (-1)^{m} \hat{\imath}^{\nu-n} \sqrt{4 \pi(2 n+1)(2 \nu+1)} \cdot \sum_{s=0}^{\infty} \hat{\imath}^{s} \sqrt{2 s+1} \cdot \\
& \left(\begin{array}{ccc}
n & \nu & s \\
0 & 0 & 0
\end{array}\right)\left(\begin{array}{ccc}
n & \nu & s \\
-m & \mu & m-\mu
\end{array}\right) Y_{s}^{m-\mu}\left(\theta_{I J}, \varphi_{I J}\right) j_{s}\left(k d_{I J}\right)
\end{aligned}
$$

A similar set of coordinate transformation relations exists for the cases when the wave number is $\kappa$, instead of $k$ :

$$
\begin{aligned}
\left\{\boldsymbol{j}\left(\kappa ; \boldsymbol{r}_{I}\right)\right\} & =\left[R g \boldsymbol{R}_{I J}\left(\kappa ; \boldsymbol{d}_{I J}\right)\right]\left\{\boldsymbol{j}\left(\kappa ; \boldsymbol{r}_{J}\right)\right\} \\
\left\{\boldsymbol{h}\left(\kappa ; \boldsymbol{r}_{I}\right)\right\} & =\left\{\begin{array}{lll}
{\left[R g \boldsymbol{R}_{I J}\left(\kappa ; \boldsymbol{d}_{I J}\right)\right]\left\{\boldsymbol{h}\left(\kappa ; \boldsymbol{r}_{J}\right)\right\},} & \text { for } & r_{J}>d_{I J} \\
{\left[\boldsymbol{R}_{I J}\left(\kappa ; \boldsymbol{d}_{I J}\right)\right]\left\{\boldsymbol{j}\left(\kappa ; \boldsymbol{r}_{J}\right)\right\},} & \text { for } & r_{J}<d_{I J}
\end{array}\right.
\end{aligned}
$$

where entries for matrix $\left[\boldsymbol{R}_{I J}\left(\kappa ; \boldsymbol{d}_{I J}\right)\right]$ and $\left[R g \boldsymbol{R}_{I J}\left(\kappa ; \boldsymbol{d}_{I J}\right)\right]$ at the $i$-th row and the $j$-th column are

$$
\left[\boldsymbol{R}_{I J}\left(\kappa ; \boldsymbol{d}_{I J}\right)\right]_{i j}=(-1)^{m} \hat{\imath}^{\nu-n} \sqrt{4 \pi(2 n+1)(2 \nu+1)} \cdot \sum_{s=0}^{\infty} \hat{\imath}^{s} \sqrt{2 s+1} .
$$




$$
\begin{aligned}
& \left(\begin{array}{lll}
n & \nu & s \\
0 & 0 & 0
\end{array}\right)\left(\begin{array}{ccc}
n & \nu & s \\
-m & \mu & m-\mu
\end{array}\right) Y_{s}^{m-\mu}\left(\theta_{I J}, \varphi_{I J}\right) h_{s}^{(1)}\left(\kappa d_{I J}\right) \\
& {\left[R g \boldsymbol{R}_{I J}\left(\kappa ; \boldsymbol{d}_{I J}\right)\right]_{i j}=(-1)^{m} \hat{\imath}^{\nu-n} \sqrt{4 \pi(2 n+1)(2 \nu+1)} \cdot \sum_{s=0}^{\infty} \hat{\imath}^{s} \sqrt{2 s+1} \text {. }} \\
& \left(\begin{array}{lll}
n & \nu & s \\
0 & 0 & 0
\end{array}\right)\left(\begin{array}{ccc}
n & \nu & s \\
-m & \mu & m-\mu
\end{array}\right) Y_{s}^{m-\mu}\left(\theta_{I J}, \varphi_{I J}\right) j_{s}\left(\kappa d_{I J}\right)
\end{aligned}
$$

\section{3-3.2 Vector Addition Theorem}

During coordinate transformation, it is expected that the displacement vector and surface traction in a new coordinate system should be the same as those in the old coordinate system. When these two coordinate systems do not share the same origins, a vector addition theorem can help the coordinate transformation. The displacement vector in Eq. (2-53) is used to introduce the vector addition theorem. Prior to introducing the vector addition theorem, the vector wave functions in Eq.(2-54) are repeated here. Let

$$
\begin{array}{ll}
\zeta_{n m}(\kappa \boldsymbol{r})=\mathcal{Z}_{n}(\kappa r) Y_{n}^{m}(\theta, \varphi) & \text { for longitudinal waves, } \\
\xi_{n m}(k \boldsymbol{r})=\mathcal{Z}_{n}(k r) Y_{n}^{m}(\theta, \varphi) & \text { for shear waves. }
\end{array}
$$

The vector wave functions are defined as ${ }^{[53]}$

$$
\begin{aligned}
\boldsymbol{L}_{n m}(\kappa r) & =\nabla \zeta_{n m}(\kappa \boldsymbol{r}) \\
\boldsymbol{M}_{n m}(k r) & =\nabla \times\left[\boldsymbol{r} \xi_{n m}(k \boldsymbol{r})\right] \\
\boldsymbol{N}_{n m}(k r) & =\frac{1}{k} \nabla \times \boldsymbol{M}_{n m}(k r)
\end{aligned}
$$

Their expressions in terms of spherical vector harmonics are introduced in Eq. (2-54), which are repeated here

$$
\boldsymbol{L}_{n m}(\kappa r)=\mathfrak{E}_{1 q}^{1 t}(r, n) \mathfrak{P}_{n m}+\mathfrak{E}_{2 q}^{1 t}(r, n) \sqrt{n(n+1)} \mathfrak{B}_{n m}
$$




$$
\begin{aligned}
& \boldsymbol{M}_{n m}(k r)=\mathfrak{E}_{2 q}^{2 t}(r, n) \sqrt{n(n+1)} \mathfrak{C}_{n m} \\
& \boldsymbol{N}_{n m}(k r)=\mathfrak{E}_{1 q}^{3 t}(r, n) \mathfrak{P}_{n m}+\mathfrak{E}_{2 q}^{3 t}(r, n) \sqrt{n(n+1)} \mathfrak{B}_{n m}
\end{aligned}
$$

The derivation from Eq. (3-16) leading to Eq. (3-17) is detailed in Appendix H. The displacement vector can then be expressed in terms of spherical vector harmonics in Eq. (2-55), which is repeated here

$$
\boldsymbol{u}=\sum_{n=0}^{\infty} \sum_{m=-n}^{n}\left\{a_{m n}^{\phi} \boldsymbol{L}_{n m}(\kappa r)+a_{m n}^{\psi} \boldsymbol{M}_{n m}(k r)+a_{m n}^{\chi} \boldsymbol{N}_{n m}(k r)\right\}
$$

Comparing Eq. (3-18) with Eq. (2-37) for the part corresponding to shear wave $\chi$,

$$
\ell \nabla \times \nabla \times\left(r \chi \boldsymbol{e}_{r}\right)=\ell \nabla \times \nabla \times(\chi \boldsymbol{r})=\sum_{n=0}^{\infty} \sum_{m=-n}^{n} a_{m n}^{\chi} \boldsymbol{N}_{n m}(k r)
$$

Following Eqs. (3-16c) and (3-16b),

$$
\begin{aligned}
\ell \nabla \times \nabla \times(\chi \boldsymbol{r}) & =\sum_{n=0}^{\infty} \sum_{m=-n}^{n} a_{m n}^{\chi} \frac{1}{k} \nabla \times \boldsymbol{M}_{n m}(k r) \\
& =\sum_{n=0}^{\infty} \sum_{m=-n}^{n} a_{m n}^{\chi} \frac{1}{k} \nabla \times \nabla \times\left[\boldsymbol{r} \xi_{n m}(k \boldsymbol{r})\right] \\
& =\frac{1}{k} \sum_{n=0}^{\infty} \sum_{m=-n}^{n} \nabla \times \nabla \times\left[\boldsymbol{r} a_{m n}^{\chi} \xi_{n m}(k \boldsymbol{r})\right] \\
& =\frac{1}{k} \nabla \times \nabla \times\left[\boldsymbol{r} \sum_{n=0}^{\infty} \sum_{m=-n}^{n} a_{m n}^{\chi} \xi_{n m}(k \boldsymbol{r})\right]
\end{aligned}
$$

Following Eq. (2-42c) that is

$$
\begin{gathered}
\chi=\sum_{n=0}^{\infty} \sum_{m=-n}^{n} a_{m n}^{\chi} \mathcal{Z}_{n}(k r) Y_{n}^{m}(\theta, \varphi)=\sum_{n=0}^{\infty} \sum_{m=-n}^{n} a_{m n}^{\chi} \xi_{n m}(k \boldsymbol{r}), \\
\ell \nabla \times \nabla \times(\chi \boldsymbol{r})=\frac{1}{k} \nabla \times \nabla \times(\boldsymbol{r} \chi) .
\end{gathered}
$$

For Eq. (3-21) to be true, the $\ell$ in Eq. (2-9) takes the value of $\frac{1}{k}$ :

$$
\ell=\frac{1}{k}
$$

Correspondingly, the $\ell$ in functions $\mathfrak{E}_{p q}^{s t}(r, n)$ in Appendix E is replaced by $\frac{1}{k}$ when the vector wave function $\boldsymbol{N}_{n m}(k r)$ is used. 
Referring to two coordinate systems in Fig. 3.3, the displacement vector in Eq. (3-18) is expressed in the global coordinate system, and it can also be expressed in the local coordinate system. In analogy to Eq. (2-42), assume three displacement potentials in the local coordinate system are

$$
\begin{aligned}
\phi^{\prime}\left(r^{\prime}, \theta^{\prime}, \varphi^{\prime}\right) & =\sum_{n=0}^{\infty} \sum_{m=-n}^{n} a_{m n}^{\phi^{\prime}} \mathcal{Z}_{n}\left(\kappa r^{\prime}\right) Y_{n}^{m}\left(\theta^{\prime}, \varphi^{\prime}\right) \\
\psi^{\prime}\left(r^{\prime}, \theta^{\prime}, \varphi^{\prime}\right) & =\sum_{n=0}^{\infty} \sum_{m=-n}^{n} a_{m n}^{\psi^{\prime}} \mathcal{Z}_{n}\left(k r^{\prime}\right) Y_{n}^{m}\left(\theta^{\prime}, \varphi^{\prime}\right) \\
\chi^{\prime}\left(r^{\prime}, \theta^{\prime}, \varphi^{\prime}\right) & =\sum_{n=0}^{\infty} \sum_{m=-n}^{n} a_{m n}^{\chi_{m}^{\prime}} \mathcal{Z}_{n}\left(k r^{\prime}\right) Y_{n}^{m}\left(\theta^{\prime}, \varphi^{\prime}\right)
\end{aligned}
$$

where $a_{m n}^{\phi^{\prime}}, a_{m n}^{\psi^{\prime}}$ and $a_{m n}^{\chi^{\prime}}$ are wave expansion coefficients. Let

$$
\begin{array}{ll}
\zeta_{n m}\left(\kappa \boldsymbol{r}^{\prime}\right)=\mathcal{Z}_{n}\left(\kappa r^{\prime}\right) Y_{n}^{m}\left(\theta^{\prime}, \varphi^{\prime}\right) & \text { for longitudinal waves, } \\
\xi_{n m}\left(k \boldsymbol{r}^{\prime}\right)=\mathcal{Z}_{n}\left(k r^{\prime}\right) Y_{n}^{m}\left(\theta^{\prime}, \varphi^{\prime}\right) & \text { for shear waves. }
\end{array}
$$

Following definitions in Eq. (3-16), the vector wave functions in the local coordinate system are

$$
\begin{aligned}
\boldsymbol{L}_{n m}\left(\kappa r^{\prime}\right) & =\nabla \zeta_{n m}\left(\kappa \boldsymbol{r}^{\prime}\right) \\
\boldsymbol{M}_{n m}\left(k r^{\prime}\right) & =\nabla \times\left[\boldsymbol{r}^{\prime} \xi_{n m}\left(k \boldsymbol{r}^{\prime}\right)\right] \\
\boldsymbol{N}_{n m}\left(k r^{\prime}\right) & =\frac{1}{k} \nabla \times \boldsymbol{M}_{n m}\left(k r^{\prime}\right)
\end{aligned}
$$

Following the same procedure of deriving Eq. (3-18) in Section 2-3, the displacement vector in the local coordinate system can be written as

$$
\boldsymbol{u}=\sum_{n=0}^{\infty} \sum_{m=-n}^{n}\left\{a_{m n}^{\phi^{\prime}} \boldsymbol{L}_{n m}\left(\kappa r^{\prime}\right)+a_{m n}^{\psi^{\prime}} \boldsymbol{M}_{n m}\left(k r^{\prime}\right)+a_{m n}^{\chi^{\prime}} \boldsymbol{N}_{n m}\left(k r^{\prime}\right)\right\}
$$

By comparing Eqs. (3-5a) and (3-5b), it is noted that $\beta_{n m, \nu \mu}\left(\boldsymbol{r}^{\prime \prime}\right)$ is a special form of $\alpha_{n m, \nu \mu}\left(\boldsymbol{r}^{\prime \prime}\right)$ when $z_{s}\left(\kappa r^{\prime \prime}\right)$ takes the form of $j_{s}\left(\kappa r^{\prime \prime}\right)$. In general, Eq. (3-4) can be written as

$$
\zeta_{n m}(\kappa \boldsymbol{r})=\sum_{\nu=0}^{\infty} \sum_{\mu=-\nu}^{\nu} \alpha_{n m, \nu \mu}\left(\boldsymbol{r}^{\prime \prime}\right) \zeta_{\nu \mu}\left(\kappa \boldsymbol{r}^{\prime}\right)
$$


The following relation is immediately obtained from Eq. $(3-27)^{[54]}$.

$$
\nabla \zeta_{n m}(\kappa \boldsymbol{r})=\sum_{\nu=0}^{\infty} \sum_{\mu=-\nu}^{\nu} \alpha_{n m, \nu \mu}\left(\boldsymbol{r}^{\prime \prime}\right) \nabla \zeta_{\nu \mu}\left(\kappa \boldsymbol{r}^{\prime}\right)
$$

Following the definition in Eq. (3-16a),

$$
\boldsymbol{L}_{n m}(\kappa r)=\sum_{\nu=0}^{\infty} \sum_{\mu=-\nu}^{\nu} \alpha_{n m, \nu \mu}\left(\boldsymbol{r}^{\prime \prime}\right) \boldsymbol{L}_{\nu \mu}\left(\kappa r^{\prime}\right)
$$

Now consider $\boldsymbol{M}_{n m}(k r)$ in Eq. (3-16b). Equation (3-16b) can be rewritten as

$$
\begin{aligned}
\boldsymbol{M}_{n m}(k r) & =\sum_{\nu=0}^{\infty} \sum_{\mu=-\nu}^{\nu}\left[\nabla \xi_{\nu \mu}\left(k \boldsymbol{r}^{\prime}\right) \times\left(\boldsymbol{r}^{\prime}+\boldsymbol{r}^{\prime \prime}\right)\right] \alpha_{n m, \nu \mu}\left(\boldsymbol{r}^{\prime \prime}\right) \\
& =\sum_{\nu=0}^{\infty} \sum_{\mu=-\nu}^{\nu}\left\{\nabla \times\left[\boldsymbol{r}^{\prime} \xi_{\nu \mu}\left(k \boldsymbol{r}^{\prime}\right)\right]+\nabla \xi_{\nu \mu}\left(k \boldsymbol{r}^{\prime}\right) \times \boldsymbol{r}^{\prime \prime}\right\} \alpha_{n m, \nu \mu}\left(\boldsymbol{r}^{\prime \prime}\right)
\end{aligned}
$$

Following the definition in Eq. (3-16b),

$$
\boldsymbol{M}_{n m}(k r)=\sum_{\nu=0}^{\infty} \sum_{\mu=-\nu}^{\nu}\left[\boldsymbol{M}_{\nu \mu}\left(k r^{\prime}\right) \alpha_{n m, \nu \mu}\left(\boldsymbol{r}^{\prime \prime}\right)+\nabla \xi_{\nu \mu}\left(k \boldsymbol{r}^{\prime}\right) \times \boldsymbol{r}^{\prime \prime} \alpha_{n m, \nu \mu}\left(\boldsymbol{r}^{\prime \prime}\right)\right] .
$$

By writing $\boldsymbol{r}^{\prime \prime}=x^{\prime \prime} \boldsymbol{e}_{x}+y^{\prime \prime} \boldsymbol{e}_{y}+z^{\prime \prime} \boldsymbol{e}_{z}$ and expanding $\nabla \xi_{\nu \mu}\left(k \boldsymbol{r}^{\prime}\right) \times \boldsymbol{r}^{\prime \prime}$, the second term at right hand side of Eq. (3-31) can be expressed as a linear combination of $\boldsymbol{M}_{\nu \mu}\left(k r^{\prime}\right)$ and $\boldsymbol{N}_{\nu \mu}\left(k r^{\prime}\right)$ functions. To the end, Eq. (3-31) can be written as

$$
\boldsymbol{M}_{n m}(k r)=\sum_{\nu=0}^{\infty} \sum_{\mu=-\nu}^{\nu}\left[\boldsymbol{M}_{\nu \mu}\left(k r^{\prime}\right) A_{n m, \nu \mu}+\boldsymbol{N}_{\nu \mu}\left(k r^{\prime}\right) B_{n m, \nu \mu}\right] .
$$

The derivation from Eq.(3-31) leading to Eq. (3-32) was obtained by Chew and Wang ${ }^{[53]}$ (equations (6) through (12) in [53]). Equations (3-31) and (3-32) were written as

$$
\begin{gathered}
\boldsymbol{M}_{n m}(\boldsymbol{r})=\sum_{\nu=0}^{\infty} \sum_{\mu=-\nu}^{\nu}\left[\boldsymbol{M}_{\nu \mu}\left(\boldsymbol{r}^{\prime}\right) \beta_{\nu \mu, n m}\left(\boldsymbol{r}^{\prime \prime}\right)+\nabla \xi_{\nu \mu}\left(\boldsymbol{r}^{\prime}\right) \times \boldsymbol{r}^{\prime \prime} \beta_{\nu \mu, n m}\right] \\
\boldsymbol{M}_{n m}(\boldsymbol{r})=\sum_{\nu=0}^{\infty} \sum_{\mu=-\nu}^{\nu}\left[\boldsymbol{M}_{\nu \mu}\left(\boldsymbol{r}^{\prime}\right) A_{\nu \mu, n m}+\boldsymbol{N}_{\nu \mu}\left(\boldsymbol{r}^{\prime}\right) B_{\nu \mu, n m}\right]
\end{gathered}
$$

in [53] respectively. During the derivation in [53], $k=1$ was used. As mentioned in [53], results for $k \neq 1$ are obtained by replacing $r^{\prime \prime}$ by $k r^{\prime \prime}$. Therefore, the coefficients 
$A_{n m, \nu \mu}$ and $B_{n m, \nu \mu}$ in Eq. (3-32) are

$$
\begin{aligned}
& A_{n m, \nu \mu}=\alpha_{n m, \nu \mu}+k r^{\prime \prime} \sin \theta^{\prime \prime} \frac{e^{-\hat{\imath} \varphi^{\prime \prime}}}{2(\nu+1)} \sqrt{\frac{(\nu-\mu+2)(\nu-\mu+1)}{(2 \nu+1)(2 \nu+3)}} \alpha_{n m, \nu+1, \mu-1} \\
&-k r^{\prime \prime} \sin \theta^{\prime \prime} \frac{e^{-\hat{\imath} \varphi^{\prime \prime}}}{2 \nu} \sqrt{\frac{(\nu+\mu-1)(\nu+\mu)}{(2 \nu-1)(2 \nu+1)}} \alpha_{n m, \nu-1, \mu-1} \\
&-k r^{\prime \prime} \sin \theta^{\prime \prime} \frac{e^{\hat{\imath} \varphi^{\prime \prime}}}{2(\nu+1)} \sqrt{\frac{(\nu+\mu+2)(\nu+\mu+1)}{(2 \nu+1)(2 \nu+3)}} \alpha_{n m, \nu+1, \mu+1} \\
&+k r^{\prime \prime} \sin \theta^{\prime \prime} \frac{e^{\hat{\imath} \theta^{\prime \prime}}}{2 \nu} \sqrt{\frac{(\nu-\mu)(\nu-\mu-1)}{(2 \nu-1)(2 \nu+1)}} \alpha_{n m, \nu-1, \mu+1} \\
&+k r^{\prime \prime} \cos \theta^{\prime \prime} \frac{1}{\nu+1} \sqrt{\frac{(\nu+\mu+1)(\nu-\mu+1)}{(2 \nu+1)(2 \nu+3)}} \alpha_{n m, \nu+1, \mu} \\
&+k r^{\prime \prime} \cos \theta^{\prime \prime} \frac{1}{\nu} \sqrt{\frac{(\nu+\mu)(\nu-\mu)}{(2 \nu-1)(2 \nu+1)}} \alpha_{n m, \nu-1, \mu}, \\
& B_{n m, \nu \mu}=k r^{\prime \prime} \cos \theta^{\prime \prime} \frac{\hat{\imath} \mu}{\nu(\nu+1)} \alpha_{n m, \nu \mu}+\frac{\hat{\imath} k r^{\prime \prime} \sin \theta^{\prime \prime}}{2 \nu(\nu+1)}[\sqrt{(\nu-\mu)(\nu+\mu+1)} \\
&\left.\cdot e^{\hat{\imath} \varphi^{\prime \prime}} \alpha_{n m, \nu, \mu+1}+\sqrt{(\nu+\mu)(\nu-\mu+1)} e^{-\hat{\imath} \varphi^{\prime \prime}} \alpha_{n m, \nu, \mu-1}\right]
\end{aligned}
$$

Now consider $\boldsymbol{N}_{n m}(k r)$ in Eq. (3-16c).

$$
\frac{1}{k} \nabla \times \boldsymbol{N}_{n m}(k r)=\frac{1}{k} \nabla \times \frac{1}{k} \nabla \times M_{n m}(k r) .
$$

Following the vector identity ${ }^{[41]} \boldsymbol{A} \times(\boldsymbol{B} \times \boldsymbol{C})=\boldsymbol{B}(\boldsymbol{A} \cdot \boldsymbol{C})-(\boldsymbol{A} \cdot \boldsymbol{B}) \boldsymbol{C}$,

$$
\frac{1}{k} \nabla \times \boldsymbol{N}_{n m}(k r)=\frac{1}{k^{2}}\left\{\nabla\left[\nabla \cdot \boldsymbol{M}_{n m}(k r)\right]-[\nabla \cdot \nabla] \boldsymbol{M}_{n m}(k r)\right\} .
$$

Since $\nabla \times \boldsymbol{r}=0$

$$
\begin{gathered}
\boldsymbol{M}_{n m}(k r)=\nabla \times\left[\boldsymbol{r} \xi_{n m}(k \boldsymbol{r})\right]=\left[\nabla \xi_{n m}(k \boldsymbol{r})\right] \times \boldsymbol{r}, \\
\nabla \cdot \boldsymbol{M}_{n m}(k r)=\nabla \cdot\left[\nabla \xi_{n m}(k \boldsymbol{r})\right] \times \boldsymbol{r}=\left[\nabla \xi_{n m}(k \boldsymbol{r})\right] \cdot[\nabla \times \boldsymbol{r}]=0 .
\end{gathered}
$$

Following Eq. (3-35),

$$
\begin{aligned}
\frac{1}{k} \nabla \times \boldsymbol{N}_{n m}(k r) & =-\frac{1}{k^{2}} \nabla^{2} \boldsymbol{M}_{n m}(k r)=\boldsymbol{M}_{n m}(k r), \\
\boldsymbol{M}_{n m}(k r) & =\frac{1}{k} \nabla \times \boldsymbol{N}_{n m}(k r)
\end{aligned}
$$


Because $\boldsymbol{N}_{n m}(k r)=\frac{1}{k} \nabla \times \boldsymbol{M}_{n m}(k r)$ (Eq. (3-16c)) and $\boldsymbol{M}_{n m}(k r)=\frac{1}{k} \nabla \times \boldsymbol{N}_{n m}(k r)$ (Eq. (3-36)), then

$$
\boldsymbol{N}_{n m}(k r)=\sum_{\nu=0}^{\infty} \sum_{\mu=-\nu}^{\nu}\left[\boldsymbol{N}_{\nu \mu}\left(k r^{\prime}\right) A_{n m, \nu \mu}+\boldsymbol{M}_{\nu \mu}\left(k r^{\prime}\right) B_{n m, \nu \mu}\right]
$$

Equations (3-32) and (3-37) are expressions of the vector addition theorem ${ }^{[42,43,53]}$.

Substituting Equations (3-32) and (3-37) into Eq. (2-55), the displacement vector $\boldsymbol{u}$ can be written as

$$
\begin{aligned}
\boldsymbol{u}= & \sum_{n=0}^{\infty} \sum_{m=-n}^{n}\left\{a_{m n}^{\phi} \boldsymbol{L}_{n m}(\kappa r)+a_{m n}^{\psi} \boldsymbol{M}_{n m}(k r)+a_{m n}^{\chi} \boldsymbol{N}_{n m}(k r)\right\} \\
= & \sum_{n=0}^{\infty} \sum_{m=-n}^{n}\left\{a_{m n}^{\phi} \sum_{\nu=0}^{\infty} \sum_{\mu=-\nu}^{\nu} \alpha_{n m, \nu \mu}\left(\boldsymbol{r}^{\prime \prime}\right) \boldsymbol{L}_{\nu \mu}\left(\kappa r^{\prime}\right)\right. \\
& +a_{m n}^{\psi} \sum_{\nu=0}^{\infty} \sum_{\mu=-\nu}^{\nu}\left[\boldsymbol{M}_{\nu \mu}\left(k r^{\prime}\right) A_{n m, \nu \mu}+\boldsymbol{N}_{\nu \mu}\left(k r^{\prime}\right) B_{n m, \nu \mu}\right] \\
& \left.+a_{m n}^{\chi} \sum_{\nu=0}^{\infty} \sum_{\mu=-\nu}^{\nu}\left[\boldsymbol{N}_{\nu \mu}\left(k r^{\prime}\right) A_{n m, \nu \mu}+\boldsymbol{M}_{\nu \mu}\left(k r^{\prime}\right) B_{n m, \nu \mu}\right]\right\} \\
= & \sum_{n=0}^{\infty} \sum_{m=-n}^{n} \sum_{\nu=0}^{\infty} \sum_{\mu=-\nu}^{\nu} a_{m n}^{\phi} \alpha_{n m, \nu \mu}\left(\boldsymbol{r}^{\prime \prime}\right) \boldsymbol{L}_{\nu \mu}\left(\kappa r^{\prime}\right) \\
& +\sum_{n=0}^{\infty} \sum_{m=-n}^{n} \sum_{\nu=0}^{\infty} \sum_{\mu=-\nu}^{\nu} a_{m n}^{\psi} A_{n m, \nu \mu} \boldsymbol{M}_{\nu \mu}\left(k r^{\prime}\right) \\
& +\sum_{n=0}^{\infty} \sum_{m=-n}^{n} \sum_{\nu=0}^{\infty} \sum_{\mu=-\nu}^{\nu} a_{m n}^{\psi} B_{n m, \nu \mu} \boldsymbol{N}_{\nu \mu}\left(k r^{\prime}\right) \\
& +\sum_{n=0}^{\infty} \sum_{m=-n}^{n} \sum_{\nu=0}^{\infty} \sum_{\mu=-\nu}^{\nu} a_{m n}^{\chi} A_{n m, \nu \mu} \boldsymbol{N}_{\nu \mu}\left(k r^{\prime}\right) \\
& +\sum_{n=0}^{\infty} \sum_{m=-n}^{n} \sum_{\nu=0}^{\infty} \sum_{\mu=-\nu}^{\nu} a_{m n}^{\chi} B_{n m, \nu \mu} \boldsymbol{M}_{\nu \mu}\left(k r^{\prime}\right) \\
= & \sum_{\nu=0}^{\infty} \sum_{\mu=-\nu}^{\nu} \sum_{n=0}^{\infty} \sum_{m=-n}^{n}\left\{a_{m n}^{\phi} \alpha_{n m, \nu \mu}\left(\boldsymbol{r}^{\prime \prime}\right) \boldsymbol{L}_{\nu \mu}\left(\kappa r^{\prime}\right)\right. \\
& +\left[a_{m n}^{\psi} A_{n m, \nu \mu}+a_{m n}^{\chi} B_{n m, \nu \mu}\right] \boldsymbol{M}_{\nu \mu}\left(k r^{\prime}\right) \\
& \left.+\left[a_{m n}^{\psi} B_{n m, \nu \mu}+a_{m n}^{\chi} A_{n m, \nu \mu}\right] \boldsymbol{N}_{\nu \mu}\left(k r^{\prime}\right)\right\}
\end{aligned}
$$


By comparing Eqs. (3-38) with (3-26), the relation between two sets of wave expansion coefficients can be written as

$$
\begin{aligned}
& \left\{a_{\nu \mu}^{\phi^{\prime}}\right\}=\left[\alpha_{n m, \nu \mu}\right]^{T}\left\{a_{n m}^{\phi}\right\} \\
& \left\{a_{\nu \mu}^{\psi^{\prime}}\right\}=\left[A_{n m, \nu \mu}\right]^{T}\left\{a_{n m}^{\psi}\right\}+\left[B_{n m, \nu \mu}\right]^{T}\left\{a_{n m}^{\chi}\right\} \\
& \left\{a_{\nu \mu}^{\psi^{\prime}}\right\}=\left[B_{n m, \nu \mu}\right]^{T}\left\{a_{n m}^{\psi}\right\}+\left[A_{n m, \nu \mu}\right]^{T}\left\{a_{n m}^{\chi}\right\}
\end{aligned}
$$

or, in matrix form

$$
\left\{\begin{array}{c}
\left\{a_{\nu \mu}^{\phi^{\prime}}\right\} \\
\left\{a_{\nu \mu}^{\psi^{\prime}}\right\} \\
\left\{a_{\nu \mu}^{\psi^{\prime}}\right\}
\end{array}\right\}=\left[\begin{array}{ccc}
{\left[\alpha_{n m, \nu \mu}\right]^{T}} & 0 & 0 \\
0 & {\left[A_{n m, \nu \mu}\right]^{T}} & {\left[B_{n m, \nu \mu}\right]^{T}} \\
0 & {\left[B_{n m, \nu \mu}\right]^{T}} & {\left[A_{n m, \nu \mu}\right]^{T}}
\end{array}\right]\left\{\begin{array}{c}
\left\{a_{n m}^{\phi}\right\} \\
\left\{a_{n m}^{\psi}\right\} \\
\left\{a_{n m}^{\chi}\right\}
\end{array}\right\}
$$

Recall Eqs. (3-11a) through (3-11b) for coordinate transformation for wave expansion basis. It is noted that two different transformation matrices $\left[R g \boldsymbol{R}_{I J}\left(k ; \boldsymbol{d}_{I J}\right)\right]$ and $\left[\boldsymbol{R}_{I J}\left(k ; \boldsymbol{d}_{I J}\right)\right]$ are used for different transformation. Similarly, the entries of $\left[\alpha_{n m, \nu \mu}\right]$, $\left[A_{n m, \nu \mu}\right]$ and $\left[B_{n m, \nu \mu}\right]$ need to be specified.

For transformation between Scatterer I's system and Scatterer J's system in Fig. 3.4, compare Figs. 3.4 and 3.3 and let

$$
\boldsymbol{r}_{I}=\boldsymbol{r}, \boldsymbol{r}_{J}=\boldsymbol{r}^{\prime}, \boldsymbol{d}_{I J}=\boldsymbol{r}^{\prime \prime}
$$

First, when the wave expansion bases $\left\{\boldsymbol{h}\left(\kappa ; \boldsymbol{r}_{I}\right)\right\}$ and $\left\{\boldsymbol{h}\left(k ; \boldsymbol{r}_{I}\right)\right\}$ in scatterer $I$ 's system are transformed as the wave expansion bases $\left\{\boldsymbol{j}\left(\kappa ; \boldsymbol{r}_{J}\right)\right\}$ and $\left\{\boldsymbol{j}\left(k ; \boldsymbol{r}_{J}\right)\right\}$ in scatterer $J$ 's system, Eqs. (3-11b) and (3-13b) are used with requirement $r_{J}<d_{I J}$. Therefore, $\left[\alpha_{n m, \nu \mu}\right]$ in Eq. $(3-40)$ takes the form of $\left[\boldsymbol{R}_{I J}\left(\kappa ; \boldsymbol{d}_{I J}\right)\right]$ in Eq. $(3-14 \mathrm{a})$, and the entries of $\left[A_{n m, \nu \mu}\right]$ and $\left[B_{n m, \nu \mu}\right]$ are based on entries of $\left[\alpha_{n m, \nu \mu}\right]$, which can be obtained by using Eq. (3-9b). In order to distinguish this case, define $\left[\boldsymbol{Q}_{I J}^{A}\left(k ; \boldsymbol{d}_{I J}\right)\right]$ and $\left[\boldsymbol{Q}_{I J}^{B}\left(k ; \boldsymbol{d}_{I J}\right)\right]$ such that

$$
\left[\boldsymbol{Q}_{I J}^{A}\left(k ; \boldsymbol{d}_{I J}\right)\right]_{i j}=A_{n m, \nu \mu}
$$




$$
\left[\boldsymbol{Q}_{I J}^{B}\left(k ; \boldsymbol{d}_{I J}\right)\right]_{i j}=B_{n m, \nu \mu}
$$

Then, transpose of the square matrix at right hand side of Eq. (3-40) can be written as

$$
\left[\mathbb{Q}_{I J}\right]=\left[\begin{array}{ccc}
{\left[\boldsymbol{R}_{I J}\left(\kappa ; \boldsymbol{d}_{I J}\right)\right]} & 0 & 0 \\
0 & {\left[\boldsymbol{Q}_{I J}^{A}\left(k ; \boldsymbol{d}_{I J}\right)\right]} & {\left[\boldsymbol{Q}_{I J}^{B}\left(k ; \boldsymbol{d}_{I J}\right)\right]} \\
0 & {\left[\boldsymbol{Q}_{I J}^{B}\left(k ; \boldsymbol{d}_{I J}\right)\right]} & {\left[\boldsymbol{Q}_{I J}^{A}\left(k ; \boldsymbol{d}_{I J}\right)\right]}
\end{array}\right]
$$

Second, when the wave expansion bases $\left\{\boldsymbol{h}\left(\kappa ; \boldsymbol{r}_{I}\right)\right\}$ and $\left\{\boldsymbol{h}\left(k ; \boldsymbol{r}_{I}\right)\right\}$ in scatterer I's system are transformed as the wave expansion bases $\left\{\boldsymbol{h}\left(\kappa ; \boldsymbol{r}_{J}\right)\right\}$ and $\left\{\boldsymbol{h}\left(k ; \boldsymbol{r}_{J}\right)\right\}$ in scatterer $J$ 's system, Eqs. (3-11b) and (3-13b) are used with requirement $r_{J}>d_{I J}$. Therefore, $\left[\alpha_{n m, \nu \mu}\right]$ in Eq. (3-40) takes the form of $\left[R g \boldsymbol{R}_{I J}\left(\kappa ; \boldsymbol{d}_{I J}\right)\right]$ in Eq. (3-14b), and the entries of $\left[A_{n m, \nu \mu}\right]$ and $\left[B_{n m, \nu \mu}\right]$ are based on entries of $\left[\alpha_{n m, \nu \mu}\right]$, which becomes the $\beta_{n m, \nu \mu}$ in Eq. (3-9a). In order to distinguish this case, define $\left[\operatorname{Rg} \boldsymbol{Q}_{I J}^{A}\left(k ; \boldsymbol{d}_{I J}\right)\right]$ and $\left[R g \boldsymbol{Q}_{I J}^{B}\left(k ; \boldsymbol{d}_{I J}\right)\right]$ such that

$$
\begin{aligned}
& {\left[R g \boldsymbol{Q}_{I J}^{A}\left(k ; \boldsymbol{d}_{I J}\right)\right]_{i j}=A_{n m, \nu \mu}} \\
& {\left[R g \boldsymbol{Q}_{I J}^{B}\left(k ; \boldsymbol{d}_{I J}\right)\right]_{i j}=B_{n m, \nu \mu}}
\end{aligned}
$$

Then, transpose of the square matrix at right hand side of Eq. (3-40) can be written as

$$
\left[R g \mathbb{Q}_{I J}\right]=\left[\begin{array}{ccc}
{\left[R g \boldsymbol{R}_{I J}\left(\kappa ; \boldsymbol{d}_{I J}\right)\right]} & 0 & 0 \\
0 & {\left[R g \boldsymbol{Q}_{I J}^{A}\left(k ; \boldsymbol{d}_{I J}\right)\right]} & {\left[R g \boldsymbol{Q}_{I J}^{B}\left(k ; \boldsymbol{d}_{I J}\right)\right]} \\
0 & {\left[R g \boldsymbol{Q}_{I J}^{B}\left(k ; \boldsymbol{d}_{I J}\right)\right]} & {\left[R g \boldsymbol{Q}_{I J}^{A}\left(k ; \boldsymbol{d}_{I J}\right)\right]}
\end{array}\right]
$$

Third, when the wave expansion bases $\left\{\boldsymbol{j}\left(\kappa ; \boldsymbol{r}_{I}\right)\right\}$ and $\left\{\boldsymbol{j}\left(k ; \boldsymbol{r}_{I}\right)\right\}$ in scatterer $I$ 's system are transformed as the wave expansion bases $\left\{\boldsymbol{j}\left(\kappa ; \boldsymbol{r}_{J}\right)\right\}$ and $\left\{\boldsymbol{j}\left(k ; \boldsymbol{r}_{J}\right)\right\}$ in scatterer $J$ 's system, Eqs. (3-11a) and (3-13a) are used. Therefore, $\left[\alpha_{n m, \nu \mu}\right]$ in Eq. $(3-40)$ takes the form of $\left[R g \boldsymbol{R}_{I J}\left(\kappa ; \boldsymbol{d}_{I J}\right)\right]$ in Eq. $(3-14 \mathrm{~b})$, and the entries of $\left[A_{n m, \nu \mu}\right]$ and $\left[B_{n m, \nu \mu}\right]$ are based on entries of $\left[\alpha_{n m, \nu \mu}\right]$, which becomes the $\beta_{n m, \nu \mu}$ in Eq. (3-9a). 
For this case, transpose of the square matrix at right hand side of Eq. (3-40) can be written as Eq. (3-45).

In analogy to Eq. (2-100) for compact notations, the wave expansion coefficients matrix in Scatterer I's system corresponding to wave expansion bases $\{\boldsymbol{j}(\cdot)\}$ can be written as $\left\{\mathbb{A}_{I}\right\}$, and the wave expansion coefficients matrix in Scatterer I's system corresponding to wave expansion bases $\{\boldsymbol{h}(\cdot)\}$ can be written as $\left\{\mathbb{B}_{I}\right\}$, where

$$
\left\{\mathbb{A}_{I}\right\}=\left\{\begin{array}{c}
\left\{\boldsymbol{A}_{I}^{\phi}\right\} \\
\left\{\boldsymbol{A}_{I}^{\psi}\right\} \\
\left\{\boldsymbol{A}_{I}^{\chi}\right\}
\end{array}\right\} \quad \text { and } \quad\left\{\mathbb{B}_{I}\right\}=\left\{\begin{array}{c}
\left\{\boldsymbol{B}_{I}^{\phi}\right\} \\
\left\{\boldsymbol{B}_{I}^{\psi}\right\} \\
\left\{\boldsymbol{B}_{I}^{\chi}\right\}
\end{array}\right\}
$$

For transformation of wave expansion bases $\{\boldsymbol{h}(\cdot)\}$ in Scatterer I's system as wave expansion bases $\{\boldsymbol{j}(\cdot)\}$ in Scatterer J's system, Eq. (3-40) can be written as

$$
\left\{\mathbb{A}_{J}\right\}=\left[\mathbb{Q}_{I J}\right]^{T}\left\{\mathbb{B}_{I}\right\}
$$

For transformation of wave expansion bases $\{\boldsymbol{h}(\cdot)\}$ in Scatterer I's system as wave expansion bases $\{\boldsymbol{h}(\cdot)\}$ in Scatterer J's system, Eq. (3-40) can be written as

$$
\left\{\mathbb{B}_{J}\right\}=\left[R g \mathbb{Q}_{I J}\right]^{T}\left\{\mathbb{B}_{I}\right\}
$$

For transformation of wave expansion bases $\{\boldsymbol{j}(\cdot)\}$ in Scatterer I's system as wave expansion bases $\{\boldsymbol{j}(\cdot)\}$ in Scatterer J's system, Eq. (3-40) can be written as

$$
\left\{\mathbb{A}_{J}\right\}=\left[R g \mathbb{Q}_{I J}\right]^{T}\left\{\mathbb{A}_{I}\right\}
$$

\section{3-4 Formulation of Multiple Scattering Solution}

The multiple scattering solution in steady-state is formulated in this section. One formulation approach is based on waves at various order in Section 3-2, while the other approach is based on single scattering problem with the incident waves composed of external incident wave and total scattered waves from all other scatterers. 


\section{3-4.1 Formulation Based on Waves at Various Orders}

Given incident waves in global coordinate system with origin $O$ can be written as,

$$
\begin{aligned}
\phi^{\text {inc }} & =\left\{\boldsymbol{A}^{\phi}\right\}^{T}\{\boldsymbol{j}(\kappa ; \boldsymbol{r})\}, \\
\psi^{\mathrm{inc}} & =\left\{\boldsymbol{A}^{\psi}\right\}^{T}\{\boldsymbol{j}(k ; \boldsymbol{r})\}, \\
\chi^{\mathrm{inc}} & =\left\{\boldsymbol{A}^{\chi}\right\}^{T}\{\boldsymbol{j}(k ; \boldsymbol{r})\} .
\end{aligned}
$$

The incident waves can be expressed in Scatterer I's local spherical coordinate system as following.

$$
\begin{array}{r}
\phi_{I}^{\text {inc }}=\left\{\boldsymbol{A}_{I}^{\phi}\right\}^{T}\left\{\boldsymbol{j}\left(\kappa ; \boldsymbol{r}_{I}\right)\right\} \\
\psi_{I}^{\text {inc }}=\left\{\boldsymbol{A}_{I}^{\psi}\right\}^{T}\left\{\boldsymbol{j}\left(k ; \boldsymbol{r}_{I}\right)\right\} \\
\chi_{I}^{\text {inc }}=\left\{\boldsymbol{A}_{I}^{\chi}\right\}^{T}\left\{\boldsymbol{j}\left(k ; \boldsymbol{r}_{I}\right)\right\}
\end{array}
$$

where

$$
\left\{\mathbb{A}_{I}\right\}=\left[R g \mathbb{Q O}_{O I}\right]^{T}\{\mathbb{A}\}
$$

and $O$ stands for the global coordinate system with origin $O$.

First Order Waves

The first order scattered waves in Scatterer I's local spherical coordinate system, $\Phi_{I}^{(1)}$, includes three components that can be expressed as

$$
\begin{aligned}
& \phi_{I}^{(1)}=\left\{\boldsymbol{B}_{I}^{(1), \phi}\right\}^{T}\left\{\boldsymbol{h}\left(\kappa ; \boldsymbol{r}_{I}\right)\right\} \\
& \psi_{I}^{(1)}=\left\{\boldsymbol{B}_{I}^{(1), \psi}\right\}^{T}\left\{\boldsymbol{h}\left(k ; \boldsymbol{r}_{I}\right)\right\} \\
& \chi_{I}^{(1)}=\left\{\boldsymbol{B}_{I}^{(1), \chi}\right\}^{T}\left\{\boldsymbol{h}\left(k ; \boldsymbol{r}_{I}\right)\right\}
\end{aligned}
$$

According to the physical process described in Section 3-2, the excitation source at first order scattering is the incident wave. Then, wave expansion coefficients of the first order scattered waves by Scatterer $I$ can be obtained by using the super 
$T$-matrix of Scatterer $I$ :

$$
\left\{\mathbb{B}_{I}^{(1)}\right\}=\left[\mathbb{T}_{I}\right]\left\{\mathbb{A}_{I}\right\}
$$

Second Order Waves

The second order scattered wave scattered by Scatterer $J$ due to the first order scattered wave scattered by Scatterer $I$ can be expressed in Scatterer $J$ 's local spherical coordinate system, such as

$$
\begin{aligned}
& \phi_{J \text { due to } \Phi_{I}^{(1)}}^{(2)}=\left\{\boldsymbol{B}_{I J}^{(2), \phi}\right\}^{T}\left\{\boldsymbol{h}\left(\kappa ; \boldsymbol{r}_{J}\right)\right\} \\
& \psi_{J \text { due to } \Phi_{I}^{(1)}}^{(2)}=\left\{\boldsymbol{B}_{I J}^{(2), \psi}\right\}^{T}\left\{\boldsymbol{h}\left(k ; \boldsymbol{r}_{J}\right)\right\} \\
& \chi_{J \text { due to } \Phi_{I}^{(1)}}^{(2)}=\left\{\boldsymbol{B}_{I J}^{(2), \chi}\right\}^{T}\left\{\boldsymbol{h}\left(k ; \boldsymbol{r}_{J}\right)\right\}
\end{aligned}
$$

Note that the first order scattered wave scattered by Scatterer $I$ in Eq. (3-53) is expressed in Scatterer I's local coordinate. In order to obtain wave expansion coefficients of the second order scattered wave, it is necessary to transform the expressions in Eq. (3-53) as incident waves into Scatterer $J$ 's local coordinate system. The later can be written as

$$
\begin{array}{r}
\phi_{J}^{(1), \text { inc }}=\left\{\boldsymbol{A}_{J}^{(1), \phi}\right\}^{T}\left\{\boldsymbol{j}\left(\kappa ; \boldsymbol{r}_{J}\right)\right\} \\
\psi_{J}^{(1), \text { inc }}=\left\{\boldsymbol{A}_{J}^{(1), \psi}\right\}^{T}\left\{\boldsymbol{j}\left(k ; \boldsymbol{r}_{J}\right)\right\} \\
\chi_{J}^{(1), \text { inc }}=\left\{\boldsymbol{A}_{J}^{(1), \chi}\right\}^{T}\left\{\boldsymbol{j}\left(k ; \boldsymbol{r}_{J}\right)\right\}
\end{array}
$$

By using Eq. (3-47), the coordinate transformation is performed such that

$$
\left\{\begin{array}{c}
\left\{\boldsymbol{A}_{J}^{(1), \phi}\right\} \\
\left\{\boldsymbol{A}_{J}^{(1), \psi}\right\} \\
\left\{\boldsymbol{A}_{J}^{(1), \chi}\right\}
\end{array}\right\}=\left[\mathbb{Q}_{I J}\right]^{T}\left\{\begin{array}{c}
\left\{\boldsymbol{B}_{I}^{(1), \phi}\right\} \\
\left\{\boldsymbol{B}_{I}^{(1), \psi}\right\} \\
\left\{\boldsymbol{B}_{I}^{(1), \chi}\right\}
\end{array}\right\}
$$

Then, the wave expansion coefficients of the second order scattered waves by Scatterer 
$J$ due to the first order scattered wave scattered by Scatterer $I$ can be obtained.

$$
\left\{\begin{array}{c}
\left\{\boldsymbol{B}_{J I}^{(2), \phi}\right\} \\
\left\{\boldsymbol{B}_{J I}^{(2), \psi}\right\} \\
\left\{\boldsymbol{B}_{J I}^{(2), \chi}\right\}
\end{array}\right\}=\left[\mathbb{T}_{J}\right]\left\{\begin{array}{c}
\left\{\boldsymbol{A}_{J}^{(1), \phi}\right\} \\
\left\{\boldsymbol{A}_{J}^{(1), \psi}\right\} \\
\left\{\boldsymbol{A}_{J}^{(1), \chi}\right\}
\end{array}\right\}
$$

or

$$
\left\{\begin{array}{c}
\left\{\boldsymbol{B}_{J I}^{(2), \phi}\right\} \\
\left\{\boldsymbol{B}_{J I}^{(2), \psi}\right\} \\
\left\{\boldsymbol{B}_{J I}^{(2), \chi}\right\}
\end{array}\right\}=\left[\mathbb{T}_{J}\right]\left[\mathbb{Q}_{I J}\right]^{T}\left\{\mathbb{B}_{I}^{(1)}\right\}
$$

Denote the total second order scattered wave scattered by Scatterer $J$ as

$$
\begin{aligned}
\phi_{J}^{(2)} & =\left\{\boldsymbol{B}_{J}^{(2), \phi}\right\}^{T}\left\{\boldsymbol{h}\left(\kappa ; \boldsymbol{r}_{J}\right)\right\} \\
\psi_{J}^{(2)} & =\left\{\boldsymbol{B}_{J}^{(2), \psi}\right\}^{T}\left\{\boldsymbol{h}\left(k ; \boldsymbol{r}_{J}\right)\right\} \\
\chi_{J}^{(2)} & =\left\{\boldsymbol{B}_{J}^{(2), \chi}\right\}^{T}\left\{\boldsymbol{h}\left(k ; \boldsymbol{r}_{J}\right)\right\}
\end{aligned}
$$

then, according to eqn. (3-1),

$$
\left\{\mathbb{B}_{J}^{(2)}\right\} \equiv\left\{\begin{array}{c}
\left\{\boldsymbol{B}_{J}^{(2), \phi}\right\} \\
\left\{\boldsymbol{B}_{J}^{(2), \psi}\right\} \\
\left\{\boldsymbol{B}_{J}^{(2), \chi}\right\}
\end{array}\right\}=\sum_{\substack{I=1 \\
I \neq J}}^{N}\left[\mathbb{T}_{J}\right]\left[\mathbb{Q}_{I J}\right]^{T}\left\{\mathbb{B}_{I}^{(1)}\right\}
$$

General p-th Order Waves

In the same manner, denote the general $p$-th order scattered wave scattered by Scatterer $J$ as

$$
\begin{aligned}
\phi_{J}^{(p)} & =\left\{\boldsymbol{B}_{J}^{(p), \phi}\right\}^{T}\left\{\boldsymbol{h}\left(\kappa ; \boldsymbol{r}_{J}\right)\right\} \\
\psi_{J}^{(p)} & =\left\{\boldsymbol{B}_{J}^{(p), \psi}\right\}^{T}\left\{\boldsymbol{h}\left(k ; \boldsymbol{r}_{J}\right)\right\} \\
\chi_{J}^{(p)} & =\left\{\boldsymbol{B}_{J}^{(p), \chi}\right\}^{T}\left\{\boldsymbol{h}\left(k ; \boldsymbol{r}_{J}\right)\right\}
\end{aligned}
$$


Then,

\section{Multiple Scattering Solution}

$$
\left\{\mathbb{B}_{J}^{(p)}\right\} \equiv\left\{\begin{array}{c}
\left\{\boldsymbol{B}_{J}^{(p), \phi}\right\} \\
\left\{\boldsymbol{B}_{J}^{(p), \psi}\right\} \\
\left\{\boldsymbol{B}_{J}^{(2), \chi}\right\}
\end{array}\right\}=\sum_{\substack{I=1 \\
I \neq J}}^{N}\left[\mathbb{T}_{J}\right]\left[\mathbb{Q}_{I J}\right]^{T}\left\{\mathbb{B}_{I}^{(p-1)}\right\}
$$

Denote the summation of all orders of the scattered wave scattered by Scatterer $J$, called the total scattered wave scattered by Scatterer J, as

$$
\begin{aligned}
& \phi_{J}^{\text {sct }}=\left\{\boldsymbol{B}_{J}^{\phi}\right\}^{T}\left\{\boldsymbol{h}\left(\kappa ; \boldsymbol{r}_{J}\right)\right\} \\
& \psi_{J}^{\text {sct }}=\left\{\boldsymbol{B}_{J}^{\psi}\right\}^{T}\left\{\boldsymbol{h}\left(k ; \boldsymbol{r}_{J}\right)\right\} \\
& \chi_{J}^{\text {sct }}=\left\{\boldsymbol{B}_{J}^{\chi}\right\}^{T}\left\{\boldsymbol{h}\left(k ; \boldsymbol{r}_{J}\right)\right\}
\end{aligned}
$$

And denote the accompanying wave expansion coefficient as $\left\{\mathbb{B}_{J}\right\}$,

$$
\left\{\mathbb{B}_{J}\right\}=\left\{\begin{array}{c}
\left\{\boldsymbol{B}_{J}^{\phi}\right\} \\
\left\{\boldsymbol{B}_{J}^{\psi}\right\} \\
\left\{\boldsymbol{B}_{J}^{\chi}\right\}
\end{array}\right\}
$$

By definition,

$$
\left\{\mathbb{B}_{J}\right\}=\sum_{p=1}^{\infty}\left\{\mathbb{B}_{J}^{(p)}\right\}
$$

Making an exhaustive listing of all equations similar to eqn. (3-67), from first order to $p$-th order of scattered waves, and summing all these equations give

$$
\sum_{q=1}^{p}\left\{\mathbb{B}_{J}^{(q)}\right\}=\left[\mathbb{T}_{J}\right]\left\{\mathbb{A}_{J}\right\}+\sum_{\substack{I=1 \\ I \neq J}}^{N}\left[\mathbb{T}_{J}\right]\left[\mathbb{Q}_{I J}\right]^{T}\left(\sum_{q=1}^{p-1}\left\{\mathbb{B}_{I}^{(q)}\right\}\right)
$$

Noting that when $p \rightarrow \infty$,

$$
\begin{gathered}
\sum_{q=1}^{p}\left\{\mathbb{B}_{J}^{(q)}\right\}=\sum_{q=1}^{p-1}\left\{\mathbb{B}_{J}^{(q)}\right\}=\left\{\mathbb{B}_{J}\right\} \\
\left\{\mathbb{B}_{J}\right\}=\left[\mathbb{T}_{J}\right]\left\{\mathbb{A}_{J}\right\}+\sum_{\substack{I=1 \\
I \neq J}}^{N}\left[\mathbb{T}_{J}\right]\left[\mathbb{Q}_{I J}\right]^{T}\left\{\mathbb{B}_{I}\right\}
\end{gathered}
$$


To solve for $\left\{\mathbb{B}_{I}\right\}$, define

$$
\{\mathfrak{B}\}=\left\{\begin{array}{c}
\left\{\mathbb{B}_{1}\right\} \\
\left\{\mathbb{B}_{2}\right\} \\
\left\{\mathbb{B}_{3}\right\} \\
\vdots \\
\left\{\mathbb{B}_{N}\right\}
\end{array}\right\}, \quad\{\mathfrak{A}\}=\left\{\begin{array}{c}
{\left[\mathbb{T}_{1}\right]\left\{\mathbb{A}_{1}\right\}} \\
{\left[\mathbb{T}_{2}\right]\left\{\mathbb{A}_{2}\right\}} \\
{\left[\mathbb{T}_{3}\right]\left\{\mathbb{A}_{3}\right\}} \\
\vdots \\
{\left[\mathbb{T}_{N}\right]\left\{\mathbb{A}_{N}\right\}}
\end{array}\right\}=\left\{\begin{array}{c}
{\left[\mathbb{T}_{1}\right]\left[R g \mathbb{Q}_{O 1}\right]^{T}} \\
{\left[\mathbb{T}_{2}\right]\left[R g \mathbb{Q}_{O 2}\right]^{T}} \\
{\left[\mathbb{T}_{3}\right]\left[R g \mathbb{Q}_{O 3}\right]^{T}} \\
\vdots \\
{\left[\mathbb{T}_{N}\right]\left[R g \mathbb{Q}_{O N}\right]^{T}}
\end{array}\right\}\{\mathbb{A}\}
$$

and

$$
[\mathfrak{L}]=\left[\begin{array}{ccccc}
{[\mathbb{I}]} & -\left[\mathbb{T}_{1}\right]\left[\mathbb{Q}_{21}\right]^{T} & -\left[\mathbb{T}_{1}\right]\left[\mathbb{Q}_{31}\right]^{T} & \ldots & -\left[\mathbb{T}_{1}\right]\left[\mathbb{Q}_{N 1}\right]^{T} \\
-\left[\mathbb{T}_{2}\right]\left[\mathbb{Q}_{12}\right]^{T} & {[\mathbb{I}]} & -\left[\mathbb{T}_{2}\right]\left[\mathbb{Q}_{32}\right]^{T} & \ldots & -\left[\mathbb{T}_{2}\right]\left[\mathbb{Q}_{N 2}\right]^{T} \\
-\left[\mathbb{T}_{3}\right]\left[\mathbb{Q}_{13}\right]^{T} & -\left[\mathbb{T}_{3}\right]\left[\mathbb{Q}_{23}\right]^{T} & {[\mathbb{I}]} & \ldots & -\left[\mathbb{T}_{3}\right]\left[\mathbb{Q}_{N 3}\right]^{T} \\
\ldots & \ldots & \ldots & & \ldots \\
-\left[\mathbb{T}_{N}\right]\left[\mathbb{Q}_{1 N}\right]^{T} & -\left[\mathbb{T}_{N}\right]\left[\mathbb{Q}_{2 N}\right]^{T} & -\left[\mathbb{T}_{N}\right]\left[\mathbb{Q}_{3 N}\right]^{T} & \ldots & {[\mathbb{I}]}
\end{array}\right]
$$

where [I] is an identity matrix, then eqn. (3-75) becomes

$$
[\mathfrak{L}]\{\mathfrak{B}\}=\{\mathfrak{A}\}
$$

which can be solved by many readily available solvers for linear equation systems.

Equation (3-75) represents the solution for the multiple scattering problem at hand. The total wave in the host medium consists of the incident wave plus total scattered waves by all scatterers in the field, that is

$$
\begin{aligned}
& \phi^{\text {total }}=\phi^{\text {inc }}+\sum_{J=1}^{N} \phi_{J}^{\text {sct }} \\
& \psi^{\text {total }}=\psi^{\text {inc }}+\sum_{J=1}^{N} \psi_{J}^{\text {sct }} \\
& \chi^{\text {total }}=\chi^{\text {inc }}+\sum_{J=1}^{N} \chi_{J}^{\text {sct }}
\end{aligned}
$$




\section{3-4.2 Formulation Based on Single Scattering Solution}

For Scatterer $J$, the incident waves include the external incident waves and total scattered waves from all other scatterers.

The wave expansion coefficients of external incident waves in Scatterer J's coordinate system is $\left\{\mathbb{A}_{J}\right\}$. The wave expansion coefficients of total scattered waves by Scatterer $I$ and in Scatterer I's local coordinate system is $\left\{\mathbb{B}_{I}\right\}$. The scattered waves by Scatterer $I$ are transformed as incident waves in Scatterer J's local system. The wave expansion coefficients of the resultant incident waves can be written as $\left[\mathbb{Q}_{I J}\right]^{T}\left\{\mathbb{B}_{I}\right\}$ according to Eq. (3-47). Therefore, the solution of single scattering problem about Scatterer $J$ can be expressed as

$$
\left\{\mathbb{B}_{J}\right\}=\left[\mathbb{T}_{J}\right]\left\{\mathbb{A}_{J}\right\}+\left[\mathbb{T}_{J}\right] \sum_{\substack{I=1 \\ I \neq J}}^{N}\left[\mathbb{Q}_{I J}\right]^{T}\left\{\mathbb{B}_{I}\right\} .
$$

Equation (3-80) is the same as Eq. (3-75).

This approach leads to the same solution as previous approach.

\section{3-4.3 Displacements and Stresses}

The total wave fields, such as displacements and stresses, in the host medium consist of the incident wave plus the total scattered waves by all scatterers. The total displacements and stresses are the summation of contributions from each wave.

In general, expressions of the incident wave fields are known. Assume the coefficients of the expansion expressions of the incident waves are known. Their contributions to displacements and stresses in spherical coordinate system are

$$
\begin{aligned}
u_{r}^{i n c} & =\sum_{n=0}^{\infty} \sum_{m=-n}^{n}\left[A_{m n}^{\phi} \mathfrak{E}_{11}^{11}(r, n)+A_{m n}^{\chi} \mathfrak{E}_{11}^{31}(r, n)\right] Y_{n}^{m} \\
u_{\theta}^{i n c} & =\sum_{n=0}^{\infty} \sum_{m=-n}^{n}\left\{\left[A_{m n}^{\phi} \mathfrak{E}_{21}^{11}(r, n)+A_{m n}^{\chi} \mathfrak{E}_{21}^{31}(r, n)\right]\left(Y_{, \theta}\right)_{n}^{m}\right.
\end{aligned}
$$




$$
\begin{aligned}
& \left.+A_{m n}^{\psi} \mathfrak{E}_{21}^{21}(r, n)\left(Y_{, \varphi}\right)_{n}^{m}\right\} \\
u_{\varphi}^{i n c}= & \sum_{n=0}^{\infty} \sum_{m=-n}^{n}\left\{\left[A_{m n}^{\phi} \mathfrak{E}_{31}^{11}(r, n)+A_{m n}^{\chi} \mathfrak{E}_{31}^{31}(r, n)\right]\left(Y_{, \varphi}\right)_{n}^{m}\right. \\
& \left.+A_{m n}^{\psi} \mathfrak{E}_{31}^{21}(r, n)\left(Y_{, \theta}\right)_{n}^{m}\right\} \\
\sigma_{r r}^{i n c}= & \sum_{n=0}^{\infty} \sum_{m=-n}^{n}\left\{A_{m n}^{\phi} \mathfrak{E}_{41}^{11}(r, n)+A_{m n}^{\chi} \mathfrak{E}_{41}^{31}(r, n)\right\} Y_{n}^{m} \\
\sigma_{r \theta}^{i n c}= & \sum_{n=0}^{\infty} \sum_{m=-n}^{n}\left\{\left[A_{m n}^{\phi} \mathfrak{E}_{71}^{11}(r, n)+A_{I m n}^{\chi} \mathfrak{E}_{71}^{31}(r, n)\right]\left(Y_{, \theta}\right)_{n}^{m}\right. \\
& \left.+A_{m n}^{\psi} \mathfrak{E}_{71}^{21}(r, n)\left(Y_{, \varphi}\right)_{n}^{m}\right\} \\
\sigma_{r \varphi}^{i n c}= & \sum_{n=0}^{\infty} \sum_{m=-n}^{n}\left\{\left[A_{m n}^{\phi} \mathfrak{E}_{81}^{11}(r, n)+A_{m n}^{\chi} \mathfrak{E}_{81}^{31}(r, n)\right]\left(Y_{, \varphi}\right)_{n}^{m}\right. \\
& \left.+A_{m n}^{\psi} \mathfrak{E}_{81}^{21}(r, n)\left(Y_{, \theta}\right)_{n}^{m}\right\} \\
\sigma_{\theta \theta}^{i n c}= & \sum_{n=0}^{\infty} \sum_{m=-n}^{n}\left\{A_{m n}^{\phi} \mathfrak{E}_{51}^{11}(\mathbf{r}, n)+A_{m n}^{\chi} \mathfrak{E}_{51}^{31}(\mathbf{r}, n)+A_{m n}^{\psi} \mathfrak{E}_{51}^{21}(\mathbf{r}, n)\right\} \\
\sigma_{\varphi \varphi}^{i n c}= & \sum_{n=0}^{\infty} \sum_{m=-n}^{n}\left\{A_{m n}^{\phi} \mathfrak{E}_{61}^{11}(\mathbf{r}, n)+A_{m n}^{\chi} \mathfrak{E}_{61}^{31}(\mathbf{r}, n)+A_{m n}^{\psi} \mathfrak{E}_{61}^{21}(\mathbf{r}, n)\right\} \\
\sigma_{\theta \varphi}^{i n c}= & \sum_{n=0}^{\infty} \sum_{m=-n}^{n}\left\{A_{m n}^{\phi} \mathfrak{E}_{91}^{11}(\mathbf{r}, n)+A_{m n}^{\chi} \mathfrak{E}_{91}^{31}(\mathbf{r}, n)+A_{m n}^{\psi} \mathfrak{E}_{91}^{21}(\mathbf{r}, n)\right\}
\end{aligned}
$$

where superscript inc denotes the contribution from the incident waves. In Cartesian coordinate system, displacements and stresses can be obtained using Eqs. (D-8a) and (D-10a).

For plane longitudinal incident wave represented by displacement potentials in Eq. (2-131), which are repeated here

$$
\phi^{i n c}=\frac{\hat{\imath}}{\kappa} e^{-\hat{\imath} \kappa r \cos (\theta)}=\frac{\hat{\imath}}{\kappa} e^{-\hat{\imath} \kappa z}, \quad \psi^{i n c}=\chi^{i n c}=0
$$

the expansion expression of $\phi$ in Eq. $(2-132)$ is repeated here

$$
\phi^{i n c}=\sum_{n=0}^{\infty} \frac{(-\hat{\imath})^{n}}{\kappa} \sqrt{4 \pi(2 n+1)} j_{n}(\kappa r) Y_{n}^{0}(\theta, \varphi)
$$

and the displacements and stresses in Section 2-6.1 are repeated here

$$
u_{r}^{i n c}=\cos \theta e^{-\hat{\imath} \kappa r \cos \theta}
$$




$$
\begin{aligned}
& u_{\theta}^{i n c}=-\sin \theta e^{-\hat{\imath} \kappa r \cos \theta}, \\
& \sigma_{r r}^{i n c}=\left[\lambda(-\hat{\imath} \kappa)+2 \mu\left(-\hat{\imath} \kappa \cos ^{2} \theta\right)\right] e^{-\hat{\imath} \kappa r \cos \theta}, \\
& \sigma_{r \theta}^{i n c}=\mu(2 \hat{\imath} \kappa \sin \theta \cos \theta) e^{-\hat{\imath} \kappa r \cos \theta}, \\
& \sigma_{\theta \theta}^{i n c}=\left[\lambda(-\hat{\imath} \kappa)+2 \mu\left(-\hat{\imath} \kappa \sin ^{2} \theta\right)\right] e^{-\hat{\imath} \kappa r \cos \theta}, \\
& \sigma_{\varphi \varphi}^{i n c}=\lambda(-\hat{\imath} \kappa) e^{-\hat{\imath} \kappa r \cos \theta} \\
& u_{\varphi}^{i n c}=\sigma_{r \varphi}^{i n c}=\sigma_{\theta \varphi}^{i n c}=0 . \\
& u_{z}^{i n c}=e^{-\hat{\imath} \kappa r \cos \theta}=e^{-\hat{\imath} \kappa z}, \\
& \sigma_{x x}^{i n c}=\lambda(-\hat{\imath} \kappa) e^{-\hat{\imath} \kappa z}, \\
& \sigma_{y y}^{i n c}=\lambda(-\hat{\imath} \kappa) e^{-\hat{\imath} \kappa z}, \\
& \sigma_{z z}^{i n c}=(\lambda+2 \mu)(-\hat{\imath} \kappa) e^{-\hat{\imath} \kappa z} . \\
& u_{x}^{i n c}=u_{y}^{i n c}=\sigma_{x y}^{i n c}=\sigma_{x z}^{i n c}=\sigma_{y z}^{i n c}=0
\end{aligned}
$$

After solving Eq. (3-75), the scattered wave expansion coefficients $\left\{\mathbb{B}_{I}\right\}$ are obtained in Scatterer I's local coordinate system. For any point $(x, y, z)$ in the global Cartesian coordinate system, its coordinates $\left(x_{I}, y_{I}, z_{I}\right)$ in local Cartesian coordinate system can be obtained through coordinate translation, and its coordinates $\left(r_{I}, \theta_{I}, \varphi_{I}\right)$ in local spherical coordinate system can be obtained using Eq. (D-2). The contribution to the displacements and stresses from the scattered waves can be expressed in each scatterer's local spherical coordinate system. For Scatterer I,

$$
\begin{aligned}
u_{I r_{I}}^{s c t}= & \sum_{n=0}^{\infty} \sum_{m=-n}^{n}\left[B_{I m n}^{\phi} \mathfrak{E}_{11}^{13}\left(r_{I}, n\right)+B_{I m n}^{\chi} \mathfrak{E}_{11}^{33}\left(r_{I}, n\right)\right] Y_{n}^{m} \\
u_{I \theta_{I}}^{s c t}= & \sum_{n=0}^{\infty} \sum_{m=-n}^{n}\left\{\left[B_{I m n}^{\phi} \mathfrak{E}_{21}^{13}\left(r_{I}, n\right)+B_{I m n}^{\chi} \mathfrak{E}_{21}^{33}\left(r_{I}, n\right)\right]\left(Y_{, \theta}\right)_{n}^{m}\right. \\
& \left.\quad+B_{I m n}^{\psi} \mathfrak{E}_{21}^{23}\left(r_{I}, n\right)\left(Y_{, \varphi}\right)_{n}^{m}\right\} \\
u_{I \varphi_{I}}^{s c t}= & \sum_{n=0}^{\infty} \sum_{m=-n}^{n}\left\{\left[B_{I m n}^{\phi} \mathfrak{E}_{31}^{13}\left(r_{I}, n\right)+B_{I m n}^{\chi} \mathfrak{E}_{31}^{33}\left(r_{I}, n\right)\right]\left(Y_{, \varphi}\right)_{n}^{m}\right.
\end{aligned}
$$




$$
\begin{aligned}
& \left.+B_{I m n}^{\psi} \mathfrak{E}_{31}^{23}\left(r_{I}, n\right)\left(Y_{, \theta}\right)_{n}^{m}\right\} \\
& \sigma_{I r_{I} r_{I}}^{s c t}=\sum_{n=0}^{\infty} \sum_{m=-n}^{n}\left\{B_{I m n}^{\phi} \mathfrak{E}_{41}^{13}\left(r_{I}, n\right)+B_{I m n}^{\chi} \mathfrak{E}_{41}^{33}\left(r_{I}, n\right)\right\} Y_{n}^{m} \\
& \sigma_{I r_{I} \theta_{I}}^{s c t}=\sum_{n=0}^{\infty} \sum_{m=-n}^{n}\left\{\left[B_{I m n}^{\phi} \mathfrak{E}_{71}^{13}\left(r_{I}, n\right)+B_{I m n}^{\chi} \mathfrak{E}_{71}^{33}\left(r_{I}, n\right)\right]\left(Y_{, \theta}\right)_{n}^{m}\right. \\
& \left.+B_{I m n}^{\psi} \mathfrak{E}_{71}^{23}\left(r_{I}, n\right)\left(Y_{, \varphi}\right)_{n}^{m}\right\} \\
& \sigma_{I r_{I} \varphi_{I}}^{s c t}=\sum_{n=0}^{\infty} \sum_{m=-n}^{n}\left\{\left[B_{I m n}^{\phi} \mathfrak{E}_{81}^{13}\left(r_{I}, n\right)+B_{I m n}^{\chi} \mathfrak{E}_{81}^{33}\left(r_{I}, n\right)\right]\left(Y_{, \varphi}\right)_{n}^{m}\right. \\
& \left.+B_{I m n}^{\psi} \mathfrak{E}_{81}^{23}\left(r_{I}, n\right)\left(Y_{, \theta}\right)_{n}^{m}\right\} \\
& \sigma_{I \theta_{I} \theta_{I}}^{s c t}=\sum_{n=0}^{\infty} \sum_{m=-n}^{n}\left\{B_{I m n}^{\phi} \mathfrak{E}_{51}^{13}\left(\mathbf{r}_{I}, n\right)+B_{I m n}^{\chi} \mathfrak{E}_{51}^{33}\left(\mathbf{r}_{I}, n\right)+B_{I m n}^{\psi} \mathfrak{E}_{51}^{23}\left(\mathbf{r}_{I}, n\right)\right\} \\
& \sigma_{I \varphi_{I} \varphi_{I}}^{s c t}=\sum_{n=0}^{\infty} \sum_{m=-n}^{n}\left\{B_{I m n}^{\phi} \mathfrak{E}_{61}^{13}\left(\mathbf{r}_{I}, n\right)+B_{I m n}^{\chi} \mathfrak{E}_{61}^{33}\left(\mathbf{r}_{I}, n\right)+B_{I m n}^{\psi} \mathfrak{E}_{61}^{23}\left(\mathbf{r}_{I}, n\right)\right\} \\
& \sigma_{I \theta_{I} \varphi_{I}}^{s c t}=\sum_{n=0}^{\infty} \sum_{m=-n}^{n}\left\{B_{I m n}^{\phi} \mathfrak{E}_{91}^{13}\left(\mathbf{r}_{I}, n\right)+B_{I m n}^{\chi} \mathfrak{E}_{91}^{33}\left(\mathbf{r}_{I}, n\right)+B_{I m n}^{\psi} \mathfrak{E}_{91}^{23}\left(\mathbf{r}_{I}, n\right)\right\}
\end{aligned}
$$

The contribution to the displacements and stresses from the scattered waves can also be expressed in each scatterer's local Cartesian coordinate system. For Scatterer $I$,

$$
\begin{aligned}
&\left\{\begin{array}{c}
u_{I x}^{s c t} \\
u_{I y}^{s c t} \\
u_{I z}^{s c t}
\end{array}\right\}= {\left[\begin{array}{ccc}
\sin \theta_{I} \cos \varphi_{I} & \sin \theta_{I} \sin \varphi_{I} & \cos \theta_{I} \\
\cos \theta_{I} \cos \varphi_{I} & \cos \theta_{I} \sin \varphi_{I} & -\sin \theta_{I} \\
-\sin \varphi_{I} & \cos \varphi_{I} & 0
\end{array}\right]\left\{\begin{array}{c}
u_{I r_{I}}^{s c t} \\
u_{I \theta_{I}}^{s c t} \\
u_{I \varphi_{I}}^{s c t}
\end{array}\right\}, } \\
& {\left[\begin{array}{cccc}
\sigma_{I x x}^{s c t} & \sigma_{I x y}^{s c t} & \sigma_{I x z}^{s c t} \\
\sigma_{I x y}^{s c t} & \sigma_{I y y}^{s c t} & \sigma_{I y z}^{s c t} \\
\sigma_{I x z}^{s c t} & \sigma_{I y z}^{s c t} & \sigma_{I z z}^{s c t}
\end{array}\right]=} {\left[\begin{array}{ccc}
\sin \theta_{I} \cos \varphi_{I} & \sin \theta_{I} \sin \varphi_{I} & \cos \theta_{I} \\
\cos \theta_{I} \cos \varphi_{I} & \cos \theta_{I} \sin \varphi_{I} & -\sin \theta_{I} \\
-\sin \varphi_{I} & \cos \varphi_{I} & 0
\end{array}\right]\left[\begin{array}{ccc}
\sigma_{I r_{I} r_{I}}^{s c t} & \sigma_{I r_{I} \theta_{I}}^{s c t} & \sigma_{I r_{I} \varphi_{I}}^{s c t} \\
\sigma_{I r_{I} \theta_{I}}^{s c t} & \sigma_{I \theta_{I} \theta_{I}}^{s c t} & \sigma_{I \theta_{I} \varphi_{I}}^{s c t} \\
\sigma_{I r_{I} \varphi_{I}}^{s c t} & \sigma_{I \theta_{I} \varphi_{I}}^{s c t} & \sigma_{I \varphi_{I} \varphi_{I}}^{s c t}
\end{array}\right] } \\
& {\left[\begin{array}{ccc}
\sin \theta_{I} \cos \varphi_{I} & \sin \theta_{I} \sin \varphi_{I} & \cos \theta_{I} \\
\cos \theta_{I} \cos \varphi_{I} & \cos \theta_{I} \sin \varphi_{I} & -\sin \theta_{I} \\
-\sin \varphi_{I} & \cos \varphi_{I} & 0
\end{array}\right] }
\end{aligned}
$$


The total displacements and stresses in the host medium in the global Cartesian system can be expressed as

$$
\begin{gathered}
u_{x}=u_{x}^{i n c}+\sum_{I=1}^{N} u_{I x}^{s c t} \\
u_{y}=u_{y}^{i n c}+\sum_{I=1}^{N} u_{I y}^{s c t} \\
u_{z}=u_{z}^{i n c}+\sum_{I=1}^{N} u_{I z}^{s c t} \\
\sigma_{x x}=\sigma_{x x}^{i n c}+\sum_{I=1}^{N} \sigma_{I x x}^{s c t} \\
\sigma_{x y}=\sigma_{x y}^{i n c}+\sum_{I=1}^{N} \sigma_{I x y}^{s c t} \\
\sigma_{x z}=\sigma_{x z}^{i n c}+\sum_{I=1}^{N} \sigma_{I x z}^{s c t} \\
\sigma_{y y}=\sigma_{y y}^{i n c}+\sum_{I=1}^{N} \sigma_{I y y}^{s c t} \\
\sigma_{z z}=\sigma_{z z}^{i n c}+\sum_{I=1}^{N} \sigma_{I z z}^{s c t} \\
\sigma_{y z}=\sigma_{y z}^{i n c}+\sum_{I=1}^{N} \sigma_{I y z}^{s c t}
\end{gathered}
$$

where $N$ is the number of scatterers.

Following Eqs. (D-8b) and (D-10b) for coordinate transformation, the total displacements and stresses in the global spherical system can be obtained from those in the global Cartesian system.

At a position inside of a scatterer, wave fields are computed from the refracted waves of the scatterer. For Scatterer $I$, the refracted wave expansion coefficients $\{\mathbb{C}\}_{I}$ are obtained from the scattered wave expansion coefficients $\left\{\mathbb{B}_{I}\right\}$ using Eq. (2-107), such as

$$
\left\{\mathbb{C}_{I}\right\}=\left[\mathbb{G}_{I}\right]\left\{\mathbb{B}_{I}\right\}
$$


where $\left[\mathbb{G}_{I}\right]$ can be obtained using Eq. (2-108). For Scatterer $I$,

$$
\begin{aligned}
& u_{I r_{I}}^{r e f}= \sum_{n=0}^{\infty} \sum_{m=-n}^{n}\left[C_{I m n}^{\phi} \mathfrak{E}_{12}^{11}\left(r_{I}, n\right)+C_{I m n}^{\chi} \mathfrak{E}_{12}^{31}\left(r_{I}, n\right)\right] Y_{n}^{m} \\
& u_{I \theta_{I}}^{r e f}= \sum_{n=0}^{\infty} \sum_{m=-n}^{n}\left\{\left[C_{I m n}^{\phi} \mathfrak{E}_{22}^{11}\left(r_{I}, n\right)+C_{I m n}^{\chi} \mathfrak{E}_{22}^{31}\left(r_{I}, n\right)\right]\left(Y_{, \theta}\right)_{n}^{m}\right. \\
&\left.+C_{I m n}^{\psi} \mathfrak{E}_{22}^{21}\left(r_{I}, n\right)\left(Y_{, \varphi}\right)_{n}^{m}\right\} \\
& u_{I \varphi_{I}}^{r e f}= \sum_{n=0}^{\infty} \sum_{m=-n}^{n}\left\{\left[C_{I m n}^{\phi} \mathfrak{E}_{32}^{11}\left(r_{I}, n\right)+C_{I m n}^{\chi} \mathfrak{E}_{32}^{31}\left(r_{I}, n\right)\right]\left(Y_{, \varphi}\right)_{n}^{m}\right. \\
&\left.+C_{I m n}^{\psi} \mathfrak{E}_{32}^{21}\left(r_{I}, n\right)\left(Y_{, \theta}\right)_{n}^{m}\right\} \\
& \sigma_{I r_{I} r_{I}}^{r e f}= \sum_{n=0}^{\infty} \sum_{m=-n}^{n}\left\{C_{I m n}^{\phi} \mathfrak{E}_{42}^{11}\left(r_{I}, n\right)+C_{I m n}^{\chi} \mathfrak{E}_{42}^{31}\left(r_{I}, n\right)\right\} Y_{n}^{m} \\
& \sigma_{I r_{I} \theta_{I}}^{r e f}= \sum_{n=0}^{\infty} \sum_{m=-n}^{n}\left\{\left[C_{I m n}^{\phi} \mathfrak{E}_{72}^{11}\left(r_{I}, n\right)+C_{I m n}^{\chi} \mathfrak{E}_{72}^{31}\left(r_{I}, n\right)\right]\left(Y_{, \theta}\right)_{n}^{m}\right. \\
&\left.+C_{I m n}^{\psi} \mathfrak{E}_{72}^{21}\left(r_{I}, n\right)\left(Y_{, \varphi}\right)_{n}^{m}\right\} \\
& \sigma_{I \varphi_{I} \varphi_{I}}^{r e f}= \sum_{n=0}^{\infty} \sum_{m=-n}^{n}\left\{C_{I m n}^{\phi} \mathfrak{E}_{62}^{11}\left(\mathbf{r}_{I}, n\right)+C_{I m n}^{\chi} \mathfrak{E}_{62}^{31}\left(\mathbf{r}_{I}, n\right)+C_{I m n}^{\psi} \mathfrak{E}_{62}^{21}\left(\mathbf{r}_{I}, n\right)\right\} \\
& \sigma_{I \theta_{I} \varphi_{I}}^{r e f}= \sum_{n=0}^{\infty} \sum_{m=-n}^{n}\left\{C_{I m n}^{\phi} \mathfrak{E}_{92}^{11}\left(\mathbf{r}_{I}, n\right)+C_{I m n}^{\chi} \mathfrak{E}_{92}^{31}\left(\mathbf{r}_{I}, n\right)+C_{I m n}^{\psi} \mathfrak{E}_{92}^{21}\left(\mathbf{r}_{I}, n\right)\right\} \\
& \sigma_{I \theta_{I} \theta_{I}}^{r e f}= \sum_{n=0}^{\infty} \sum_{m=-n}^{n}\left\{C_{I m n}^{\phi} \mathfrak{E}_{52}^{11}\left(\mathbf{r}_{I}, n\right)+C_{I m n}^{\chi} \mathfrak{E}_{52}^{31}\left(\mathbf{r}_{I}, n\right)+C_{I m n}^{\psi} \mathfrak{E}_{52}^{21}\left(\mathbf{r}_{I}, n\right)\right\} \\
&= \sum_{n=0}^{n}\left\{\left[C_{I m n}^{\phi} \mathfrak{E}_{82}^{11}\left(r_{I}, n\right)+C_{I m n}^{\chi} \mathfrak{E}_{82}^{31}\left(r_{I}, n\right)\right]\left(Y_{, \varphi}\right)_{n}^{m}\right. \\
&\left.\left.\sigma_{I r_{I} \varphi_{I}}, n\right)\left(Y_{, \theta}\right)_{n}^{m}\right\}
\end{aligned}
$$

and

$$
\left\{\begin{array}{c}
u_{I x}^{r e f} \\
u_{I y}^{r e f} \\
u_{I z}^{r e f}
\end{array}\right\}=\left[\begin{array}{ccc}
\sin \theta_{I} \cos \varphi_{I} & \sin \theta_{I} \sin \varphi_{I} & \cos \theta_{I} \\
\cos \theta_{I} \cos \varphi_{I} & \cos \theta_{I} \sin \varphi_{I} & -\sin \theta_{I} \\
-\sin \varphi_{I} & \cos \varphi_{I} & 0
\end{array}\right]^{T}\left\{\begin{array}{c}
u_{I r_{I}}^{r e f} \\
u_{I \theta_{I}}^{r e f} \\
u_{I \varphi_{I}}^{r e f}
\end{array}\right\},
$$




$$
\begin{aligned}
{\left[\begin{array}{ccc}
\sigma_{I x x}^{r e f} & \sigma_{I x y}^{r e f} & \sigma_{I x z}^{r e f} \\
\sigma_{I x y}^{r e f} & \sigma_{I y y}^{r e f} & \sigma_{I y z}^{r e f} \\
\sigma_{I x z}^{r e f} & \sigma_{I y z}^{r e f} & \sigma_{I z z}^{r e f}
\end{array}\right]=} & {\left[\begin{array}{ccc}
\sin \theta_{I} \cos \varphi_{I} & \sin \theta_{I} \sin \varphi_{I} & \cos \theta_{I} \\
\cos \theta_{I} \cos \varphi_{I} & \cos \theta_{I} \sin \varphi_{I} & -\sin \theta_{I} \\
-\sin \varphi_{I} & \cos \varphi_{I} & 0
\end{array}\right]\left[\begin{array}{ccc}
\sigma_{I r_{I} r_{I}}^{r e f} & \sigma_{I r_{I} \theta_{I}}^{r e f} & \sigma_{I r_{I} \varphi_{I}}^{r e f} \\
\sigma_{I r_{I} \theta_{I}}^{r e f} & \sigma_{I \theta_{I} \theta_{I}}^{r e f} & \sigma_{I \theta_{I} \varphi_{I}}^{r e f} \\
\sigma_{I r_{I} \varphi_{I}}^{r e f} & \sigma_{I \theta_{I} \varphi_{I}}^{r e f} & \sigma_{I \varphi_{I} \varphi_{I}}^{r e f}
\end{array}\right] } \\
& {\left[\begin{array}{cccc}
\sin \theta_{I} \cos \varphi_{I} & \sin \theta_{I} \sin \varphi_{I} & \cos \theta_{I} \\
\cos \theta_{I} \cos \varphi_{I} & \cos \theta_{I} \sin \varphi_{I} & -\sin \theta_{I} \\
-\sin \varphi_{I} & \cos \varphi_{I} & 0
\end{array}\right] }
\end{aligned}
$$

Wave fields in a local Cartesian system are the same as in the global Cartesian system. 


\section{Chapter 4}

\section{Implementation of Multiple Scattering Solution}

\section{4-1 Introduction}

In this chapter, issues concerning computer implementation of the multiple scattering solution are addressed. Since the addition theorems are important parts of the multiple scattering solution, issues concerning their implementation are also considered in this chapter.

In this thesis, the multiple-scattering solution, which is represented by a set of linear equations, Eq. (3-75), is implemented. In Section 4-2, numerical examples of computing wave fields are used to examine implementation of the addition theorems. In Section 4-3, the relation of truncation number and truncation error for the multiple scattering solution is discussed, and the multiple-scattering solution is verified. The verifications are based on three approaches: (1) published results, (2) energy conservation, and (3) boundary continuities. 


\section{4-2 Implementation of Addition Theorems}

Figure 4.1, which is the same as Fig. 3.3, is referred to in this section. The origin of the global coordinate system is the position $O$, and the origin of the local coordinate system is the position $O^{\prime}$. Wave expansion bases in both coordinate systems can be transformed from each other with help of the scalar addition theorems. Expressions of a vector, such as a displacement vector and a surface traction, in both coordinate systems can be transformed with help of the vector addition theorem. The scalar addition theorem is used in the vector addition theorem. Wave fields, such as displacements and stresses, can be computed in both coordinate systems. Each field, such as $u_{x}$, computed in both coordinate systems is expected to be same. Implementation of the addition theorems are verified through comparison of wave fields in two coordinate systems.

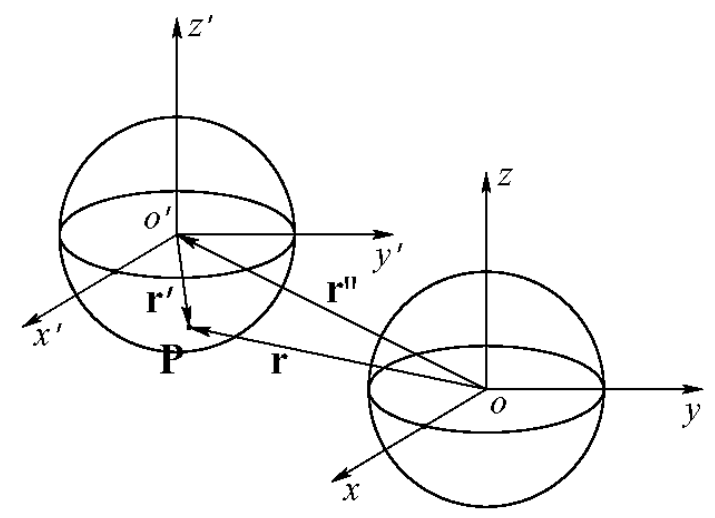

Figure 4.1: Vectors $\boldsymbol{r}=\boldsymbol{r}^{\prime}+\boldsymbol{r}^{\prime \prime}$ for addition theorem.

Ten positions at a spherical surface of unit radius are chosen for computing displacements and stresses. The spherical surface is centered at position $O^{\prime}$, the origin of the local coordinate system in Fig. 4.1. In the local coordinate system, spherical and Cartesian coordinates are listed in Table 4.1. The spherical coordinates in Table 4.1 
are chosen according to the following Fibonacci grids:

$$
r_{i}^{\prime}=R=1, \theta_{i}^{\prime}=\operatorname{acos}\left[-1+0.4 i+\frac{\sin (\pi(-1+0.4 i))}{\pi}\right], \text { and } \varphi_{i}=0.6 i \pi .
$$

Equation (4-1) is obtained by letting $F=5$ and $F^{\prime}=3$ in Eq. (2-129). The spherical coordinates of the first five positions in Table 4.1 are $\left(r_{i}^{\prime}, \theta_{i}^{\prime}, \varphi_{i}^{\prime}\right)$ with $1 \leq i \leq 5$, and the spherical coordinates of the last five positions $\left(r_{i}^{\prime}, \theta_{i}^{\prime}, \pi+\varphi_{i}^{\prime}\right)$ with $1 \leq i \leq$ 5. The Cartesian coordinates in Table 4.1 are obtained from spherical coordinates

Table 4.1: Coordinates of Ten Positions in the Local System.

\begin{tabular}{|c|c|rrr|}
\hline Position & $\begin{array}{c}\text { Spherical coordinates } \\
r^{\prime}, \theta^{\prime} \text { (radians) }, \varphi^{\prime} \text { (radians) }\end{array}$ & \multicolumn{3}{|c|}{ Cartesian coordinates } \\
\hline 1 & $1,2.696872,1.884956$ & -0.132941, & \multicolumn{1}{c|}{0.409150,} & -0.902731 \\
2 & $1,1.968278,3.769911$ & -0.745945, & -0.541961, & -0.387098 \\
3 & $1,1.173314,5.654867$ & 0.745945, & -0.541961, & 0.387098 \\
4 & $1,0.444721,1.256637$ & 0.132941, & 0.409150, & 0.902731 \\
5 & $1,0.100000,3.141593$ & -0.099833, & $1.223108 \times 10^{-17}$, & 0.995004 \\
6 & $1,2.696872,5.026548$ & 0.132941, & -0.409150, & -0.902731 \\
7 & $1,1.968278,0.628319$ & 0.745945, & 0.541961, & -0.387098 \\
8 & $1,1.173314,2.513274$ & -0.745945, & 0.541961, & 0.387098 \\
9 & $1,0.444721,4.398230$ & -0.132941, & -0.409150, & 0.902731 \\
10 & $1,0.100000,6.283185$ & 0.099833, & $-2.446215 \times 10^{-17}$, & 0.995004 \\
\hline
\end{tabular}

using Eq. (D-1). Since $\boldsymbol{r}\left(r_{i}, \theta_{i}, \varphi_{i}\right)=\boldsymbol{r}^{\prime}\left(r_{i}^{\prime}, \theta_{i}^{\prime}, \varphi_{i}^{\prime}\right)+\boldsymbol{r}^{\prime \prime}\left(r_{i}^{\prime \prime}, \theta_{i}^{\prime \prime}, \varphi_{i}^{\prime \prime}\right)$ in Fig. 4.1, global coordinates $\left(r_{i}, \theta_{i}, \varphi_{i}\right)$ can be determined for these ten positions when $\boldsymbol{r}^{\prime \prime}$ is specified.

The number of significant figures is used to describe the accuracy of a numerical value compared with another value. In scientific notation $a \times 10^{b}$, coefficient $a$ is chosen such that $1 \leq a<10$ and exponent $b$ is an integer. For comparison of $a_{1} \times 10^{b_{1}}$ with $a_{2} \times 10^{b_{2}}$ in general, the number of significant figures is zero when $b_{1} \neq b_{2}$, and the number of significant figures is the number of digits which are the same between $a_{1}$ and $a_{2}$. Here, a reference value is used as an extra condition to the definition of significant figures. The new definition follows through three numerical 
values: $a_{1} \times 10^{b_{1}}, a_{2} \times 10^{b_{2}}$ and $a_{3} \times 10^{b_{3}}$. When the value $a_{3} \times 10^{b_{3}}$ is used as the reference to compare $a_{1} \times 10^{b_{1}}$ with $a_{2} \times 10^{b_{2}}$, the number of significant figures is:

(1) the number of digits which are the same between $a_{1}$ and $a_{2}$, when $b_{1}=b_{2}$ and $b_{1} \geq b_{3}$

(2) zero, when $b_{1} \neq b_{2}$ and $b_{1} \geq b_{3}$ or $b_{2} \geq b_{3}$;

(3) the number of same digits between $a_{1}$ and $a_{2}$, plus $b_{3}-b_{1}$, when $b_{1}=b_{2}$ and $b_{1}<b_{3}$;

(4) $b_{3}-b_{1}$, when $b_{1} \neq b_{2}, b_{1}<b_{3}$ and $b_{2}<b_{3}$.

Examples are given bellow to specify the reason for this definition.

For comparison of displacement $u_{x}, u_{y}$ or $u_{z}$ at ten positions, the magnitude of $u_{x}, u_{y}$ and $u_{z}$ at the positions may make a big difference. The maximum value of $\sqrt{u_{x}^{2}+u_{y}^{2}+u_{z}^{2}}$ among the ten positions is chosen as the reference to compare values at each of the ten positions. For example, assume the reference value is $1.000000 \times 10^{1}$ to compare two values of $u_{x}$ in global and local coordinate systems. That is $a_{3} \times 10^{b_{3}}=$ $1.000000 \times 10^{1}$. Then,

(1) at one position, $a_{1} \times 10^{b_{1}}=9.091254 \times 10^{0}$ and $a_{2} \times 10^{b_{2}}=9.091252 \times 10^{0}$ so that the number of significant figures is 6 ;

(2) at one position, $a_{1} \times 10^{b_{1}}=8.919245 \times 10^{0}$ and $a_{2} \times 10^{b_{2}}=9.291053 \times 10^{0}$ so that the number of significant figures is 0 ;

(3) at one position, $a_{1} \times 10^{b_{1}}=2.092145 \times 10^{-6}$ and $a_{2} \times 10^{b_{2}}=2.130426 \times 10^{-6}$ so that the number of significant figures is $1+1-(-6)=8$;

(4) at one position, $a_{1} \times 10^{b_{1}}=2.091254 \times 10^{-14}$ and $a_{2} \times 10^{b_{2}}=1.103462 \times 10^{-7}$ so that the number of significant figures is $0+1-(-7)=8$.

The comparison of small values is highlighted in this definition of significant figures.

In this section, a steel sphere and an ice matrix are chosen, because the ice matrix is going to be used in next section and the elastic properties and mass density of steel 
are much larger than the corresponding ones of ice. The material properties are given in Table 4.2. For scattering of elastic waves by a steel sphere embedded in ice matrix, wave fields are computed in the global and local coordinate systems in Fig 4.1. The steel sphere of unit radius sits at origin $O$ of the global coordinate system. The wave frequency is $10^{5} \mathrm{hz}$. In ice, the longitudinal wave number is $\kappa a=1.5787$ (with $a$ as the radius of the steel sphere) and shear wave number is $k a=3.1574$. In the steel, the longitudinal wave number is $\kappa a=16.8740$ and shear wave number is $k a=31.2248$.

Table 4.2: Material Properties of Low Carbon Steel (After [2]) and Ice (After [3]).

\begin{tabular}{|c|c|c|}
\hline Property & Matrix (Ice) & Sphere (Steel) \\
\hline Lamé constant $\lambda(\mathrm{GPa})$ & 7.92 & 113.9 \\
Lamé constant $\mu(\mathrm{GPa})$ & 3.96 & 80. \\
Density $\left(\mathrm{kg} / \mathrm{m}^{3}\right)$ & 1000. & 7800. \\
$\mathrm{P}$ wave speed $(\mathrm{km} / \mathrm{s})$ & 3.98 & 5.926 \\
$\mathrm{~S}$ wave speed $(\mathrm{km} / \mathrm{s})$ & 1.99 & 3.203 \\
\hline
\end{tabular}

\section{4-2.1 Transformation of Incident Wave Fields}

In this section, incident wave fields at ten positions are computed in the global and local coordinate systems in Fig. 4.1. Analytical expressions of the incident wave fields are given in the global coordinate system. The vector addition theorem is used in computation of incident wave fields in the local coordinate systems. Physically, the incident wave fields at the same position should be the same. Then, comparison of incident wave fields between two coordinate systems can be used to verify the vector addition theorem.

The local coordinates of the ten positions are specified in Table 4.1. Two cases in which $\boldsymbol{r}^{\prime \prime}=2.1 a \boldsymbol{e}_{x}+2.1 a \boldsymbol{e}_{y}+2.1 a \boldsymbol{e}_{z}$ and $\boldsymbol{r}^{\prime \prime}=21 a \boldsymbol{e}_{x}+21 a \boldsymbol{e}_{y}+21 a \boldsymbol{e}_{z}$ are considered. An incident wave is represented by displacement potentials $\phi, \psi$ and $\chi$. Three types 
of incident waves are used in this section.

First, an incident wave is taken as $\psi=\chi=0$, and $\phi$ in Eq. (2-131). Equation $(2-131)$ is repeated as

$$
\phi=\frac{\hat{\imath}}{\kappa} e^{-\hat{\imath} \kappa r \cos (\theta)}=\frac{\hat{\imath}}{\kappa} e^{-\hat{\imath} \kappa z} .
$$

The analytical expressions of displacements and stresses of this wave in the global Cartesian coordinate systems are given in Eq. (2-138).

The wave expansion expression of $\phi$ in Eq. (2-132) is repeated as Eq. (4-3).

$$
\phi^{i n c}=\sum_{n=0}^{\infty} \frac{(-\hat{\imath})^{n}}{\kappa} \sqrt{4 \pi(2 n+1)} j_{n}(\kappa r) Y_{n}^{0}(\theta, \varphi)
$$

The wave expansion coefficients of the incident wave in the global coordinate system are obtained and written as $\left\{\mathbb{A}_{O}\right\}$. The wave expansion coefficients of the incident wave in local coordinate system, $\left\{\mathbb{A}_{O^{\prime}}\right\}$, can be obtained from $\left\{\mathbb{A}_{O}\right\}$ by applying the vector addition theorem (through Eq. (3-49)). Equation (3-49) is written as

$$
\left\{\mathbb{A}_{O^{\prime}}\right\}=\left[R g \mathbb{Q}_{O O^{\prime}}\right]^{T}\left\{\mathbb{A}_{O}\right\}
$$

The incident wave fields in the local spherical system can be computed through wave expansion expressions in Eqs. (2-43) and (2-44). The incident wave fields in the local Cartesian coordinate system can be obtained from those in the local spherical system by using coordinate transformation of Eqs. (D-8a) and (D-10a). Each pair of incident wave field in the local and global Cartesian systems, such as $u_{x}$ and $u_{x^{\prime}}$, should be the same.

The maximum value of $\sqrt{u_{x}^{2}+u_{y}^{2}+u_{z}^{2}}$ among ten positions is taken as the reference for the comparison of each displacement component $u_{x}, u_{y}$ or $u_{z}$. The number of significant figures of displacement $u_{z}$ at one position is obtained by comparing the two values in both coordinate systems. The smallest number of significant figures of $u_{z}$ at all ten positions is taken as the number of significant figures of $u_{z}$. The smallest number of significant figures of displacements $u_{x}, u_{y}$ and $u_{z}$ is taken as the number of 
significant figures of displacements. The number of significant figures for the stresses is obtained in the same way.

Figure 4.2 shows the relation between the truncation number and significant figures of displacements and the relation between the truncation number and significant figures of stresses. In Fig. 4.2, $r^{\prime \prime}=3.64$ represents that origin $O^{\prime}$ has Cartesian coordinates $x=y=z=2.1 a$ in global Cartesian system and $r^{\prime \prime}=36.4$ represents that origin $O^{\prime}$ has Cartesian coordinates $x=y=z=21 a$ in global Cartesian system.

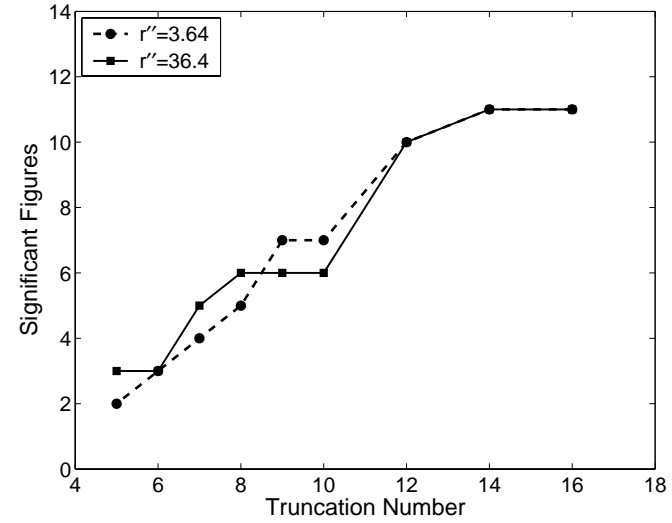

(a) For displacements

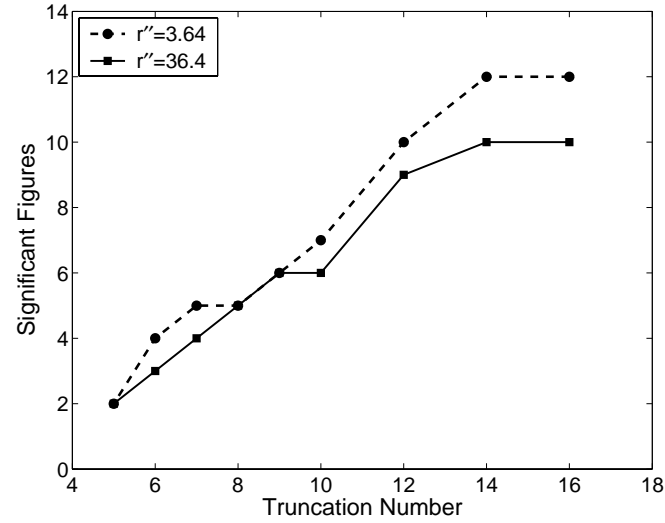

(b) For stresses

Figure 4.2: Significant figures of displacements and stresses due to incident wave $\phi$ after coordinate transformation. $\kappa a=1.5757$ and $r^{\prime}=a=1$. (a) Significant figures of displacements vs. truncation number. (b) Significant figures of stresses vs. truncation number.

Second, an incident wave is taken as $\phi=\chi=0$ and

$$
\psi=\frac{\hat{\imath}}{k} e^{-\hat{\imath} k r \cos (\theta)}=\frac{\hat{\imath}}{k} e^{-\hat{\imath} k z} .
$$

In analogy to Eq. (4-3), $\psi$ can be expanded as

$$
\psi^{i n c}=\sum_{n=0}^{\infty} \frac{(-\hat{\imath})^{n}}{k} \sqrt{4 \pi(2 n+1)} j_{n}(k r) Y_{n}^{0}(\theta, \varphi) .
$$

Following relation between displacements, strains and stresses and displacement potentials, analytical expressions of displacements and stresses can be obtained for this 
incident wave. In spherical coordinate system,

$$
\begin{aligned}
u_{\varphi} & =r \sin \theta e^{-\hat{\imath} k r \cos \theta} \\
\sigma_{r \varphi} & =\mu[-\hat{\imath} k r \sin \theta \cos \theta)] e^{-\hat{\imath} k r \cos \theta} \\
\sigma_{\theta \varphi} & \left.=\mu\left[\hat{\imath} k r \sin ^{2} \theta\right)\right] e^{-\hat{\imath} k r \cos \theta} \\
u_{r} & =u_{\theta}=\sigma_{r r}=\sigma_{\theta \theta}=\sigma_{\varphi \varphi}=\sigma_{r \theta}=0 .
\end{aligned}
$$

In Cartesian coordinate system

$$
\begin{aligned}
u_{x} & =-r \sin \theta \sin \varphi e^{-\hat{\imath} k r \cos \theta}=-y e^{-\hat{\imath} k z} \\
u_{y} & =r \sin \theta \cos \varphi e^{-\hat{\imath} k r \cos \theta}=x e^{-\hat{\imath} k z} \\
\sigma_{x z} & =\mu(\hat{\imath} k y) e^{-\hat{\imath} k z} \\
\sigma_{y z} & =\mu(-\hat{\imath} k x) e^{-\hat{\imath} k z} \\
u_{z} & =\sigma_{x x}=\sigma_{x y}=\sigma_{y y}=\sigma_{z z}=0
\end{aligned}
$$

By following the procedure in the first case, displacements and stresses in the local Cartesian coordinate system are obtained. The relations between truncation number and numbers of significant figures of displacements and stresses are shown in Fig. 4.3.

Third, an incident wave is taken as $\phi=\psi=0$ and

$$
\chi=\frac{\hat{\imath}}{k} e^{-\hat{\imath} k r \cos (\theta)}=\frac{\hat{\imath}}{k} e^{-\hat{\imath} k z} .
$$

In analogy to Eq. (4-3), $\chi$ can be expanded as

$$
\chi^{i n c}=\sum_{n=0}^{\infty} \frac{(-\hat{\imath})^{n}}{k} \sqrt{4 \pi(2 n+1)} j_{n}(k r) Y_{n}^{0}(\theta, \varphi) .
$$

Following relation between displacements, strains and stresses and displacement potentials, analytical expressions of displacements and stresses can be obtained for this incident wave. In spherical coordinate system,

$$
u_{r}=\frac{1}{k}\left[2 \frac{\hat{\imath}}{k}(-\hat{\imath} k \cos \theta)+r \cos \theta(-\hat{\imath} k \cos \theta)+r k^{2} \frac{\hat{\imath}}{k}\right] e^{-\hat{\imath} k r \cos \theta}
$$




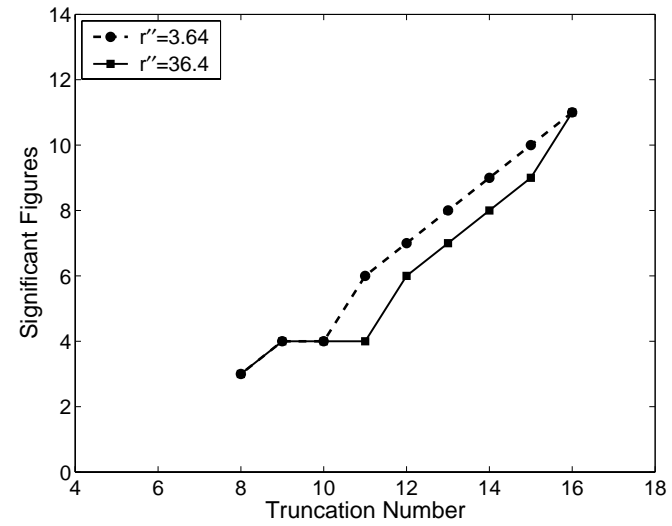

(a) For displacements

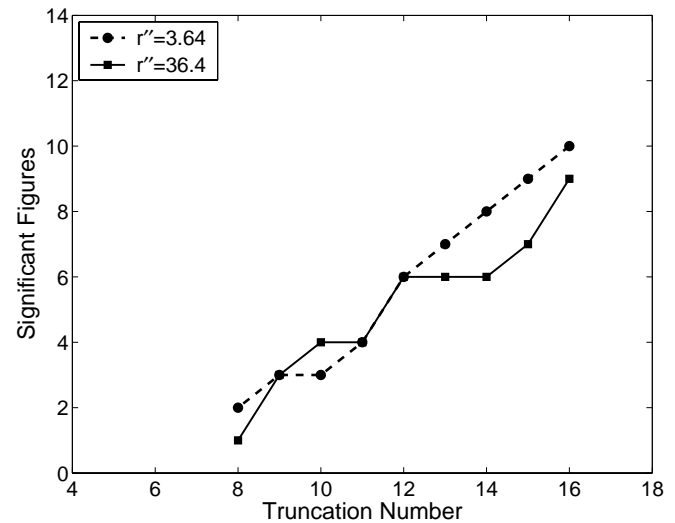

(b) For stresses

Figure 4.3: Significant figures of displacements and stresses due to incident wave $\psi$ after coordinate transformation. $k a=3.1574$ and $r^{\prime}=a=1$. (a) Significant figures of displacements vs. truncation number. (b) Significant figures of stresses vs. truncation number.

$$
\begin{aligned}
= & \frac{1}{k}\left[2 \cos \theta+\hat{\imath} k r \sin ^{2} \theta\right] e^{-\hat{\imath} k r \cos \theta} \\
u_{\theta}= & \frac{1}{k}\left[\frac{1}{r} \frac{\hat{\imath}}{k}(\hat{\imath} k r \sin \theta)+(-\sin \theta)+\cos \theta(\hat{\imath} k r \sin \theta)\right] e^{-\hat{\imath} k r \cos \theta} \\
= & \frac{1}{k}[-2 \sin \theta+\hat{\imath} k r \sin \theta \cos \theta] \\
\sigma_{r r}= & 2 \mu\left[k r \sin ^{2} \theta \cos \theta+\hat{\imath}\left(\sin ^{2} \theta-2 \cos ^{2} \theta\right)\right] e^{-\hat{\imath} k r \cos \theta} \\
\sigma_{r \theta}= & \frac{\mu}{k}\left[\frac{1}{r}\left(-2 \sin \theta-k^{2} r^{2} \sin ^{3} \theta+\hat{\imath} 4 k r \sin \theta \cos \theta\right)-\frac{1}{r}(-2 \sin \theta+\hat{\imath} k r \sin \theta \cos \theta)\right. \\
& \left.+k^{2} r \sin \theta \cos ^{2} \theta+\hat{\imath} 3 k \sin \theta \cos \theta\right] e^{-\hat{\imath} k r \cos \theta} \\
= & \mu\left[k r \sin \theta\left(\cos { }^{2} \theta-\sin ^{2} \theta\right)+\hat{\imath} 6 \sin \theta \cos \theta\right] e^{-\hat{\imath} k r \cos \theta} \\
\sigma_{\theta \theta}= & \frac{2 \mu}{k r}\left[2 \cos ^{2} \theta+\hat{\imath} k r \sin ^{2} \theta-2 \cos \theta-k^{2} r^{2} \sin { }^{2} \theta \cos \theta\right. \\
& \left.+\hat{\imath} k r\left(\cos ^{2} \theta-3 \sin ^{2} \theta\right)\right] e^{-\hat{\imath} k r \cos \theta} \\
= & 2 \mu\left[\hat{\imath}\left(\cos ^{2} \theta-2 \sin ^{2} \theta\right)-k r \sin { }^{2} \theta \cos \theta\right] e^{-\hat{\imath} k r \cos \theta} \\
\sigma_{\varphi \varphi}= & \frac{2 \mu}{k r}\left[0+2 \cos \theta+\hat{\imath} k r \sin ^{2} \theta+\frac{\cos \theta}{\sin \theta}(-2 \sin \theta+\hat{\imath} k r \sin \theta \cos \theta)\right] e^{-\hat{\imath} k r \cos \theta} \\
= & 2 \mu(\hat{\imath}) e^{-\hat{\imath} k r \cos \theta} \\
u_{\varphi}= & \sigma_{r \varphi}=\sigma_{\theta \varphi}=0
\end{aligned}
$$


In Cartesian coordinate system,

$$
\begin{aligned}
u_{x} & =\hat{\imath} r \sin \theta \sin \varphi e^{-\hat{\imath} k r \cos \theta}=\hat{\imath} x e^{-\hat{\imath} k z} \\
u_{y} & =\hat{\imath} r \sin \theta \sin \varphi e^{-\hat{\imath} k r \cos \theta}=\hat{\imath} y e^{-\hat{\imath} k z} \\
u_{z} & =\frac{2}{k} e^{-\hat{\imath} k z} \\
\sigma_{x x} & =2 \mu\left(\hat{\imath} e^{-\hat{\imath} k z}\right) \\
\sigma_{y y} & =2 \mu\left(\hat{\imath} e^{-\hat{\imath} k z}\right) \\
\sigma_{x x} & =2 \mu\left(-\hat{\imath} 2 e^{-\hat{\imath} k z}\right) \\
\sigma_{x z} & =\mu(k x) e^{-\hat{\imath} k z} \\
\sigma_{y z} & =\mu(k y) e^{-\hat{\imath} k z} \\
\sigma_{x y} & =0
\end{aligned}
$$

By following the procedure in the first case, displacements and stresses in the local Cartesian coordinate system are obtained. The relations between truncation number and numbers of significant figures of displacements and stresses are shown in Fig. 4.4.

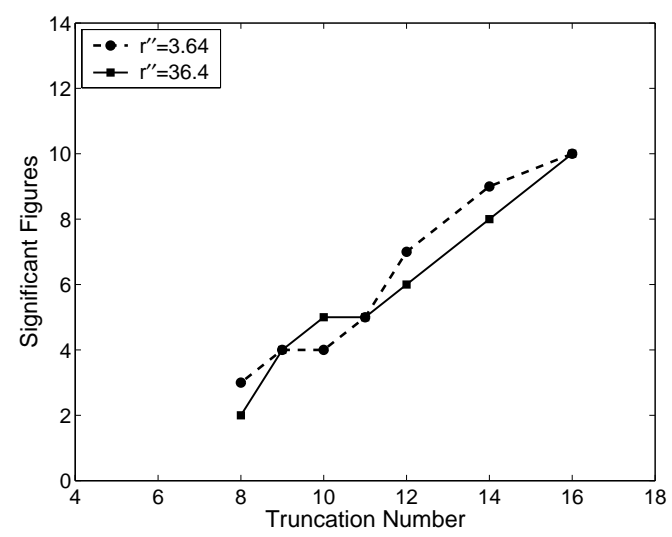

(a) For displacements

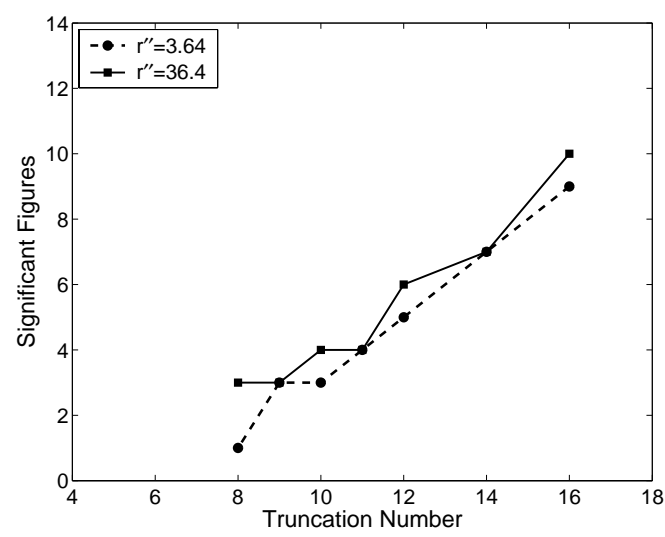

(b) For stresses

Figure 4.4: Significant figures of displacements and stresses due to incident wave $\psi$ after coordinate transformation. $k a=3.1574$ and $r^{\prime}=a$. (a) Significant figures of displacements vs. truncation number. (b) Significant figures of stresses vs. truncation number. 
In Fig. 4.2, displacements and stresses (due to incident wave $\phi$ ) computed in two coordinate systems have 5 significant figures when the truncation number is 5 , and the number of significant figures generally increases when the truncation number increases. In Fig. 4.3, displacements and stresses (due to incident wave $\psi$ ) computed in two coordinate systems have 3 or more significant figures when the truncation number is 9 , and the number of significant figures generally increases when the truncation number increases. In Fig. 4.4, displacements and stresses (due to incident wave $\chi$ ) computed in two coordinate systems have 3 or more significant figures when the truncation number is 9, and the number of significant figures generally increases when the truncation number increases. Because the vector addition theorem is used to compute the incident wave fields in the local coordinate system that agree with the incident wave fields in the global coordinate system, the vector addition theorem is verified.

In Figs. 4.2 through 4.4, for the same distance $r^{\prime \prime}$, the number of significant figures of shear wave fields is smaller than that of longitudinal wave fields after transformation, since the shear wave number is larger than the longitudinal wave number for same wave frequency. The truncation number should be not less than 14 in order to obtain 6 or more significant figures of incident wave fields for the case of $r^{\prime \prime} \leq 36.4$.

\section{4-2.2 Transformation of Scattered Wave Fields}

Scattered wave fields in one coordinate system can be re-expressed in another coordinate system with help of the vector addition theorem. Physically, the scattered wave fields at the same position should be the same. Then, comparison of scattered wave fields between two coordinate systems can be used to verify the vector addition theorem.

Single scattering is considered in this section. One scatterer is centered at position 
$O$, the origin of global coordinate system in Fig. 4.1. The scattered wave fields at ten positions are computed in the global and local coordinate systems in Fig. 4.1. The vector addition theorem is used in computation of the scattered wave fields in the local coordinate system. Comparison of the scattered wave fields between global and local coordinate systems can be used to verify the vector addition theorem.

The local coordinates of the ten positions are specified in Table 4.1. Two cases in which $\boldsymbol{r}^{\prime \prime}=2.1 a \boldsymbol{e}_{x}+2.1 a \boldsymbol{e}_{y}+2.1 a \boldsymbol{e}_{z}$ and $\boldsymbol{r}^{\prime \prime}=21 a \boldsymbol{e}_{x}+21 a \boldsymbol{e}_{y}+21 a \boldsymbol{e}_{z}$ are considered. An incident wave is represented by displacement potentials $\phi, \psi$ and $\chi$. Three types of incident waves are used in this section.

First, an incident wave is taken as $\psi=\chi=0$, and $\phi$ in Eq. (4-2). The wave expansion coefficients of the incident wave in the global coordinate system are obtained by using Eq. (4-3) and written as $\left\{\mathbb{A}_{O}\right\}$. The wave expansion coefficients of the scattered waves in the global coordinate system can be obtained by solving the single scattering problem, and can be written as $\left\{\mathbb{B}_{O}\right\}$. The displacements and stresses due to the scattered waves in the global coordinate system can be computed through wave expansion expressions in Eqs. (2-43) and (2-44). The scattered wave fields in the global Cartesian coordinate system can be obtained from those in the global spherical system by using coordinate transformation of Eqs. (D-8a) and (D-10a).

When the wave expansion bases $\{\boldsymbol{h}(\cdot)\}$ in the global coordinate system are transformed as $\{\boldsymbol{j}(\cdot)\}$, the wave expansion coefficients $\left\{\mathbb{A}_{O^{\prime}}\right\}$ corresponding to $\{\boldsymbol{j}(\cdot)\}$, can be obtained by applying vector addition theorem through Eq. (3-47). Equation (3-47) can be written as

$$
\left\{\mathbb{A}_{O^{\prime}}\right\}=\left[\mathbb{Q}_{O O^{\prime}}\right]^{T}\left\{\mathbb{B}_{O}\right\}
$$

The displacements and stresses due to the scattered waves in the local coordinate system can be computed through wave expansion expressions in Eqs. (2-43) and (2-44). The scattered wave fields in the local Cartesian coordinate system can be 
obtained from those in the local spherical system by using coordinate transformation of Eqs. (D-8a) and (D-10a). Each pair of scattered wave field in the local and global Cartesian systems, such as $u_{x}$ and $u_{x^{\prime}}$, should be the same.

The maximum value of $\sqrt{u_{x}^{2}+u_{y}^{2}+u_{z}^{2}}$ among ten positions is taken as the reference for the comparison of each displacement component $u_{x}, u_{y}$ or $u_{z}$. The number of significant figures of displacement $u_{z}$ at one position is obtained by comparing the two values in both coordinate systems. The smallest number of significant figures of $u_{z}$ at all ten positions is taken as the number of significant figures of $u_{z}$. The smallest number of significant figures of displacements $u_{x}, u_{y}$ and $u_{z}$ is taken as the number of significant figures of displacements. The number of significant figures for the stresses is obtained in the same way.

Figure 4.5 shows the relation between the truncation number and significant figures of displacements and the relation between the truncation number and significant figures of stresses. In Fig. 4.5, $r^{\prime \prime}=3.64$ represents that origin $O^{\prime}$ has Cartesian coordinates $x=y=z=2.1 a$ in the global Cartesian system and $r^{\prime \prime}=36.4$ represents that origin $O^{\prime}$ has Cartesian coordinates $x=y=z=21 a$ in the global Cartesian system.

Similarly, when the incident wave is taken as $\phi=\chi=0$ and $\psi$ in Eq. (4-5), the relation of truncation number and the significant figures of scattered wave fields are shown in Fig. 4.6. When the incident wave is taken as $\phi=\psi=0$ and $\chi$ in Eq. (4-9), the significant figures of scattered wave fields are shown in Fig. 4.7. In these figures, $r^{\prime \prime}=3.64$ represents that origin $O^{\prime}$ has Cartesian coordinates $x=y=z=2.1 a$ in the global Cartesian system and $r^{\prime \prime}=36.4$ represents that origin $O^{\prime}$ has Cartesian coordinates $x=y=z=21 a$ in the global Cartesian system.

The results of comparison are shown in Figs. 4.5 through 4.7. In Fig. 4.5, the transformed results have 2 or more significant figures when the truncation number 


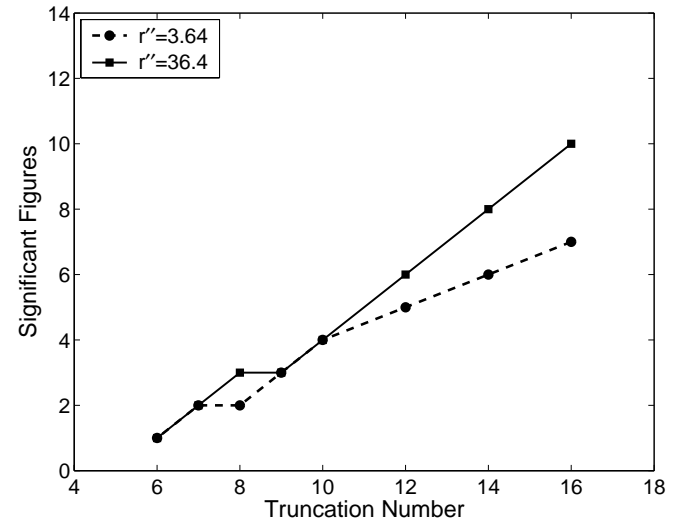

(a)

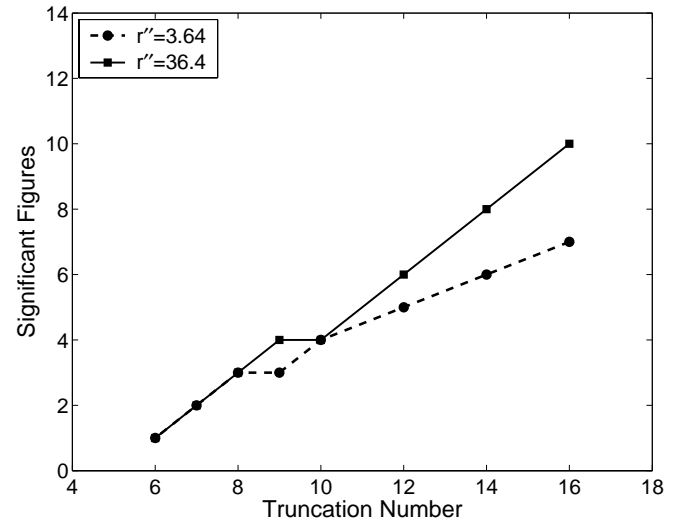

(b)

Figure 4.5: Significant figures of the transformed results of displacement and stress due to the scattered waves for incident wave $\phi . \kappa a=1.5757, k a=3.1574$, and $r^{\prime}=a$. (a) Significant figures of displacements vs. truncation number. (b) Significant figures of stresses vs. truncation number.

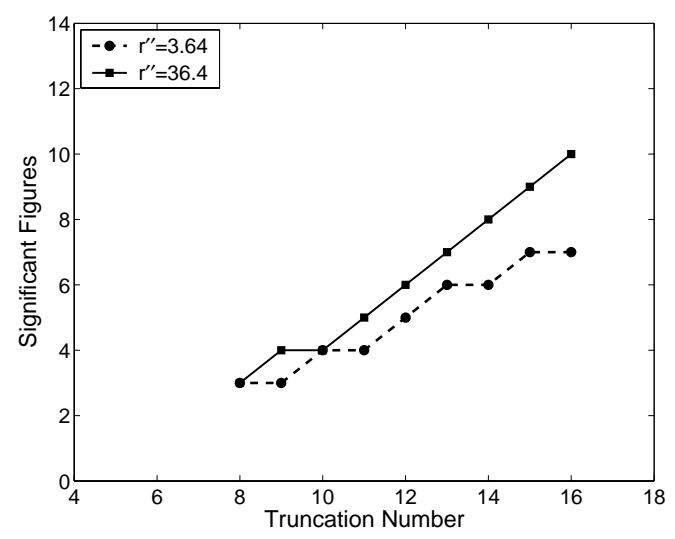

(a)

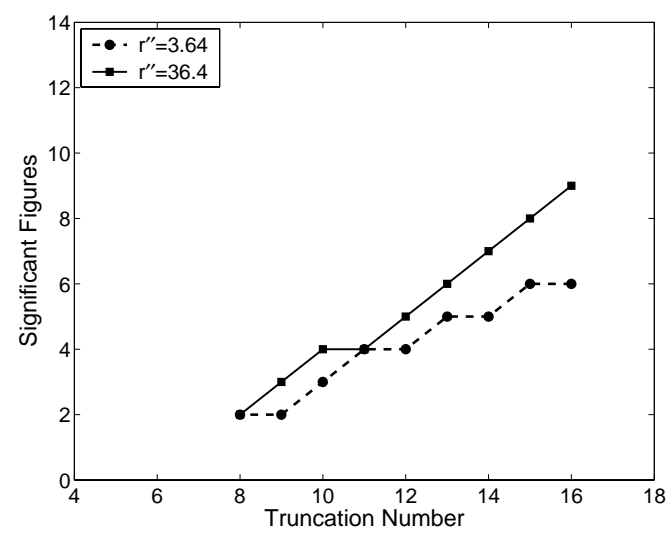

(b)

Figure 4.6: Significant figures of the transformed results of displacement and stress due to scattered waves for incident wave $\psi$. $\kappa a=1.5757, k a=3.1574$, and $r^{\prime}=a$. (a) Significant figures of displacements vs. truncation number. (b) Significant figures of stresses vs. truncation number. 


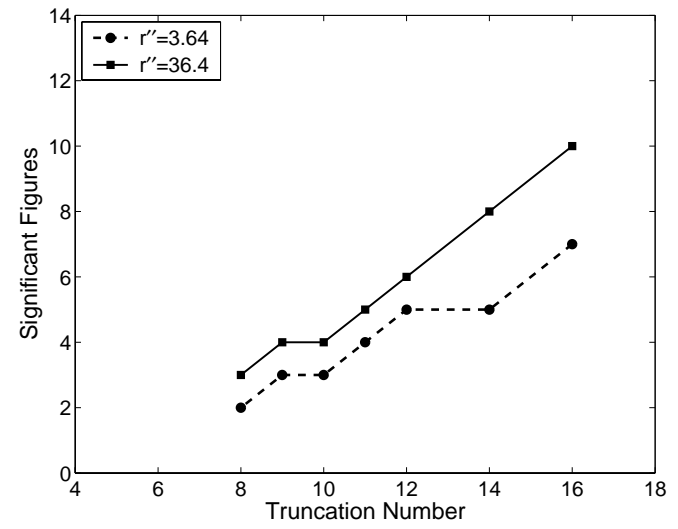

(a)

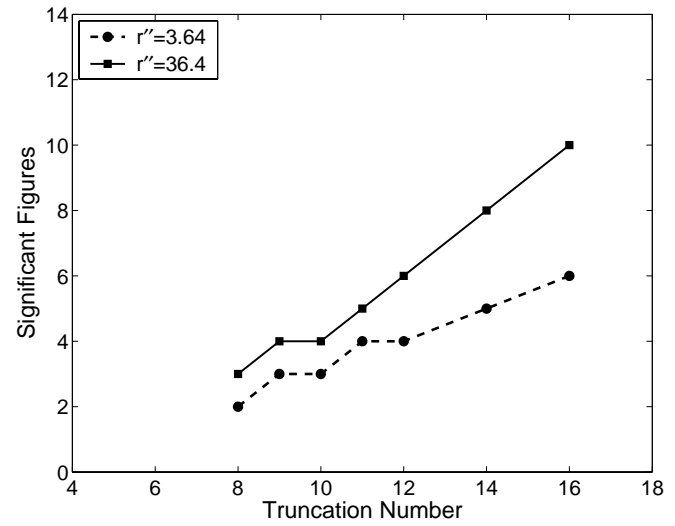

(b)

Figure 4.7: Significant figures of the transformed results of displacement and stress due to the scattered waves for incident wave $\chi . \kappa a=1.5757, k a=3.1574$, and $r^{\prime}=a$. (a) Significant figures of displacements vs. truncation number. (b) Significant figures of stresses vs. truncation number.

is 7 or larger. In Fig. 4.6, the transformed results have 2 or more significant figures when the truncation number is 8 or larger. In Fig. 4.7, the transformed results have 2 or more significant figures when the truncation number is 8 or larger. Generally, the number of significant figures of the scattered wave fields increases when the distance $r^{\prime \prime}$ increases for the same truncation number, which agrees with the discussion about the truncation criterion in Section 2-6.2. The truncation number should be not smaller than 14 in order to have 6 or more significant figures of scattered wave fields when $r^{\prime \prime} \geq 3.64$. The vector addition theorem is also verified by comparison of scattered wave fields in two coordinate systems, because the vector addition theorem is used in the computation of the transformed scattered wave fields.

By comparing Figs. 4.2 with 4.5, comparing Figs. 4.3 with 4.6, and comparing Figs. 4.4 with 4.7 , it is observed that the number of significant figures of the incident wave fields (due to coordinate transformation) is larger than that of the scattered wave fields (due to coordinate transformation) for the same truncation number and the same distance $r^{\prime \prime}$. 


\section{4-3 Program Verification}

In this section, the truncation criterion for the multiple scattering solution is discussed and implementation of the multiple scattering solution is verified. The verification is first through a published result, then through the fundamental physical principle of energy conservation, and finally, through boundary continuities.

\section{4-3.1 Truncation Error}

In this section, the truncation error of the multiple scattering solution in Eq. (3-75) is discussed. Recall Eq. (3-75). It is noticed that the truncation error comes from incident wave expansion coefficients matrix $\left\{\mathbb{A}_{J}\right\}$, coordinate transformation matrix $\left[\mathbb{Q}_{I J}\right]$, and the super $T$-matrix $\left[\mathbb{T}_{J}\right]$. By comparing the multiple scattering solution in Eq. (3-75) and the single scattering solution in Eq. (2-101), coordinate transformation matrix $\left[\mathbb{Q}_{I J}\right]$ provides one more source for truncation error.

Generally, an incident wave is given in the global coordinate system. The incident wave expansion coefficients matrix $\left\{\mathbb{A}_{J}\right\}$ in the local coordinate system of scatterer $J$ is obtained from that in the global coordinate system through coordinate transformation. The transformation of the wave fields due to longitudinal plane incident waves is discussed in Section 4-2.1. In Eq. (3-75), the coordinate transformation matrix $\left[\mathbb{Q}_{I J}\right]$ provide the coordinate transformation of the wave expansion bases $\{\boldsymbol{h}(\cdot)\}$ in one coordinate system as $\{\boldsymbol{j}(\cdot)\}$ in another coordinate system. The transformation of the scattered wave fields is discussed in Section 4-2.2. It is concluded in Section 4-2 that the number of significant figures of the incident wave fields (due to coordinate transformation) is larger than that of the scattered wave fields (due to coordinate transformation) for the same truncation number and the same distance $r^{\prime \prime}$. Therefore, the error of scattered wave fields due to coordinate transformation using matrix 
$\left[\mathbb{Q}_{I J}\right]$ is treated as the truncation error in the multiple scattering solution in this thesis.

Recall Eq. (3-45), which is the definition of the coordinate transformation matrix $\left[\mathbb{Q}_{I J}\right]$. The entries in $\left[\mathbb{Q}_{I J}\right]$ are based on the first equation in Eq. (3-4) with the requirement that $r^{\prime}<r^{\prime \prime}$. The first equation in Eq. (3-4) is repeated here for convenience.

$$
h_{n}^{(1)}(k r) Y_{n}^{m}(\theta, \varphi)=\sum_{p=0}^{20} \sum_{q=-p}^{p} \alpha_{n m, p q}\left(\boldsymbol{r}^{\prime \prime}\right) j_{p}\left(k r^{\prime}\right) Y_{p}^{q}\left(\theta^{\prime}, \varphi^{\prime}\right) \quad \text { for } \quad r^{\prime}<r^{\prime \prime} .
$$

The $r^{\prime \prime}$ plays an important role in determining the relation between the truncation number and the truncation error, because the truncation error increases when the $r^{\prime \prime}$ decreases in Figs. 4.5 through 4.7.

The coordinate $\varphi^{\prime}$ also plays an important role in determining the relation between the truncation number and the truncation error. When the truncation number is 20 , examples of the number of significant figures of the right hand side (RHS) of Eq. (4-22) are shown in Fig. 4.8. For $n=1$ and $m=0$, the RHS has eleven significant figures at $\varphi^{\prime}=\pi$ but has no significant figures at $\varphi^{\prime}=0.5 \pi$. For $n=20$ and $m=0$, the RHS has no significant figures at $\varphi^{\prime}=\pi$ but has seven significant figures at $\varphi^{\prime}=0.5 \pi$. There is no explanation yet for the cases that the RHS has no significant figures.

\section{4-3.2 Comparison with a Published Result}

By using an iterative method to solve multiple scattering problems, Doyle [24] shows images of wave fields for multiple scattering by two spherical quartz scatterers in ice. The material properties of quartz and ice are listed in Table 4.3, which are

provided by Doyle ${ }^{[55]}$. In [24], the incident longitudinal plane wave propagates along the positive direction of $Z$-axis, and the two scatterers are spheres of $0.5 \mathrm{~mm}$ radius and centered at $Z$-axis with $z=-1 \mathrm{~mm}$ and $z=1 \mathrm{~mm}$. The incident wave has 


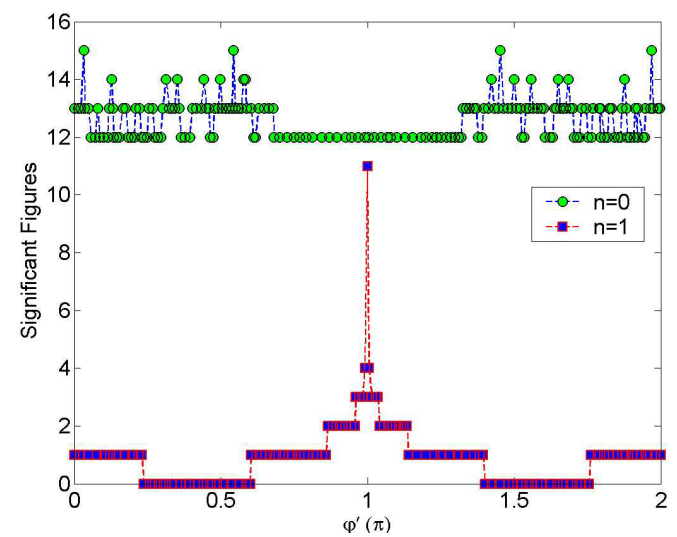

(a)

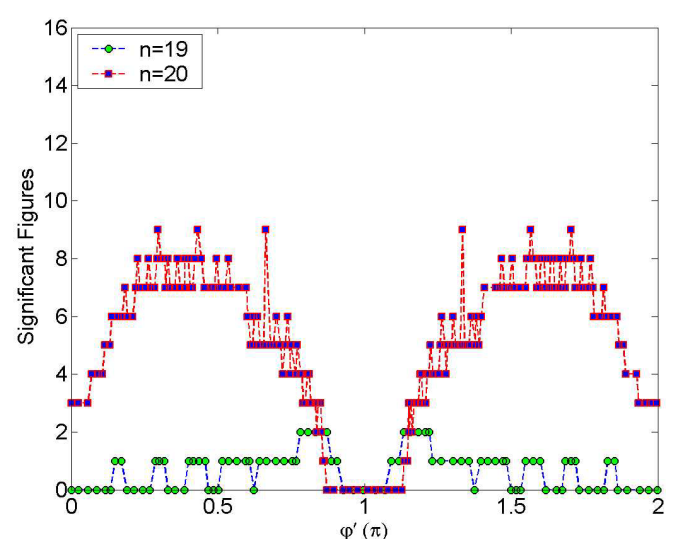

(b)

Figure 4.8: Significant figures of the right hand side of Eq. (4-22) with truncation number 20 and $k=1$. In spherical coordinates, $\boldsymbol{r}^{\prime}=\left(1.0, \pi / 2, \varphi^{\prime}\right), \boldsymbol{r}^{\prime \prime}=$ (3.81, $\pi / 2, \pi)$. (a) $m=0: n=0$ and $n=1$. (b) $m=0: n=19$ and $n=20$.

frequency of $5 \mathrm{MHz}$ and displacement of unit amplitude. At this frequency, the longitudinal wave number is $\kappa a=1.5787$ (with $a$ as the radius of the spheres) and shear wave number is $k a=3.1574$; in the steel, the longitudinal wave number is $\kappa a=1.1780$ and shear wave number is $k a=1.7850$.

Table 4.3: Material Properties of Quartz and Ice.

\begin{tabular}{|c|c|c|}
\hline Property & Matrix (Ice) & Sphere (Quartz) \\
\hline Lamé constant $\lambda(\mathrm{GPa})$ & 7.92 & 16.235 \\
Lamé constant $\mu(\mathrm{GPa})$ & 3.96 & 32.215 \\
Density $\left(\mathrm{kg} / \mathrm{m}^{3}\right)$ & 1000. & 2600. \\
$\mathrm{P}$ wave speed $(\mathrm{km} / \mathrm{s})$ & 3.98 & 5.57 \\
$\mathrm{~S}$ wave speed $(\mathrm{km} / \mathrm{s})$ & 1.99 & 3.52 \\
\hline
\end{tabular}

The real part of the displacement $u_{z}$ of total waves in $x=0$ plane is shown in Fig.6(a) in [24]. Figure 6(a) in [24] is duplicated here as Fig. 4.9(a). The image is $400 \times 400$ pixels with a pixel size of $0.01 \times 0.01 \mathrm{~mm}$ and the gray scale ranges are -1.8 to $1.29^{[55]}$. The truncation number is 12 and the truncation error is on the order of $1 \%[24]$. 


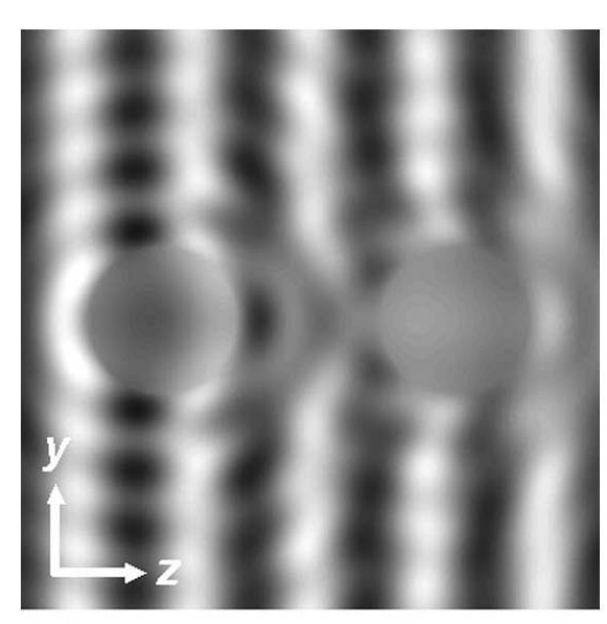

(a) $u_{z}$ of total waves, by Doyle ${ }^{[24]}$

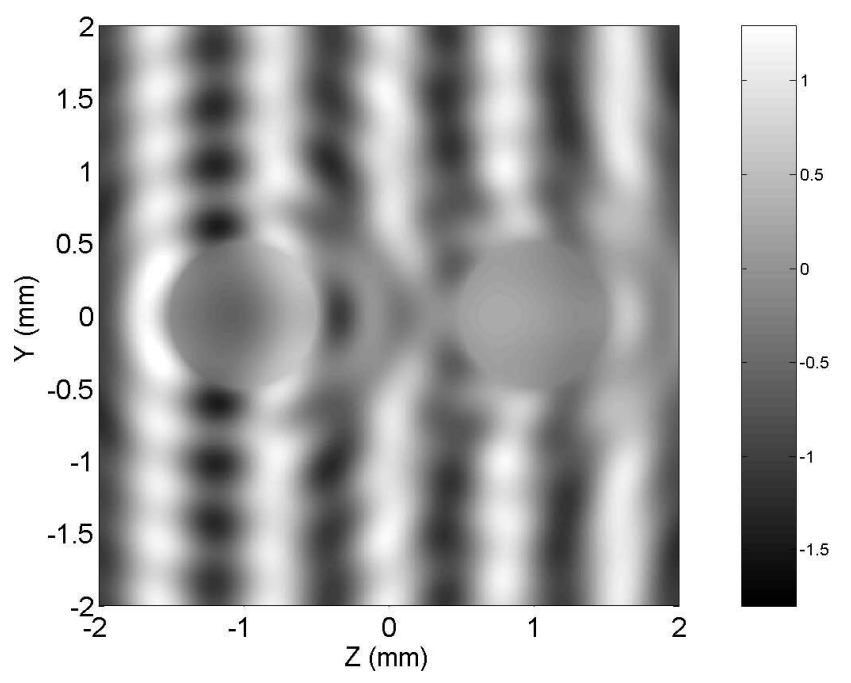

(b) $u_{z}$ of total waves, by the author

Figure 4.9: Comparison with published result of multiple scattering of 5-MHz longitudinal plane wave incident on a pair of 1.0-mm diameter quartz spheres in ice. Two spheres are $2.0-\mathrm{mm}$ away and sit at $Z$-axis symmetrically about origin.

The multiple scattering problem of two spherical quartz scatterers in ice is solved through Eq. (3-75). Following the procedure of computation in Section 3-4.3, the displacement $u_{z}$ of total waves can be calculated. The displacement $u_{z}$ of total waves in $x=0$ plane is shown in Fig. 4.9(b). Images in Figs. 4.9(a) and 4.9(b) have the same size, the same resolution, and the same gray scale ranges. Figure 4.9(b) agrees with Figure 4.9(a).

As mentioned in Section 4-3.1, the truncation error of scattered wave fields due to coordinate transformation can serve as the truncation error in a multiple scattering solution. Figure 4.10 shows the number of significant figures of incident and scattered wave fields due to coordinate transformation for the computation of producing Fig. 4.9(b). Incident wave fields (over spherical surface of a scatterer) computed in the local coordinate system of a scatterer are compared with those computed in the global coordinate system. Scattered wave fields of one scatterer are transformed as wave fields in the coordinate system of the other scatterer. The fields are computed 
over the spherical surface of the second scatterer from both coordinate systems. The number of significant figures is obtained in the same way as in Section 4-2.

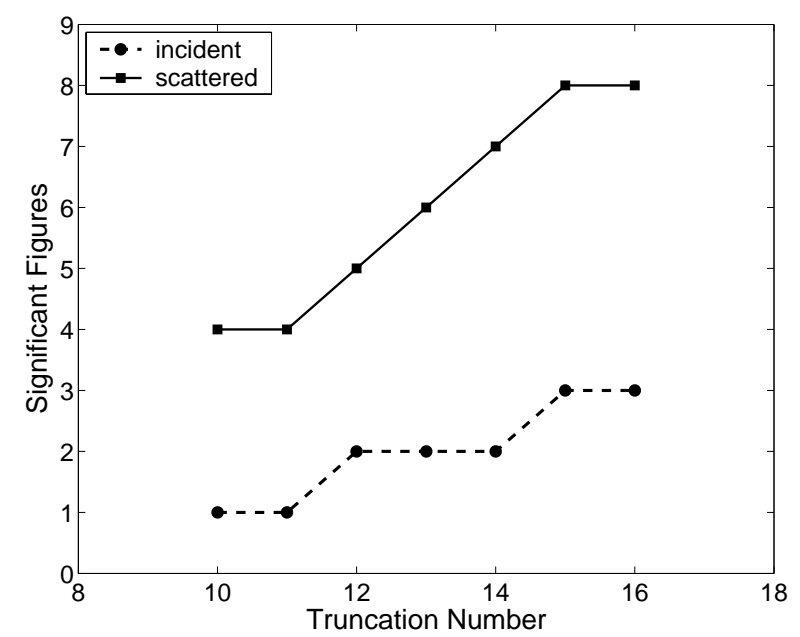

Figure 4.10: Significant figures of incident and scattered wave fields due to coordinate transformation. A 5-MHz longitudinal plane wave incident on a pair of $1.0-\mathrm{mm}$ diameter quartz spheres in ice.

In Fig. 4.10, the number of significant figures of the scattered wave fields is larger than that of the incident wave fields. When analytical expressions of incident wave fields are used, the truncation error of the scattered wave fields is the truncation error in the multiple scattering solution. For the computation of producing Fig. 4.9(b) (with truncation number 12), the truncation error is $1 \times 10^{-5}$ that corresponds to five significant figures in the solution.

\section{4-3.3 Energy Conservation}

The energy flux across a closed spherical surface is checked for the multiple scattering solution of producing Fig. 4.9(b). These two scatterers are centered on the $Z$-axis and symmetric about the origin, with each being $1 \mathrm{~mm}$ away from the origin. The energy flux is evaluated using the normalized average energy flux $\langle\dot{E}\rangle_{N}$ in 
Eq. (2-149). In Eq. (2-149), $\dot{e}_{\text {inc }}$ can be found in Eq. $(2-148)$ and $\langle\dot{E}\rangle$ can be computed through Eq. (2-130) for a spherical surface of radius R. And in Eq. (2-130), stresses and displacements are the fields of total waves and computed by following the process in Section 3-4.3.

The normalized average energy flux $\langle\dot{E}\rangle_{N}$ in Eq. (2-149) is evaluated for three cases: (1) scatterers are outside the spherical surface of radius $r=1 \mathrm{~mm}$, which is centered at Cartesian coordinates $(x=y=z=2 \mathrm{~mm})$; (2) a spherical surface is concentric with the scatterer that is centered at Cartesian coordinates $(x=y=$ $0 \mathrm{~mm}, z=1 \mathrm{~mm}$ ) and the radius of the spherical surface is $r=0.75 \mathrm{~mm}$ or $r=$ $3.75 \mathrm{~mm}$; (3) both scatterers are inside the spherical surface, which is centered at the origin or the middle position between two scatterers and a radius is chosen in the range of $2 \leq r \leq 55$. The results are shown in Table 4.4 .

Table 4.4: Normalized Average Energy Flux through Closed Surfaces

\begin{tabular}{|c|c|c|}
\hline & $r(\mathrm{~mm})$ & $\langle\dot{E}\rangle_{N}$ \\
\hline Case-1 & 1 & $1.47825 \times 10^{-7}$ \\
\hline Case-2 & 0.75 & $5.70025 \times 10^{-12}$ \\
& 3.75 & $6.62623 \times 10^{-13}$ \\
\hline & 2 & $4.35270 \times 10^{-13}$ \\
& 3 & $1.37697 \times 10^{-14}$ \\
Case-3 & 4 & $4.33971 \times 10^{-14}$ \\
& 5 & $4.35133 \times 10^{-16}$ \\
& 55 & $1.26702 \times 10^{-13}$ \\
\hline
\end{tabular}

Note: Truncation number is $12, \kappa=7893.5 \mathrm{~m}^{-1}$.

The normalized average energy flux $\langle\dot{E}\rangle_{N}$ is supposed to be zero, since there is no energy generation or dissipation inside the spherical surface in each of the cases. The values in Table 4.4 show that energy conservation is satisfied and the numerical errors are on the order of $10^{-7}$ or less. 


\section{4-3.4 Field Continuity}

Continuities of displacement and surface traction at the interface between the host medium and each inclusion should be satisfied in the local coordinate system of each inclusion. In local and global coordinate systems, displacement vector $\boldsymbol{u}$ at a position can be written as

$$
\begin{aligned}
\boldsymbol{u} & =u_{r^{\prime}} \boldsymbol{e}_{r^{\prime}}+u_{\theta^{\prime}} \boldsymbol{e}_{\theta^{\prime}}+u_{\varphi^{\prime}} \boldsymbol{e}_{\varphi^{\prime}}=u_{x^{\prime}} \boldsymbol{e}_{x^{\prime}}+u_{y^{\prime}} \boldsymbol{e}_{y^{\prime}}+u_{z^{\prime}} \boldsymbol{e}_{z^{\prime}} \\
& =u_{x} \boldsymbol{e}_{y}+u_{y} \boldsymbol{e}_{y}+u_{z} \boldsymbol{e}_{z}=u_{r} \boldsymbol{e}_{r}+u_{\theta} \boldsymbol{e}_{\theta}+u_{\varphi} \boldsymbol{e}_{\varphi}
\end{aligned}
$$

Therefore, the displacement continuity is satisfied in the global and all local coordinate systems.

For the multiple scattering solution of producing Fig. 4.9(b), continuity of displacement is shown through continuity of each displacement component in the global coordinate system. Following the computation process in Section 3-4.3, at a point outside the scatterer, the displacements are computed from the incident and all scattered waves; at a point inside the scatterer, the displacements are computed from the refracted waves of the scatterer. In Fig.4.11, modulus of $u_{x}, u_{y}$ and $u_{z}$ in the $y=0$ plane are shown. A black circle represents a scatterer's boundary. At every point on the boundary of one of the two scatterers, each displacement component from both sides of the scatterer are the same, which is shown in Fig.4.11.

Similarly, the continuities of $u_{r}, u_{\theta}$ and $u_{\varphi}$ are shown through modulus of each displacement component in Fig.4.12. The displacement continuity is satisfied.

For a multiple scattering solution, the continuity of surface traction requires continuities of stresses $\sigma_{r_{I} r_{I}}, \sigma_{r_{I} \theta_{I}}$ and $\sigma_{r_{I} \varphi_{I}}$ in the local spherical coordinate system of Scatterer $I$. The process of computing wave fields is stated in Section 3-4.3. For the multiple scattering solution of producing Fig. 4.9(b), the local spherical coordinate system of the scatterer with positive $Z$ coordinate is chosen as an example. Let " 1 " 


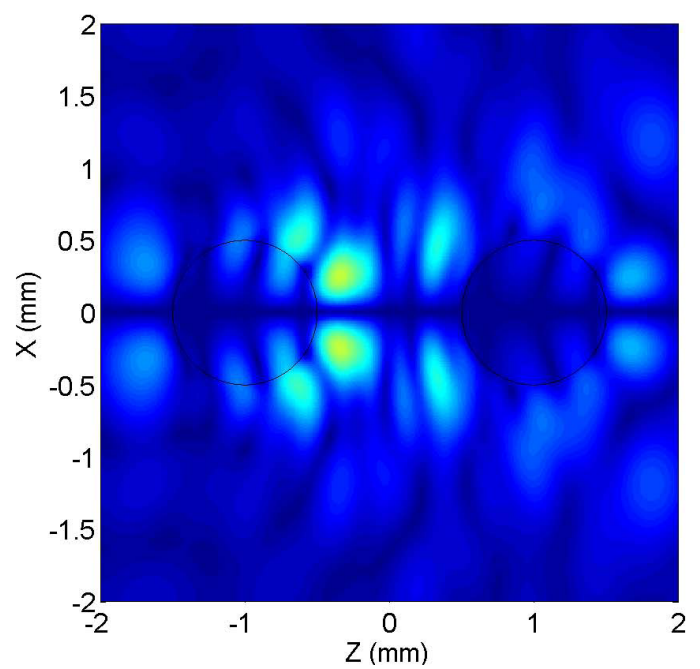

(a)

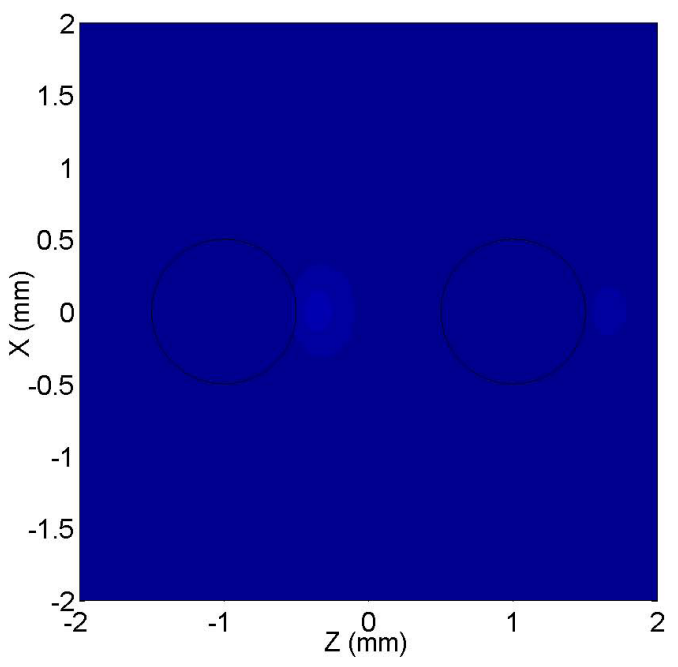

(b)

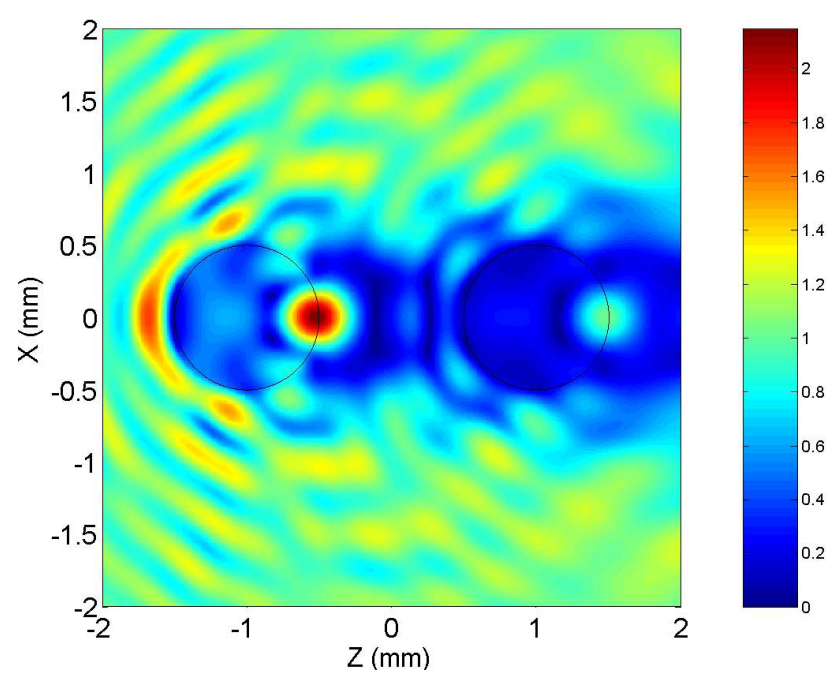

(c)

Figure 4.11: Displacement continuity is shown through $\left|u_{x}\right|,\left|u_{y}\right|$ and $\left|u_{z}\right|$ for the multiple scattering of a longitudinal plane wave incident by a pair of quartz spheres in ice. In ice, $\kappa a=3.94675$ and $k a=7.8935$; In quartz, $\kappa a=2.82008$ and $k a=4.46247$. Two identical spheres of radius $a$ are $4 a$ away and sit at $Z$-axis symmetrically about the origin. A black circle represents a scatterer's boundary. (a) $\left|u_{x}\right|$ in the $y=0$ plane, (b) $\left|u_{y}\right|$ in the $y=0$ plane, (c) $\left|u_{z}\right|$ in the $y=0$ plane. 


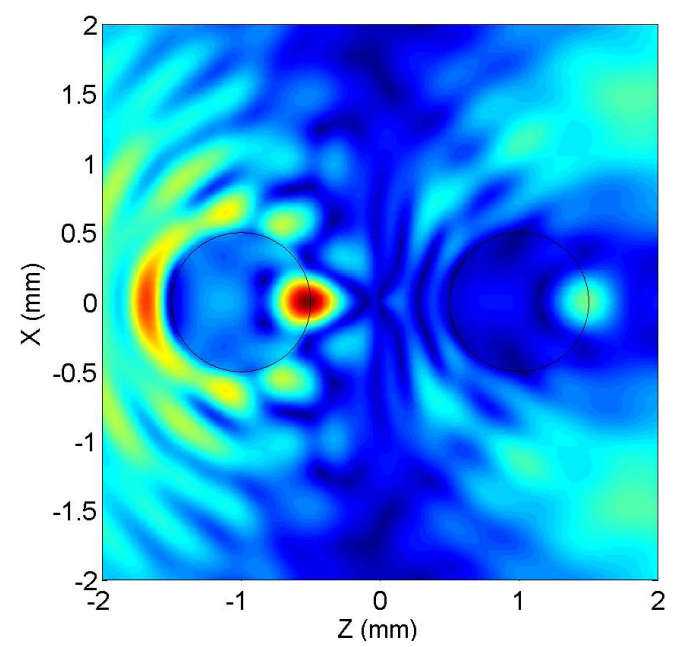

(a)

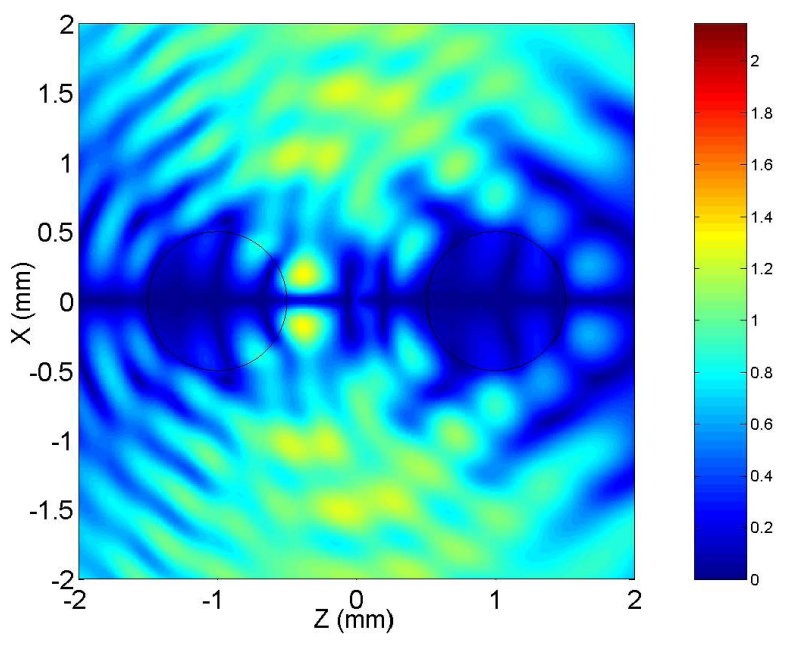

(b)

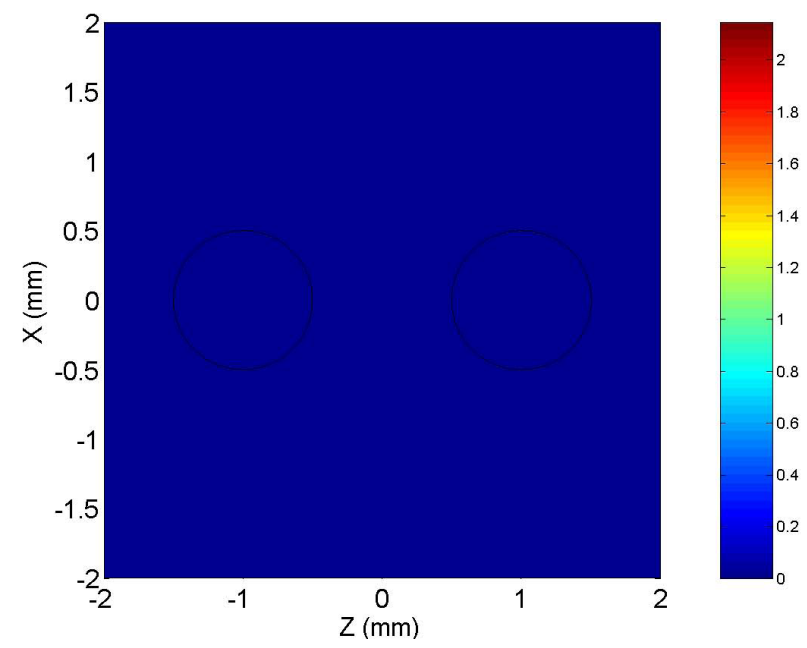

(c)

Figure 4.12: Displacement continuity is shown through $\left|u_{r}\right|,\left|u_{\theta}\right|$ and $\left|u_{\varphi}\right|$ in the $y=0$ plane for the multiple scattering of a longitudinal plane wave incident by a pair of quartz spheres in ice. In ice, $\kappa a=3.94675$ and $k a=7.8935$; In quartz, $\kappa a=2.82008$ and $k a=4.46247$. Two identical spheres of radius $a$ are $4 a$ away and sit at $Z$-axis symmetrically about the origin. A black circle represents a scatterer's boundary. (a) $\left|u_{r}\right|$ in the $y=0$ plane, (b) $\left|u_{\theta}\right|$ in the $y=0$ plane, (c) $\left|u_{\varphi}\right|$ in the $y=0$ plane. 
denote the local coordinate system. In Fig. 4.13, modulus of $\sigma_{r_{1} r_{1}}, \sigma_{r_{1} \theta_{1}}$ and $\sigma_{r_{1} \varphi_{1}}$ are shown. A black circle represents a scatterer's boundary. At a point on the black circle centered at the right side of Fig. 4.13(a), modulus of $\sigma_{r_{1} r_{1}}$ obtained from both sides of the scatterer are the same. But at a point on the black circle centered at the left side of Fig. 4.13(a), modulus of $\sigma_{r_{1} r_{1}}$ obtained from both sides of the scatterer are not the same. Similar observation can be made for $\sigma_{r_{1} \theta_{1}}$ in Fig. 4.13(b) and $\sigma_{r_{1} \varphi_{1}}$ in Fig. 4.13(c). The continuity of surface traction is satisfied in the local coordinate system of the local scatterer with positive $Z$ coordinate.

The local spherical coordinate system of the scatterer with negative $Z$ coordinate is chosen as another example. Let "2" denote the local coordinate system. In Fig. 4.14, modulus of $\sigma_{r_{2} r_{2}}, \sigma_{r_{2} \theta_{2}}$ and $\sigma_{r_{2} \varphi_{2}}$ are shown. A black circle represents a scatterer's boundary. At a point on the black circle centered at the left side of Fig. 4.14(a),

modulus of $\sigma_{r_{2} r_{2}}$ obtained from both sides of the scatterer are the same. But at a point on the black circle centered at the right side of Fig. 4.14(a), modulus of $\sigma_{r_{2} r_{2}}$ obtained from both sides of the scatterer are not the same. Similar observation can be made for $\sigma_{r_{2} \theta_{2}}$ in Fig. 4.14(b) and $\sigma_{r_{2} \varphi_{2}}$ in Fig. 4.14(c). The continuity of surface traction is also satisfied in the local coordinate system of the local scatterer with negative $Z$ coordinate. It is concluded that the continuity of surface traction, or continuities of stresses $\sigma_{r_{I} r_{I}}, \sigma_{r_{I} \theta_{I}}$ and $\sigma_{r_{I} \varphi_{I}}$, in the local spherical coordinate system of Scatterer $I$ are satisfied.

\section{4-4 Concluding Remarks}

The implementation of the multiple scattering solution has been verified through computation of multiple scattering of $5-\mathrm{MHz}$ longitudinal plane incident wave by a pair of 1.0-mm diameter quartz spheres in ice. Figure. 6(a) in [24] is reproduced in 


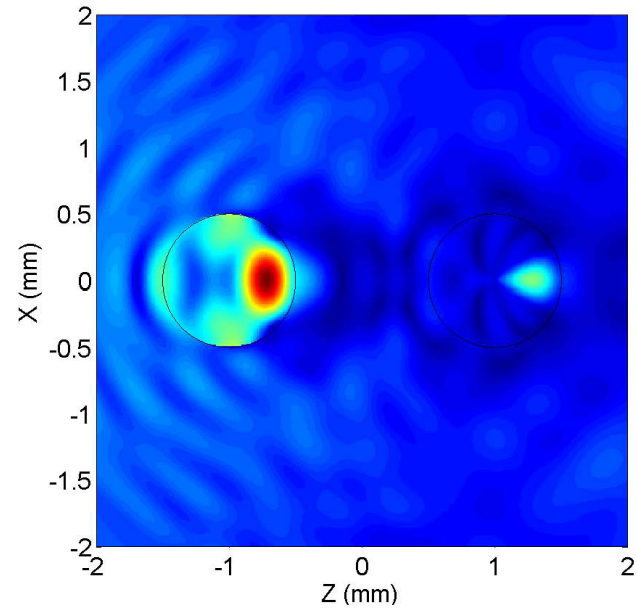

(a)

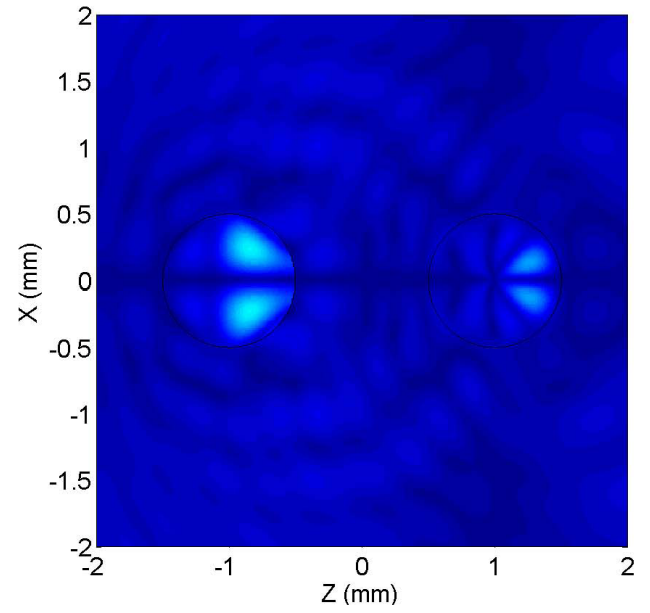

(b)

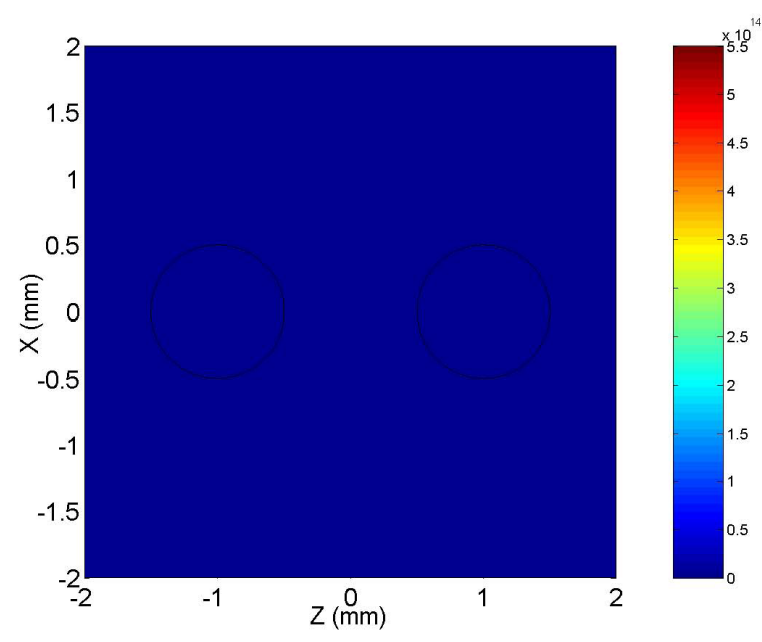

(c)

Figure 4.13: Surface traction continuity is shown through $\left|\sigma_{r_{1} r_{1}}\right|,\left|\sigma_{r_{1} \theta_{1}}\right|$ and $\left|\sigma_{r_{1} \varphi_{1}}\right|$ in the $y=0$ plane for the multiple scattering of a longitudinal plane wave incident by a pair of quartz spheres in ice. Stresses $\left|\sigma_{r_{1} r_{1}}\right|,\left|\sigma_{r_{1} \theta_{1}}\right|$ and $\left|\sigma_{r_{1} \varphi_{1}}\right|$ are in the local spherical coordinate system of the scatterer at positive part of the $Z$-axis. In ice, $\kappa a=3.94675$ and $k a=7.8935$; In quartz, $\kappa a=2.82008$ and $k a=4.46247$. Two identical spheres of radius $a$ are $4 a$ away and sit at $Z$-axis symmetrically about the origin. A black circle represents a scatterer's boundary. (a) $\left|\sigma_{r_{1} r_{1}}\right|$ in the $y=0$ plane, (b) $\left|\sigma_{r_{1} \theta_{1}}\right|$ in the $y=0$ plane, (c) $\left|\sigma_{r_{1} \varphi_{1}}\right|$ in the $y=0$ plane. At a point on the black circle centered at the right side of Fig. 4.13(a), modulus of $\sigma_{r_{1} r_{1}}$ obtained from both sides of the scatterer are the same. But at a point on the black circle centered at the left side of Fig. 4.13(a), modulus of $\sigma_{r_{1} r_{1}}$ obtained from both sides of the scatterer are not the same. Similar observation can be made for $\sigma_{r_{1} \theta_{1}}$ in Fig. 4.13(b) and $\sigma_{r_{1} \varphi_{1}}$ in Fig. 4.13(c). The continuity of surface traction is satisfied in the local coordinate system of the scatterer with positive $Z$ coordinate. 


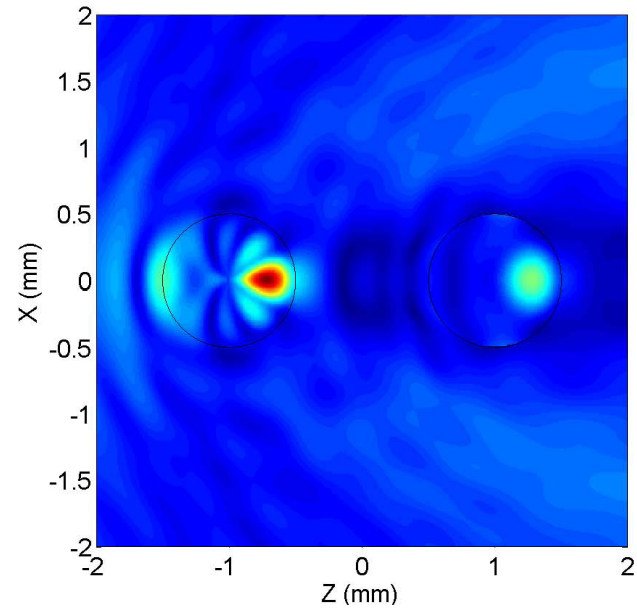

(a)

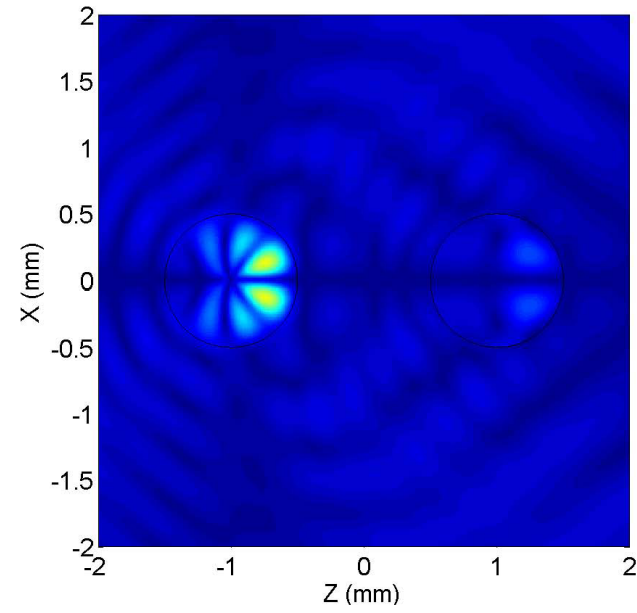

(b)

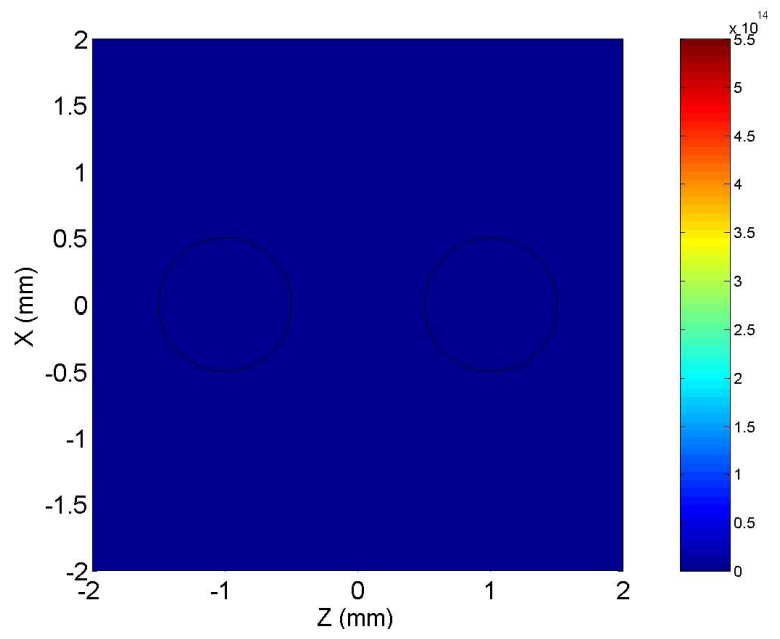

(c)

Figure 4.14: Surface traction continuity is shown through $\left|\sigma_{r_{2} r_{2}}\right|,\left|\sigma_{r_{2} \theta_{2}}\right|$ and $\left|\sigma_{r_{2} \varphi_{2}}\right|$ in the $y=0$ plane for the multiple scattering of a longitudinal plane wave incident by a pair of quartz spheres in ice. Stresses $\left|\sigma_{r_{2} r_{2}}\right|,\left|\sigma_{r_{2} \theta_{2}}\right|$ and $\left|\sigma_{r_{2} \varphi_{2}}\right|$ are in the local spherical coordinate system of the scatterer at negative part of the $Z$-axis. In ice, $\kappa a=3.94675$ and $k a=7.8935$; In quartz, $\kappa a=2.82008$ and $k a=4.46247$. Two identical spheres of radius $a$ are $4 a$ away and sit at $Z$-axis symmetrically about the origin. A black circle represents a scatterer's boundary. (a) $\left|\sigma_{r_{2} r_{2}}\right|$ in the $y=0$ plane, (b) $\left|\sigma_{r_{2} \theta_{2}}\right|$ in the $y=0$ plane, (c) $\left|\sigma_{r_{2} \varphi_{2}}\right|$ in the $y=0$ plane. At a point on the black circle centered at the left side of Fig. 4.14(a), modulus of $\sigma_{r_{2} r_{2}}$ obtained from both sides of the scatterer are the same. But at a point on the black circle centered at the right side of Fig. 4.14(a), modulus of $\sigma_{r_{2} r_{2}}$ obtained from both sides of the scatterer are not the same. Similar observation can be made for $\sigma_{r_{2} \theta_{2}}$ in Fig. 4.14(b) and $\sigma_{r_{2} \varphi_{2}}$ in Fig. 4.14(c). The continuity of surface traction is also satisfied in the local coordinate system of the scatterer with negative $Z$ coordinate. 
Fig. 4.9(b), and energy conservation and boundary continuity are also satisfied.

The truncation criterion for the multiple scattering solution is discussed. For a particular computation, the convergence of scattered wave fields after translation can serve as truncation criterion. The truncation criterion needs to be simplified in future work. 


\section{Chapter 5}

\section{Scatterer Polymerization}

\section{5-1 Introduction}

Equation (3-75) represents the multiple scattering solution, which is repeated here as

$$
\begin{gathered}
\sum_{q=1}^{p}\left\{\mathbb{B}_{J}^{(q)}\right\}=\sum_{q=1}^{p-1}\left\{\mathbb{B}_{J}^{(q)}\right\}=\left\{\mathbb{B}_{J}\right\} \\
\left\{\mathbb{B}_{J}\right\}=\left[\mathbb{T}_{J}\right]\left\{\mathbb{A}_{J}\right\}+\sum_{\substack{I=1 \\
I \neq J}}^{N}\left[\mathbb{T}_{J}\right]\left[\mathbb{Q}_{I J}\right]^{T}\left\{\mathbb{B}_{I}\right\}
\end{gathered}
$$

For notational simplicity, Eq. (3-75) is written as Eq. (3-78) and repeated here as

$$
[\mathfrak{L}]\{\mathfrak{B}\}=\{\mathfrak{A}\}
$$

where $\{\mathfrak{A}\},\{\mathfrak{B}\}$ and $[\mathfrak{L}]$ are defined in Eqs. $(3-76)$ and $(3-77)$.

The $[\mathfrak{L}]$ matrix is square and of order $(3 N \mathcal{M})$ for $N$ scatterers when the truncation size of $T$-matrix of a scatterer is $\mathcal{M}$. The size of computer memory required to solve Eq. (5-3) grows at a rate of $(3 N \mathcal{M})^{2}$. With such a growth rate, the number of scatterers in a multiple scattering problem to be solved is limited by computer 
memory. As a way to overcome the limitation due to size of $[\mathfrak{L}]$, the scatterer polymerization methodology ${ }^{[50]}$ is used to reformulate the multiple scattering solution for an arbitrary number of scatterers.

The scatterer polymerization methodology treats an assemblage of scatterers as an abstract scatterer. In the single scattering solution, a T-matrix is defined for an actual scatterer to relate the scattered wave expansion coefficients to the incident wave expansion coefficients. The scatterer polymerization methodology finds a super T-matrix for an abstract scatterer. Thus, the number of actual scatterers in solving Eq. (3-78) is replaced by a lesser number of abstract scatterers.

This chapter describes the idea and the algorithm of the scatterer polymerization methodology for three-dimensional multiple scattering of elastic waves. A numerical example follows to verify this method by using different approaches, with or without scatterer polymerization, to solve a physically identical multiple scattering problem.

\section{5-2 Idea of Scatterer Polymerization Methodology}

The idea of scatterer polymerization methodology is first introduced in two-

dimensional space ${ }^{[34,50]}$. In three-dimensional space, the idea is the same. This section and the next section are based on [34, 50].

In the single scattering problem, the scattered waves are valid outside the scatterer and the refracted waves are valid inside the scatterer. The territory of the scatterer is defined as the interior region of the scatterer. A T-matrix is defined for the scatterer. When the T-matrix is obtained, the single scattering problem is solved, since the T-matrix relates scattered wave expansion coefficients to incident wave expansion coefficients in the local coordinate system of the scatterer.

In a multiple scattering problem with $N$ scatterers in the host medium, the $N$ 
scatterers can be assembled as a group. If a T-matrix for the assemblage of scatterers can be defined and obtained to relate the scattered wave expansion coefficients to the incident wave coefficients in the local coordinate system of the group, the assemblage of scatterers can be treated as a single abstract scatterer and the multiple scattering problem can be solved just like a single scattering problem. In comparison with the single scatterer, the territory for the group can be defined to specify regions in which the scattered and refracted waves of the group are valid.

When the number of scatterers is large, these scatterers can be divided into $M$ groups. Each of these groups can be assembled as an abstract scatterer. And then the $M$ abstract scatterers can be assembled as an abstract scatterer to solve the scattering problem.

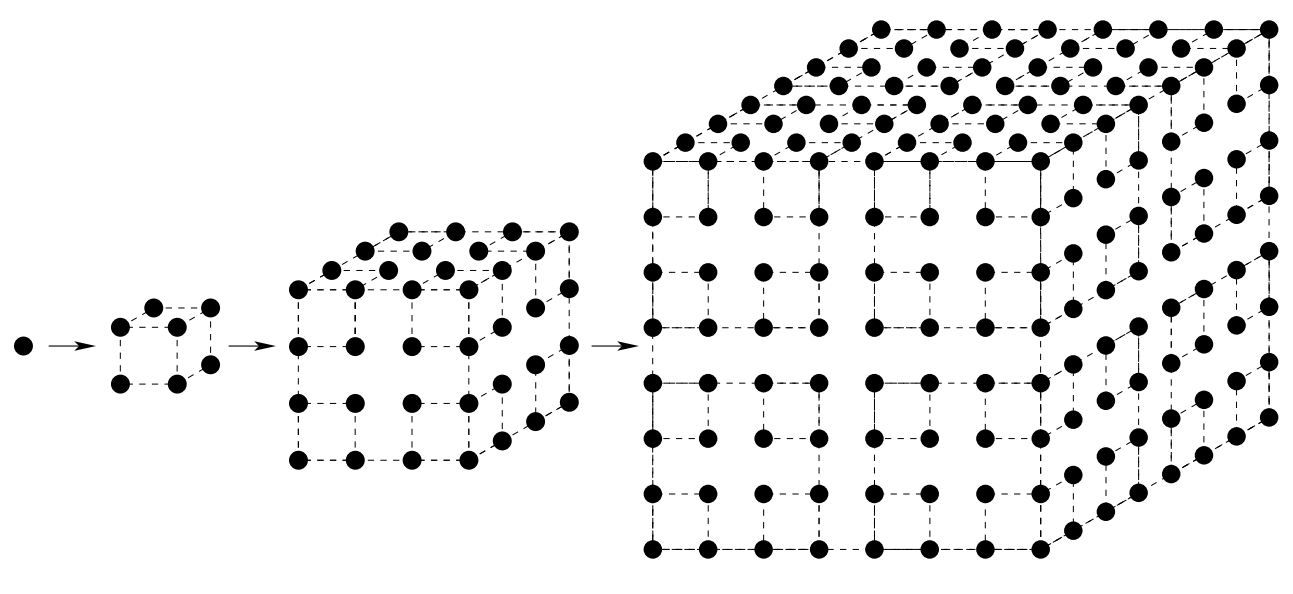

Stage 1

Stage 2

Stage 3

Figure 5.1: An example of scatterer polymerization

Figure 5.1 illustrates this strategy as an example with 3 stages. At the left end stands an actual scatterer. Then, an abstract scatterer in the first stage, in the second stage, and in the third stage follow from left to right. In the first stage, the abstract scatterer consists of 8 actual scatterers. In the second stage, the abstract scatterer consists of 64 actual scatterers or 8 abstract scatterers (each one is formed in the first 
stage). In the third stage, the abstract scatterer at right side in Fig. 5.1 consists of 512 actual scatterers, 64 abstract scatterers (each one is formed in the first stage), or 8 abstract scatterers (each one is formed in the second stage). The process of such assemblage can be repeated until an expected number of scatterers is reached in a multiple scattering problem. Each step of assembling a group of scatterers is called scatterer polymerization in analogy to polymer material science ${ }^{[50]}$. The abstract scatterer built by a scatterer polymerization process is called a molecule. In the first stage, the abstract scatterer is a molecule and each of the actual scatterers is called an element. In general, an assemblage results in a molecule and a T-matrix for the molecule can be found in the process of scatterer polymerization.

As stated in Section 5-1, matrix $[\mathfrak{L}]$ in the multiple scattering solution is a square matrix of order $(3 N \mathcal{M})$ for $N$ scatterers when truncation size of $T$-matrix of a scatterer is $\mathcal{M}$. The scatterer polymerization methodology splits actual scatterers into small groups so that the number of scatterers in each stage of scatterer polymerization process is reduced. Let $[\mathfrak{T}]$ be the matrix of linear equations in one stage of the scatterer polymerization process. The matrix $[\mathfrak{T}]$ has size of $(3 \mathcal{N} \mathcal{M})^{2}$ when there are $\mathcal{N}$ elements or molecules to be assembled in a scatterer polymerization process. For example, 8 elements are assembled in the first stage of scatterer polymerization process in Fig. 5.1 so that $\mathcal{N}=8$ in the first stage, and 8 molecules are assembled in the second stage of scatterer polymerization process in Fig. 5.1 so that $\mathcal{N}=8$ in the second stage. The sizes of matrices $[\mathfrak{L}]$ and $[\mathfrak{T}]$ for solving the multiple scattering problems in Fig. 5.1 are estimated and compared in Table. 5.1. In Table. 5.1, $N$ are the number of actual scatterers. In the first stage, the problem sizes are the same. When the scatterer polymerization method is used, the problem sizes in the second and third stages are significantly smaller than those without using the scatterer polymerization method. 
It should be noted that the number of identical actual scatterers or molecules to be assembled in each stage in Fig. 5.1 happens to be 8 . There is no such a restriction for the scatterer polymerization methodology. In a stage of scatterer polymerization process, a group of actual scatterers, abstract scatterers or a mixture of actual and abstract scatterers can be assembled as a molecule. It depends on the geometrical position of actual scatterers in the host medium.

Table 5.1: Comparison of Problem Sizes of Scatterer Polymerization Methodology and Multiple Scattering

\begin{tabular}{|c|c|c|c|c|c|}
\hline & \multicolumn{3}{|c|}{ Scatterer Polymerization } & \multicolumn{2}{c|}{ Multiple Scattering } \\
\cline { 2 - 6 } & $\mathcal{N}$ & $N$ & Size $\left(\sim(3 \mathcal{N} \mathcal{M})^{2}\right)$ & $N$ & Size $\left(\sim(3 N \mathcal{M})^{2}\right)$ \\
\hline Stage 1 & 8 & 8 & $\sim 576 \mathcal{M}^{2}$ & 8 & $\sim 576 \mathcal{M}^{2}$ \\
\hline Stage 2 & 8 & 64 & $\sim 576 \mathcal{M}^{2}$ & 64 & $\sim 36864 \mathcal{M}^{2}$ \\
\hline Stage 3 & 8 & 512 & $\sim 576 \mathcal{M}^{2}$ & 512 & $\sim 2359296 \mathcal{M}^{2}$ \\
\hline
\end{tabular}

\section{5-3 Algorithm of Scatterer Polymerization}

The algorithm of scatterer polymerization is based on the multiple scattering solution. Recall Eq. (3-79). Recall in Eq. (3-79), the displacement potentials of total waves are expressed in global and local coordinate systems. The displacement potentials of total waves are required to be expressed in only one coordinate system in the process of scatterer polymerization such that

$$
\begin{array}{r}
\phi=\left\{\boldsymbol{A}^{\phi}\right\}^{T}\{\boldsymbol{j}(\kappa, \boldsymbol{r})\}+\left\{\boldsymbol{B}^{\phi}\right\}^{T}\{\boldsymbol{h}(\kappa, \boldsymbol{r})\} \\
\psi=\left\{\boldsymbol{A}^{\psi}\right\}^{T}\{\boldsymbol{j}(k, \boldsymbol{r})\}+\left\{\boldsymbol{B}^{\psi}\right\}^{T}\{\boldsymbol{h}(k, \boldsymbol{r})\} \\
\chi=\left\{\boldsymbol{A}^{\chi}\right\}^{T}\{\boldsymbol{j}(k, \boldsymbol{r})\}+\left\{\boldsymbol{B}^{\chi}\right\}^{T}\{\boldsymbol{h}(k, \boldsymbol{r})\}
\end{array}
$$




\section{5-3.1 Mathematics of Scatterer Polymerization}

Multiple scattering solution Eq. (5-3) can be written as

$$
\{\mathfrak{B}\}=[\mathfrak{L}]^{-1}\{\mathfrak{A}\},
$$

where matrices $\{\mathfrak{B}\}$ and $\{\mathfrak{B}\}$ in Eq. (3-76) are repeated here,

$$
\{\mathfrak{B}\}=\left\{\begin{array}{c}
\left\{\mathbb{B}_{1}\right\} \\
\left\{\mathbb{B}_{2}\right\} \\
\left\{\mathbb{B}_{3}\right\} \\
\vdots \\
\left\{\mathbb{B}_{N}\right\}
\end{array}\right\}, \quad\{\mathfrak{A}\}=\left\{\begin{array}{c}
{\left[\mathbb{T}_{1}\right]\left\{\mathbb{A}_{1}\right\}} \\
{\left[\mathbb{T}_{2}\right]\left\{\mathbb{A}_{2}\right\}} \\
{\left[\mathbb{T}_{3}\right]\left\{\mathbb{A}_{3}\right\}} \\
\vdots \\
{\left[\mathbb{T}_{N}\right]\left\{\mathbb{A}_{N}\right\}}
\end{array}\right\}=\left\{\begin{array}{c}
{\left[\mathbb{T}_{1}\right]\left[R g \mathbb{Q}_{O 1}\right]^{T}} \\
{\left[\mathbb{T}_{2}\right]\left[R g \mathbb{Q}_{O 2}\right]^{T}} \\
{\left[\mathbb{T}_{3}\right]\left[R g \mathbb{Q}_{O 3}\right]^{T}} \\
\vdots \\
{\left[\mathbb{T}_{N}\right]\left[R g \mathbb{Q}_{O N}\right]^{T}}
\end{array}\right\}\{\mathbb{A}\}
$$

The matrix $\left\{\mathbb{B}_{J}\right\}$ is the scattered wave expansion coefficients in scatterer $J$ 's local coordinate system. The matrix $[\mathfrak{L}]$ is square and of order $(3 N \mathcal{M})$ for $N$ scatterers when the truncation size of matrix $\left[\mathbb{T}_{I}\right]$ of Scatterer $I$ is $3 \mathcal{M}$.

Define a matrix $[\mathfrak{K}]$ such that

$$
[\mathfrak{K}]=[\mathfrak{L}]^{-1}=\left[\begin{array}{ccccc}
{\left[\mathbb{K}_{11}\right]} & {\left[\mathbb{K}_{12}\right]} & {\left[\mathbb{K}_{13}\right]} & \ldots & {\left[\mathbb{K}_{1 N}\right]} \\
{\left[\mathbb{K}_{21}\right]} & {\left[\mathbb{K}_{22}\right]} & {\left[\mathbb{K}_{23}\right]} & \ldots & {\left[\mathbb{K}_{1 N}\right]} \\
{\left[\mathbb{K}_{31}\right]} & {\left[\mathbb{K}_{32}\right]} & {\left[\mathbb{K}_{33}\right]} & \ldots & {\left[\mathbb{K}_{1 N}\right]} \\
\ldots & \ldots & \ldots & & \ldots \\
{\left[\mathbb{K}_{N 1}\right]} & {\left[\mathbb{K}_{N 2}\right]} & {\left[\mathbb{K}_{N 3}\right]} & \ldots & {\left[\mathbb{K}_{N N}\right]}
\end{array}\right]
$$

where the matrix $\left[\mathbb{K}_{J I}\right]$ is defined as a square matrix of order $3 \mathcal{M}$. Then, the solution structure of $\{\mathfrak{B}\}$ suggests that

$$
\left\{\mathbb{B}_{J}\right\}=\sum_{I=1}^{N}\left[\mathbb{K}_{J I}\right]\left[\mathbb{T}_{I}\right]\left\{\mathbb{A}_{I}\right\}=\sum_{I=1}^{N}\left[\mathbb{K}_{J I}\right]\left[\mathbb{T}_{I}\right]\left[R g \mathbb{Q}_{O I}\right]^{T}\{\mathbb{A}\}
$$

The scattered wave expansion coefficients $\left\{\mathbb{B}_{J}\right\}$ is in the local coordinate system of Scatterer $J$. Referring to Fig. 5.2, the scattered waves in the local coordinate system of scatterer $J$ (with origin at $O_{J}$ ) can be transformed as scattered waves in the global coordinate system (with origin at $O$ ). The wave expansion coefficients in the global 
coordinate system $\left\{\mathbb{B}_{\text {Odueto } J}\right\}$ from the contribution of Scatterer $J$ can be obtained according to Eq. (3-48),

$$
\left\{\mathbb{B}_{\text {O due to } J}\right\}=\left[R g \mathbb{Q}_{J O}\right]^{T}\left\{\mathbb{B}_{J}\right\}
$$

The summation of contributions from all scatterers forms the total scattered wave expansion coefficients in the global coordinate system, such as

$$
\{\mathbb{B}\}=\sum_{J=1}^{N}\left[R g \mathbb{Q}_{J O}\right]^{T}\left\{\mathbb{B}_{J}\right\}
$$

Substituting Eq. (5-8) into Eq. (5-10) renders

$$
\{\mathbb{B}\}=\sum_{J=1}^{N}\left[R g \mathbb{Q}_{J O}\right]^{T} \sum_{I=1}^{N}\left[K_{J I}\right]\left[\mathbb{T}_{I}\right]\left[R g \mathbb{Q}_{O I}\right]^{T}\{\mathbb{A}\}
$$

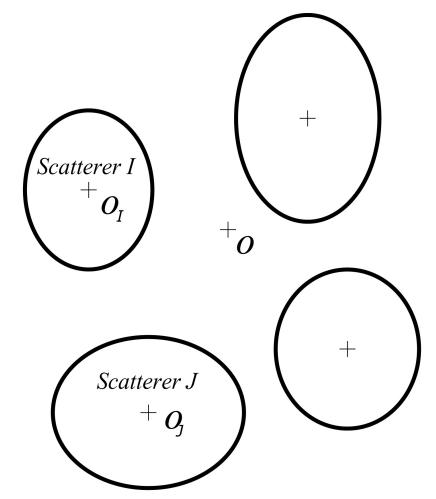

Figure 5.2: Position $O$ is chosen as the origin of the global coordinate system for 4 scatterers.

In comparison with Eq. (2-101), which is repeated as

$$
\{\mathbb{B}\}=[\mathbb{T}]\{\mathbb{A}\}
$$

Eq. (5-11) can be written as

$$
\{\mathbb{B}\}=\left[\mathbb{T}_{O}^{N}\right]\{\mathbb{A}\}
$$

where $\left[\mathbb{T}_{O}^{N}\right]$ is the super $T$-matrix for this group of $N$ scatterers with position $O$ as 
origin of the coordinate system and

$$
\left[\mathbb{T}_{O}^{N}\right]=\sum_{J=1}^{N}\left[R g \mathbb{Q}_{J O}\right]^{T} \sum_{I=1}^{N}\left[K_{J I}\right]\left[\mathbb{T}_{I}\right]\left[R g \mathbb{Q}_{O I}\right]^{T}
$$

Then, $\left\{\boldsymbol{B}^{\phi}\right\},\left\{\boldsymbol{B}^{\psi}\right\}$ and $\left\{\boldsymbol{B}^{\phi}\right\}$ can be obtained from $\{\mathbb{B}\}$ according to Eq. (2-100). The wave fields can be computed just like those of a single scattering problem. In details, displacements and stresses due to the scattered waves can be computed using Eqs. (2-109a) through (2-109i).

\section{5-3.2 Core Region and Territory of An Abstract Scatterer}

As shown in Section 5-3.1, the super T-matrix for an assemblage of scatterers are obtained through Eq. (5-14). The assemblage of scatterers are treated as an abstract scatterer. As an example in Fig.5.3, four scatterers are treated as an abstract scatterer.

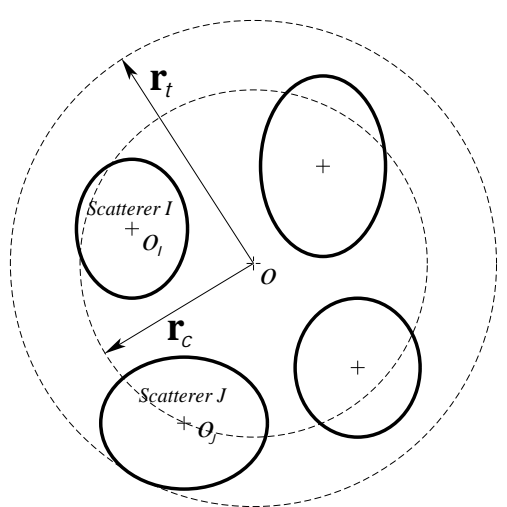

Figure 5.3: Core and territory of an abstract scatterer made up of 4 scatterers. The core is defined as the spherical region of radius $r_{c}$, where $r_{c}=\left|O O_{J}\right|$ and $\left|O O_{J}\right|$ is the largest distance from the origin $O$ to each local origin. The territory is the smallest spherical surface that circumscribes all four scatterers, whose radius is $r_{t}$.

Addition theorems are used to formulate Eq. (5-14). Validity conditions of applying addition theorems can be found in Eqs. (3-11b) or (3-13b). In Fig. 5.3, the validity condition of transforming scattered waves in the local coordinate system of 
scatterer $I$ to scattered waves in the global coordinate system is $r>\left|O O_{I}\right|$, where $\left|O O_{I}\right|$ is the distance between origins of the global coordinate system and the local coordinate system of scatterer $I$. Among the distances of the global origin $O$ to the local origins in Fig. 5.3, $\left|O O_{J}\right|$ is the largest one. Therefore, the validity condition for applying Eq. (5-14) in Fig. 5.3 is $r>\left|O O_{J}\right|$. Let $r_{C}=\left|O O_{J}\right|$ in Fig. 5.3. The region of $r \leq r_{C}$ is defined as the core of an abstract scatterer.

The territory of an actual scatterer is its boundary surface. In analogy, the territory of an abstract scatterer is defined as the smallest spherical surface that circumscribes all scatterers that make up the abstract scatterer. In Fig. 5.3, $r_{t}$ is the radius of the spherical surface that circumscribes all four scatterers. Therefore, the territory of the abstract scatterer in Fig. 5.3 is the spherical surface of radius $r_{t}$. The scattered wave of the actual scatterer $J$ exists outside its territory. In analogy, scattered waves of an abstract scatterer are referred to those outside territory of the abstract scatterer.

The definition of the core, the territory and their radii apply to an assemblage of abstract scatterers. Figure 5.4 shows such an example with three abstract scatterers. $O$ is the origin of global coordinate system, $O_{1}, O_{2}$ and $O_{3}$ are origins of local coordinate systems. The core region is inside of the spherical surface of radius $r_{C}$ and the territory is the spherical surface of radius $r_{t}$.

It is noticed that information of wave fields inside the core region is lost in the scatterer polymerization process. The information is lost when Eq. (5-11) is obtained from Eq. (5-10) and the scattered wave coefficients $\left\{\mathbb{B}_{J}\right\}$ are not saved. If the scattered wave coefficients $\left\{\mathbb{B}_{J}\right\}$ are saved, the lost information can be recovered. However, such a recovering process would soon become tedious within a few stages of polymerization. 


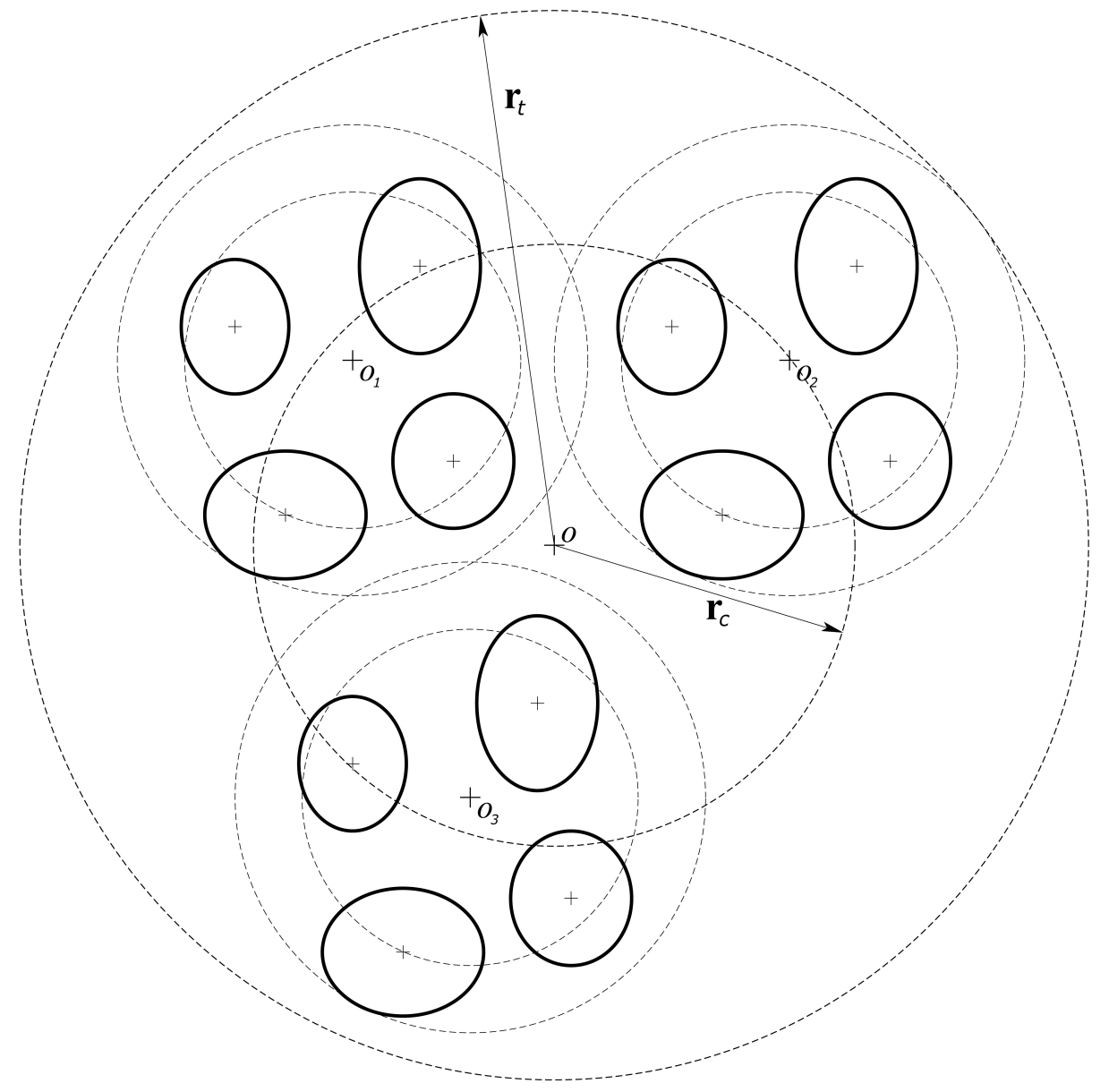

Figure 5.4: Core and territory of an abstract scatterer made up of 3 abstract scatterers. The core is defined as the spherical region of radius $r_{c}$, where $r_{c}=\left|O O_{2}\right|$, $\left|O O_{2}\right|>\left|O O_{3}\right|$, and $\left|O O_{2}\right|>\left|O O_{1}\right|$. The territory is the smallest spherical surface that circumscribes all three abstract scatterers, whose radius is $r_{t}$. 


\section{5-4 Verification}

The verification for the scatterer polymerization methodology is through solving a physically identical multiple scattering problem from different approaches, with or without scatterer polymerization.

\section{5-4.1 Description of Numerical Procedure}

As shown in Fig. 5.5, eight scatterers embedded in epoxy matrix are in cubical lattice arrangement. They are low-carbon spheres of unit diameter. They are labeled with number "1" through "8" and their locations in the global coordinate system are detailed in Fig. 5.5(b).

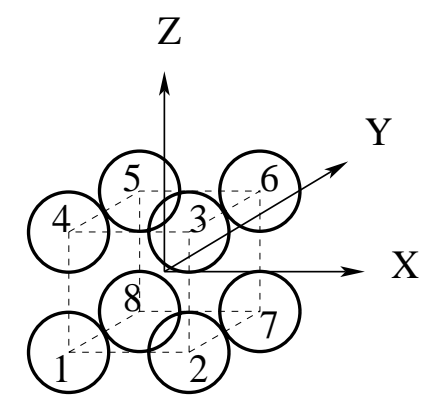

(a) Lattice structure

$$
\begin{array}{ll}
1:(-d,-d,-d) & 2:(d,-d,-d) \\
3:(d,-d, d) & 4:(-d,-d, d) \\
5:(-d, d, d) & 6:(d, d, d) \\
7:(d, d,-d) & 8:(-d, d,-d)
\end{array}
$$

(b) Cartesian coordinates $(x, y, z)$ of scatterers of radius $a, d=1.5 a=0.75$

Figure 5.5: Cubical lattice of 8 scatterers

In this section, a low-carbon steel sphere and an epoxy matrix are chosen, because epoxy is commonly used as matrix in composites, low-carbon steel is a common material, and the elastic properties and mass density of steel are much larger than the corresponding ones of epoxy. Material properties of epoxy and low-carbon steel are listed in Table 5.2. For epoxy, Young's modulus E, Poisson's ratio $\nu$ and density $\rho$ are taken from [4], while Lamé constants are derived from $E$ and $\nu$ using Eqs. (5-15a) and $(5-15 b)^{[41]}$. For low-carbon steel, Young's modulus $E$, shear modulus $\mu$ and 
Table 5.2: Material Properties of Low-Carbon Steel and Epoxy (after [2, 4])

\begin{tabular}{|c|c|c|}
\hline Property & Matrix (Epoxy) & Sphere (Low-Carbon Steel) \\
\hline Young's modulus $E(\mathrm{GPa})$ & 3.12 & 207. \\
Poisson's ratio $\nu$ & 0.38 & 0.294 \\
Density $\rho\left(\mathrm{kg} / \mathrm{m}^{3}\right)$ & 1200. & 7800. \\
Lamé constant $\lambda(\mathrm{GPa})$ & 3.58 & 113.939 \\
Lamé constant $\mu(\mathrm{GPa})$ & 1.13 & 80. \\
$\mathrm{P}$ Wave Speed $(\mathrm{km} / \mathrm{s})$ & 2.206 & 5.926 \\
S Wave Speed $(\mathrm{km} / \mathrm{s})$ & 0.971 & 3.202 \\
\hline
\end{tabular}

density $\rho$ are taken from [2], while Poisson's ratio $\nu$ and Lamé constant are derived from $E$ and $\mu$ using Eqs. (5-15c) and (5-15a) ${ }^{[41]}$.

$$
\begin{aligned}
\lambda & =\frac{E \nu}{(1+\nu)(1-2 \nu)} \\
\mu & =\frac{E}{2(1+\nu)} \\
\nu & =\frac{E}{2 \mu}-1
\end{aligned}
$$

For the cubical arrangement in Fig. 5.5, the multiple scattering problem is solved using five approaches. These five approaches are designated as "NP", "P1" "Px", "Py", and "Pz" in Fig. 5.6. These approaches are specified as following, with help of Figs. 5.5 and 5.6.

In "NP" approach in Fig. 5.6(a), the scatterer polymerization method is not used.

In "P1" approach, all eight scatterers are assembled as an abstract scatterer using the scatterer polymerization method, and the spherical surface in light green color in Fig. 5.6(b) represents the territory of the abstract scatterer. Then, the multiple scattering problem of eight actual scatterers becomes a single scattering problem of one abstract scatterers.

In "Px" approach, scatterers 1, 2, 7 and 8 are assembled as an abstract scatterer, scatterers 3, 4, 5 and 6 are assembled as another abstract scatterer using the scatterer 


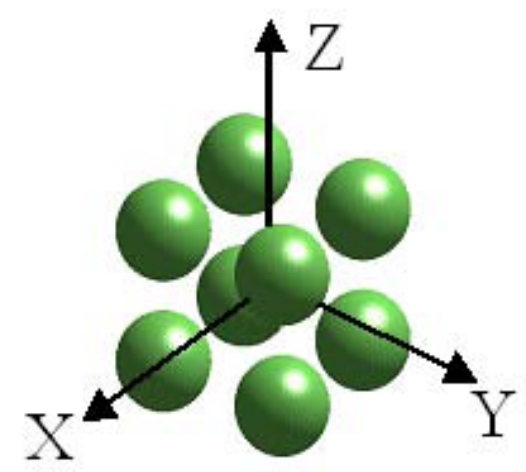

(a) NP

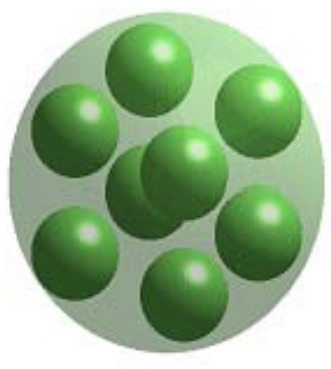

(b) P1

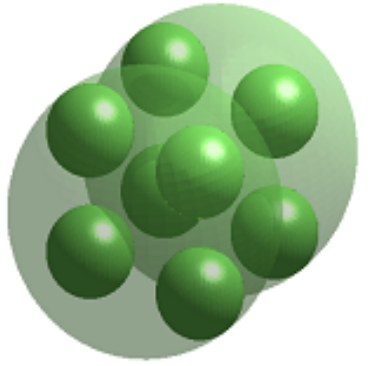

(c) $\mathrm{Px}$

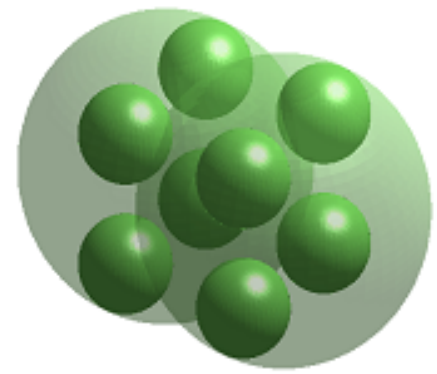

(d) Py

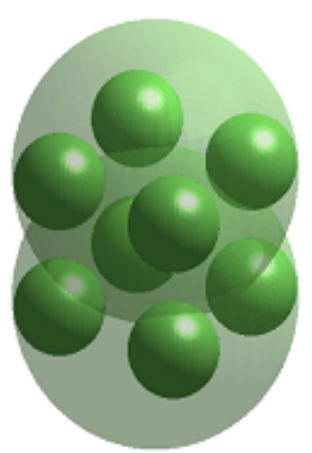

(e) $\mathrm{Pz}$

Figure 5.6: Five approaches to solve the multiple scattering problem of 8 scatterers. (a) NP: the scatterer polymerization method is not used. (b) P1: eight scatterers are assembled as an abstract scatterer, the spherical surface in light green color represents the territory of the abstract scatterer. (c) Px: eight scatterers are assembled as two abstract scatterers in the $X$-axis, each abstract encloses four actual scatterers, and each spherical surface in light green color represents the territory of an abstract scatterer. (d) Py: eight scatterers are assembled as two abstract scatterers in the $Y$ axis, each abstract encloses four actual scatterers, and each spherical surface in light green color represents the territory of an abstract scatterer. (c) Pz: eight scatterers are assembled as two abstract scatterers in the $Z$-axis, each abstract encloses four actual scatterers, and each spherical surface in light green color represents the territory of an abstract scatterer. 
polymerization method, and each spherical surface in light green color in Fig. 5.6(c) represents the territory of an abstract scatterer. Then, the multiple scattering problem of eight actual scatterers becomes a multiple scattering problem of two abstract scatterers in the $X$-axis.

In "Py" approach, scatterers 1, 4, 5 and 8 are assembled as an abstract scatterer, scatterers 2, 3, 6 and 7 are assembled as another abstract scatterer using the scatterer polymerization method, and each spherical surface in light green color in Fig. 5.6(c) represents the territory of an abstract scatterer. Then, the multiple scattering problem of eight actual scatterers becomes a multiple scattering problem of two abstract scatterers in the $Y$-axis.

In "Pz" approach, scatterers 1, 2, 3 and 4 are assembled as an abstract scatterer, scatterers 5, 6, 7 and 8 are assembled as another abstract scatterer using the scatterer polymerization method, and each spherical surface in light green color in Fig. 5.6(c) represents the territory of an abstract scatterer. Then the multiple scattering problem of eight actual scatterers becomes a multiple scattering problem of two abstract scatterers in the $Z$-axis.

\section{5-4.2 Comparison of Numerical Results}

The incident wave is chosen as a longitudinal plane wave propagating along $Z$ axis with displacement of unit amplitude. In the host medium, the longitudinal wave number is $\kappa a=0.5$ and transverse wave number is $k a=1.136515$. In scatterers, the longitudinal wave number is $\kappa a=0.0296242$ and the transverse wave number is $k a=0.054819$. The truncation number for computation is 14 .

For the same physical case, the same numerical results are expected through different approaches. In this section, comparison is made using displacements and stresses in Cartesian coordinate system of the scattered waves in the $y=0$ plane, and using 
the energy flux across a closed spherical surface.

The modulus of displacements $u_{x}, u_{y}$ and $u_{z}$ are compared in Figs. 5.7 through 5.9 , where $x / a \in[-10,10]$ and $z / a \in[-10,10]$. Table 5.3 through 5.5 show numerical results at three positions in Fig. 5.7 through 5.9. The Cartesian coordinates of these three positions are $(x=-3.35 a, y=0, z=-9.05 a),(x=3.5 a, y=0, z=3.5 a)$, and $(x=5.8 a, y=0, z=-6.9 a)$.

Table 5.3: Modulus of $u_{x}$ of scattered waves from five computational approaches

\begin{tabular}{|c|c|c|c|}
\hline & Position-1 & Position-2 & Position-3 \\
\cline { 2 - 4 } & $x=-3.35, y=0, z=-9.05$ & $x=3.5, y=0, z=3.5$ & $x=5.8, y=0, z=-6.9$ \\
\hline NP & $1.182724 \times 10^{-01}$ & $9.008451 \times 10^{-02}$ & $6.717650 \times 10^{-02}$ \\
\hline P1 & $1.182724 \times 10^{-01}$ & $9.008449 \times 10^{-02}$ & $6.717651 \times 10^{-02}$ \\
\hline Px & $1.184284 \times 10^{-01}$ & $8.553823 \times 10^{-02}$ & $6.625936 \times 10^{-02}$ \\
\hline Py & $1.166038 \times 10^{-01}$ & $8.669063 \times 10^{-02}$ & $6.534989 \times 10^{-02}$ \\
\hline Pz & $1.219427 \times 10^{-01}$ & $8.345876 \times 10^{-02}$ & $6.724638 \times 10^{-02}$ \\
\hline
\end{tabular}

Table 5.4: Modulus of $u_{y}$ of scattered waves from five computational approaches

\begin{tabular}{|c|c|c|c|}
\hline & Position-1 & Position-2 & Position-3 \\
\cline { 2 - 4 } & $x=-3.35, y=0, z=-9.05$ & $x=3.5, y=0, z=3.5$ & $x=5.8, y=0, z=-6.9$ \\
\hline $\mathrm{NP}$ & $2.428191 \times 10^{-07}$ & $1.083972 \times 10^{-06}$ & $2.865438 \times 10^{-07}$ \\
\hline $\mathrm{P} 1$ & $2.488501 \times 10^{-07}$ & $1.079419 \times 10^{-06}$ & $2.773200 \times 10^{-07}$ \\
\hline $\mathrm{Px}$ & $5.062927 \times 10^{-07}$ & $5.192732 \times 10^{-03}$ & $1.402860 \times 10^{-03}$ \\
\hline $\mathrm{Py}$ & $1.235001 \times 10^{-07}$ & $4.693196 \times 10^{-04}$ & $6.454594 \times 10^{-04}$ \\
\hline $\mathrm{Pz}$ & $1.990963 \times 10^{-07}$ & $1.925139 \times 10^{-04}$ & $1.922226 \times 10^{-04}$ \\
\hline
\end{tabular}

In Figs. 5.7 through 5.9 and Table 5.3 through 5.5, for the approach of "Px" (forming two abstract scatterers in the $X$-axis), scattered wave expansion coefficients of two abstract scatterers are obtained after solving the multiple scattering problem of two abstract scatterers. By using Eqs. (3-85a) through (3-85c), displacements $u_{r}, u_{\theta}$ and $u_{\varphi}$ due to the scattered waves in each local coordinate system can be computed. Then, displacements $u_{x}, u_{y}$ and $u_{z}$ in each local coordinate system can be obtained 


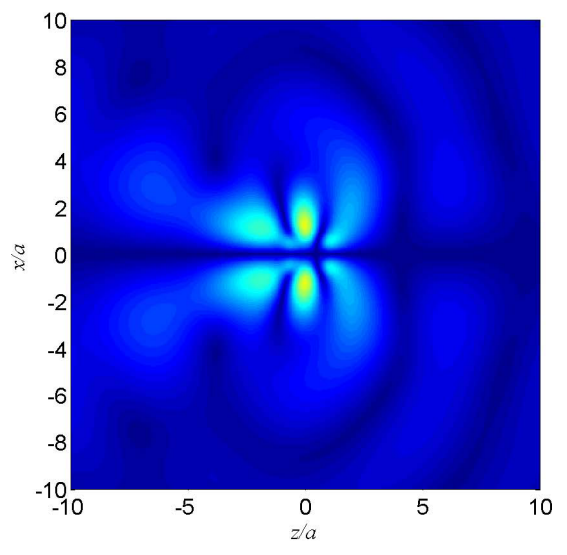

(a)

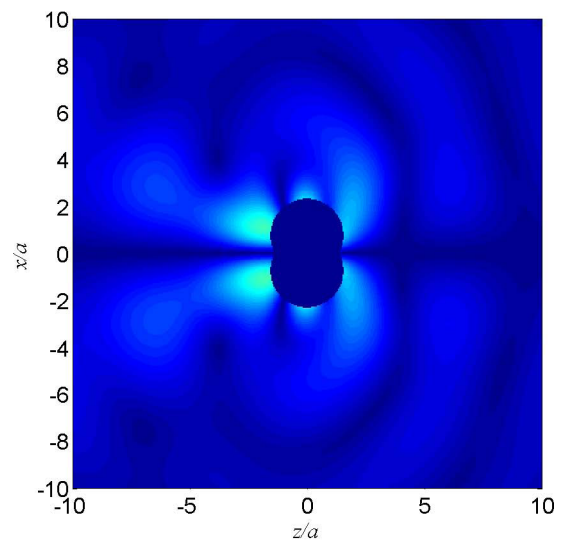

(c)

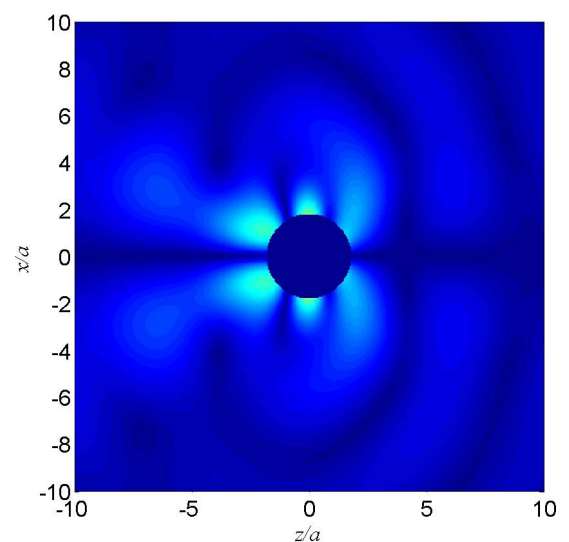

(b)

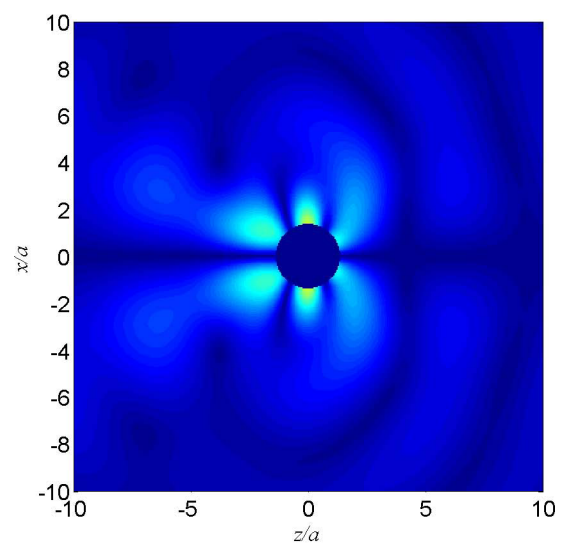

(d)

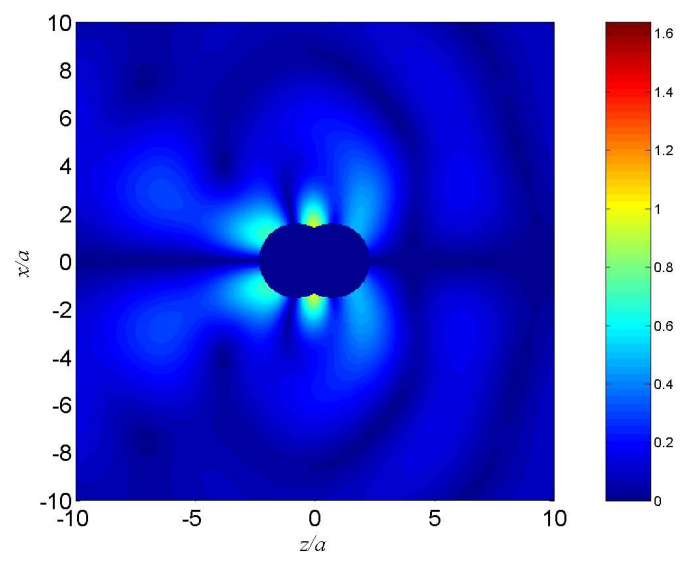

(e)

Figure 5.7: Modulus of $u_{x}$ of scattered waves at the $y=0$ plane, for the multiple scattering problem of a longitudinal plane wave $(\kappa a=0.5)$ by 8 scatterers of low-carbon steel spheres in epoxy matrix. The problem is solved in 5 approaches designated as: (a) NP, (b) P1, (c) Px, (d) Py and (c) Pz, which are specified in Section 5-4.1. 


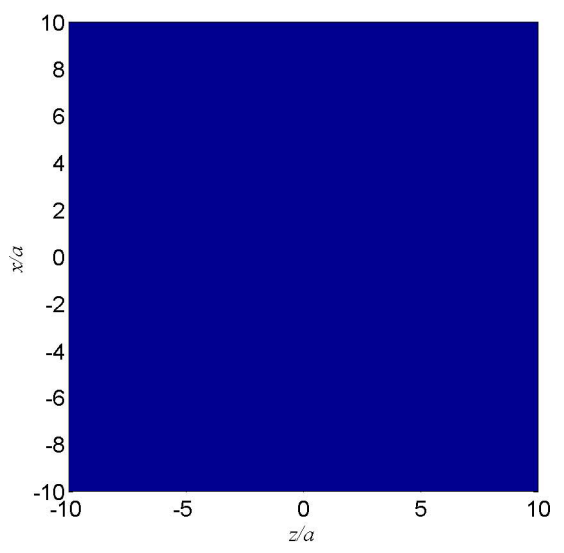

(a)

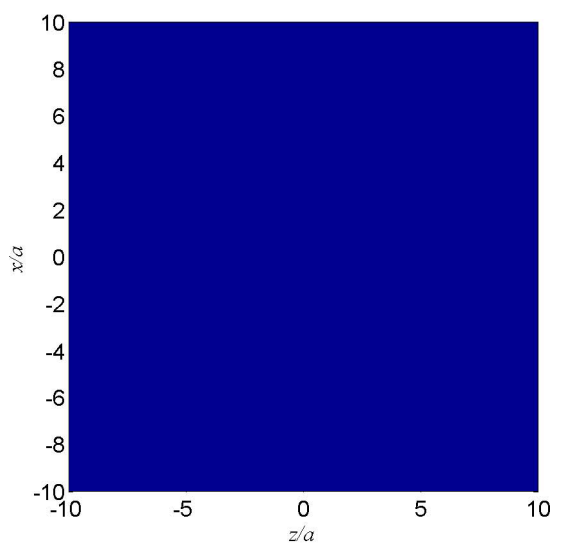

(c)

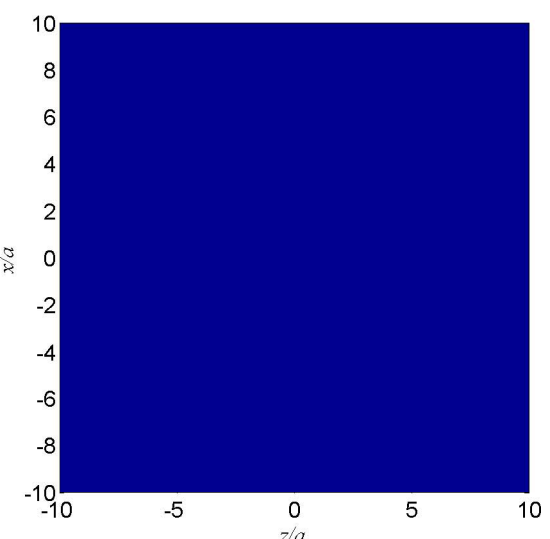

(b)

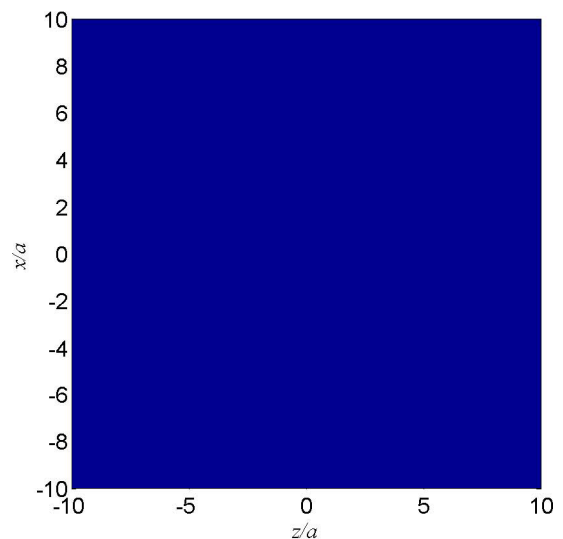

(d)

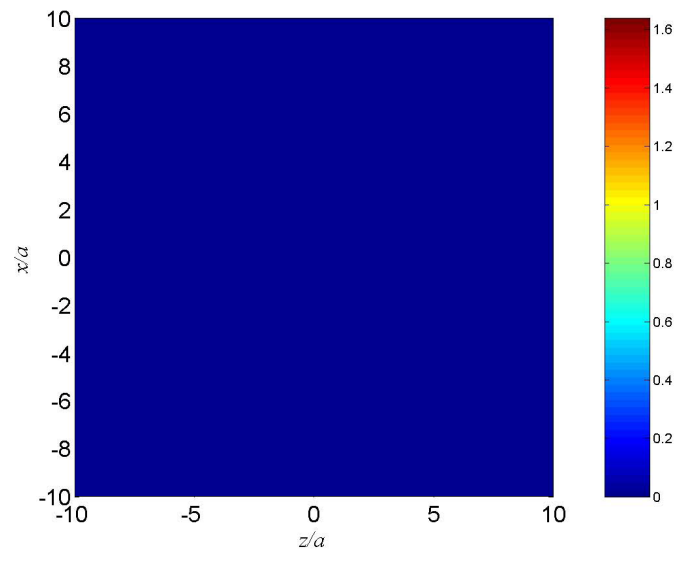

(e)

Figure 5.8: Modulus of $u_{y}$ of scattered waves at the $y=0$ plane, for the multiple scattering problem of a longitudinal plane wave $(\kappa a=0.5)$ by 8 scatterers of low-carbon steel spheres in epoxy matrix. The problem is solved in 5 approaches designated as: (a) NP, (b) P1, (c) Px, (d) Py and (c) Pz, which are specified in Section 5-4.1. 


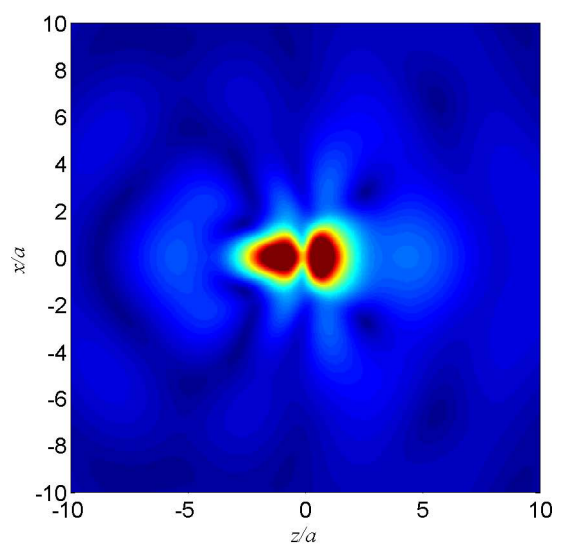

(a)

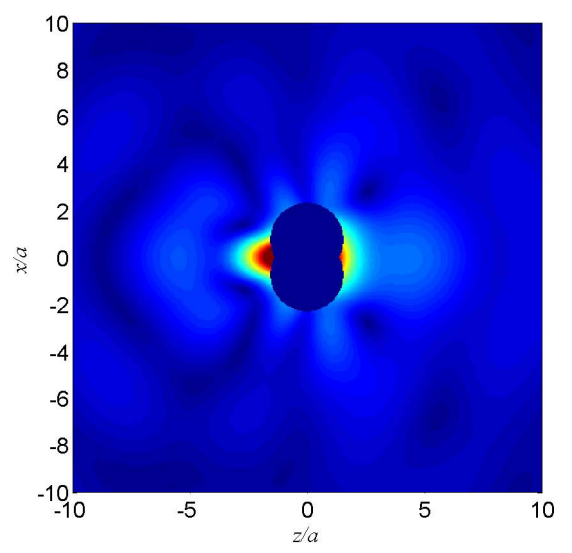

(c)

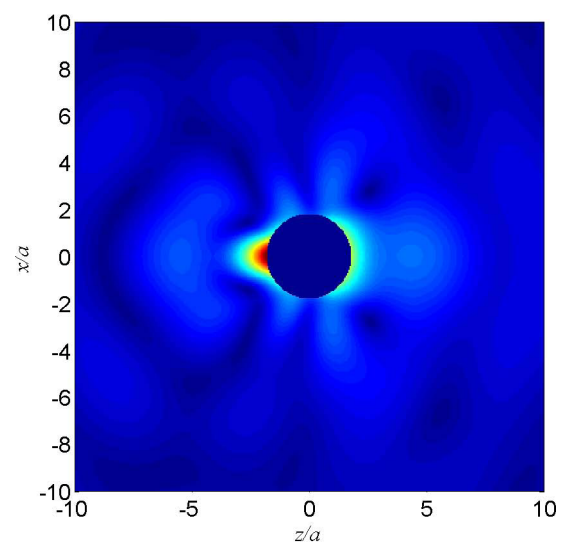

(b)

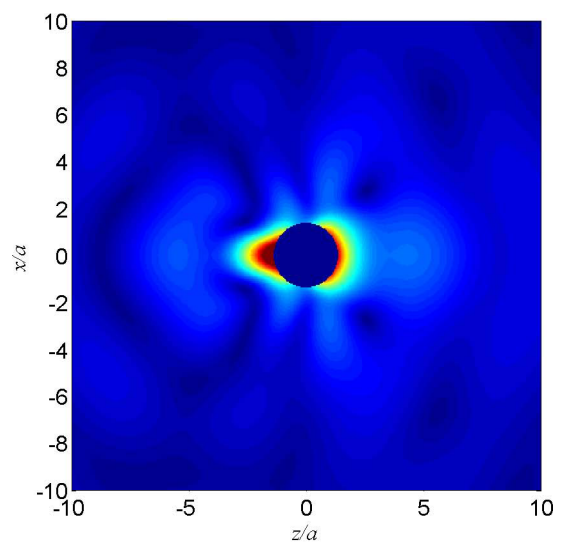

(d)

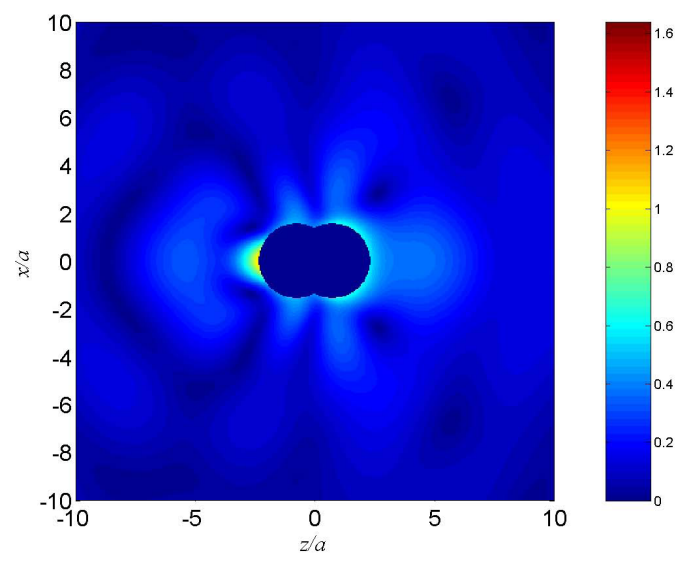

(e)

Figure 5.9: Modulus of $u_{z}$ of scattered waves at the $y=0$ plane, for the multiple scattering problem of a longitudinal plane wave $(\kappa a=0.5)$ by 8 scatterers of low-carbon steel spheres in epoxy matrix. The problem is solved in 5 approaches designated as: (a) NP, (b) P1, (c) Px, (d) Py and (c) Pz, which are specified in Section 5-4.1. 
Table 5.5: Modulus of $u_{z}$ of scattered waves from five computational approaches

\begin{tabular}{|c|c|c|c|}
\hline & Position-1 & Position-2 & Position-3 \\
\cline { 2 - 4 } & $x=-3.35, y=0, z=-9.05$ & $x=3.5, y=0, z=3.5$ & $x=5.8, y=0, z=-6.9$ \\
\hline $\mathrm{NP}$ & $9.220057 \times 10^{-02}$ & $1.374787 \times 10^{-01}$ & $6.264184 \times 10^{-02}$ \\
\hline $\mathrm{P} 1$ & $9.220089 \times 10^{-02}$ & $1.374802 \times 10^{-01}$ & $6.264148 \times 10^{-02}$ \\
\hline $\mathrm{Px}$ & $9.001175 \times 10^{-02}$ & $1.368788 \times 10^{-01}$ & $6.860185 \times 10^{-02}$ \\
\hline $\mathrm{Py}$ & $9.029301 \times 10^{-02}$ & $1.369531 \times 10^{-01}$ & $6.860185 \times 10^{-02}$ \\
\hline $\mathrm{Pz}$ & $9.667429 \times 10^{-02}$ & $1.327075 \times 10^{-01}$ & $6.254342 \times 10^{-02}$ \\
\hline
\end{tabular}

from corresponding $u_{r}, u_{\theta}$ and $u_{\varphi}$ using Eq. (3-86). The displacements $u_{x}, u_{y}$ and $u_{z}$ in each local coordinate system can directly be used as $u_{x}, u_{y}$ and $u_{z}$ in the global coordinate system. At each position, summation of $u_{x}$ due to the scattered waves of two abstract scatterers is the $u_{x}$ due to the total scattered waves. Displacement $u_{y}$ and $u_{z}$ are obtained in the same way. For other approaches, the processes of computing $u_{x}, u_{y}$ and $u_{z}$ are similar.

Wave information inside an abstract scatterer is lost and wave fields inside an abstract scatterer are set to zero when the scatterer polymerization method is used. No information is lost in the "NP" approach, where actual scatterers are not assembled as abstract scatterer(s). For $u_{x}$ at each position where wave information is not lost, the five approaches produce similar results with errors on the order of $10^{-2}$ or less, which can be supported by numerical results in Table 5.3. For comparison of displacements, the maximum value of displacement amplitude is used as a reference according to the definition of number of significant figures in Section 4-2. The reference value is chosen as $1.64 \times 10^{0}$ according to the color range in Figs. 5.7 through 5.9. The number of significant figures of $u_{y}$ in Table 5.4 are 2 or more. In other words, the errors are on the order of $10^{-2}$ or less. These errors can not be distinguished in Fig. 5.7. Similar observation can be made for $u_{y}$ and $u_{z}$. At each position where wave information is not lost, the five approaches produce similar results with errors on the order of $10^{-2}$ 
or less.

The modulus of stresses $\sigma_{x x}, \sigma_{y y}, \sigma_{z z}, \sigma_{x y}, \sigma_{x z}$, and $\sigma_{y z}$ are compared in Figs. 5.10 through 5.15, where $x / a \in[-10,10]$ and $z / a \in[-10,10]$. Table 5.6 through 5.11 show numerical results at three positions in Fig. 5.7 through 5.9. The Cartesian coordinates of these three positions are $(x=-3.35 a, y=0, z=-9.05 a),(x=3.5 a, y=0, z=$ $3.5 a)$, and $(x=5.8 a, y=0, z=-6.9 a)$.

Table 5.6: Modulus of $\sigma_{x x}$ of scattered waves from five computational approaches

\begin{tabular}{|c|c|c|c|}
\hline & Position-1 & Position-2 & Position-3 \\
\cline { 2 - 4 } & $x=-3.35, y=0, z=-9.05$ & $x=3.5, y=0, z=3.5$ & $x=5.8, y=0, z=-6.9$ \\
\hline NP & $6.237409 \times 10^{8}$ & $1.400086 \times 10^{8}$ & $3.023054 \times 10^{8}$ \\
\hline P1 & $6.237409 \times 10^{8}$ & $1.400086 \times 10^{8}$ & $3.023054 \times 10^{8}$ \\
\hline Px & $6.232012 \times 10^{8}$ & $1.466921 \times 10^{8}$ & $2.945396 \times 10^{8}$ \\
\hline Py & $6.164791 \times 10^{8}$ & $1.343874 \times 10^{8}$ & $2.964330 \times 10^{8}$ \\
\hline Pz & $6.432925 \times 10^{8}$ & $1.792969 \times 10^{8}$ & $3.013180 \times 10^{8}$ \\
\hline
\end{tabular}

Table 5.7: Modulus of $\sigma_{y y}$ of scattered waves from five computational approaches

\begin{tabular}{|c|c|c|c|}
\hline & Position-1 & Position-2 & Position-3 \\
\cline { 2 - 4 } & $x=-3.35, y=0, z=-9.05$ & $x=3.5, y=0, z=3.5$ & $x=5.8, y=0, z=-6.9$ \\
\hline NP & $2.343165 \times 10^{8}$ & $5.950781 \times 10^{8}$ & $2.715825 \times 10^{8}$ \\
\hline P1 & $2.343165 \times 10^{8}$ & $5.950781 \times 10^{8}$ & $2.715825 \times 10^{8}$ \\
\hline Px & $2.347803 \times 10^{8}$ & $5.927061 \times 10^{8}$ & $2.716409 \times 10^{8}$ \\
\hline Py & $2.260833 \times 10^{8}$ & $5.895308 \times 10^{8}$ & $2.698674 \times 10^{8}$ \\
\hline Pz & $2.336593 \times 10^{8}$ & $6.031881 \times 10^{8}$ & $2.783104 \times 10^{8}$ \\
\hline
\end{tabular}

In Figs. 5.10 through 5.15 and Table 5.6 through 5.11, for the approach of "Px" (forming two abstract scatterers in the $X$-axis), scattered wave expansion coefficients of two abstract scatterers are obtained after solving the multiple scattering problem of two abstract scatterers. By using Eqs. (3-85d) through (3-85i), stresses $\sigma_{r r}, \sigma_{r \theta}$, $\sigma_{r \varphi}, \sigma_{\theta \theta}, \sigma_{\theta \varphi}$, and $\sigma_{\varphi \varphi}$ due to the scattered waves in each local coordinate system can be computed. Then, stresses $\sigma_{x x}, \sigma_{y y}, \sigma_{z z}, \sigma_{x y}, \sigma_{x z}$, and $\sigma_{y z}$ in each local coordinate 


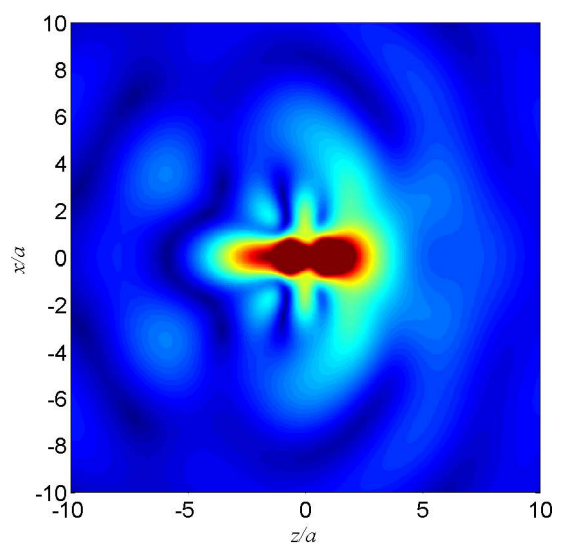

(a)

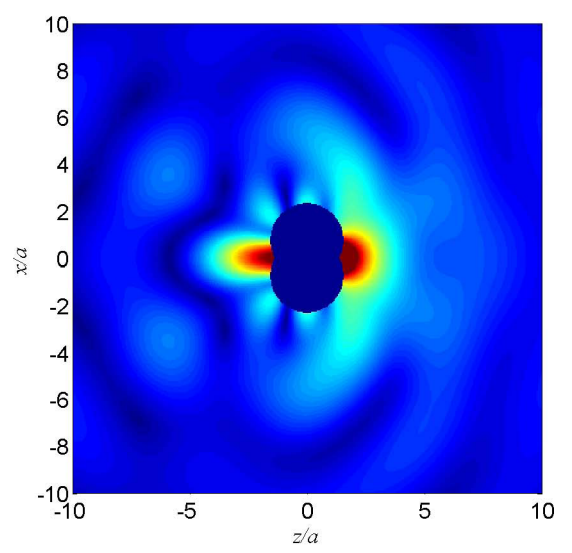

(c)

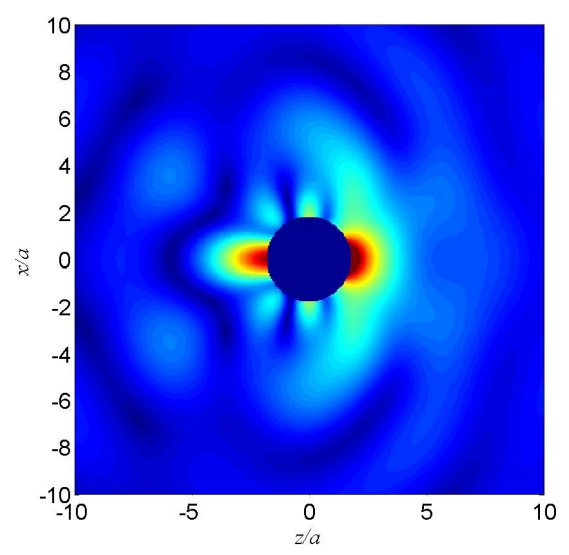

(b)

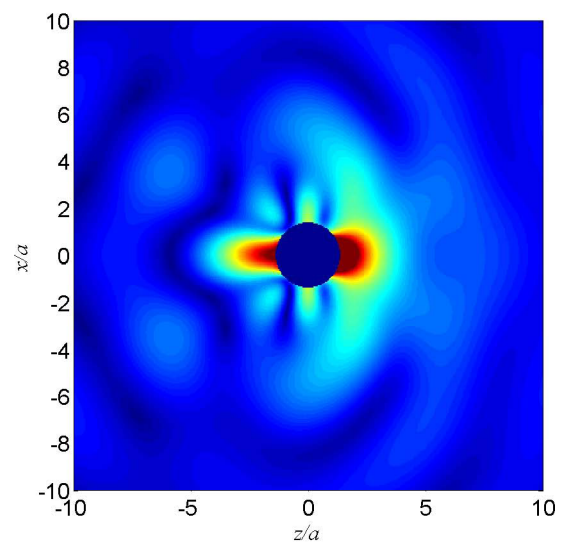

(d)

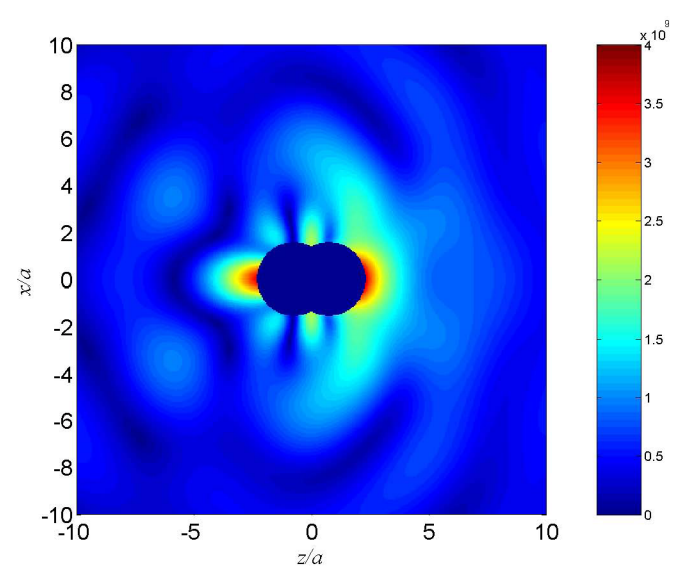

(e)

Figure 5.10: Modulus of $\sigma_{x x}$ of scattered waves at the $y=0$ plane, for the multiple scattering problem of a longitudinal plane wave $(\kappa a=0.5)$ by 8 scatterers of low-carbon steel spheres in epoxy matrix. The problem is solved in 5 approaches designated as: (a) NP, (b) P1, (c) Px, (d) Py and (c) Pz, which are specified in Section 5-4.1. 


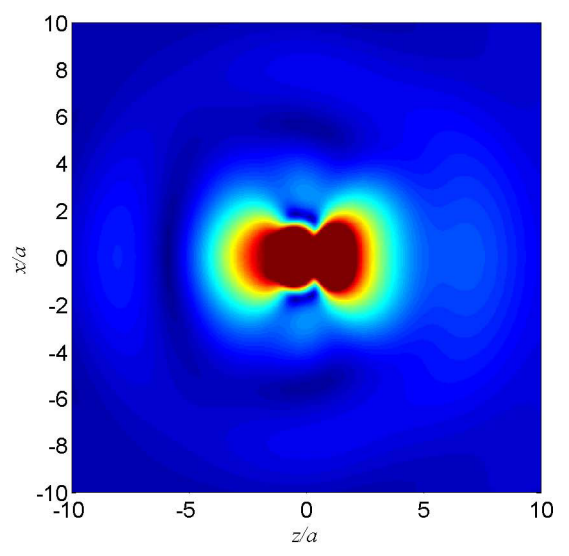

(a)

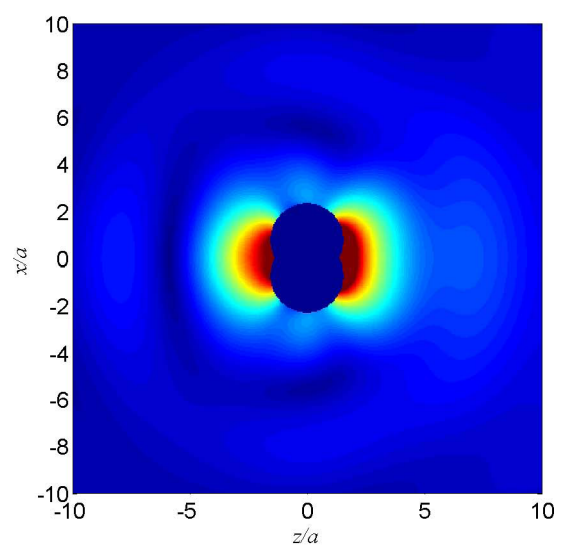

(c)

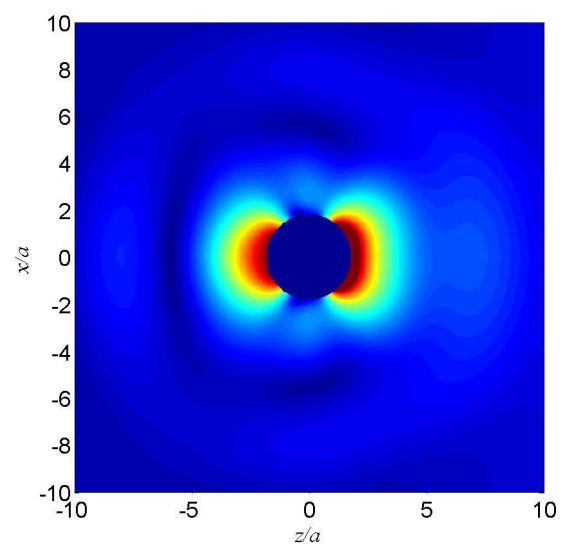

(b)

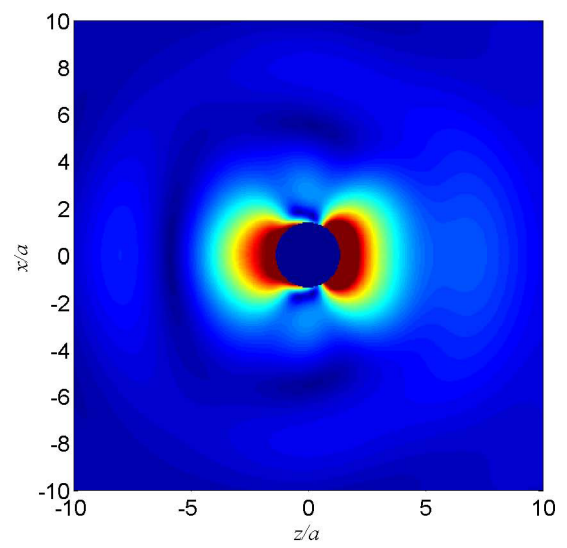

(d)

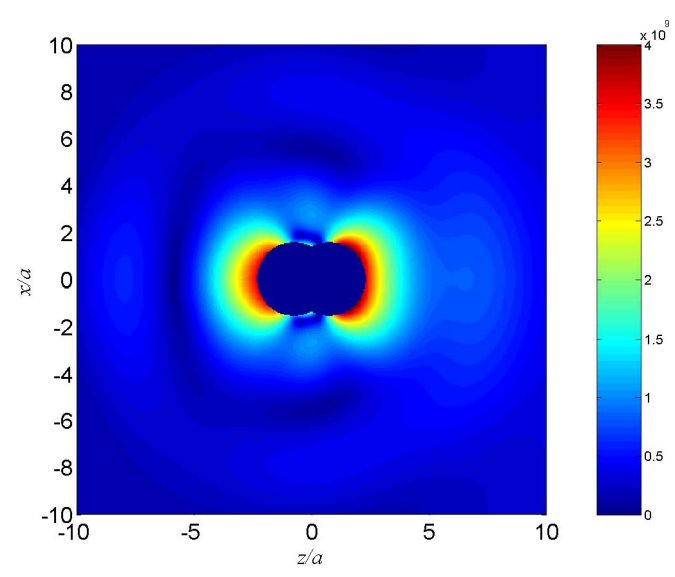

(e)

Figure 5.11: Modulus of $\sigma_{y y}$ of scattered waves at the $y=0$ plane, for the multiple scattering problem of a longitudinal plane wave $(\kappa a=0.5)$ by 8 scatterers of low-carbon steel spheres in epoxy matrix. The problem is solved in 5 approaches designated as: (a) NP, (b) P1, (c) Px, (d) Py and (c) Pz, which are specified in Section 5-4.1. 


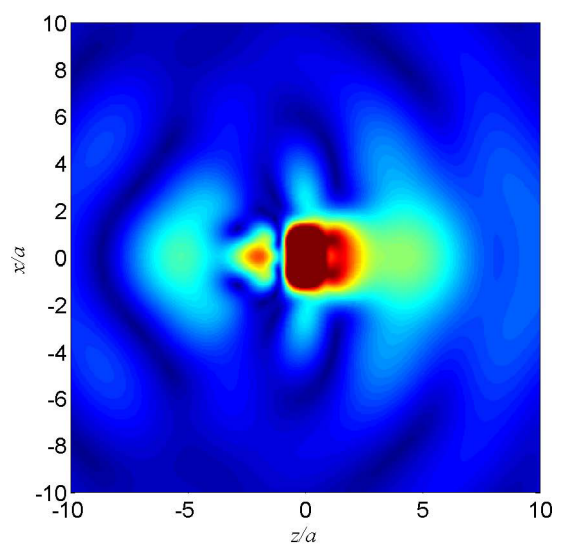

(a)

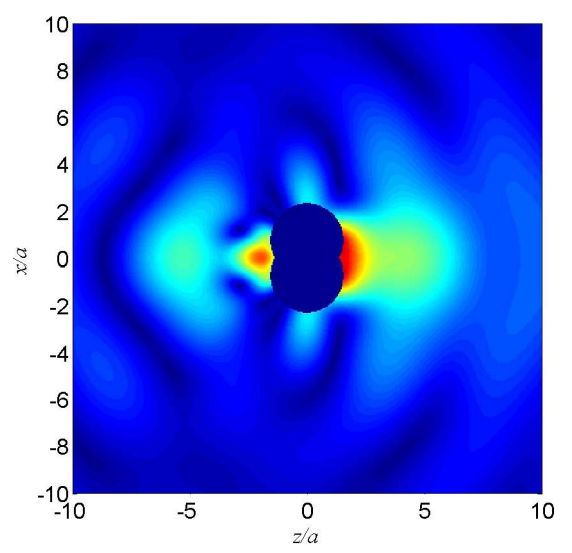

(c)

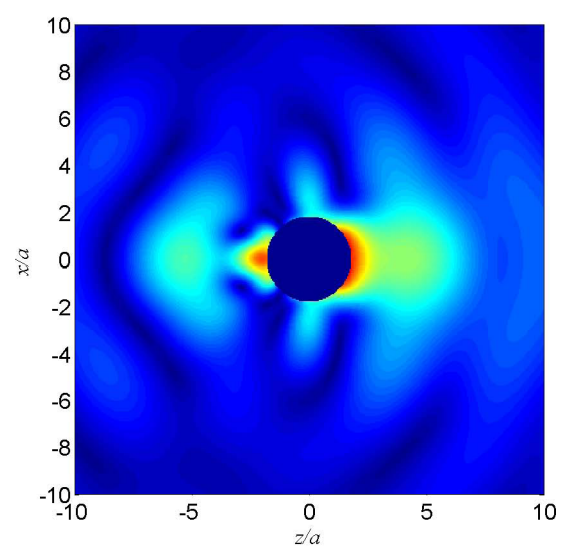

(b)

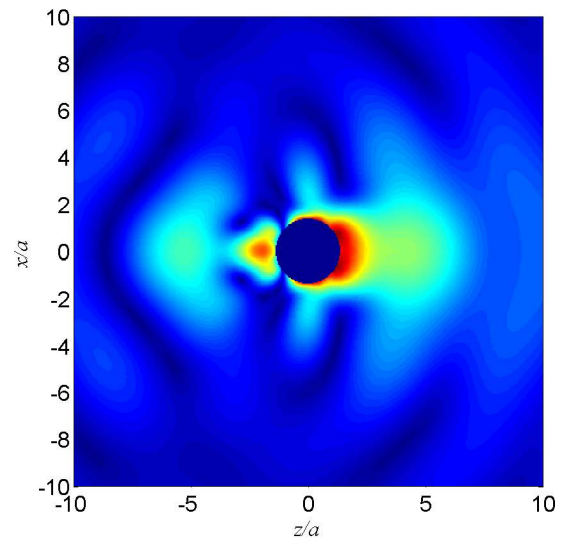

(d)

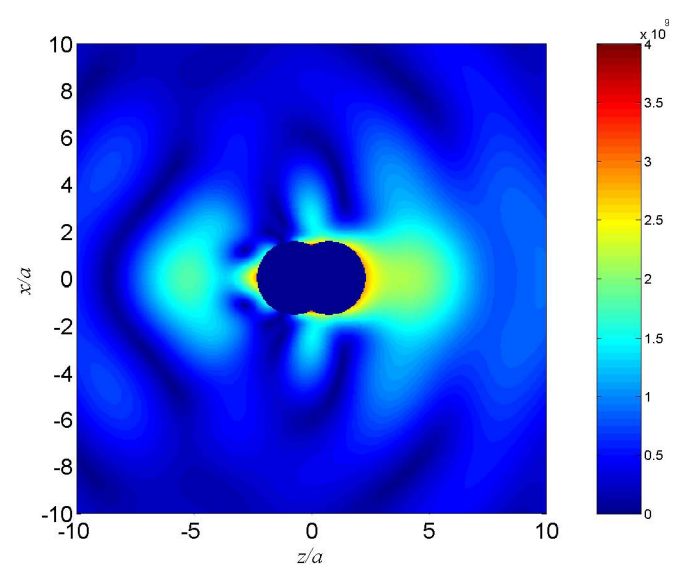

(e)

Figure 5.12: Modulus of $\sigma_{z z}$ of scattered waves at the $y=0$ plane, for the multiple scattering problem of a longitudinal plane wave $(\kappa a=0.5)$ by 8 scatterers of low-carbon steel spheres in epoxy matrix. The problem is solved in 5 approaches designated as: (a) NP, (b) P1, (c) Px, (d) Py and (c) Pz, which are specified in Section 5-4.1. 


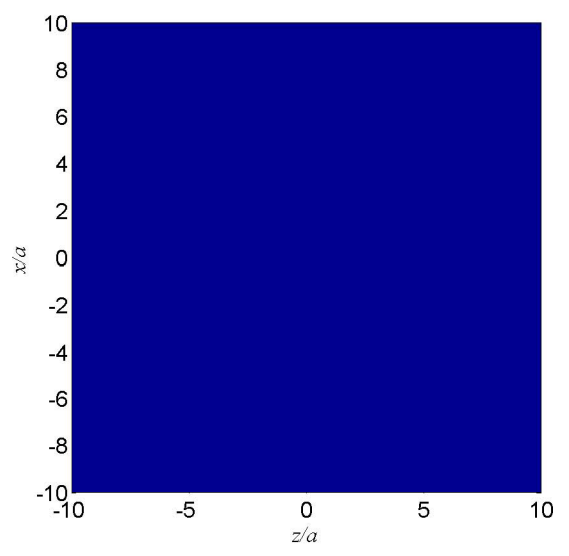

(a)

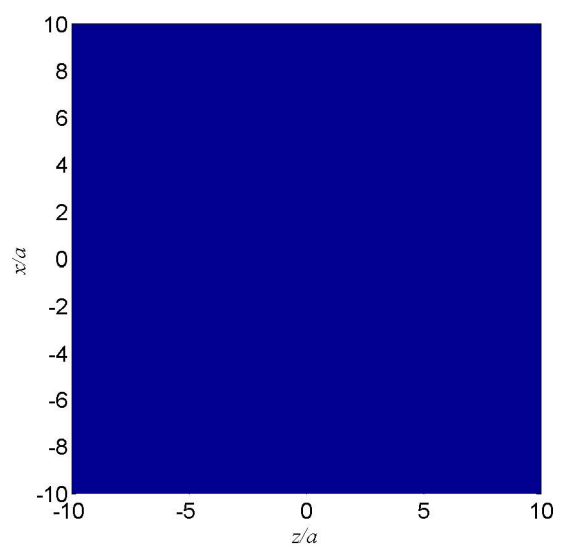

(c)

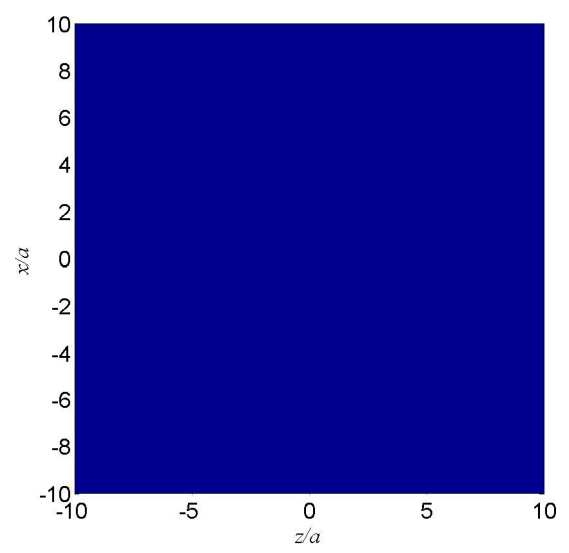

(b)

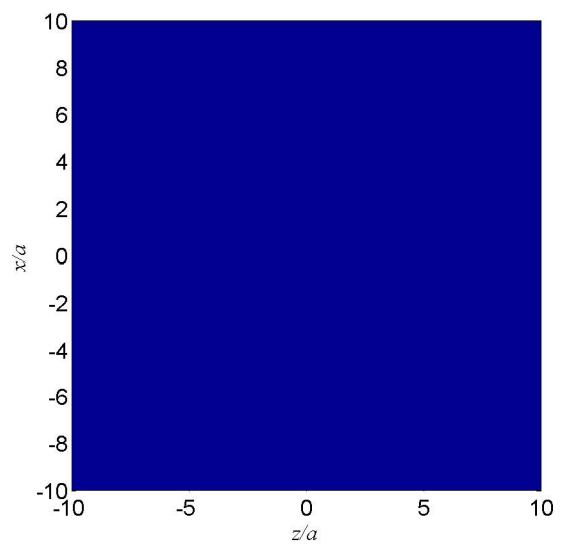

(d)

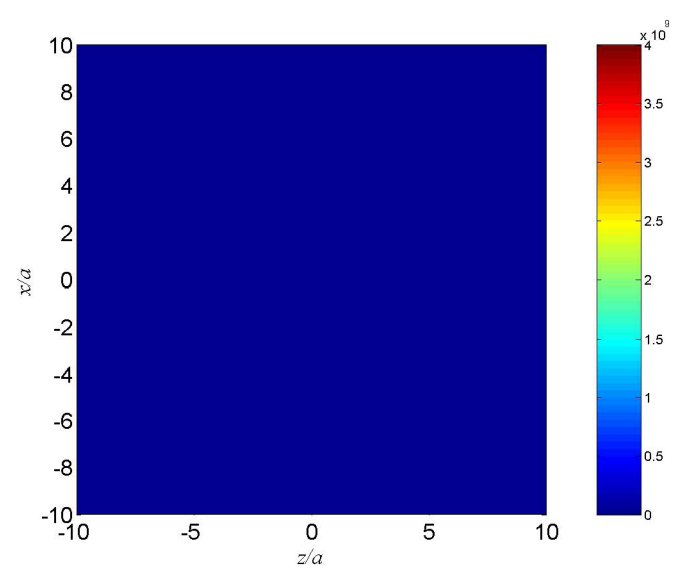

(e)

Figure 5.13: Modulus of $\sigma_{x y}$ of scattered waves at the $y=0$ plane, for the multiple scattering problem of a longitudinal plane wave $(\kappa a=0.5)$ by 8 scatterers of low-carbon steel spheres in epoxy matrix. The problem is solved in 5 approaches designated as: (a) NP, (b) P1, (c) Px, (d) Py and (c) Pz, which are specified in Section 5-4.1. 


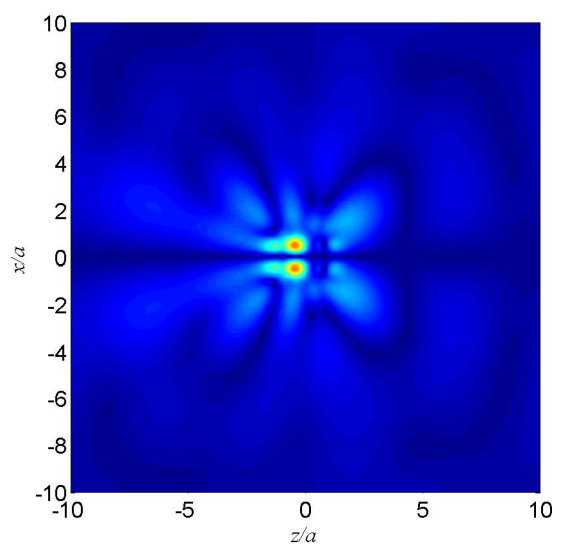

(a)

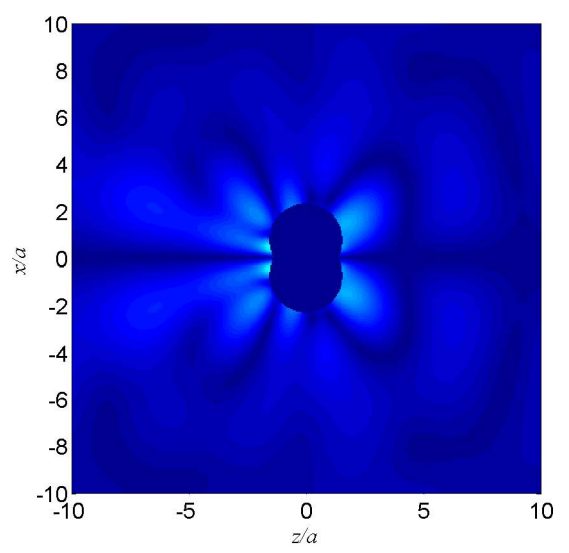

(c)

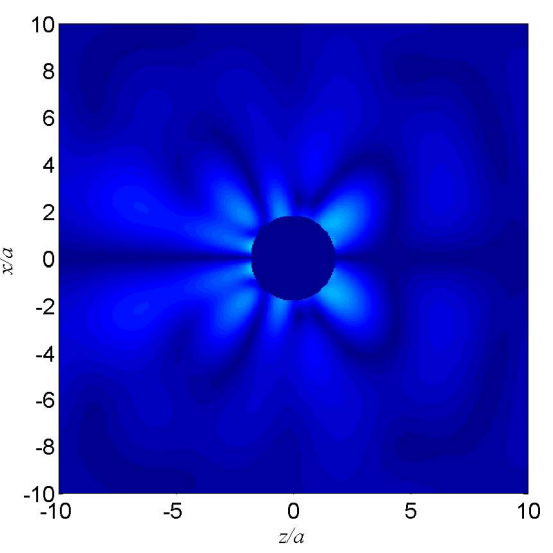

(b)

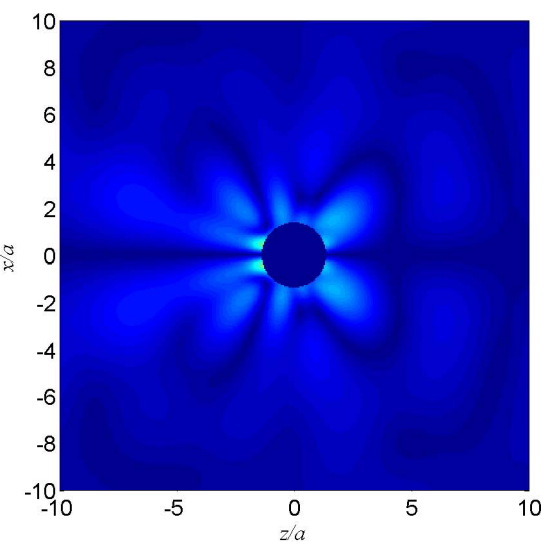

(d)

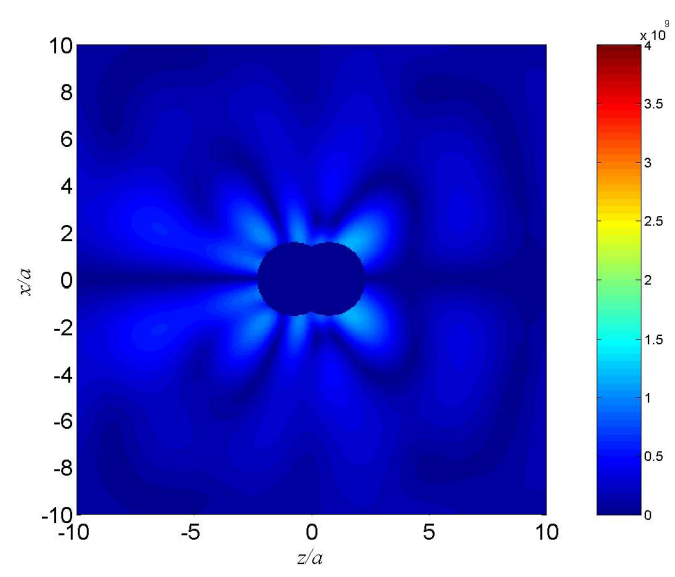

(e)

Figure 5.14: Modulus of $\sigma_{x z}$ of scattered waves at the $y=0$ plane, for the multiple scattering problem of a longitudinal plane wave $(\kappa a=0.5)$ by 8 scatterers of low-carbon steel spheres in epoxy matrix. The problem is solved in 5 approaches designated as: (a) NP, (b) P1, (c) Px, (d) Py and (c) Pz, which are specified in Section 5-4.1. 


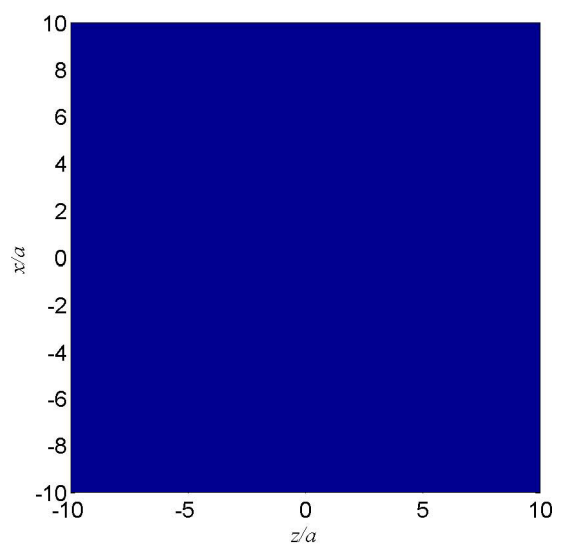

(a)

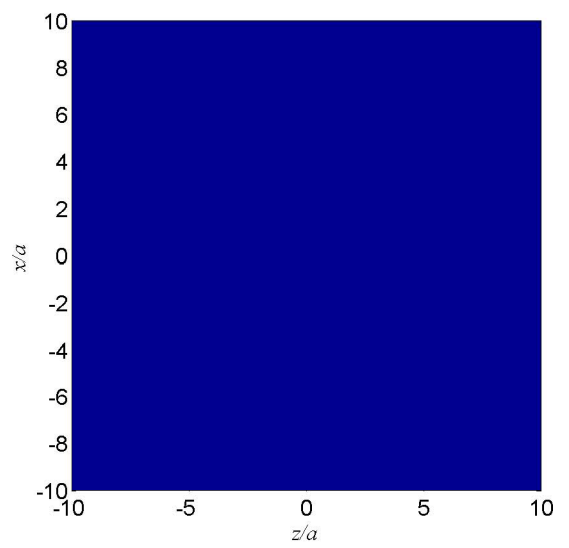

(c)

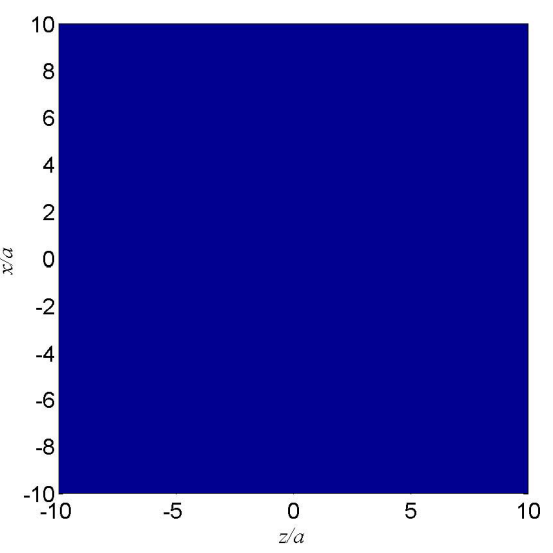

(b)

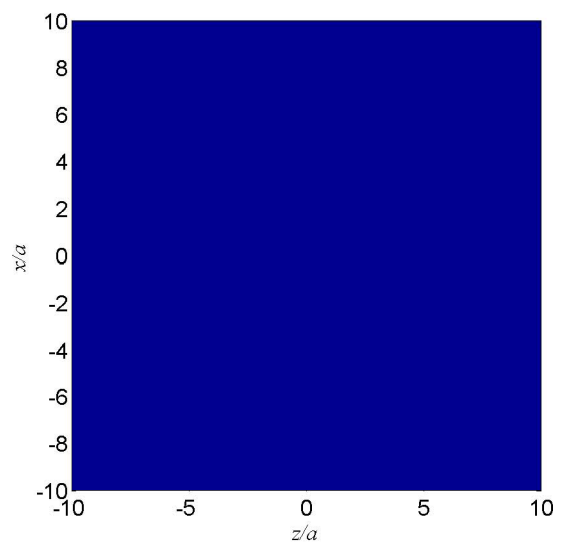

(d)

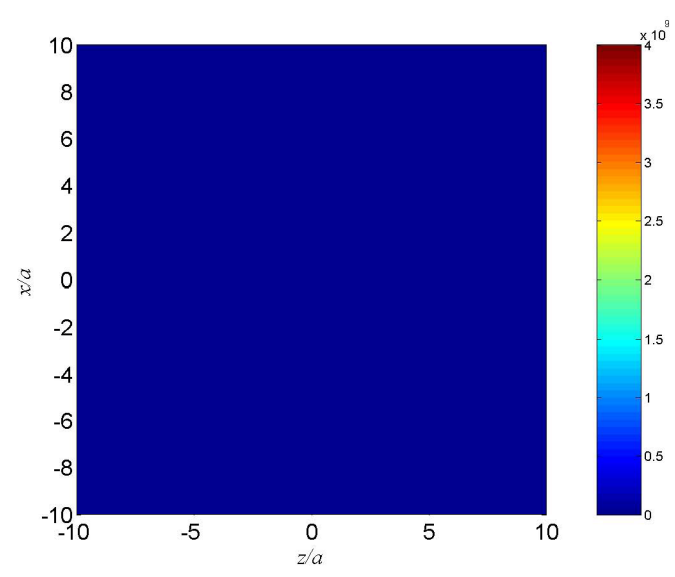

(e)

Figure 5.15: Modulus of $\sigma_{y z}$ of scattered waves at the $y=0$ plane, for the multiple scattering problem of a longitudinal plane wave $(\kappa a=0.5)$ by 8 scatterers of low-carbon steel spheres in epoxy matrix. The problem is solved in 5 approaches designated as: (a) NP, (b) P1, (c) Px, (d) Py and (c) Pz, which are specified in Section 5-4.1. 
Table 5.8: Modulus of $\sigma_{z z}$ of scattered waves from five computational approaches

\begin{tabular}{|c|c|c|c|}
\hline & Position-1 & Position-2 & Position-3 \\
\cline { 2 - 4 } & $x=-3.35, y=0, z=-9.05$ & $x=3.5, y=0, z=3.5$ & $x=5.8, y=0, z=-6.9$ \\
\hline NP & $6.062854 \times 10^{7}$ & $7.837257 \times 10^{8}$ & $2.756721 \times 10^{8}$ \\
\hline P1 & $6.062854 \times 10^{7}$ & $7.837257 \times 10^{8}$ & $2.756721 \times 10^{8}$ \\
\hline Px & $5.119138 \times 10^{7}$ & $7.751203 \times 10^{8}$ & $2.891229 \times 10^{8}$ \\
\hline $\mathrm{Py}$ & $5.938014 \times 10^{7}$ & $7.757372 \times 10^{8}$ & $2.749133 \times 10^{8}$ \\
\hline $\mathrm{Pz}$ & $4.320920 \times 10^{7}$ & $7.782864 \times 10^{8}$ & $2.785462 \times 10^{8}$ \\
\hline
\end{tabular}

Table 5.9: Modulus of $\sigma_{x y}$ of scattered waves from five computational approaches

\begin{tabular}{|c|c|c|c|}
\hline & Position-1 & Position-2 & Position-3 \\
\cline { 2 - 4 } & $x=-3.35, y=0, z=-9.05$ & $x=3.5, y=0, z=3.5$ & $x=5.8, y=0, z=-6.9$ \\
\hline $\mathrm{NP}$ & $4.318454 \times 10^{2}$ & $2.383794 \times 10^{3}$ & $6.107322 \times 10^{2}$ \\
\hline $\mathrm{P} 1$ & $4.270854 \times 10^{2}$ & $2.393021 \times 10^{3}$ & $6.124678 \times 10^{2}$ \\
\hline $\mathrm{Px}$ & $4.754982 \times 10^{5}$ & $.059688 \times 10^{6}$ & $4.114855 \times 10^{5}$ \\
\hline $\mathrm{Py}$ & $3.501932 \times 10^{5}$ & $9.197228 \times 10^{5}$ & $5.474268 \times 10^{5}$ \\
\hline $\mathrm{Pz}$ & $2.264993 \times 10^{5}$ & $3.483181 \times 10^{5}$ & $2.402719 \times 10^{5}$ \\
\hline
\end{tabular}

system can be obtained from corresponding $\sigma_{r r}, \sigma_{r \theta}, \sigma_{r \varphi}, \sigma_{\theta \theta}, \sigma_{\theta \varphi}$, and $\sigma_{\varphi \varphi}$ using Eq. (3-87). The stresses $\sigma_{x x}, \sigma_{y y}, \sigma_{z z}, \sigma_{x y}, \sigma_{x z}$, and $\sigma_{y z}$ in each local coordinate system can directly be used as stresses $\sigma_{x x}, \sigma_{y y}, \sigma_{z z}, \sigma_{x y}, \sigma_{x z}$, and $\sigma_{y z}$ in the global coordinate system. At each position, summation of $\sigma_{x x}$ due to the scattered waves of two abstract scatterers is the $\sigma_{x x}$ due to the total scattered waves. Other stress components are obtained in the same way. For other approaches, the processes of computing stresses $\sigma_{x x}, \sigma_{y y}, \sigma_{z z}, \sigma_{x y}, \sigma_{x z}$, and $\sigma_{y z}$ are similar.

Wave information inside an abstract scatterer is lost and wave fields inside an abstract scatterer are set to zero when the scatterer polymerization method is used. No information is lost in the "NP" approach, where actual scatterers are not assembled as abstract scatterer(s). Similar to displacements, at each position where wave information is not lost, the five approaches produce similar results with errors on the 
Table 5.10: Modulus of $\sigma_{x z}$ of scattered waves from five computational approaches

\begin{tabular}{|c|c|c|c|}
\hline & Position-1 & Position-2 & Position-3 \\
\cline { 2 - 4 } & $x=-3.35, y=0, z=-9.05$ & $x=3.5, y=0, z=3.5$ & $x=5.8, y=0, z=-6.9$ \\
\hline $\mathrm{NP}$ & $1.555784 \times 10^{8}$ & $4.311858 \times 10^{8}$ & $1.976730 \times 10^{8}$ \\
\hline $\mathrm{P} 1$ & $1.555784 \times 10^{8}$ & $4.311858 \times 10^{8}$ & $1.976700 \times 10^{8}$ \\
\hline $\mathrm{Px}$ & $1.503968 \times 10^{8}$ & $4.338592 \times 10^{8}$ & $1.992560 \times 10^{8}$ \\
\hline $\mathrm{Py}$ & $1.510910 \times 10^{8}$ & $4.103324 \times 10^{8}$ & $1.910052 \times 10^{8}$ \\
\hline $\mathrm{Pz}$ & $1.626996 \times 10^{8}$ & $4.304978 \times 10^{8}$ & $2.016705 \times 10^{8}$ \\
\hline
\end{tabular}

Table 5.11: Modulus of $\sigma_{y z}$ of scattered waves from five computational approaches

\begin{tabular}{|c|c|c|c|}
\hline & Position-1 & Position-2 & Position-3 \\
\cline { 2 - 4 } & $x=-3.35, y=0, z=-9.05$ & $x=3.5, y=0, z=3.5$ & $x=5.8, y=0, z=-6.9$ \\
\hline NP & $6.010242 \times 10^{2}$ & $1.493683 \times 10^{3}$ & $4.043581 \times 10^{2}$ \\
\hline P1 & $5.987692 \times 10^{2}$ & $1.502433 \times 10^{3}$ & $4.050177 \times 10^{2}$ \\
\hline Px & $1.105292 \times 10^{5}$ & $1.554891 \times 10^{6}$ & $4.550483 \times 10^{5}$ \\
\hline $\mathrm{Py}$ & $1.693275 \times 10^{5}$ & $4.569725 \times 10^{5}$ & $3.373687 \times 10^{5}$ \\
\hline $\mathrm{Pz}$ & $8.423181 \times 10^{4}$ & $3.077861 \times 10^{5}$ & $1.898931 \times 10^{5}$ \\
\hline
\end{tabular}

order of $10^{-2}$ or less, which can be supported by numerical results.

The energy flux across a closed spherical surface enclosing all eight scatterers should be zero, when there is no energy dissipation or generation inside the closed surface. Following Eq. (2-149), the normalized average energy flux across the spherical surface of $R=3.7 a$ (centered at the origin of the global coordinate system) is computed for the scattering problem in this section. The results from the five approaches are listed in Table 5.12. In computation of using Eq. (2-149), displacements and stresses at each position on the spherical surface are the summation of contributions of incident and scattered waves. The analytical expressions in Eq. (2-136) are used for the incident wave. For scattered waves of each scatterer, displacements and stresses in each local spherical coordinate system are computed using Eqs. (2-43a) through (2-44f), then displacements and stresses in each local Cartesian coordinate 
system are obtained using transformation Eqs. (D-8a) and (D-10a), and then displacements and stresses in the global spherical coordinate system are obtained using transformation Eqs. (D-8b) and (D-10b). In Table 5.12, the normalized average energy flux from five approaches approximately equal to zero with errors on the order of $10^{-4}$ or less. Therefore, energy conservation is satisfied with the errors on the order of $10^{-4}$ or less.

Table 5.12: Comparison of normalized average energy flux(NAEF) from five approaches

\begin{tabular}{|c|c|c|}
\hline$\dot{e}_{i n c}(\mathrm{~J} / \mathrm{s})$ & Approach & NAEF \\
\hline \multirow{6}{*}{$6.442614 \times 10^{12}$} & $\mathrm{NP}$ & $5.664487 \times 10^{-05}$ \\
\cline { 2 - 3 } & $\mathrm{P} 1$ & $1.570881 \times 10^{-10}$ \\
\cline { 2 - 3 } & $\mathrm{Px}$ & $1.546582 \times 10^{-04}$ \\
\cline { 2 - 3 } & $\mathrm{Py}$ & $1.909286 \times 10^{-04}$ \\
\cline { 2 - 3 } & $\mathrm{Pz}$ & $1.496299 \times 10^{-05}$ \\
\hline
\end{tabular}

\section{5-5 Concluding Remarks}

The scatterer polymerization method assembles a group of scatterers as an abstract scatterers, so that the number of actual scatterers is reduced to a lesser number of abstract scatterers in computation. In this chapter, the multiple scattering solution is reformulated by using the scatterer polymerization method. The reformulated solution and implementation are verified by solving a multiple scattering problem of eight low-carbon steel scatterers in an epoxy matrix.

The truncation criterion has not been discussed yet in this chapter. When all eight scatterers in Fig. 5.5 are assembled as one abstract scatterer, the truncation error of using the scatterer polymerization method should be the same as that of using the multiple scattering solution. Because no extra computation is involved. When eight 
scatterers are separated and assembled as two abstract scatterers, translation between the coordinate systems of two abstract scatterers causes additional errors. In other words, the truncation error increases when the scatterer polymerization method is used to solve the multiple scattering problem with the same truncation number. 


\section{Chapter 6}

\section{Application Example}

\section{6-1 Introduction}

As shown in the previous chapter, the scatterer polymerization method is based on the analytically exact solution, it can be used to reduce the number of actual scatterers to a lesser number of abstract scatterers, and it is extended to a three-dimensional multiple scattering problem. An application example of using the scatterer polymerization method is given in this chapter. In this example, the band gap formation process for elastic wave propagation in a cubical lattice arrangement of spherical scatterers is observed through a series of numerical simulations.

In this chapter, the numerical procedure of this numerical application example is first described. Then, the relationship among the truncation error, the truncation number and the wave number is explored. Thereafter, simulation results are shown. 


\section{6-2 Numerical Procedure}

There are a total of sixty-four scatterers in a cubical lattice arrangement in the application example. The scatterers are identical spheres of low-carbon steel and of unity radius. The host medium is epoxy. Material properties are listed in Table 5.2. A longitudinal plane incident waves propagate along positive $Z$-axis direction with displacement of unity amplitude, whose analytical expressions are shown in Section 26.1.

\section{6-2.1 Sixteen Scatterers in A Layer}

The sixteen scatterers are in square arrangement in the $z=0$ plane, as shown in Fig. 6.1. The square arrangement is symmetrical about $X$-axis and $Y$-axis. The actual scatterers 1,2, 5 and 6 are assembled as an abstract scatterer; the actual scatterers 3, 4, 7 and 8 are assembled as an abstract scatterer; the scatterers 9, 10, 13, and 14 are assembled as an abstract scatterer; and the actual scatterers 11, 12, 15 and 16 are assembled as an abstract scatterer. Three-dimensional and two-dimensional views are shown in Fig. 6.1. In Fig. 6.1(a), each sphere in light green color represents an abstract scatterer, each sphere in green color represents an actual scatterer, and an abstract scatterer encloses four actual scatterers. In Fig. 6.1(b), each dashed circle represents the territory of an abstract scatterer and each solid circle represents an actual scatterer. There are four identical abstract scatterers in the $z=0$ plane. Then, the multiple scattering problem of 16 actual scatterers becomes a multiple scattering problem of 4 abstract scatterers. 


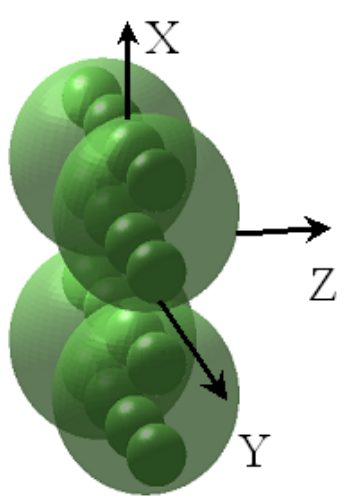

(a)

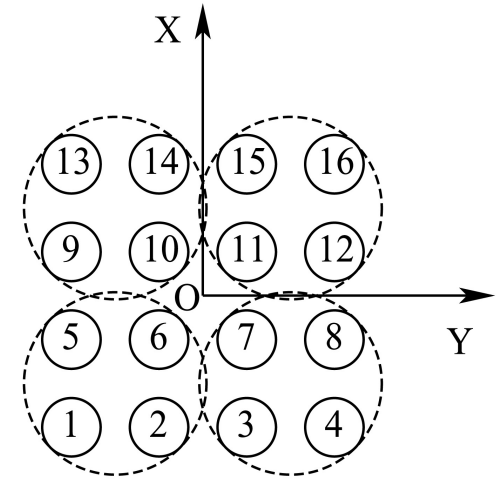

(b)

Figure 6.1: Sixteen actual scatterers in a square arrangement in a layer. (a) Threedimensional view: each sphere in light green color represents an abstract scatterer, each sphere in green color represents an actual scatterer, and an abstract scatterer encloses four actual scatterers. (b) Two-dimensional view: each dashed circle represents an abstract scatterer and each solid circle represents an actual scatterer.

\section{6-2.2 Thirty-Two Scatterers in Two Layers}

The thirty-two scatterers are in a cubic lattice arrangement, where sixteen scatterers are in the $z=0$ plane and another sixteen scatterers are in a plane with negative $z$-axis, as shown in Fig. 6.2. The sixteen scatterers in the $z=0$ plane are in exactly the same arrangement as that in Section 6-2.1. The other sixteen scatterers are in the similar arrangement as the sixteen scatterers in the $z=0$ plane, so that only sixteen actual scatterers are shown in the two-dimensional view in Fig. 6.2(b).

As shown in Fig.6.2(a), eight actual scatterers are assembled as an abstract scatterer and four identical abstract scatterers are obtained. Each spherical surface in light green color represents an abstract scatterer in Fig. 6.2(a) and each dashed circle represents an abstract scatterer in Fig. 6.2(b). The center of each abstract scatterer in at the geometric center of the involved actual scatterers. The centers of four abstract scatterers are in the plane at the middle of the two layers. Then, the multiple scattering problem of thirty-two actual scatterers becomes a multiple scattering problem 
of four abstract scatterers.

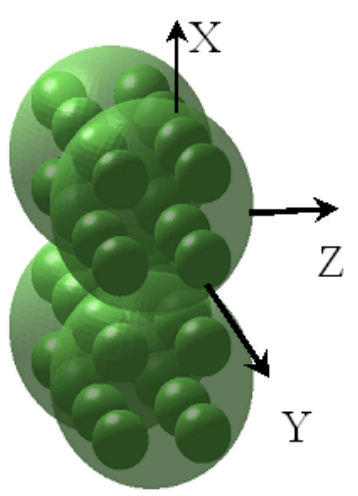

(a)

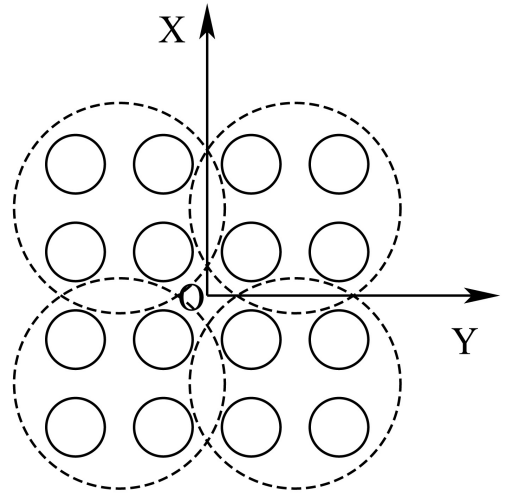

(b)

Figure 6.2: Thirty-two actual scatterers in a cubic arrangement. (a) Threedimensional view: each sphere in light green color represents an abstract scatterer, each sphere in green color represents an actual scatterer, and an abstract scatterer encloses eight actual scatterers. (b) Two-dimensional view: each dashed circle represents an abstract scatterer and each solid circle represents an actual scatterer.

\section{6-2.3 Sixty-Four Scatterers in Four Layers}

The sixty-four scatterers are in a cubic lattice arrangement, with thirty-two scatterers in the same arrangement as in Fig. 6.2, and another thirty-two scatterers in the similar arrangement, as shown in Fig. 6.3. Therefore, only sixteen actual scatterers are shown in the two-dimensional view in Fig. 6.3(b).

As shown in Fig.6.2(a), eight actual scatterers are assembled as an abstract scatterer and four identical abstract scatterers are obtained. Each spherical surface in light green color represents an abstract scatterer in Fig. 6.2(a) and each dashed circle represents an abstract scatterer in Fig. 6.2(b). The center of each abstract scatterer is in the geometrical center of the involved actual scatterers. Then, the multiple scattering problem of sixty-four actual scatterers becomes a multiple scattering problem of eight abstract scatterers. 


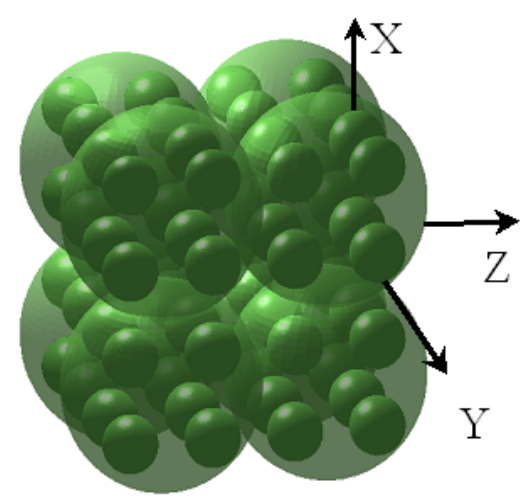

(a)

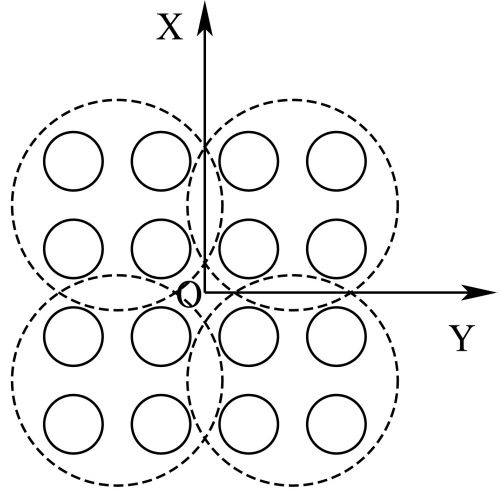

(b)

Figure 6.3: Sixty-four actual scatterers in a cubic arrangement. (a) Threedimensional view: each sphere in light green color represents an abstract scatterer, each sphere in green color represents an actual scatterer, and an abstract scatterer encloses eight actual scatterers. (b) Two-dimensional view: each dashed circle represents an abstract scatterer and each solid circle represents an actual scatterer.

Along the propagation direction of the incident wave, scatterer arrangements in Sections 6-2.1 through 6-2.3 are viewed as comprising layers of scatterers, within which scatterers form a square grid. Starting from one layer, which is in the $z=0$ plane, and by increasing the number of layers along negative $Z$ direction, multiple scattering problems are set up. For each multiple scattering problem, the scatterer polymerization method is used to reduce the number of actual scatterers to a lesser number of abstract scatterers. Each multiple scattering problem of actual scatterers becomes a multiple scattering problem of abstract scatterers.

After solving a multiple scattering problem of abstract scatterers, scattered wave expansion coefficients of the abstract scatterers are obtained. By using Eqs. (3-85a) through (3-85i), displacements and stresses due to the scattered waves in each local spherical coordinate system can be computed. Then, displacements and stresses in each local Cartesian coordinate system can be obtained from corresponding wave fields in local spherical coordinate system using Eq. (3-87). The displacements and 
stresses in each local Cartesian coordinate system can directly be used as those in the global Cartesian coordinate system. At each position, summation of $\sigma_{x x}$ due to the scattered waves of two abstract scatterers is the $\sigma_{x x}$ due to the total scattered waves. Other wave field components are obtained in the same way.

The displacements and stresses due to the longitudinal plane incident waves, which are used in this chapter, are expressed in Eqs. (2-136) through (2-138). As the system is linear, at a position outside the territories of all abstract scatterers, displacements and stresses of total waves are summations of contributions of incident waves and scattered waves.

\section{6-3 Truncation Number and the Error due to Co- ordinate Transformation}

From the polymerization process in the numerical procedure in Section 6-2, it is known that wave field transformation occurs not only between local coordinate systems of two actual scatterers, but also between local coordinate systems of any two abstract scatterers. The relation of the truncation number and the error during wave field transformation needs to be found prior to solving the multiple scattering

problem. Plane longitudinal waves, propagating along $Z$-axis with a displacement of unity amplitude, are used as the incident waves in this section. Analytical expressions of the incident wave can be found in Eqs. (2-136) through (2-138).

Figure 6.4, the same as Fig. 3.3, is referred in this section. For wave field transformation between the two local coordinate systems in Fig. 6.4, scattered waves of the scatterer centered at $O$ are obtained by solving the single scattering problem. The scattered waves can be re-expressed as waves in another local coordinate system with its origin at $O^{\prime}$ and the expansion coefficients after the transformation can be 
obtained using Eq. (3-47). The scattered wave fields at positions on the spherical surface of the scatterer centered at $O^{\prime}$ can be computed in two local coordinate systems. The scattered wave fields obtained in the two local coordinate systems are compared, in the way as follows, to find how many significant figures remain after the transformation.

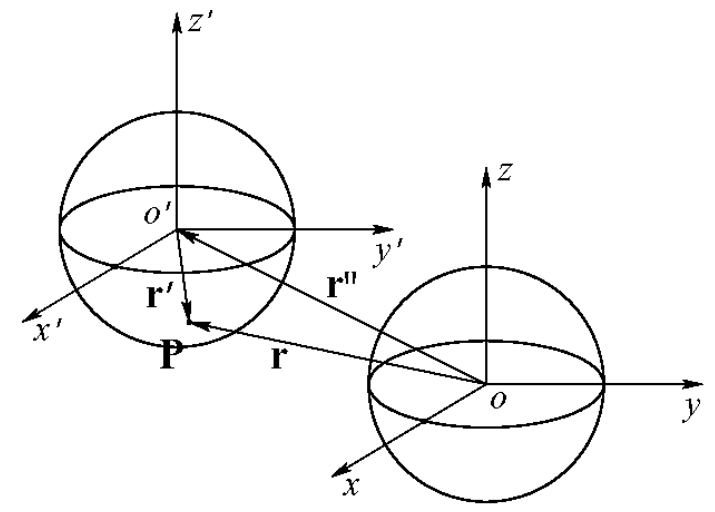

Figure 6.4: Vectors $\boldsymbol{r}=\boldsymbol{r}^{\prime}+\boldsymbol{r}^{\prime \prime}$ for addition theorem

When the two scatterers in Fig. 6.4 are actual scatterers used in Section 6-2, the spacing between them is chosen as three times scatterer's radius as an example. Let $R$ be the radius of the actual scatterers $\left(R=1\right.$ here). Then, $r^{\prime}=R$ and $r^{\prime \prime} \geq 3 R$. Ten positions in the local coordinate system, as shown in Table 4.1, are chosen for computing wave fields. For a normalized longitudinal wave number $\kappa r^{\prime}=1.5$, the relation among the truncation number, the number of significant figures, the ratio $r^{\prime \prime} / r^{\prime}$ and three orientations of vector $\mathbf{r}^{\prime \prime}$ during coordinate transformation are shown in Fig. 6.5, in which the number of significant figures is the smallest number of significant figures among all displacement and stress components due to the scattered wave at all selected positions. Figure 6.5 shows that transformation along $Z$-axis direction results in lesser significant figures than transformation along $X$-axis or $Y$ axis direction.

When two scatterers are abstract scatterers, two cases need to be considered. 


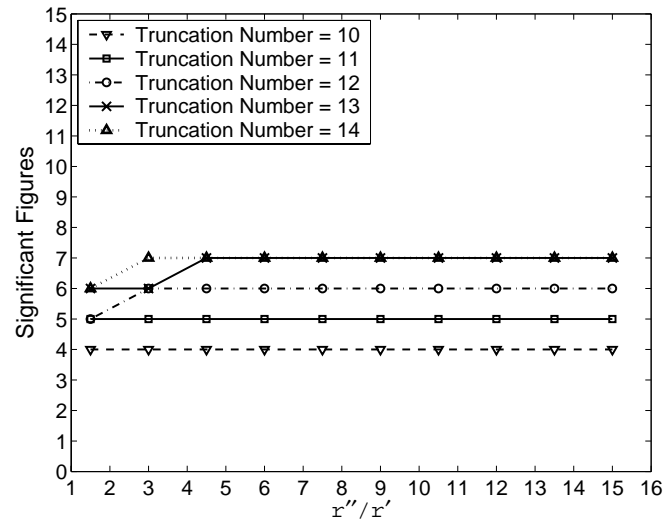

(a) Transformation along $X$ direction

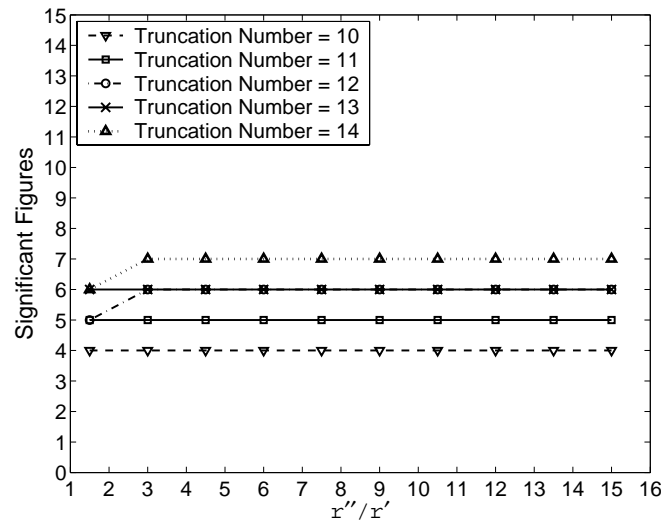

(b) Transformation along $Y$ direction

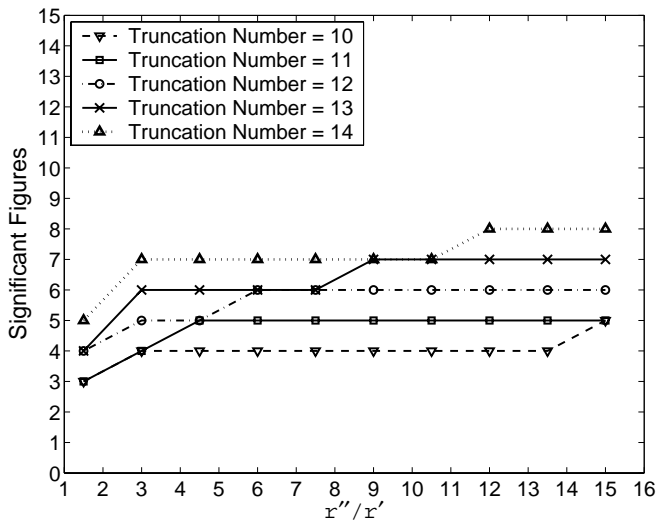

(c) Transformation along $Z$ direction

Figure 6.5: Relation of truncation number, significant figures, the ratio $r^{\prime \prime} / r^{\prime}$ and three orientations of vector $\mathbf{r}^{\prime \prime}$ during coordinate transformation. The wave number is $\kappa r^{\prime}=1.5$. 
For one layer of sixteen scatterers in Fig. 6.1, the numerical procedure in Section 62 implies that the smallest distance between centers of two abstract scatterers is six times the radius of actual scatterers, and the radius of abstract scatterers is $\left(1+\frac{3 \sqrt{2}}{2}\right) R$. In other words, $r^{\prime}=\left(1+\frac{3 \sqrt{2}}{2}\right) R$ and $r^{\prime \prime} \geq 6 R$ in this case. For two layers or four layers of scatterers, the numerical procedure in Section 6-2 implies that the smallest distance between centers of two abstract scatterers is six times the radius of actual scatterers, and the radius of abstract scatterers is $\left(1+\frac{3 \sqrt{3}}{2}\right) R$. In other words, $r^{\prime}=\left(1+\frac{3 \sqrt{3}}{2}\right) R$ and $r^{\prime \prime} \geq 6 R$ in this case.

Generally, the number of significant figures of the scattered wave fields increases when the ratio $r^{\prime \prime} / r^{\prime}$ increases for the same truncation number, which is concluded from the comparison in Figs. 4.5 through 4.7 and which agrees with the discussion about the truncation criterion in Section 2-6.2. Therefore, relations among the truncation number, the number of significant figures and the distance for coordinate transformation are explored in the case of four layers of scatterers, rather than in the case of one layer of scatterers. Since transformation along $Z$-axis direction results in lesser significant figures than transformation along $X$-axis or $Y$-axis direction (Fig. 6.5), the transformation along $Z$-axis direction is explored.

When $r^{\prime}=\left(1+\frac{3 \sqrt{3}}{2}\right) \approx 3.598, r^{\prime \prime} \geq 6 R=6$ and $\mathbf{r}^{\prime \prime}$ is along $Z$-axis direction, ten positions in the local coordinate system, as shown in Table 6.1, are chosen for computing displacements and stresses due to the scattered waves. As stated at beginning in this section, scattered waves of the scatterer centered at $O$ are obtained by solving the single scattering problem. The scattered waves can be re-expressed as waves in the local coordinate system with its origin at $O^{\prime}$ and the expansion coefficients after the transformation can be obtained using Eq. (3-47). The scattered wave fields at the ten positions (see Table 6.1) on the spherical surface of the scatterer centered at $O^{\prime}$ can be computed in two local coordinate systems. The scattered wave fields obtained 
in the two local coordinate systems are compared.

Table 6.1: Coordinates of Ten Positions in the Local System.

\begin{tabular}{|c|c|c|c|c|}
\hline Position & $\begin{array}{c}\text { Spherical coordinates } \\
r^{\prime}, \theta^{\prime} \text { (radians), } \varphi^{\prime} \text { (radians) }\end{array}$ & \multicolumn{3}{|c|}{$\begin{array}{c}\text { Cartesian coordinates } \\
x^{\prime}, y^{\prime}, z^{\prime} \\
\end{array}$} \\
\hline 1 & $3.598,2.696872,1.884956$ & -0.478322, & 1.472122 & -3.248026 \\
\hline 2 & $3.598,1.968278,3.769911$ & -2.683910 , & -1.949976 & -1.392779 \\
\hline 3 & $3.598,1.173314,5.654867$ & 2.683910 , & -1.949976, & 1.392779 \\
\hline 4 & $3.598,0.444721,1.256637$ & 0.478322 , & 1.472122, & 3.248026 \\
\hline 5 & $3.598,0.100000,3.141593$ & -0.359199 & 0.000000 & 3.580024 \\
\hline 6 & $3.598,2.696872,5.026548$ & 0.478322 & -1.472122 & -3.248026 \\
\hline 7 & $3.598,1.968278,0.628319$ & 2.683910, & 1.949976, & -1.392779 \\
\hline 8 & $3.598,1.173314,2.513274$ & -2.683910 , & 1.949976, & 1.392779 \\
\hline 9 & $3.598,0.444721,4.398230$ & -0.478322 , & -1.472122 & 3.248026 \\
\hline 10 & $3.598,0.100000,6.283185$ & 0.359199, & 0.000000 & 3.580024 \\
\hline
\end{tabular}

Figure 6.6 shows the relation between the normalized wave number and the significant figures for truncation number 14 and ratio $r^{\prime \prime} / r^{\prime}=1.66756\left(r^{\prime \prime}=6 R\right.$, $\left.r^{\prime}=\left(1+\frac{3 \sqrt{3}}{2}\right) R\right)$. The number of significant figures is the smallest number of significant figures among all displacement and stress components due to the scattered wave at all selected positions. The wave number is normalized by radius of actual spherical scatterer $(a=R)$. The normalized wave number $\kappa a$ is in the range from 0.025 to 1.5 . The number of significant figures is 2 or larger when $\kappa a \leq 1.475$. The number of significant figures is 1 when $\kappa a=1.5$. 


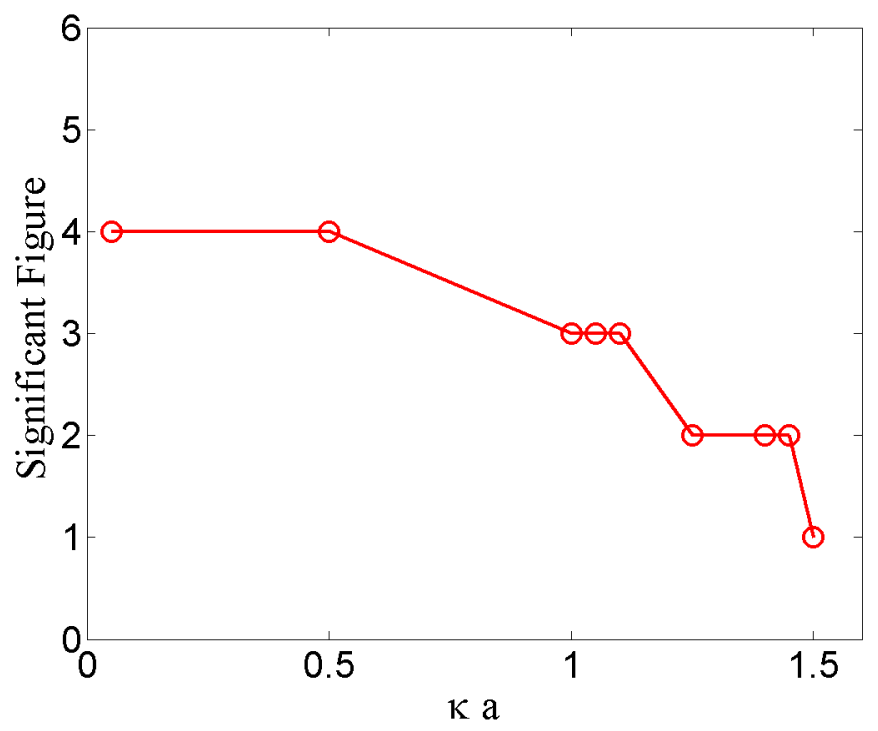

Figure 6.6: Relation of wave number and significant figure after transformation between two abstract scatterers. The truncation number is $14 . r^{\prime \prime} / r^{\prime}=1.66756$.

\section{6-4 Simulation Results}

The incident waves are plane longitudinal waves propagating along positive $Z$-axis direction, as stated in Section 6-2. The normalized wave number $\kappa a$ for the application example is taken in the range from 0.25 to 1.5. According to Fig. 6.6, the number of significant figures is 2 or larger for $\kappa a \leq 1.475$ when truncation number is 14 . Generally, when $\kappa a$ is very small, a truncation number smaller than $14 \mathrm{cab}$ be used to achieve 2 significant figures. The relation of truncation number and wave number $\kappa a$ for achieving 2 significant figures is not investigated. For simple selection, the truncation number is taken as 14 for computation. The numerical procedure of computing wave fields is stated in Section 6-2.

There are three subsections in this section: the first is to display the transmission ratio, the next is to display displacement components $u_{x}, u_{y}$ and $u_{z}$, and the last is to display stress components in Cartesian coordinate system. 


\section{6-4.1 Transmission Ratio of $u_{z}$}

Since $\left|u_{z}\right|$ of incident waves has unity value, $\left|u_{z}\right|$ of total waves at a forward position, which is the position behind the last layer of scatterers along forward direction (incident wave propagation direction), also represents the transmission ratio of $\left|u_{z}\right|$ with respect to that of the incident waves. Measurement of $\left|u_{z}\right|$ is made at positions on the $Z$-axis in order to reduce the effect of the edge of the region in which scatterers occupy, since the arrangements of scatterers are symmetrical about the $Z$-axis. An average value of $\left|u_{z}\right|$ over a small area in the $X-Y$ plane around a position on the $Z$-axis is used as the $\left|u_{z}\right|$ value at the position. In the calculation, the small area is taken as $-1.5 a \leq x \leq 1.5 a,-1.5 a \leq y \leq 1.5 a$, and centered at an interested position on the $Z$-axis. One hundred positions evenly distributed in the square area are chosen to compute the average value of $u_{z}$.

For the cases of one, two and four layers, values of $\left|u_{z}\right|$ at position $z=4 a$ are compared in Fig. 6.7(a). In Fig. 6.7(a), D, which is defined as the distance from an interested position to the plane where the last layer of scatterers (along forward direction) are located, is used instead of $z$ because $D=z$ in this example and $D$ does not depend on selection of a coordinate system. The $\kappa a$ is in the range from 0.25 to 1.5. Similarly, values of $\left|u_{z}\right|$ at positions $D=8 a, 12 a, 16 a$, and $20 a$ are compared in Figs. 6.7(b) through 6.7(e). In the calculation for Figs. 6.7, the spacing between two scatterers is three time of the scatterer's radius. In Figs. 6.7(a) through 6.7(e), when $\kappa a$ is in the range from 0.95 to $1.5,\left|u_{z}\right| \leq 0.8$, it decreases when the number of layers increases, and it generally increases when the distance increases; when $\kappa a$ is in a small range around $0.75,\left|u_{z}\right|<1$, it decreases when the number of layers increases, and it generally increases when the distance increases.

When the spacing between two scatterers changes, the transmission ratio of $\left|u_{z}\right|$ in the forward direction is affected. As an example to observe the effects of spacing 


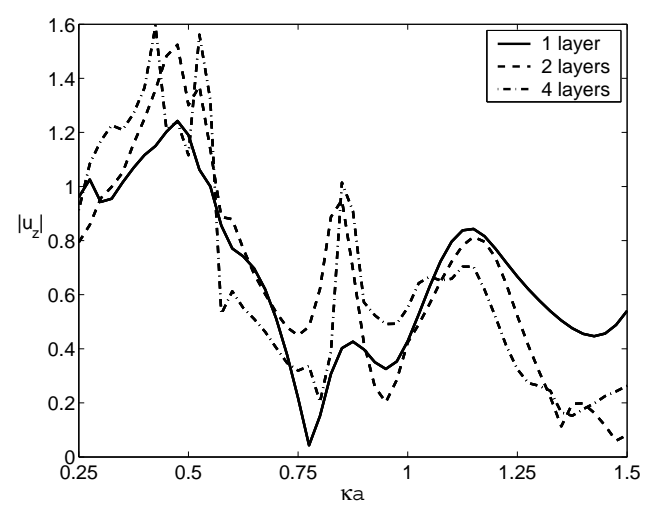

(a) At $D / a=4$

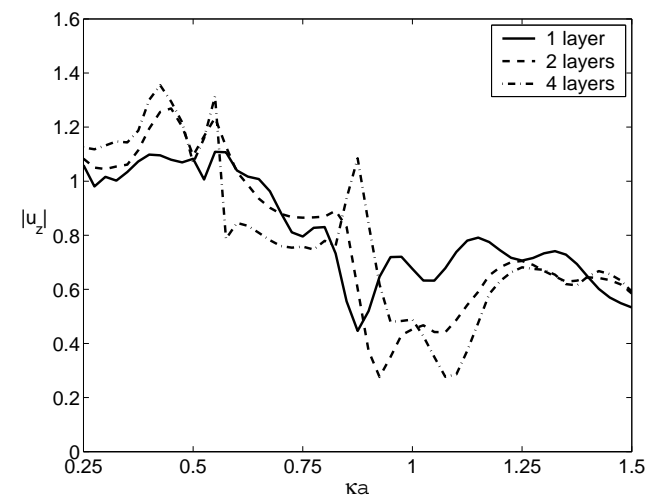

(c) At $D / a=12$

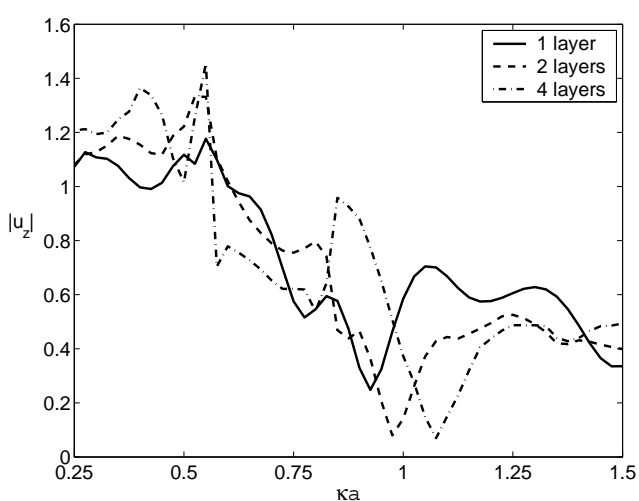

(b) At $D / a=8$

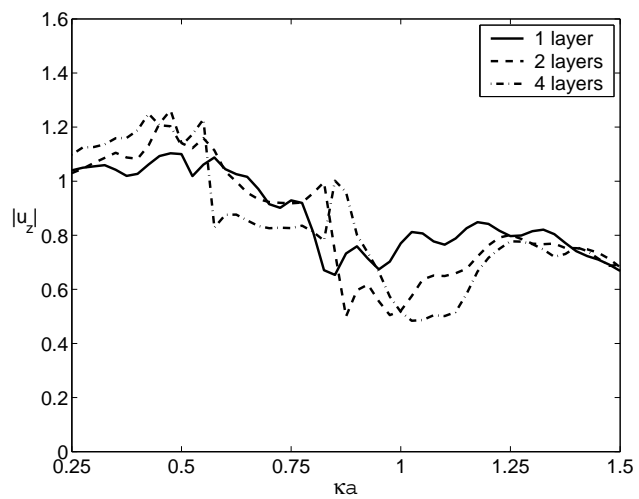

(d) At $D / a=16$

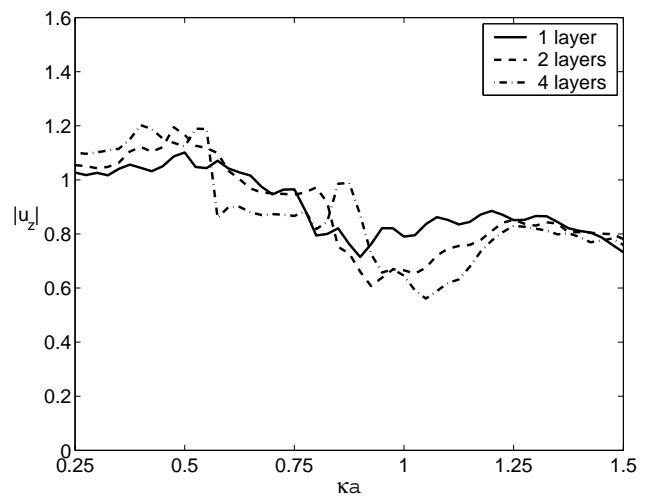

(e) At $D / a=20$

Figure 6.7: Comparison of $u_{z}$ modulus for different layers 
change, $\left|u_{z}\right|$ at each spacing $d$ and $\kappa a$ is computed for the case of thirty-two scatterers. The values of $u_{z}$ are calculated at the positions $D(=z)=6 a, 8 a, 10 a$, and $12 a$. In order to observe the effects of continuously varying parameters, $\left|u_{z}\right|$ at each spacing $d$ and $\kappa a$ is converted and assembled into a colorized spectrum map. Figure 6.8 shows the forward spectrum map for the case of thirty-two scatterers. In the small region where $d / a<2.9$ and $\kappa a<0.4$, there exists numerical unsteady for the truncation number of fourteen.

Observe Fig. 6.8(b) first because some results for $d / a=3$ have been shown in Fig. 6.7(b). For $\kappa a=1.5$, transmission ratio is less than unity when spacing ratio $d / a \geq 2.3$. For $\kappa a=1$, transmission ratio is less than unity when $d / a \geq 2.75$. But when $\kappa a$ is around 0.5 , transmission ratio is about unity or larger than unity when $3.35 \geq d / a \geq 2.25$. Possibly, constructive interferences occur between the incident wave and the scattered waves at the observation positions. When the observation position is away from the scatterers, the effect of constructive interference generally decreases, as shown in four subfigures in Fig. 6.8.

For $d / a=3$, transmission ratios in Fig. 6.8(b) are less than unity in the ranges of $0.65 \leq \kappa a \leq 0.8$ and $0.95 \leq \kappa a \leq 1.5$. The transmission ratios in the range of $0.65 \leq \kappa a \leq 0.8$ are generally larger than those in the range of $0.95 \leq \kappa a \leq 1.5$, which has also been shown in Fig. 6.7(b). For $d / a=3.3$, transmission ratios in the ranges of $0.6 \leq \kappa a \leq 0.7$ and $0.75 \leq \kappa a \leq 1.5$ are less than unity. The transmission ratios in the range of $0.6 \leq \kappa a \leq 0.7$ are generally smaller than those in the range of $0.75 \leq \kappa a \leq$ 1.5. Similar phenomena are observed for other values of $d / a$, except $d / a=2.25$. For $d / a=2.25$, the transmission ratios are about unity. When $d / a$ decreases, the limits of the range of $\kappa a$ where transmission ratios are less than unity, increase in Fig. 6.8(b). The limits for each $d / a$ appear the same for different observation positions in Fig. 6.8. There are slightly changes of values of transmission ratio at each $d / a$ and $\kappa a$ among 


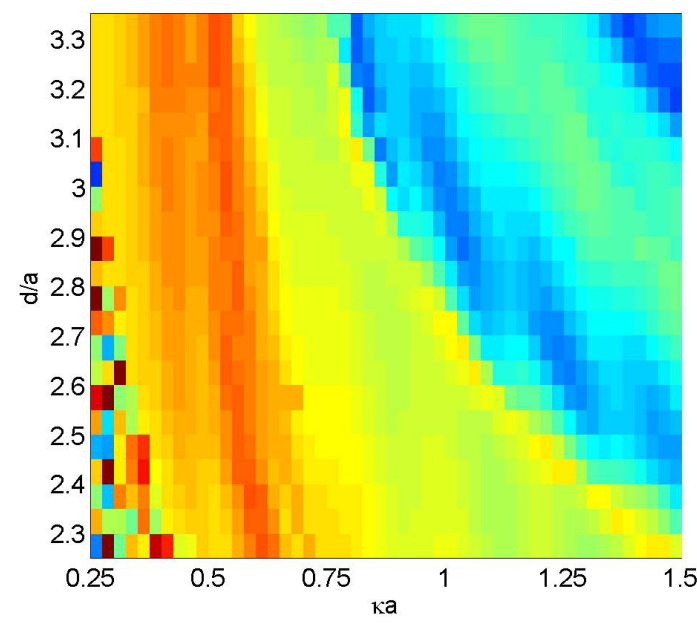

(a) At $D / a=6$

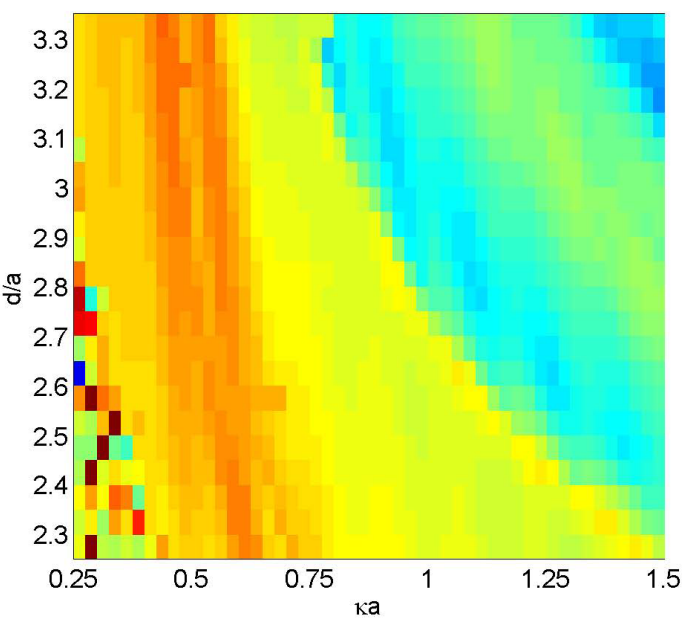

(c) At $D / a=10$

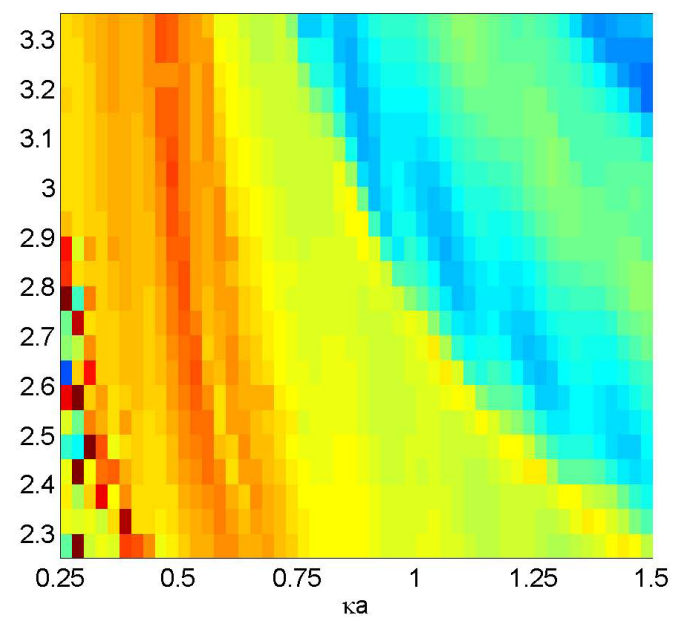

(b) At $D / a=8$

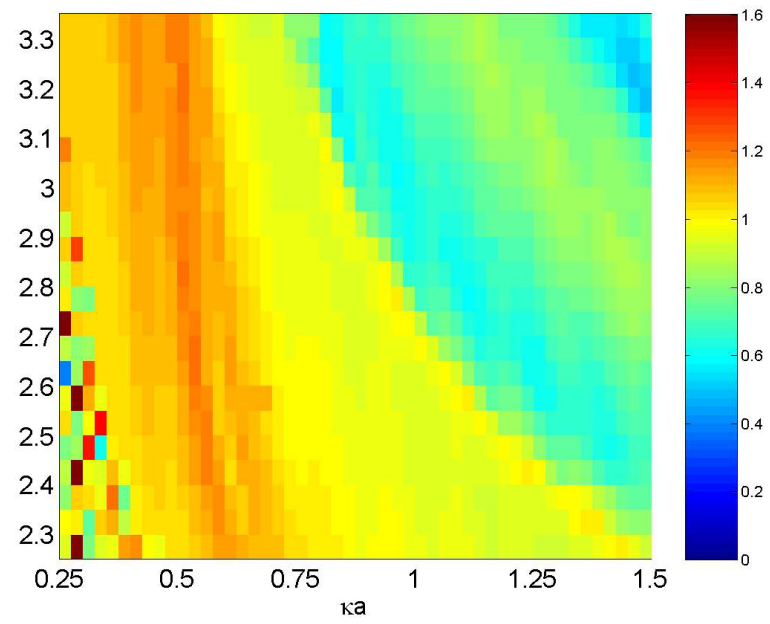

(d) At $D / a=12$

Figure 6.8: Forward spectrum map

(The discrete texture is due to numerical steps.) 
these subfigures. When the observation position is away from the scatterers, values of transmission ratio at each $d / a$ and $\kappa a$ slightly approach to unity.

\section{6-4.2 Displacements for Different Number of Layers}

In this section, displacement components in Cartesian coordinate system in the $y=0$ plane are illustrated as a part of the simulation results. In Fig. 6.9, the geometric relation of scatterer arrangement and the $y=0$ plane is shown. In the calculation, the spacing between two scatterers is three time of the scatterer's radius. The scatterers located in the $z=0$ plane (Section 6-2.1) are shown and the incident wave propagates along $Z$-axis with displacement $u_{z}$. According to the displacements due to the incident waves in Eqs. (2-138a) and (2-138e), $u_{x}^{i n c}$ and $u_{y}^{\text {inc }}$ are zero, and $u_{z}^{i n c}=e^{-\hat{\imath} \kappa z}$.

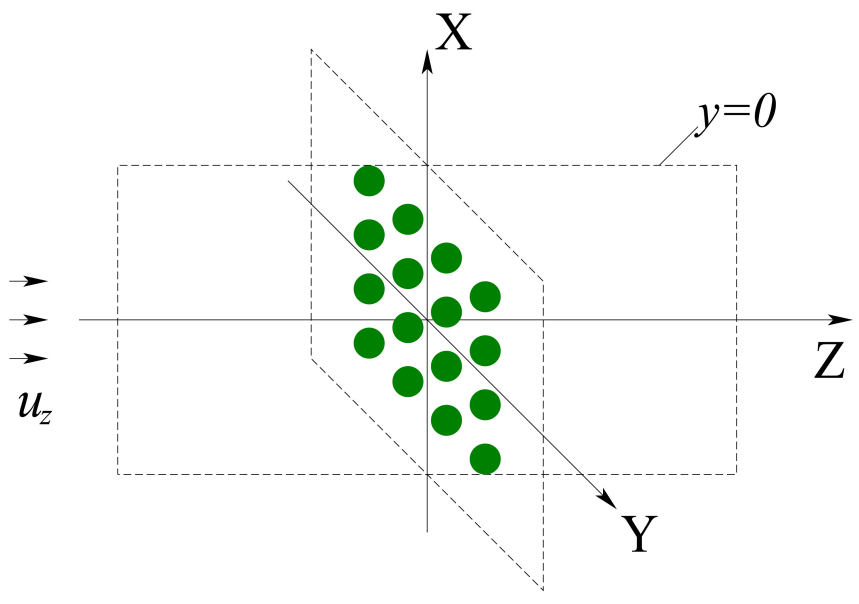

Figure 6.9: Geometric relation of scatterer arrangement and the $y=0$ plane. The scatterers located in the $z=0$ plane (Section 6-2.1) are shown. The incident wave propagates along $Z$-axis with displacement $u_{z}$.

By checking numerical values of $u_{y}$ in this application example, the displacement component $u_{y}$ in the $y=0$ plane is on the order of $10^{-5}$ or smaller. For example, modulus of $u_{y}$ in the $y=0$ plane when $\kappa a=1.35$ and there are sixty-four scatterers 
is shown in Fig. 6.10. The values of $\left|u_{y}\right|$ in Fig. 6.10 are smaller than $3 \times 10^{-5}$, which is approximately zero in comparison with unity value of $\left|u_{z}^{\text {inc }}\right|$. Therefore, the displacement component $u_{y}$ in the $y=0$ plane for other cases is not displayed.

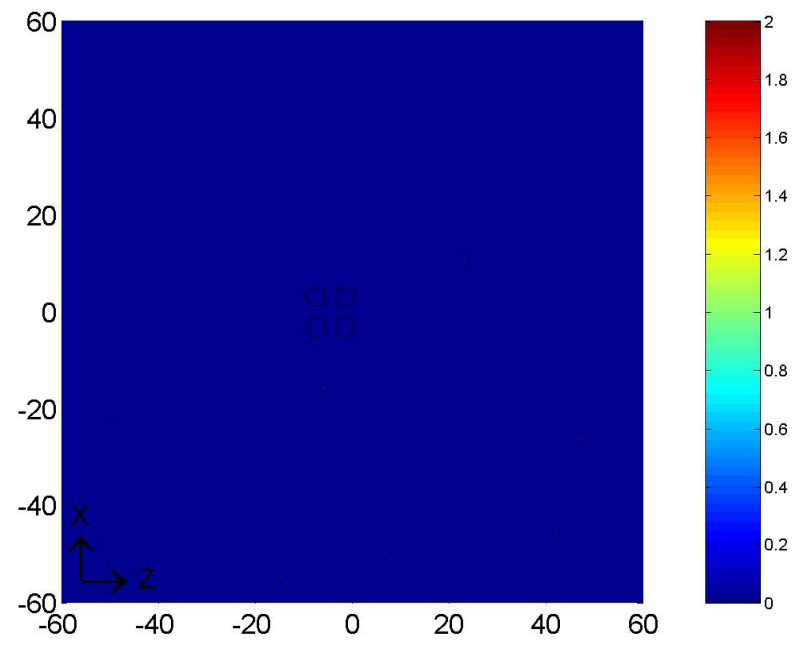

Figure 6.10: modulus of $u_{y}$ in the $y=0$ plane when $\kappa a=1.35$ and there are sixty-four scatterers. The values in this figure are smaller than $3 \times 10^{-5}$.

Figures are arranged in the following series. The displacement component $u_{z}$ is displayed before $u_{x}$. For the case of one layer, modulus of $u_{z}$ due to the total waves is displayed first and followed by real part of $u_{z}$ due to the total waves, modulus of $u_{z}$ due to the scattered waves, and the real part of $u_{z}$ due to the scattered waves; modulus of $u_{x}$ due to the total waves is displayed first and followed by real part of $u_{x}$ due to the total waves, modulus of $u_{x}$ due to the scattered waves, and the real part of $u_{x}$ due to the scattered waves. After $u_{z}$ and $u_{x}$ are displayed for the case of one layer, figures of $u_{z}$ and $u_{x}$ for the case of two layers and then for the case of four layers are followed in the same order as for the case of one layer.

For the case of one layer of scatterers, $u_{z}$ at various wave frequencies are put in one figure in order to show the effect of wave frequency. For example, Fig. 6.11 shows the modulus of displacement $u_{z}$ of total waves in $y=0$ plane at evenly distributed wave frequencies, in which a wave frequency is represented by normalized longitudinal 
wave number in the host medium. The displacement $u_{z}$ inside black circles are not computed during scatterer polymerization process and are set to zero. In a similar way, the real part of $u_{z}$, the modulus and the real part of $u_{x}$ of total waves and scattered waves are shown in Figs. 6.12 through 6.16.

In Fig. 6.11, the distribution of modulus of $u_{z}$ is symmetric about $x=0$ axis in each sub-figure and the pattern of the distribution changes with respect to the orientation and wave frequency. The edge of the shadow region, the region right behind the scatterers, form an angle with $Z$-axis. The angle decreases as wave number increases. Outside of the shadow region, periodic peaks appear as contour lines. The spacing between contour lines corresponds to the incident wavelength, which decreases as the linear reciprocal of the frequency. When $\kappa a$ increases, the spacing between contour lines decreases. In Fig. 6.11(e), $\kappa a=0.9$ and the value of periodic peaks along the direction perpendicular to $Z$-axis is larger than that along the $Z$ axis. Similarly, the value of periodic peaks is larger along a certain direction than that along the other directions in the sub-figures in Fig. 6.11. Specifically, in Fig. 6.11(i) the value of periodic peaks is larger along negative $Z$-axis and along the edge of the shadow region.

In Fig. 6.12, the distribution of real part of $u_{z}$ is symmetric about $x=0$ axis in each sub-figure and the pattern of the distribution changes with respect to the orientation and wave frequency. Periodic peaks appear like straight lines that parallel to each other and to $X$-axis. The spacing between parallel lines corresponds to the incident wavelength, which decreases as the linear reciprocal of the frequency. When $\kappa a$ increases, the spacing between parallel lines decreases. The edge of the shadow region form an angle with $Z$-axis. The angle decreases as wave number increases. The edge of the illuminated region, the region opposite the shadow region, also form an angle with $Z$-axis in sub-figures $6.13(\mathrm{~b})$ through $6.13(\mathrm{i})$. The angle does not change 


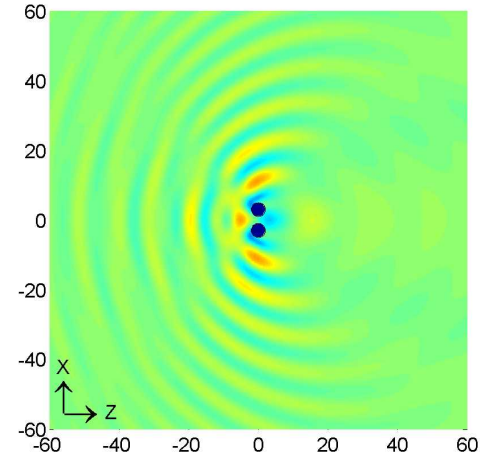

(a) $\kappa a=0.3$

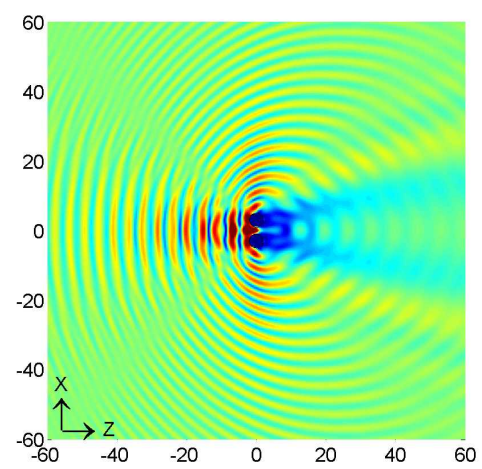

(d) $\kappa a=0.75$

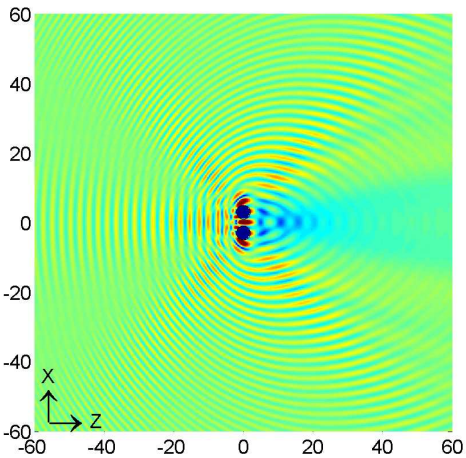

(g) $\kappa a=1.2$

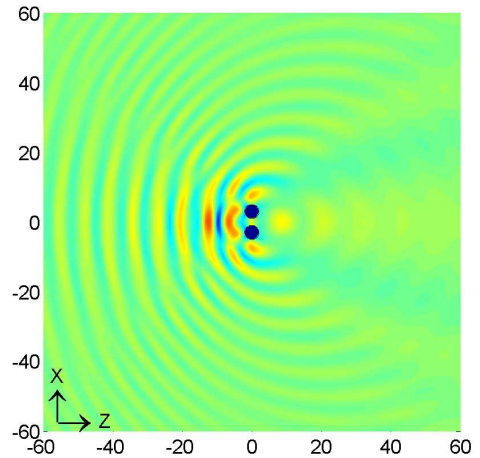

(b) $\kappa a=0.45$

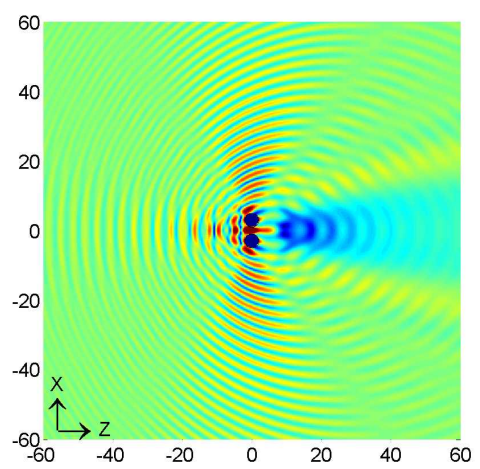

(e) $\kappa a=0.9$

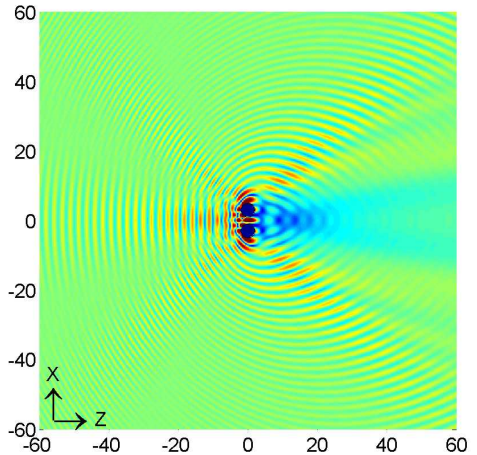

(h) $\kappa a=1.35$

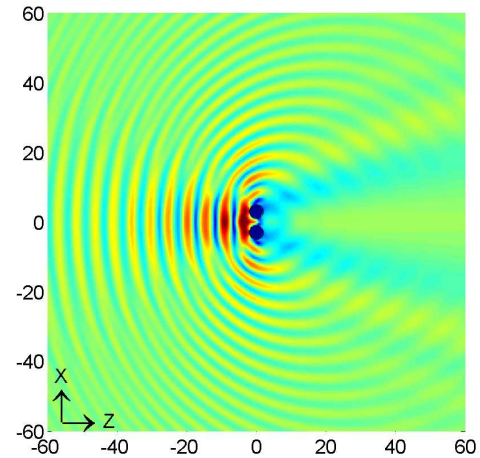

(c) $\kappa a=0.6$

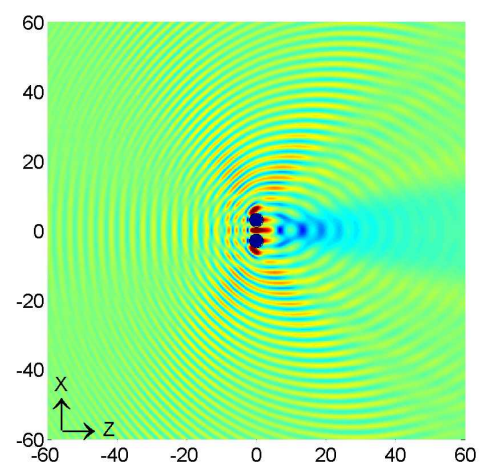

(f) $\kappa a=1.05$

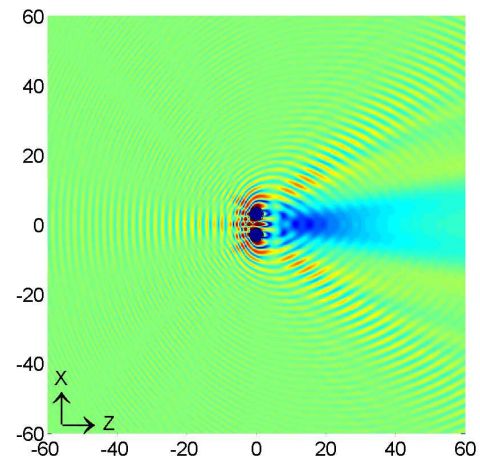

(i) $\kappa a=1.5$

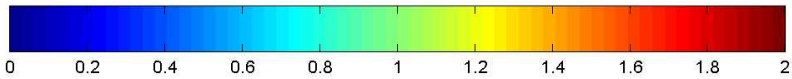

(j) Colorbar

Figure 6.11: Modulus of $u_{z}$ in the $y=0$ plane of total waves for the case of one layer of 16 scatterers. For different longitudinal wave numbers in epoxy matrix, normalized wave number $\kappa a$ is used and $a$ is radius of spherical scatterer. Wave fields inside black circles are not computed during scatterer polymerization procedure and set to zero. 


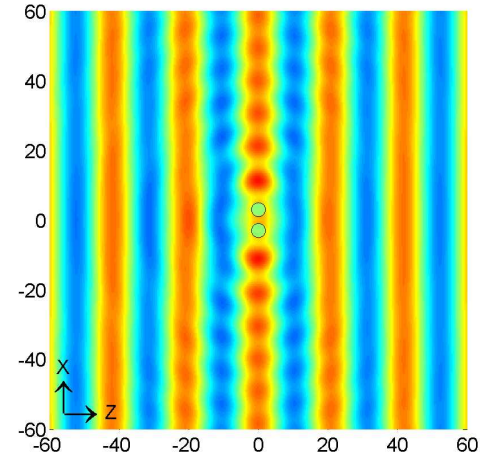

(a) $\kappa a=0.3$

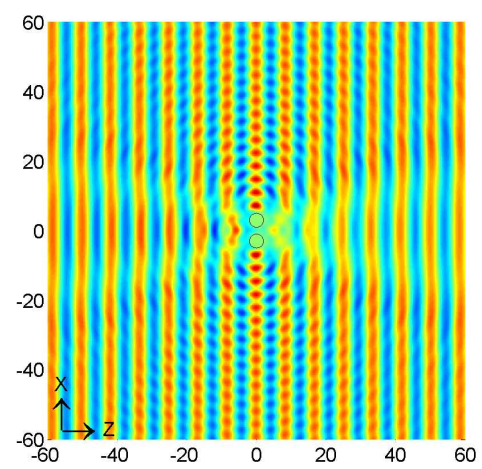

(d) $\kappa a=0.75$

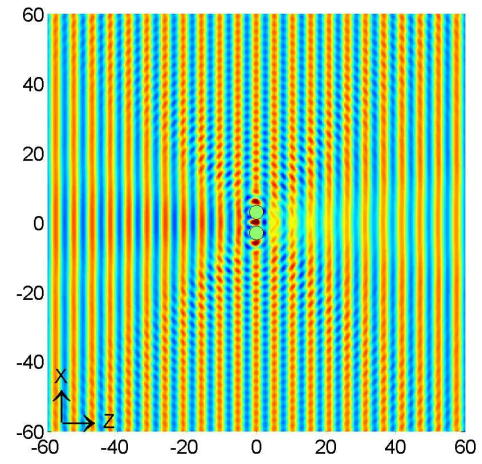

(g) $\kappa a=1.2$

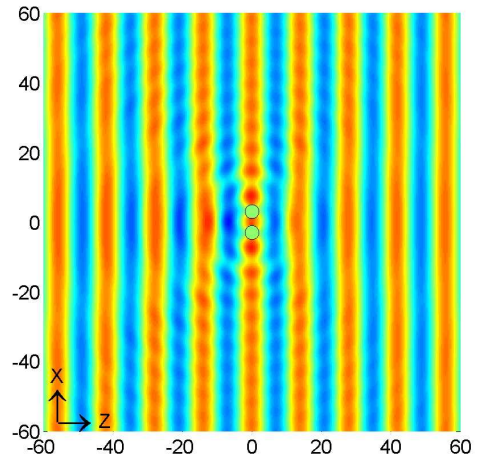

(b) $\kappa a=0.45$

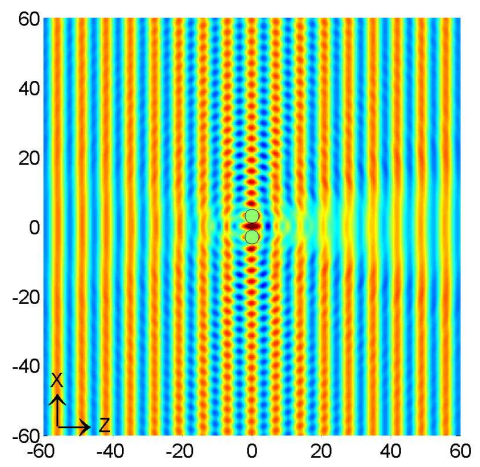

(e) $\kappa a=0.9$

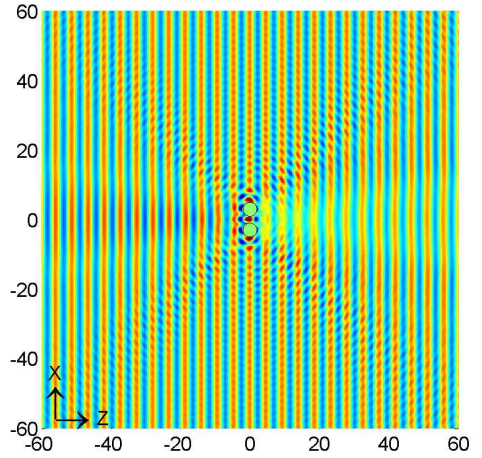

(h) $\kappa a=1.35$

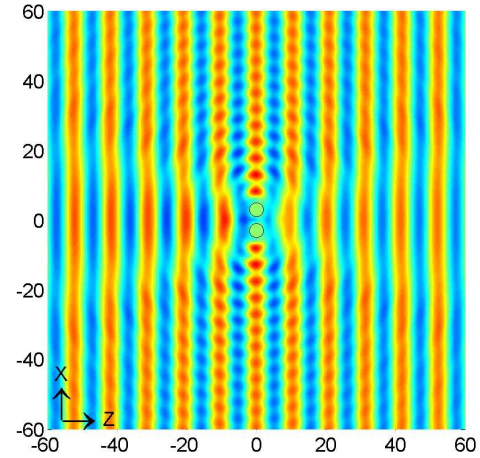

(c) $\kappa a=0.6$

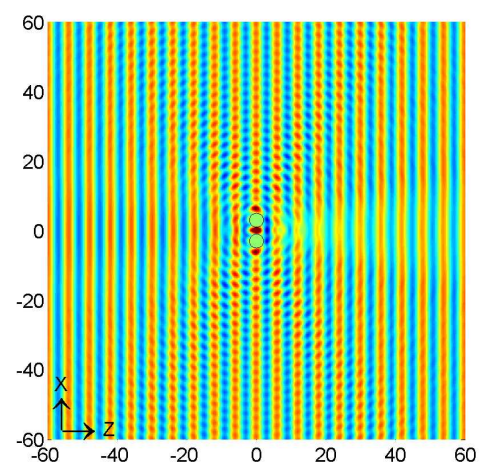

(f) $\kappa a=1.05$

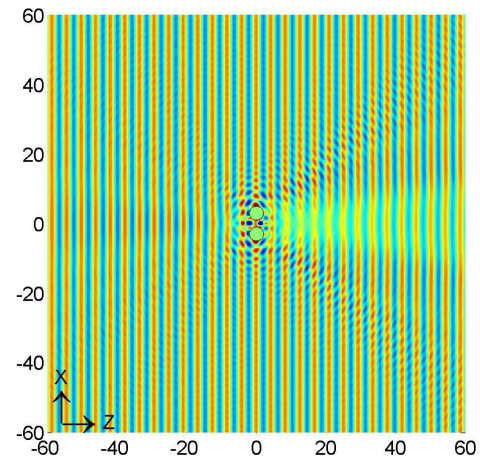

(i) $\kappa a=1.5$

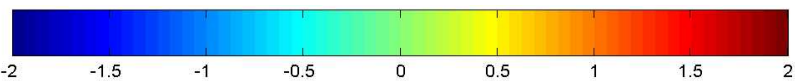

(j) Colorbar

Figure 6.12: Real part of $u_{z}$ in the $y=0$ plane of total waves for the case of one layer of 16 scatterers. For different longitudinal wave numbers in epoxy matrix, normalized wave number $\kappa a$ is used and $a$ is radius of spherical scatterer. Wave fields inside black circles are not computed during scatterer polymerization procedure and set to zero. 
with respect to wave frequency.

In Fig. 6.13, the distribution of modulus of $u_{z}$ is symmetric about $x=0$ axis in each sub-figure and the pattern of the distribution changes with respect to the orientation and wave frequency. The distribution of modulus of $u_{z}$ in the $y=0$ plane appears like several sectors, where a em sector (in analogy to the definition of circular sector) is an area bounded by two radii and the edge of an image. The number of sectors increases when wave number increases. Periodic peaks in these sectors appear like contour lines. When $\kappa a$ increases, the spacing between contour lines decreases.

In Fig. 6.14, the distribution of real part of $u_{z}$ is symmetric about $x=0$ axis in each sub-figure and the pattern of the distribution changes with respect to the orientation and wave frequency. The distribution pattern in each sub-figure appears like four sectors. In each sector, periodic peaks appear like contour lines. The spacing between periodic peaks in the shadow region and the illuminated region is double of the spacing between periodic peaks in the other regions. When $\kappa a$ increases, the spacing between contour lines decreases.

In Fig. 6.15, the distribution of modulus of $u_{x}$ is symmetric about $x=0$ axis in each sub-figure and the pattern of the distribution changes with respect to the orientation and wave frequency. Along $X$ and $Z$ axes, modulus of $u_{x}$ is zero. In Figs. 6.15(a) through 6.15(c), the distribution pattern appears like four sectors. In Figs. 6.15(d) through 6.15(i), the distribution pattern appears as more than four sectors. Periodic peaks in these sectors appear like contour lines. When $\kappa a$ increases, the spacing between contour lines decreases. In Figs. 6.15(g) and 6.15(h), values of periodic peaks along directions, which form an angle of roughly $45^{\circ}$ with respect to $Z$-axis, are larger than those along other directions. In Figs. 6.15(i), values of periodic peaks along directions, which form an angle of roughly $45^{\circ}$ with respect to positive $Z$-axis, are larger than those along other directions. 


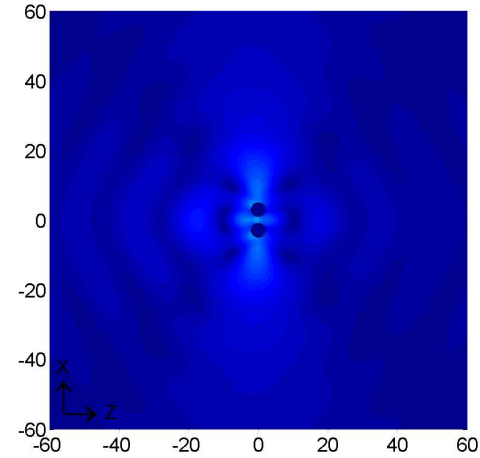

(a) $\kappa a=0.3$

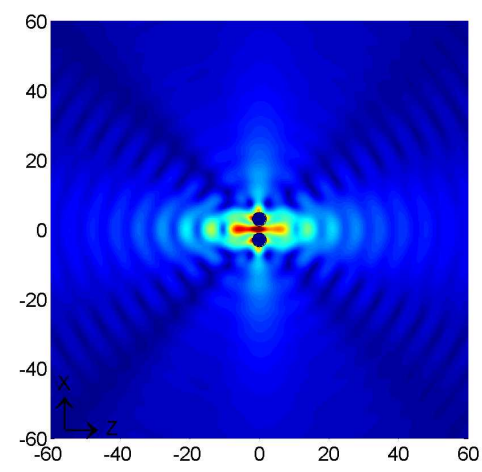

(d) $\kappa a=0.75$

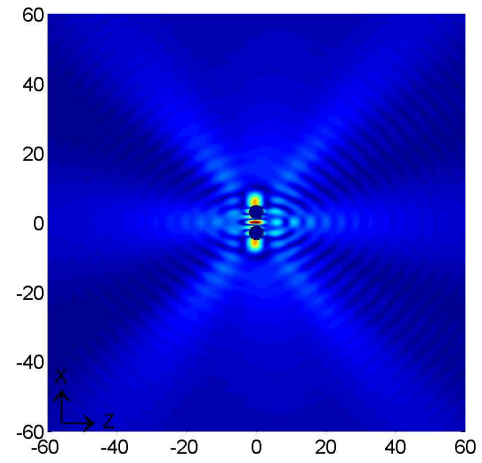

(g) $\kappa a=1.2$

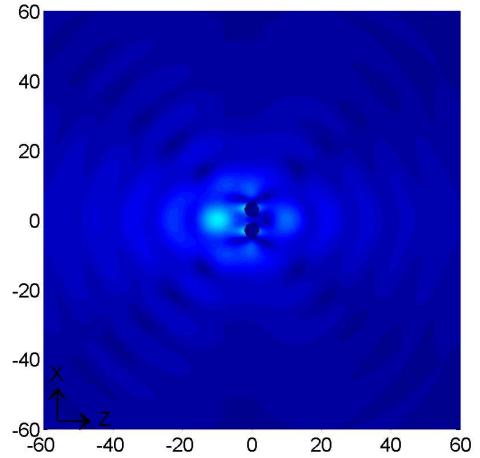

(b) $\kappa a=0.45$

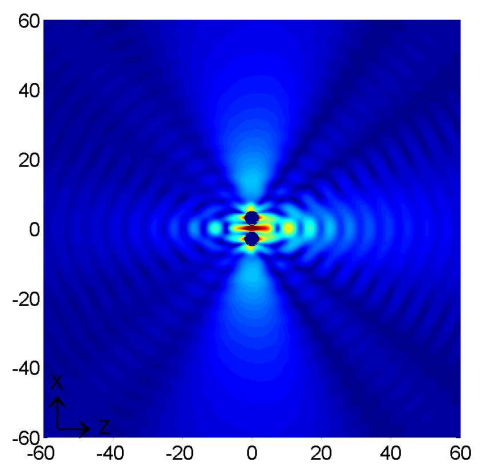

(e) $\kappa a=0.9$

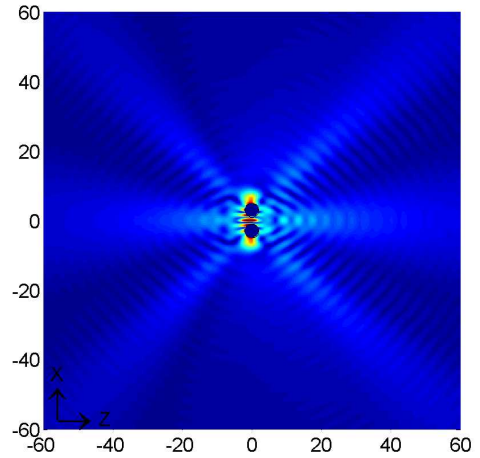

(h) $\kappa a=1.35$

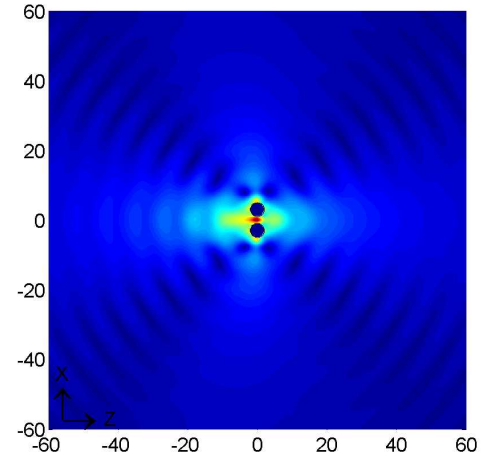

(c) $\kappa a=0.6$

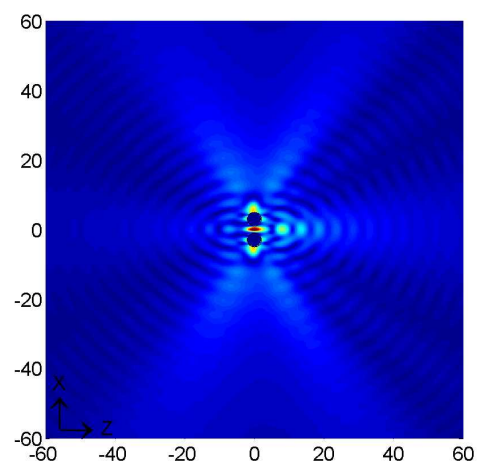

(f) $\kappa a=1.05$

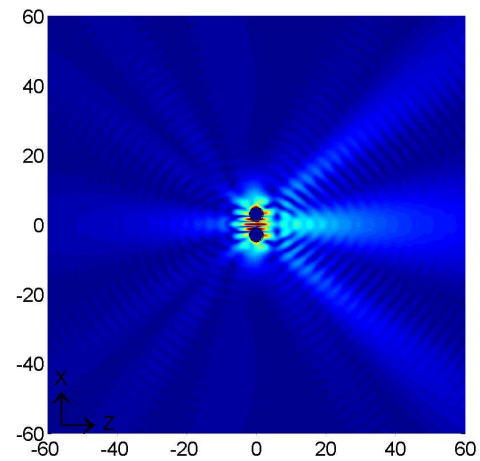

(i) $\kappa a=1.5$

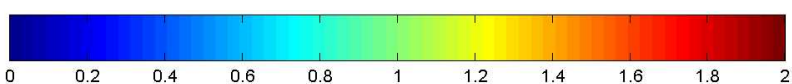

(j) Colorbar

Figure 6.13: Modulus of $u_{z}$ in the $y=0$ plane of scattered waves for the case of one layer of 16 scatterers. For different longitudinal wave numbers in epoxy matrix, normalized wave number $\kappa a$ is used and $a$ is radius of spherical scatterer. Wave fields inside black circles are not computed during scatterer polymerization procedure and set to zero. 


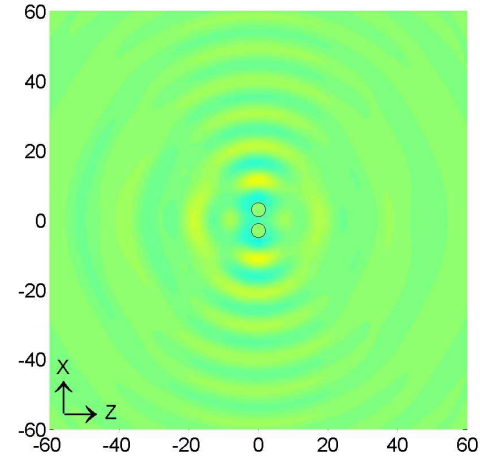

(a) $\kappa a=0.3$

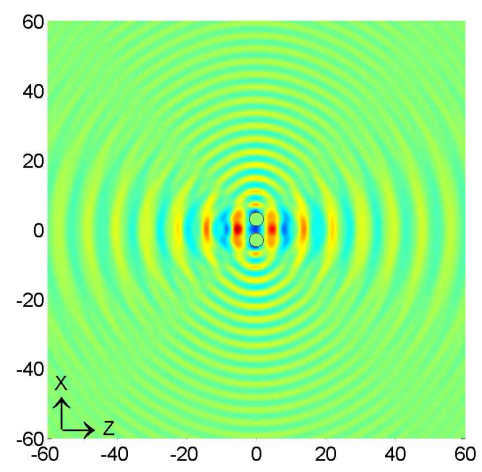

(d) $\kappa a=0.75$

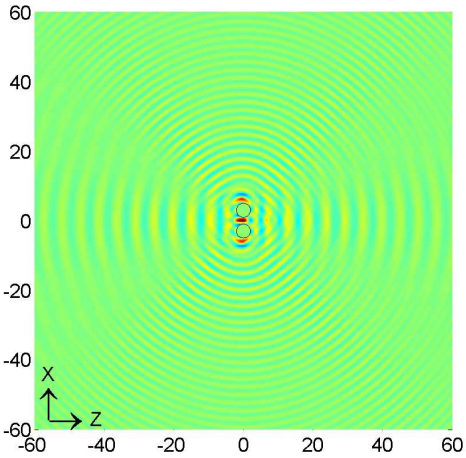

(g) $\kappa a=1.2$

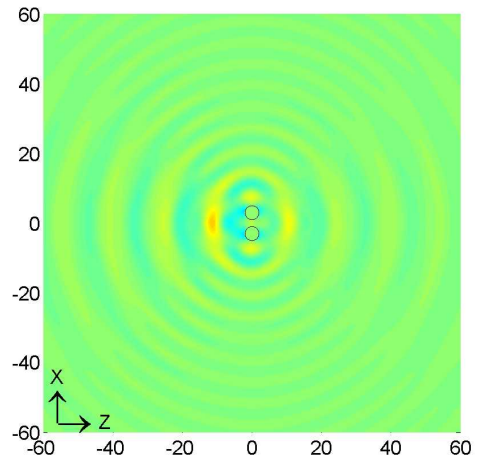

(b) $\kappa a=0.45$

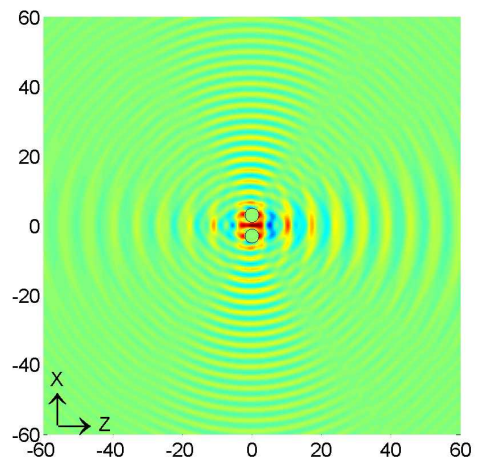

(e) $\kappa a=0.9$

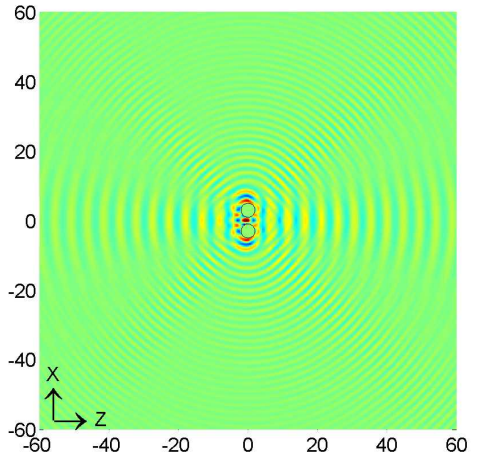

(h) $\kappa a=1.35$

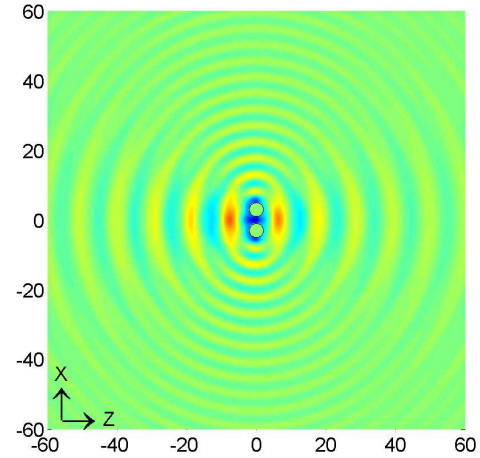

(c) $\kappa a=0.6$

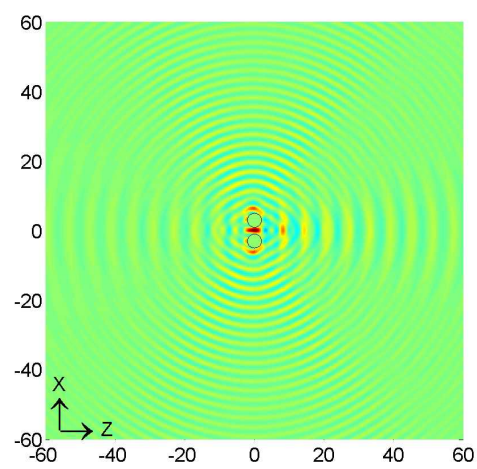

(f) $\kappa a=1.05$

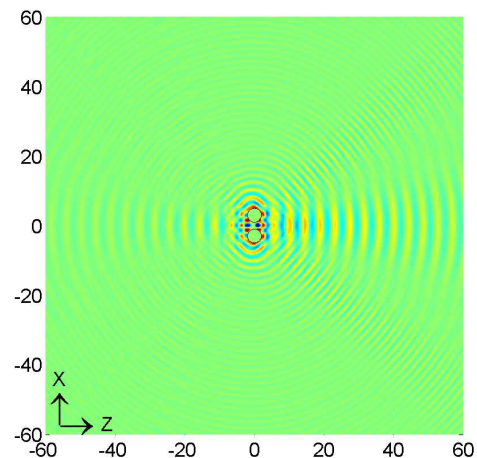

(i) $\kappa a=1.5$

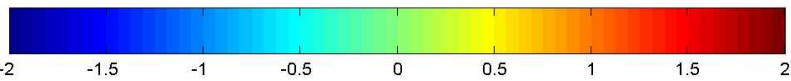

(j) Colorbar

Figure 6.14: Real part of $u_{z}$ in the $y=0$ plane of scattered waves for the case of one layer of 16 scatterers. For different longitudinal wave numbers in epoxy matrix, normalized wave number $\kappa a$ is used and $a$ is radius of spherical scatterer. Wave fields inside black circles are not computed during scatterer polymerization procedure and set to zero. 


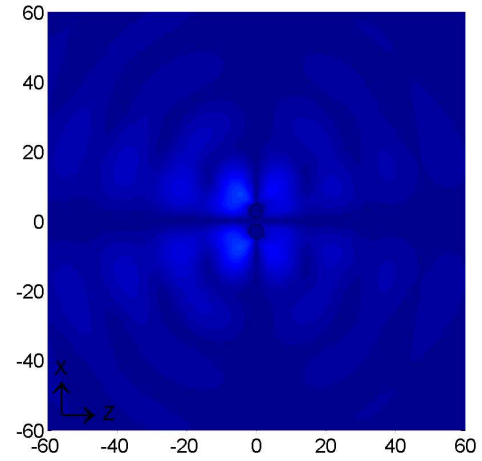

(a) $\kappa a=0.3$

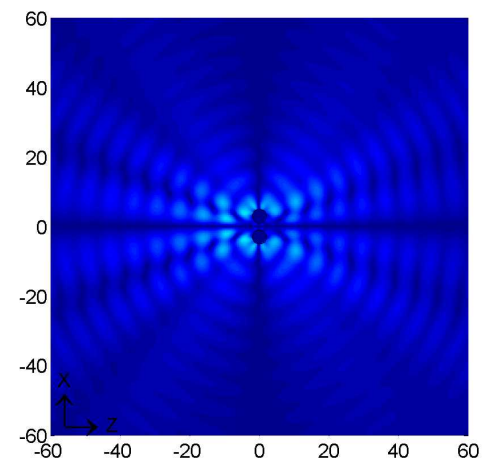

(d) $\kappa a=0.75$

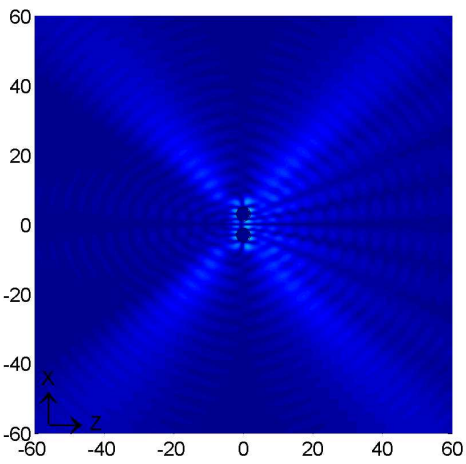

(g) $\kappa a=1.2$

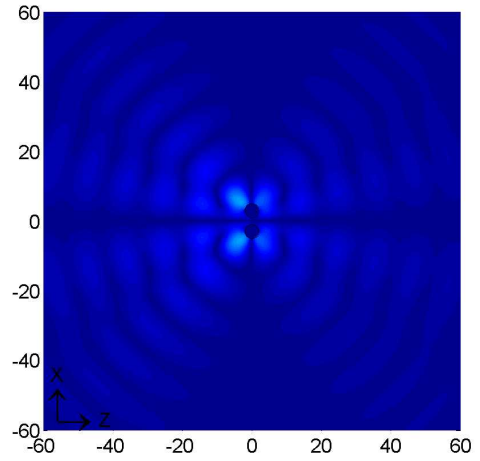

(b) $\kappa a=0.45$

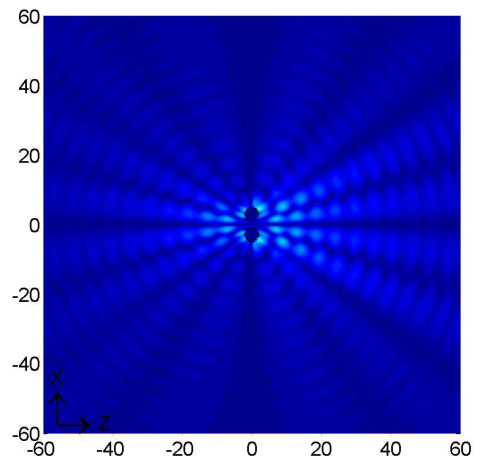

(e) $\kappa a=0.9$

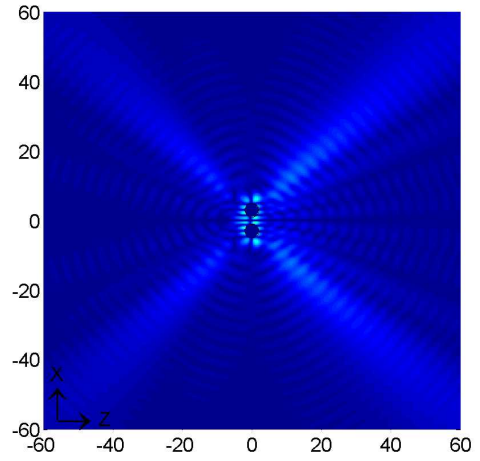

(h) $\kappa a=1.35$

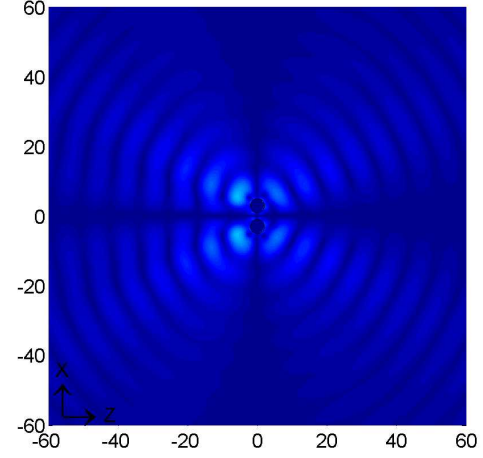

(c) $\kappa a=0.6$

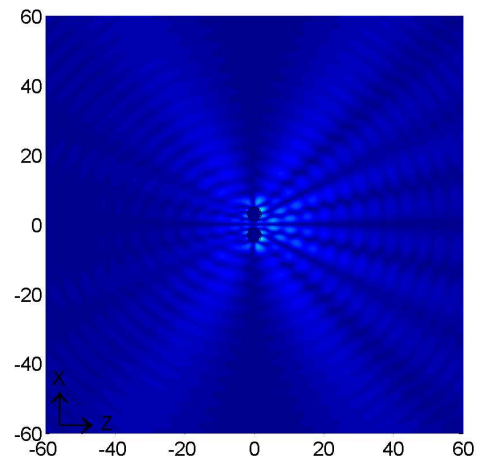

(f) $\kappa a=1.05$

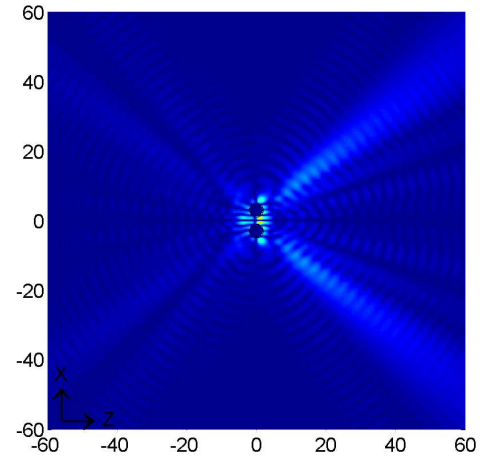

(i) $\kappa a=1.5$

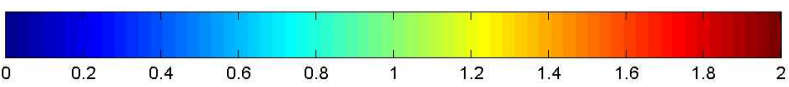

(j) Colorbar

Figure 6.15: Modulus of $u_{x}$ in the $y=0$ plane of total waves for the case of one layer of 16 scatterers. For different longitudinal wave numbers in epoxy matrix, normalized wave number $\kappa a$ is used and $a$ is radius of spherical scatterer. Wave fields inside black circles are not computed during scatterer polymerization procedure and set to zero. Values of $u_{x}$ of longitudinal incident waves are zero. 


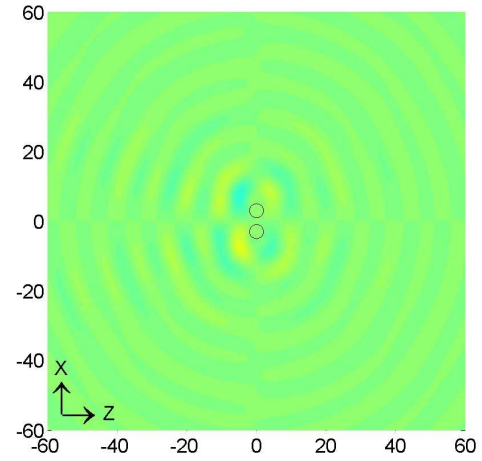

(a) $\kappa a=0.3$

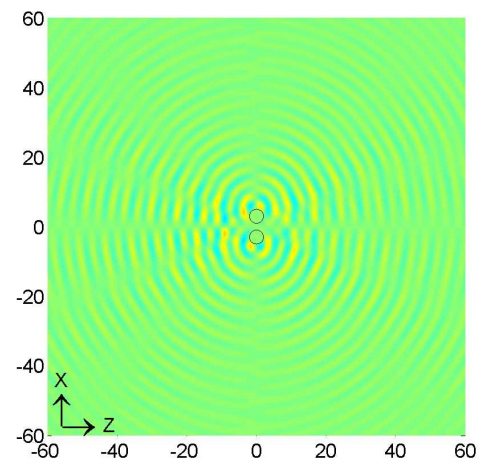

(d) $\kappa a=0.75$

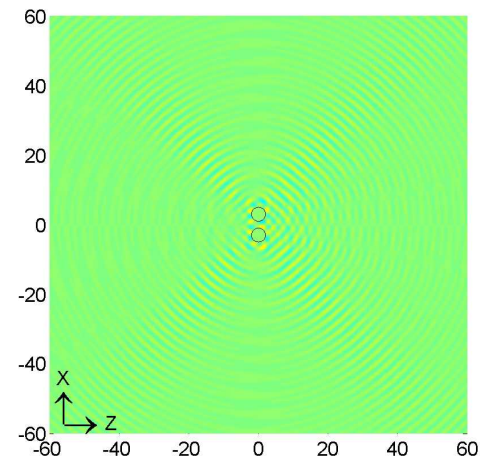

(g) $\kappa a=1.2$

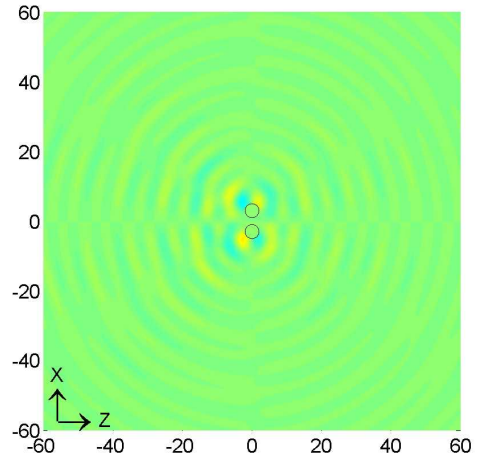

(b) $\kappa a=0.45$

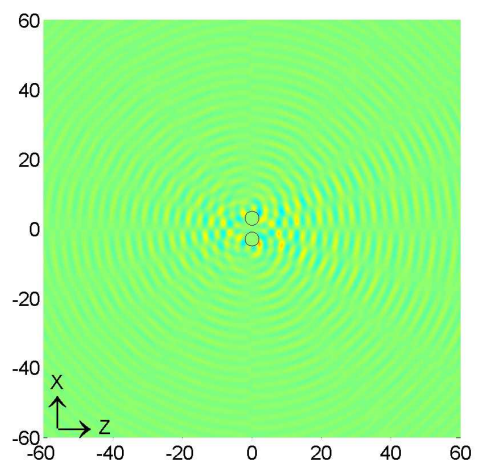

(e) $\kappa a=0.9$

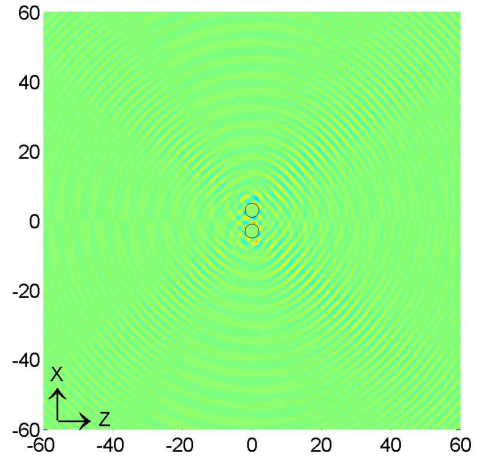

(h) $\kappa a=1.35$

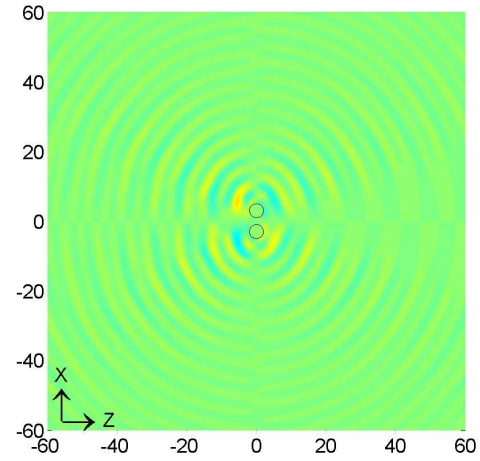

(c) $\kappa a=0.6$

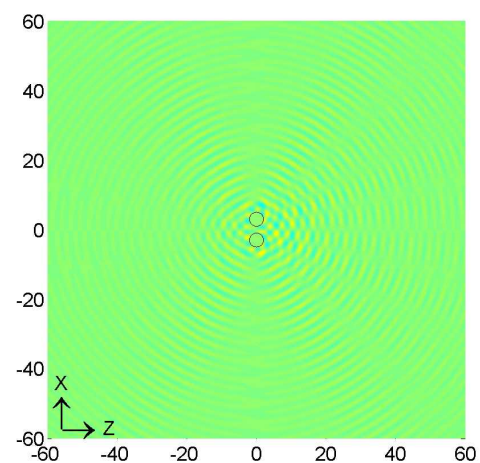

(f) $\kappa a=1.05$

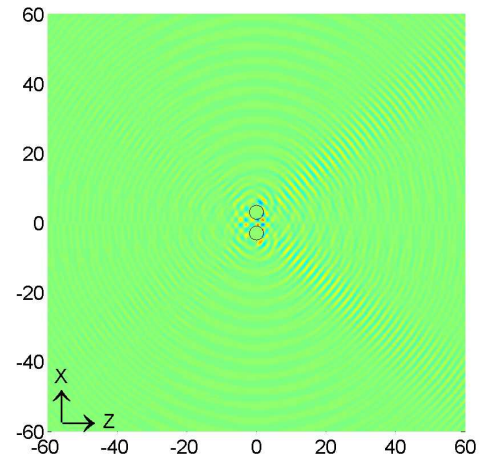

(i) $\kappa a=1.5$

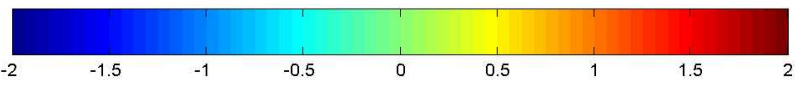

(j) Colorbar

Figure 6.16: Real part of $u_{x}$ in the $y=0$ plane of total waves for the case of one layer of 16 scatterers. For different longitudinal wave numbers in epoxy matrix, normalized wave number $\kappa a$ is used and $a$ is radius of spherical scatterer. Wave fields inside black circles are not computed during scatterer polymerization procedure and set to zero. Values of $u_{x}$ of longitudinal incident waves are zero. 
In Fig. 6.16, the distribution of modulus of $u_{x}$ is not symmetric about $x=0$ axis in each sub-figure and the pattern of the distribution changes with respect to the orientation and wave frequency. Along $X$ and $Z$ axes, real part of $u_{x}$ is zero. In each sub-figure, the distribution pattern appears as four sectors. Periodic peaks in each sector appear like contour lines. When $\kappa a$ increases, the spacing between contour lines decreases. In Figs. 6.16(d) and 6.16(e), values of periodic peaks in the shadow region and illuminated region are larger than those in other regions. In Figs. 6.16(f) and 6.16(g), values of periodic peaks along directions, which form an angle of roughly $45^{\circ}$ with respect to $Z$-axis, are larger than those along other directions. In Figs. 6.16(i), values of periodic peaks along directions, which form an angle of roughly $45^{\circ}$ with respect to positive $Z$-axis, are larger than those along other directions.

The pattern of distribution of modulus of $u_{z}$ in Fig. 6.17 is similar as that in Fig. 6.11. In the shadow region, value of modulus of $u_{z}$ in Fig. 6.17 is slightly smaller than that in Fig. 6.11 (through comparison of color in two figures). In the illuminated region, values of periodic peaks in Fig. 6.17 are slightly larger than those in Fig. 6.11. Directions, along which values of periodic peaks appear larger than those along the $Z$-axis, in Fig. 6.17 are different than those in Fig. 6.11.

The pattern of distribution of real part of $u_{z}$ in Fig. 6.18 is similar as that in Fig. 6.12. In the shadow region, real part of $u_{z}$ in Fig. 6.17 approaches to zero in larger area than that in Fig. 6.11 (through comparison of color in two figures).

The pattern of distribution of modulus of $u_{z}$ in Fig. 6.19 is similar as that in Fig. 6.13. In the shadow region and illuminated region, modulus of $u_{z}$ in Fig. 6.19 appears larger than that in Fig. 6.13 (through comparison of color in two figures). Outside the shadow region and illuminated region, the number of sectors in Fig. 6.19 is larger than that in Fig. 6.13.

The pattern of distribution of real part of $u_{z}$ in Fig. 6.20 is similar as that in 


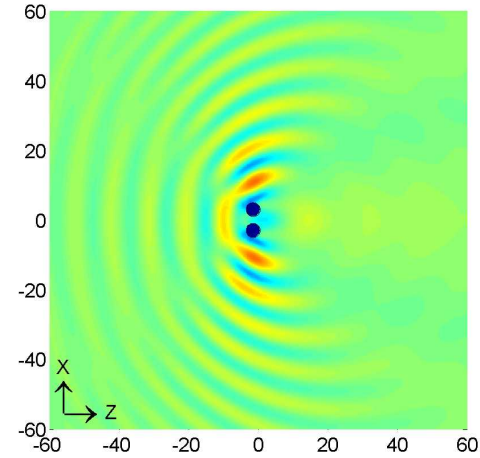

(a) $\kappa a=0.3$

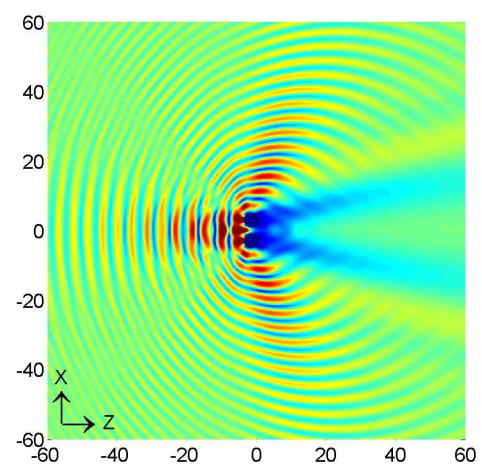

(d) $\kappa a=0.75$

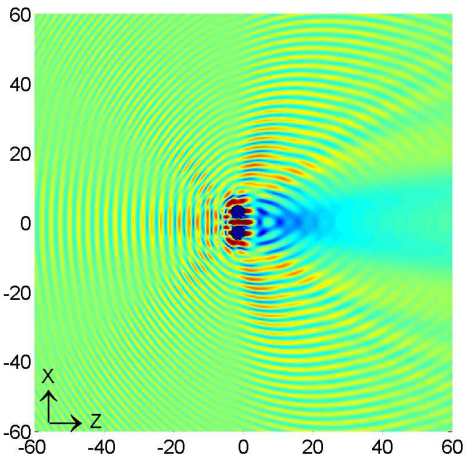

(g) $\kappa a=1.2$

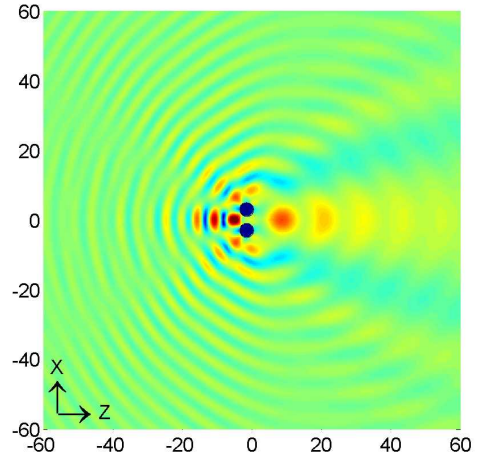

(b) $\kappa a=0.45$

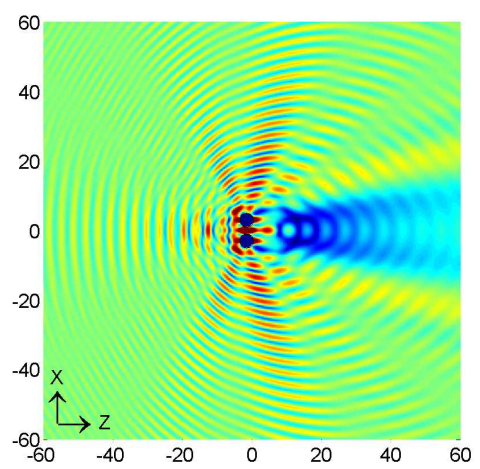

(e) $\kappa a=0.9$

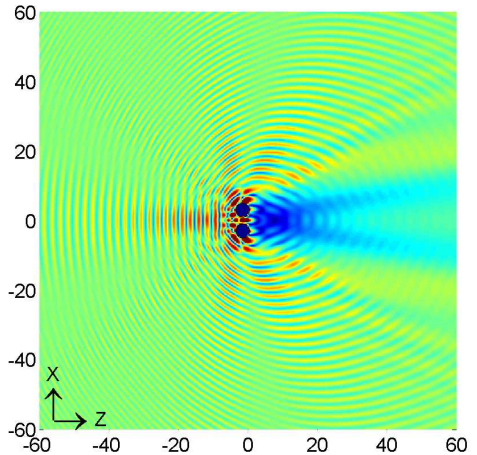

(h) $\kappa a=1.35$

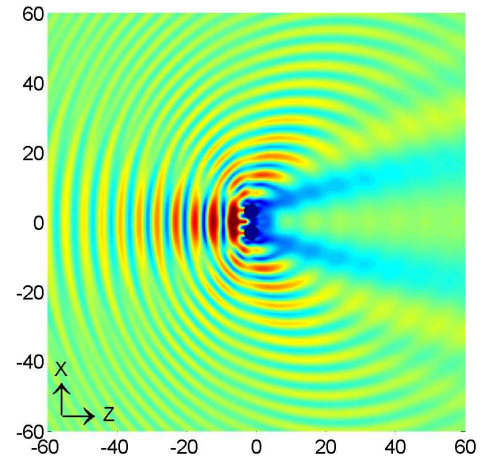

(c) $\kappa a=0.6$

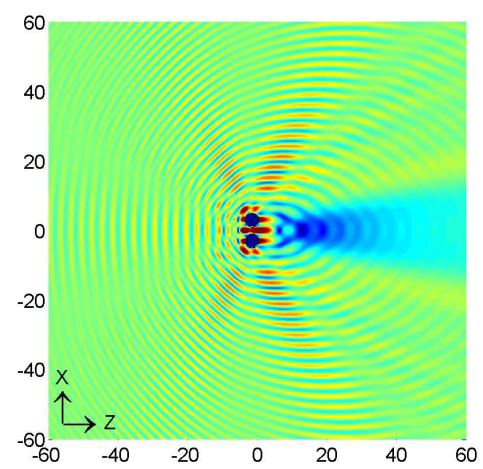

(f) $\kappa a=1.05$

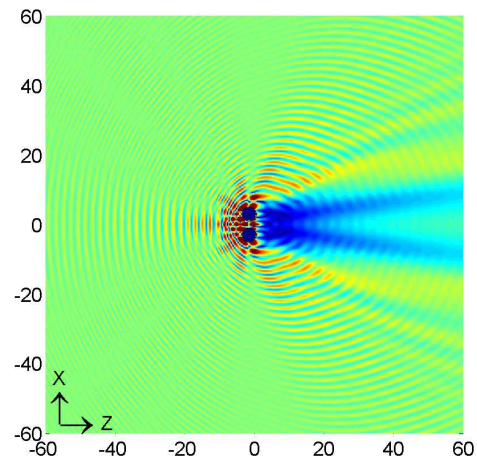

(i) $\kappa a=1.5$

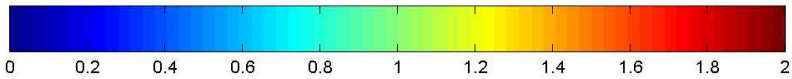

(j) Colorbar

Figure 6.17: Modulus of $u_{z}$ in the $y=0$ plane of total waves for the case of two layers of scatterers. For different longitudinal wave numbers in epoxy matrix, normalized wave number $\kappa a$ is used and $a$ is radius of spherical scatterer. Wave fields inside black circles are not computed during scatterer polymerization procedure and set to zero. 


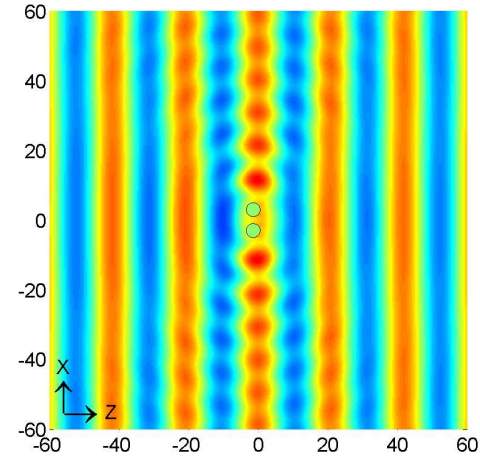

(a) $\kappa a=0.3$

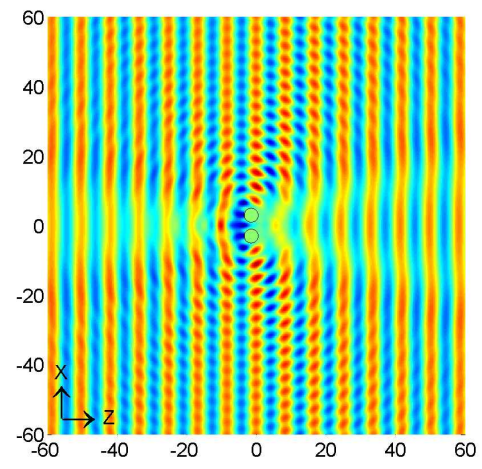

(d) $\kappa a=0.75$

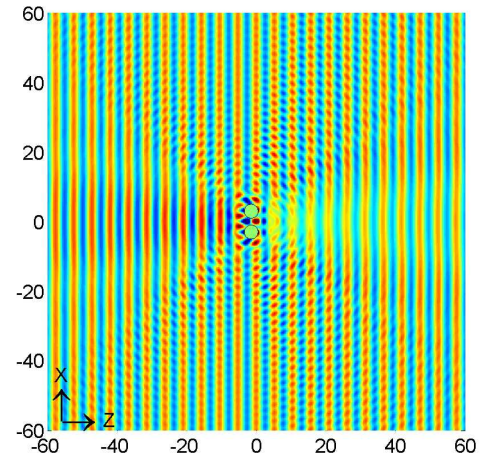

(g) $\kappa a=1.2$

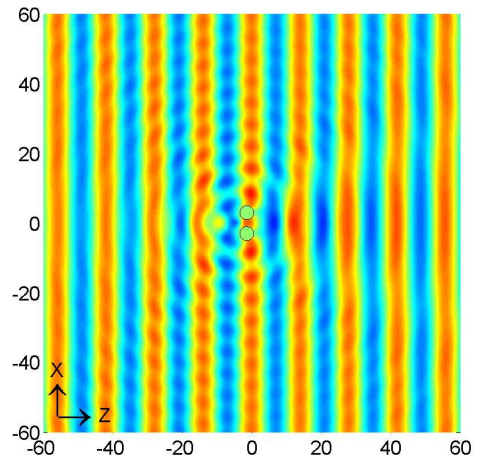

(b) $\kappa a=0.45$

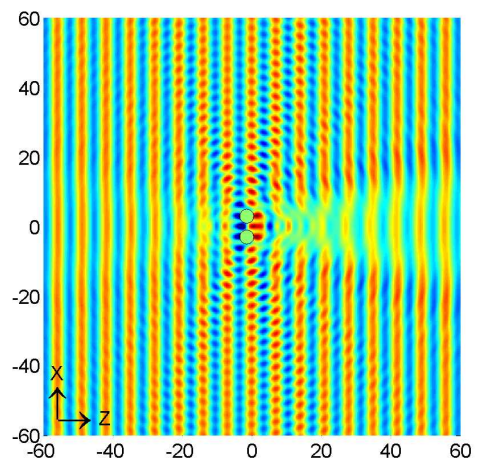

(e) $\kappa a=0.9$

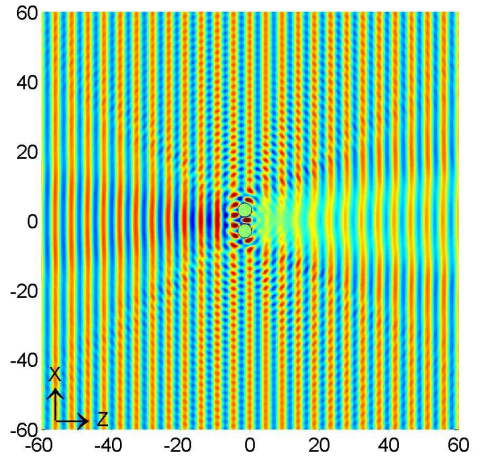

(h) $\kappa a=1.35$

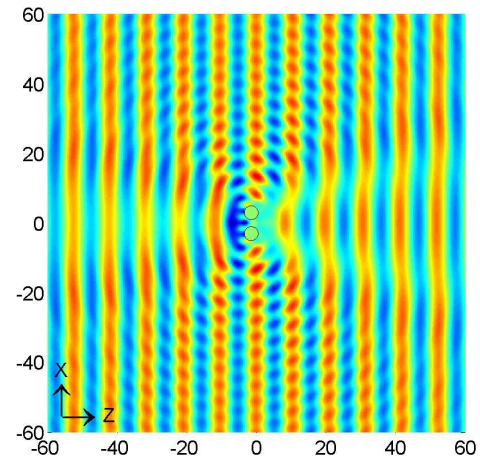

(c) $\kappa a=0.6$

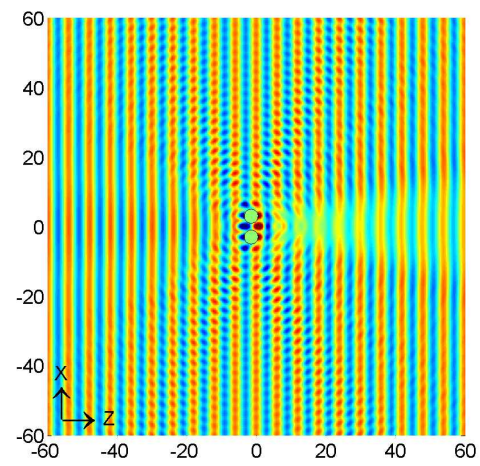

(f) $\kappa a=1.05$

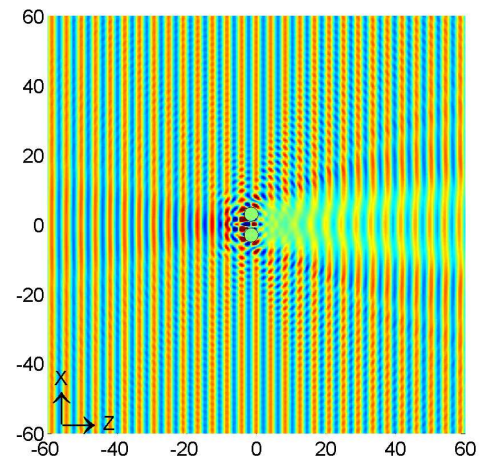

(i) $\kappa a=1.5$

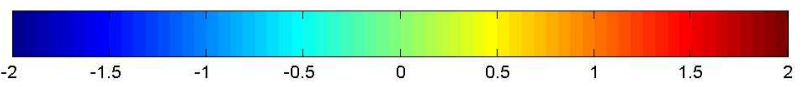

(j) Colorbar

Figure 6.18: Real part of $u_{z}$ in the $y=0$ plane of total waves for the case of two layers of scatterers. For different longitudinal wave numbers in epoxy matrix, normalized wave number $\kappa a$ is used and $a$ is radius of spherical scatterer. Wave fields inside black circles are not computed during scatterer polymerization procedure and set to zero. 


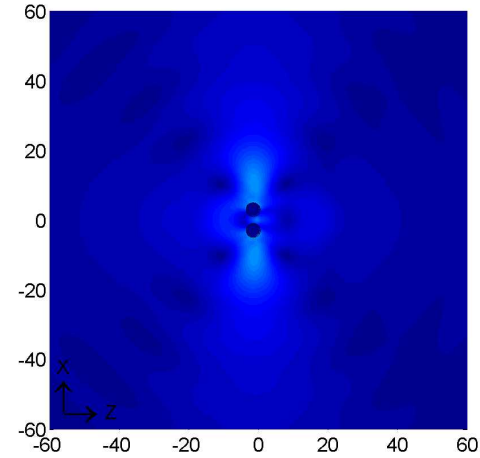

(a) $\kappa a=0.3$

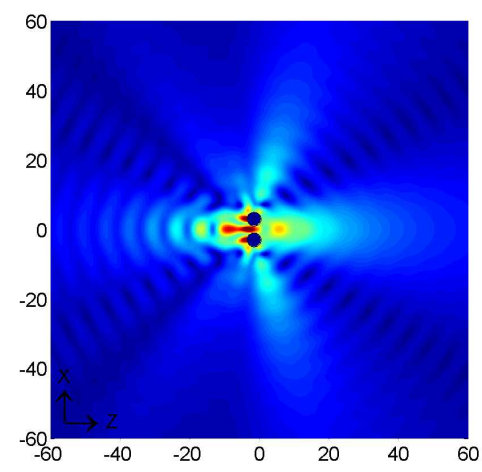

(d) $\kappa a=0.75$

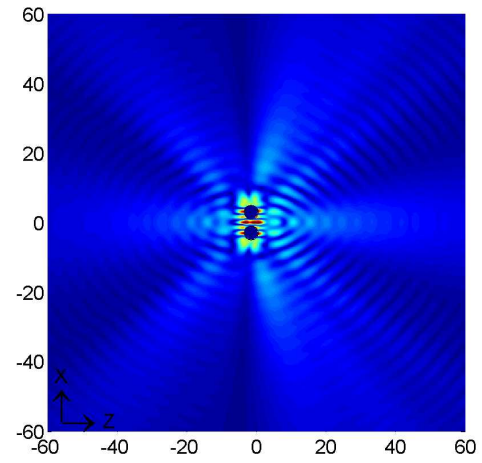

(g) $\kappa a=1.2$

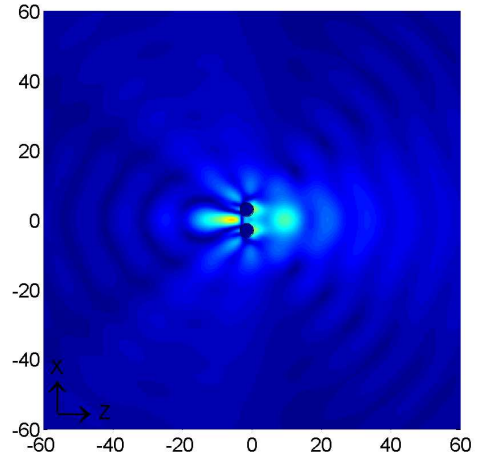

(b) $\kappa a=0.45$

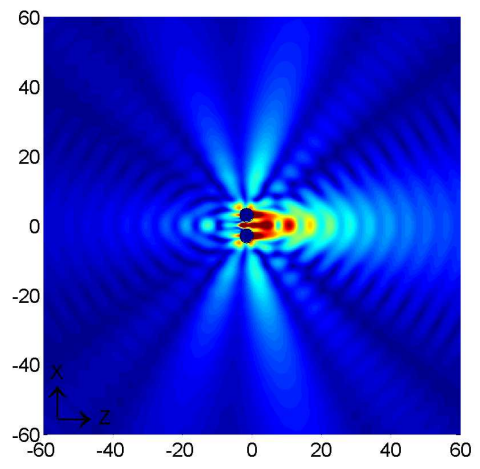

(e) $\kappa a=0.9$

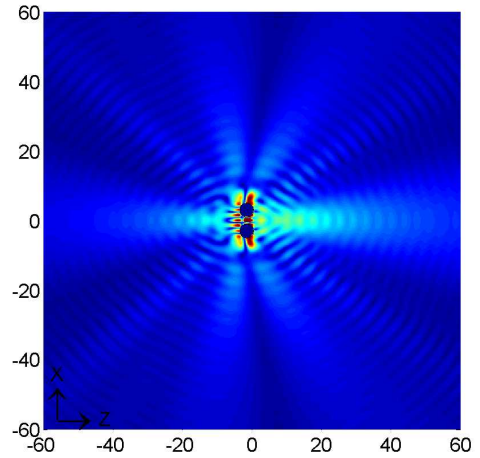

(h) $\kappa a=1.35$

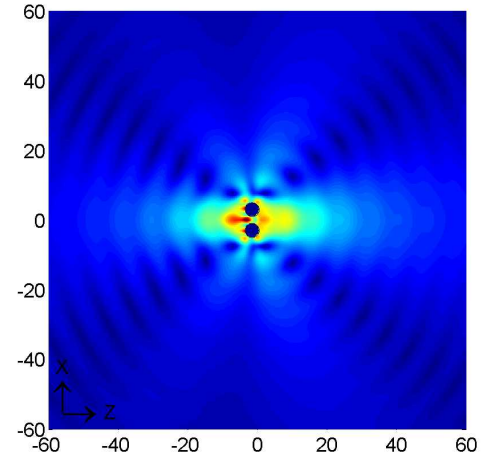

(c) $\kappa a=0.6$

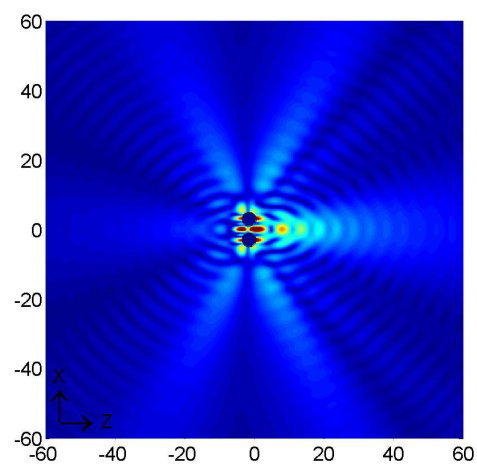

(f) $\kappa a=1.05$

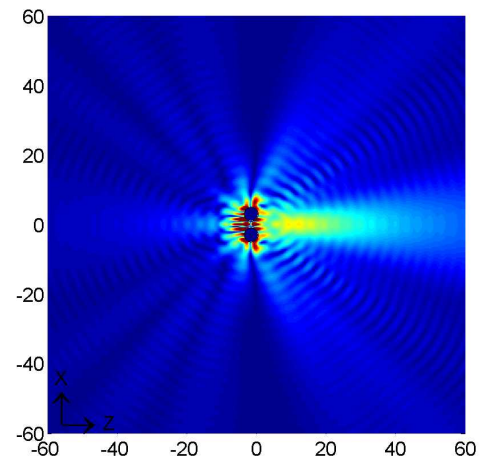

(i) $\kappa a=1.5$

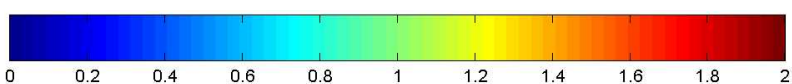

(j) Colorbar

Figure 6.19: Modulus of $u_{z}$ in the $y=0$ plane of scattered waves for the case of two layers of scatterers. For different longitudinal wave numbers in epoxy matrix, normalized wave number $\kappa a$ is used and $a$ is radius of spherical scatterer. Wave fields inside black circles are not computed during scatterer polymerization procedure and set to zero. 


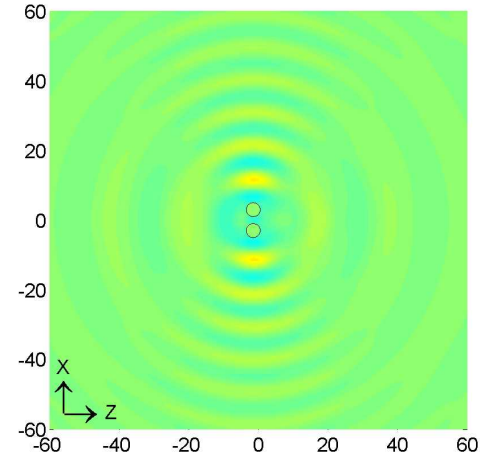

(a) $\kappa a=0.3$

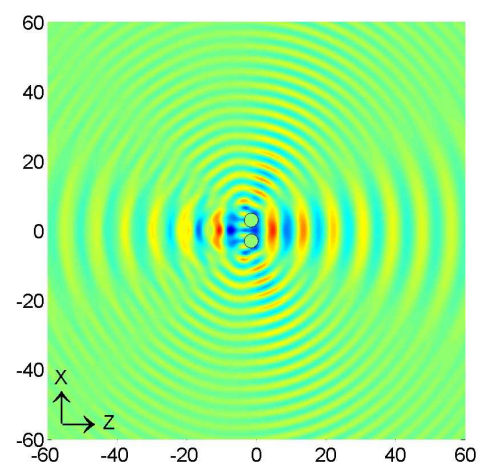

(d) $\kappa a=0.75$

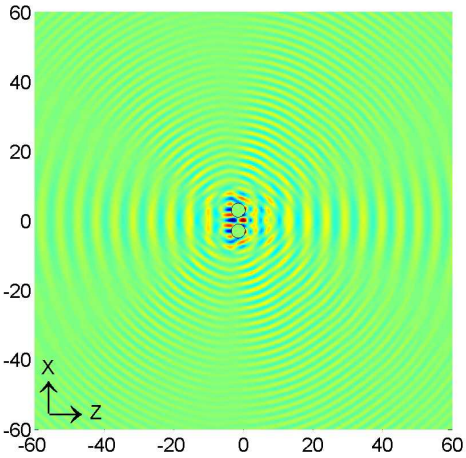

(g) $\kappa a=1.2$

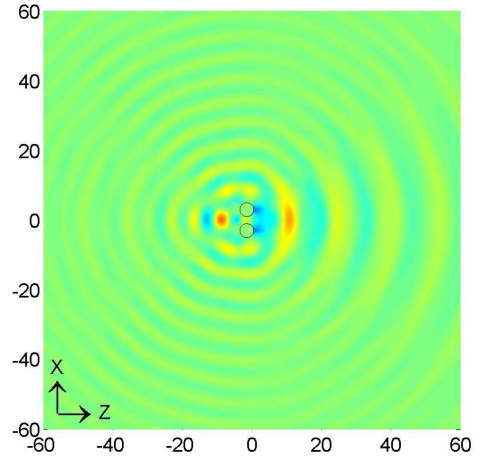

(b) $\kappa a=0.45$

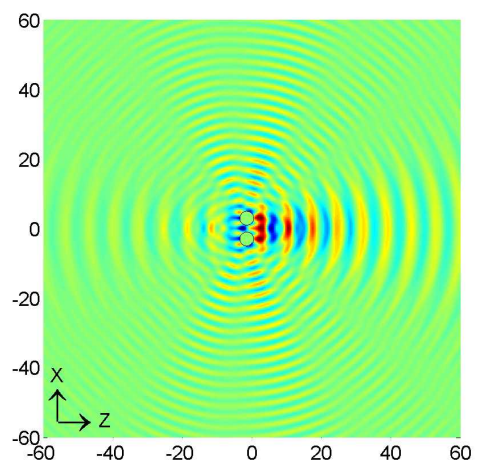

(e) $\kappa a=0.9$

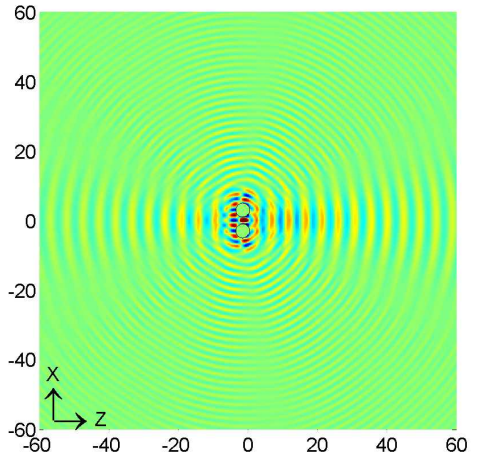

(h) $\kappa a=1.35$

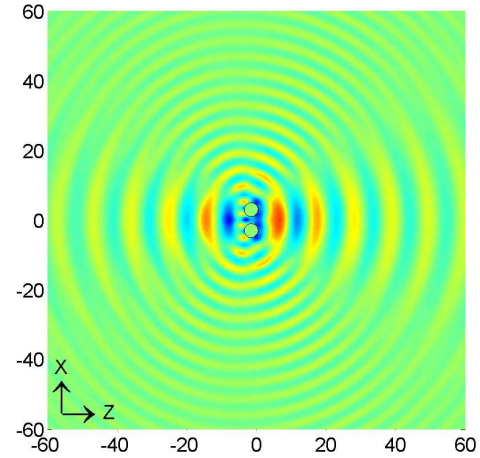

(c) $\kappa a=0.6$

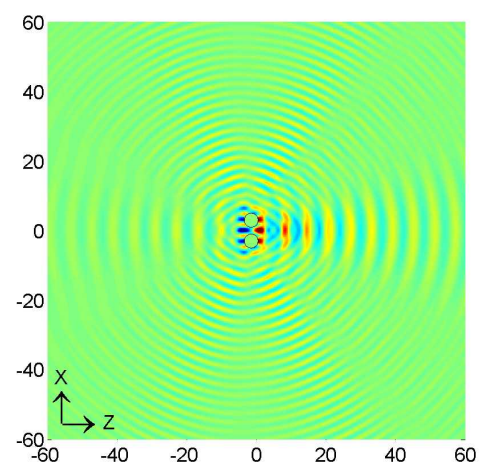

(f) $\kappa a=1.05$

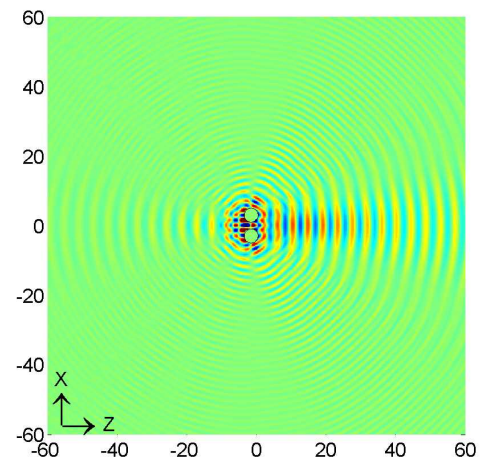

(i) $\kappa a=1.5$

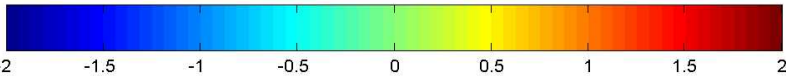

(j) Colorbar

Figure 6.20: Real part of $u_{z}$ in the $y=0$ plane of scattered waves for the case of two layers of scatterers. For different longitudinal wave numbers in epoxy matrix, normalized wave number $\kappa a$ is used and $a$ is radius of spherical scatterer. Wave fields inside black circles are not computed during scatterer polymerization procedure and set to zero. 
Fig. 6.14. In the shadow region and illuminated region, values of periodic peaks in Fig. 6.20 appear slightly larger than those in Fig. 6.14 (through comparison of color in two figures).

The pattern of distribution of modulus of $u_{x}$ in Fig. 6.21 is similar as that in Fig. 6.15. Directions, along which values of periodic peaks are larger than those in other directions, in Fig. 6.21 are different from those in Fig. 6.15. Values of periodic peaks in Fig. 6.21 appear slightly larger than those in Fig. 6.15 (through comparison of color in two figures).

The pattern of distribution of real part of $u_{x}$ in Fig. 6.22 is similar as that in Fig. 6.16. Values of periodic peaks in Fig. 6.22 appear slightly larger than those in Fig. 6.16 (through comparison of color in two figures).

The pattern of distribution of modulus of $u_{z}$ in Fig. 6.23 is similar as that in Fig. 6.17. In the shadow region, value of modulus of $u_{z}$ in Fig. 6.17 is slightly smaller than that in Fig. 6.17 (through comparison of color in two figures). In the illuminated region, values of periodic peaks in Fig. 6.23 are slightly larger than those in Fig. 6.17. Directions, along which values of periodic peaks appear larger than those along the Z-axis, in Fig. 6.23 are different from those in Fig. 6.17.

The pattern of distribution of real part of $u_{z}$ in Fig. 6.24 is similar as that in Fig. 6.18. In the shadow region, real part of $u_{z}$ in Fig. 6.23 approaches to zero in larger area than that in Fig. 6.17 (through comparison of color in two figures).

The pattern of distribution of modulus of $u_{z}$ in Fig. 6.25 is similar as that in Fig. 6.19. In the shadow region and illuminated region, modulus of $u_{z}$ in Fig. 6.25 appears larger than that in Fig. 6.19 (through comparison of color in two figures). Outside the shadow region and illuminated region, the number of sectors in Fig. 6.25 is larger than that in Fig. 6.19.

The pattern of distribution of real part of $u_{z}$ in Fig. 6.26 is similar as that in 


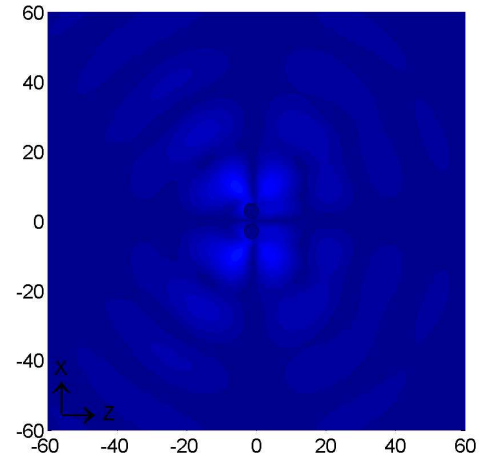

(a) $\kappa a=0.3$

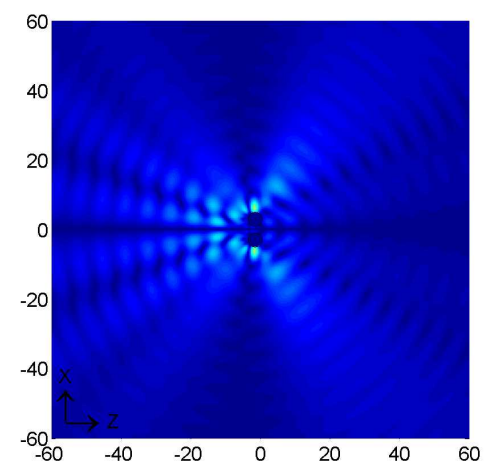

(d) $\kappa a=0.75$

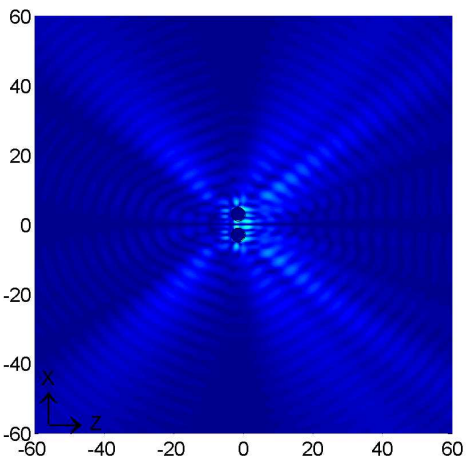

(g) $\kappa a=1.2$

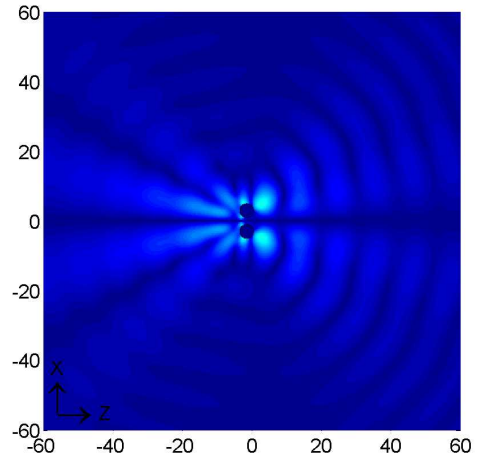

(b) $\kappa a=0.45$

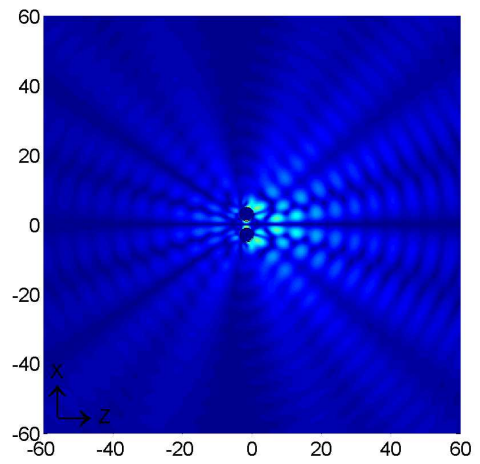

(e) $\kappa a=0.9$

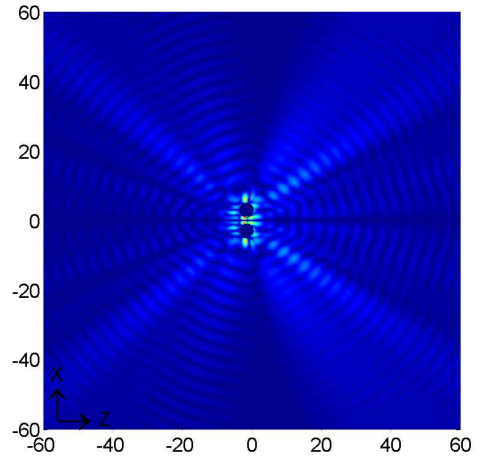

(h) $\kappa a=1.35$

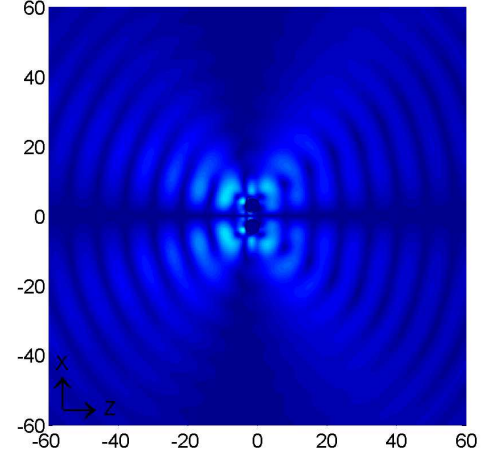

(c) $\kappa a=0.6$

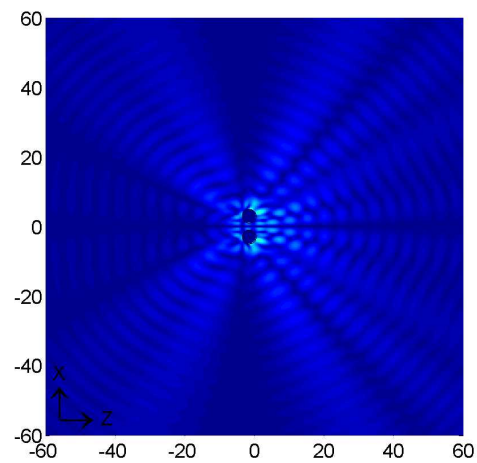

(f) $\kappa a=1.05$

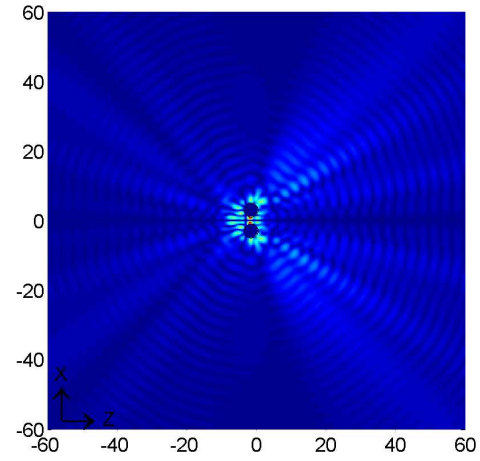

(i) $\kappa a=1.5$

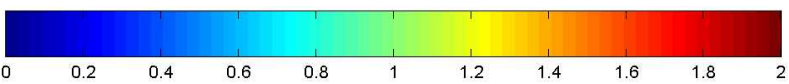

(j) Colorbar

Figure 6.21: Modulus of $u_{x}$ in the $y=0$ plane of total waves for the case of two layers of scatterers. For different longitudinal wave numbers in epoxy matrix, normalized wave number $\kappa a$ is used and $a$ is radius of spherical scatterer. Wave fields inside black circles are not computed during scatterer polymerization procedure and set to zero. Values of $u_{x}$ of longitudinal incident waves are zero. 


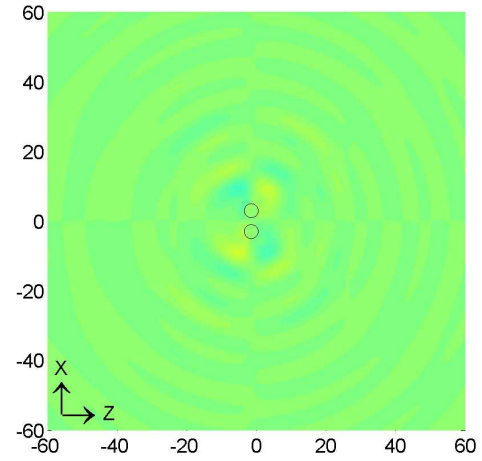

(a) $\kappa a=0.3$

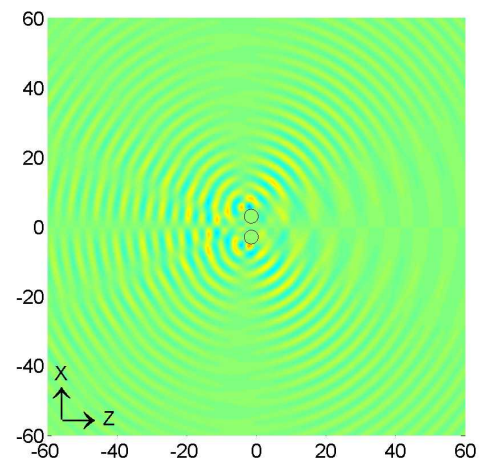

(d) $\kappa a=0.75$

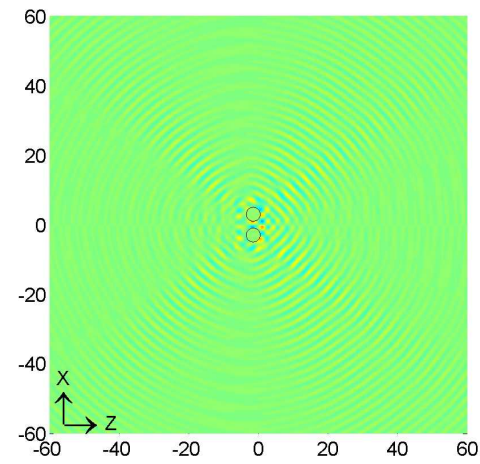

(g) $\kappa a=1.2$

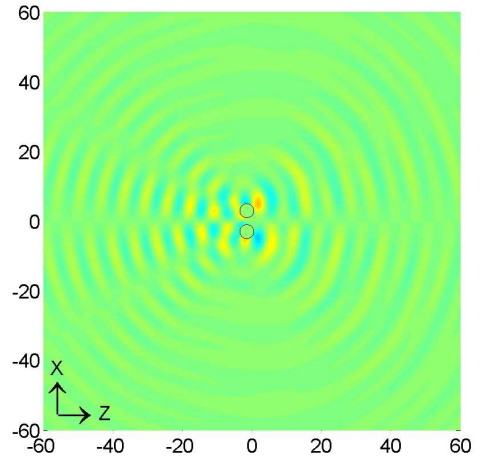

(b) $\kappa a=0.45$

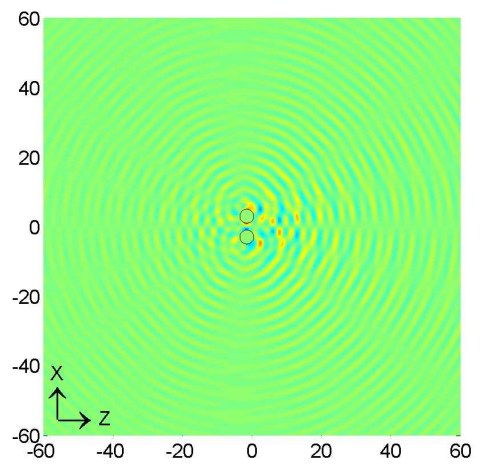

(e) $\kappa a=0.9$

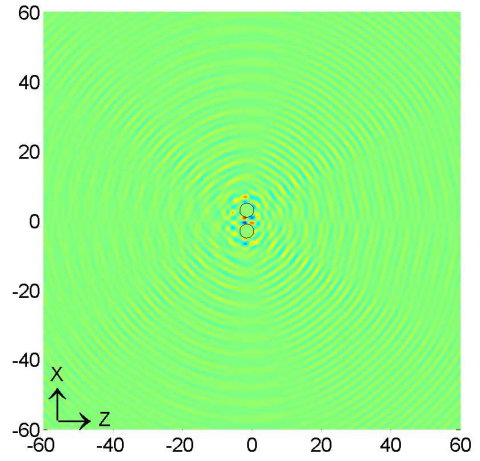

(h) $\kappa a=1.35$

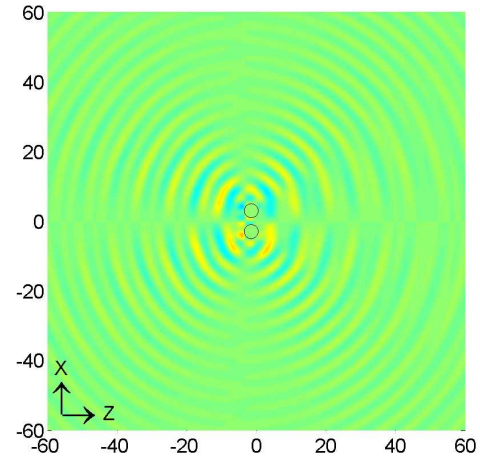

(c) $\kappa a=0.6$

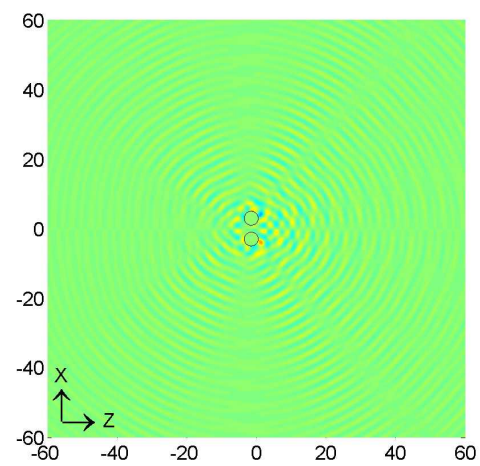

(f) $\kappa a=1.05$

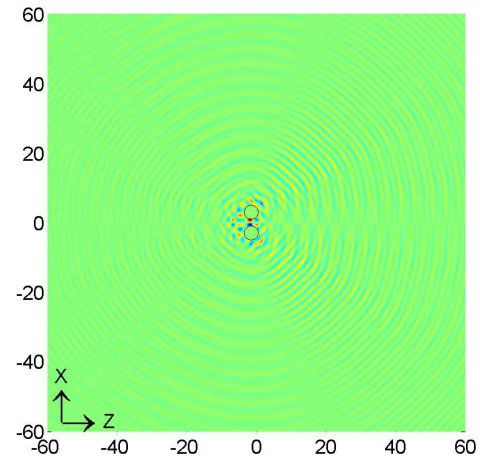

(i) $\kappa a=1.5$

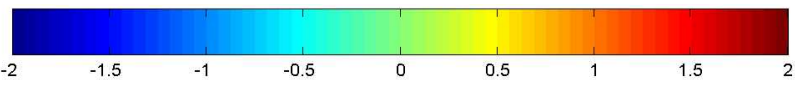

(j) Colorbar

Figure 6.22: Real part of $u_{x}$ in the $y=0$ plane of total waves for the case of two layers of scatterers. For different longitudinal wave numbers in epoxy matrix, normalized wave number $\kappa a$ is used and $a$ is radius of spherical scatterer. Wave fields inside black circles are not computed during scatterer polymerization procedure and set to zero. Values of $u_{x}$ of longitudinal incident waves are zero. 


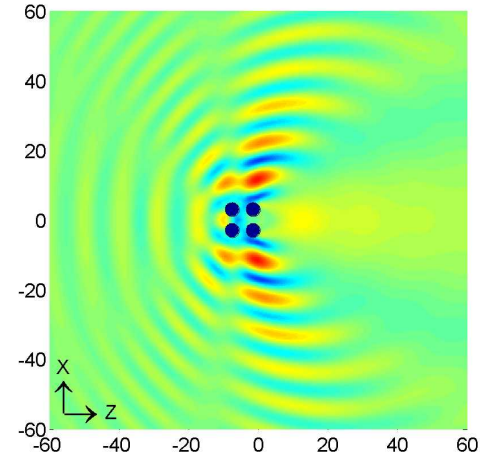

(a) $\kappa a=0.3$

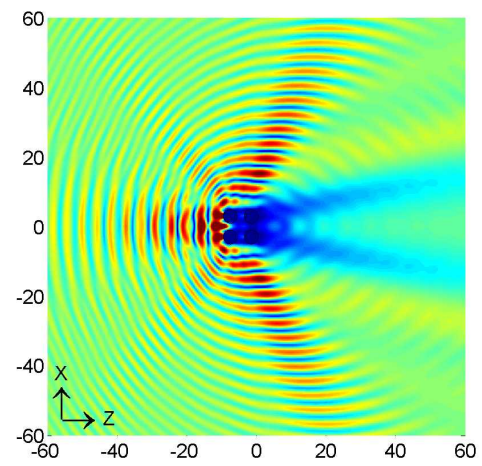

(d) $\kappa a=0.75$

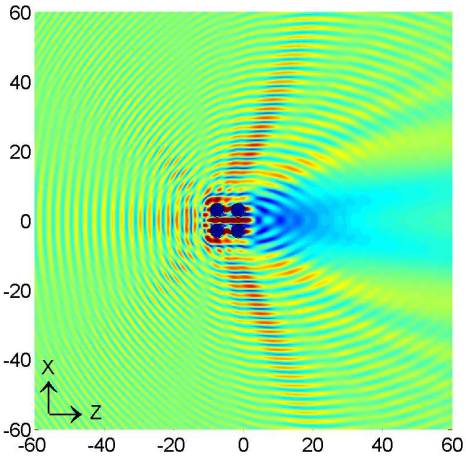

(g) $\kappa a=1.2$

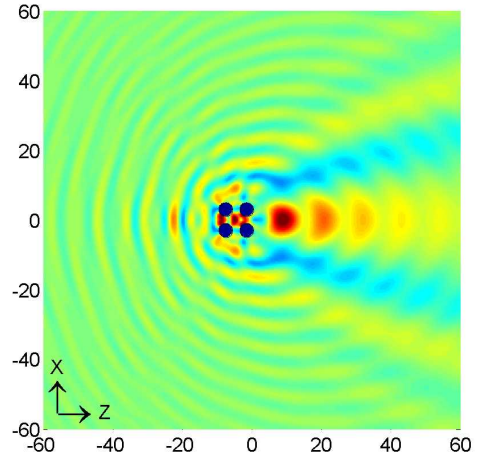

(b) $\kappa a=0.45$

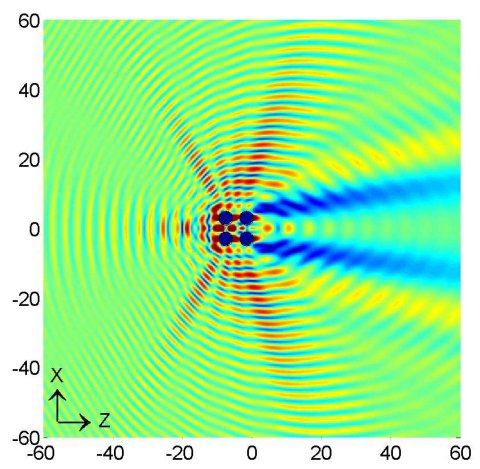

(e) $\kappa a=0.9$

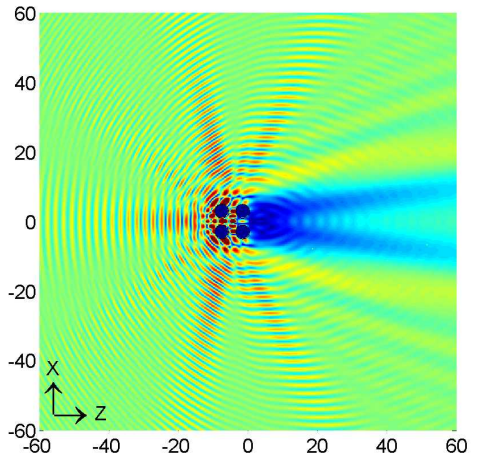

(h) $\kappa a=1.35$

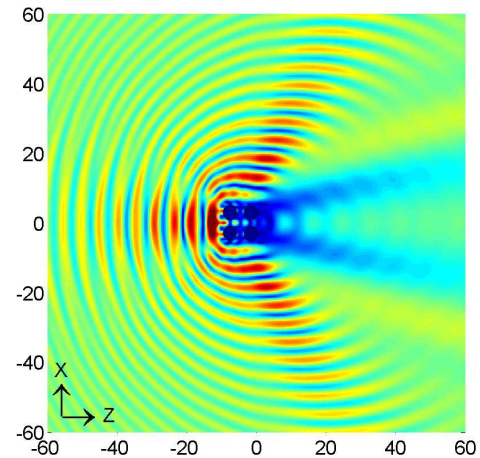

(c) $\kappa a=0.6$

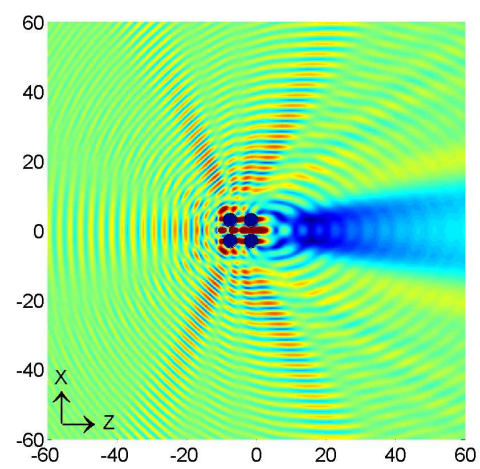

(f) $\kappa a=1.05$

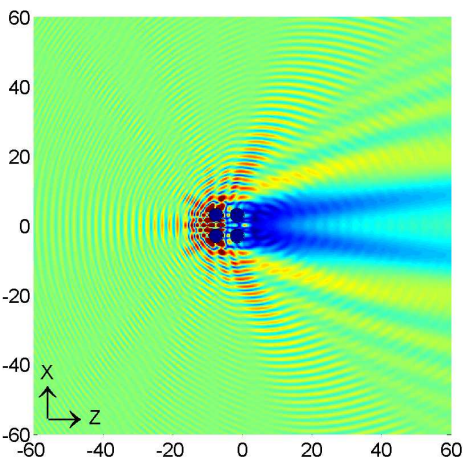

(i) $\kappa a=1.5$

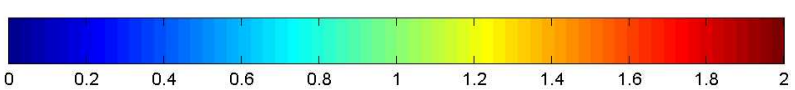

(j) Colorbar

Figure 6.23: Modulus of $u_{z}$ in the $y=0$ plane of total waves for the case of four layers of 64 scatterers. For different longitudinal wave numbers in epoxy matrix, normalized wave number $\kappa a$ is used and $a$ is radius of spherical scatterer. Wave fields inside black circles are not computed during scatterer polymerization procedure and set to zero. 


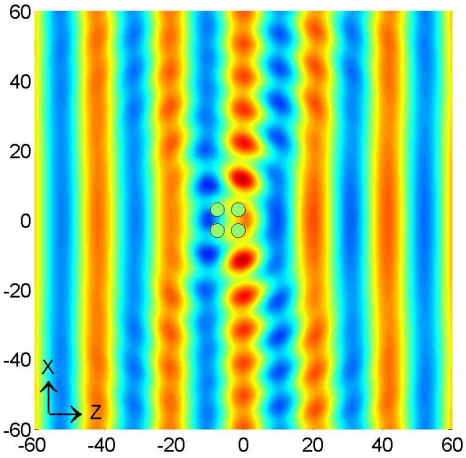

(a) $\kappa a=0.3$

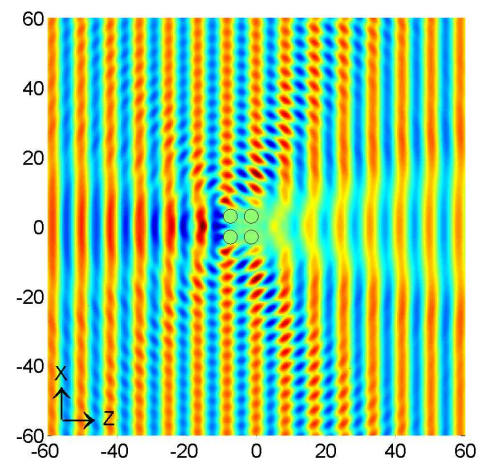

(d) $\kappa a=0.75$

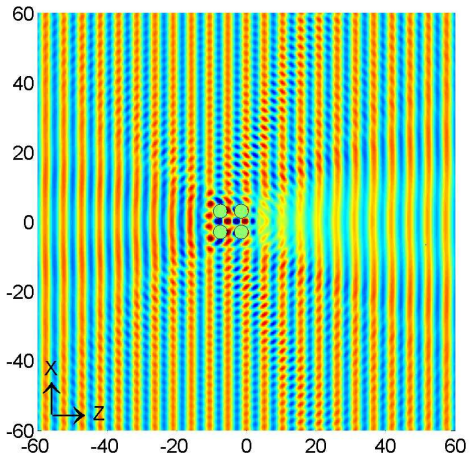

(g) $\kappa a=1.2$

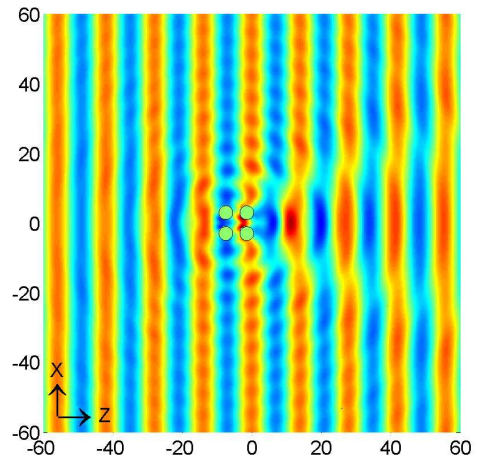

(b) $\kappa a=0.45$

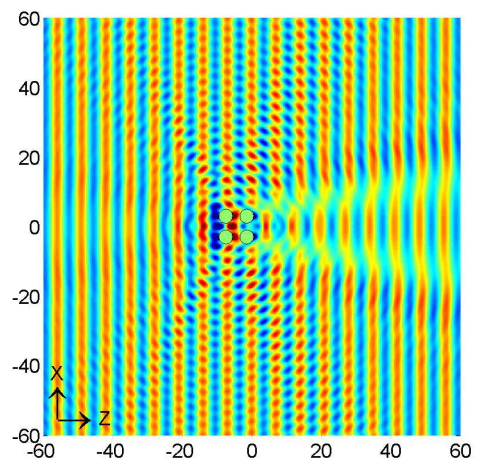

(e) $\kappa a=0.9$

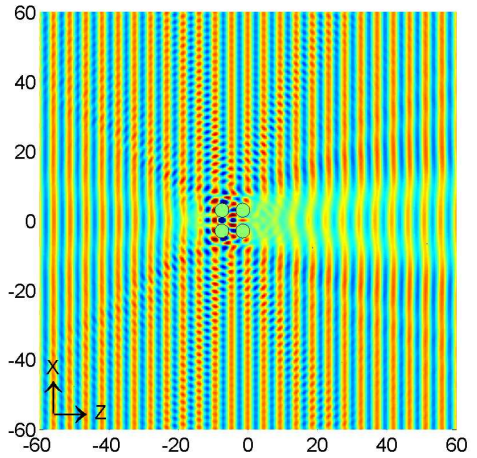

(h) $\kappa a=1.35$

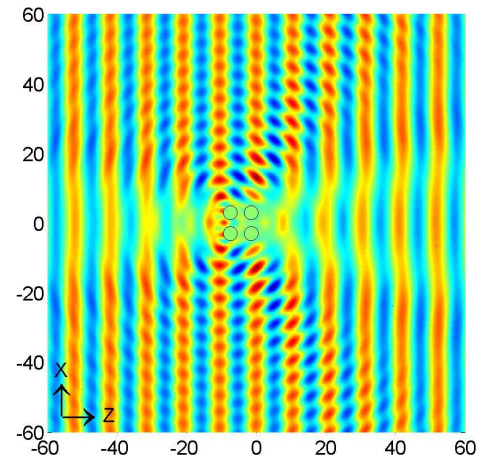

(c) $\kappa a=0.6$

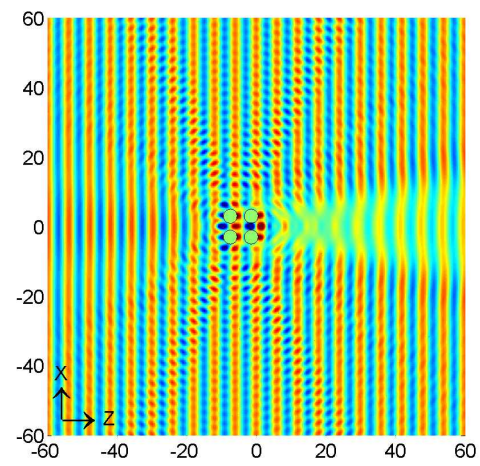

(f) $\kappa a=1.05$

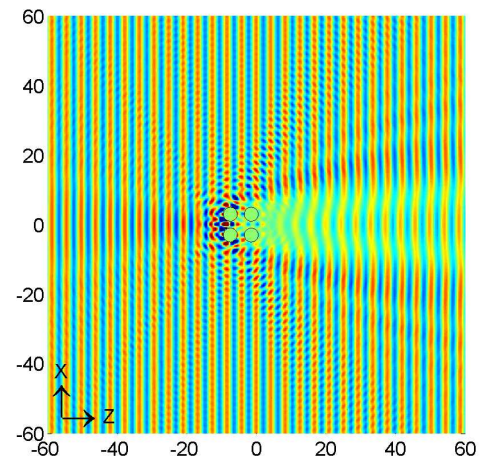

(i) $\kappa a=1.5$

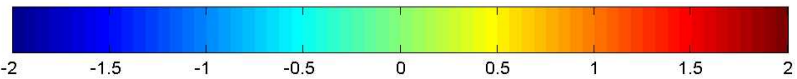

(j) Colorbar

Figure 6.24: Real part of $u_{z}$ in the $y=0$ plane of total waves for the case of four layers of 64 scatterers. For different longitudinal wave numbers in epoxy matrix, normalized wave number $\kappa a$ is used and $a$ is radius of spherical scatterer. Wave fields inside black circles are not computed during scatterer polymerization procedure and set to zero. 


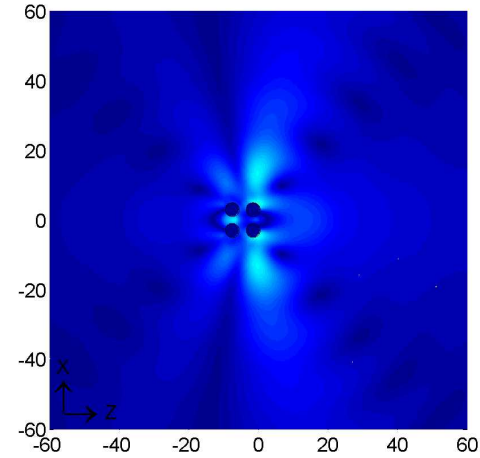

(a) $\kappa a=0.3$

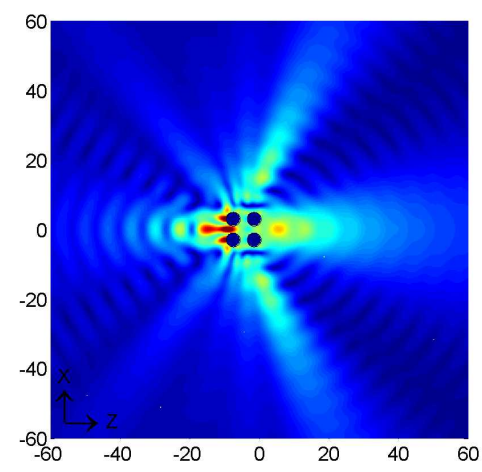

(d) $\kappa a=0.75$

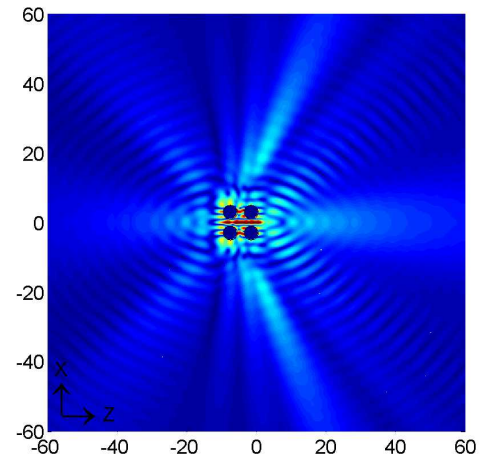

(g) $\kappa a=1.2$

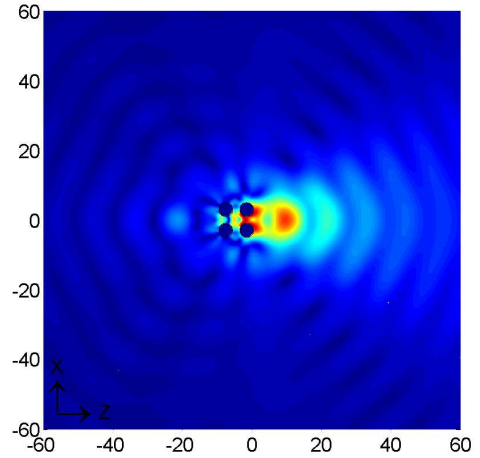

(b) $\kappa a=0.45$

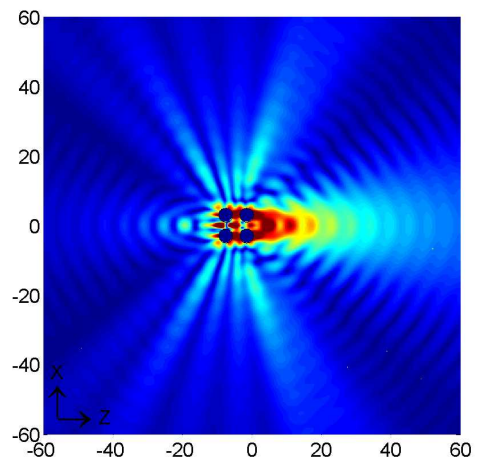

(e) $\kappa a=0.9$

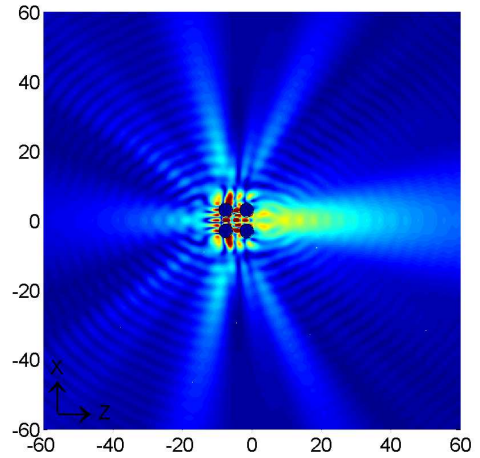

(h) $\kappa a=1.35$

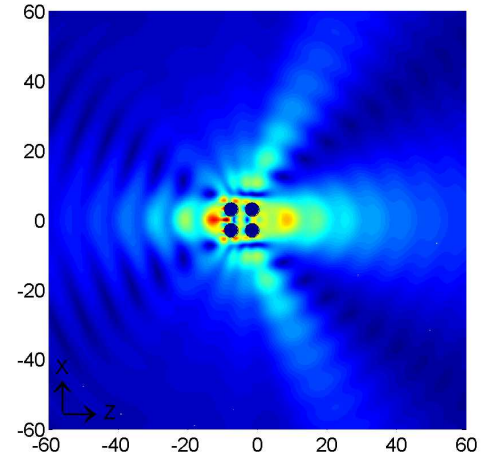

(c) $\kappa a=0.6$

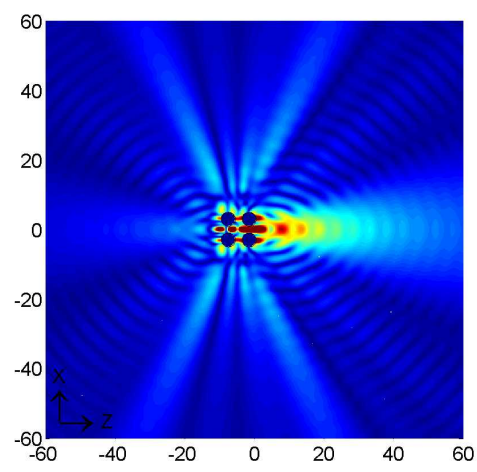

(f) $\kappa a=1.05$

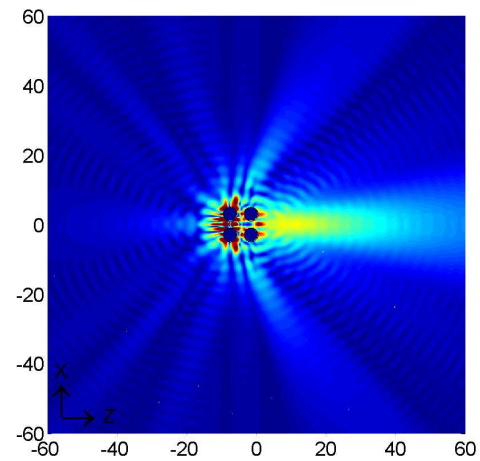

(i) $\kappa a=1.5$

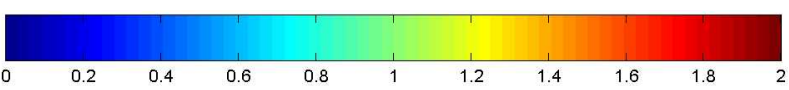

(j) Colorbar

Figure 6.25: Modulus of $u_{z}$ in the $y=0$ plane of scattered waves for the case of four layers of 64 scatterers. For different longitudinal wave numbers in epoxy matrix, normalized wave number $\kappa a$ is used and $a$ is radius of spherical scatterer. Wave fields inside black circles are not computed during scatterer polymerization procedure and set to zero. 


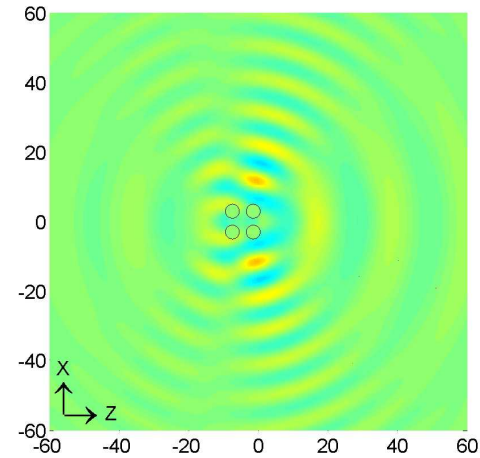

(a) $\kappa a=0.3$

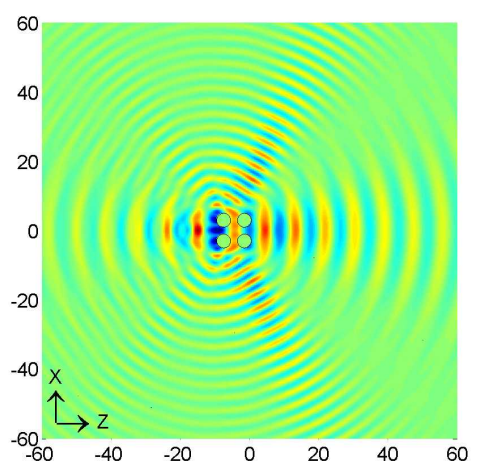

(d) $\kappa a=0.75$

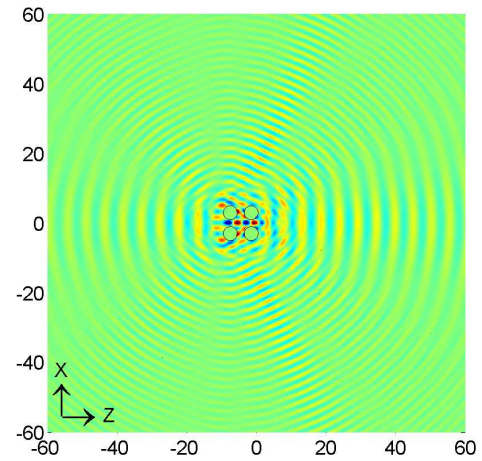

(g) $\kappa a=1.2$

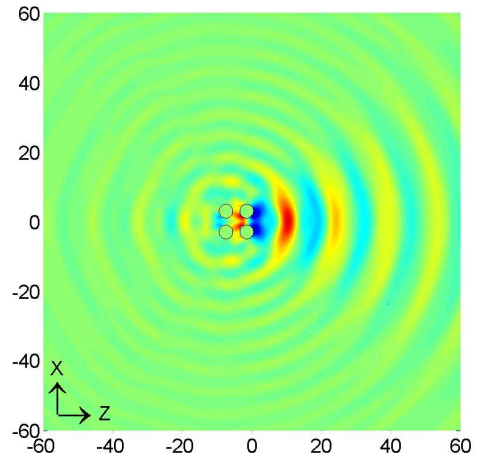

(b) $\kappa a=0.45$

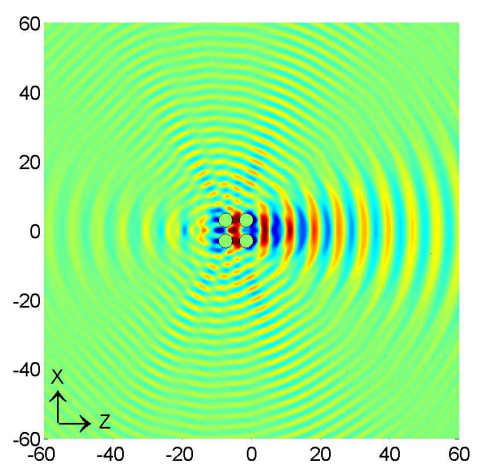

(e) $\kappa a=0.9$

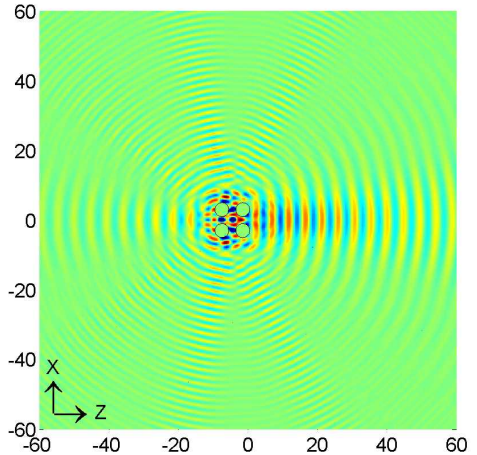

(h) $\kappa a=1.35$

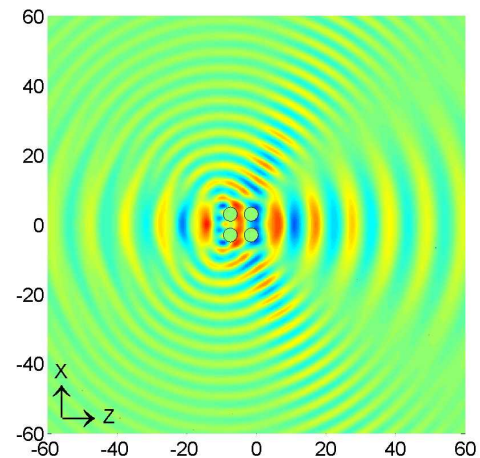

(c) $\kappa a=0.6$

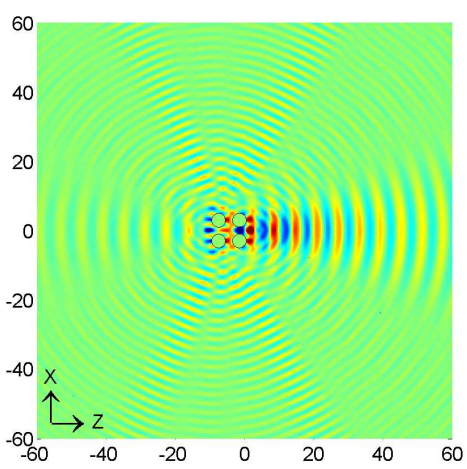

(f) $\kappa a=1.05$

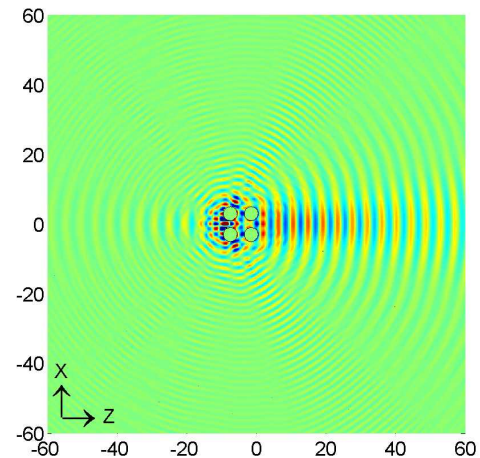

(i) $\kappa a=1.5$

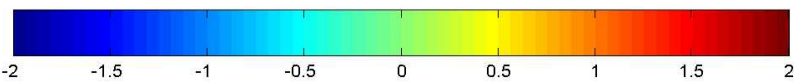

(j) Colorbar

Figure 6.26: Real part of $u_{z}$ in the $y=0$ plane of scattered waves for the case of four layers of 64 scatterers. For different longitudinal wave numbers in epoxy matrix, normalized wave number $\kappa a$ is used and $a$ is radius of spherical scatterer. Wave fields inside black circles are not computed during scatterer polymerization procedure and set to zero. 
Fig. 6.20. In the shadow region and illuminated region, values of periodic peaks in Fig. 6.20 appear slightly larger than those in Fig. 6.20 (through comparison of color in two figures).

The pattern of distribution of modulus of $u_{x}$ in Fig. 6.27 is similar as that in Fig. 6.21. Directions, along which values of periodic peaks are larger than those in other directions, in Fig. 6.27 are different from those in Fig. 6.21. Values of periodic peaks in Fig. 6.27 appear slightly larger than those in Fig. 6.21 (through comparison of color in two figures).

The pattern of distribution of real part of $u_{x}$ in Fig. 6.28 is similar as that in Fig. 6.22. Values of periodic peaks in Fig. 6.28 appear slightly larger than those in Fig. 6.22 (through comparison of color in two figures). 


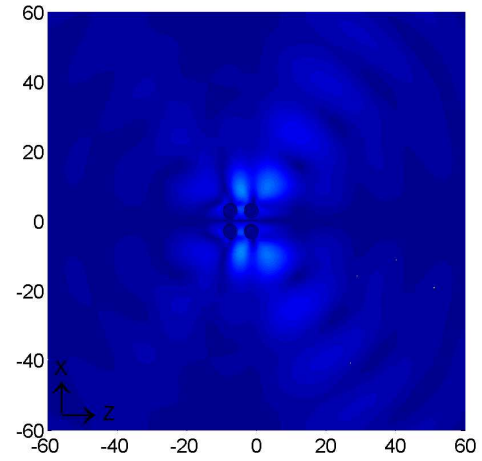

(a) $\kappa a=0.3$

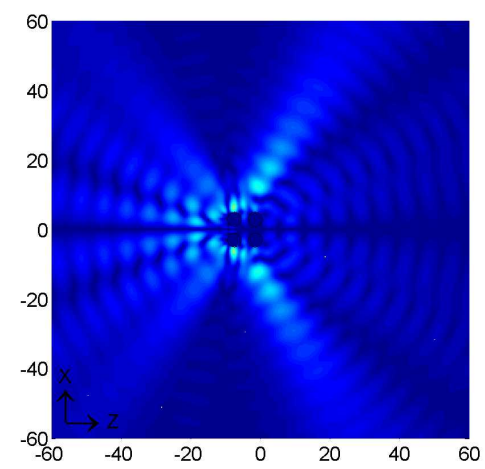

(d) $\kappa a=0.75$

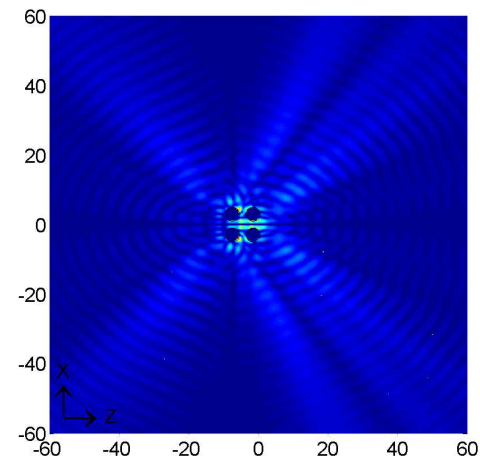

(g) $\kappa a=1.2$

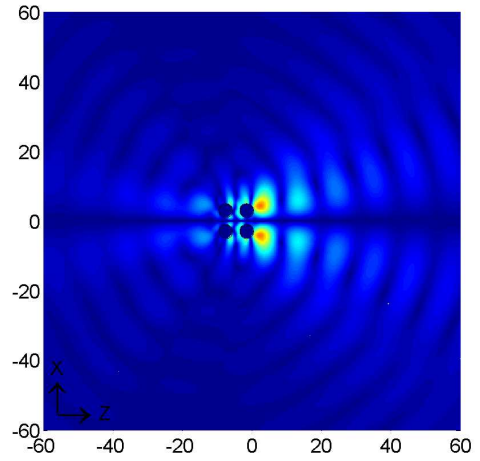

(b) $\kappa a=0.45$

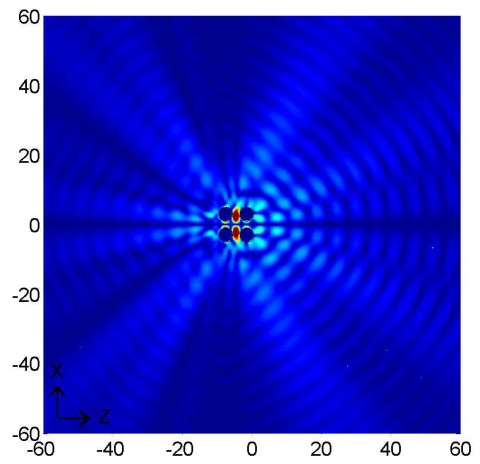

(e) $\kappa a=0.9$

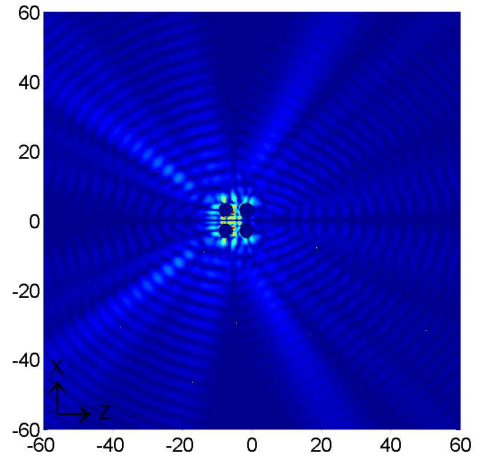

(h) $\kappa a=1.35$

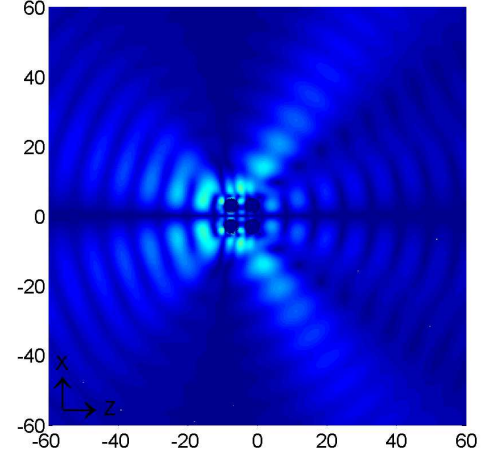

(c) $\kappa a=0.6$

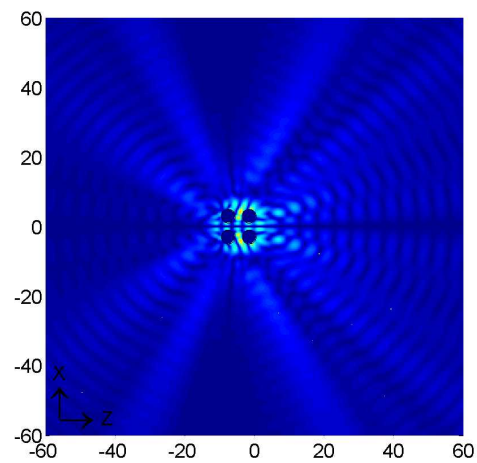

(f) $\kappa a=1.05$

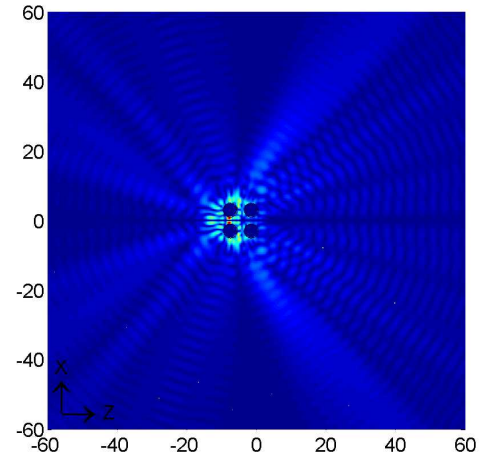

(i) $\kappa a=1.5$

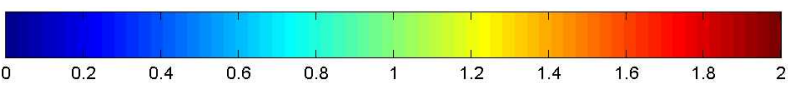

(j) Colorbar

Figure 6.27: Modulus of $u_{x}$ in the $y=0$ plane of total waves for the case of four layers of 64 scatterers. For different longitudinal wave numbers in epoxy matrix, normalized wave number $\kappa a$ is used and $a$ is radius of spherical scatterer. Wave fields inside black circles are not computed during scatterer polymerization procedure and set to zero. Values of $u_{x}$ of longitudinal incident waves are zero. 


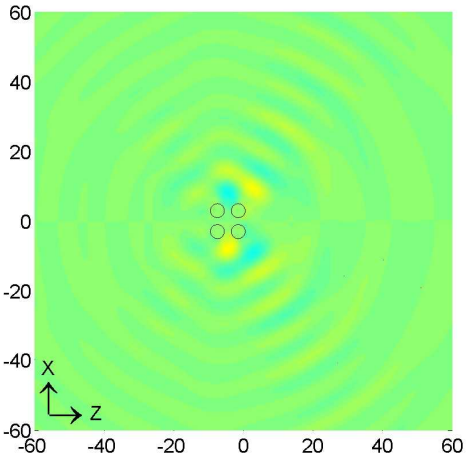

(a) $\kappa a=0.3$

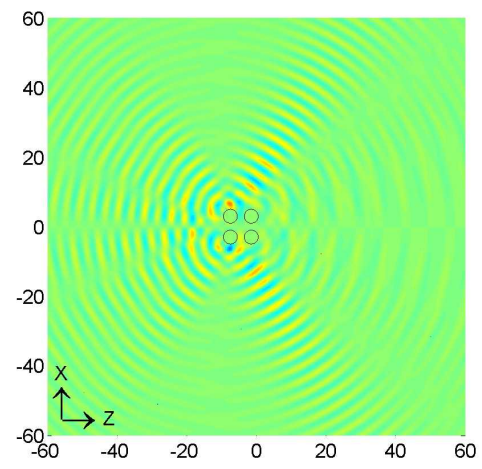

(d) $\kappa a=0.75$

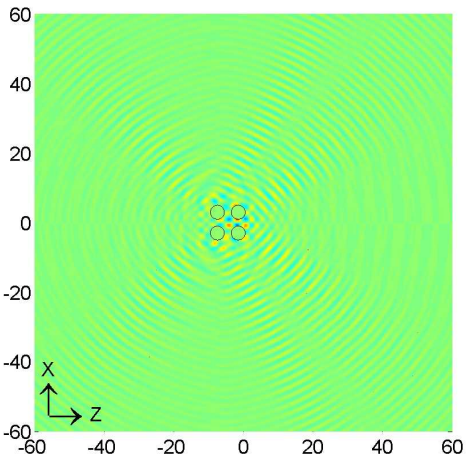

(g) $\kappa a=1.2$

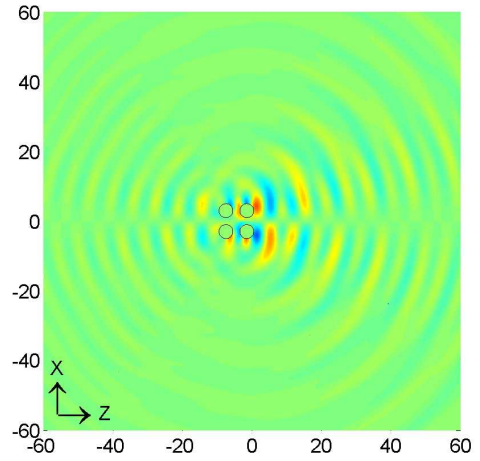

(b) $\kappa a=0.45$

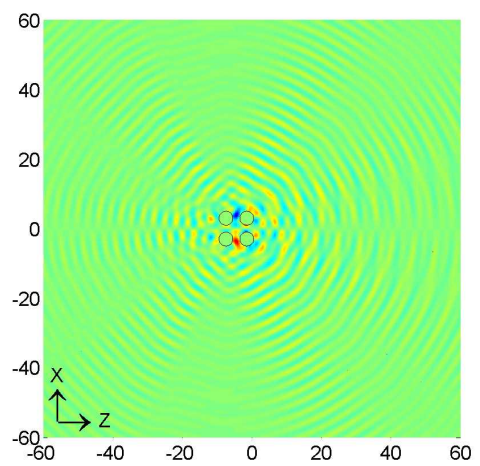

(e) $\kappa a=0.9$

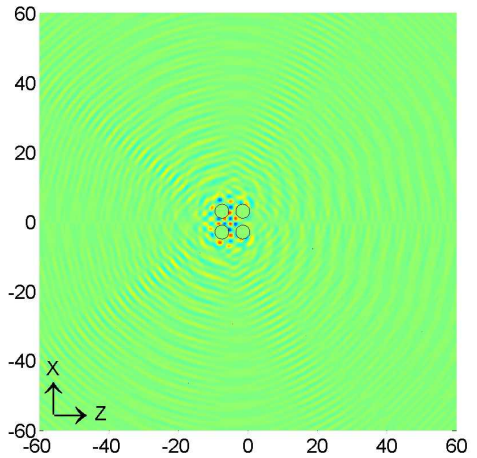

(h) $\kappa a=1.35$

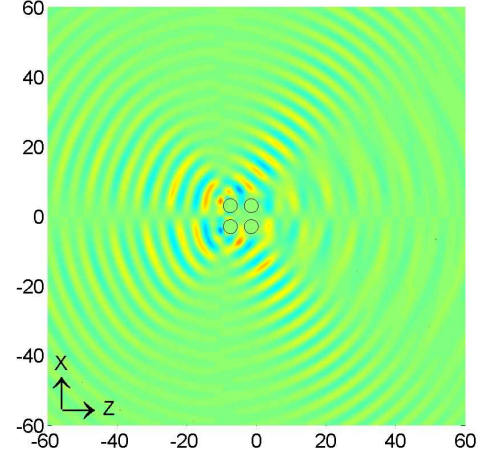

(c) $\kappa a=0.6$

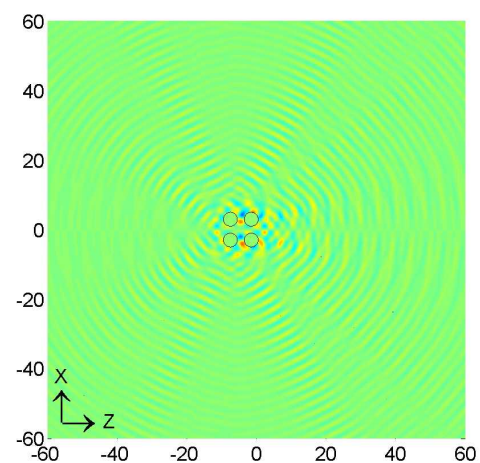

(f) $\kappa a=1.05$

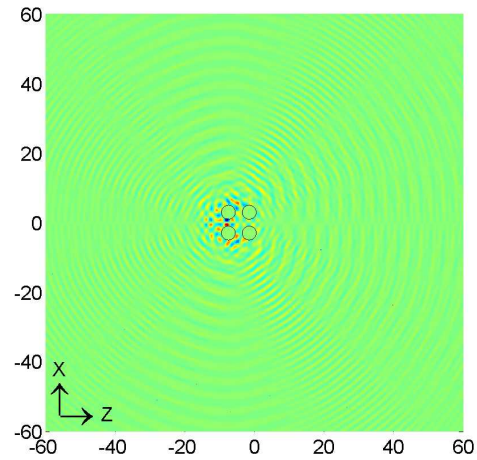

(i) $\kappa a=1.5$

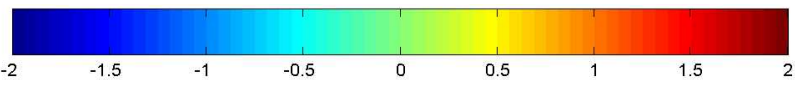

(j) Colorbar

Figure 6.28: Real part of $u_{x}$ in the $y=0$ plane of total waves for the case of four layers of 64 scatterers. For different longitudinal wave numbers in epoxy matrix, normalized wave number $\kappa a$ is used and $a$ is radius of spherical scatterer. Wave fields inside black circles are not computed during scatterer polymerization procedure and set to zero. Values of $u_{x}$ of longitudinal incident waves are zero. 


\section{6-4.3 Stresses for Different Number of Layers}

In this section, stress components in Cartesian coordinate system in the $y=0$ plane are illustrated as a part of the simulation results. In Fig. 6.9, the geometric relation of scatterer arrangement and the $y=0$ plane is shown. In the calculation, the spacing distance between two scatterers is three time of the scatterer's radius. According to the stresses due to the incident waves in Eqs. (2-138b) through (2-138e), $\sigma_{x y}^{i n c}, \sigma_{x z}^{i n c}$ and $\sigma_{y z}^{i n c}$ are zero, and other stresses are

$$
\begin{aligned}
& \sigma_{x x}^{i n c}=\lambda(-\hat{\imath} \kappa) e^{-\hat{\imath} \kappa z}, \\
& \sigma_{y y}^{i n c}=\lambda(-\hat{\imath} \kappa) e^{-\hat{\imath} \kappa z}, \\
& \sigma_{z z}^{i n c}=(\lambda+2 \mu)(-\hat{\imath} \kappa) e^{-\hat{\imath} \kappa z} .
\end{aligned}
$$

Magnitude of $\sigma_{x x}^{i n c}$ and $\sigma_{y y}^{i n c}$ are approximately half of that of $\sigma_{z z}^{i n c}$. The stress magnitudes of an incident wave are proportional to its wave number. It is not convenient to compare stress components among different wave frequencies. In stead, stress components of same wave frequency are compared among the cases of one layer, two layers and four layers of scatterers. Normalized wave number $\kappa a$ is used to indicate wave frequency. As examples, figures are given for $\kappa a=0.3,0.75$, and 1.2 .

For each wave number, stresses $\sigma_{x x}, \sigma_{y y}$ and $\sigma_{z z}$ are put together for comparison and are arranged in the following series: modulus of $\sigma_{x x}, \sigma_{y y}$ and $\sigma_{z z}$ of total waves are followed by real part of $\sigma_{x x}, \sigma_{y y}$ and $\sigma_{z z}$ of total waves, and followed by modulus of $\sigma_{x x}, \sigma_{y y}$ and $\sigma_{z z}$ of scattered waves, and followed by real part of $\sigma_{x x}, \sigma_{y y}$ and $\sigma_{z z}$ of scattered waves. The stress $\sigma_{x z}$ is displayed separately. Figures for $\kappa a=0.3$ is followed by figures for $\kappa a=0.75$ and then followed by figures for $\kappa a=1.2$. (The stress $\sigma_{x y}$ and $\sigma_{y z}$ are not displayed. For example, when $\kappa a=1.05$ the maximum value of $\left|\sigma_{x y}\right|$ and $\left|\sigma_{y z}\right|$ is smaller than $4 \times 10^{5}$. In comparison with modulus of other

stresses that are on the order of $10^{9}, \sigma_{x y}$ and $\sigma_{y z}$ are approximately zero. Therefore, 
the stress $\sigma_{x y}$ and $\sigma_{y z}$ are not displayed. )

In Fig. 6.29, $\left|\sigma_{x x}\right|$ and $\left|\sigma_{y y}\right|$ are approximately half of $\left|\sigma_{z z}\right|$, and distribution of modulus of $\sigma_{x x}, \sigma_{y y}$ and $\sigma_{z z}$ are symmetric about $x=0$ axis. In the shadow region, values of $\left|\sigma_{x x}\right|,\left|\sigma_{y y}\right|$ and $\left|\sigma_{z z}\right|$ increase when the number of layers increases. The distribution patterns of $\left|\sigma_{x x}\right|$ and $\left|\sigma_{z z}\right|$ are similar. In the sub-figures of modulus of $\sigma_{y y}$, the edge of the illuminated region form an angle with $Z$-axis. The angle in Fig. 6.29(e) is larger than that in Fig. 6.29(d) but smaller than that in Fig. 6.29(f).

In Fig. 6.30, real part of $\sigma_{x x}$ and $\sigma_{y y}$ are approximately half of real part of $\sigma_{z z}$, and distribution of real part of $\sigma_{x x}, \sigma_{y y}$ and $\sigma_{z z}$ are symmetric about $x=0$ axis. The stresses of incident wave dominate the field.

In Fig. 6.31, distribution of modulus of $\sigma_{x x}, \sigma_{y y}$ and $\sigma_{z z}$ are symmetric about $x=0$ axis. In the shadow region, values of modulus of $\sigma_{x x}, \sigma_{y y}$ and $\sigma_{z z}$ increase slightly when the number of layers increases. The distribution patterns of modulus of $\sigma_{x x}$ and $\sigma_{z z}$ are similar. In the sub-figures of modulus of $\sigma_{y y}$, the distribution appear like two bulbs in the shadow region and the illuminated region. The size of the bulb in the shadow region increases when the number of layers in creases.

In Fig. 6.32, distribution of real part of $\sigma_{x x}, \sigma_{y y}$ and $\sigma_{z z}$ are symmetric about $x=0$ axis. In the shadow region, values of real part of $\sigma_{x x}, \sigma_{y y}$ and $\sigma_{z z}$ increase slightly when the number of layers increases. The distribution patterns of real part of $\sigma_{x x}$ and $\sigma_{z z}$ are similar, and the spacing between periodic peaks in the shadow region and the illuminated region are two times of that in other regions. The spacing between periodic peaks in the sub-figures of real part of $\sigma_{y y}$, is the same as that in the shadow region in the sub-figures of real part of $\sigma_{x x}$ and $\sigma_{z z}$.

The stress component $\sigma_{x z}$ due to the incident wave is zero, so that the nonzero result of $\sigma_{x z}$ is due to scattered waves. The distribution of modulus of $\sigma_{x z}$ is symmetric about $x=0$ axis. In Fig. 6.33, values of $\left|\sigma_{x z}\right|$ in the shadow region and 


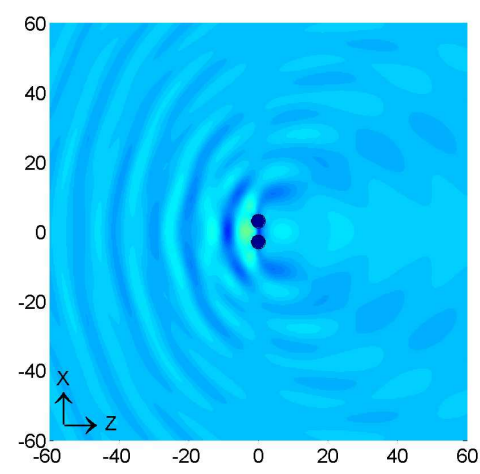

(a) $\left|\sigma_{x x}\right|: 1$ layer

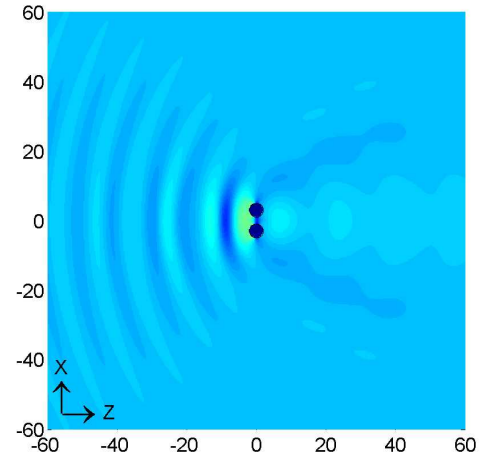

(d) $\left|\sigma_{y y}\right|: 1$ layer

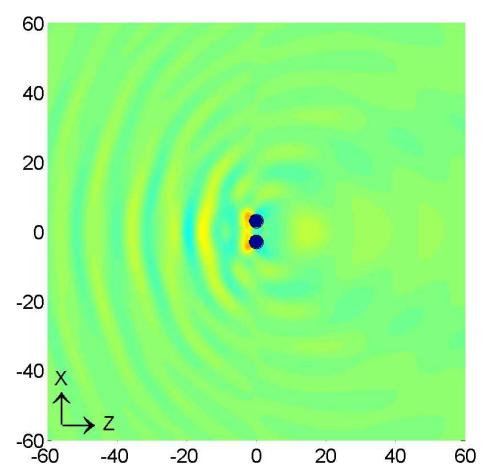

(g) $\left|\sigma_{z z}\right|: 1$ layer

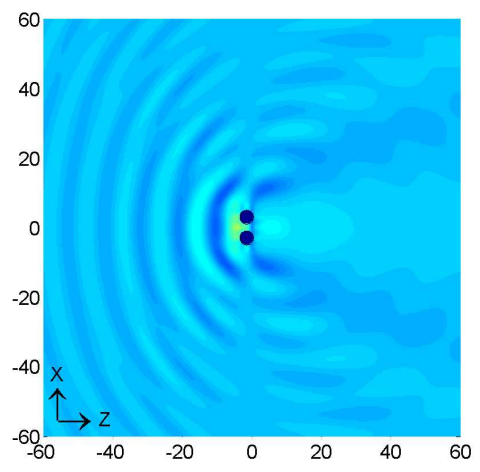

(b) $\left|\sigma_{x x}\right|: 2$ layers

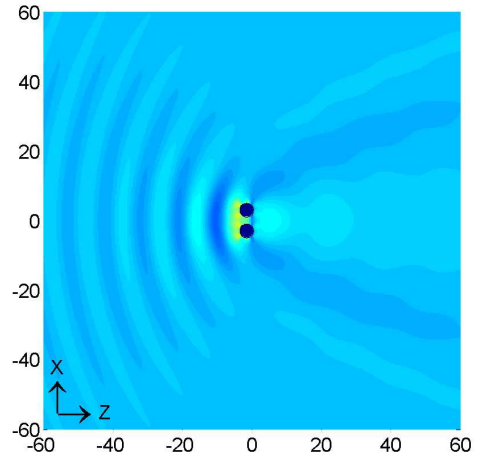

(e) $\left|\sigma_{y y}\right|: 2$ layers

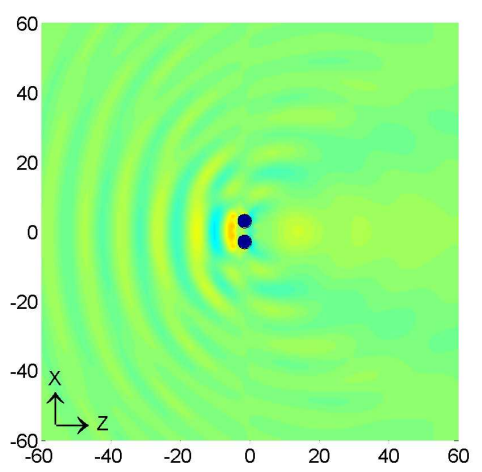

(h) $\left|\sigma_{z z}\right|: 2$ layers

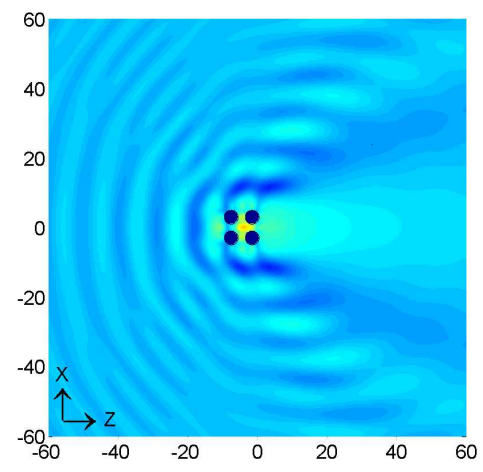

(c) $\left|\sigma_{x x}\right|: 4$ layers

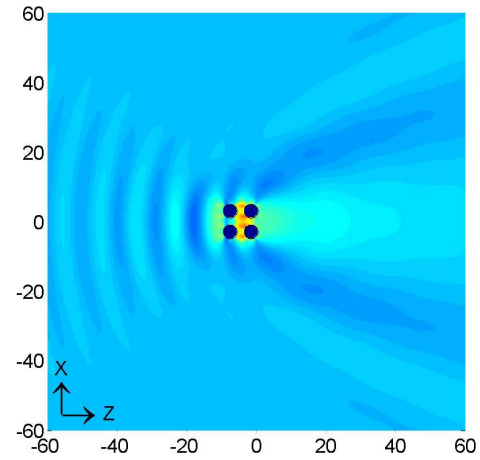

(f) $\left|\sigma_{y y}\right|: 4$ layers

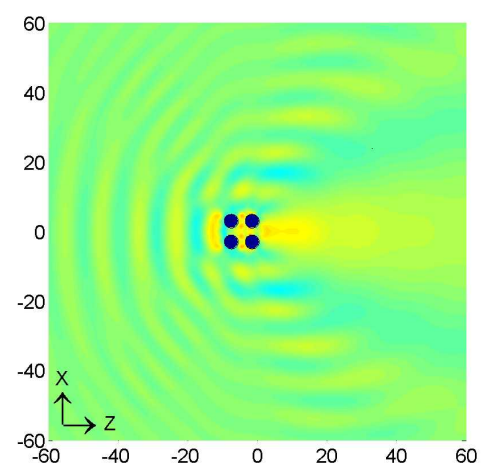

(i) $\left|\sigma_{z z}\right|: 4$ layers

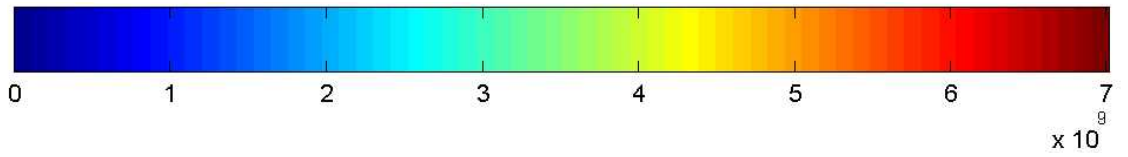

(j) Colorbar

Figure 6.29: Modulus of stresses $\sigma_{x x}, \sigma_{y y}$ and $\sigma_{z z}$ in the $y=0$ plane of total waves at $\kappa a=0.3$ and for three cases of one layer, two and four layers. Wave fields inside black circles are not computed during scatterer polymerization procedure and set to zero. 


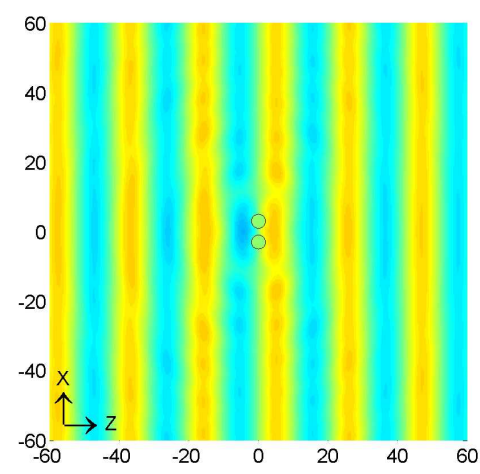

(a) $\sigma_{x x}: 1$ layer

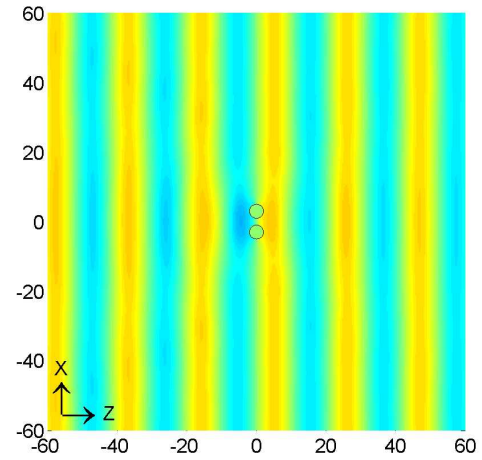

(d) $\sigma_{y y}: 1$ layer

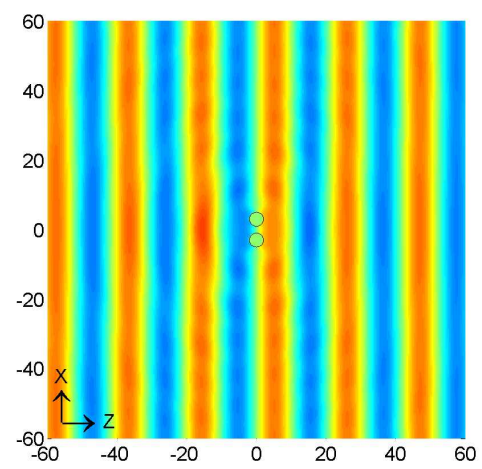

(g) $\sigma_{z z}: 1$ layer

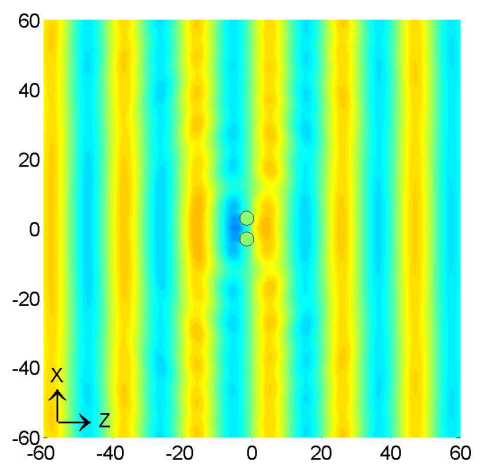

(b) $\sigma_{x x}: 2$ layers

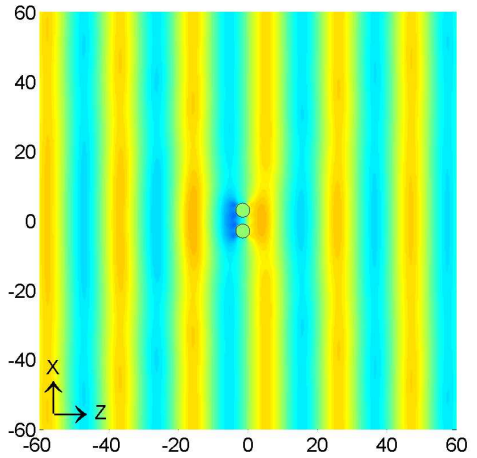

(e) $\sigma_{y y}: 2$ layers

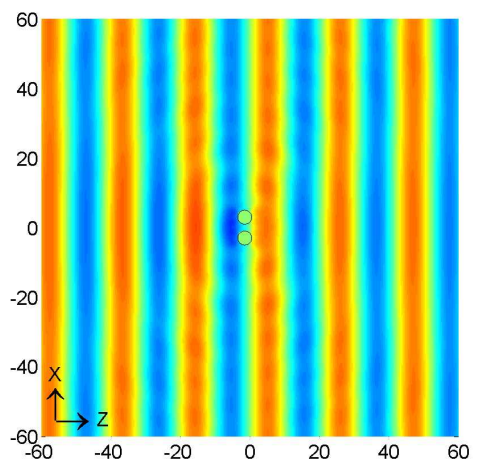

(h) $\sigma_{z z}: 2$ layers

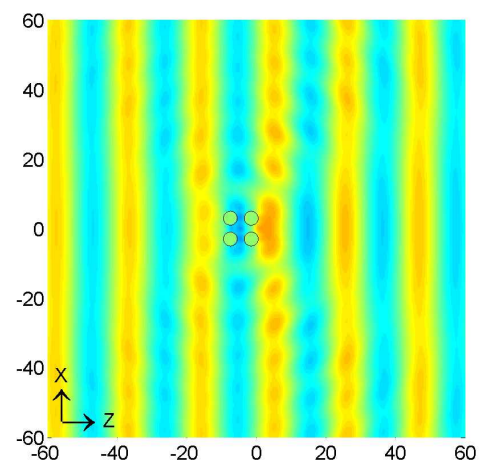

(c) $\sigma_{x x}: 4$ layers

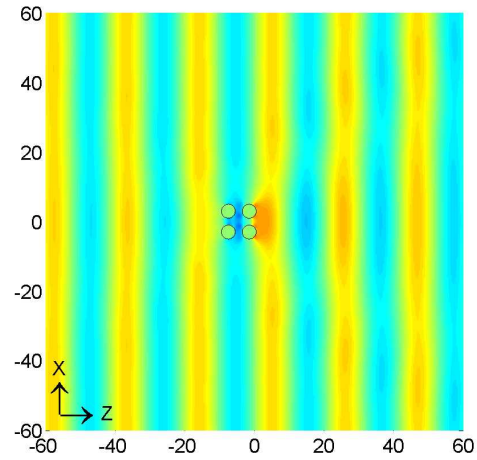

(f) $\sigma_{y y}: 4$ layers

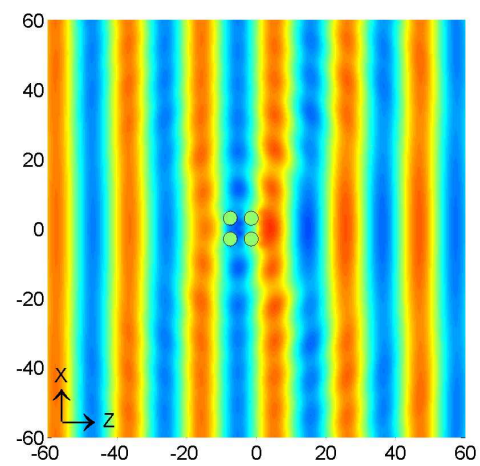

(i) $\sigma_{z z}: 4$ layers

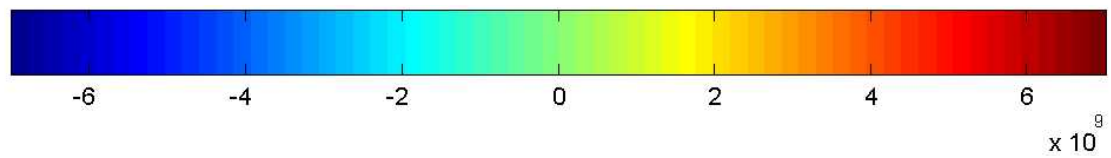

(j) Colorbar

Figure 6.30: Real part of stresses $\sigma_{x x}, \sigma_{y y}$ and $\sigma_{z z}$ in the $y=0$ plane of total waves at $\kappa a=0.3$ and for three cases of one layer, two and four layers. Wave fields inside black circles are not computed during scatterer polymerization procedure and set to zero. 


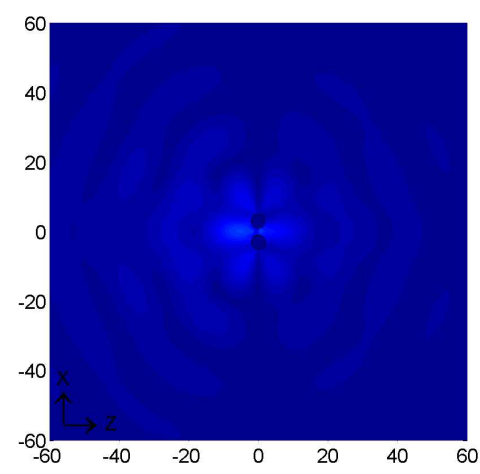

(a) $\left|\sigma_{x x}\right|: 1$ layer

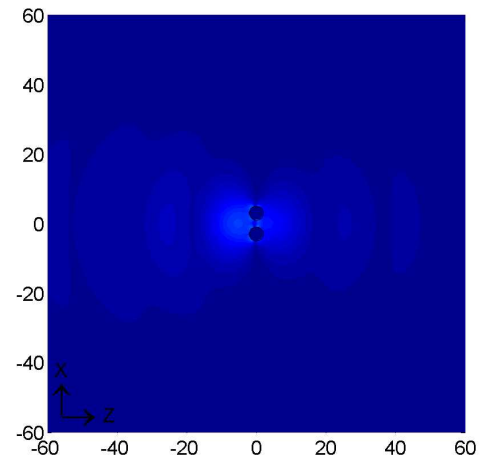

(d) $\left|\sigma_{y y}\right|: 1$ layer

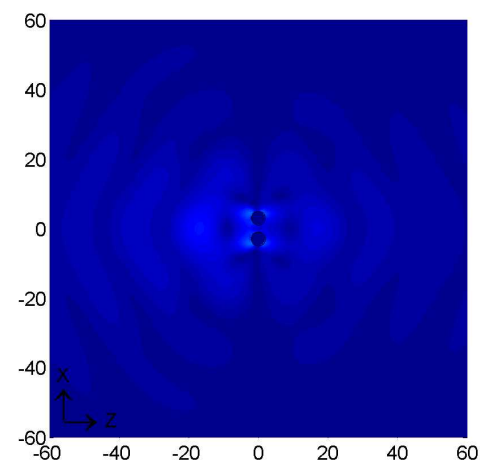

(g) $\left|\sigma_{z z}\right|: 1$ layer

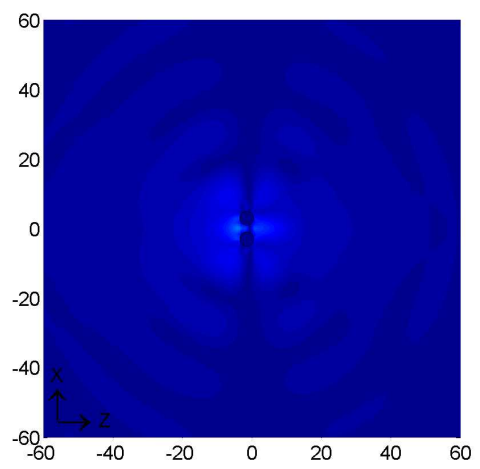

(b) $\left|\sigma_{x x}\right|: 2$ layers

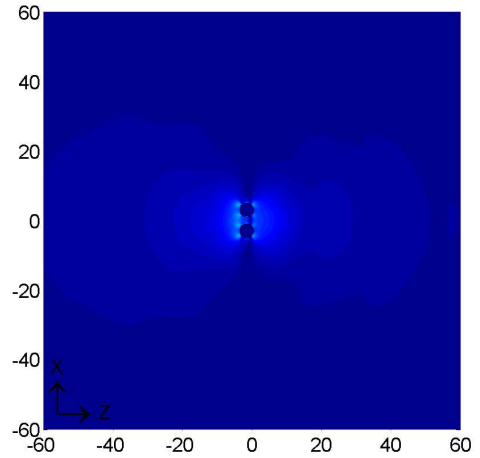

(e) $\left|\sigma_{y y}\right|: 2$ layers

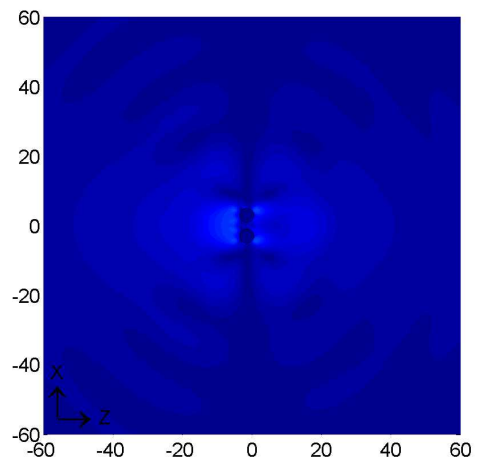

(h) $\left|\sigma_{z z}\right|: 2$ layers

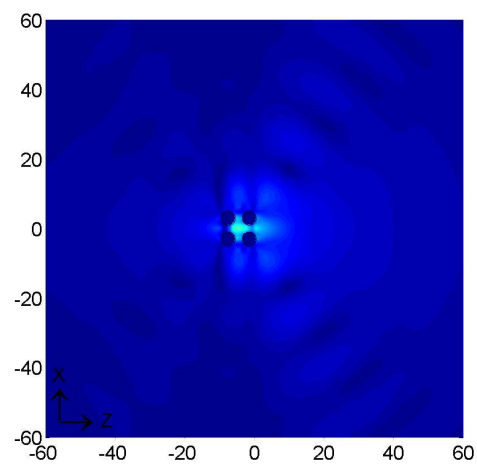

(c) $\left|\sigma_{x x}\right|: 4$ layers

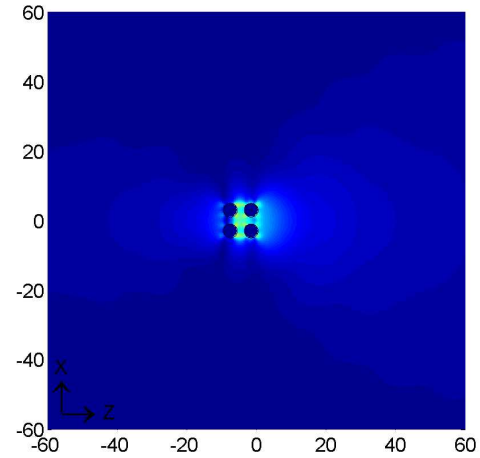

(f) $\left|\sigma_{y y}\right|: 4$ layers

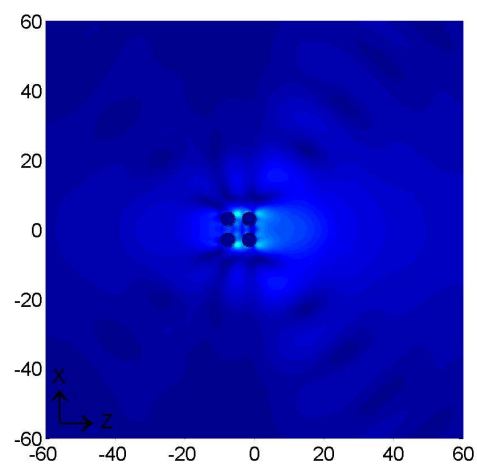

(i) $\left|\sigma_{z z}\right|: 4$ layers

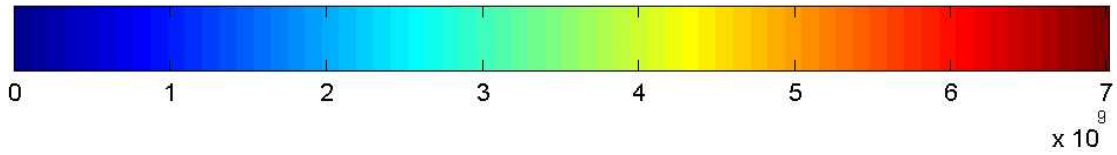

(j) Colorbar

Figure 6.31: Modulus of stresses $\sigma_{x x}, \sigma_{y y}$ and $\sigma_{z z}$ in the $y=0$ plane of scattered waves at $\kappa a=0.3$ and for three cases of one layer, two and four layers. Wave fields inside black circles are not computed during scatterer polymerization procedure and set to zero. 


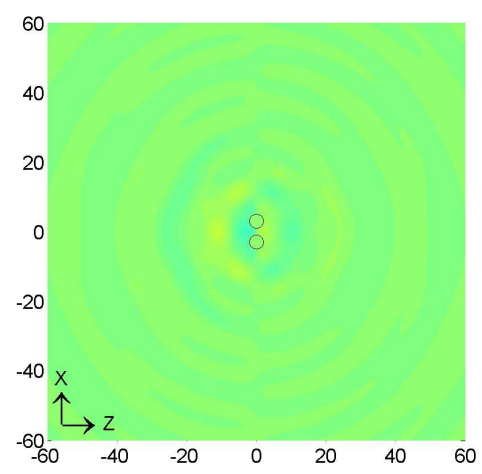

(a) $\sigma_{x x}: 1$ layer

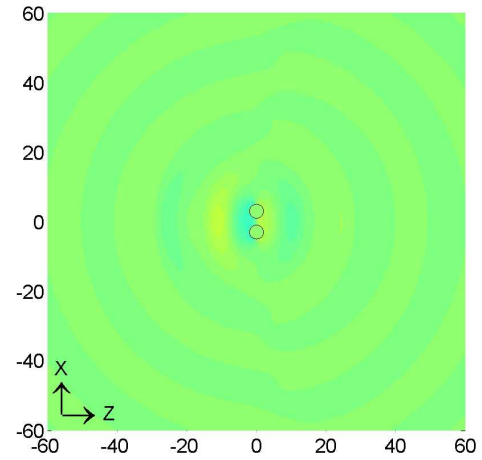

(d) $\sigma_{y y}: 1$ layer

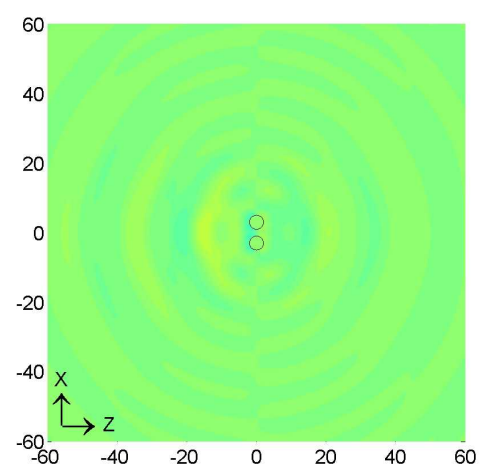

(g) $\sigma_{z z}: 1$ layer

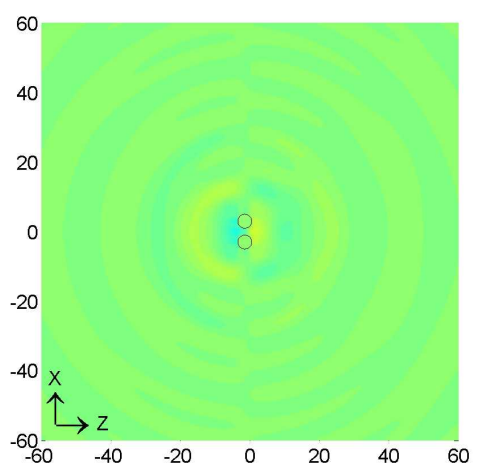

(b) $\sigma_{x x}: 2$ layers

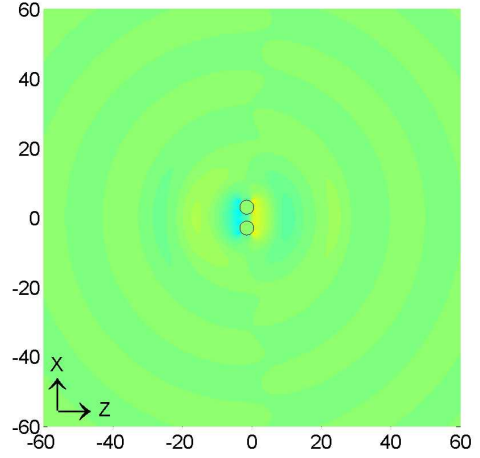

(e) $\sigma_{y y}: 2$ layers

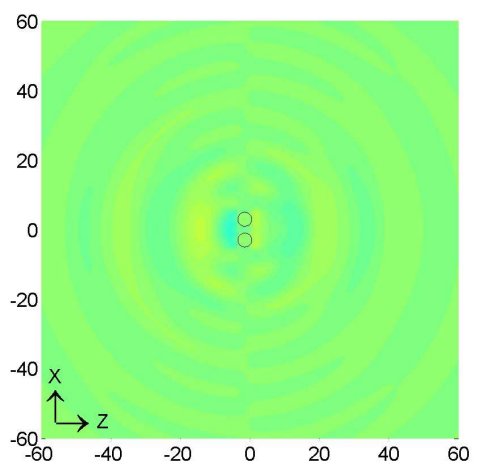

(h) $\sigma_{z z}: 2$ layers

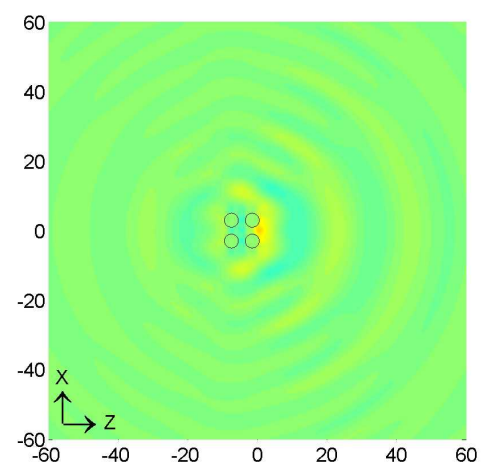

(c) $\sigma_{x x}: 4$ layers

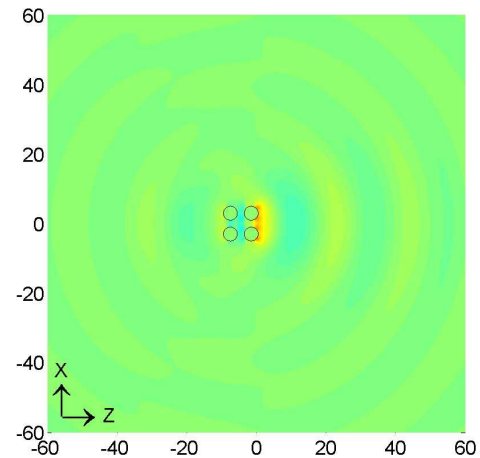

(f) $\sigma_{y y}: 4$ layers

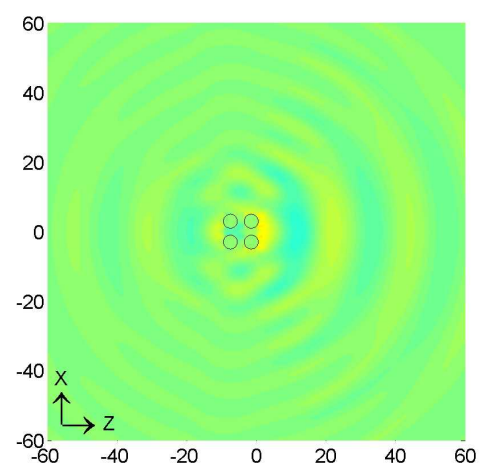

(i) $\sigma_{z z}: 4$ layers

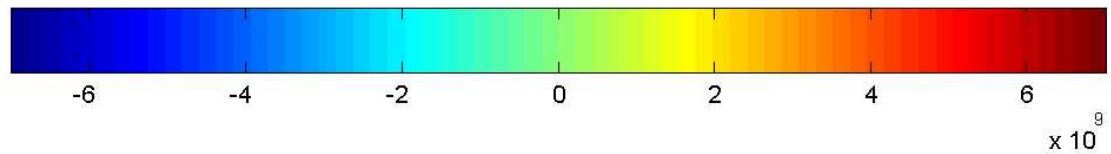

(j) Colorbar

Figure 6.32: Real part of stresses $\sigma_{x x}, \sigma_{y y}$ and $\sigma_{z z}$ in the $y=0$ plane of scattered waves at $\kappa a=0.3$ and for three cases of one layer, two and four layers. Wave fields inside black circles are not computed during scatterer polymerization procedure and set to zero. 


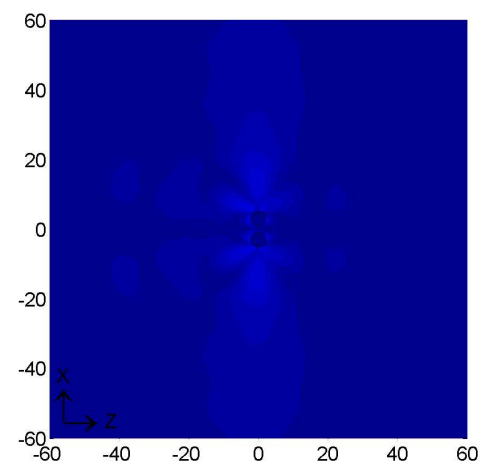

(a) $\left|\sigma_{x z}\right|: 1$ layer

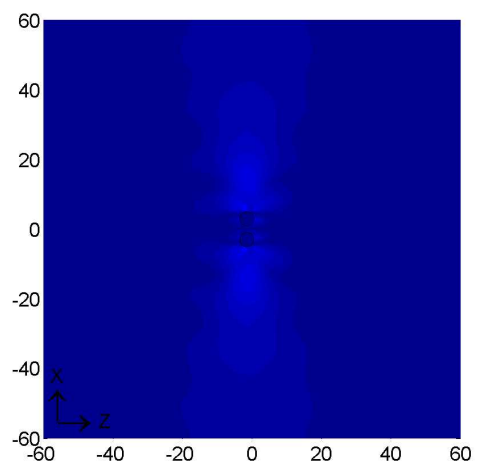

(b) $\left|\sigma_{x z}\right|: 2$ layers

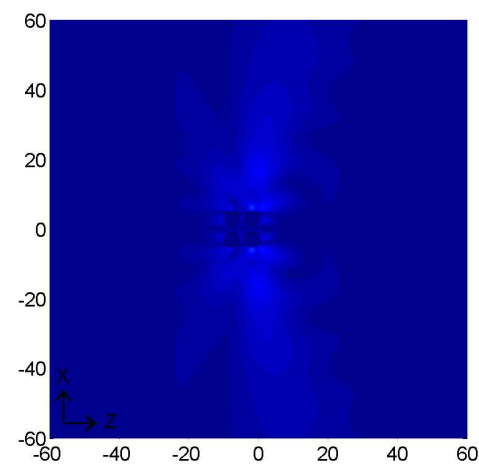

(c) $\left|\sigma_{x z}\right|: 4$ layers

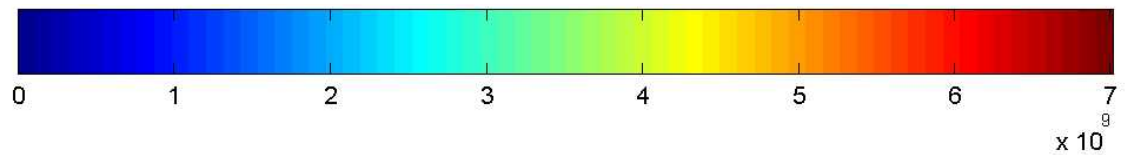

(d) Colorbar

Figure 6.33: Modulus of stress $\sigma_{x z}$ in the $y=0$ plane of total waves at $\kappa a=0.3$ and for three cases of one layer, two and four layers. Stress $\sigma_{x z}$ due to the incident wave is zero. Wave fields inside black circles are not computed during scatterer polymerization procedure and set to zero.

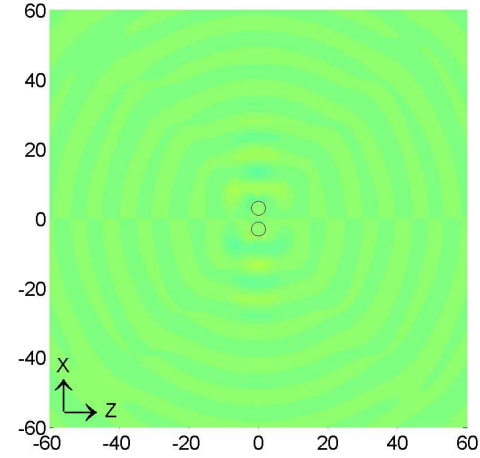

(a) $\sigma_{x z}: 1$ layer

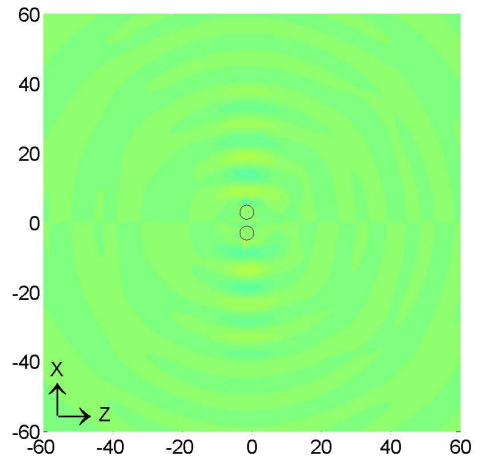

(b) $\sigma_{x z}: 2$ layers

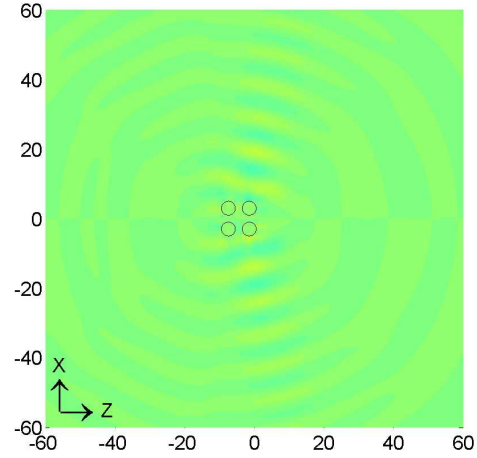

(c) $\sigma_{x z}: 4$ layers

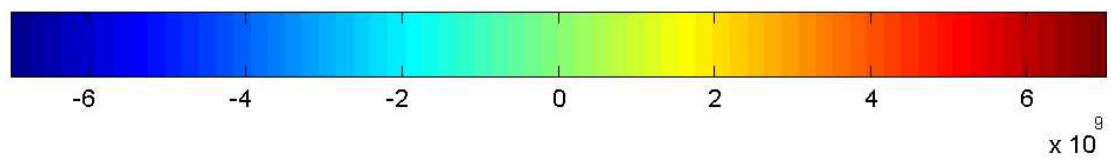

(d) Colorbar

Figure 6.34: Real part of stress $\sigma_{x z}$ in the $y=0$ plane of total waves at $\kappa a=0.3$ and for three cases of one layer, two and four layers. Stress $\sigma_{x z}$ due to the incident wave is zero. Wave fields inside black circles are not computed during scatterer polymerization procedure and set to zero. 
the illuminated region are smaller than those in other regions. The values of $\left|\sigma_{x z}\right|$ in regions other than the shadow region and the illuminated region increase when the number of layers increases. In Fig. 6.34, values of real part of $\sigma_{x z}$ in the shadow region and the illuminated region are smaller than those in other regions. The values of real part of $\sigma_{x z} \mid$ in regions other than the shadow region and the illuminated region increase when the number of layers increases.

In Fig. 6.35, $\left|\sigma_{x x}\right|$ and $\left|\sigma_{y y}\right|$ are approximately half of $\left|\sigma_{z z}\right|$, and distribution of modulus of $\sigma_{x x}, \sigma_{y y}$ and $\sigma_{z z}$ are symmetric about $x=0$ axis. The edge of the shadow region forms an angle with the $Z$-axis. In the shadow region, values of $\left|\sigma_{x x}\right|,\left|\sigma_{y y}\right|$ and $\left|\sigma_{z z}\right|$ decrease when the number of layers increases. The edge of the illuminated region form an angle with the $Z$-axis. In the illuminated region, values and distribution patterns of $\left|\sigma_{x x}\right|,\left|\sigma_{y y}\right|$ and $\left|\sigma_{z z}\right|$ do not appear changed when the number of layers increases.

In Fig. 6.36, values of real part of $\sigma_{x x}$ and $\sigma_{y y}$ are approximately half of that of $\sigma_{z z}$, and distribution of real part of $\sigma_{x x}, \sigma_{y y}$ and $\sigma_{z z}$ are symmetric about $x=0$ axis. The edge of the shadow region forms an angle with the $Z$-axis. In the shadow region, values of real part of $\sigma_{x x}, \sigma_{y y}$ and $\sigma_{z z}$ decrease when the number of layers increases. The edge of the illuminated region form an angle with the $Z$-axis. In the illuminated region, values of periodic peaks of $\sigma_{x x}, \sigma_{y y}$ and $\sigma_{z z}$ in the case of two layers are smaller than those in the cases of one and four layers.

In Fig. 6.37, distribution of modulus of $\sigma_{x x}, \sigma_{y y}$ and $\sigma_{z z}$ are symmetric about $x=0$ axis, and values of modulus of $\sigma_{x x}, \sigma_{y y}$ are approximately half of modulus of $\sigma_{z z}$. In the shadow region, values of modulus of $\sigma_{x x}, \sigma_{y y}$ and $\sigma_{z z}$ increase slightly when the number of layers increases. The distribution patterns of modulus of $\sigma_{x x}$ and $\sigma_{z z}$ are similar and appear as several sectors. In the sub-figures of modulus of $\sigma_{y y}$, the distribution appear like two sectors in the shadow region and the illuminated 


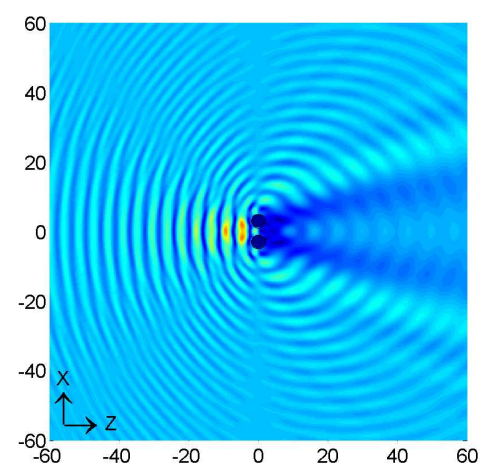

(a) $\left|\sigma_{x x}\right|: 1$ layer

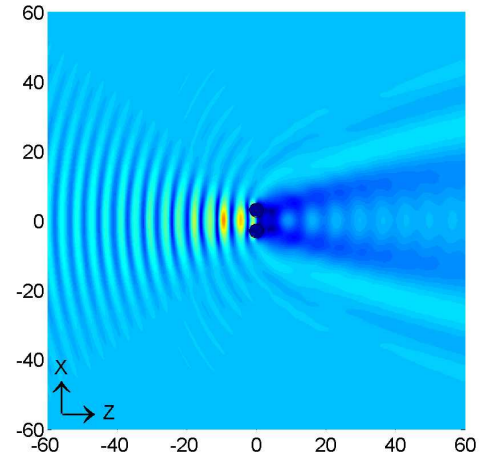

(d) $\left|\sigma_{y y}\right|: 1$ layer

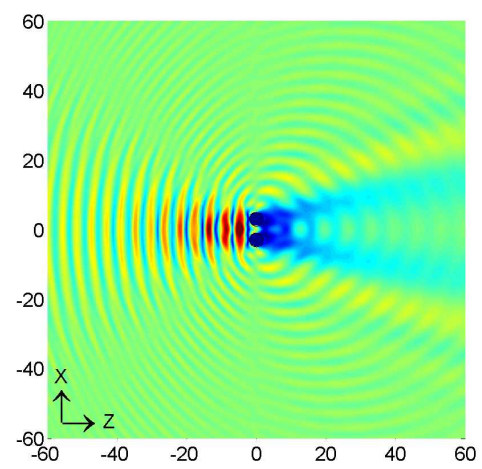

(g) $\left|\sigma_{z z}\right|: 1$ layer

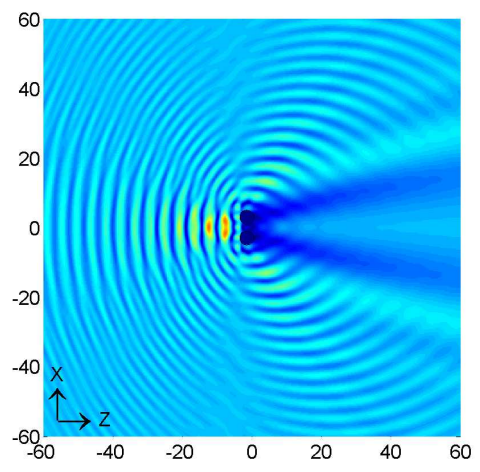

(b) $\left|\sigma_{x x}\right|: 2$ layers

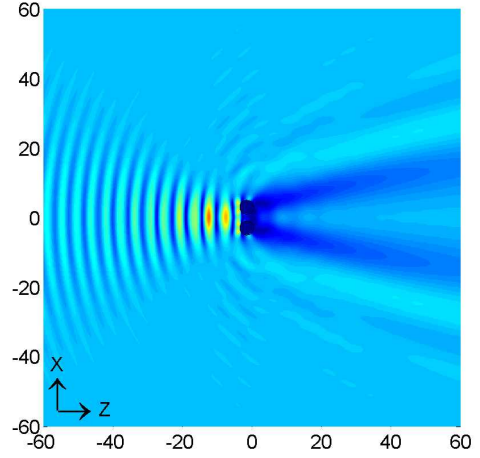

(e) $\left|\sigma_{y y}\right|: 2$ layers

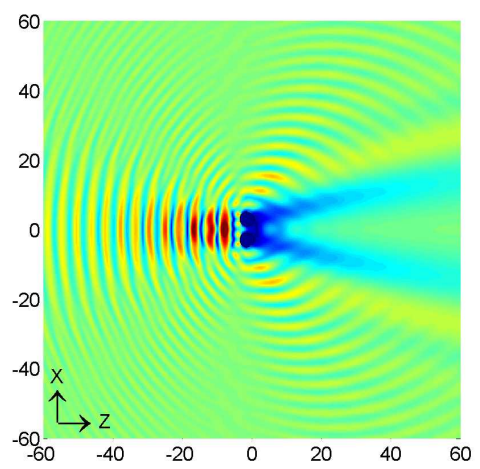

(h) $\left|\sigma_{z z}\right|: 2$ layers

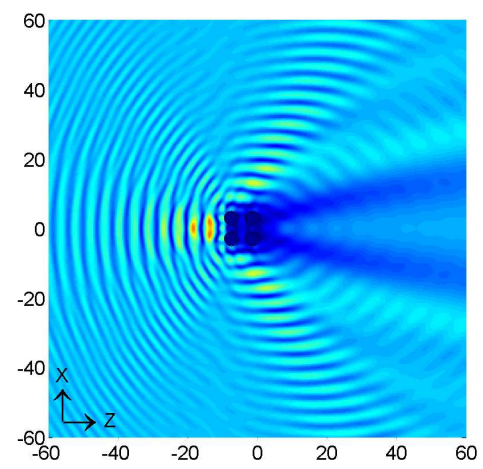

(c) $\left|\sigma_{x x}\right|: 4$ layers

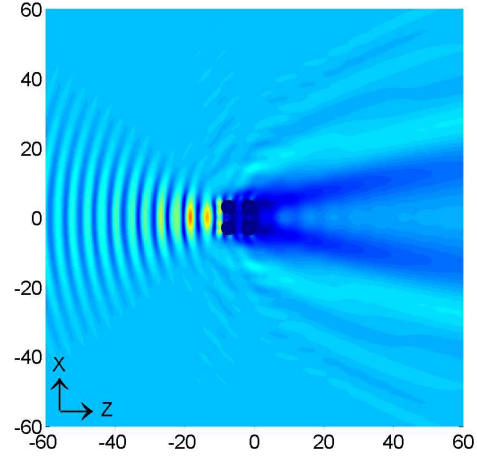

(f) $\left|\sigma_{y y}\right|: 4$ layers

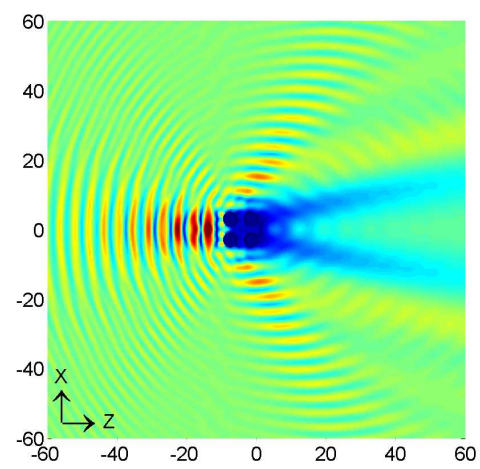

(i) $\left|\sigma_{z z}\right|: 4$ layers

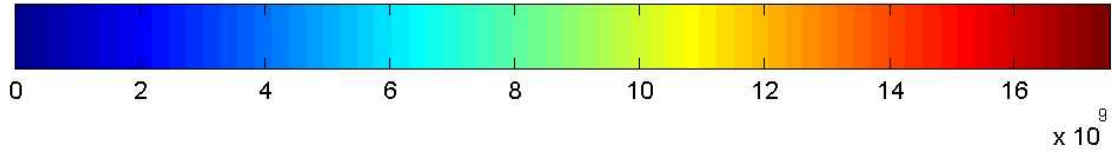

(j) Colorbar

Figure 6.35: Modulus of stresses $\sigma_{x x}, \sigma_{y y}$ and $\sigma_{z z}$ in the $y=0$ plane of total waves at $\kappa a=0.75$ and for three cases of one layer, two and four layers. Wave fields inside black circles are not computed during scatterer polymerization procedure and set to zero. 


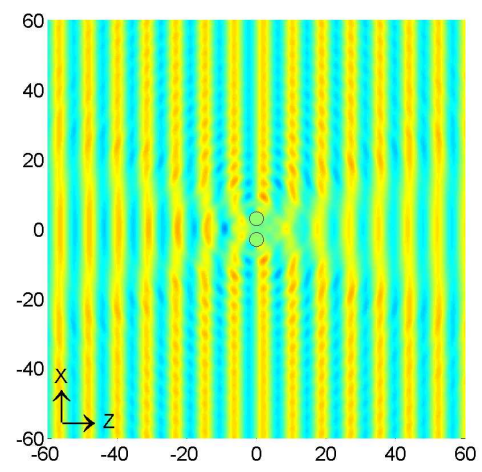

(a) $\sigma_{x x}: 1$ layer

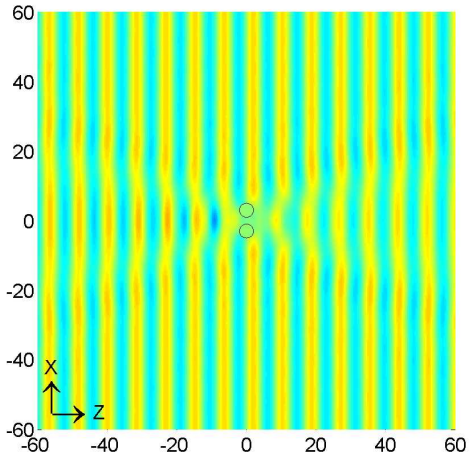

(d) $\sigma_{y y}: 1$ layer

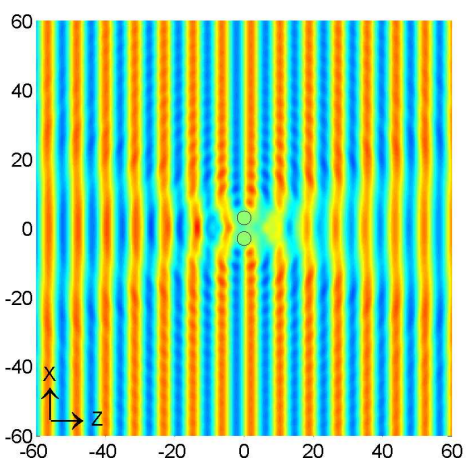

(g) $\sigma_{z z}: 1$ layer

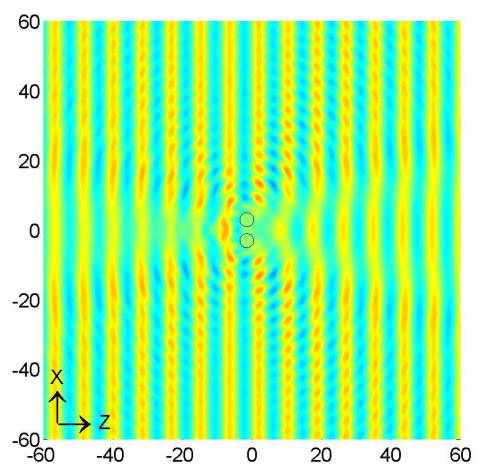

(b) $\sigma_{x x}: 2$ layers

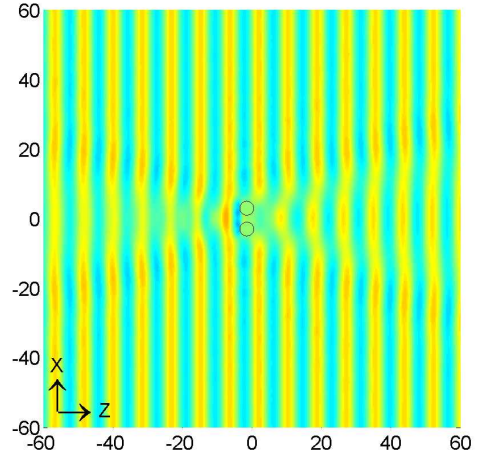

(e) $\sigma_{y y}: 2$ layers

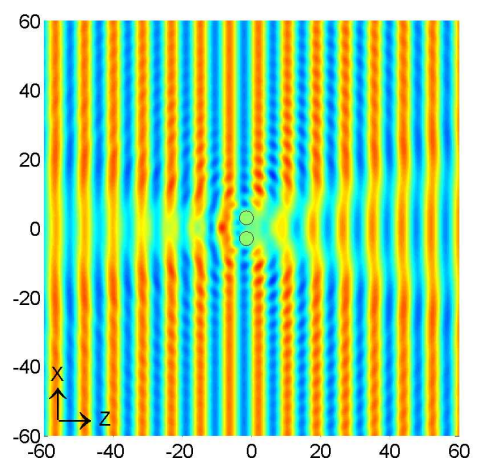

(h) $\sigma_{z z}: 2$ layers

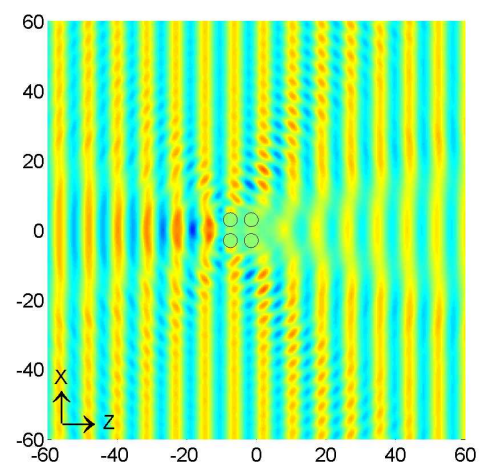

(c) $\sigma_{x x}: 4$ layers

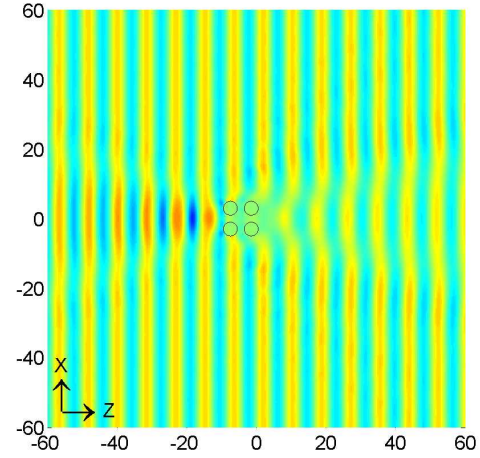

(f) $\sigma_{y y}: 4$ layers

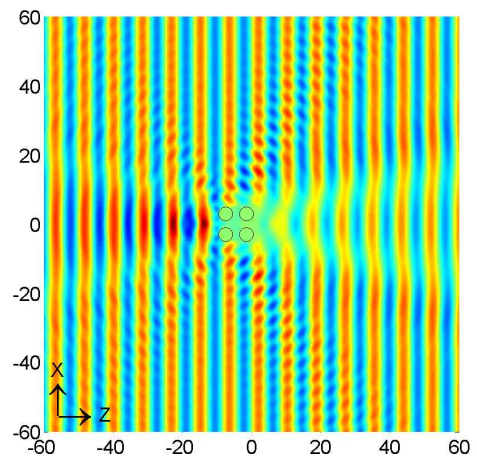

(i) $\sigma_{z z}: 4$ layers

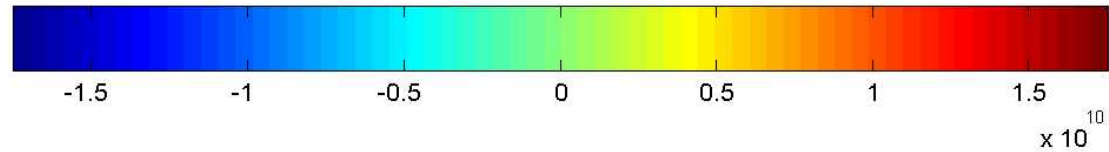

(j) Colorbar

Figure 6.36: Real part of stresses $\sigma_{x x}, \sigma_{y y}$ and $\sigma_{z z}$ in the $y=0$ plane of total waves at $\kappa a=0.75$ and for three cases of one layer, two and four layers. Wave fields inside black circles are not computed during scatterer polymerization procedure and set to zero. 


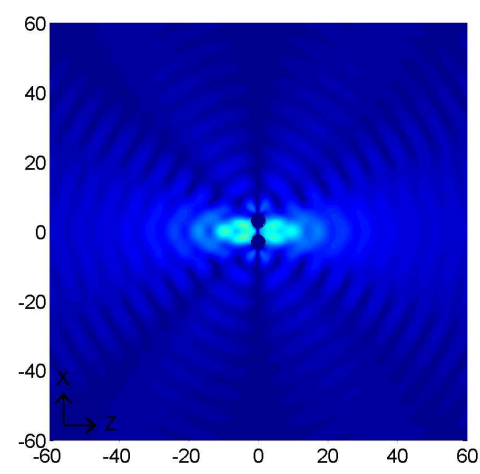

(a) $\left|\sigma_{x x}\right|: 1$ layer

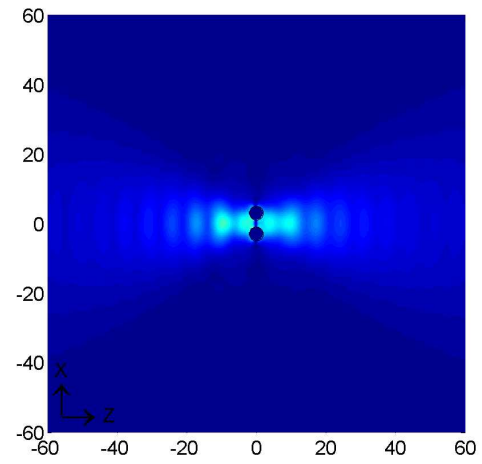

(d) $\left|\sigma_{y y}\right|: 1$ layer

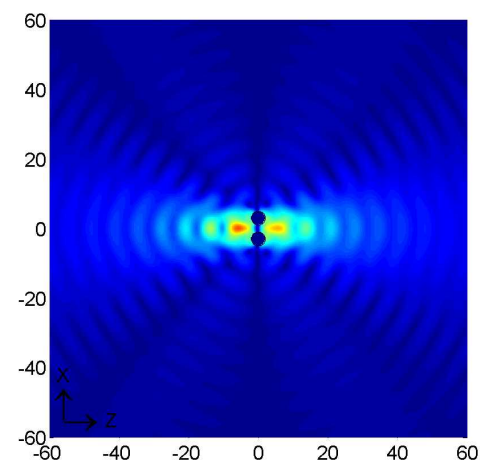

(g) $\left|\sigma_{z z}\right|: 1$ layer

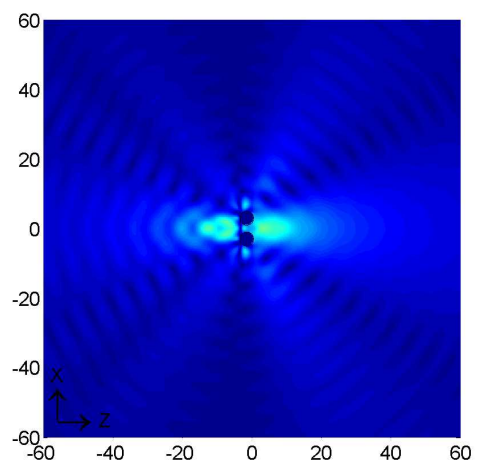

(b) $\left|\sigma_{x x}\right|: 2$ layers

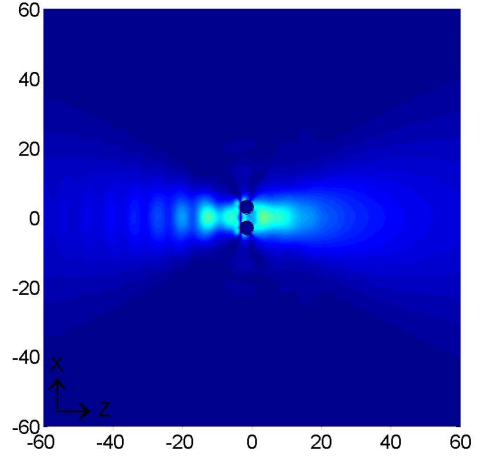

(e) $\left|\sigma_{y y}\right|: 2$ layers

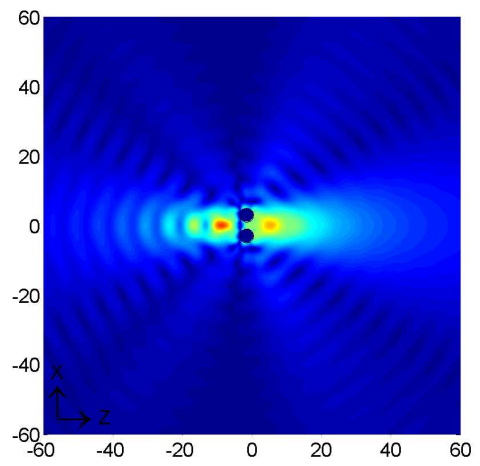

(h) $\left|\sigma_{z z}\right|: 2$ layers

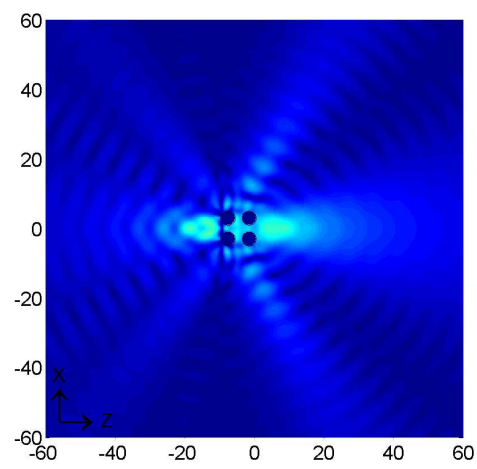

(c) $\left|\sigma_{x x}\right|: 4$ layers

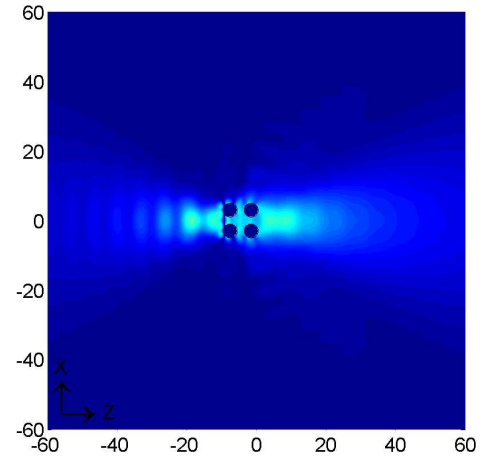

(f) $\left|\sigma_{y y}\right|: 4$ layers

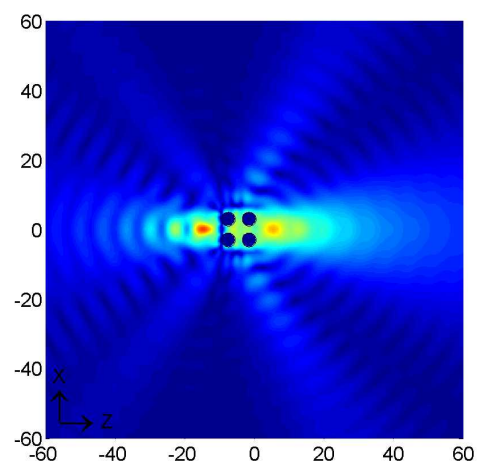

(i) $\left|\sigma_{z z}\right|: 4$ layers

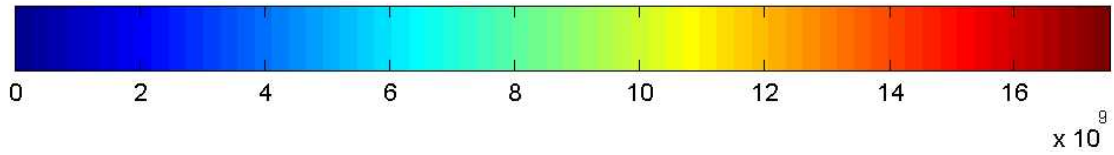

(j) Colorbar

Figure 6.37: Modulus of stresses $\sigma_{x x}, \sigma_{y y}$ and $\sigma_{z z}$ in the $y=0$ plane of scattered waves at $\kappa a=0.75$ and for three cases of one layer, two and four layers. Wave fields inside black circles are not computed during scatterer polymerization procedure and set to zero. 
region. The size of the sector in the shadow region slightly increases when the number of layers increases.

In Fig. 6.38, distribution of real part of $\sigma_{x x}, \sigma_{y y}$ and $\sigma_{z z}$ are symmetric about $x=0$ axis, and values of real part of $\sigma_{x x}, \sigma_{y y}$ are approximately half of real part of $\sigma_{z z}$. In the shadow region, values of real part of $\sigma_{x x}, \sigma_{y y}$ and $\sigma_{z z}$ increase slightly when the number of layers increases. The distribution patterns of real part of $\sigma_{x x}$ and $\sigma_{z z}$ are similar, and the spacing between periodic peaks in the shadow region and the illuminated region are two times of that in other regions. The spacing between periodic peaks in the sub-figures of real part of $\sigma_{y y}$, is the same as that in the shadow region in the sub-figures of real part of $\sigma_{x x}$ and $\sigma_{z z}$.

The stress component $\sigma_{x z}$ due to the incident wave is zero, so that the non-zero result of $\sigma_{x z}$ is due to scattered waves. The distribution of modulus of $\sigma_{x z}$ is symmetric about $x=0$ axis. In Fig. 6.39, values of $\left|\sigma_{x z}\right|$ in the shadow region and the illuminated region are smaller than those in the regions along the $z=0$ axis. The values of $\left|\sigma_{x z}\right|$ in the regions along $z=0$ axis increase when the number of layers increases. In Fig. 6.40, values of real part of $\sigma_{x z}$ in the shadow region and the illuminated region are smaller than those in the regions along the $z=0$ axis. The values of real part of $\sigma_{x z} \mid$ in regions along the $z=0$ axis increase when the number of layers increases.

In Fig. 6.41, $\left|\sigma_{x x}\right|$ and $\left|\sigma_{y y}\right|$ are approximately half of $\left|\sigma_{z z}\right|$, and distribution of modulus of $\sigma_{x x}, \sigma_{y y}$ and $\sigma_{z z}$ are symmetric about $x=0$ axis. The edge of the shadow region forms an angle with the $Z$-axis. In the shadow region, values of $\left|\sigma_{x x}\right|,\left|\sigma_{y y}\right|$ and $\left|\sigma_{z z}\right|$ decrease when the number of layers increases. In the illuminated region, periodic peaks of $\left|\sigma_{x x}\right|,\left|\sigma_{y y}\right|$ and $\left|\sigma_{z z}\right|$ appear like contour lines and values of the periodic peaks increase slightly when the number of layers increases.

In Fig. 6.42, values of real part of $\sigma_{x x}$ and $\sigma_{y y}$ are approximately half of that of $\sigma_{z z}$, and distribution of real part of $\sigma_{x x}, \sigma_{y y}$ and $\sigma_{z z}$ are symmetric about $x=0$ axis. 


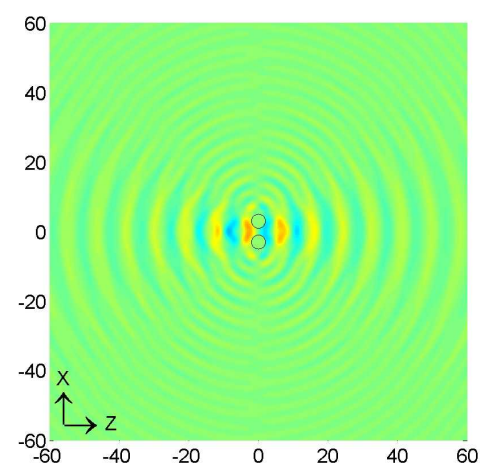

(a) $\sigma_{x x}: 1$ layer

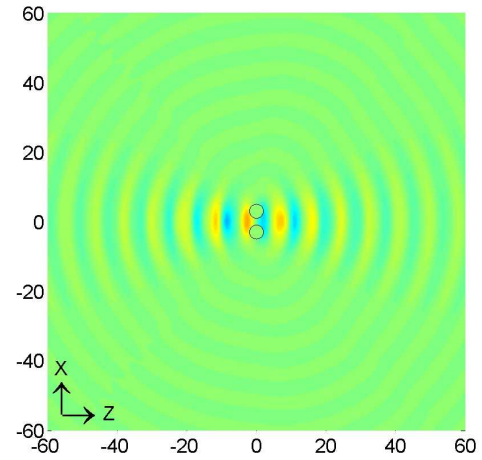

(d) $\sigma_{y y}: 1$ layer

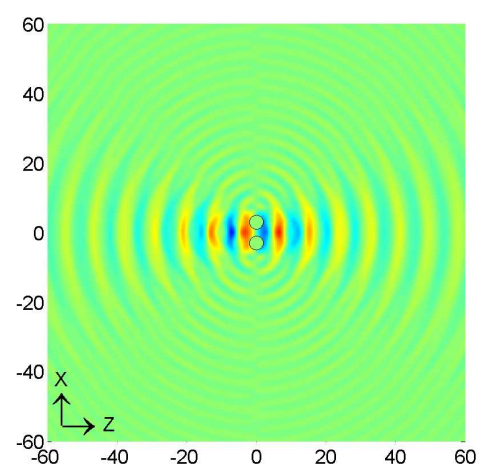

(g) $\sigma_{z z}: 1$ layer

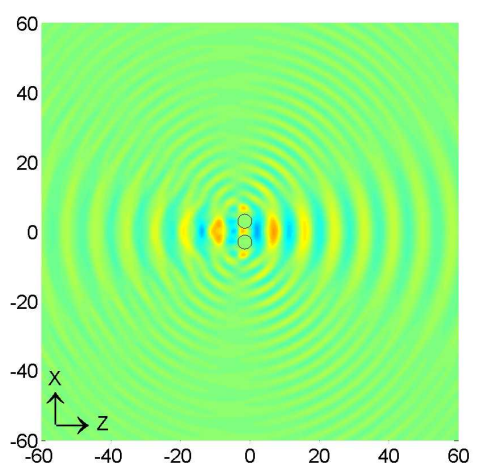

(b) $\sigma_{x x}: 2$ layers

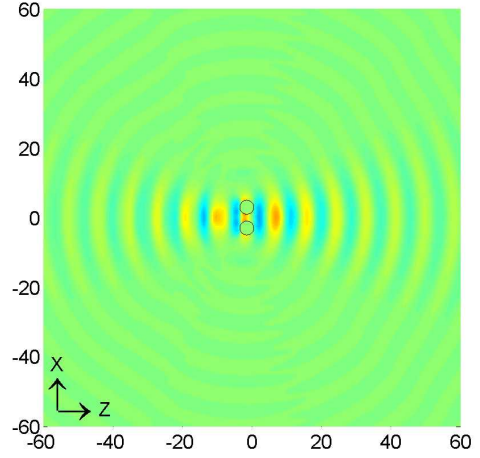

(e) $\sigma_{y y}: 2$ layers

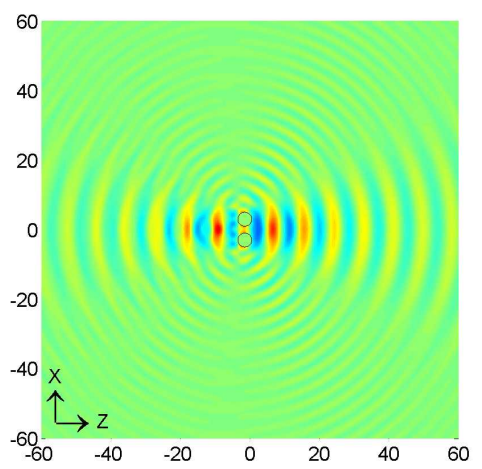

(h) $\sigma_{z z}: 2$ layers

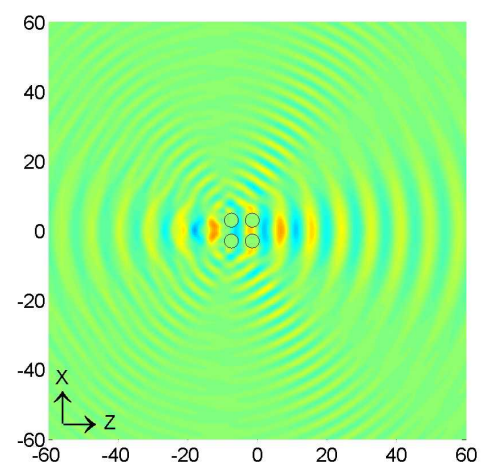

(c) $\sigma_{x x}: 4$ layers

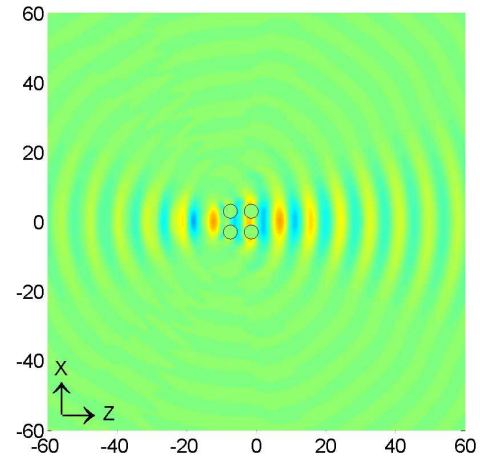

(f) $\sigma_{y y}: 4$ layers

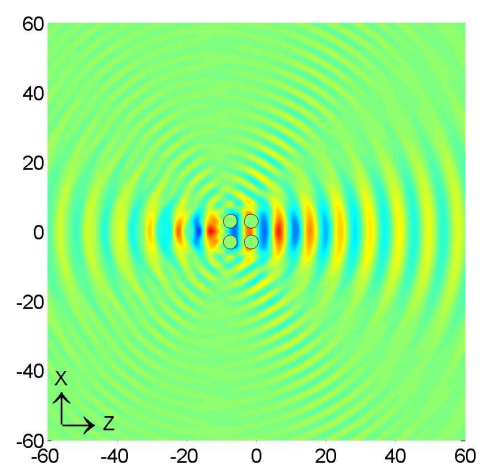

(i) $\sigma_{z z}: 4$ layers

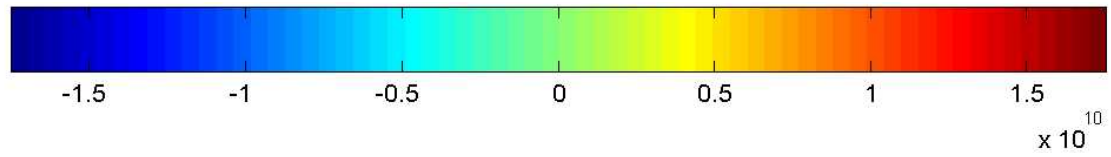

(j) Colorbar

Figure 6.38: Real part of stresses $\sigma_{x x}, \sigma_{y y}$ and $\sigma_{z z}$ in the $y=0$ plane of scattered waves at $\kappa a=0.75$ and for three cases of one layer, two and four layers. Wave fields inside black circles are not computed during scatterer polymerization procedure and set to zero. 


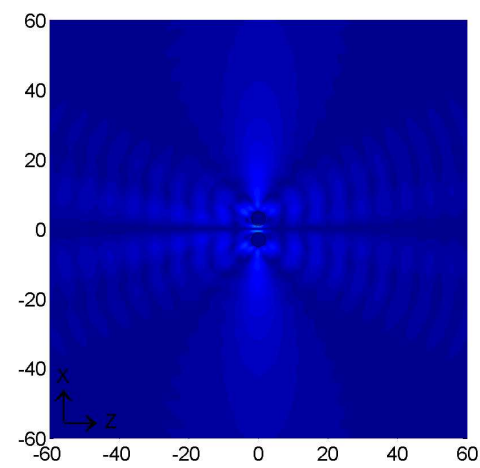

(a) $\left|\sigma_{x z}\right|: 1$ layer

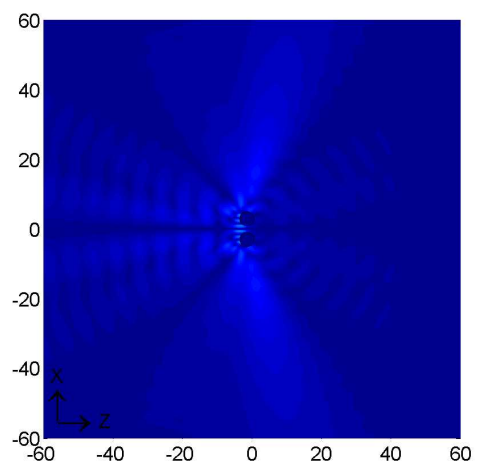

(b) $\left|\sigma_{x z}\right|: 2$ layers

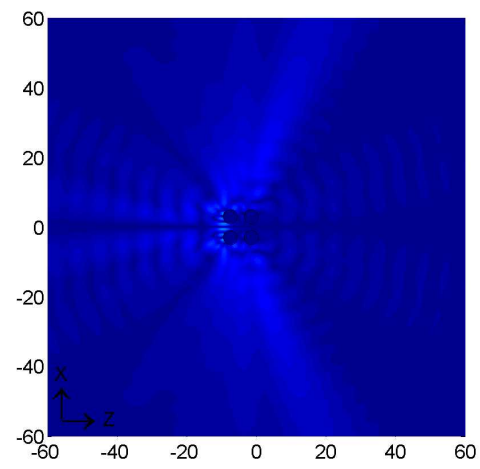

(c) $\left|\sigma_{x z}\right|: 4$ layers

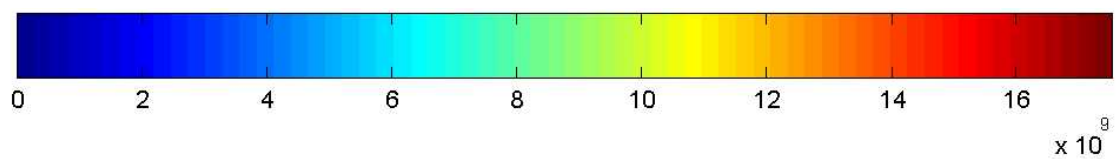

(d) Colorbar

Figure 6.39: Modulus of stress $\sigma_{x z}$ in the $y=0$ plane of total waves at $\kappa a=0.75$ and for three cases of one layer, two and four layers. Stress $\sigma_{x z}$ due to the incident wave is zero. Wave fields inside black circles are not computed during scatterer polymerization procedure and set to zero.

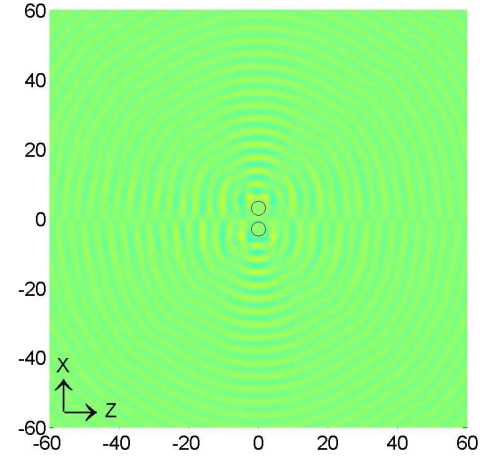

(a) $\sigma_{x z}: 1$ layer

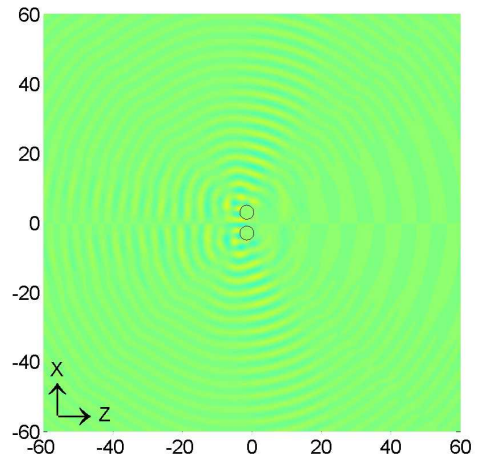

(b) $\sigma_{x z}: 2$ layers

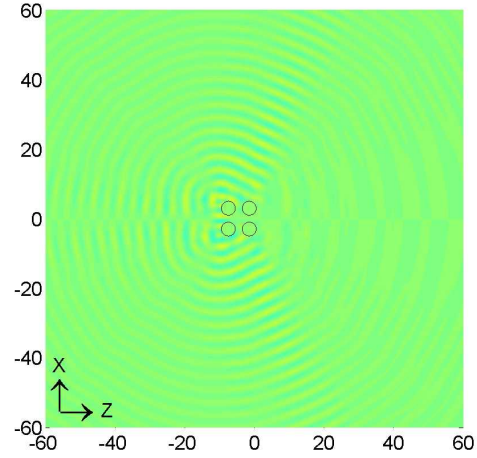

(c) $\sigma_{x z}: 4$ layers

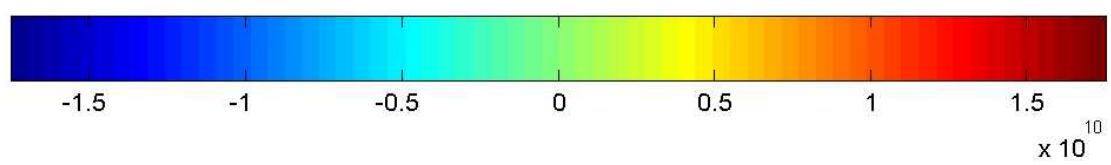

(d) Colorbar

Figure 6.40: Real part of stress $\sigma_{x z}$ in the $y=0$ plane of total waves at $\kappa a=0.75$ and for three cases of one layer, two and four layers. Stress $\sigma_{x z}$ due to the incident wave is zero. Wave fields inside black circles are not computed during scatterer polymerization procedure and set to zero. 


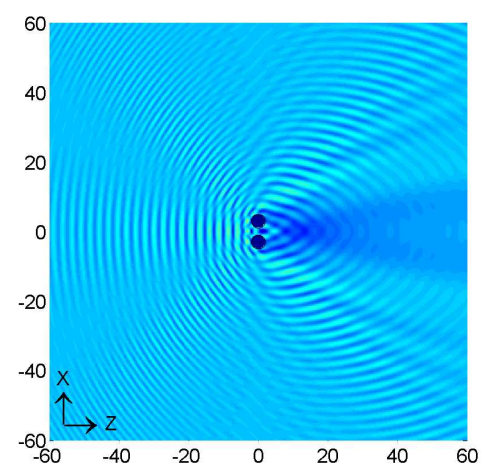

(a) $\left|\sigma_{x x}\right|: 1$ layer

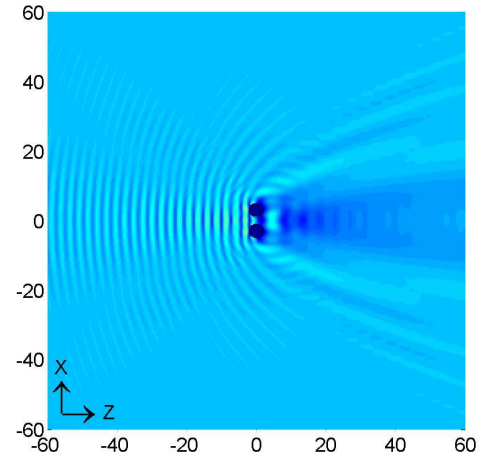

(d) $\left|\sigma_{y y}\right|: 1$ layer

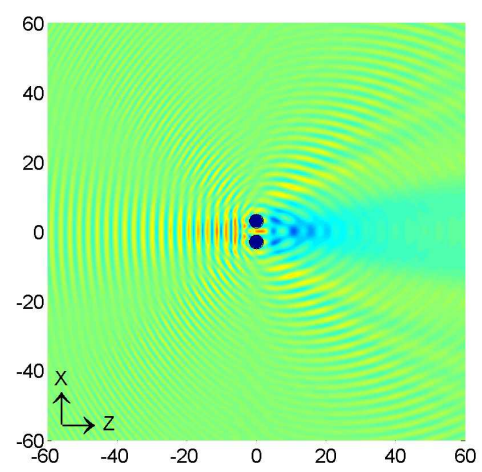

(g) $\left|\sigma_{z z}\right|: 1$ layer

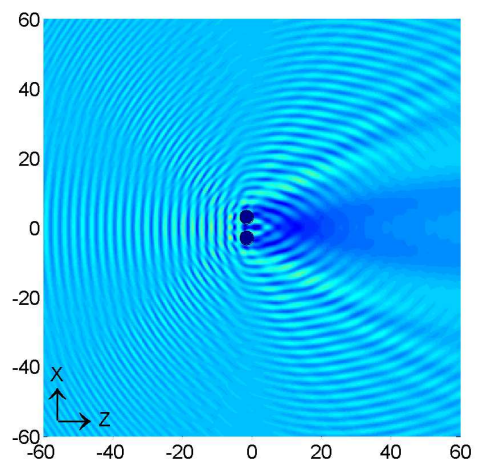

(b) $\left|\sigma_{x x}\right|: 2$ layers

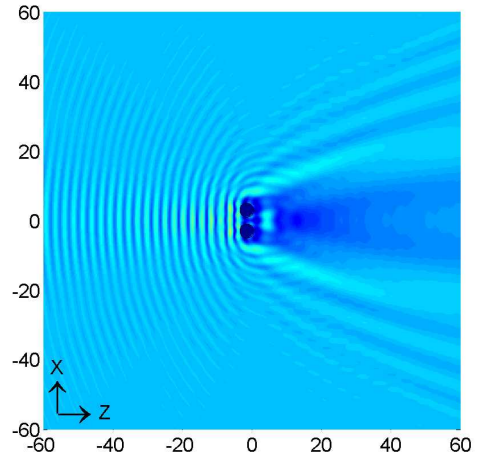

(e) $\left|\sigma_{y y}\right|: 2$ layers

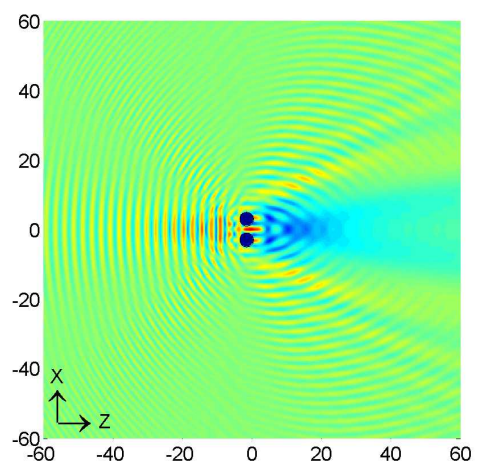

(h) $\left|\sigma_{z z}\right|: 2$ layers

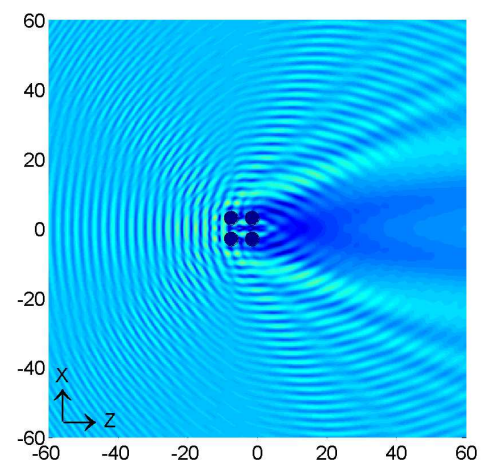

(c) $\left|\sigma_{x x}\right|: 4$ layers

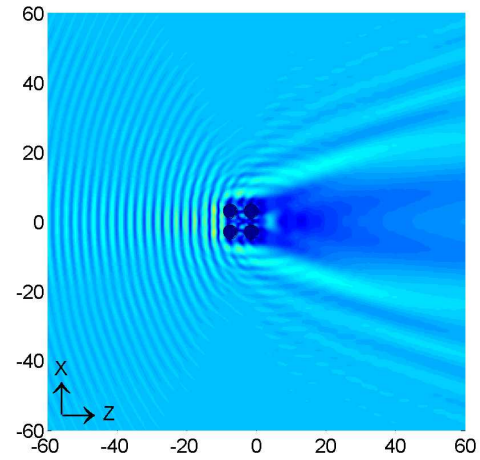

(f) $\left|\sigma_{y y}\right|: 4$ layers

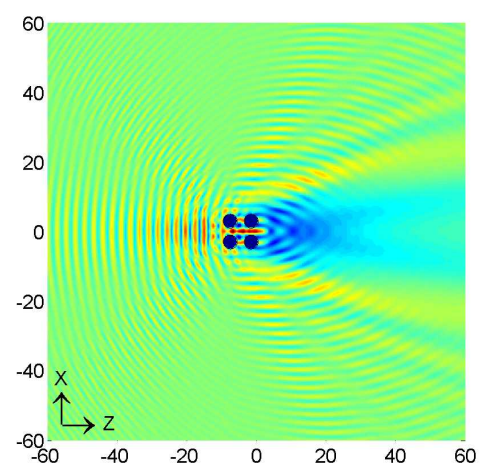

(i) $\left|\sigma_{z z}\right|: 4$ layers

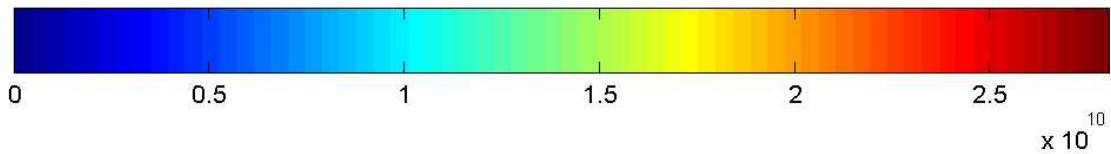

(j) Colorbar

Figure 6.41: Modulus of stresses $\sigma_{x x}, \sigma_{y y}$ and $\sigma_{z z}$ in the $y=0$ plane of total waves at $\kappa a=1.2$ and for three cases of one layer, two and four layers. Wave fields inside black circles are not computed during scatterer polymerization procedure and set to zero. 


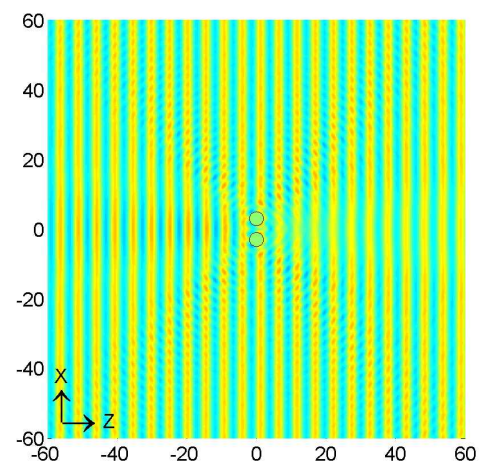

(a) $\sigma_{x x}: 1$ layer

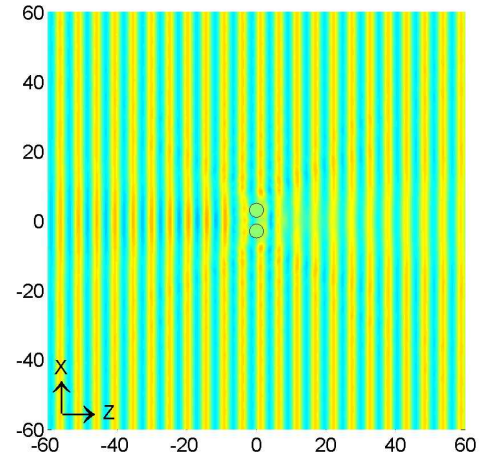

(d) $\sigma_{y y}: 1$ layer

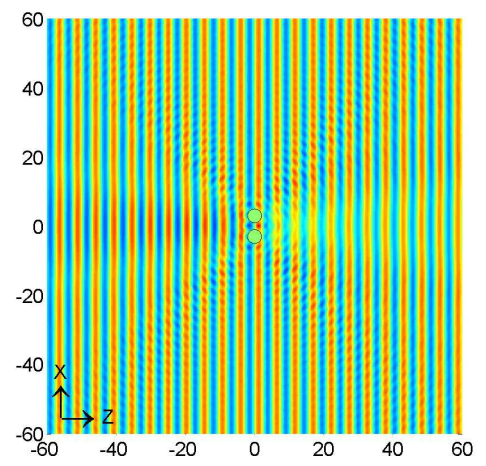

(g) $\sigma_{z z}: 1$ layer

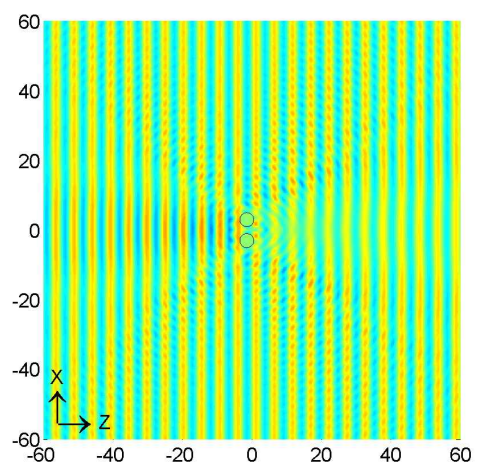

(b) $\sigma_{x x}: 2$ layers

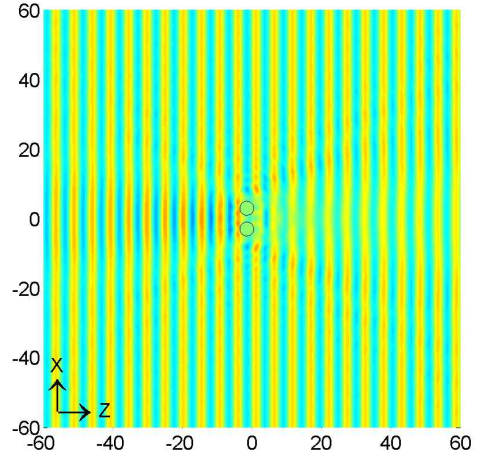

(e) $\sigma_{y y}: 2$ layers

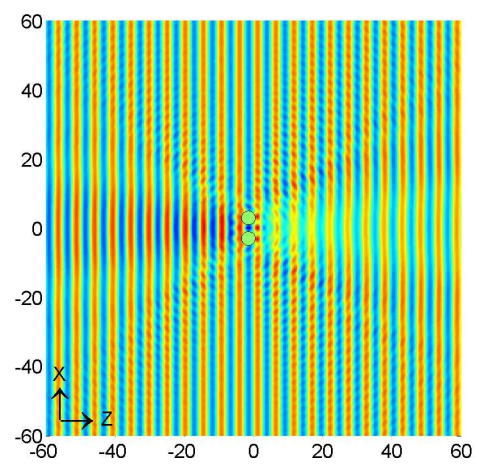

(h) $\sigma_{z z}: 2$ layers

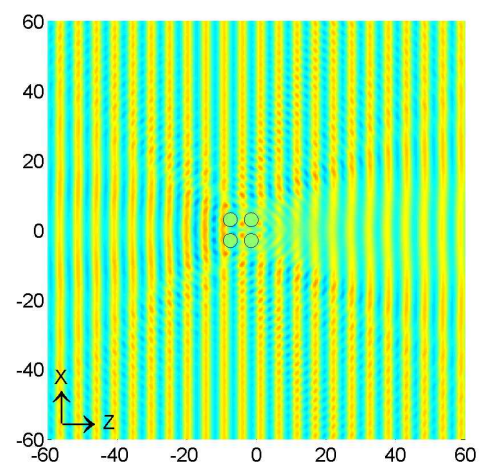

(c) $\sigma_{x x}: 4$ layers

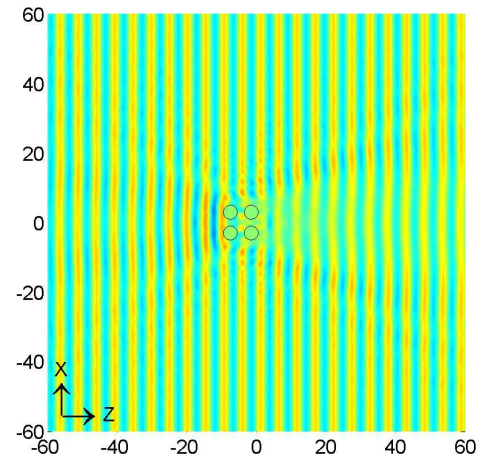

(f) $\sigma_{y y}: 4$ layers

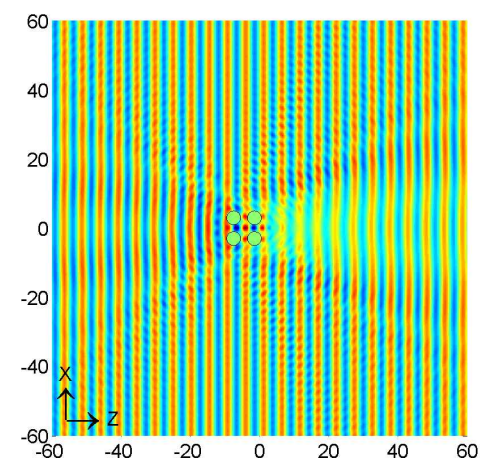

(i) $\sigma_{z z}: 4$ layers

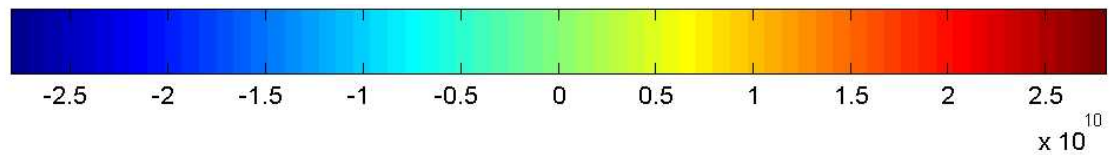

(j) Colorbar

Figure 6.42: Real part of stresses $\sigma_{x x}, \sigma_{y y}$ and $\sigma_{z z}$ in the $y=0$ plane of total waves at $\kappa a=1.2$ and for three cases of one layer, two and four layers. Wave fields inside black circles are not computed during scatterer polymerization procedure and set to zero. 
The edge of the shadow region forms an angle with the $Z$-axis. In the shadow region, values of real part of $\sigma_{x x}, \sigma_{y y}$ and $\sigma_{z z}$ decrease when the number of layers increases. The edge of the illuminated region form an angle with the $Z$-axis. In the illuminated region, values of periodic peaks of $\sigma_{x x}, \sigma_{y y}$ and $\sigma_{z z}$ in the case of two layers are larger than those in the cases of one and four layers.

In Fig. 6.43, distribution of modulus of $\sigma_{x x}, \sigma_{y y}$ and $\sigma_{z z}$ are symmetric about $x=0$ axis, and values of modulus of $\sigma_{x x}, \sigma_{y y}$ are approximately half of modulus of $\sigma_{z z}$. In the shadow region, values of modulus of $\sigma_{x x}, \sigma_{y y}$ and $\sigma_{z z}$ increase slightly when the number of layers increases. The distribution patterns of modulus of $\sigma_{x x}$ and $\sigma_{z z}$ are similar and appear as several sectors. The number of sectors in the region where $z>0$ increases when the number of layers increases. In the sub-figures of modulus of $\sigma_{y y}$, the distribution appear like several sectors in the shadow region and the illuminated region. The size of the sectors increases when the number of layers increases.

In Fig. 6.44, distribution of real part of $\sigma_{x x}, \sigma_{y y}$ and $\sigma_{z z}$ are symmetric about $x=0$ axis. In the shadow region, values of real part of $\sigma_{x x}, \sigma_{y y}$ and $\sigma_{z z}$ increase slightly when the number of layers increases. The distribution patterns of real part of $\sigma_{x x}$ and $\sigma_{z z}$ are similar, and the spacing between periodic peaks in the shadow region and the illuminated region are two times of that in other regions. The spacing between periodic peaks in the sub-figures of real part of $\sigma_{y y}$, is the same as that in the shadow region in the sub-figures of real part of $\sigma_{x x}$ and $\sigma_{z z}$.

In Fig. 6.39, the distribution of modulus of $\sigma_{x z}$ is symmetric about $x=0$ axis. Values of $\left|\sigma_{x z}\right|$ in the shadow region and the illuminated region are smaller than those in the regions along the $z=0$ axis. The values of $\left|\sigma_{x z}\right|$ in the regions along $z=0$ axis increase when the number of layers increases. In Fig. 6.40, values of real part of $\sigma_{x z}$ in the shadow region and the illuminated region are smaller than those in the regions 


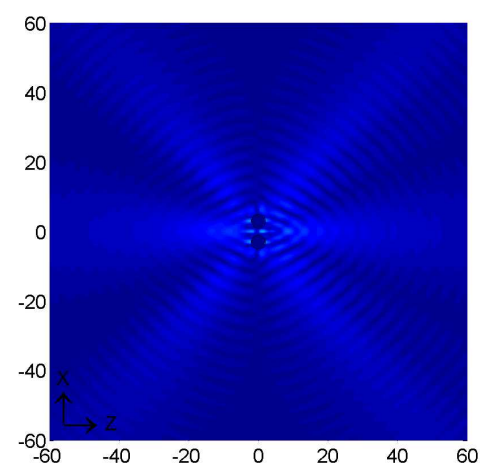

(a) $\left|\sigma_{x x}\right|: 1$ layer

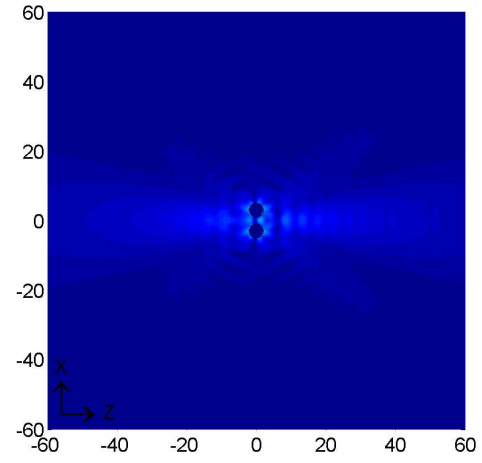

(d) $\left|\sigma_{y y}\right|: 1$ layer

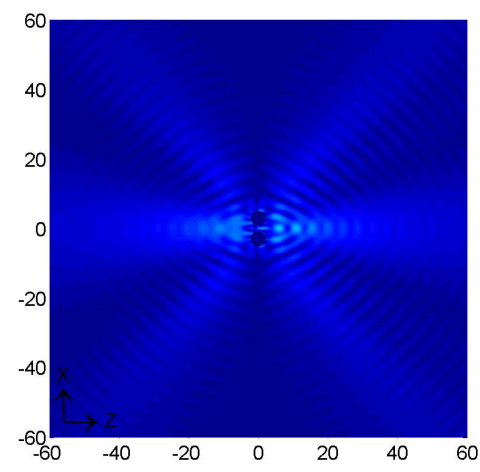

(g) $\left|\sigma_{z z}\right|: 1$ layer

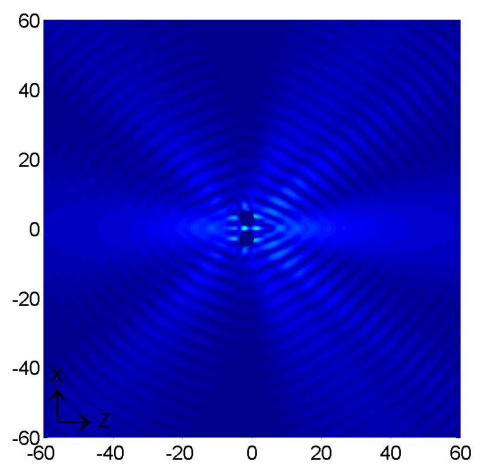

(b) $\left|\sigma_{x x}\right|: 2$ layers

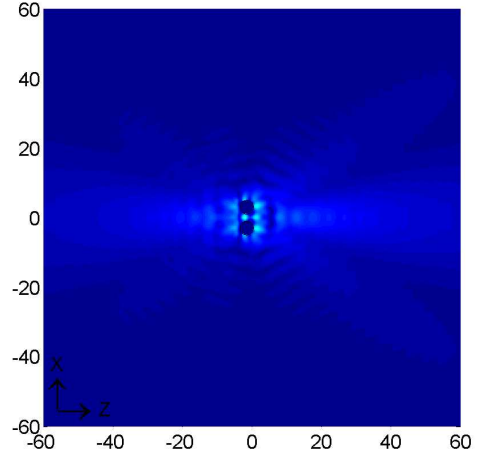

(e) $\left|\sigma_{y y}\right|: 2$ layers

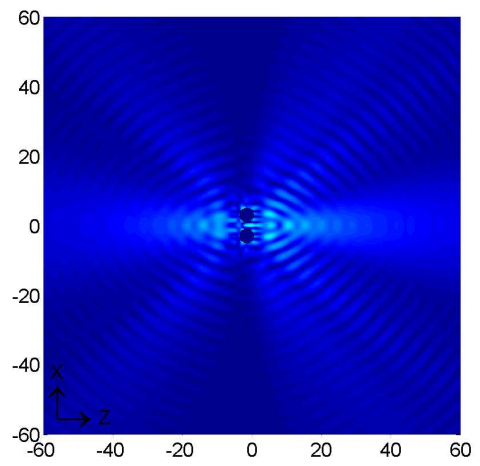

(h) $\left|\sigma_{z z}\right|: 2$ layers

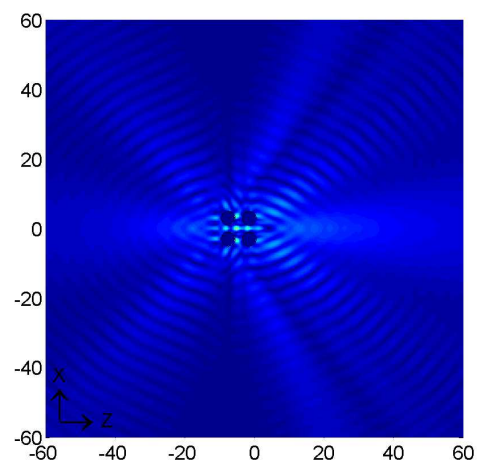

(c) $\left|\sigma_{x x}\right|: 4$ layers

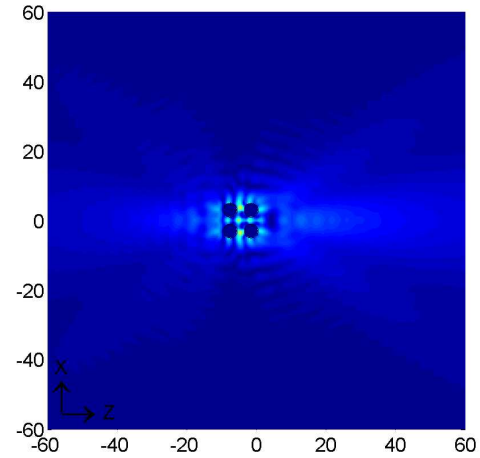

(f) $\left|\sigma_{y y}\right|: 4$ layers

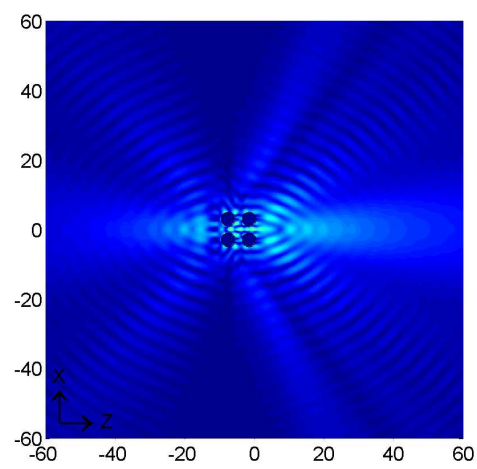

(i) $\left|\sigma_{z z}\right|: 4$ layers

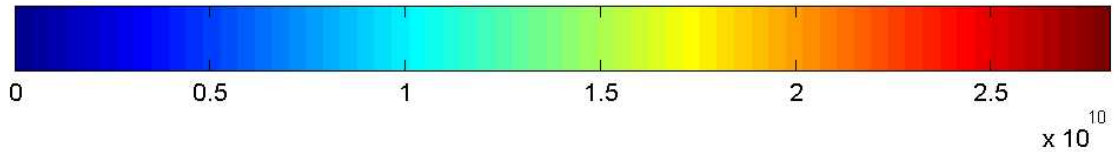

(j) Colorbar

Figure 6.43: Modulus of stresses $\sigma_{x x}, \sigma_{y y}$ and $\sigma_{z z}$ in the $y=0$ plane of scattered waves at $\kappa a=1.2$ and for three cases of one layer, two and four layers. Wave fields inside black circles are not computed during scatterer polymerization procedure and set to zero. 


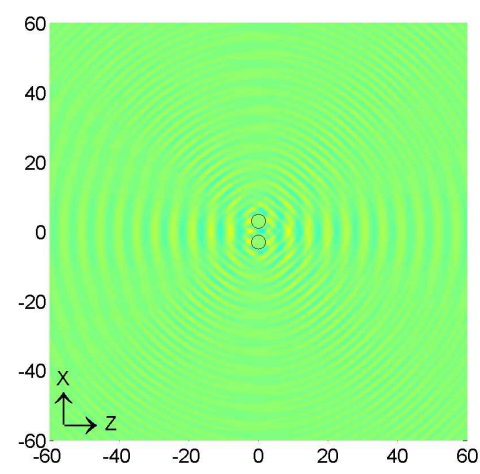

(a) $\sigma_{x x}: 1$ layer

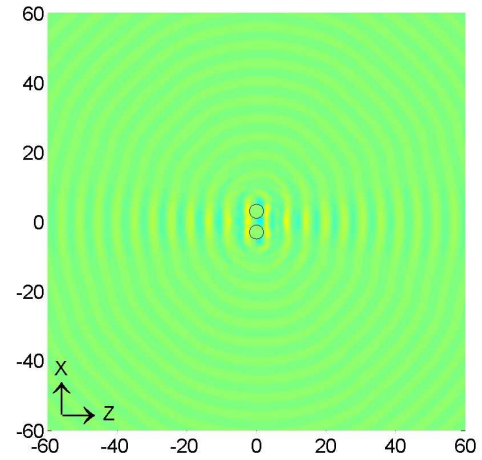

(d) $\sigma_{y y}: 1$ layer

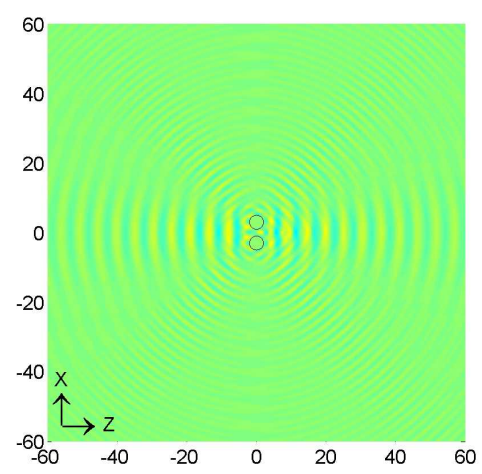

(g) $\sigma_{z z}: 1$ layer

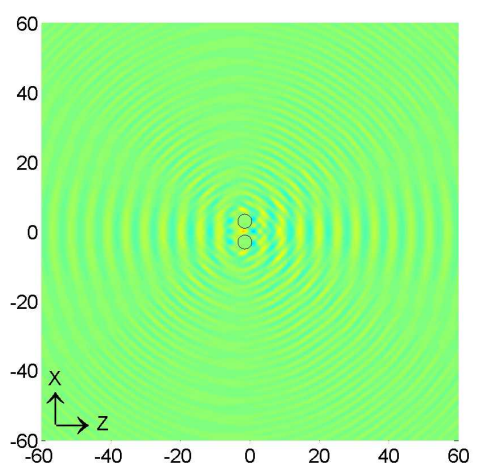

(b) $\sigma_{x x}: 2$ layers

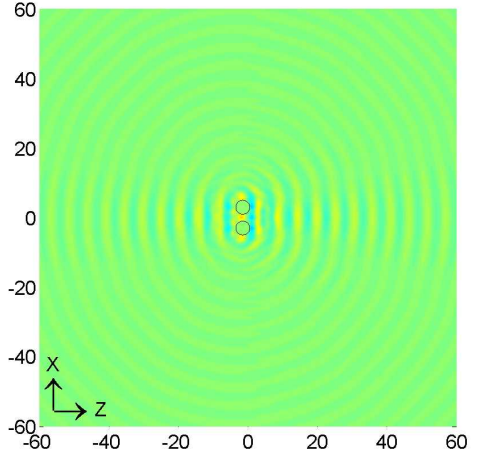

(e) $\sigma_{y y}: 2$ layers

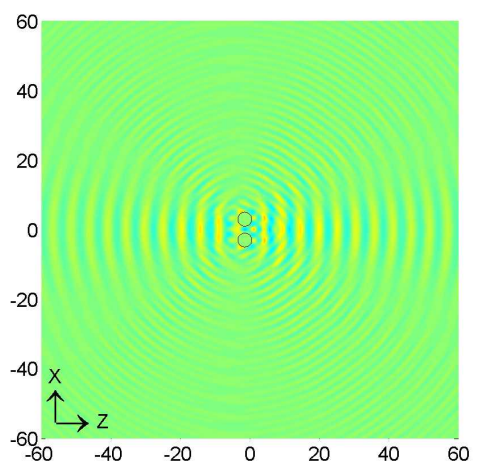

(h) $\sigma_{z z}: 2$ layers

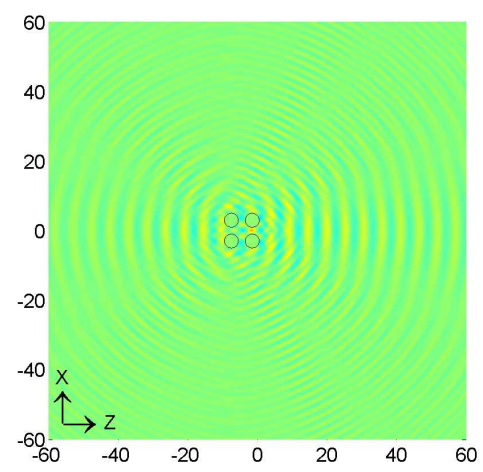

(c) $\sigma_{x x}: 4$ layers

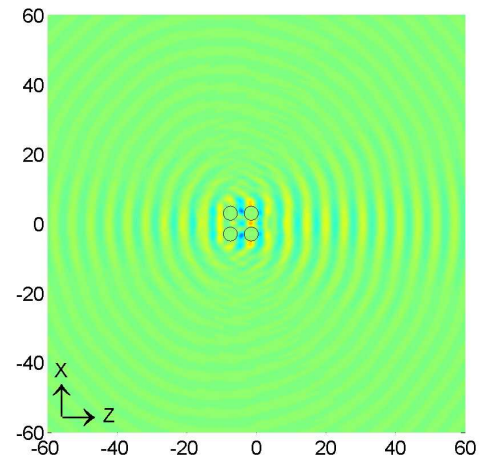

(f) $\sigma_{y y}: 4$ layers

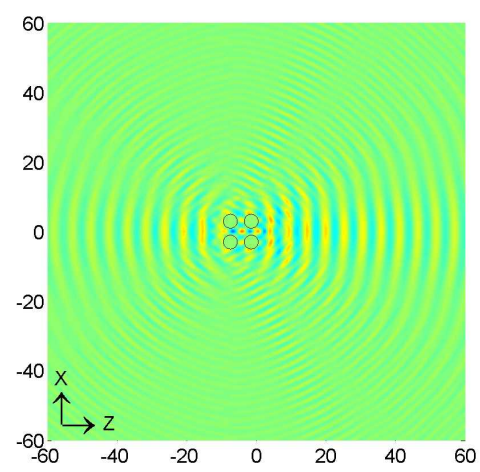

(i) $\sigma_{z z}: 4$ layers

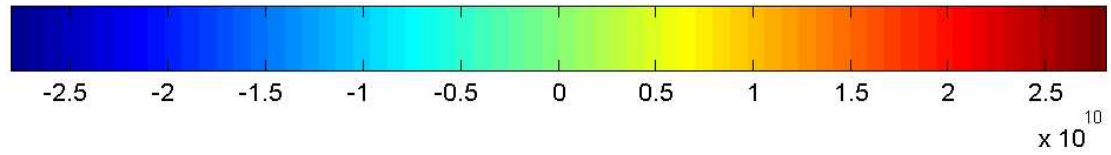

(j) Colorbar

Figure 6.44: Real part of stresses $\sigma_{x x}, \sigma_{y y}$ and $\sigma_{z z}$ in the $y=0$ plane of scattered waves at $\kappa a=1.2$ and for three cases of one layer, two and four layers. Wave fields inside black circles are not computed during scatterer polymerization procedure and set to zero. 


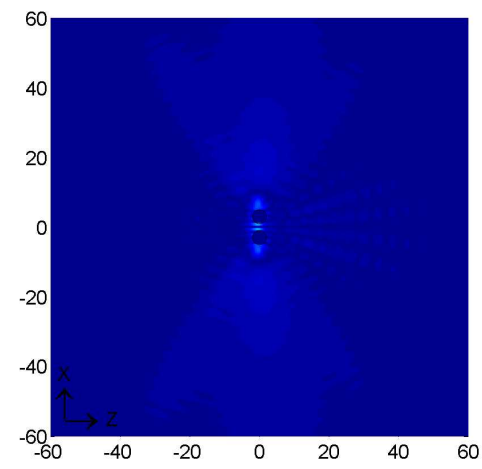

(a) $\left|\sigma_{x z}\right|: 1$ layer

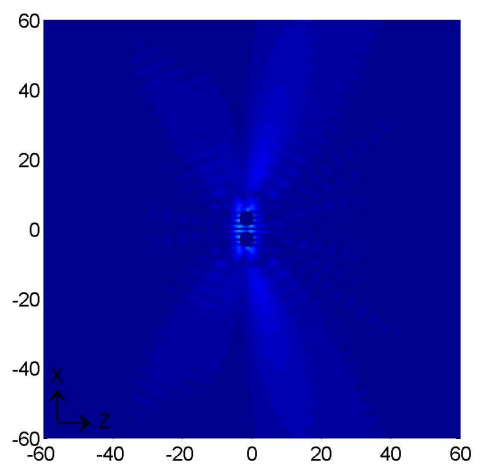

(b) $\left|\sigma_{x z}\right|: 2$ layers

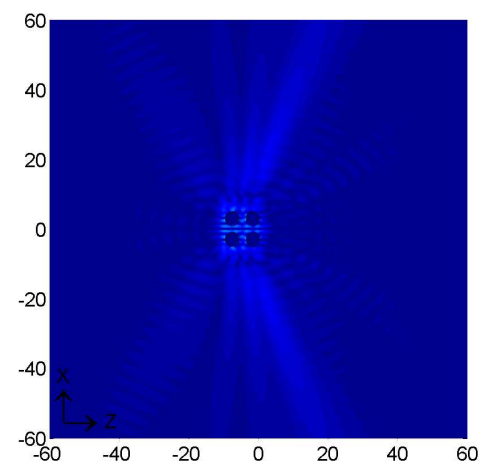

(c) $\left|\sigma_{x z}\right|: 4$ layers

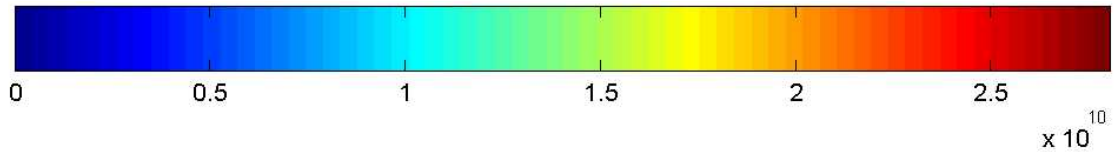

(d) Colorbar

Figure 6.45: Modulus of stress $\sigma_{x z}$ in the $y=0$ plane of total waves at $\kappa a=1.2$ and for three cases of one layer, two and four layers. Stress $\sigma_{x z}$ due to the incident wave is zero. Wave fields inside black circles are not computed during scatterer polymerization procedure and set to zero.

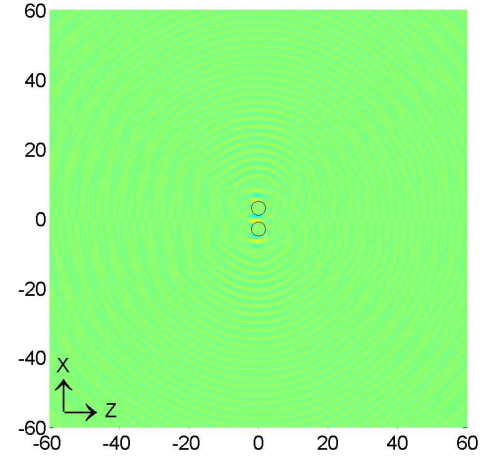

(a) $\sigma_{x z}: 1$ layer

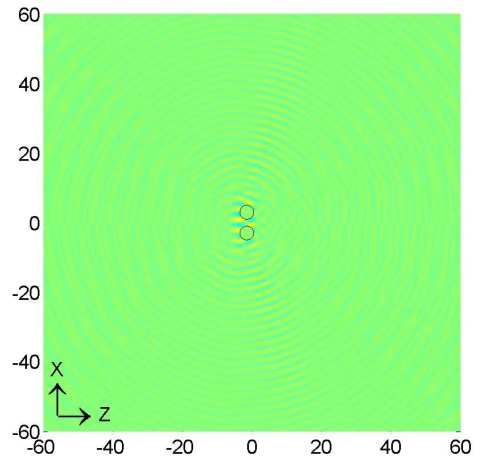

(b) $\sigma_{x z}: 2$ layers

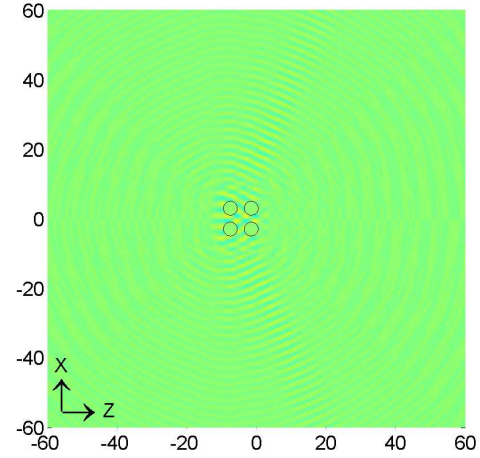

(c) $\sigma_{x z}: 4$ layers

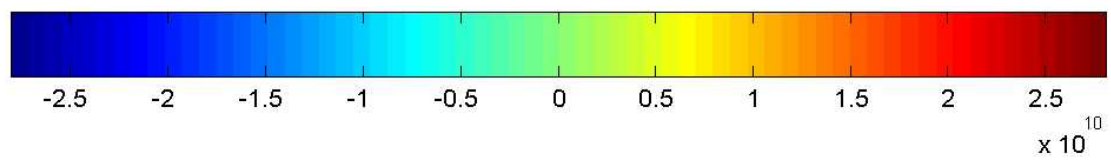

(d) Colorbar

Figure 6.46: Real part of stress $\sigma_{x z}$ in the $y=0$ plane of total waves at $\kappa a=1.2$ and for three cases of one layer, two and four layers. Stress $\sigma_{x z}$ due to the incident wave is zero. Wave fields inside black circles are not computed during scatterer polymerization procedure and set to zero. 
along the $z=0$ axis. The values of real part of $\sigma_{x z} \mid$ in regions along the $z=0$ axis increase when the number of layers increases. The stress component $\sigma_{x z}$ due to the incident wave is zero, so that the non-zero result of $\sigma_{x z}$ is due to scattered waves. In comparison with $\sigma_{x y}$ or $\sigma_{y z}$ in Figs. 6.43, $\sigma_{x z}$ is smaller than $\sigma_{x y}$ and $\sigma_{y z}$. 


\section{6-5 Concluding Remarks}

In this chapter, the application example showed that the transmission ratio of $u_{z}$ decreases in certain range of wave frequencies as the number of layers increases. It illustrates the process of band gap formation, in which the $u_{z}$ transmission ratio does not decrease to zero in the example.

In this application example, wave fields of scattered waves and total waves of the multiple scattering problems are displayed. Physical phenomena of the multiple scattering problems are observed.

This application example also demonstrated that the present computational system has the capability to simulate three-dimensional multiple scattering of elastic waves. 


\section{Chapter 7}

\section{Thesis Conclusion}

\section{7-1 Summary}

The subject of multiple scattering of elastic waves in a solid medium containing particles is not new. Multiple scattering solutions include approximate solutions, solutions from numerical methods and exact solutions. An exact solution provides more details than an approximate solution for same scattering problem. Analytically exact solutions are the focus in the thesis.

For the multiple scattering solution with iterative approach, the computation and storage of the matrix for coordinate translation between coordinate systems of every two scatterers is a limiting factor ${ }^{[24]}$. For the multiple scattering solution with $T$ matrix approach, the computation and storage of the matrix of the linear equation system is a limiting factor.

This thesis uses scatterer polymerization methodology to reduce the number of actual scatterers to a lesser number of abstract scatterers, so that the limiting factor due to the matrix size is removed. The major works that have been finished in this thesis include the following: 
1. Analytical expression of elastic wave fields in terms of the scalar and vector spherical harmonics.

2. Analytically exact solution of single scattering of elastic waves by a spherical inclusion in a solid medium is obtained, implemented and verified.

3. Analytically exact solution of three-dimensional multiple scattering of elastic waves in a solid medium containing arbitrary number and distribution of spherical scatterers is obtained, implemented and verified.

4. The scatterer polymerization methodology is extended to three-dimensional multiple scattering of elastic waves. This methodology is verified by using five approaches to solve the same scattering problem.

5. A band gap formation process is observed. An application example demonstrates that the computational system has the capability to simulate multiple scattering solutions of elastic waves in three-dimension.

\section{7-2 Future Work}

One computational system is built for three-dimensional multiple scattering of elastic waves by arbitrary number and arrangement of scatterers. But some issues have not yet been explored in detail. For example, it is known that the abstract scatterer territory size and the distance between two scatterers affect the accuracy of results and the truncation number, but the relation between the scatterer territory size, the distance between two scatterers, the total number of scatterers, the accuracy of results and the truncation number has not been detailed. These need to be explored in future work. 
The computational system has been used to study scattering cases of finite scatterers of regular arrangement. It can be used to study scattering cases of more scatterers of regular and irregular arrangements in future work. 


\section{Bibliography}

[1] C. F. Ying and R. Truell, Scattering of a plane longitudinal wave by a spherical obstacle in an isotropically elastic solid,, Journal of Applied Physics v27 n9, 1086-1097 (1956).

[2] E. J. Hearn, Mechanics of Materials, Volume 1 - An Introduction to the Mechanics of Elastic and Plastic Deformation of Solids and Structural Materials (3rd Edition), Elsevier, 1997.

[3] D. Ensminger, Ultrasonics: Fundamentals, Technology, Applications, 2nd Edition, Marcel Dekker, New York, 1988.

[4] E. J. Barbero, Introduction To Composite Materials Design, Taylor and Francis, pp.29, 1999.

[5] O. R. Geriche, Determination of the geometry of hidden defects by ultrasonics pulse analysis testing, The Journal of the Acoustic Society of America v35, 364-368 (1963).

[6] F. Cohen-Tenoudji, B. R. Tittmann, and G. Quentin, Technique for the inversion of backscattered elastic wave data to extract the geometrical parameters of defects with varing shape, Applied Physics Letters v41, 574-576 (1982).

[7] M. W. Ewing, W. S. Jardetzky, and F. Press, Elastic waves in layered media, New York, McGraw-Hill, 1957. 
[8] L.-W. Cai and J. H. Williams Jr., Nde via stop band formation in fiber reinforced composites having square fiber arrangements, Ultrasonics v37, 483-492 (1999).

[9] Z. Liu, C. T. Chan, and P. Sheng, Elastic wave scattering by periodic structures of spherical objects: Theory and experiment, Physical Review B v62 n4, 2446-2457 (2000).

[10] J. W. Strutt, Investigation of the disturbance produced by a spherical obstacle on the waves of sound, Proceedings of the London Mathematical Society v4, $253-283(1872)$.

[11] K. Sezawa, Scattering of elastic waves and some allied problems, Bull. Earthquake Res. Inst. (Tokyo Imperial University) v3, 19-41 (1927).

[12] Y.-H. Pao and C.-C. Mow, Scattering of plane compressional waves by a spherical obstacle, Journal of Applied Physics v34 n3, 493-499 (1963).

[13] N. G. Einspruch, E. J. Witterholt, and R. Truell, Scattering of a plane transverse wave by a spherical obstacle in an elastic medium,, Journal of Applied Physics v31 n5, 806-818 (1960).

[14] P. M. Morse and H. Feshbach, Methods of Theoretical Physics, McGraw-Hill Book Company, Inc., New York, 1953.

[15] A. N. Norris, Scattering of elastic waves by spherical inclusions with applications to low frequency wave propagation in composites, International Journal of Engineering Science v24 n8, 1271-1282 (1986).

[16] M. K. Hinders, Plane-elastic-wave scattering from an elastic sphere, Il Nuovo Climento v106B n7, 799-818 (1991). 
[17] P. C. Waterman, New formulation of acoustic scattering, The Journal of the Acoustic Society of America v45 n6, 1417-1429 (1969).

[18] V. Varatharajulu and Y.-H. Pao, Scattering matrix for elastic waves i. theory, The Journal of the Acoustic Society of America v60 n3, 556-566 (1976).

[19] P. C. Waterman, Matrix theory of elastic wave scattering, The Journal of the Acoustic Society of America v60 n3, 567-580 (1976).

[20] C. M. Sayers and R. L. Smith, Ultrasonic velocity and attenuation in an epoxy matrix containing lead inclusions, Journal of Physics D: Applied Physics v16, 1189-1194 (1983).

[21] V. K. Kinra, E. Ker, and S. K. Datta, Influence of particle resonance on wave propagation in a random particulate composite, Mechanics Research Communications v9, 109-114 (1982).

[22] V. Twersky, Multiple scattering of radiation by an arbitrary planar configuration of cylinders and by two parallel cylinders, Journal of Applied Physics v23 n4, 407-414 (1952).

[23] V. Twersky, Multiple scattering by arbitrary configurations in three dimensions, Journal of Mathematical Physics v3 n1, 83-91 (1962).

[24] T. E. Doyle, Iterative simulation of elastic wave scattering in arbitrary dispersions of spherical particles, The Journal of the Acoustic Society of America v119 n5, 2599-2610 (2006).

[25] A. Boström, Multiple scattering of elastic waves by bounded obstacles, The Journal of the Acoustical Society of America v67 n2, 399-413 (1980). 
[26] I. E. Psarobas, N. Stefanou, and A. Modinos, Scattering of elastic waves by periodic arrays of spherical bodies, Physical Review B v62 n1, 278-291 (2000).

[27] F. Ihlenburg, Finite element analysis of acoustic scattering, Springer-Verlag, New York, 1998.

[28] W. Axmann and P. Kuchment, An efficient finite element method for computing spectra of photonic and acoustic band-gap materials, Journal of Computational Physics v150, 468-481 (1999).

[29] P. Yang and K. N. Liou, Finite difference time domain method for light scattering by nonspherical and inhomogeneous particles, in Light Scattering by Nonspherical Particls, edited by M.I. Mishchenko, J.W.Hovenier, and L.D.Travis (Academic Press, San Diego),173-221, (2000).

[30] N. A. Gumerov and R. Duraiswami, Computation of scattering from $n$ spheres using multipole reexpansion, The Journal of the Acoustic Society of America v112 n6, 2688-2701 (2002).

[31] P. C. Waterman and R. Truell, Multiple scattering of waves, Journal of Mathematical Physics v2 n4, 512-537 (1961).

[32] L. L. Foldy, Multiple scattering of waves i. general theory of isotropic scattering distributed scatterers, Physical Review v67 n3-4, 107-119 (1945).

[33] G. C. Gaunaurd and H. Überall, Resonance effects and the ultrasonic effective properties of particulate composites, The Journal of the Acoustical Society of America v74 n1, 305-313 (1983).

[34] L.-W. Cai and J. H. Williams Jr., Large scale multiple scattering problems, Ultrasonics v37, 453-462 (1999). 
[35] L.-W. Cai and J. H. Williams Jr., Full-scale simulations of elastic wave scattering in fiber-reinforced composites, Ultrasonics v37, 463-482 (1999).

[36] Y.-H. Pao and C.-C. Mow, Diffraction of Elastic Waves and Dynamic Stress Concentrations, Crane Russak, New York, NY, 1971.

[37] K. F. Graff, Wave Motion in Elastic Solids, Dover Publications, Inc., New York, 1975.

[38] M. Abramowitz and I. A. Stegun, Handbook of Mathematical Functions with Formulas, Graphs, and Mathematical Tables, volume 10th Printing, United States Department of Commerce, 1972.

[39] A. R. Edmonds, Angular Momentum in Quantum Mechanics, Princeton University Press, Princeton, New Jersey, 1957.

[40] A. Ben-Menahem and S. J. Singh, Seismic Waves and Sources, Springer-Verlag New York Inc., Heidelberg Berlin, 1981.

[41] Y. C. Fung and P. Tong, Classical and Computational Solid Mechanics, World Scientific Publishing Co. Pte. Ltd., 2001.

[42] W. C. Chew, Addition theorem for spherical wave functions, Quarterly of Applied Mathematics V19 n1, 15-24 (1961).

[43] O. R. Cruzan, Translational addition theorem for spherical vector wave functions, Quarterly of Applied Mathematics V20 n1, 33-40 (1962).

[44] J. A. Stratton, Electromagnetic Theory, McGraw-Hill, New York, 1941.

[45] W. C. Chew, A derivation of the vector addition theorem, Microwave and Optical Technology Letters V3 n7, 256-260 (1990). 
[46] J. H. Hannay and J. F. Nye, Fibonacci numerical integration on a sphere, Journal of Physics A: Mathematical and General v37, 11591-11601 (2004).

[47] R. Swinbank and R. J. Purser, Numerical weather prediction (fibonacci grids: a novel approach to global modelling), Forecasting Research Technical Report No.468 (2006).

[48] G. Johnson and R. Truell, Numerical computations of elastic scattering cross section, Journal of Applied Physics v36 n11, 3466-3475 (1965).

[49] Thunderhead Engineering, Digxy version 1.0, Thunderhead Engineering, 403 Poyntz Ave, Suite B, Manhattan, KS 66502-6081, USA.

[50] L.-W. Cai, Full-Scale Simulation of Multiple Scattering of Elastic Waves in Fiber Reinforced Composites, PhD thesis, Massachusetts Institute of Technology, Cambrige, MA, June 1998.

[51] W. C. Chew, Waves and Fields in Inhomogeneous Media, Van Nostrand Reinhold, 1990.

[52] E. Condon and G.H.Shortley, The Theory of Atomic Spectra, Cambridge University Press, 1935.

[53] W. C. Chew and Y. M. Wang, Efficient ways to compute the vector addition theorem, Journal of Electromagnetic Waves and Applications v7 n5, 651-665 (1993).

[54] R. J. A. Tough, The transformation properties of vector multipole fields under a translation of coordinate origin, Journal of Physics A: Mathematical and General 10, 1079-1087 (1977). 
[55] T. E. Doyle, Utah State University, Logan, Utah, 2006, (Private Communication).

[56] J. D. Achenbach, Wave Propagations in Elastic Solids, North-Holland, Amsterdam, 1975.

[57] D. R. Bland, Solutions of Laplace's Equation, volume 2nd impression, Routledge and Kegan Paul Ltd, London, 1961. 


\section{Appendix A}

\section{Orthogonal Curvilinear Coordinate}

\section{System}

Without lose of generality, assume an orthogonal curvilinear coordinate system with coordinates $\left(\xi_{1}, \xi_{2}, \xi_{3}\right)$, with unit vectors $\boldsymbol{e}_{1}, \boldsymbol{e}_{2}$ and $\boldsymbol{e}_{2}$. For reference, coordinates in a Cartesian coordinate system are denoted as $\left(x_{1}, x_{2}, x_{3}\right)$ with unit vectors $\boldsymbol{n}_{1}, \boldsymbol{n}_{2}$ and $\boldsymbol{n}_{2}$. The curvilinear coordinate system is orthogonal and hence

$$
\boldsymbol{e}_{i} \cdot \boldsymbol{e}_{j}=\delta_{i j} \quad \text { and } \quad \boldsymbol{e}_{i} \times \boldsymbol{e}_{j}=\varepsilon_{i j k} \boldsymbol{e}_{k}
$$

where $\delta_{i j}$ is the Kronecker delta, and $\varepsilon_{i j k}$ is the permutation symbol.

Furthermore, an infinitesimal vector can be represented as

$$
d \boldsymbol{r}=h_{1} d \xi_{1} \boldsymbol{e}_{1}+h_{2} d \xi_{2} \boldsymbol{e}_{2}+h_{3} d \xi_{3} \boldsymbol{e}_{3}
$$

where $h_{i}$ is called the scale factor for coordinate $\xi_{i}(i=1,2,3)$, which can be computed by

$$
h_{i}=\sqrt{\left(\frac{\partial x_{1}}{\partial \xi_{i}}\right)^{2}+\left(\frac{\partial x_{2}}{\partial \xi_{i}}\right)^{2}+\left(\frac{\partial x_{3}}{\partial \xi_{i}}\right)^{2}}=\frac{1}{\sqrt{\left(\frac{\partial \xi_{i}}{\partial x_{1}}\right)^{2}+\left(\frac{\partial \xi_{i}}{\partial x_{2}}\right)^{2}+\left(\frac{\partial \xi_{i}}{\partial x_{3}}\right)^{2}}}
$$


From eqn. (A-2), we can write, for any $i$,

$$
\frac{\partial \boldsymbol{r}}{\partial \xi_{i}}=h_{i} \boldsymbol{e}_{i}
$$

Since, for any $i$ and $j(i \neq j)$,

$$
\frac{\partial}{\partial \xi_{j}}\left(\frac{\partial \boldsymbol{r}}{\partial \xi_{i}}\right)=\frac{\partial}{\partial \xi_{i}}\left(\frac{\partial \boldsymbol{r}}{\partial \xi_{j}}\right)
$$

we have

$$
\frac{\partial\left(h_{i} \boldsymbol{e}_{i}\right)}{\partial \xi_{j}}=\frac{\partial\left(h_{j} \boldsymbol{e}_{j}\right)}{\partial \xi_{i}}
$$

or

$$
h_{i} \frac{\partial \boldsymbol{e}_{i}}{\partial \xi_{j}}+\frac{\partial h_{i}}{\partial \xi_{j}} \boldsymbol{e}_{i}=h_{j} \frac{\partial \boldsymbol{e}_{j}}{\partial \xi_{i}}+\frac{\partial h_{j}}{\partial \xi_{i}} \boldsymbol{e}_{j}
$$

Applying dot-product with $\boldsymbol{e}_{i}$ to both sides of eqn. (A-5) gives

$$
h_{i} \boldsymbol{e}_{i} \cdot \frac{\partial \boldsymbol{e}_{i}}{\partial \xi_{j}}+\frac{\partial h_{i}}{\partial \xi_{j}}=\boldsymbol{e}_{i} \cdot h_{j} \frac{\partial \boldsymbol{e}_{j}}{\partial \xi_{i}}
$$

where $\boldsymbol{e}_{i} \cdot \boldsymbol{e}_{i}=1$ and $\boldsymbol{e}_{i} \cdot \boldsymbol{e}_{j}=0$ have been used. Since

$$
\frac{\partial\left(\boldsymbol{e}_{i} \cdot \boldsymbol{e}_{i}\right)}{\partial \xi_{j}}=0=2 \boldsymbol{e}_{i} \cdot \frac{\partial \boldsymbol{e}_{i}}{\partial \xi_{j}}
$$

eqn. (A-7) implies that $\partial \boldsymbol{e}_{i} / \partial \xi_{j}$ is normal to $\boldsymbol{e}_{i}$. Furthermore, eqn. (A-6) becomes

$$
\frac{\partial h_{i}}{\partial \xi_{j}}=\boldsymbol{e}_{i} \cdot h_{j} \frac{\partial \boldsymbol{e}_{j}}{\partial \xi_{i}}
$$

Applying dot-product with $\boldsymbol{e}_{k}(k \neq i, j)$ to both sides of eqn. (A-5) gives

$$
h_{i} \boldsymbol{e}_{k} \cdot \frac{\partial \boldsymbol{e}_{i}}{\partial \xi_{j}}=h_{j} \boldsymbol{e}_{k} \cdot \frac{\partial \boldsymbol{e}_{j}}{\partial \xi_{i}}
$$

Also, since $\boldsymbol{e}_{i} \cdot \boldsymbol{e}_{k}=0$,

$$
\frac{\partial\left(\boldsymbol{e}_{i} \cdot \boldsymbol{e}_{k}\right)}{\partial \xi_{j}}=0=\boldsymbol{e}_{i} \cdot \frac{\partial \boldsymbol{e}_{k}}{\partial \xi_{j}}+\boldsymbol{e}_{k} \cdot \frac{\partial \boldsymbol{e}_{i}}{\partial \xi_{j}}
$$

Note that similar relations as in eqns. $(\mathrm{A}-9)$ and $(\mathrm{A}-10)$ exist, with appropriate permutations of indices $i, j$ and $k$.

From eqn. (A-10),

$$
\boldsymbol{e}_{k} \cdot \frac{\partial \boldsymbol{e}_{i}}{\partial \xi_{j}}=-\boldsymbol{e}_{i} \cdot \frac{\partial \boldsymbol{e}_{k}}{\partial \xi_{j}}=-\frac{h_{j}}{h_{k}} \boldsymbol{e}_{i} \cdot \frac{\partial \boldsymbol{e}_{j}}{\partial \xi_{k}} \quad \text { (using eqn. (A-9) with } i \text { and } k \text { exchanged) }
$$




$$
\begin{aligned}
& =\frac{h_{j}}{h_{k}} \boldsymbol{e}_{j} \cdot \frac{\partial \boldsymbol{e}_{i}}{\partial \xi_{k}} \quad \text { (using eqn. (A-10) with } j \text { and } k \text { exchanged) } \\
& =\frac{h_{j}}{h_{k}} \frac{h_{k}}{h_{i}} \boldsymbol{e}_{j} \cdot \frac{\partial \boldsymbol{e}_{k}}{\partial \xi_{j}} \quad \text { (using eqn. (A-9) with } j \text { and } k \text { exchanged) } \\
& =-\frac{h_{j}}{h_{i}} \boldsymbol{e}_{k} \cdot \frac{\partial \boldsymbol{e}_{j}}{\partial \xi_{i}} \quad \text { (using eqn. (A-10) with } i \text { and } j \text { exchanged) } \\
& =-\frac{h_{j}}{h_{i}} \frac{h_{i}}{h_{j}} \boldsymbol{e}_{k} \cdot \frac{\partial \boldsymbol{e}_{i}}{\partial \xi_{j}} \quad \text { (using eqn. (A-9) with } i \text { and } j \text { exchanged) } \\
& =-\boldsymbol{e}_{k} \cdot \frac{\partial \boldsymbol{e}_{i}}{\partial \xi_{j}}
\end{aligned}
$$

Equation (A-11) implies

$$
\boldsymbol{e}_{i} \cdot \frac{\partial \boldsymbol{e}_{k}}{\partial \xi_{j}}=0
$$

That is, $\partial \boldsymbol{e}_{i} / \partial \xi_{j}$ is normal to $\boldsymbol{e}_{k}$.

Therefore, $\partial \boldsymbol{e}_{i} / \partial \xi_{j}$ is normal to both $\boldsymbol{e}_{i}$ and $\boldsymbol{e}_{k}$, hence, $\partial \boldsymbol{e}_{i} / \partial \xi_{j}$ is along the direction of $\boldsymbol{e}_{j}$. Thus, eqn. (A-5) gives, for $i \neq j$,

$$
\frac{\partial \boldsymbol{e}_{i}}{\partial \xi_{j}}=\frac{\boldsymbol{e}_{j}}{h_{i}} \frac{\partial h_{j}}{\partial \xi_{i}}
$$

For $i, j, k$ in cyclic order,

$$
\begin{aligned}
\frac{\partial \boldsymbol{e}_{i}}{\partial \xi_{i}} & =\frac{\partial\left(\boldsymbol{e}_{j} \times \boldsymbol{e}_{k}\right)}{\partial \xi_{i}}=\boldsymbol{e}_{j} \times \frac{\partial \boldsymbol{e}_{k}}{\partial \xi_{i}}+\frac{\partial \boldsymbol{e}_{j}}{\partial \xi_{i}} \times \boldsymbol{e}_{k} \\
& =\boldsymbol{e}_{j} \times \frac{\boldsymbol{e}_{i}}{h_{k}} \frac{\partial h_{i}}{\partial \xi_{k}}+\frac{\boldsymbol{e}_{i}}{h_{j}} \frac{\partial h_{i}}{\partial \xi_{j}} \times \boldsymbol{e}_{k}=-\frac{\boldsymbol{e}_{j}}{h_{j}} \frac{\partial h_{i}}{\partial \xi_{j}}-\frac{\boldsymbol{e}_{k}}{h_{k}} \frac{\partial h_{i}}{\partial \xi_{k}}
\end{aligned}
$$

Equations $(\mathrm{A}-13)$ and $(\mathrm{A}-14)$ form the bases for obtaining the vectorial operators in the curvilinear coordinate system. Details can be found in [56]. Expressions for the vectorial operators in the orthogonal curvilinear coordinate systems are summarized as following

$$
\begin{aligned}
\nabla & =\frac{\boldsymbol{e}_{1}}{h_{1}} \frac{\partial}{\partial \xi_{1}}+\frac{\boldsymbol{e}_{2}}{h_{2}} \frac{\partial}{\partial \xi_{2}}+\frac{\boldsymbol{e}_{3}}{h_{3}} \frac{\partial}{\partial \xi_{3}} \\
\nabla \cdot \boldsymbol{V} & =\frac{1}{h_{1} h_{2} h_{3}}\left[\frac{\partial\left(V_{1} h_{2} h_{3}\right)}{\partial \xi_{1}}+\frac{\partial\left(h_{1} V_{2} h_{3}\right)}{\partial \xi_{2}}+\frac{\partial\left(h_{1} h_{2} V_{3}\right)}{\partial \xi_{3}}\right]
\end{aligned}
$$




$$
\begin{aligned}
\nabla \times \boldsymbol{V}= & \frac{1}{h_{1} h_{2} h_{3}}\left|\begin{array}{ccc}
h_{1} \boldsymbol{e}_{1} & h_{2} \boldsymbol{e}_{2} & h_{3} \boldsymbol{e}_{3} \\
\frac{\partial}{\partial \xi_{1}} & \frac{\partial}{\partial \xi_{2}} & \frac{\partial}{\partial \xi_{3}} \\
h_{1} V_{1} & h_{2} V_{2} & h_{3} V_{3}
\end{array}\right| \\
\nabla^{2} v= & \frac{1}{h_{1} h_{2} h_{3}}\left[\frac{\partial}{\partial \xi_{1}}\left(\frac{h_{2} h_{3}}{h_{1}} \frac{\partial v}{\partial \xi_{1}}\right)+\frac{\partial}{\partial \xi_{2}}\left(\frac{h_{1} h_{3}}{h_{2}} \frac{\partial v}{\partial \xi_{2}}\right)\right. \\
& \left.+\frac{\partial}{\partial \xi_{3}}\left(\frac{h_{1} h_{2}}{h_{3}} \frac{\partial v}{\partial \xi_{3}}\right)\right]
\end{aligned}
$$




\section{Appendix B}

\section{Reduce Navier's Equation to Three Scalar Wave Equations}

The Navier's equation, for the motion of a particle in an elastic medium, is

$$
\rho \ddot{\boldsymbol{u}}=\mu \nabla^{2} \boldsymbol{u}+(\lambda+\mu) \nabla(\nabla \cdot \boldsymbol{u})
$$

Details of reducing the Navier's equation eqn. (B-1) to the following three scalar wave equations are introduced in this Appendix.

$$
\begin{aligned}
& \ddot{\phi}=c_{L}^{2} \nabla^{2} \phi \\
& \ddot{\psi}=c_{S}^{2} \nabla^{2} \psi \\
& \ddot{\chi}=c_{S}^{2} \nabla^{2} \chi
\end{aligned}
$$

According to Helmholtz's theorem of decomposition of a vector field ${ }^{[14]}$, displacement vector $\boldsymbol{u}$ can be written in terms of displacement potentials, as follows:

$$
\boldsymbol{u}=\nabla \phi+\nabla \times \boldsymbol{\psi}
$$


where $\phi$ is called the scalar displacement potential, and $\boldsymbol{\psi}$ is called the vector displacement potential of $\boldsymbol{u}$. Substituting eqn. (B-3) into eqn. (B-1) gives

$$
\begin{aligned}
\rho \nabla \ddot{\phi}+\rho \nabla \times \ddot{\boldsymbol{\psi}}= & \mu \nabla^{2}(\nabla \phi)+\mu \nabla^{2}(\nabla \times \boldsymbol{\psi}) \\
& +(\lambda+\mu)\{\nabla(\nabla \cdot \nabla \phi)+\nabla[\nabla \cdot(\nabla \times \boldsymbol{\psi})]\}
\end{aligned}
$$

Since

$$
\begin{gathered}
\nabla \cdot \nabla=\nabla^{2}, \quad \nabla^{2}(\nabla \phi)=\nabla\left(\nabla^{2} \phi\right) \\
\nabla \cdot(\nabla \times \boldsymbol{\psi})=0, \quad \nabla^{2}(\nabla \times \boldsymbol{\psi})=\nabla \times\left(\nabla^{2} \boldsymbol{\psi}\right)
\end{gathered}
$$

eqn. (B-4) can be written as

$$
\rho \nabla \ddot{\phi}+\rho \nabla \times \ddot{\boldsymbol{\psi}}=(\lambda+2 \mu) \nabla\left(\nabla^{2} \phi\right)+\mu \nabla \times\left(\nabla^{2} \boldsymbol{\psi}\right)
$$

or

$$
\nabla\left[\rho \ddot{\phi}-(\lambda+2 \mu) \nabla^{2} \phi\right]+\nabla \times\left[\rho \ddot{\boldsymbol{\psi}}-\mu \nabla^{2} \boldsymbol{\psi}\right]=0
$$

For the general case, eqn. (B-6) gives rise to two simultaneous equations

$$
\ddot{\phi}=c_{L}^{2} \nabla^{2} \phi \quad \text { and } \quad \ddot{\boldsymbol{\psi}}=c_{S}^{2} \nabla^{2} \boldsymbol{\psi}
$$

where

$$
c_{L}=\sqrt{\frac{\lambda+2 \mu}{\rho}} \quad \text { and } \quad c_{S}=\sqrt{\frac{\mu}{\rho}}
$$

are two different wave speeds, representing two different types of waves in the solids. Furthermore, since $\nabla \cdot(\nabla \times \boldsymbol{\psi})=0$, waves due to $\boldsymbol{\psi}$ do not cause volume change and are thus called the shear waves; and since $\nabla \times(\nabla \phi)=0$, waves due to $\phi$ are called the longitudinal wave. Note that the two wave equations are uncoupled.

Also note that the above equations are reached via replacing the displacement vector by the displacement potentials, the process replaces 3 scalar functions $(3 \mathrm{com}$ ponents of displacement) by 4 scalar functions ( 1 scalar potential plus 1 vector potential), this redundancy indicates that the 4 scalar components are not independent. 
Usually components of the vectorial potential are restricted by the condition

$$
\nabla \cdot \boldsymbol{\psi}=0
$$

Because of this condition, the 3 components of the vectorial potential can be replaced by 2 of them. Combined with the scalar potential $\phi$, the wave equation can be completely solved by using 3 displacement potentials.

We choose $\psi$ and $\chi$ such that each term in eqn. (2-9) satisfies eqn. (B-6). For $\psi$, this is

$$
\nabla \times\left[c_{S}^{2} \nabla^{2}\left(w \psi \boldsymbol{e}_{3}\right)-w \ddot{\psi} \boldsymbol{e}_{3}\right]=0
$$

Recall that, for any vector

$$
\nabla^{2} \boldsymbol{f}=\nabla(\nabla \cdot \boldsymbol{f})-\nabla \times \nabla \times \boldsymbol{f}
$$

Using vector operators for a general curvilinear coordinate system $\left(\xi_{1}, \xi_{2}, \xi_{3}\right)$ (with scale factor $h_{1}, h_{2}$ and $h_{3}$ and unit vectors $\boldsymbol{e}_{1}, \boldsymbol{e}_{2}$ and $\left.\boldsymbol{e}_{3}\right)$ as detailed in Appendix A, we have

$$
\begin{aligned}
\nabla \cdot\left(w \psi \boldsymbol{e}_{3}\right)= & \frac{1}{h_{1} h_{2} h_{3}} \frac{\partial\left(h_{1} h_{2} w \psi\right)}{\partial \xi_{3}}=\frac{1}{h_{3}}\left[\frac{w \psi}{h_{1} h_{2}} \frac{\partial\left(h_{1} h_{2}\right)}{\partial \xi_{3}}+\frac{\partial(w \psi)}{\partial \xi_{3}}\right] \\
\nabla \times\left(w \psi \boldsymbol{e}_{3}\right)= & \frac{\boldsymbol{e}_{1}}{h_{2} h_{3}} \frac{\partial\left(h_{3} w \psi\right)}{\partial \xi_{2}}-\frac{\boldsymbol{e}_{2}}{h_{1} h_{3}} \frac{\partial\left(h_{3} w \psi\right)}{\partial \xi_{1}} \\
\nabla\left[\nabla \cdot\left(w \psi \boldsymbol{e}_{3}\right)\right]= & \frac{\boldsymbol{e}_{1}}{h_{1}} \frac{\partial}{\partial \xi_{1}}\left\{\frac{1}{h_{3}}\left[\frac{w \psi}{h_{1} h_{2}} \frac{\partial\left(h_{1} h_{2}\right)}{\partial \xi_{3}}+\frac{\partial(w \psi)}{\partial \xi_{3}}\right]\right\} \\
& +\frac{\boldsymbol{e}_{2}}{h_{2}} \frac{\partial}{\partial \xi_{2}}\left\{\frac{1}{h_{3}}\left[\frac{w \psi}{h_{1} h_{2}} \frac{\partial\left(h_{1} h_{2}\right)}{\partial \xi_{3}}+\frac{\partial(w \psi)}{\partial \xi_{3}}\right]\right\} \\
& +\frac{\boldsymbol{e}_{3}}{h_{3}} \frac{\partial}{\partial \xi_{3}}\left\{\frac{1}{h_{3}}\left[\frac{w \psi}{h_{1} h_{2}} \frac{\partial\left(h_{1} h_{2}\right)}{\partial \xi_{3}}+\frac{\partial(w \psi)}{\partial \xi_{3}}\right]\right\} \\
\nabla \times \nabla \times\left(w \psi \boldsymbol{e}_{3}\right)= & \frac{\boldsymbol{e}_{1}}{h_{2} h_{3}} \frac{\partial}{\partial \xi_{3}}\left[\frac{h_{2}}{h_{1} h_{3}} \frac{\partial\left(h_{3} w \psi\right)}{\partial \xi_{1}}\right]+\frac{\boldsymbol{e}_{2}}{h_{1} h_{3}} \frac{\partial}{\partial \xi_{3}}\left[\frac{h_{1}}{h_{2} h_{3}} \frac{\partial\left(h_{3} w \psi\right)}{\partial \xi_{2}}\right] \\
& -\frac{\boldsymbol{e}_{3}}{h_{1} h_{2}}\left\{\frac{\partial}{\partial \xi_{1}}\left[\frac{h_{2}}{h_{1} h_{3}} \frac{\partial\left(h_{3} w \psi\right)}{\partial \xi_{1}}\right]+\frac{\partial}{\partial \xi_{2}}\left[\frac{h_{1}}{h_{2} h_{3}} \frac{\partial\left(h_{3} w \psi\right)}{\partial \xi_{2}}\right]\right\}
\end{aligned}
$$

Assume: $h_{3}$ is a constant, and $h_{2} / h_{1}$ is independent of $\xi_{3}$, then

$$
\nabla\left[\nabla \cdot\left(w \psi \boldsymbol{e}_{3}\right)\right]=\frac{\boldsymbol{e}_{1}}{h_{1} h_{3}}\left\{\frac{\partial}{\partial \xi_{1}}\left[\frac{w \psi}{h_{1} h_{2}} \frac{\partial\left(h_{1} h_{2}\right)}{\partial \xi_{3}}\right]+\frac{\partial^{2}(w \psi)}{\partial \xi_{1} \partial \xi_{3}}\right\}
$$




$$
\begin{aligned}
& +\frac{\boldsymbol{e}_{2}}{h_{2} h_{3}}\left\{\frac{\partial}{\partial \xi_{2}}\left[\frac{w \psi}{h_{1} h_{2}} \frac{\partial\left(h_{1} h_{2}\right)}{\partial \xi_{3}}\right]+\frac{\partial^{2}(w \psi)}{\partial \xi_{2} \partial \xi_{3}}\right\} \\
& +\frac{\boldsymbol{e}_{3}}{h_{3}^{2}}\left\{\frac{\partial}{\partial \xi_{3}}\left[\frac{w \psi}{h_{1} h_{2}} \frac{\partial\left(h_{1} h_{2}\right)}{\partial \xi_{3}}\right]+\frac{\partial^{2}(w \psi)}{\partial \xi_{3}^{2}}\right\} \\
\nabla \times \nabla \times\left(w \psi \boldsymbol{e}_{3}\right)= & \frac{\boldsymbol{e}_{1}}{h_{1} h_{3}} \frac{\partial^{2}(w \psi)}{\partial \xi_{1} \partial \xi_{3}}+\frac{\boldsymbol{e}_{2}}{h_{2} h_{3}} \frac{\partial^{2}(w \psi)}{\partial \xi_{2} \partial \xi_{3}} \\
& -\frac{\boldsymbol{e}_{3}}{h_{1} h_{2}}\left\{\frac{\partial}{\partial \xi_{1}}\left[\frac{h_{2}}{h_{1}} \frac{\partial(w \psi)}{\partial \xi_{1}}\right]+\frac{\partial}{\partial \xi_{2}}\left[\frac{h_{1}}{h_{2}} \frac{\partial(w \psi)}{\partial \xi_{2}}\right]\right\}
\end{aligned}
$$

Therefore

$$
\begin{aligned}
\nabla^{2}\left(w \psi \boldsymbol{e}_{3}\right)= & \frac{1}{h_{3}}\left\{\frac{\boldsymbol{e}_{1}}{h_{1}} \frac{\partial}{\partial \xi_{1}}\left[\frac{w \psi}{h_{1} h_{2}} \frac{\partial\left(h_{1} h_{2}\right)}{\partial \xi_{3}}\right]+\frac{\boldsymbol{e}_{2}}{h_{2}} \frac{\partial}{\partial \xi_{2}}\left[\frac{w \psi}{h_{1} h_{2}} \frac{\partial\left(h_{1} h_{2}\right)}{\partial \xi_{3}}\right]\right. \\
& \left.+\frac{\boldsymbol{e}_{3}}{h_{3}} \frac{\partial}{\partial \xi_{3}}\left[\frac{w \psi}{h_{1} h_{2}} \frac{\partial\left(h_{1} h_{2}\right)}{\partial \xi_{3}}\right]\right\}+\frac{\boldsymbol{e}_{3}}{h_{3}^{2}} \frac{\partial^{2}(w \psi)}{\partial \xi_{3}^{2}} \\
& +\frac{\boldsymbol{e}_{3}}{h_{1} h_{2}}\left\{\frac{\partial}{\partial \xi_{1}}\left[\frac{h_{2}}{h_{1}} \frac{\partial(w \psi)}{\partial \xi_{1}}\right]+\frac{\partial}{\partial \xi_{2}}\left[\frac{h_{1}}{h_{2}} \frac{\partial(w \psi)}{\partial \xi_{2}}\right]\right\}
\end{aligned}
$$

Also recall that

$$
\begin{aligned}
\nabla^{2}(w \psi)= & \frac{1}{h_{1} h_{2} h_{3}}\left[\frac{\partial}{\partial \xi_{1}}\left(\frac{h_{2} h_{3}}{h_{1}} \frac{\partial(w \psi)}{\partial \xi_{1}}\right)+\frac{\partial}{\partial \xi_{2}}\left(\frac{h_{1} h_{3}}{h_{2}} \frac{\partial(w \psi)}{\partial \xi_{2}}\right)\right. \\
& \left.+\frac{\partial}{\partial \xi_{3}}\left(\frac{h_{1} h_{2}}{h_{3}} \frac{\partial(w \psi)}{\partial \xi_{3}}\right)\right] \\
= & \frac{1}{h_{1} h_{2}} \frac{\partial}{\partial \xi_{1}}\left[\frac{h_{2}}{h_{1}} \frac{\partial(w \psi)}{\partial \xi_{1}}\right]+\frac{1}{h_{1} h_{2}} \frac{\partial}{\partial \xi_{2}}\left[\frac{h_{1}}{h_{2}} \frac{\partial(w \psi)}{\partial \xi_{2}}\right] \\
& +\frac{1}{h_{1} h_{2} h_{3}^{2}} \frac{\partial}{\partial \xi_{3}}\left[h_{1} h_{2} \frac{\partial(w \psi)}{\partial \xi_{3}}\right]
\end{aligned}
$$

we have

$$
\nabla^{2}\left(w \psi \boldsymbol{e}_{3}\right)=\frac{1}{h_{3}} \nabla\left(\frac{w \psi}{h_{1} h_{2}} \frac{\partial\left(h_{1} h_{2}\right)}{\partial \xi_{3}}\right)+\left[\nabla^{2}(w \psi)-\frac{1}{h_{1} h_{2} h_{3}^{2}} \frac{\partial\left(h_{1} h_{2}\right)}{\partial \xi_{3}} \frac{\partial(w \psi)}{\partial \xi_{3}}\right] \boldsymbol{e}_{3}
$$

For cylindrical coordinate systems (including circular, elliptical and parabolic cylindrical systems), $h_{1} h_{2}$ is independent of $\xi_{3}$, eqn. (B-19) becomes

$$
\nabla^{2}\left(w \psi \boldsymbol{e}_{3}\right)=\nabla^{2}(w \psi) \boldsymbol{e}_{3}
$$

For spherical and conical coordinate systems, $h_{1} h_{2}=\xi_{3}^{2} f\left(\xi_{1}, \xi_{2}\right)$, hence

$$
\frac{\partial\left(h_{1} h_{2}\right)}{\partial \xi_{3}}=2 \xi_{3} f\left(\xi_{1}, \xi_{2}\right)=2 \frac{h_{1} h_{2}}{\xi_{3}}
$$


Also,

$$
\nabla^{2}(w \psi)=w \nabla^{2} \psi+2(\nabla w) \cdot(\nabla \psi)+\psi \nabla^{2} w
$$

eqn. (B-19) becomes

$$
\begin{aligned}
\nabla^{2}\left(w \psi \boldsymbol{e}_{3}\right)= & \frac{2}{h_{3}} \nabla\left(\frac{w \psi}{\xi_{3}}\right)+\left[w \nabla^{2} \psi+2(\nabla w) \cdot(\nabla \psi)\right. \\
& \left.+\psi \nabla^{2} w-\frac{2}{\xi_{3} h_{3}^{2}} \frac{\partial(w \psi)}{\partial \xi_{3}}\right] \boldsymbol{e}_{3}
\end{aligned}
$$

If we choose $w=\xi_{3}$, then

$$
(\nabla w) \cdot(\nabla \psi)=\frac{1}{h_{3}^{2}} \frac{\partial w}{\partial \xi_{3}} \frac{\partial \psi}{\partial \xi_{3}} \quad \text { and } \quad \nabla^{2} w=\frac{1}{h_{1} h_{2} h_{3}} \frac{\partial}{\partial \xi_{3}}\left(\frac{h_{1} h_{2}}{h_{3}}\right)=\frac{2}{\xi_{3} h_{3}^{2}}
$$

eqn. (B-21) becomes

$$
\begin{aligned}
\nabla^{2}\left(w \psi \boldsymbol{e}_{3}\right) & =\frac{2}{h_{3}} \nabla \psi+\left[w \nabla^{2} \psi+\frac{2}{h_{3}^{2}} \frac{\partial \psi}{\partial \xi_{3}}+\frac{2 \psi}{\xi_{3} h_{3}^{2}}-\frac{2}{h_{3}^{2}} \frac{\partial \psi}{\partial \xi_{3}}-\frac{2}{\xi_{3} h_{3}^{2}} \psi\right] \boldsymbol{e}_{3} \\
& =\frac{2}{h_{3}} \nabla \psi+w \nabla^{2} \psi \boldsymbol{e}_{3}
\end{aligned}
$$

Since $\nabla \times(\nabla \psi) \equiv 0$, for cylindrical, spherical and conical coordinate systems that have been considered, the equation for $\psi$ is

$$
c_{S}^{2} \nabla^{2} \psi=\ddot{\psi}
$$

Following the similar procedure, it can be shown that the equation for $\chi$ is formally the same.

To summarize, the assumptions made about the coordinate system include

- $h_{3}$ is a constant;

- $h 2 / h 1$ is independent of $\xi_{3}$;

- $h_{1} h_{2}$ is either independent of $\xi_{3}$ (cylindrical coordinate systems), or $h_{1} h_{2}=$ $\xi_{3}^{2} f\left(\xi_{1}, \xi_{2}\right)$ (spherical and conical coordinate systems);

- $w$ is constant (cylindrical coordinate systems) or $w=\xi_{3}$ (spherical and conical coordinate systems). 
These conditions are satisfied by the following 6 coordinate systems: Cartesian coordinate system, circular cylindrical coordinate system, elliptical cylindrical coordinate system, parabolic cylindrical coordinate system, spherical coordinate system and conical coordinate system. For these coordinate systems, the elastic wave equation in eqn. (2-6) can be reduced to the following three uncoupled equations of scalar displacement potentials:

$$
\begin{aligned}
& \ddot{\phi}=c_{L}^{2} \nabla^{2} \phi \\
& \ddot{\psi}=c_{S}^{2} \nabla^{2} \psi \\
& \ddot{\chi}=c_{S}^{2} \nabla^{2} \chi
\end{aligned}
$$




\section{Appendix C}

\section{Spherical Coordinate System and Field Operators}

Suppose the spherical coordinates $(r, \theta, \varphi)$ of a point $P$ in Fig. C.1 are given, where $\varphi(0 \leq \varphi \leq 2 \pi)$ is the azimuthal angle measured from positive direction of $x$-axis in the $x-y$ plane, and $\theta(0 \leq \theta \leq \pi)$ is the zenith angle measured from positive direction of $z$-axis. Then, the Cartesian coordinates $(x, y, z)$ for $P$ are

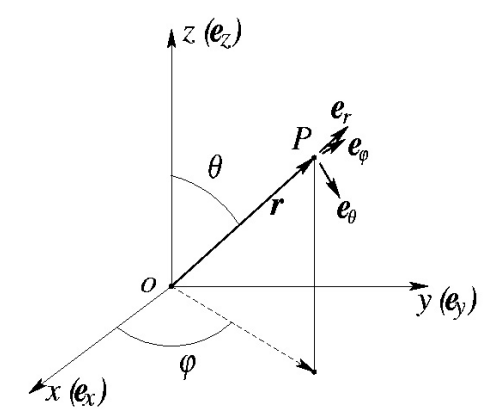

Figure C.1: Spherical and Cartesian Coordinate Systems.

$$
x=r \sin \theta \cos \varphi, \quad y=r \sin \theta \sin \varphi, \quad \text { and } \quad z=r \cos \theta
$$

In general, the inverse is

$$
r=\sqrt{x^{2}+y^{2}+z^{2}}
$$




$$
\begin{aligned}
& \theta=\cos ^{-1}(z / r), \\
& \varphi=\tan ^{-1}(y / x)
\end{aligned}
$$

and the unit vectors are

$$
\begin{aligned}
& \boldsymbol{e}_{r}=\sin \theta \cos \varphi \boldsymbol{e}_{x}+\sin \theta \sin \varphi \boldsymbol{e}_{y}+\cos \theta \boldsymbol{e}_{z} \\
& \boldsymbol{e}_{\theta}=\cos \theta \cos \varphi \boldsymbol{e}_{x}+\cos \theta \sin \varphi \boldsymbol{e}_{y}-\sin \theta \boldsymbol{e}_{z} \\
& \boldsymbol{e}_{\varphi}=-\sin \varphi \boldsymbol{e}_{x}+\cos \varphi \boldsymbol{e}_{y}
\end{aligned}
$$

An infinitesimal vector can be written as

$$
d \boldsymbol{r}=d r \boldsymbol{e}_{r}+r \sin \theta d \varphi \boldsymbol{e}_{\varphi}+r d \theta \boldsymbol{e}_{\theta}
$$

Thus

$$
\xi_{1}=r, \quad \xi_{2}=\varphi, \quad \xi_{3}=\theta
$$

and

$$
h_{1}=1, \quad h_{2}=r \sin \theta, \quad h_{3}=r
$$

The operators are

$$
\begin{aligned}
\nabla a= & \frac{\partial a}{\partial r} \boldsymbol{e}_{r}+\frac{1}{r \sin \theta} \frac{\partial a}{\partial \varphi} \boldsymbol{e}_{\varphi}+\frac{1}{r} \frac{\partial a}{\partial \theta} \boldsymbol{e}_{\theta} \\
\nabla \cdot \boldsymbol{V}= & \frac{1}{r^{2} \sin \theta}\left[\frac{\partial\left(r^{2} \sin \theta V_{r}\right)}{\partial r}+\frac{\partial\left(r V_{\varphi}\right)}{\partial \varphi}+\frac{\partial\left(r \sin \theta V_{\theta}\right)}{\partial \theta}\right] \\
= & \frac{1}{r^{2}} \frac{\partial\left(r^{2} V_{r}\right)}{\partial r}+\frac{1}{r \sin \theta} \frac{\partial V_{\varphi}}{\partial \varphi}+\frac{1}{r \sin \theta} \frac{\partial\left(\sin \theta V_{\theta}\right)}{\partial \theta} \\
\nabla \times \boldsymbol{V}= & \frac{1}{r^{2} \sin \theta}\left|\begin{array}{l}
\frac{\partial}{\partial r} \frac{\partial}{\partial \theta} \\
\boldsymbol{e}_{r} r V_{\theta} r \sin \theta V_{\varphi}
\end{array}\right| \\
= & \frac{1}{r^{2} \sin \theta}\left[\begin{array}{l}
\left(-\frac{\partial\left(r V_{\theta}\right)}{\partial \varphi}+\frac{\partial\left(r \sin \theta V_{\varphi}\right)}{\partial \theta}\right) \\
\end{array}\right) \boldsymbol{e}_{r}-\left(\frac{\partial V_{r}}{\partial \theta}-\frac{\partial\left(r V_{\theta}\right)}{\partial r}\right) r \sin \theta \boldsymbol{e}_{\varphi} \\
& \left.-\left(\frac{\partial\left(r \sin \theta V_{\varphi}\right)}{\partial r}-\frac{\partial V_{r}}{\partial \varphi}\right) r \boldsymbol{e}_{\theta}\right] \\
= & \frac{-1}{r \sin \theta}\left(\frac{\partial V_{\theta}}{\partial \varphi}-\frac{\partial\left(\sin \theta V_{\varphi}\right)}{\partial \theta}\right) \boldsymbol{e}_{r}-\left(\frac{1}{r} \frac{\partial V_{r}}{\partial \theta}-\frac{\partial V_{\theta}}{\partial r}-\frac{V_{\theta}}{r}\right) \boldsymbol{e}_{\varphi}
\end{aligned}
$$




$$
\begin{aligned}
& -\left(\frac{\partial V_{\varphi}}{\partial r}+\frac{V_{\varphi}}{r}-\frac{1}{r \sin \theta} \frac{\partial V_{r}}{\partial \varphi}\right) \boldsymbol{e}_{\theta} \\
\nabla^{2} v= & \frac{1}{r^{2} \sin \theta}\left[\frac{\partial}{\partial r}\left(r^{2} \sin \theta \frac{\partial v}{\partial r}\right)+\frac{\partial}{\partial \varphi}\left(\frac{1}{\sin \theta} \frac{\partial v}{\partial \varphi}\right)+\frac{\partial}{\partial \theta}\left(\sin \theta \frac{\partial v}{\partial \theta}\right)\right] \\
= & \frac{1}{r^{2}} \frac{\partial}{\partial r}\left(r^{2} \frac{\partial v}{\partial r}\right)+\frac{1}{r^{2} \sin ^{2} \theta} \frac{\partial^{2} v}{\partial \varphi^{2}}+\frac{1}{r^{2} \sin \theta} \frac{\partial}{\partial \theta}\left(\sin \theta \frac{\partial v}{\partial \theta}\right)
\end{aligned}
$$




\section{Appendix D}

\section{Coordinate Transformation for}

\section{Displacements, Strains and}

\section{Stresses between Spherical and}

\section{Cartesian Coordinate Systems}

Figure D.1 is referred here for coordinate transformation.

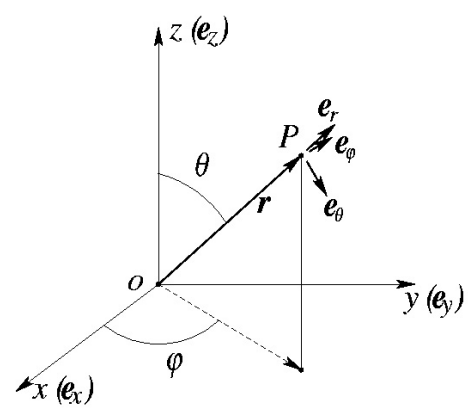

Figure D.1: Spherical and Cartesian Coordinate Systems.

Suppose the spherical coordinates $(r, \theta, \varphi)$ of a point $P$ in Fig. D.1 are given, where $\varphi(0 \leq \varphi \leq 2 \pi)$ is the azimuthal angle measured from positive direction of $x$-axis in 
the $x$ - $y$ plane, and $\theta(0 \leq \theta \leq \pi)$ is the zenith angle measured from positive direction of $z$-axis. Then, the Cartesian coordinates $(x, y, z)$ for $P$ are

$$
\begin{aligned}
& x=r \sin \theta \cos \varphi \\
& y=r \sin \theta \sin \varphi \\
& z=r \cos \theta
\end{aligned}
$$

When the Cartesian coordinates $(x, y, z)$ of a point $P$ are given, then the spherical coordinates $(r, \theta, \varphi)$ for $P$ are

$$
\begin{aligned}
& r=\sqrt{x^{2}+y^{2}+z^{2}} \\
& \theta=\arccos (z / r) \\
& \varphi=\left\{\begin{array}{l}
0 \text { if } x=0 \text { and } y=0 \\
\pi / 2 \text { if } x=0 \text { and } y>0 \\
3 \pi / 2 \text { if } x=0 \text { and } y<0 \\
\pi+\arctan (y / x) \text { if } x<0 \\
\arctan (y / x) \quad \text { if } \quad x>0 \text { and } y \geq 0 \\
2 \pi+\arctan (y / x) \text { if } x>0 \text { and } y<0
\end{array}\right.
\end{aligned}
$$

where $0 \leq \theta \leq \pi$ when $0 \leq \arccos (z / r) \leq \pi$, and $0 \leq \varphi<2 \pi$ when $-\pi / 2<$ $\arctan (y / x)<\pi / 2$. When the length of a vector is zero, it is zero vector that has all components equal to zero. Equation (D-2c) is essentially the same as Eq. (C-2c), except that the coordinate $\varphi$ in Eq. (D-2c) is confined in the range $[0,2 \pi]$ and the coordinate $\varphi$ in Eq. $(\mathrm{C}-2 \mathrm{c})$ is not confined.

The $\boldsymbol{e}_{x}, \boldsymbol{e}_{y}$ and $\boldsymbol{e}_{z}$ are unit vectors of $x, y$ and $z$ axes respectively. And the $\boldsymbol{e}_{r}, \boldsymbol{e}_{\theta}$ and $\boldsymbol{e}_{\varphi}$ are unit vectors of $r, \theta$ and $\varphi$ respectively. Then,

$$
\begin{aligned}
& \boldsymbol{e}_{r}=\left(\boldsymbol{e}_{x} \cos \varphi+\boldsymbol{e}_{y} \sin \varphi\right) \sin \theta+\boldsymbol{e}_{z} \cos \theta \\
& \boldsymbol{e}_{\theta}=\left(\boldsymbol{e}_{x} \cos \varphi+\boldsymbol{e}_{y} \sin \varphi\right) \cos \theta-\boldsymbol{e}_{z} \sin \theta \\
& \boldsymbol{e}_{\varphi}=-\boldsymbol{e}_{x} \sin \varphi+\boldsymbol{e}_{y} \cos \varphi
\end{aligned}
$$


By multiplying Eq. (D-3a) with $\boldsymbol{e}_{x}$ to form dot product,

$$
\begin{aligned}
\boldsymbol{e}_{r} \cdot \boldsymbol{e}_{x} & =\boldsymbol{e}_{x} \cdot \boldsymbol{e}_{x} \cos \varphi \sin \theta+\boldsymbol{e}_{y} \cdot \boldsymbol{e}_{x} \sin \varphi \sin \theta+\boldsymbol{e}_{z} \cdot \boldsymbol{e}_{x} \cos \theta \\
& =\cos \varphi \sin \theta
\end{aligned}
$$

Write $\cos \left(\boldsymbol{e}_{r}, \boldsymbol{e}_{x}\right)=\boldsymbol{e}_{r} \cdot \boldsymbol{e}_{x}$ since $\boldsymbol{e}_{r} \cdot \boldsymbol{e}_{x}$ represents the cosine of the angle between vectors $\boldsymbol{e}_{r}$ and $\boldsymbol{e}_{x}$. Similarly, by multiplying Eqs. (D-3a) through (D-3c) with $\boldsymbol{e}_{x}, \boldsymbol{e}_{y}$, or $\boldsymbol{e}_{z}$ to form dot products, the following relationship can be obtained:

$$
\begin{array}{rrr}
\cos \left(\boldsymbol{e}_{r}, \boldsymbol{e}_{x}\right)=\sin \theta \cos \varphi, & \cos \left(\boldsymbol{e}_{r}, \boldsymbol{e}_{y}\right)=\sin \theta \sin \varphi, & \cos \left(\boldsymbol{e}_{r}, \boldsymbol{e}_{z}\right)=\cos \theta, \\
\cos \left(\boldsymbol{e}_{\theta}, \boldsymbol{e}_{x}\right)=\cos \theta \cos \varphi, & \cos \left(\boldsymbol{e}_{\theta}, \boldsymbol{e}_{y}\right)=\cos \theta \sin \varphi, & \cos \left(\boldsymbol{e}_{\theta}, \boldsymbol{e}_{z}\right)=-\sin \theta, \\
\cos \left(\boldsymbol{e}_{\varphi}, \boldsymbol{e}_{x}\right)=-\sin \varphi, & \cos \left(\boldsymbol{e}_{\varphi}, \boldsymbol{e}_{y}\right)=\cos \varphi, & \cos \left(\boldsymbol{e}_{\varphi}, \boldsymbol{e}_{z}\right)=0,
\end{array}
$$

where $\left.\cos \left(\boldsymbol{e}_{1}, \boldsymbol{e}_{2}\right)\left(=\boldsymbol{e}_{1} \cdot \boldsymbol{e}_{2}\right)\right)$ stands for cosine of the angle between vectors $\boldsymbol{e}_{1}$ and $\boldsymbol{e}_{2}$. A matrix describes the rotation operation based on the cosines.

$$
\boldsymbol{T}_{r}=\left[\begin{array}{ccc}
\sin \theta \cos \varphi & \sin \theta \sin \varphi & \cos \theta \\
\cos \theta \cos \varphi & \cos \theta \sin \varphi & -\sin \theta \\
-\sin \varphi & \cos \varphi & 0
\end{array}\right]
$$

where subscript $r$ denotes the transformation is due to rotation of a coordinate system (since the spherical and Cartesian coordinate systems share the same origin).

At point $P$, the displacement vector can be expressed in Cartesian coordinate system and in spherical system, in equation,

$$
\boldsymbol{u}=u_{x} \boldsymbol{e}_{x}+u_{y} \boldsymbol{e}_{y}+u_{z} \boldsymbol{e}_{z}=u_{r} \boldsymbol{e}_{r}+u_{\theta} \boldsymbol{e}_{\theta}+u_{\varphi} \boldsymbol{e}_{\varphi}
$$

By multiplying Eq. (D-6) with $\boldsymbol{e}_{x}$ to form dot product,

$$
\begin{aligned}
u_{x} & =u_{r} \boldsymbol{e}_{r} \cdot \boldsymbol{e}_{x}+u_{\theta} \boldsymbol{e}_{\theta} \cdot \boldsymbol{e}_{x}+u_{\varphi} \boldsymbol{e}_{\varphi} \cdot \boldsymbol{e}_{x} \\
& =u_{r} \sin \theta \cos \varphi+u_{\theta} \cos \theta \cos \varphi+u_{\varphi}(-\sin \varphi)
\end{aligned}
$$

Similarly, $u_{y}$ and $u_{z}$ can be written in terms of $u_{r}, u_{\theta}$ and $u_{\varphi}$. And similarly, $u_{r}, u_{\theta}$ 
and $u_{\varphi}$ can also be written in terms of $u_{x}, u_{y}$ and $u_{z}$. In other words, the displacement components in two coordinate systems can be transformed from each other and transformation can be written in the following equations.

$$
\begin{aligned}
& \left\{\begin{array}{l}
u_{x} \\
u_{y} \\
u_{z}
\end{array}\right\}=\left[\begin{array}{ccc}
\sin \theta \cos \varphi & \cos \theta \cos \varphi & -\sin \varphi \\
\sin \theta \sin \varphi & \cos \theta \sin \varphi & \cos \varphi \\
\cos \theta & -\sin \theta & 0
\end{array}\right]\left\{\begin{array}{c}
u_{r} \\
u_{\theta} \\
u_{\varphi}
\end{array}\right\}=\left[\boldsymbol{T}_{r}\right]^{T}\left\{\begin{array}{c}
u_{r} \\
u_{\theta} \\
u_{\varphi}
\end{array}\right\}, \\
& \left\{\begin{array}{l}
u_{r} \\
u_{\theta} \\
u_{\varphi}
\end{array}\right\}=\left[\begin{array}{ccc}
\sin \theta \cos \varphi & \sin \theta \sin \varphi & \cos \theta \\
\cos \theta \cos \varphi & \cos \theta \sin \varphi & -\sin \theta \\
-\sin \varphi & \cos \varphi & 0
\end{array}\right]\left\{\begin{array}{l}
u_{x} \\
u_{y} \\
u_{z}
\end{array}\right\}=\left[\boldsymbol{T}_{r}\right]\left\{\begin{array}{l}
u_{x} \\
u_{y} \\
u_{z}
\end{array}\right\} .
\end{aligned}
$$

Also at point $P$, the strain or stress tensors in Cartesian system and in spherical system can be transformed from each other. Let $\boldsymbol{S}_{s}$ and $\boldsymbol{S}_{c}$ represent a tensor in spherical system and Cartesian system respectively, and

$$
\begin{aligned}
& \boldsymbol{S}_{s}=\left[\begin{array}{lll}
S_{r r} & S_{r \theta} & S_{r \varphi} \\
S_{\theta r} & S_{\theta \theta} & S_{\theta \varphi} \\
S_{\varphi r} & S_{\varphi \theta} & S_{\varphi \varphi}
\end{array}\right] \\
& \boldsymbol{S}_{c}=\left[\begin{array}{lll}
S_{x x} & S_{x y} & S_{x z} \\
S_{y x} & S_{y y} & S_{y z} \\
S_{z x} & S_{z y} & S_{z z}
\end{array}\right]
\end{aligned}
$$

where $S$ can be either strain $\epsilon$ or stress $\sigma$. The tensor transformation can be written as (Fung and Tong, 2001, pp538, eqn.(16.7:22))

$$
\begin{aligned}
\boldsymbol{S}_{c} & =\boldsymbol{T}_{r}^{T} \boldsymbol{S}_{s} \boldsymbol{T}_{r} \\
\boldsymbol{S}_{s} & =\boldsymbol{T}_{r} \boldsymbol{S}_{c} \boldsymbol{T}_{r}^{T}
\end{aligned}
$$


Explicitly,

$$
\begin{aligned}
& {\left[\begin{array}{lll}
S_{x x} & S_{x y} & S_{x z} \\
S_{y x} & S_{y y} & S_{y z} \\
S_{z x} & S_{z y} & S_{z z}
\end{array}\right]=\left[\begin{array}{ccc}
\sin \theta \cos \varphi & \cos \theta \cos \varphi & -\sin \varphi \\
\sin \theta \sin \varphi & \cos \theta \sin \varphi & \cos \varphi \\
\cos \theta & -\sin \theta & 0
\end{array}\right]\left[\begin{array}{ccc}
S_{r r} & S_{r \theta} & S_{r \varphi} \\
S_{\theta r} & S_{\theta \theta} & S_{\theta \varphi} \\
S_{\varphi r} & S_{\varphi \theta} & S_{\varphi \varphi}
\end{array}\right]} \\
& {\left[\begin{array}{ccc}
\sin \theta \cos \varphi & \sin \theta \sin \varphi & \cos \theta \\
\cos \theta \cos \varphi & \cos \theta \sin \varphi & -\sin \theta \\
-\sin \varphi & \cos \varphi & 0
\end{array}\right]} \\
& {\left[\begin{array}{lll}
S_{r r} & S_{r \theta} & S_{r \varphi} \\
S_{\theta r} & S_{\theta \theta} & S_{\theta \varphi} \\
S_{\varphi r} & S_{\varphi \theta} & S_{\varphi \varphi}
\end{array}\right]=\left[\begin{array}{ccc}
\sin \theta \cos \varphi & \sin \theta \sin \varphi & \cos \theta \\
\cos \theta \cos \varphi & \cos \theta \sin \varphi & -\sin \theta \\
-\sin \varphi & \cos \varphi & 0
\end{array}\right]\left[\begin{array}{ccc}
S_{x x} & S_{x y} & S_{x z} \\
S_{y x} & S_{y y} & S_{y z} \\
S_{z x} & S_{z y} & S_{z z}
\end{array}\right]} \\
& {\left[\begin{array}{ccc}
\sin \theta \cos \varphi & \cos \theta \cos \varphi & -\sin \varphi \\
\sin \theta \sin \varphi & \cos \theta \sin \varphi & \cos \varphi \\
\cos \theta & -\sin \theta & 0
\end{array}\right]}
\end{aligned}
$$

Displacements, strains and stresses in Cartesian coordinate system can be transformed from those in spherical coordinate system, and vice versa. 


\section{Appendix E}

\section{Definition of Functions $\mathfrak{E}_{p q}^{s t}(r, n)$ and $\mathfrak{E}_{p q}^{s t}(\mathbf{r}, n)$}

By following Pao and Mow ${ }^{[36]}$, the $\mathfrak{E}_{p q}^{s t}(r, n)$ is defined such as

$$
\begin{aligned}
& \mathfrak{E}_{1 q}^{1 t}(r, n)=\kappa \mathcal{Z}_{n}^{\prime}(\kappa r) \\
& \mathfrak{E}_{1 q}^{2 t}(r, n)=0 \\
& \mathfrak{E}_{1 q}^{3 t}(r, n)=\frac{\ell}{r} n(n+1) \mathcal{Z}_{n}(k r) \\
& \mathfrak{E}_{2 q}^{1 t}(r, n)=\frac{1}{r} \mathcal{Z}_{n}(\kappa r) \\
& \mathfrak{E}_{2 q}^{2 t}(r, n)=\mathcal{Z}_{n}(k r) \\
& \mathfrak{E}_{2 q}^{3 t}(r, n)=\frac{\ell}{r}\left(\mathcal{Z}_{n}(k r)+k r \mathcal{Z}_{n}^{\prime}(k r)\right) \\
& \mathfrak{E}_{3 q}^{1 t}(r, n)=\frac{1}{r} \mathcal{Z}_{n}(\kappa r) \\
& \mathfrak{E}_{3 q}^{2 t}(r, n)=-\mathcal{Z}_{n}(k r) \\
& \mathfrak{E}_{3 q}^{3 t}(r, n)=\frac{\ell}{r}\left(\mathcal{Z}_{n}(k r)+k r \mathcal{Z}_{n}^{\prime}(k r)\right) \\
& \mathfrak{E}_{4 q}^{1 t}(r, n)=\frac{2 \mu}{r^{2}}\left[\left(n^{2}-n-\frac{1}{2} k^{2} r^{2}\right) \mathcal{Z}_{n}(\kappa r)+2 \kappa r \mathcal{Z}_{n+1}(\kappa r)\right] \\
& \mathfrak{E}_{4 q}^{2 t}(r, n)=0
\end{aligned}
$$




$$
\begin{aligned}
\mathfrak{E}_{4 q}^{3 t}(r, n) & =\frac{2 \mu \ell}{r^{2}} n(n+1)\left[(n-1) \mathcal{Z}_{n}(k r)-k r \mathcal{Z}_{n+1}(k r)\right] \\
\mathfrak{E}_{7 q}^{1 t}(r, n) & =\frac{2 \mu}{r^{2}}\left[(n-1) \mathcal{Z}_{n}(\kappa r)-\kappa r \mathcal{Z}_{n+1}(\kappa r)\right] \\
\mathfrak{E}_{7 q}^{2 t}(r, n) & =\frac{\mu}{r}\left[(n-1) \mathcal{Z}_{n}(k r)-k r \mathcal{Z}_{n+1}(k r)\right] \\
\mathfrak{E}_{7 q}^{3 t}(r, n) & =\frac{2 \mu}{r^{2}} \ell\left[\left(n^{2}-1-\frac{1}{2} k^{2} r^{2}\right) \mathcal{Z}_{n}(k r)+k r \mathcal{Z}_{n+1}(k r)\right] \\
\mathfrak{E}_{8 q}^{1 t}(r, n) & =\frac{2 \mu}{r^{2}}\left[(n-1) \mathcal{Z}_{n}(\kappa r)-\kappa r \mathcal{Z}_{n+1}(\kappa r)\right] \\
\mathfrak{E}_{8 q}^{2 t}(r, n) & =-\frac{\mu}{r}\left[(n-1) \mathcal{Z}_{n}(k r)-k r \mathcal{Z}_{n+1}(k r)\right] \\
\mathfrak{E}_{8 q}^{3 t}(r, n) & =\frac{2 \mu}{r^{2}} \ell\left[\left(n^{2}-1-\frac{1}{2} k^{2} r^{2}\right) \mathcal{Z}_{n}(k r)+k r \mathcal{Z}_{n+1}(k r)\right]
\end{aligned}
$$

The $\mathfrak{E}_{p q}^{s t}(\mathbf{r}, n)$ is defined as following,

$$
\begin{aligned}
& \mathfrak{E}_{5 q}^{1 t}(\mathbf{r}, n)=\left(-\lambda \kappa^{2} \mathcal{Z}_{n}(\kappa r)+\frac{2 \mu}{r} \kappa \mathcal{Z}_{n}^{\prime}(\kappa r)\right) Y_{n}^{m}(\theta, \varphi) \\
& +\frac{2 \mu}{r^{2}} \mathcal{Z}_{n}(\kappa r) \frac{\partial^{2} Y_{n}^{m}(\theta, \varphi)}{\partial \theta^{2}} \\
& \mathfrak{E}_{5 q}^{2 t}(\mathbf{r}, n)=-\frac{2 \mu}{r} \mathcal{Z}_{n}(k r) \frac{1}{\sin \theta} \frac{\partial^{2} Y_{n}^{m}(\theta, \varphi)}{\partial \theta \partial \varphi}+\frac{2 \mu}{r} \mathcal{Z}_{n}(k r) \frac{\cos \theta}{\sin ^{2} \theta} \frac{\partial Y_{n}^{m}(\theta, \varphi)}{\partial \varphi} \\
& \mathfrak{E}_{5 q}^{3 t}(\mathbf{r}, n)=\frac{2 \mu}{r^{2}} \ell\left(\mathcal{Z}_{n}(k r)+k r \mathcal{Z}_{n}^{\prime}(k r)\right) \frac{\partial^{2} Y_{n}^{m}(\theta, \varphi)}{\partial \theta^{2}} \\
& +\frac{2 \mu}{r^{2}} \ell n(n+1) \mathcal{Z}_{n}(k r) Y_{n}^{m}(\theta, \varphi) \\
& \mathfrak{E}_{6 q}^{1 t}(\mathbf{r}, n)=\left(-\lambda \kappa^{2} \mathcal{Z}_{n}(\kappa r)+\frac{2 \mu}{r} \kappa \mathcal{Z}_{n}^{\prime}(\kappa r)\right) Y_{n}^{m}(\theta, \varphi)+\frac{2 \mu}{r^{2}} \mathcal{Z}_{n}(\kappa r) \frac{1}{\sin ^{2} \theta} \frac{\partial^{2} Y_{n}^{m}(\theta, \varphi)}{\partial \varphi^{2}} \\
& +\frac{2 \mu}{r^{2}} \mathcal{Z}_{n}(\kappa r) \frac{\cos \theta}{\sin \theta} \frac{\partial Y_{n}^{m}(\theta, \varphi)}{\partial \varphi} \\
& \mathfrak{E}_{6 q}^{2 t}(\mathbf{r}, n)=-\frac{2 \mu}{r} \mathcal{Z}_{n}(k r) \frac{1}{\sin \theta} \frac{\partial^{2} Y_{n}^{m}(\theta, \varphi)}{\partial \theta \partial \varphi}+\frac{2 \mu}{r} \mathcal{Z}_{n}(k r) \frac{\cos \theta}{\sin ^{2} \theta} \frac{\partial Y_{n}^{m}(\theta, \varphi)}{\partial \varphi} \\
& \mathfrak{E}_{6 q}^{3 t}(\mathbf{r}, n)=\frac{2 \mu}{r^{2}} \ell\left(\mathcal{Z}_{n}(k r)+k r \mathcal{Z}_{n}^{\prime}(k r)\right) \frac{1}{\sin ^{2} \theta} \frac{\partial^{2} Y_{n}^{m}(\theta, \varphi)}{\partial \varphi^{2}} \\
& +\frac{2 \mu}{r^{2}} \ln (n+1) \mathcal{Z}_{n}(k r) Y_{n}^{m}(\theta, \varphi) \\
& \mathfrak{E}_{9 q}^{1 t}(\mathbf{r}, n)=\frac{\mu}{r^{2}} \mathcal{Z}_{n}(\kappa r)\left(\frac{2}{\sin \theta} \frac{\partial^{2} Y_{n}^{m}(\theta, \varphi)}{\partial \theta \partial \varphi}-\frac{\cos \theta}{\sin ^{2} \theta} \frac{\partial Y_{n}^{m}(\theta, \varphi)}{\partial \varphi}\right) \\
& \mathfrak{E}_{9 q}^{2 t}(\mathbf{r}, n)=\frac{\mu}{r} \mathcal{Z}_{n}(k r)\left[\frac{\cos \theta}{\sin \theta} \frac{\partial Y_{n}^{m}(\theta, \varphi)}{\partial \theta}-\frac{\partial^{2} Y_{n}^{m}(\theta, \varphi)}{\partial \theta^{2}}+\frac{1}{\sin ^{2} \theta} \frac{\partial^{2} Y_{n}^{m}(\theta, \varphi)}{\partial \varphi^{2}}\right]
\end{aligned}
$$




$$
\mathfrak{E}_{9 q}^{3 t}(\mathbf{r}, n)=\frac{\mu}{r^{2}} \ell\left(\mathcal{Z}_{n}(k r)+\mathcal{Z}_{n}^{\prime}(k r)\right)\left(\frac{2}{\sin \theta} \frac{\partial^{2} Y_{n}^{m}(\theta, \varphi)}{\partial \theta \partial \varphi}-\frac{\cos \theta}{\sin ^{2} \theta} \frac{\partial Y_{n}^{m}(\theta, \varphi)}{\partial \varphi}\right)
$$

The subscript $p$ runs from 1 to 9 and is used to denote the component of displacement and stress. Particularly, $p=1$ for displacement $u_{r}, p=2$ for $u_{\theta}, p=3$ for $u_{\varphi}$, $p=4$ for $\sigma_{r r}, p=5$ for $\sigma_{\theta \theta}, p=6$ for $\sigma_{\varphi \varphi}, p=7$ for $\sigma_{r \theta}, p=8$ for $\sigma_{r \varphi}$ and $p=9$ for $\sigma_{\theta \varphi}$. The subscript $q=1,2$, refers to medium type, 1 for host medium and 2 for scatterer. It's noted that, $\kappa$ and $k$ are for host medium when $q=1$, while $\kappa$ and $k$ are for scatterer when $q=2$. The superscript $s$ runs from 1 to 3 and denotes wave-type $\phi, \psi$ and $\chi$ respectively. The superscript $t$, which equals 1 or 3 , is used to denote the kind of spherical Bessel function, $\mathcal{Z}_{n}(\cdot) . \mathcal{Z}_{n}(\cdot)=j_{n}(\cdot)$ corresponding to $t=1$ for incident and refracted waves. $\mathcal{Z}_{n}(\cdot)=h_{n}^{(1)}(\cdot)$ corresponding to $t=3$ for scattered wave.

For the same wave in the same medium, there exist

$$
\begin{aligned}
& \mathfrak{E}_{2 q}^{1 t}(r, n)=\mathfrak{E}_{3 q}^{1 t}(r, n) \\
& \mathfrak{E}_{2 q}^{2 t}(r, n)=-\mathfrak{E}_{3 q}^{2 t}(r, n) \\
& \mathfrak{E}_{2 q}^{3 t}(r, n)=\mathfrak{E}_{3 q}^{3 t}(r, n) \\
& \mathfrak{E}_{7 q}^{1 t}(r, n)=\mathfrak{E}_{8 q}^{1 t}(r, n) \\
& \mathfrak{E}_{7 q}^{2 t}(r, n)=-\mathfrak{E}_{8 q}^{2 t}(r, n) \\
& \mathfrak{E}_{7 q}^{3 t}(r, n)=\mathfrak{E}_{8 q}^{3 t}(r, n)
\end{aligned}
$$




\section{Appendix F}

\section{Vector Spherical Harmonics}

The vector spherical harmonics $\mathbb{P}_{m n}, \mathbb{B}_{m n}$ and $\mathbb{C}_{m n}$ are defined such that ${ }^{[14]}$

$$
\begin{aligned}
\mathbb{P}_{m n}(\theta, \varphi) & =\mathbf{e}_{r} X_{n}^{m}(\theta, \varphi) \\
\mathbb{B}_{m n}(\theta, \varphi) & =\frac{r}{\sqrt{n(n+1)}} \nabla\left[X_{n}^{m}(\theta, \varphi)\right] \\
& =\frac{\sqrt{n(n+1)}}{(2 n+1) \sin \theta}\left\{\mathbf{e}_{\theta}\left[\frac{n-m+1}{n+1} X_{n+1}^{m}-\frac{n+m}{n} X_{n-1}^{m}\right]+\mathbf{e}_{\varphi} \frac{m(2 n+1)}{n(n+1)} \hat{\imath} X_{n}^{m}\right\} \\
& =\frac{1}{\sqrt{n(n+1)}} \mathbf{e}_{\theta}\left(X_{, \theta}\right)_{n}^{m}+\frac{1}{\sqrt{n(n+1)}} \mathbf{e}_{\varphi}\left(X_{, \varphi}\right)_{n}^{m} \\
\mathbb{C}_{m n}(\theta, \varphi) & =\frac{1}{\sqrt{n(n+1)}} \nabla \times\left[\mathbf{e}_{r} r X_{n}^{m}(\theta, \varphi)\right] \\
& =\frac{\sqrt{n(n+1)}}{(2 n+1) \sin \theta}\left\{\mathbf{e}_{\theta} \frac{m(2 n+1)}{n(n+1)} \hat{\imath} X_{n}^{m}-\mathbf{e}_{\varphi}\left[\frac{n-m+1}{n+1} X_{n+1}^{m}-\frac{n+m}{n} X_{n-1}^{m}\right]\right\} \\
& =\frac{1}{\sqrt{n(n+1)}} \mathbf{e}_{\theta}\left(X_{, \varphi}\right)_{n}^{m}-\frac{1}{\sqrt{n(n+1)}} \mathbf{e}_{\varphi}\left(X_{, \theta}\right)_{n}^{m}
\end{aligned}
$$

where $\hat{\imath}=\sqrt{-1}, n>0$ for eqns. $(\mathrm{F}-1 \mathrm{~b})$ and $(\mathrm{F}-1 \mathrm{c}), n \geq m \geq 0, \mathbf{e}_{r}, \mathbf{e}_{\theta}$ and $\mathbf{e}_{\varphi}$ are three unit vectors in spherical coordinates, $X_{n}^{m},\left(X_{, \theta}\right)_{n}^{m}$ and $\left(X_{, \varphi}\right)_{n}^{m}$ are defined as,

$$
\begin{aligned}
X_{n}^{m} & =X_{n}^{m}(\theta, \varphi)=P_{n}^{m}(\cos \theta) \mathrm{e}^{\hat{\imath} m \varphi} \\
\left(X_{, \theta}\right)_{n}^{m} & =\frac{\partial X_{n}^{m}(\theta, \varphi)}{\partial \theta} \\
\left(X_{, \varphi}\right)_{n}^{m} & =\frac{1}{\sin \theta} \frac{\partial X_{n}^{m}(\theta, \varphi)}{\partial \varphi}
\end{aligned}
$$


where $P_{n}^{m}(\cos \theta)$ is the associated Legendré polynomial and the vector operator $\nabla$ in spherical coordinates is given in Appendix B.

Extend $m$ limit to $|m| \leq n$, and extend the limit of $n$ to $n \geq 0$ with

$$
\mathbb{B}_{00}=\mathbb{C}_{00}=0
$$

The vector spherical harmonics $\mathbb{P}_{m n}, \mathbb{B}_{m n}$ and $\mathbb{C}_{m n}$ have the following properties:

$$
\begin{gathered}
\mathbb{P}_{m n} \cdot \mathbb{B}_{m n}=\mathbb{P}_{m n} \cdot \mathbb{C}_{m n}=\mathbb{B}_{m n} \cdot \mathbb{C}_{m n}=0 \\
\int_{\Omega} \mathbb{P}_{m n} \cdot \mathbb{B}_{\mu \nu} d \Omega=\int_{\Omega} \mathbb{P}_{m n} \cdot \mathbb{C}_{\mu \nu} d \Omega=\int_{\Omega} \mathbb{B}_{m n} \cdot \mathbb{C}_{\mu \nu} d \Omega=0 \\
\int_{\Omega} \mathbb{P}_{m n} \cdot \tilde{\mathbb{B}}_{\mu \nu} d \Omega=\int_{\Omega} \mathbb{P}_{m n} \cdot \tilde{\mathbb{C}}_{\mu \nu} d \Omega=\int_{\Omega} \mathbb{B}_{m n} \cdot \tilde{\mathbb{C}}_{\mu \nu} d \Omega=0 \\
\int_{\Omega} \mathbb{P}_{m n} \cdot \tilde{\mathbb{P}}_{\mu \nu} d \Omega=\frac{8 \pi / \epsilon_{m}}{2 n+1} \frac{(n+m) !}{(n-m) !} \delta_{m \mu} \delta_{n \nu}
\end{gathered}
$$

for $\nu=0$

$$
\int_{\Omega} \mathbb{B}_{m n} \cdot \tilde{\mathbb{B}}_{00} d \Omega=\int_{\Omega} \mathbb{C}_{m n} \cdot \tilde{\mathbb{C}}_{00} d \Omega=0
$$

and for $\nu \geq 1$

$$
\int_{\Omega} \mathbb{B}_{m n} \cdot \tilde{\mathbb{B}}_{\mu \nu} d \Omega=\int_{\Omega} \mathbb{C}_{m n} \cdot \tilde{\mathbb{C}}_{\mu \nu} d \Omega=\frac{8 \pi / \epsilon_{m}}{2 n+1} \frac{(n+m) !}{(n-m) !} \delta_{m \mu} \delta_{n \nu}
$$

where $\Omega$ denotes the spherical surface of unit radius, $d \Omega=\sin \theta d \theta d \varphi$, the integration is over the ranges $0 \leq \theta \leq \pi, 0 \leq \varphi \leq 2 \pi$, the tilde indicates a complex conjugate and $\epsilon_{m}$ is a factor with values of $\epsilon_{0}=1, \epsilon_{m}=2(m \neq 0)$. Property eqn. (F-4) can be proved directly from definition of spherical vector harmonics. For instance,

$$
\begin{aligned}
\mathbb{B}_{m n} \cdot \mathbb{C}_{m n} & =\frac{1}{n(n+1)}\left[\mathbf{e}_{\theta}\left(X_{, \theta}\right)_{n}^{m}+\mathbf{e}_{\varphi}\left(X_{, \varphi}\right)_{n}^{m}\right] \cdot\left[\mathbf{e}_{\theta}\left(X_{, \varphi}\right)_{n}^{m}-\mathbf{e}_{\varphi}\left(X_{, \theta}\right)_{n}^{m}\right] \\
& =\frac{1}{n(n+1)}\left[\left(X_{, \theta}\right)_{n}^{m}\left(X_{, \varphi}\right)_{n}^{m} \mathbf{e}_{\theta} \cdot \mathbf{e}_{\theta}-\left(X_{, \varphi}\right)_{n}^{m}\left(X_{, \theta}\right)_{n}^{m} \mathbf{e}_{\varphi} \cdot \mathbf{e}_{\varphi}\right] \\
& =0
\end{aligned}
$$

The properties of eqns. $(\mathrm{F}-5)$ through $(\mathrm{F}-9)$ will be proved at the following. 
B.1 For $n \geq 1$ and $n \geq m \geq 0$

Define

$$
\begin{aligned}
& X_{n m}^{e}(\theta, \varphi)=\cos (m \varphi) P_{n}^{m}(\cos \theta) \\
& X_{n m}^{o}(\theta, \varphi)=\sin (m \varphi) P_{n}^{m}(\cos \theta)
\end{aligned}
$$

And let $\mathbf{P}_{m n}(\theta, \varphi), \mathbf{B}_{m n}(\theta, \varphi)$ and $\mathbf{C}_{m n}(\theta, \varphi)$ be the originally defined vector spherical harmonics by Morse and Feshbach ${ }^{[14]}$. Because

$$
\begin{aligned}
X_{n}^{m}(\theta, \varphi) & =P_{n}^{m}(\cos \theta) e^{\hat{\imath} m \varphi}=\cos (m \varphi) P_{n}^{m}(\cos \theta)+\hat{\imath} \sin (m \varphi) P_{n}^{m}(\cos \theta) \\
& =X_{n m}^{e}(\theta, \varphi)+\hat{\imath} X_{n m}^{o}(\theta, \varphi)
\end{aligned}
$$

the real part of a vector function is denoted by the superscript $e$ (for even) and the imaginary part is denoted by the superscript $o$ (for odd); that is

$$
\begin{aligned}
\mathbf{P}_{m n}(\theta, \varphi) & =\mathbf{P}_{m n}^{e}(\theta, \varphi)+\hat{\imath} \mathbf{P}_{m n}^{o}(\theta, \varphi) \\
\mathbf{B}_{m n}(\theta, \varphi) & =\mathbf{B}_{m n}^{e}(\theta, \varphi)+\imath \mathbf{B}_{m n}^{o}(\theta, \varphi) \\
\mathbf{C}_{m n}(\theta, \varphi) & =\mathbf{C}_{m n}^{e}(\theta, \varphi)+\imath \mathbf{C}_{m n}^{o}(\theta, \varphi)
\end{aligned}
$$

where

$$
\begin{aligned}
\mathbf{P}_{m n}^{e}(\theta, \varphi)= & \mathbf{e}_{r} X_{n m}^{e}(\theta, \varphi) \\
\mathbf{P}_{m n}^{o}(\theta, \varphi)= & \mathbf{e}_{r} X_{n m}^{o}(\theta, \varphi) \\
\mathbf{B}_{m n}^{e}(\theta, \varphi)= & \frac{r}{\sqrt{n(n+1)}} \nabla\left[X_{n m}^{e}(\theta, \varphi)\right]=\mathbf{e}_{r} \times \mathbf{C}_{m n}^{e} \\
= & \frac{\sqrt{n(n+1)}}{(2 n+1) \sin \theta}\left\{\mathbf{e}_{\theta}\left[\frac{n-m+1}{n+1} X_{(n+1) m}^{e}-\frac{n+m}{n} X_{(n-1) m}^{e}\right]\right. \\
& \left.\quad+\mathbf{e}_{\varphi} \frac{m(2 n+1)}{n(n+1)} \hat{\imath} X_{n m}^{e}\right\}
\end{aligned}
$$




$$
\begin{aligned}
\mathbf{C}_{m n}^{e}(\theta, \varphi)=\frac{1}{\sqrt{n(n+1)}} \nabla \times\left[\mathbf{e}_{r} r X_{n m}^{e}(\theta, \varphi)\right]=-\mathbf{e}_{r} \times \mathbf{B}_{m n}^{e} \\
=\frac{\sqrt{n(n+1)}}{(2 n+1) \sin \theta}\left\{\mathbf{e}_{\theta} \frac{m(2 n+1)}{n(n+1)} \hat{\imath} X_{n m}^{e}-\mathbf{e}_{\varphi}\left[\frac{n-m+1}{n+1} X_{(n+1) m}^{e}\right.\right. \\
\left.\left.\quad-\frac{n+m}{n} X_{(n-1) m}^{e}\right]\right\} \\
\mathbf{C}_{m n}^{o}(\theta, \varphi)=\frac{1}{\sqrt{n(n+1)}} \nabla \times\left[\mathbf{e}_{r} r X_{n m}^{o}(\theta, \varphi)\right]=-\mathbf{e}_{r} \times \mathbf{B}_{m n}^{o} \\
=\frac{\sqrt{n(n+1)}}{(2 n+1) \sin \theta}\left\{\mathbf{e}_{\theta} \frac{m(2 n+1)}{n(n+1)} \hat{\imath} X_{n m}^{o}-\mathbf{e}_{\varphi}\left[\frac{n-m+1}{n+1} X_{(n+1) m}^{o}\right.\right. \\
\left.\left.\quad-\frac{n+m}{n} X_{(n-1) m}^{o}\right]\right\}
\end{aligned}
$$

According to Morse and Feshbach [14], the vector spherical harmonics are orthogonal to each other,

$$
\begin{gathered}
\mathbf{P}_{m n}^{s} \cdot \mathbf{B}_{m n}^{s}=\mathbf{P}_{m n}^{s} \cdot \mathbf{C}_{m n}^{s}=\mathbf{B}_{m n}^{s} \cdot \mathbf{C}_{m n}^{s}=0 \\
\int_{\Omega} \mathbf{P}_{m n}^{s} \cdot \mathbf{B}_{\mu \nu}^{\sigma}=\int_{\Omega} \mathbf{P}_{m n}^{s} \cdot \mathbf{C}_{\mu \nu}^{\sigma}=\int_{\Omega} \mathbf{B}_{m n}^{s} \cdot \mathbf{C}_{\mu \nu}^{\sigma}=0
\end{gathered}
$$

and

$$
\begin{aligned}
\int_{\Omega} \mathbf{P}_{m n}^{s} \cdot \mathbf{P}_{\mu \nu}^{\sigma} d \Omega & =\int_{\Omega} \mathbf{B}_{m n}^{s} \cdot \mathbf{B}_{\mu \nu}^{\sigma} d \Omega \\
& =\int_{\Omega} \mathbf{C}_{m n}^{s} \cdot \mathbf{C}_{\mu \nu}^{\sigma} d \Omega=\frac{4 \pi / \epsilon_{m}}{2 n+1} \frac{(n+m) !}{(n-m) !} \delta_{s \sigma} \delta_{m \mu} \delta_{n \nu}
\end{aligned}
$$

where $\Omega$ is spherical surface with unit radius, $d \Omega=\sin \theta d \theta d \varphi$ and the integration is over the ranges $0 \leq \theta \leq \pi, 0 \leq \varphi \leq 2 \pi(s, \sigma=e, o ; \nu=1,2,3, \ldots, \mu=0,1, \ldots, \nu ; n=$ $1,2,3, \ldots, m=0,1, \ldots, n)$, and $\epsilon_{m}$ is the Neumann factor with values of $\epsilon_{0}=1, \epsilon_{m}=$ $2(m=1,2,3, \ldots)$.

Therefore,

$$
\begin{aligned}
\int_{\Omega} \mathbb{P}_{m n} \cdot \tilde{\mathbb{P}}_{\mu \nu} d \Omega & =\int_{\Omega}\left\{\mathbb{P}_{m n}^{e} \cdot \mathbb{P}_{\mu \nu}^{e}+\mathbb{P}_{m n}^{o} \cdot \mathbb{P}_{\mu \nu}^{o}+\hat{\imath} \mathbb{P}_{m n}^{e} \cdot \mathbb{P}_{\mu \nu}^{o}-\hat{\imath} \mathbb{P}_{m n}^{o} \cdot \mathbb{P}_{\mu \nu}^{e}\right\} d \Omega \\
& =\int_{\Omega}\left\{\mathbb{P}_{m n}^{e} \cdot \mathbb{P}_{\mu \nu}^{e}+\mathbb{P}_{m n}^{o} \cdot \mathbb{P}_{\mu \nu}^{o}\right\} d \Omega \\
& =\frac{8 \pi / \epsilon_{m}}{2 n+1} \frac{(n+m) !}{(n-m) !} \delta_{m \mu} \delta_{n \nu}
\end{aligned}
$$




$$
\begin{aligned}
\int_{\Omega} \mathbb{P}_{m n} \cdot \mathbb{B}_{\mu \nu} d \Omega & =\int_{\Omega}\left\{\mathbb{P}_{m n}^{e} \cdot \mathbb{B}_{\mu \nu}^{e}-\mathbb{P}_{m n}^{o} \cdot \mathbb{B}_{\mu \nu}^{o}+\hat{\imath} \mathbb{P}_{m n}^{e} \cdot \mathbb{B}_{\mu \nu}^{o}+\hat{\imath} \mathbb{P}_{m n}^{o} \cdot \mathbb{B}_{\mu \nu}^{e}\right\} d \Omega \\
& =0
\end{aligned}
$$

Similarly,

$$
\int_{\Omega} \mathbb{B}_{m n} \cdot \tilde{\mathbb{B}}_{\mu \nu} d \Omega=\int_{\Omega} \mathbb{C}_{m n} \cdot \tilde{\mathbb{C}}_{\mu \nu} d \Omega=\frac{8 \pi / \epsilon_{m}}{(2 n+1)} \frac{(n+m) !}{(n-m) !} \delta_{m \mu} \delta_{n \nu}
$$

and

$$
\int_{\Omega} \mathbb{P}_{m n} \cdot \mathbb{C}_{\mu \nu} d \Omega=\int_{\Omega} \mathbb{B}_{m n} \cdot \mathbb{C}_{\mu \nu} d \Omega=0
$$

B.2 For $n \geq 1$ and $n \geq m \geq-n$

\section{B.2.1 $\mathbb{P}_{m n}$}

Assuming $m \geq 0$, it is known that ${ }^{[38]}$

$$
P_{n}^{-m}(\cos \theta)=\frac{(n-m) !}{(n+m) !} P_{n}^{m}(\cos \theta)
$$

and

$$
\begin{aligned}
\mathbb{P}_{(-m) n} & =\mathbf{e}_{r}\left[\cos (-m \varphi) P_{n}^{-m}(\cos \theta)+\hat{\imath} \sin (-m \varphi) P_{n}^{-m}(\cos \theta)\right] \\
& =\mathbf{e}_{r}\left[\cos (m \varphi) P_{n}^{-m}(\cos \theta)-\hat{\imath} \sin (m \varphi) P_{n}^{-m}(\cos \theta)\right] \\
& =\mathbf{e}_{r}\left[\frac{(n-m) !}{(n+m) !} \cos (m \varphi) P_{n}^{m}(\cos \theta)-\frac{(n-m) !}{(n+m) !} \hat{\sin }(m \varphi) P_{n}^{m}(\cos \theta)\right] \\
& =\mathbf{e}_{r}\left[\frac{(n-m) !}{(n+m) !} \mathbb{P}_{m n}^{e}-\hat{\imath} \frac{(n-m) !}{(n+m) !} \mathbb{P}_{m n}^{o}\right]
\end{aligned}
$$

Comparing eqn. (F-23) with eqn. $(\mathrm{F}-13)$ gives

$$
\mathbb{P}_{(-m) n}^{e}=\frac{(n-m) !}{(n+m) !} \mathbb{P}_{m n}^{e}, \quad \mathbb{P}_{(-m) n}^{o}=-\frac{(n-m) !}{(n+m) !} \mathbb{P}_{m n}^{o}
$$

or

$$
\mathbb{P}_{(-m) n}^{s}=(-1)^{s} \frac{(n-m) !}{(n+m) !} \mathbb{P}_{m n}^{s}, \quad \text { with }(-1)^{\mathrm{e}}=1,(-1)^{\mathrm{o}}=-1, \mathrm{~s}=\mathrm{e}, \mathrm{o}
$$


Therefore, by assuming $\mu \geq 0$,

$$
\begin{aligned}
\int_{\Omega} \mathbb{P}_{(-m) n}^{s} \cdot \mathbb{P}_{(-\mu) \nu}^{\sigma} d \Omega & =\int_{\Omega}(-1)^{s+\sigma} \frac{(n-m) !}{(n+m) !} \frac{(\nu-\mu) !}{(\nu+\mu) !} \mathbb{P}_{m n}^{s} \cdot \mathbb{P}_{\mu \nu}^{\sigma} d \Omega \\
& =(-1)^{s+\sigma} \frac{(n-m) !}{(n+m) !} \frac{(n-m) !}{(n+m) !} \frac{4 \pi / \epsilon_{m}}{2 n+1} \frac{(n+m) !}{(n-m) !} \delta_{s \sigma} \delta_{m \mu} \delta_{n \nu} \\
& =(-1)^{s+\sigma} \frac{4 \pi / \epsilon_{m}}{2 n+1} \frac{(n-m) !}{(n+m) !} \delta_{s \sigma} \delta_{m \mu} \delta_{n \nu} \\
& =(-1)^{s+\sigma} \frac{4 \pi / \epsilon_{m}}{2 n+1} \frac{(n+(-m)) !}{(n-(-m)) !} \delta_{s \sigma} \delta_{(-m)(-\mu)} \delta_{n \nu}
\end{aligned}
$$

Similarly,

$$
\int_{\Omega} \mathbb{P}_{(-m) n}^{s} \cdot \mathbb{P}_{\mu \nu}^{\sigma} d \Omega=(-1)^{s} \frac{4 \pi / \epsilon_{m}}{2 n+1} \delta_{s \sigma} \delta_{m \mu} \delta_{n \nu}
$$

Since

$$
\begin{aligned}
\mathbb{P}_{(-m) n} \cdot \tilde{\mathbb{P}}_{\mu \nu}= & \mathbb{P}_{(-m) n}^{e} \cdot \mathbb{P}_{\mu \nu}^{e}+\mathbb{P}_{(-m) n}^{o} \cdot \mathbb{P}_{\mu \nu}^{o} \\
& +\hat{\imath}\left[-\mathbb{P}_{(-m) n}^{e} \cdot \mathbb{P}_{\mu \nu}^{o}+\mathbb{P}_{(-m) n}^{o} \cdot \mathbb{P}_{\mu \nu}^{e}\right]
\end{aligned}
$$

there is

$$
\begin{aligned}
\int_{\Omega} \mathbb{P}_{(-m) n} \cdot \tilde{\mathbb{P}}_{\mu \nu} d \Omega= & \int_{\Omega} \mathbb{P}_{(-m) n}^{e} \cdot \mathbb{P}_{\mu \nu}^{e} d \Omega+\int_{\Omega} \mathbb{P}_{(-m) n}^{o} \cdot \mathbb{P}_{\mu \nu}^{o} d \Omega \\
& +\hat{\imath} \int_{\Omega} \mathbb{P}_{(-m) n}^{e} \cdot \mathbb{P}_{\mu \nu}^{o} d \Omega+\hat{\imath} \int_{\Omega} \mathbb{P}_{(-m) n}^{o} \cdot \mathbb{P}_{\mu \nu}^{e} d \Omega \\
= & \frac{4 \pi / \epsilon_{m}}{2 n+1} \delta_{e e} \delta_{m \mu} \delta_{n \nu}-\frac{4 \pi / \epsilon_{m}}{2 n+1} \delta_{o o} \delta_{m \mu} \delta_{n \nu}=0
\end{aligned}
$$

Similarly,

$$
\int_{\Omega} \mathbb{P}_{(-m) n} \cdot \tilde{\mathbb{P}}_{(-\mu) \nu} d \Omega=\frac{8 \pi / \epsilon_{m}}{2 n+1} \frac{(n+(-m)) !}{(n-(-m)) !} \delta_{(-m)(-\mu)} \delta_{n \nu}
$$

and

$$
\int_{\Omega} \mathbb{P}_{m n} \cdot \tilde{\mathbb{P}}_{\mu \nu} d \Omega=\frac{8 \pi \epsilon_{m}}{2 n+1} \frac{(n+m) !}{(n-m) !} \delta_{m \mu} \delta_{n \nu}
$$

In general, the orthogonality can be written as

$$
\int_{\Omega} \mathbb{P}_{m n} \cdot \tilde{\mathbb{P}}_{\mu \nu} d \Omega=\frac{8 \pi / \epsilon_{m}}{2 n+1} \frac{(n+m) !}{(n-m) !} \delta_{m \mu} \delta_{n \nu}
$$

where $n \geq 1, n \geq m \geq-n$; and $\nu \geq 1, \nu \geq \mu \geq-\nu$. 


\section{B.2.2 $\mathbb{B}_{m n}$}

Assuming $m \geq 0$, then

$$
\begin{aligned}
\mathbb{B}_{(-m) n}(\theta, \varphi) & =\frac{r}{\sqrt{n(n+1)}} \nabla\left[X_{n}^{-m}(\theta, \varphi)\right] \\
& =\frac{r}{\sqrt{n(n+1)}} \nabla\left[\frac{(n-m) !}{(n+m) !} P_{n}^{m}(\cos \theta) e^{-\hat{\imath} m \varphi}\right] \\
& =\frac{(n-m) !}{(n+m) !} \frac{r}{\sqrt{n(n+1)}} \nabla\left[P_{n}^{m}(\cos \theta) e^{-\hat{\imath} m \varphi}\right] \\
& =\frac{(n-m) !}{(n+m) !} \frac{r}{\sqrt{n(n+1)}} \nabla\left[X_{n m}^{e}(\theta, \varphi)-\hat{\imath} X_{n m}^{o}(\theta, \varphi)\right] \\
& =\frac{(n-m) !}{(n+m) !}\left(\mathbf{B}_{m n}^{e}-\hat{\imath} \mathbf{B}_{m n}^{o}\right)
\end{aligned}
$$

Hence,

$$
\mathbb{B}_{(-m) n}^{e}=\mathbb{B}_{m n}^{e}, \mathbb{B}_{(-m) n}^{o}=-\mathbb{B}_{m n}^{s}
$$

or

$$
\mathbb{B}_{(-m) n}^{s}=(-1)^{s} \frac{(n-m) !}{(n+m) !} \mathbb{B}_{m n}^{s}, \quad \text { with }(-1)^{\mathrm{e}}=1,(-1)^{\mathrm{o}}=-1, \mathrm{~s}=\mathrm{e}, \mathrm{o}
$$

Therefore, by assuming $\mu \geq 0$,

$$
\begin{aligned}
\int_{\Omega} \mathbb{B}_{(-m) n}^{s} \cdot \mathbb{B}_{(-\mu) \nu}^{\sigma} d \Omega & =\int_{\Omega}(-1)^{s+\sigma} \frac{(n-m) !}{(n+m) !} \frac{(\nu-\mu) !}{(\nu+\mu) !} \mathbb{B}_{m n}^{s} \cdot \mathbb{B}_{\mu \nu}^{\sigma} d \Omega \\
& =(-1)^{s+\sigma} \frac{(n-m) !}{(n+m) !} \frac{(n-m) !}{(n+m) !} \frac{4 \pi / \epsilon_{m}}{2 n+1} \frac{(n+m) !}{(n-m) !} \delta_{s \sigma} \delta_{m \mu} \delta_{n \nu} \\
& =(-1)^{s+\sigma} \frac{4 \pi / \epsilon_{m}}{2 n+1} \frac{(n-m) !}{(n+m) !} \delta_{s \sigma} \delta_{m \mu} \delta_{n \nu} \\
& =(-1)^{s+\sigma} \frac{4 \pi / \epsilon_{m}}{2 n+1} \frac{(n+(-m)) !}{(n-(-m)) !} \delta_{s \sigma} \delta_{(-m)(-\mu)} \delta_{n \nu}
\end{aligned}
$$

Similarly,

$$
\int_{\Omega} \mathbb{B}_{(-m) n}^{s} \cdot \mathbb{B}_{\mu \nu}^{\sigma} d \Omega=(-1)^{s} \frac{4 \pi / \epsilon_{m}}{2 n+1} \delta_{s \sigma} \delta_{m \mu} \delta_{n \nu}
$$

Since

$$
\mathbb{B}_{(-m) n} \cdot \tilde{\mathbb{B}}_{\mu \nu}=\mathbb{B}_{(-m) n}^{e} \cdot \mathbb{B}_{\mu \nu}^{e}+\mathbb{B}_{(-m) n}^{o} \cdot \mathbb{B}_{\mu \nu}^{o}
$$




$$
+\hat{\imath}\left[-\mathbb{B}_{(-m) n}^{e} \cdot \mathbb{B}_{\mu \nu}^{o}+\mathbb{B}_{(-m) n}^{o} \cdot \mathbb{B}_{\mu \nu}^{e}\right]
$$

there is

$$
\begin{aligned}
\int_{\Omega} \mathbb{B}_{(-m) n} \cdot \tilde{\mathbb{B}}_{\mu \nu} d \Omega= & \int_{\Omega} \mathbb{B}_{(-m) n}^{e} \cdot \mathbb{B}_{\mu \nu}^{e} d \Omega+\int_{\Omega} \mathbb{B}_{(-m) n}^{o} \cdot \mathbb{B}_{\mu \nu}^{o} d \Omega \\
& +\hat{\imath} \int_{\Omega} \mathbb{B}_{(-m) n}^{e} \cdot \mathbb{B}_{\mu \nu}^{o} d \Omega+\hat{\imath} \int_{\Omega} \mathbb{B}_{(-m) n}^{o} \cdot \mathbb{B}_{\mu \nu}^{e} d \Omega \\
= & \frac{4 \pi / \epsilon_{m}}{2 n+1} \delta_{e e} \delta_{m \mu} \delta_{n \nu}-\frac{4 \pi / \epsilon_{m}}{2 n+1} \delta_{o o} \delta_{m \mu} \delta_{n \nu}=0
\end{aligned}
$$

Similarly,

$$
\int_{\Omega} \mathbb{B}_{(-m) n} \cdot \tilde{\mathbb{B}}_{(-\mu) \nu} d \Omega=\frac{8 \pi / \epsilon_{m}}{2 n+1} \frac{(n+(-m)) !}{(n-(-m)) !} \delta_{(-m)(-\mu)} \delta_{n \nu}
$$

and

$$
\int_{\Omega} \mathbb{B}_{m n} \cdot \tilde{\mathbb{B}}_{\mu \nu} d \Omega=\frac{8 \pi / \epsilon_{m}}{2 n+1} \frac{(n+m) !}{(n-m) !} \delta_{m \mu} \delta_{n \nu}
$$

In general, the orthogonality can be written as

$$
\int_{\Omega} \mathbb{B}_{m n} \cdot \tilde{\mathbb{B}}_{\mu \nu} d \Omega=\frac{8 \pi / \epsilon_{m}}{2 n+1} \frac{(n+m) !}{(n-m) !} \delta_{m \mu} \delta_{n \nu}
$$

where $n \geq 1, n \geq m \geq-n$; and $\nu \geq 1, \nu \geq \mu \geq-\nu$.

Similarly, there is

$$
\int_{\Omega} \mathbb{C}_{m n} \cdot \tilde{\mathbb{C}}_{\mu \nu} d \Omega=\frac{8 \pi / \epsilon_{m}}{(2 n+1)} \frac{(n+m) !}{(n-m) !} \delta_{m \mu} \delta_{n \nu}
$$

and

$$
\int_{\Omega} \mathbb{P}_{m n} \cdot \mathbb{B}_{\mu \nu} d \Omega=\int_{\Omega} \mathbb{P}_{m n} \cdot \mathbb{C}_{\mu \nu} d \Omega=\int_{\Omega} \mathbb{B}_{m n} \cdot \mathbb{C}_{\mu \nu} d \Omega=0
$$

with $n \geq 1, n \geq m \geq-n$; and $\nu \geq 1, \nu \geq \mu \geq-\nu$.

\section{B.3 For $n=0$}

Since $\mathbb{B}_{00}=\mathbb{C}_{00}=0$ (see eqn $\left.\mathrm{F}-3\right)$, there is

$$
\int_{\Omega} \mathbb{P}_{m n} \cdot \mathbb{B}_{00} d \Omega=\int_{\Omega} \mathbb{P}_{m n} \cdot \mathbb{B}_{00} d \Omega=\int_{\Omega} \mathbb{B}_{m n} \cdot \mathbb{C}_{00} d \Omega=0
$$


and

$$
\int_{\Omega} \mathbb{B}_{m n} \cdot \tilde{\mathbb{B}}_{00} d \Omega=\int_{\Omega} \mathbb{C}_{m n} \cdot \tilde{\mathbb{C}}_{00} d \Omega=0
$$

Meanwhile, eqn. (F-1a) gives $\mathbb{P}_{00}=\mathbf{e}_{r}$. It's known that $\int_{-1}^{1} P_{n}(x) d x=0$, if $n \neq 0$ (Eq.(166) in [57]). Hence, for $\nu=0$

$$
\begin{aligned}
\int_{\Omega} \mathbb{P}_{m n} \cdot \tilde{\mathbb{P}}_{00} d \Omega & =\int_{\Omega} \mathbf{e}_{r} X_{n}^{m}(\theta, \varphi) \cdot \mathbf{e}_{r} d \Omega \\
& =\int_{0}^{2 \pi} e^{\hat{\imath} m \varphi} d \varphi \int_{0}^{\pi} P_{n}^{m}(\cos \theta) \sin \theta d \theta \\
& =4 \pi \delta_{m 0} \delta_{n 0} \\
& =\frac{8 \pi / \epsilon_{m}}{2 n+1} \frac{(n+m) !}{(n-m) !} \delta_{m 0} \delta_{n 0}
\end{aligned}
$$




\section{Appendix G}

\section{Normalized Vector Spherical}

\section{Harmonics}

In comparison with vector spherical harmonics $\mathbb{P}_{m n}, \mathbb{B}_{m n}$ and $\mathbb{C}_{m n}$ in Appendix $\mathrm{F}$, the normalized vector spherical harmonics $\mathfrak{P}_{m n}, \mathfrak{B}_{m n}$ and $\mathfrak{C}_{m n}$ are defined such that

$$
\begin{aligned}
\mathfrak{P}_{m n}(\theta, \varphi) & =\mathbf{e}_{r} Y_{n}^{m}(\theta, \varphi) \\
\mathfrak{B}_{m n}(\theta, \varphi) & =\frac{r}{\sqrt{n(n+1)}} \nabla\left[Y_{n}^{m}(\theta, \varphi)\right] \\
& =\frac{\sqrt{n(n+1)}}{(2 n+1) \sin \theta}\left\{\mathbf{e}_{\theta}\left[\frac{n-m+1}{n+1} Y_{n+1}^{m}-\frac{n+m}{n} Y_{n-1}^{m}\right]+\mathbf{e}_{\varphi} \frac{m(2 n+1)}{n(n+1)} \hat{\imath} Y_{n}^{m}\right\} \\
& =\frac{1}{\sqrt{n(n+1)}} \mathbf{e}_{\theta}\left(Y_{, \theta}\right)_{n}^{m}+\frac{1}{\sqrt{n(n+1)}} \mathbf{e}_{\varphi}\left(Y_{, \varphi}\right)_{n}^{m} \\
\mathfrak{C}_{m n}(\theta, \varphi) & =\frac{1}{\sqrt{n(n+1)}} \nabla \times\left[\mathbf{e}_{r} r Y_{n}^{m}(\theta, \varphi)\right] \\
& =\frac{\sqrt{n(n+1)}}{(2 n+1) \sin \theta}\left\{\mathbf{e}_{\theta} \frac{m(2 n+1)}{n(n+1)} \hat{\imath} Y_{n}^{m}-\mathbf{e}_{\varphi}\left[\frac{n-m+1}{n+1} Y_{n+1}^{m}-\frac{n+m}{n} Y_{n-1}^{m}\right]\right\} \\
& =\frac{1}{\sqrt{n(n+1)}} \mathbf{e}_{\theta}\left(Y_{, \varphi}\right)_{n}^{m}-\frac{1}{\sqrt{n(n+1)}} \mathbf{e}_{\varphi}\left(Y_{, \theta}\right)_{n}^{m}
\end{aligned}
$$

where $\hat{\imath}=\sqrt{-1}, n \geq 0, n \geq m \geq-n ; \mathbf{e}_{r}, \mathbf{e}_{\theta}$ and $\mathbf{e}_{\varphi}$ are three unit vectors in a spherical coordinate system (Appendix C); $Y_{n}^{m},\left(Y_{, \theta}\right)_{n}^{m}$ and $\left(Y_{, \varphi}\right)_{n}^{m}$ are defined in 
Eq. (2-47) and are repeated here

$$
\begin{aligned}
Y_{n}^{m} & =Y_{n}^{m}(\theta, \varphi)=\sqrt{\frac{2 n+1}{4 \pi} \frac{(n-m) !}{(n+m) !}} P_{n}^{m}(\cos \theta) \mathrm{e}^{\hat{\imath} m \varphi}, \\
\left(Y_{, \theta}\right)_{n}^{m} & =\frac{\partial Y_{n}^{m}(\theta, \varphi)}{\partial \theta} \\
\left(Y_{, \varphi}\right)_{n}^{m} & =\frac{1}{\sin \theta} \frac{\partial Y_{n}^{m}(\theta, \varphi)}{\partial \varphi}
\end{aligned}
$$

where $P_{n}^{m}(\cos \theta)$ is the associated Legendré polynomial.

The normalized spherical harmonics $\mathfrak{P}_{m n}, \mathfrak{B}_{m n}$ and $\mathfrak{C}_{m n}$ have following properties (the derivation is followed after the list of properties).

$$
\begin{gathered}
\mathfrak{P}_{m n} \cdot \mathfrak{B}_{m n}=\mathfrak{P}_{m n} \cdot \mathfrak{C}_{m n}=\mathfrak{B}_{m n} \cdot \mathfrak{C}_{m n}=0 \\
\int_{\Omega} \mathfrak{P}_{m n} \cdot \mathfrak{B}_{\mu \nu} d \Omega=\int_{\Omega} \mathfrak{P}_{m n} \cdot \mathfrak{C}_{\mu \nu} d \Omega=\int_{\Omega} \mathfrak{B}_{m n} \cdot \mathfrak{C}_{\mu \nu} d \Omega=0 \\
\int_{\Omega} \mathfrak{P}_{m n} \cdot \tilde{\mathfrak{B}}_{\mu \nu} d \Omega=\int_{\Omega} \mathfrak{P}_{m n} \cdot \tilde{\mathfrak{C}}_{\mu \nu} d \Omega=\int_{\Omega} \mathfrak{B}_{m n} \cdot \tilde{\mathfrak{C}}_{\mu \nu} d \Omega=0 \\
\int_{\Omega} \mathfrak{P}_{m n} \cdot \tilde{\mathfrak{P}}_{\mu \nu} d \Omega=\frac{2}{\epsilon_{m}} \delta_{m \mu} \delta_{n \nu}
\end{gathered}
$$

for $\nu=0$

$$
\int_{\Omega} \mathfrak{B}_{m n} \cdot \tilde{\mathfrak{B}}_{00} d \Omega=\int_{\Omega} \mathfrak{C}_{m n} \cdot \tilde{\mathfrak{C}}_{00} d \Omega=0
$$

and for $\nu \geq 1$

$$
\int_{\Omega} \mathfrak{B}_{m n} \cdot \tilde{\mathfrak{B}}_{\mu \nu} d \Omega=\int_{\Omega} \mathfrak{C}_{m n} \cdot \tilde{\mathfrak{C}}_{\mu \nu} d \Omega=\delta_{m \mu} \delta_{n \nu}
$$

where $\Omega$ denotes the spherical surface of unit radius, $d \Omega=\sin \theta d \theta d \varphi$, the integration is over the ranges $0 \leq \theta \leq \pi, 0 \leq \varphi \leq 2 \pi$, the tilde indicates a complex conjugate and $\epsilon_{m}$ is a factor with values of $\epsilon_{0}=1, \epsilon_{m}=2(m \neq 0)$.

By comparing $\mathfrak{P}_{m n}$ in Eq. $(\mathrm{G}-1 \mathrm{a})$ with $\mathbb{P}_{m n}$ in Eq. $(\mathrm{F}-1 \mathrm{a})$, it is found that

$$
\mathfrak{P}_{m n}=\sqrt{\frac{2 n+1}{4 \pi} \frac{(n-m) !}{(n+m) !}} \mathbb{P}_{m n}
$$


Similarly,

$$
\begin{aligned}
& \mathfrak{B}_{m n}=\sqrt{\frac{2 n+1}{4 \pi} \frac{(n-m) !}{(n+m) !}} \mathbb{B}_{m n}, \\
& \mathfrak{C}_{m n}=\sqrt{\frac{2 n+1}{4 \pi} \frac{(n-m) !}{(n+m) !}} \mathbb{C}_{m n} .
\end{aligned}
$$

Following Eq. (F-4),

$$
\mathfrak{P}_{m n} \cdot \mathfrak{B}_{m n}=\frac{2 n+1}{4 \pi} \frac{(n-m) !}{(n+m) !} \mathbb{P}_{m n} \cdot \mathbb{B}_{m n}=0 .
$$

Similarly,

$$
\mathfrak{P}_{m n} \cdot \mathfrak{C}_{m n}=\mathfrak{B}_{m n} \cdot \mathfrak{C}_{m n}=0
$$

Following Eq. (F-5),

$$
\int_{\Omega} \mathfrak{P}_{m n} \cdot \mathfrak{B}_{\mu \nu} d \Omega=\frac{2 n+1}{4 \pi} \frac{(n-m) !}{(n+m) !} \int_{\Omega} \mathbb{P}_{m n} \cdot \mathbb{B}_{\mu \nu} d \Omega=0 .
$$

Similarly,

$$
\int_{\Omega} \mathfrak{P}_{m n} \cdot \mathfrak{C}_{\mu \nu} d \Omega=\int_{\Omega} \mathfrak{B}_{m n} \cdot \mathfrak{C}_{\mu \nu} d \Omega=0
$$

Following Eq. $(\mathrm{F}-6)$,

$$
\int_{\Omega} \mathfrak{P}_{m n} \cdot \tilde{\mathfrak{B}}_{\mu \nu} d \Omega=\frac{2 n+1}{4 \pi} \frac{(n-m) !}{(n+m) !} \int_{\Omega} \mathbb{P}_{m n} \cdot \tilde{\mathbb{B}}_{\mu \nu} d \Omega=0 .
$$

Similarly,

$$
\int_{\Omega} \mathfrak{P}_{m n} \cdot \tilde{\mathfrak{C}}_{\mu \nu} d \Omega=\int_{\Omega} \mathfrak{B}_{m n} \cdot \tilde{\mathfrak{C}}_{\mu \nu} d \Omega=0 .
$$

Following Eq. $(\mathrm{F}-7)$,

$$
\begin{aligned}
\int_{\Omega} \mathfrak{P}_{m n} \cdot \tilde{\mathfrak{P}}_{\mu \nu} d \Omega & =\frac{2 n+1}{4 \pi} \frac{(n-m) !}{(n+m) !} \int_{\Omega} \mathbb{P}_{m n} \cdot \tilde{\mathbb{P}}_{\mu \nu} d \Omega \\
& =\frac{2 n+1}{4 \pi} \frac{(n-m) !}{(n+m) !} \frac{8 \pi / \epsilon_{m}}{2 n+1} \frac{(n+m) !}{(n-m) !} \delta_{m \mu} \delta_{n \nu} \\
& =\frac{2}{\epsilon_{m}} \delta_{m \mu} \delta_{n \nu} .
\end{aligned}
$$

Following Eq. (F-8),

$$
\int_{\Omega} \mathfrak{B}_{m n} \cdot \tilde{\mathfrak{B}}_{00} d \Omega=\frac{2 n+1}{4 \pi} \frac{(n-m) !}{(n+m) !} \int_{\Omega} \mathbb{B}_{m n} \cdot \tilde{\mathbb{B}}_{00} d \Omega=0 .
$$


Similarly,

$$
\int_{\Omega} \mathfrak{C}_{m n} \cdot \tilde{\mathfrak{C}}_{00} d \Omega=0 .
$$

Following Eq. (F-9), for $\nu \geq 1$

$$
\begin{aligned}
\int_{\Omega} \mathfrak{B}_{m n} \cdot \tilde{\mathfrak{B}}_{\mu \nu} d \Omega & =\frac{2 n+1}{4 \pi} \frac{(n-m) !}{(n+m) !} \int_{\Omega} \mathbb{B}_{m n} \cdot \tilde{\mathbb{B}}_{\mu \nu} d \Omega \\
& =\frac{2 n+1}{4 \pi} \frac{(n-m) !}{(n+m) !} \frac{8 \pi / \epsilon_{m}}{2 n+1} \frac{(n+m) !}{(n-m) !} \delta_{m \mu} \delta_{n \nu} \\
& =\frac{2}{\epsilon_{m}} \delta_{m \mu} \delta_{n \nu} .
\end{aligned}
$$

Similarly, for $\nu \geq 1$

$$
\int_{\Omega} \mathfrak{B}_{m n} \cdot \tilde{\mathfrak{B}}_{\mu \nu} d \Omega=\frac{2}{\epsilon_{m}} \delta_{m \mu} \delta_{n \nu}
$$




\section{Appendix $\mathbf{H}$}

\section{Vector Wave Functions in Terms of Vector Spherical Harmonics}

Let

$$
\begin{array}{ll}
\zeta_{n m}(\kappa \boldsymbol{r})=\mathcal{Z}_{n}(\kappa r) Y_{n}^{m}(\theta, \varphi) & \text { for longitudinal waves, } \\
\xi_{n m}(k \boldsymbol{r})=\mathcal{Z}_{n}(k r) Y_{n}^{m}(\theta, \varphi) & \text { for shear waves. }
\end{array}
$$

where $\mathcal{Z}_{n}(\cdot)$ denotes a spherical Bessel function, either $j_{n}(\cdot)$ or $h_{n}^{(1)}(\cdot)$, and $Y_{n}^{m}(\theta, \varphi)$ is the normalized spherical harmonics defined in Eq. (2-35) and repeated here as

$$
Y_{n}^{m}(\theta, \varphi)=\sqrt{\frac{2 n+1}{4 \pi} \frac{(n-m) !}{(n+m) !}} P_{n}^{m}(\cos \theta) \mathrm{e}^{\hat{\imath} m \varphi}
$$

where $P_{n}^{m}(\cos \theta)$ is the associated Legendré polynomial.

In spherical coordinates, definition of a set of vector wave functions can be found in [42-45]. Following the definition in [45], vector wave functions are modified such that

$$
\begin{aligned}
\boldsymbol{L}_{n m}(\kappa r) & =\nabla \zeta_{n m}(\kappa \boldsymbol{r}), \\
\boldsymbol{M}_{n m}(k r) & =\nabla \times\left[\boldsymbol{r} \xi_{n m}(k \boldsymbol{r})\right],
\end{aligned}
$$




$$
\boldsymbol{N}_{n m}(k r)=\frac{1}{k} \nabla \times \boldsymbol{M}_{n m}(k r) .
$$

The following two operators (Appendix C) are needed to obtain vector wave functions in terms of vector spherical harmonics.

$$
\begin{aligned}
\nabla a= & \frac{\partial a}{\partial r} \boldsymbol{e}_{r}+\frac{1}{r} \frac{\partial a}{\partial \theta} \boldsymbol{e}_{\theta}+\frac{1}{r \sin \theta} \frac{\partial a}{\partial \varphi} \boldsymbol{e}_{\varphi} \\
\nabla \times \boldsymbol{V}= & \frac{1}{r \sin \theta}\left(\frac{\partial\left(\sin \theta V_{\varphi}\right)}{\partial \theta}-\frac{\partial V_{\theta}}{\partial \varphi}\right) \boldsymbol{e}_{r}-\left(\frac{\partial V_{\varphi}}{\partial r}+\frac{V_{\varphi}}{r}-\frac{1}{r \sin \theta} \frac{\partial V_{r}}{\partial \varphi}\right) \boldsymbol{e}_{\theta} \\
& -\left(\frac{1}{r} \frac{\partial V_{r}}{\partial \theta}-\frac{\partial V_{\theta}}{\partial r}-\frac{V_{\theta}}{r}\right) \boldsymbol{e}_{\varphi}
\end{aligned}
$$

Since

$$
\begin{aligned}
\nabla \zeta_{n m}(\kappa \boldsymbol{r})= & \boldsymbol{e}_{r} \frac{\partial \zeta_{n m}(\kappa \boldsymbol{r})}{\partial r}+\boldsymbol{e}_{\theta} \frac{1}{r} \frac{\partial \zeta_{n m}(\kappa \boldsymbol{r})}{\partial \theta}+\boldsymbol{e}_{\varphi} \frac{1}{r \sin \theta} \frac{\partial \zeta_{n m}(\kappa \boldsymbol{r})}{\partial \varphi} \\
= & \boldsymbol{e}_{r} \frac{\partial \mathcal{Z}_{n}(\kappa r)}{\partial r} Y_{n}^{m}(\theta, \varphi)+\boldsymbol{e}_{\theta} \frac{\mathcal{Z}_{n}(\kappa r)}{r} \frac{\partial Y_{n}^{m}(\theta, \varphi)}{\partial \theta}+\boldsymbol{e}_{\varphi} \frac{\mathcal{Z}_{n}(\kappa r)}{r \sin \theta} \frac{\partial Y_{n}^{m}(\theta, \varphi)}{\partial \varphi} \\
= & \boldsymbol{e}_{r} \kappa \mathcal{Z}_{n}^{\prime}(\kappa r) Y_{n}^{m}(\theta, \varphi) \\
& +\frac{\mathcal{Z}_{n}(\kappa r)}{r}\left(\boldsymbol{e}_{\theta} \frac{\partial Y_{n}^{m}(\theta, \varphi)}{\partial \theta}+\boldsymbol{e}_{\varphi} \frac{1}{\sin \theta} \frac{\partial Y_{n}^{m}(\theta, \varphi)}{\partial \varphi}\right)
\end{aligned}
$$

then,

$$
\boldsymbol{L}_{n m}(\kappa r)=\kappa \mathcal{Z}_{n}^{\prime}(\kappa r) \mathfrak{P}_{m n}+\frac{\mathcal{Z}_{n}(\kappa r)}{r} \sqrt{n(n+1)} \mathfrak{B}_{m n}
$$

where $\mathfrak{P}_{m n}$ and $\mathfrak{B}_{m n}$ are defined in Eqs. $(\mathrm{G}-1 \mathrm{a})$ and $(\mathrm{G}-1 \mathrm{~b})$ and are repeated here

$$
\begin{aligned}
\mathfrak{P}_{m n}(\theta, \varphi) & =\mathbf{e}_{r} Y_{n}^{m}(\theta, \varphi) \\
\mathfrak{B}_{m n}(\theta, \varphi) & =\frac{r}{\sqrt{n(n+1)}} \nabla\left[Y_{n}^{m}(\theta, \varphi)\right] \\
& =\frac{1}{\sqrt{n(n+1)}} \mathbf{e}_{\theta} \frac{\partial Y_{n}^{m}(\theta, \varphi)}{\partial \theta}+\frac{1}{\sqrt{n(n+1)}} \mathbf{e}_{\varphi} \frac{1}{\sin \theta} \frac{\partial Y_{n}^{m}(\theta, \varphi)}{\partial \varphi}
\end{aligned}
$$

Since

$$
\begin{aligned}
\nabla \times\left[\boldsymbol{r} \xi_{n m}(k \boldsymbol{r})\right] & =\nabla \times\left[\boldsymbol{e}_{r} r \xi_{n m}(k \boldsymbol{r})\right]=\nabla\left[r \xi_{n m}(k \boldsymbol{r})\right] \times \boldsymbol{e}_{r} \\
& =\frac{1}{r \sin \theta} \frac{\partial\left(r \xi_{n m}(k \boldsymbol{r})\right)}{\partial \varphi} \boldsymbol{e}_{\theta}-\frac{1}{r} \frac{\partial\left(r \xi_{n m}(k \boldsymbol{r})\right)}{\partial \theta} \boldsymbol{e}_{\varphi} \\
& =\frac{1}{\sin \theta} \frac{\partial\left(\xi_{n m}(k \boldsymbol{r})\right)}{\partial \varphi} \boldsymbol{e}_{\theta}-\frac{\partial\left(\xi_{n m}(k \boldsymbol{r})\right)}{\partial \theta} \boldsymbol{e}_{\varphi}
\end{aligned}
$$




$$
\begin{aligned}
& =\mathcal{Z}_{n}(k r) \frac{1}{\sin \theta} \frac{\partial Y_{n}^{m}(\theta, \varphi)}{\partial \varphi} \boldsymbol{e}_{\theta}-\mathcal{Z}_{n}(k r) \frac{\partial Y_{n}^{m}(\theta, \varphi)}{\partial \theta} \boldsymbol{e}_{\varphi} \\
& =\mathcal{Z}_{n}(k r) \sqrt{n(n+1)} \mathfrak{C}_{n m}
\end{aligned}
$$

Then,

$$
\boldsymbol{M}_{n m}(k r)=\mathcal{Z}_{n}(k r) \sqrt{n(n+1)} \mathfrak{C}_{n m}
$$

where $\mathfrak{C}_{n m}$ is defined in Eq. $(\mathrm{G}-1 \mathrm{c})$ and repeated here

$$
\begin{aligned}
\mathfrak{C}_{m n}(\theta, \varphi) & =\frac{1}{\sqrt{n(n+1)}} \nabla \times\left[\mathbf{e}_{r} r Y_{n}^{m}(\theta, \varphi)\right] \\
& =\frac{1}{\sqrt{n(n+1)}} \mathbf{e}_{\theta} \frac{1}{\sin \theta} \frac{\partial Y_{n}^{m}(\theta, \varphi)}{\partial \varphi}-\frac{1}{\sqrt{n(n+1)}} \mathbf{e}_{\varphi} \frac{\partial Y_{n}^{m}(\theta, \varphi)}{\partial \theta}
\end{aligned}
$$

Meanwhile,

$$
\begin{aligned}
\nabla \times \boldsymbol{M}_{n m}(k r)= & \boldsymbol{e}_{r} \frac{1}{r \sin \theta}\left\{\frac{\partial}{\partial \theta}\left[-\sin \theta \mathcal{Z}_{n}(k r) \frac{\partial Y_{n}^{m}(\theta, \varphi)}{\partial \theta}\right]\right. \\
& \left.-\frac{\partial}{\partial \varphi}\left[\mathcal{Z}_{n}(k r) \frac{1}{\sin \theta} \frac{\partial Y_{n}^{m}(\theta, \varphi)}{\partial \varphi}\right]\right\} \\
& -\boldsymbol{e}_{\theta}\left\{\frac{\partial}{\partial r}\left[-\mathcal{Z}_{n}(k r) \frac{\partial Y_{n}^{m}(\theta, \varphi)}{\partial \theta}\right]+\frac{1}{r}\left[-\mathcal{Z}_{n}(k r) \frac{\partial Y_{n}^{m}(\theta, \varphi)}{\partial \theta}\right]\right\} \\
& +\boldsymbol{e}_{\varphi}\left\{\frac{\partial}{\partial r}\left[\mathcal{Z}_{n}(k r) \frac{1}{\sin \theta} \frac{\partial Y_{n}^{m}(\theta, \varphi)}{\partial \varphi}\right]+\frac{\mathcal{Z}_{n}(k r)}{r} \frac{1}{\sin \theta} \frac{\partial Y_{n}^{m}(\theta, \varphi)}{\partial \varphi}\right\} \\
= & \boldsymbol{e}_{r}\left\{\frac{-\mathcal{Z}_{n}(k r)}{r \sin \theta} \frac{\partial}{\partial \theta}\left[\sin \theta \frac{\partial Y_{n}^{m}(\theta, \varphi)}{\partial \theta}\right]-\frac{\mathcal{Z}_{n}(k r)}{r \sin ^{2} \theta} \frac{\partial^{2} Y_{n}^{m}(\theta, \varphi)}{\partial \varphi^{2}}\right\} \\
& +\boldsymbol{e}_{\theta}\left\{k \mathcal{Z}_{n}^{\prime}(k r) \frac{\partial Y_{n}^{m}(\theta, \varphi)}{\partial \theta}+\frac{\mathcal{Z}_{n}(k r)}{r} \frac{\partial Y_{n}^{m}(\theta, \varphi)}{\partial \theta}\right\} \\
& +\boldsymbol{e}_{\varphi}\left\{k \mathcal{Z}_{n}^{\prime}(k r) \frac{1}{\sin \theta} \frac{\partial Y_{n}^{m}(\theta, \varphi)}{\partial \varphi}+\frac{\mathcal{Z}_{n}(k r)}{r} \frac{1}{\sin ^{2}} \frac{\partial Y_{n}^{m}(\theta, \varphi)}{\partial \varphi}\right\} \\
= & \boldsymbol{e}_{r}\left\{\frac{-\mathcal{Z}_{n}(k r)}{r \sin \theta} \frac{\partial}{\partial \theta}\left[\sin \theta \frac{\partial Y_{n}^{m}(\theta, \varphi)}{\partial \theta}\right]+\frac{\mathcal{Z}_{n}(k r)}{r} \frac{m^{2}}{\sin ^{2} \theta} Y_{n}^{m}(\theta, \varphi)\right\} \\
& +k \mathcal{Z}_{n}^{\prime}(k r)\left\{\boldsymbol{e}_{\theta} \frac{\partial Y_{n}^{m}(\theta, \varphi)}{\partial \theta}+\boldsymbol{e}_{\varphi} \frac{1}{\sin \theta} \frac{\partial Y_{n}^{m}(\theta, \varphi)}{\partial \varphi}\right\} \\
& +\frac{\mathcal{Z}_{n}(k r)}{r}\left\{\boldsymbol{e}_{\theta} \frac{\partial Y_{n}^{m}(\theta, \varphi)}{\partial \theta}+\boldsymbol{e}_{\varphi} \frac{1}{\sin \theta} \frac{\partial Y_{n}^{m}(\theta, \varphi)}{\partial \varphi}\right\} \\
= & \boldsymbol{e}_{r}\left\{\frac{-\mathcal{Z}_{n}(k r)}{r \sin \theta} \frac{\partial}{\partial \theta}\left[\sin \theta \frac{\partial Y_{n}^{m}(\theta, \varphi)}{\partial \theta}\right]+\frac{\mathcal{Z}_{n}(k r)}{r} \frac{m^{2}}{\sin ^{2} \theta} Y_{n}^{m}(\theta, \varphi)\right\} \\
& +\left[k \mathcal{Z}_{n}^{\prime}(k r)+\frac{\mathcal{Z}_{n}(k r)}{r}\right] \sqrt{n(n+1)} \mathfrak{B}_{n m} \\
& {[(1)) }
\end{aligned}
$$


Since

$$
\begin{aligned}
\frac{d P_{n}^{m}(\cos \theta)}{d \theta} & =\frac{1}{\sin \theta}\left[n \cos \theta P_{n}^{m}(\cos \theta)-(n+m) P_{n-1}^{m}(\cos \theta)\right] \\
& =\frac{1}{\sin \theta}\left[(n-m+1) P_{n+1}^{m}(\cos \theta)-(n+1) P_{n}^{m}(\cos \theta)\right]
\end{aligned}
$$

Then,

$$
\begin{aligned}
\frac{d}{d \theta}\left[\sin \theta \frac{d P_{n}^{m}(\theta, \varphi)}{d \theta}\right]= & \frac{d}{d \theta}\left[n \cos \theta P_{n}^{m}(\cos \theta)-(n+m) P_{n-1}^{m}(\cos \theta)\right] \\
= & -n \sin \theta P_{n}^{m}(\cos \theta)+n \cos \theta \frac{d P_{n}^{m}(\cos \theta)}{d \theta} \\
& -(n+m) \frac{d P_{n-1}^{m}(\cos \theta)}{d \theta} \\
= & -n \sin \theta P_{n}^{m}(\cos \theta)+\frac{n \cos \theta}{\sin \theta}\left[n \cos \theta P_{n}^{m}(\cos \theta)\right. \\
& \left.-(n+m) P_{n-1}^{m}(\cos \theta)\right] \\
& -\frac{(n+m)}{\sin \theta}\left[(n-m) P_{n}^{m}(\cos \theta)-n P_{n-1}^{m}(\cos \theta)\right] \\
= & P_{n}^{m}(\cos \theta)\left[-n \sin \theta+\frac{n^{2} \cos { }^{2} \theta}{\sin \theta}-\frac{\left(n^{2}-m^{2}\right)}{\sin \theta}\right] \\
& +P_{n-1}^{m}(\cos \theta)\left[-\frac{n(n+m) \cos \theta}{\sin \theta}+\frac{n(n+m) \cos \theta}{\sin \theta}\right] \\
= & P_{n}^{m}(\cos \theta)\left[-n \sin \theta-n^{2} \sin \theta+\frac{m^{2}}{\sin \theta}\right]
\end{aligned}
$$

Then,

$$
\frac{1}{\sin \theta} \frac{\partial}{\partial \theta}\left[\sin \theta \frac{\partial P_{n}^{m}(\theta, \varphi)}{d \theta}\right]=P_{n}^{m}(\cos \theta)\left[-n-n^{2}+\frac{m^{2}}{\sin ^{2} \theta}\right]
$$

and

$$
\frac{1}{\sin \theta} \frac{\partial}{\partial \theta}\left[\sin \theta \frac{\partial Y_{n}^{m}(\theta, \varphi)}{d \theta}\right]=Y_{n}^{m}(\cos \theta)\left[-n-n^{2}+\frac{m^{2}}{\sin ^{2} \theta}\right]
$$

therefore,

$$
\begin{aligned}
& \boldsymbol{e}_{r}\left\{\frac{-\mathcal{Z}_{n}(k r)}{r \sin \theta} \frac{\partial}{\partial \theta}\left[\sin \theta \frac{\partial Y_{n}^{m}(\theta, \varphi)}{\partial \theta}\right]+\frac{\mathcal{Z}_{n}(k r)}{r} \frac{m^{2}}{\sin ^{2} \theta} Y_{n}^{m}(\theta, \varphi)\right\} \\
= & \boldsymbol{e}_{r} \frac{\mathcal{Z}_{n}(k r)}{r} n(n+1) Y_{n}^{m}(\theta, \varphi) \\
= & \frac{\mathcal{Z}_{n}(k r)}{r} n(n+1) \mathfrak{P}_{n m}
\end{aligned}
$$


and

$$
\nabla \times M_{n m}(k r)=\frac{\mathcal{Z}_{n}(k r)}{r} n(n+1) \mathfrak{P}_{n m}+\left[k \mathcal{Z}_{n}^{\prime}(k r)+\frac{\mathcal{Z}_{n}(k r)}{r}\right] \sqrt{n(n+1)} \mathfrak{B}_{n m}
$$

Finally,

$$
\begin{aligned}
\boldsymbol{N}_{n m}(k r) & =\frac{1}{k} \nabla \times \boldsymbol{M}_{n m}(k r) \\
& =\frac{\mathcal{Z}_{n}(k r)}{k r} n(n+1) \mathfrak{P}_{n m}+\left[\mathcal{Z}_{n}^{\prime}(k r)+\frac{\mathcal{Z}_{n}(k r)}{k r}\right] \sqrt{n(n+1)} \mathfrak{B}_{n m}
\end{aligned}
$$

